\title{
2017 Program of Studies: Ice-Ocean Interactions
}

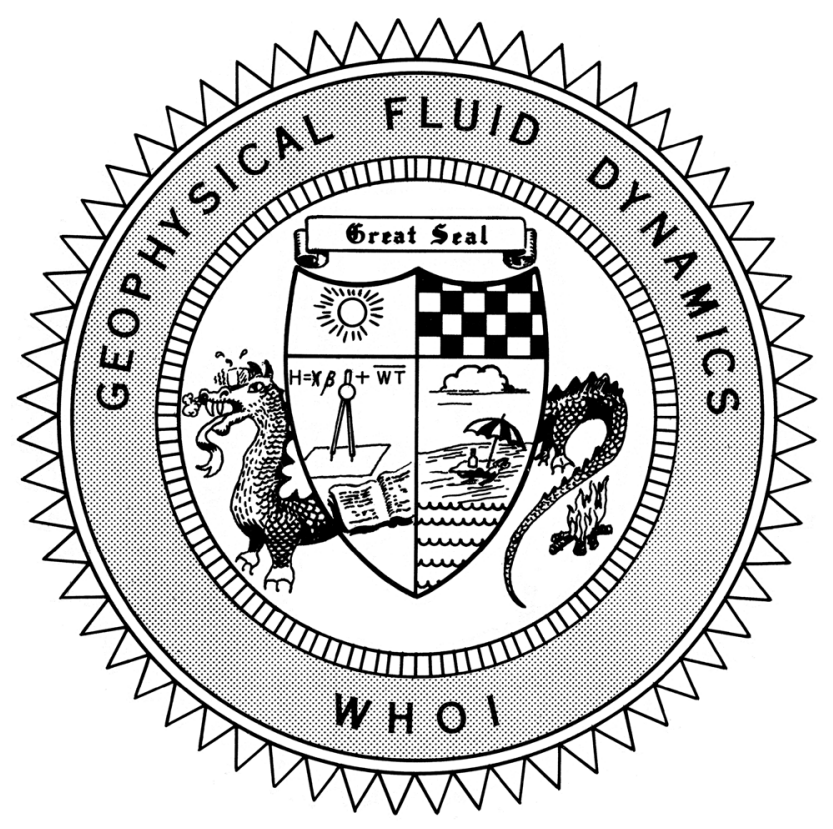

Course Lectures

Fellows' Project Reports 


\section{WHOI-2021-05}

\section{Program of Study: Ice-Ocean Interactions}

by

Claudia Cenedese and Mary-Louise Timmermans

Woods Hole Oceanographic Institution

Woods Hole, Massachusetts 02543

November 2018

\section{Technical Report}

National Science Foundation under Grant No. OCE-1829864.

Reproduction in whole or in part is permitted for any purpose of the United States Government. This report should be cited as Woods Hole

Oceanographic Institution Technical Report, WHOI-2021-05.

Approved for public release; distribution unlimited.

Approved for Distribution:

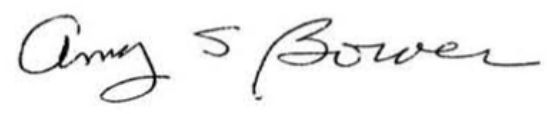

Amy Bower, Chair

Department of Physical Oceanography 


\section{Preface}

The 2017 Geophysical Fluid Dynamics Summer Study Program theme was Ice-Ocean Interactions. Three principal lecturers, Andrew Fowler (Oxford), Adrian Jenkins (British Antarctic Survey) and Fiamma Straneo (WHOI/Scripps Institution of Oceanography) were our expert guides for the first two weeks. Their captivating lectures covered topics ranging from the theoretical underpinnings of ice-sheet dynamics, to models and observations of ice-ocean interactions and high-latitude ocean circulation, to the role of the cryosphere in climate change. These icy topics did not end after the first two weeks. Several of the Fellows' projects related to ice-ocean dynamics and thermodynamics, and many visitors gave talks on these themes.

The first ten chapters of this volume document these lectures, each prepared by pairs of the summer's GFD fellows. Following the principal lecture notes are the written reports of the fellows' own research projects. This summer's fellows were:

- Robert Fajber, University of Toronto

- Margaret Lindeman, Scripps Institution of Oceanography

- Madeleine Youngs, Massachusetts Institute of Technology

- Federico Fuentes, University of Texas-Austin

- Thomasina Ball, University of Cambridge

- Jessica Kenigson, University of Colorado-Boulder

- Eric Hester, University of Sydney

- Agostino Meroni, University of Milan-Bicocca

- Guillaume Michel, Ecole Normale Suprieure

- Earle Wilson, University of Washington

- Madelaine Gamble Rosevear, University of Tasmania

The 2017 Sears Public Lecture was given by Professor Richard Alley (Pennsylvania State University) on Ice Sheets and Sea Level Rise. For the conclusion of his exceptional presentation Professor Alley played guitar and sang a lively tune about climate change. His talk drew a big crowd of GFD folks and Woods Hole locals who kept the conversation going at the reception afterwards.

Claudia Cenedese and Mary-Louise Timmermans were co-directors, and the steadystream of visitors, plus large number of long-term staff members around for the summer, ensured that the fellows never lacked for guidance. Andrew Wells (Oxford) was awarded the GFD Distinguished Scholar Award for outstanding contributions to mentoring and research in the program.

The various laboratory experiments were facilitated by able support from Anders Jensen who had the challenge of making icebergs of different shapes and sizes. Julie Hildebrandt and Janet Fields kept the whole program running smoothly with efficient administration in all aspects. We continue to be indebted to WHOI Education, and are grateful for the opportunity to share scientific ideas on the porch at Walsh, the picnic tables on the lawn, and in the bustle of Woods Hole village. 


\section{Table of Contents}

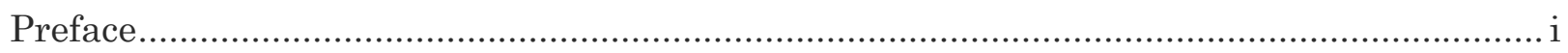

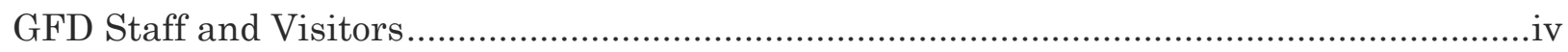

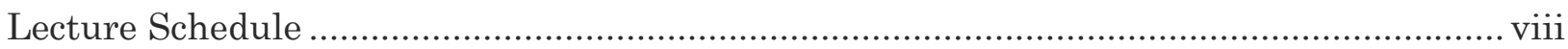

\section{Principal Lectures}

$\underline{\text { Week } 1}$

Lecture 1: Introduction to Ice-Ocean Interactions

Introduction to Glaciers and Ice Sheets (Andrew Fowler) ............................................. 1

The Antarctic Ice Sheet and the Southern Ocean: An Introduction (Adrian Jenkins)....... 2

Greenland Ice Sheet Changes:The Ocean as a Trigger and Receiver (Fiamma Straneo)..... 8

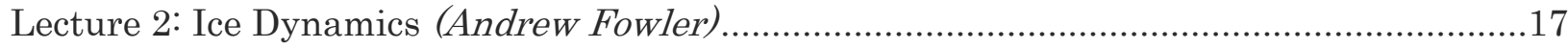

Lecture 3: Subglacial Control of Ice Flow (Andrew Fowler) ............................................29

Lecture 4: Processes at the Ice-Ocean Interface (Andrew Fowler) ....................................44

Lecture 5: Basic Theory of Ice-Ocean Interaction (Adrian Jenkins) .................................55

Lecture 6: Ocean Circulation beneath Ice Shelves (Adrian Jenkins) .................................73

Lecture 7: Ice-Ocean Interactions around Antarctica (Adrian Jenkins) ............................95

Lecture 8: Testing the Ocean Trigger Hypothesis for Greenland's Recent Glacier

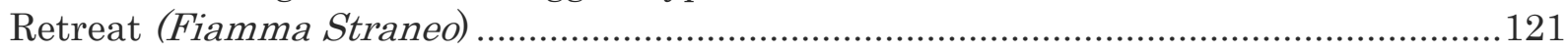

Lecture 9: Greenland Glacier-Ocean Interaction, Part I (Fiamma Straneo) .....................134

Lecture 10: Greenland Glacier-Ocean Interaction, Part II (Fiamma Straneo)..................150

\section{Fellows' Reports}

Seeing the Ocean through Sea Ice

Robert A. Fajber, University of Toronto.

A Mechanism for Secondary Sea Ice Formation Driven by Double-diffusive Supercooling Margaret $R$. Lindeman, Scripps Institution of Oceanography..... 
Eddy Energy Fluxes in Mixed Barotropic-Baroclinic Instability: Upgradient or Downgradient?

Madeleine K. Youngs, MIT/WHOI Joint Program.

Global Stability of 2D Plane Couette Flow Beyond the Energy Stability Limit

Federico Fuentes, The University of Texas at Austin

Indentation of Deformable Plastic Layers

Thomasina Ball, University of Cambridge.

A Simple Adiabatic Model for Vertical Variation of Halocline Slope in the Beaufort Gyre Jessica Kenigson, University of Colorado at Boulder.

Investigating the Effect of Iceberg Aspect Ratio on Submarine Melting

Eric W. Hester, The University of Sydney

Nonlinear Influence of Background Rotation on Iceberg Melting

Agostino N. Meroni, University of Milan-Bicocca....

Cooling via Baroclinic Acoustic Streaming Guillaume Michel, Ecole Normale Supérieure

The Dynamics of Subglacial Plume Lift-off Earle Wilson, University of Washington.

Turbid Tales: Where do Subglacial Sediments Go?

Madelaine Gamble Rosevear, University of Tasmania 428 


\section{Geophysical Fluid Dynamics Participants}

\section{FELLOWS}

Thomasina Ball

Robert Fajber

Federico Fuentes

Madelaine Gamble Rosevear

Eric Hester

Jessica Kenigson

Margaret Lindeman

Agostino Meroni

Guillaume Michel

Earle Wilson

Madeleine Youngs

\section{STAFF AND VISITORS}

\author{
Ryan Abernathy \\ James Anderson \\ Neil Balmforth \\ Nicholas Beaird \\ Yana Bebieva \\ Graham Benham \\ Luca Brandt \\ Keaton Burns \\ Colm-cille Caulfield \\ Claudia Cenedese \\ Vamsi Krishna Chalamalla \\ Gregory Chini \\ Timothy Creyts \\ Predrag Cvitanovic \\ Megan Davies Wykes \\ Charles Doering \\ Ekaterina Ezhova \\ Giovanni Fantuzzi \\ Anna Fitzmaurice \\ Glenn Flierl \\ Andrew Fowler \\ Pascale Garaud \\ Renske Gelderloos \\ David Goluskin \\ Pedram Hassanzadeh \\ Ian Hewitt \\ Joseph Hitchen
}

\author{
University of Cambridge \\ University of Toronto \\ University of Texas at Austin \\ University of Tasmania \\ University of Sydney \\ University of Colorado at Boulder \\ Scripps Institution of Oceanography \\ University of Milan - Bicocca \\ Ecole Normale Supérieure \\ University of Washington \\ MIT/WHOI Joint Program
}

Columbia University

Stevens Institute of Technology

University of British Columbia

Woods Hole Oceanographic Institution

Yale University

University of Oxford

KTH Mechanics

Massachusetts Institute of Technology

University of Cambridge

Woods Hole Oceanographic Institution

University of North Carolina

University of New Hampshire

Columbia University

Georgia Institute of Technology

University of Cambridge

University of Michigan

University of Helsinki

Imperial College London

Princeton University

Massachusetts Institute of Technology

University of Limerick

University of California, Santa Cruz

Johns Hopkins University

University of Victoria

Rice University

University of Oxford

Oxford University 
Rebecca Jackson

Malte Jansen

Adrian Jenkins

Alexis Kaminski

Norman Lebovitz

Daniel Lecoanet

Christopher MacMackin

Georgy Manucharyan

Craig McConnochie

Colin Meyer

Carlos Moffat

M. Nicholas Moore

Philip Morrison

Morven Muilwijk

Sara Nasab

Jerome Neufeld

James Parkinson

Pierre-Yves Passaggia

Joseph Pedlosky

Samuel Pegler

Bhargav Rallabandi

James Rice

Roberta Sciascia

Stefanie Semper

Donald Slater

Kate Snow

Edward Spiegel

Erik Stenberg

Fiammetta Straneo

Zhan Su

Bruce Sutherland

Kevin Thielen

Jean-Luc Thiffeault

Andreas Thurnherr

Mary-Louise Timmermans

Srikanth Toppaladoddi

George Veronis

Brian von Herzen

Scott Weady

Andrew Wells

Jack Whitehead

Andrew Woods

Grae Worster

Carl Wunsch

Philip Yecko

Mengnan Zhao
Oregon State University

University of Chicago

British Antarctic Survey

University of Washington

University of Chicago

Princeton University

University of Oxford

California Institute of Technology

Woods Hole Oceanographic Institution

Massachusetts Institute of Technology

University of Delaware

Florida State University

University of Texas at Austin

University of Bergen

University of California, Santa Cruz

University of Cambridge

University of Oxford

University of North Carolina

Woods Hole Oceanographic Institution

University of Leeds

Princeton University

Harvard University

Italian Natl. Research Council ISMAR

University of Bergen

University of Edinburgh

University of Edinburgh

Columbia University

KTH Royal Institute of Technology

Woods Hole Oceanographic Institution

California Institute of Technology

University of Alberta

University of Oxford

University of Wisconsin, Madison

Columbia University

Yale University

Yale University

Yale University

The Climate Foundation

Yale University

University of Oxford

Woods Hole Oceanographic Institution

University of Cambridge

University of Cambridge

Massachusetts Institute of Technology

Cooper Union

Yale University 


\section{Principal Lecturers}

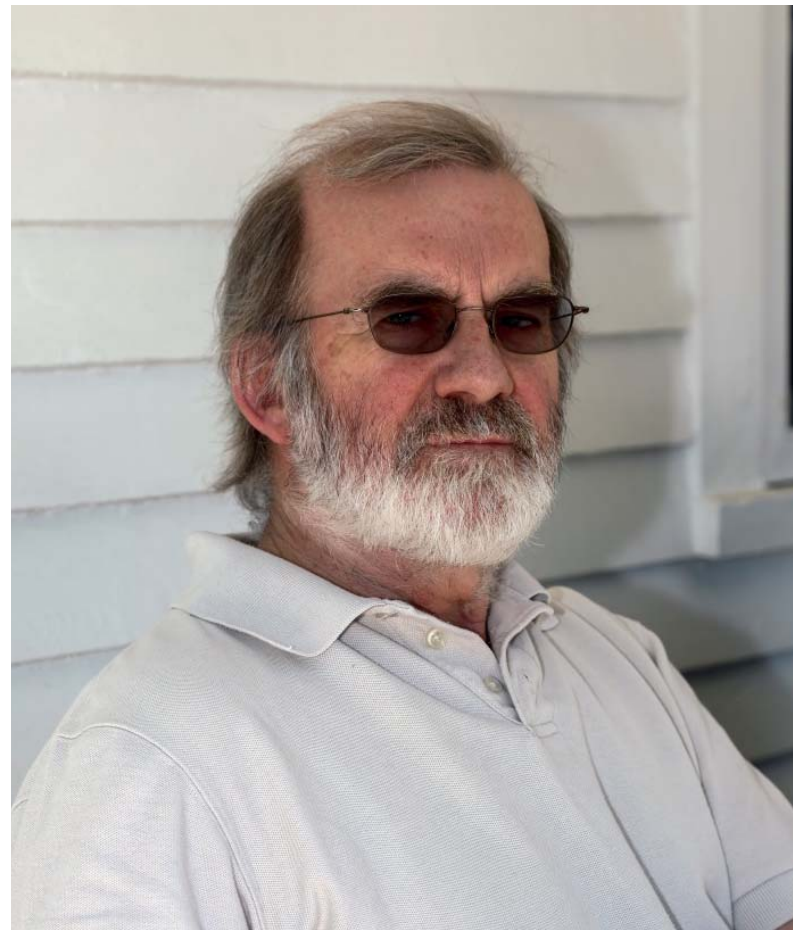

Andrew Fowler

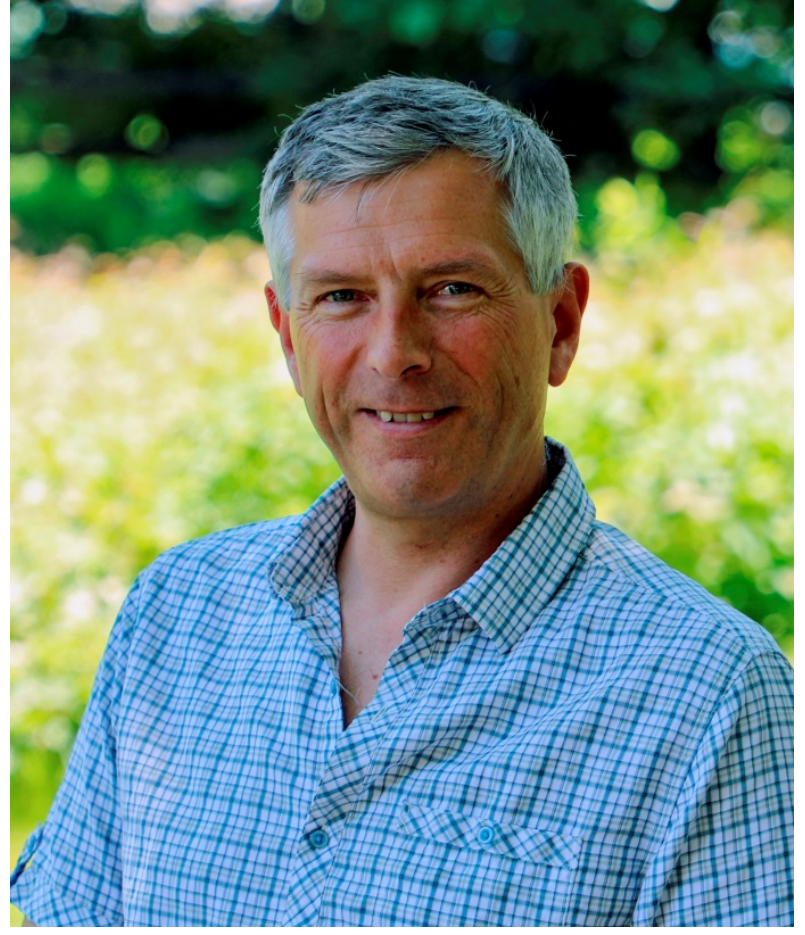

Andrew Jenkins

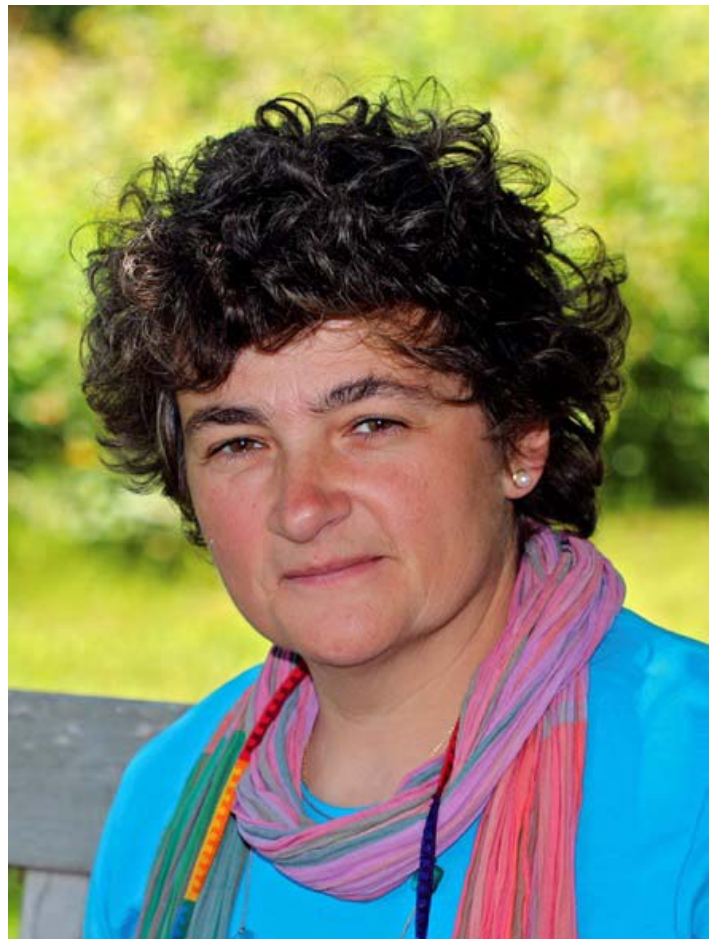

Fiammetta Straneo 


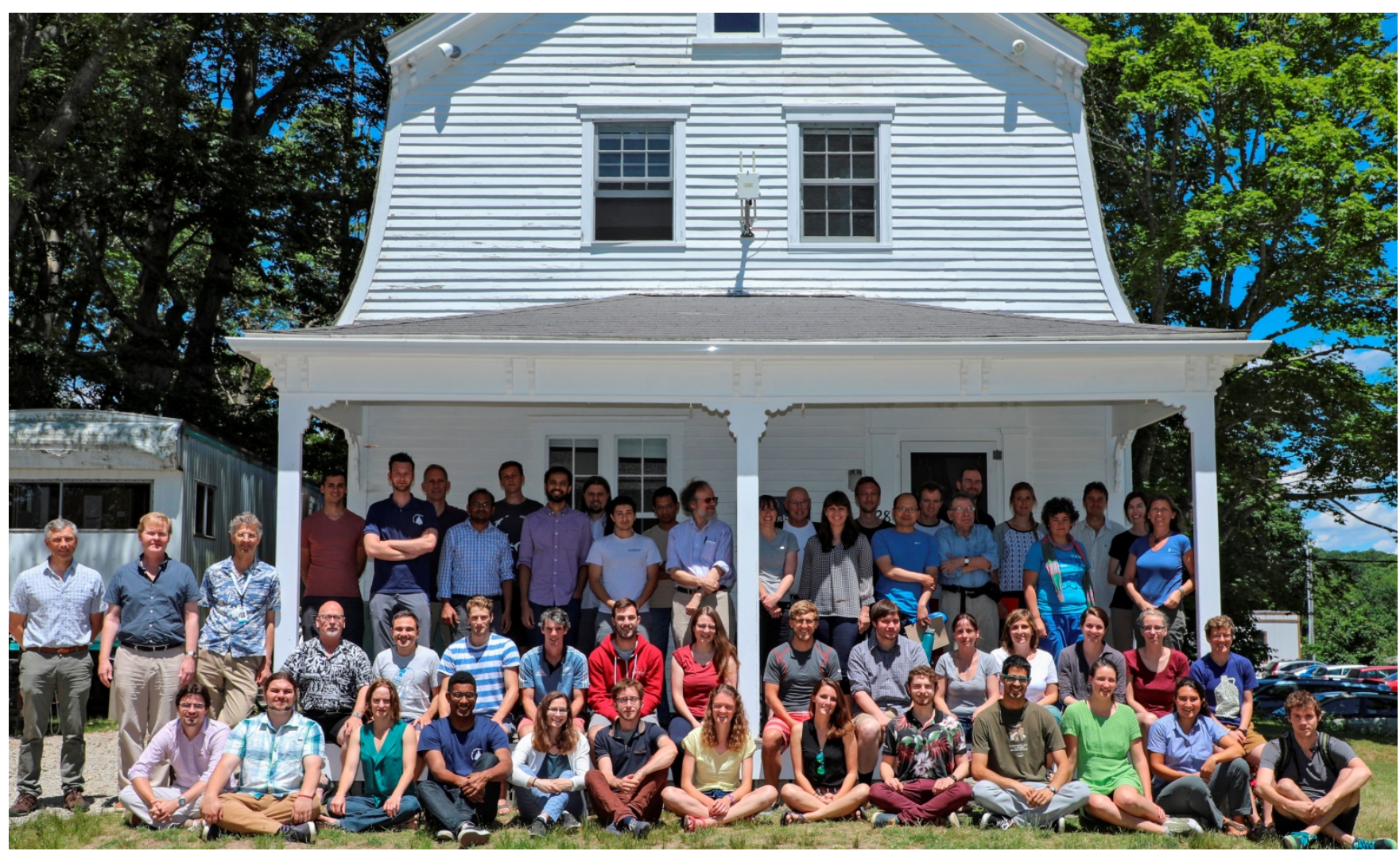

\section{Geophysical Fluid Dynamics Summer School Participants}

First Row (L-R): (All seated) Federico Fuentes, Robert Fajber, Jessica Kenigson, Earle Wilson, Madeleine Youngs, Guillaume Michel, Thomasina Ball, Margaret Lindeman, Eric Hester, Agostino Meroni, Madeleine Gamble Rosevear, Isabela LeBras, Henri Drake

Second Row (L-R): (Standing) Adrian Jenkins, Bruce Sutherland, Brian von Herzen (Seated) Charlie Doering, Greg Chini, David Goluskin, Craig McConnochie, Keaton Burns, Stefanie Semper, Colin Meyer, Sam Pegler, Pascale Garaud, Roberta Sciascia, Rebecca Jackson, Renske Gelderloos, Laura Stevens

Third Row (L-R): Pierre-Yves Passagia, Morven Muilwijk, Karl Helfrich, Vamsi Krishna Chalamalla, Kevin Thielen, Bhargav Rallabandi, Andrew Wells, Georgy Manucharyan, Srikanth Toppaladoddi, Glenn Flierl, Kate Snow, Jack Whitehead, Yana Bebieva, Erik Stenberg, Gordon Zhang, Donald Slater, Carl Wunsch, Nick Beaird, Maike Sonnewald, Fiamma Straneo, Neil Balmforth, Mary-Louise Timmermans, Claudia Cenedese

Missing from photo: Ryan Abernathey, Luca Brandt, Colm-cille Calufield, Megan DaviesWykes, Ekaterina Ezhova, Giovanni Fantuzzi, Anna FitzMaurice, Andrew Fowler, Pedram Hassanzadeh, Ian Hewitt, Joseph Hitchen, Malte Jansen, Alexis Kaminski, Daniel Lecoanet, Christopher MacMackin, Carlos Moffat, Nick Moore, Phil Morrison, Sara Nasab, Jerome Neufeld, Jamie Parkinson, Joe Pedlosky, Ed Speigel, Jean-Luc Thiffeault, Andrew Thompson, Andreas Thurnherr, Andrew Woods, Grae Worster, Phil Yecko, Megnan Zhao 


\section{Lecture Schedule}

\section{PRINCIPAL LECTURES}

Monday, June 19

Introduction to Ice-Ocean Interactions

Part I: Introduction to Glaciers and Ice Sheets Andrew Fowler

Part II: The Antarctic Ice Sheet and the Southern Ocean: An Introduction Adrian Jenkins

Part III: Greenland Ice Sheet Changes: The Ocean as a Trigger and Receiver Fiamma Straneo

Tuesday, June 20

Ice Dynamics

Andrew Fowler

Wednesday, June 21

Subglacial Control of Ice Flow Andrew Fowler

Thursday, June 22

Processes at the Ice-Ocean Interface Andrew Fowler

Friday, June 23

Basic Theory of Ice-Ocean Interaction Adrian Jenkins

Monday, June 26

Ocean Circulation beneath Ice Shelves Adrian Jenkins

Tuesday, June 27

Ice-Ocean Interactions around Antarctica Adrian Jenkins

Wednesday, June 28

Testing the Ocean Trigger Hypothesis for Greenland's Recent Glacier Retreat Fiamma Straneo

Thursday, June 29

Greenland Glacier-Ocean Interaction: Part I

Fiamma Straneo 
Friday, June 30

Greenland Glacier-Ocean Interaction: Part II

Fiamma Straneo

\section{SEMINARS}

Monday, July 3

When is a Meltwater Plume not a Plume (and Other Murky Questions)?

Andrew Wells, University of Oxford

Tuesday, July 4

HOLIDAY

Wednesday, July 5

Numerical Simulations of Turbulent Buoyant Plumes: Effects of Complex Stratifications and Wall Presence on Plume Dynamics

Ekaterina Ezhova, University of Helsinki

Thursday, July 6

Modeling Submarine Melting at Tidewater Glaciers in Greenland

Donald Slater, University of Edinburgh

Atmospheric vs. Oceanic Control of Labrador Sea Water Formation

Renske Gelderloos, Johns Hopkins University

Friday, July 7

Water Mass Transformation under Southern Ocean Sea Ice

Ryan Abernathey, Columbia University

Monday, July 10

Transport through Straits and Observed from HF Radar Measurements

Roberta Sciascia, Institute of Marine Sciences, National Research Council

Tuesday, July 11

A Mechanism for Deep Ocean Circulation Changes between Warm and Glacial Climates

Malte Jansen, University of Chicago

Wednesday, July 12

Accurate Linear Response Function of Climate Models: Calculations and Applications

Pedram Hassanzadeh, Rice University

Thursday, July 13

Extremal Time Averages and Optimal Bounds in Dynamical Systems

David Goluskin, University of Michigan

Some Strong Effects of Weak Rotation on Nonlinear Internal Waves

Karl Helfrich, WHOI 
Friday, July 14

Shooting for the MOOM? (Some of the) Mysteries of Ocean Mixing

Com-cille Caulfield, University of Cambridge

Monday, July 17

Double-diffusive Mixing in the Arctic Ocean

Yana Bebieva, Yale University

Tuesday, July 18

Self-sculpting of a Dissolvable Body due to Gravitational Convection

Megan Davies Wykes, DAMTP

Wednesday, July 19

Measuring Core Stellar Magnetic Fields using Wave Conversion

Daniel Lecoanet, Princeton University

Thursday, July 20

Bounds on Heat Transfer in Bénard-Marangoni Convection

Giovanni Fantuzzi, Imperial College London

The Influence of Memory in Mesoscale Eddy Field on the Variability of Large-scale Ocean Circulation

Georgy Manucharyan, California Institute of Technology

Friday, July 21

Exact Coherent Structures in Wall-bounded Shear Flows at Extreme Reynolds Number

Greg Chini, University of New Hampshire

Monday, July 24

Fjord Processes and the Melting of Tidewater Glaciers

Carlos Moffat, University of Delaware

Tuesday, July 25

Transient Stability of Shear Layers Forced by Standing Internal Waves

Alexis Kaminski, Oregon State University

Wednesday, July 26

Projecting Sea-level Rise: New Insights from History and Physics

Richard Alley, Pennsylvania State University

SEARS PUBLIC LECTURE - Redfield Auditorium

How High Will the Tide Be? Ice Sheets and Sea Level Rise

Richard Alley, Pennsylvania State University

Thursday, July 27

Lagrangian Transport and Superharmonic Instability of Internal Modes

Bruce Sutherland, University of Alberta 
Available Potential Energy for Ocean Convection, Abrupt Climate Change, Mesoscale Eddies, and Submesoscale Eddies

Zhan Su, California Institute of Technology

Friday, July 28

Transport and Mixing by Viscous Vortex Rings

Jean-Luc Thiffeault, University of Wisconsin-Madison

Monday, July 31

Optimal Stirring for Maximal Mixing

Charlie Doering, University of Michigan

Tuesday, August 1

Thesis Defense: Influence of Meltwater on Greenland Ice Sheet Dynamics

Laura Stevens, WHOI/MIT Joint Program

Wednesday, August 2

Double-diffusive Staircases in Giant Planets

Pascale Garaud, University of California-Santa Cruz

Thursday, August 3

Fluid Deformation of the Solid Earth: Bending and Breaking Rock and Ice Jerome Neufeld, University of Cambridge

Examining Ice-Ocean Interactions with Laboratory Experiments

Craig McConnochie, WHOI

Friday, August 4

Effects of Subglacial Lubrication on Ice-sheet Dynamics and Mass Loss Ian Hewitt, University of Oxford

Monday, August 7

Simple Models of Marine Ice Sheets

Grae Worster, University of Cambridge

Tuesday, August 8

GEMPIC: An Exact Poisson Integrator for the Full Vlasov-Maxwell System

Phil Morrison, University of Texas-Austin

Wednesday, August 9

Formation of Stable Margins to Streams of Fast-flowing Ice in Antarctica

Jim Rice, Harvard University

Thursday, August 10

The Control of Marine Ice Sheet Dynamics and Stability by Ice-shelf Buttressing

Sam Pegler, University of Leeds

Friday, August 11

How Focused Flexibility Maximizes the Thrust Production of Flapping Wings

Nick Moore, Florida State University 


\section{FELLOWS' PRESENTATIONS}

Monday, August 21

Seeing Ocean through Sea Ice

Robert Fajber, University of Toronto

A Mechanism for Secondary Sea Ice Formation Driven by Double-diffusive Supercooling Margaret Lindeman, Scripps Institution of Oceanography

Tuesday, August 22

Eddy Energy Fluxes in Mixed Barotropic-Baroclinic Instability: Upgradient or Downgradient?

Madeleine Youngs, Massachusetts Institute of Technology

Global Stability of 2D Plane Couette Flow beyond the Energy Stability Limit

Federico Fuentes, University of Texas-Austin

Indentation of Deformable Plastic Layers

Thomasina Ball, University of Cambridge

Wednesday, August 23

A Simple Adiabatic Model for Vertical Variation of Halocline Slope in the Beaufort Gyre Jessica Kenigson, University of Colorado-Boulder

Investigating the Effect of Iceberg Aspect Ratio on Submarine Melting

Eric Hester, University of Sydney

Nonlinear Influence of Background Rotation on Iceberg Melting

Agostino Meroni, University of Milan-Bicocca

Cooling via Baroclinic Acoustic Streaming

Guillaume Michel, Ecole Normale Supérieure

Thursday, August 24

The Dynamics of Subglacial Plume Lift-off

Earle Wilson, University of Washington

Turbid Tales: Where Do Sub-glacial Sediments Go?

Madelaine Gamble Rosevear, University of Tasmania 


\title{
GFD 2017 Lecture 1: Introduction to Ice-Ocean Interactions
}

\author{
Andrew Fowler, Adrian Jenkins and Fiamma Straneo; \\ notes compiled by Thomasina Ball and Robert Fajber
}

June 19, 2017

\section{Introduction to Glaciers and Ice Sheets, Andrew Fowler}

\section{$1.1 \quad$ The long view of polar caps}

The ice caps that we have today indicate that Earth is currently in the middle of an ice age. There are two ice sheets, one on Antarctica in the Southern hemisphere, and one on Greenland in the Northern hemisphere. Both cover almost the entire landmass that they are on. However, the ice sheets that we see today are not permanent features of the Earth system. There have been many times in Earth's history when the caps were altogether missing, and many times when the caps expanded to be much larger than they are today and fill the entire globe.

The Milanković cycles describe the different variations in solar insolation due to variations in the orbit of the planet. There are several cycles: a cycle of obliquity with a period of 41,000 years, a cycle of axial precession with with a period of 22,000 years and a cycle of apsidal precession with a period of 100,000 years. The exact details of the cycles are unimportant; what is important is the apparent synchronization between the paleoproxy records of temperature and insolation values (figure 1). The paleotemperature record is characterized by large sawtooth oscillations with sudden onsets and slow declines, with an approximate periodicity of $10^{5}$ years.

This observation suggests a simple theory of ice ages - when there is relatively high insolation the ice sheets melt and when there is relatively low insolation the ice sheets grow. The observed cycles are somewhat at odds with this theory however (figure 1). Although there is a $10^{5}$ year cycle present in the insolation, it is not the strongest cycle. This suggests that the Milanković cycles may not be the driving force behind the changing ice cover, but instead may set the phase of an oscillation which already exists. Further support comes from comparing the temperature record with the $\mathrm{CO}_{2}$, which shows a strong correlation. This implies that the sawtooth oscillations in temperature that we observe could be an oscillation of the coupled climate system. Interestingly, the paleo temperature record also reveals a coupling between Greenland and Antarctica near the transition, according to

$$
G=-\frac{d A}{d t}
$$




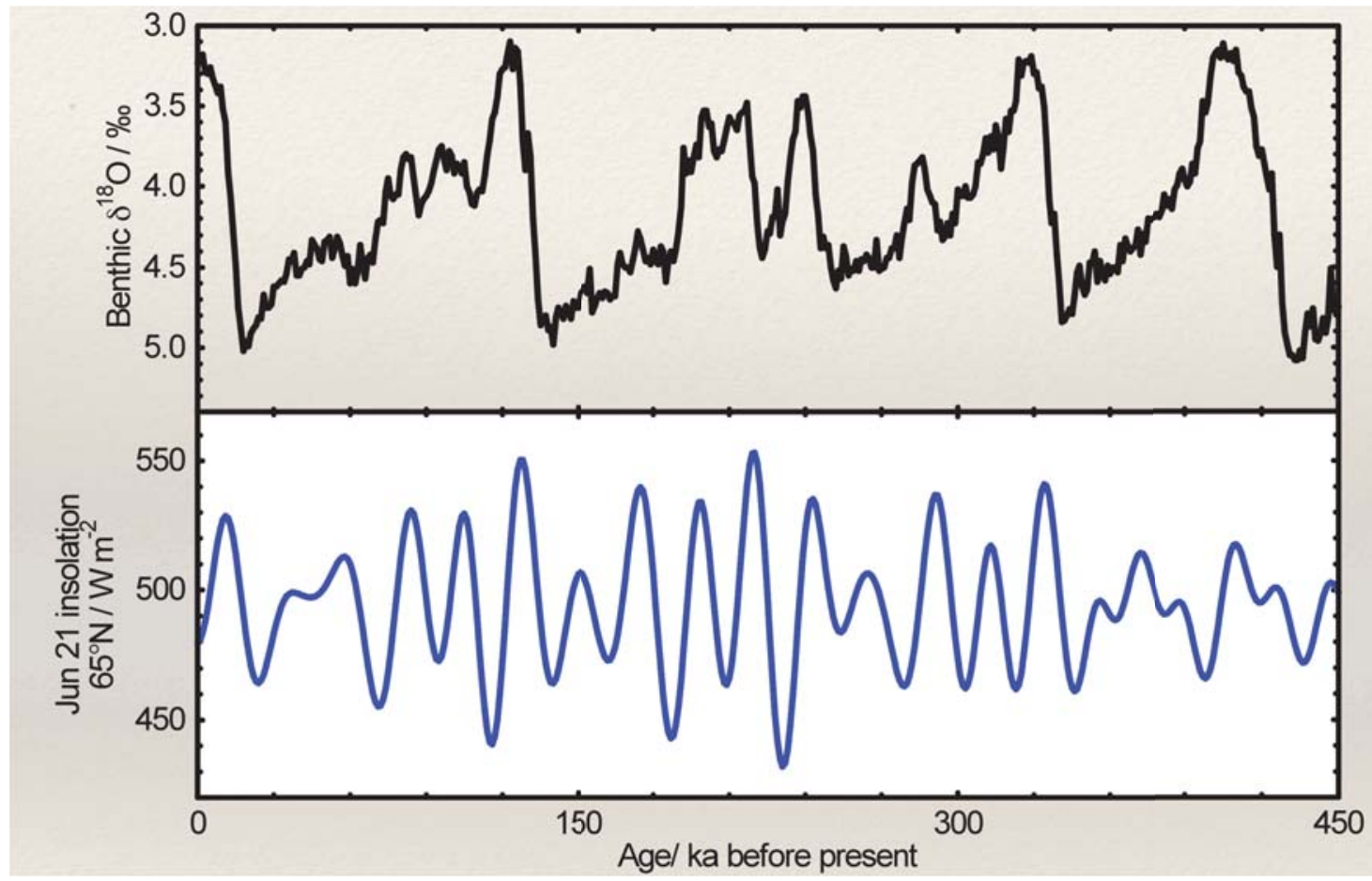

Figure 1: Top: a paleo oxygen isotope record constructed from sediments. Since $O^{16}$ evaporates more rapidly and condenses more slowly than $\mathrm{O}^{18}$, it is preferentially deposited into ice masses, which leaves the ocean enriched in $\mathrm{O}^{18}$. A higher $\mathrm{O}^{18}$ value therefore represents more land ice. This isotopic signature is incorporated into the shells of living organisms and turned into sediment. Thus, relatively high $\delta \mathrm{O}^{18}$ values correspond to cold periods and relatively low $\delta \mathrm{O}^{18}$ to warm periods. Bottom: the variations in insolation calculated using the Milanković cycles. 
where $G$ is the temperature over Greenland and $A$ is the temperature over Antarctica. Apparently the two ice masses are able to communicate, either through an atmospheric teleconnection or ocean heat transport.

It is believed that northeastern North America has been previously covered in ice, referred to as the Laurentide ice sheet. When this sheet collapses large quantities of icebergs raft sediments into the North Atlantic ocean, referred to as Heinreich events. Some of these sediments have been shown to originate in Hudson Bay, suggesting that there was an ice dome over Hudson bay. There is some partial synchronization between the Heinrich events and the Dansgaard-Oeschger events, sudden change in Greenland surface temperature over decades, suggesting that these sudden changes in air temperature could be linked to the collapse of the ice sheet.

The initiation process of an ice sheet has also been studied, particularly for Antarctica. The Earth has been cooling since $50 \mathrm{Ma}$ (although this is being opposed currently by anthropogenic climate change), and the Antarctic ice sheet began growing at approximately 34 Ma. Modeling studies suggest that the ice sheet begins as mountain glaciers, which grow until they are able to join together and form an ice sheet over the eastern half of the continent.

\subsection{A taxonomy of ice flows}

Ice can be modeled as a viscous fluid. Frequently the ice will move quite rapidly; in a glacier this causes surges to occur and in an ice sheet it causes ice streams to form. Glaciers and ice sheets both refer to masses of grounded ice; the difference is in their size. Ice sheets cover entire landmasses while glaciers can be any size. Parts of an ice sheet can be referred to as glaciers.

The ice flow moves ice from areas of accumulation to areas of ablation. If the ablation is sufficient to terminate the ice sheet over land, the boundary of the sheet is referred to as a dry margin. If the ablation is not enough to remove all of the ice over land, the ice will flow into the ocean. The boundary between the ice and the ocean is called a wet margin in this case. Wet margins can further be categorized into tidewater glaciers, where the glacier rests on the bed, and ice shelves, where the ice extends itself by floating into the water. The endpoint of an ice mass determines some of the behavior of the ice motion, and so it is advantageous to classify glaciers by the nature of their termination.

Antarctica is split between the west and the east by the trans-antarctic mountains. The East Antarctic Ice Sheet is a continental ice sheet: if it were removed, the ground would be largely above sea level. The West Antarctic Ice Sheet, on the other hand is a submarine ice sheet; most of its base is below sea level, and it contains large ice shelves. Antarctica is also characterized by large numbers of ice streams (figure 2), which accomplish a large fraction

of the ice transport, which brings mass from the central mass accretion zone to the outlying ablation and loss zones. 


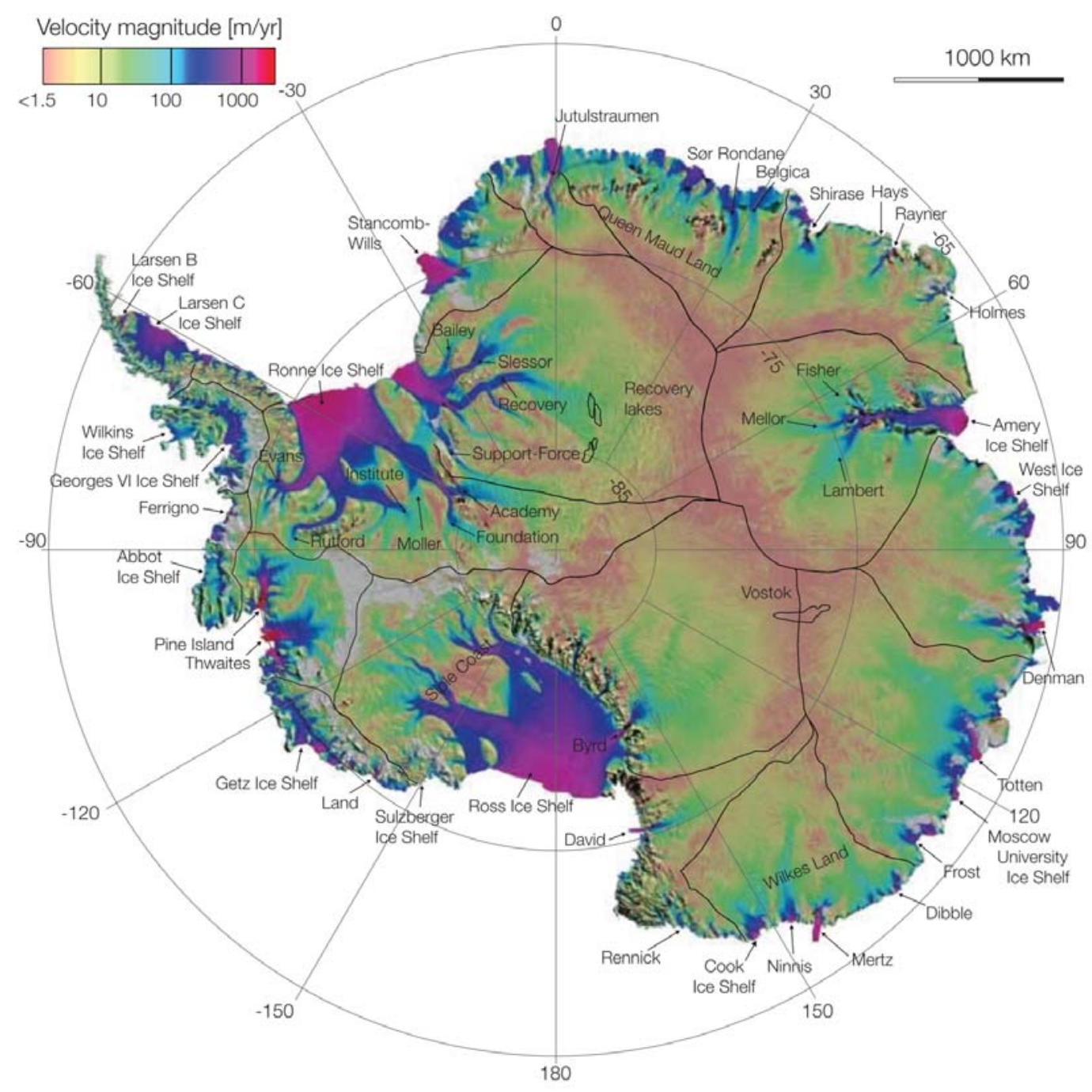

Figure 2: Ice speeds in Antarctica, from [13] 


\section{The Antarctic Ice Sheet and the Southern Ocean: An Introduction, Adrian Jenkins}

\subsection{Marine ice sheets and sea level}

Marine ice sheets sit on bedrock that falls below sea level. At the edges of these ice sheets, floating ice shelves form where the ice is not thick enough to maintain contact with the bed. Antarctica sits on average $500 \mathrm{~m}$ below sea level due to several factors: Antarctic topography, the weight of the ice sheet, tectonics and erosion of the continental shelf by ice flow. As a result, most ice shelves are found in Antarctica, where they cover an area comparable in size to the Greenland ice sheet $>1.561$ million $\mathrm{km}^{2}$. These are particularly found in West Antarctica where the ice is grounded in water $2 \mathrm{~km}$ deep, in contrast to East Antarctica which is mainly sitting on a bed above sea level. Ice shelves form only $11 \%$ of the Antarctic ice sheet but control $80 \%$ of the outflow from the continent. Therefore, understanding the behavior of ice shelves is important for understanding the $0.5 \mathrm{~mm} / \mathrm{yr}$ contribution Antarctica makes to global mean sea level, $97 \%$ of which is contributed when ice crosses the grounding line.

Unlike terrestrial ice sheets where accumulation of snow directly balances the melting of ice at lower elevations, for marine ice sheets the mass balance is intimately linked with ice dynamics, where ice is lost to the ocean through iceberg calving and basal melting. Figure 3 shows the basal melt rates of Antarctic ice shelves where, in total, basal melting is the largest ablation process with basal melt of $1325 \pm 235$ gigatons per year (Gt/yr) versus $1089 \pm 235 \mathrm{Gt} / \mathrm{yr}$ through calving [10]. Most of the current mass loss is balanced by the accumulation due to snow fall with the excess driving thinning of ice shelves, for example West Antarctica's ice shelves experienced a 134 Gt/yr mass loss during the period 2010-2013 [7]. Reductions in ice shelf thickness reduce the buttressing of the grounded ice allowing flow across the grounding line to accelerate and hence increase the rate of ice sheet mass loss [8]. The observed rate of ice loss is highest near the grounding line. This suggests ice shelf thinning in response to an increase in ocean-induced basal melting due to increased flux of Circumpolar Deep Water (CDW) onto the continental shelf. This increase in ocean-forced melting may have caused grounding lines to retreat onto a reverse slope which can trigger a runaway Marine Ice Sheet Instability (MISI).

Another possible process of ice sheet retreat is the Marine Ice Cliff Instability (MICI) [4] driven instead by atmospheric warming. Increased surface meltwater and summer rainfall on low topography can form ponds on the surface of the ice which drain into existing crevasses. It is thought that this allows water to penetrate into the ice causing it to fracture and eventually break off. An example of this is the Antarctic Peninsula's Larsen B ice shelf during its sudden break up in 2002. The Antarctic Peninsula is also one of the fastest warming regions in the world with an observed rise of $+0.53 \mathrm{C} / 10 \mathrm{yr}$ pushing the surface temperature close to a critical threshold where the surface is warm enough to melt during the summer. Although most of the Antarctic sheet sees surface temperatures well below

this threshold, trends of decadal atmospheric warming threaten to push the zone of melting further south towards larger ice shelves. 


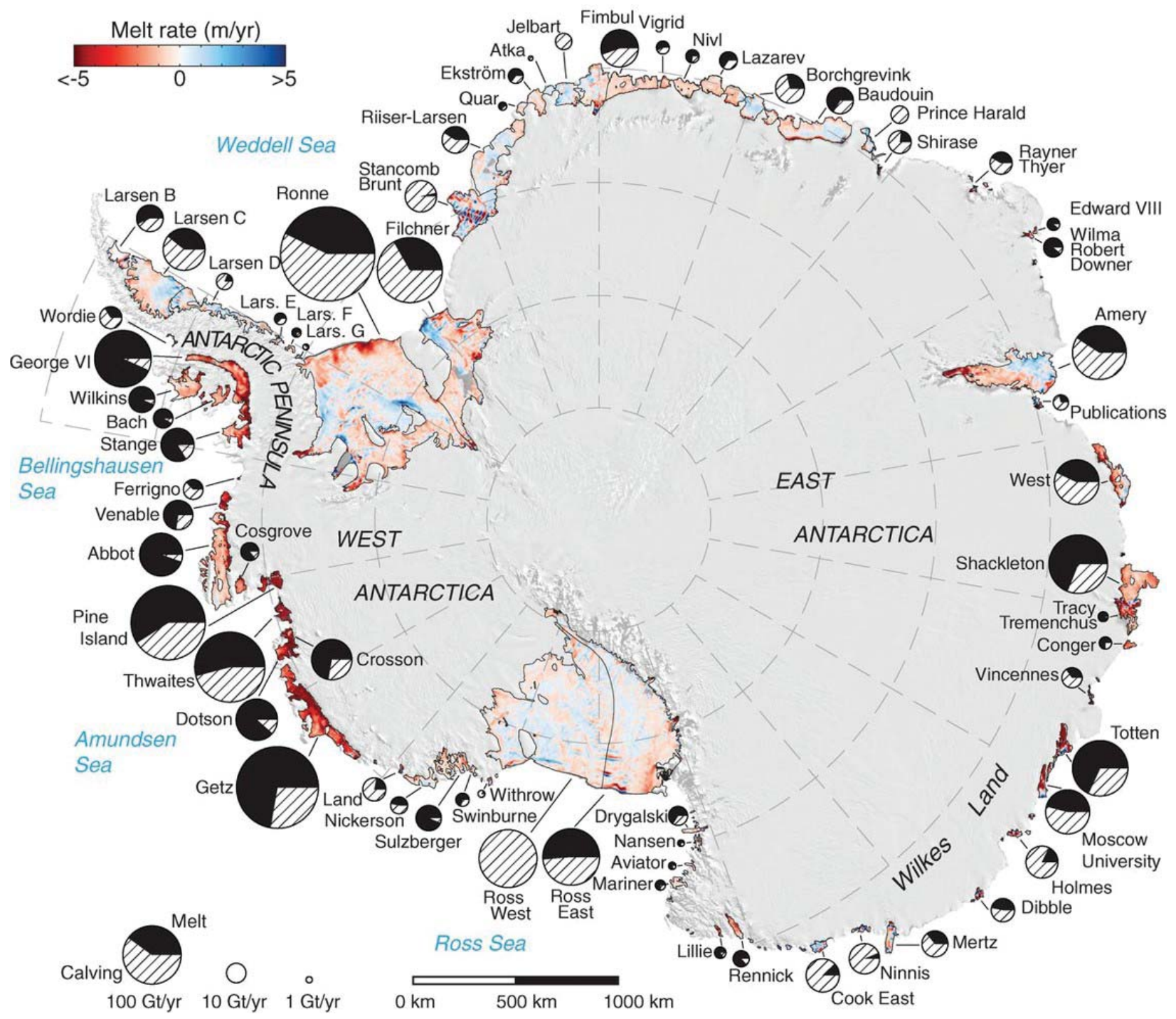

Figure 3: Basal melt rates of Antarctic ice shelves. Each circle graph is proportional in area to the mass loss from each shelf through iceberg calving (hatch fill) and basal melting (black fill) $[10]$.

The collapse of ice sheets due to atmospheric warming could lead to an instability when marine ice sheets have a depth of around $1 \mathrm{~km},>90 \mathrm{~m}$ of which is above sea level. As a result, the longitudinal stresses of the cliff face would exceed the yield strength of the ice (1MPa) leading to continued collapse until a reduction in temperature allows buttressing to reform. Again, if retreat moved the grounding line onto a reverse slope the MISI could take hold. This has been seen in Helheim and Jakobshavn glaciers in Greenland and Crane Glacier on the Antarctic Peninsula highlighting the importance of understanding the combination of MISI and MICI on an ice sheet. Figure 4 demonstrates these two methods of ice sheet retreat. 


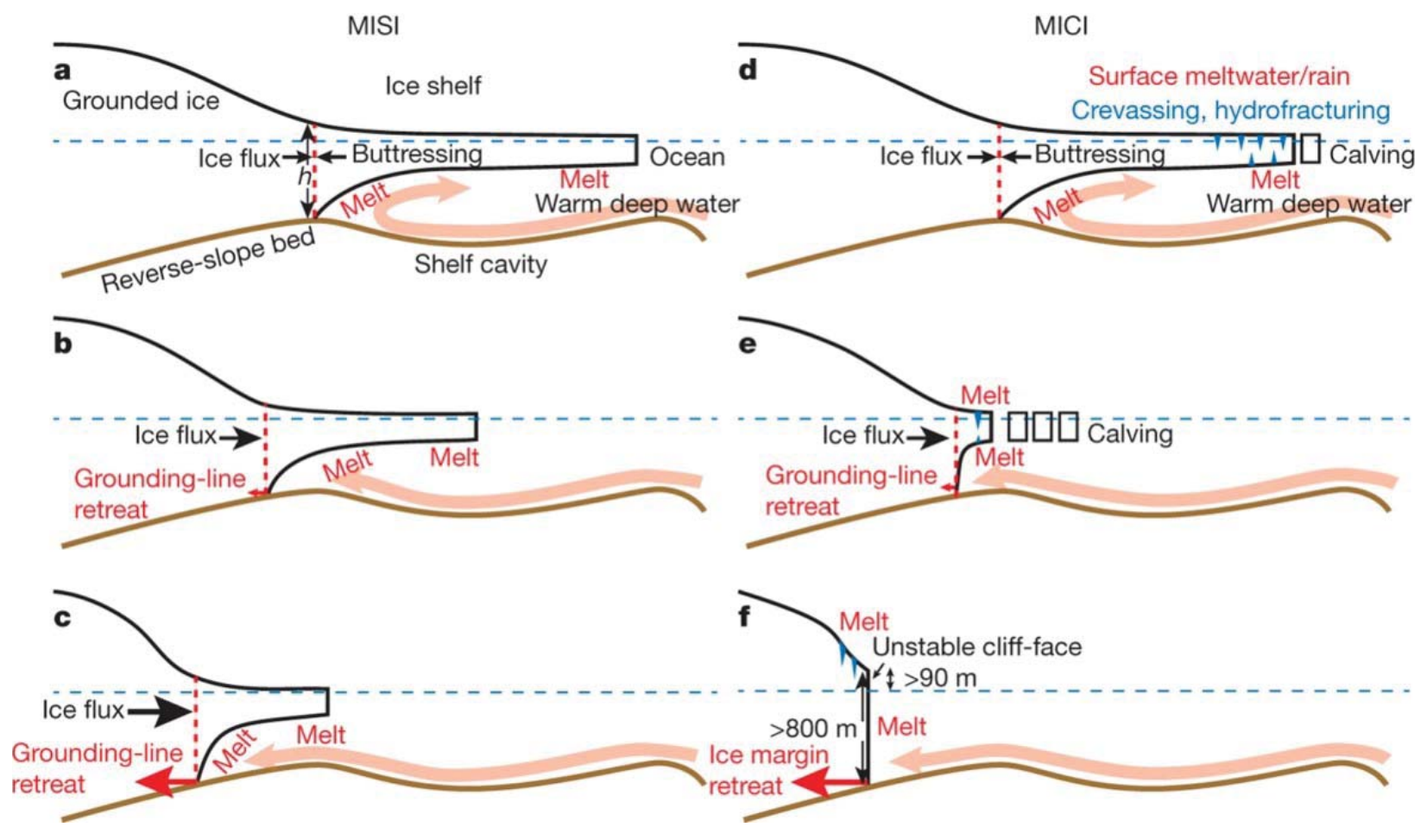

Figure 4: Schematic of MISI and MICI processes. (a-c) and (d-f) show ice retreat due to oceanic and atmospheric warming respectively. (a) Stable marine ice sheet with buttressing. Sub-ice melt rates increase with ocean warming and increased flux of the CDW onto the continental shelf. (b) Thinning of ice shelves due to sub-ice melting forcing the grounding line to retreat onto a reverse slope. (c) Grounding line positioned on a reverse slope triggers a runaway MISI. (d) Increased surface meltwater causes crevasses to fill up and eventually break off. (e) Increased calving provides another method of mass loss which, alongside MISI, causes the grounding line to retreat. (f) When continued calving breaks off the entire ice shelf, ice shelves with a height $>800 \mathrm{~m}$ with cliff face $>90 \mathrm{~m}$ become unstable and could collapse leading to further grounding line retreat leading to the MICI [4] 


\subsection{Ocean circulation near the Antarctic ice sheet and meridional overturning circulation}

Differential solar heating causes vertical convection in the atmosphere which helps drive horizontal wind patterns with easterlies near the poles and in the tropics and westerlies at mid-latitudes. The westerly winds over the Southern Ocean are uninterrupted by land and so can drive the zonally continuous Antarctic Circumpolar Current (ACC), the largest wind-driven current on Earth, and the only current that connects the Atlantic, Pacific and Indian Oceans. These westerly winds coupled with the easterlies near the poles south of a minimum in mean sea level pressure drive surface divergence and upwelling of Circumpolar Deep Water (CDW). This water mass supplies heat and nutrients to the surface playing a key role in marine ecosystems in the Antarctic region. Similarly, north of these westerlies, there is a surface convergence which drives downwelling of fresh Antarctic Intermediate Water (AAIW). South of the ACC, salty water formed beneath sea ice then cooled beneath ice shelves sinks to form the Antarctic Bottom Water (AABW), the coldest, deepest water in the ocean, see Figure 5.

Similar to the Antarctic, cold North Atlantic Water sinks as North Atlantic Deep Water (NADW), which is transformed into CDW in the Southern Ocean. This upwelling brings saline water to the surface, which either freshens to form the downwelling AAIW, seen most notably on figure 6 , or cools to join the fresh water from the continental shelf. However, further salt input from sea ice formed in the western Ross and Weddell seas is needed to increase the density of water sufficiently for the AABW to form.

North of the ACC surface water surrounding the Antarctic follows the meridional gradient, however south of the ACC subsurface temperatures on the shelf itself range significantly: from fresh water formed below floating ice shelves with temperatures below the surface freezing point, to warm waters from intruding CDW along the Pacific coast of West Antarctica with temperatures $3 \mathrm{C}$ above the surface freezing point.

\section{Greenland Ice Sheet Changes: The Ocean as a Trigger and a Receiver, Fiamma Straneo}

Greenland is changing rapidly and is losing mass at twice the rate of Antarctica. Observations from satellites have shown a loss of $2700 \pm 930 \mathrm{Gt}$ of ice between 1992 and 2011 from Greenland compared with $1350 \pm 1010$ Gt for Antarctica, contributing to a cumulative sea level rise of $7 \mathrm{~mm}$ and $4 \mathrm{~mm}$ respectively [14]. It is important to understand contributions to sea level rise and their uncertainties for informing future predictions (such as the IPCC report, which currently does not include ice sheet dynamics).

Greenland's increasing ice loss is also effecting many others processes. Maps of temperature data over the last century have shown an anomalous sub-polar North Atlantic cooling converse to the warming seen globally. This region of cooling compares well with climate models subject to a strong reduction in Atlantic meridional overturning circulation (AMOC) induced by adding a fresh water anomaly in the North Atlantic. If the climate models are forced further, an extension of the cooling region is seen causing a shutdown of the Labrador Sea convection, which has only briefly occurred so far [9]. Böning et al. 2016 [3] argue that 


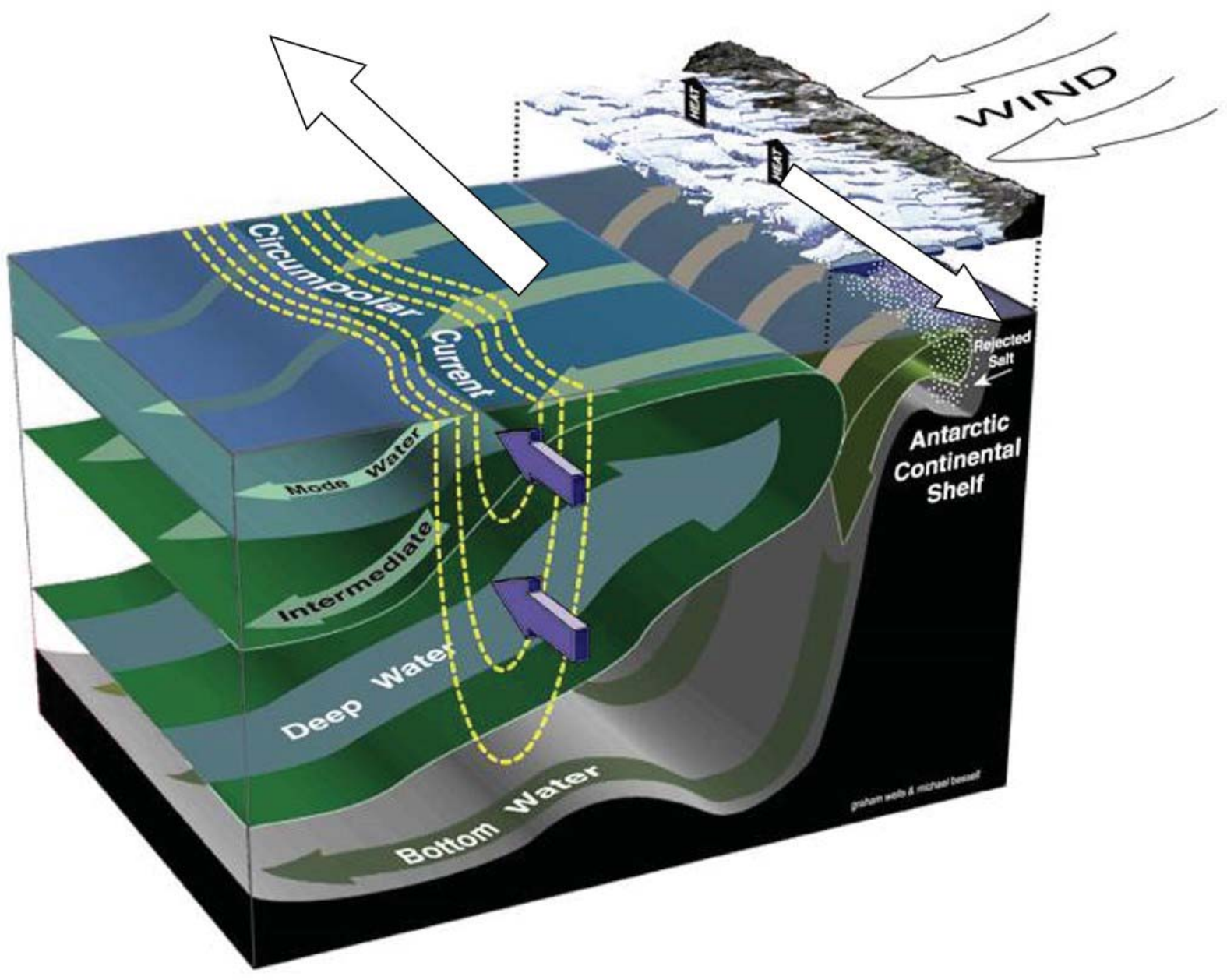

Figure 5: Schematic showing Ocean Circulation near the Antarctic Ice Sheet, particularly the upwelling of the CDW and downwelling of the AAIW and AABW in relation to the westerlies driving the ACC. 

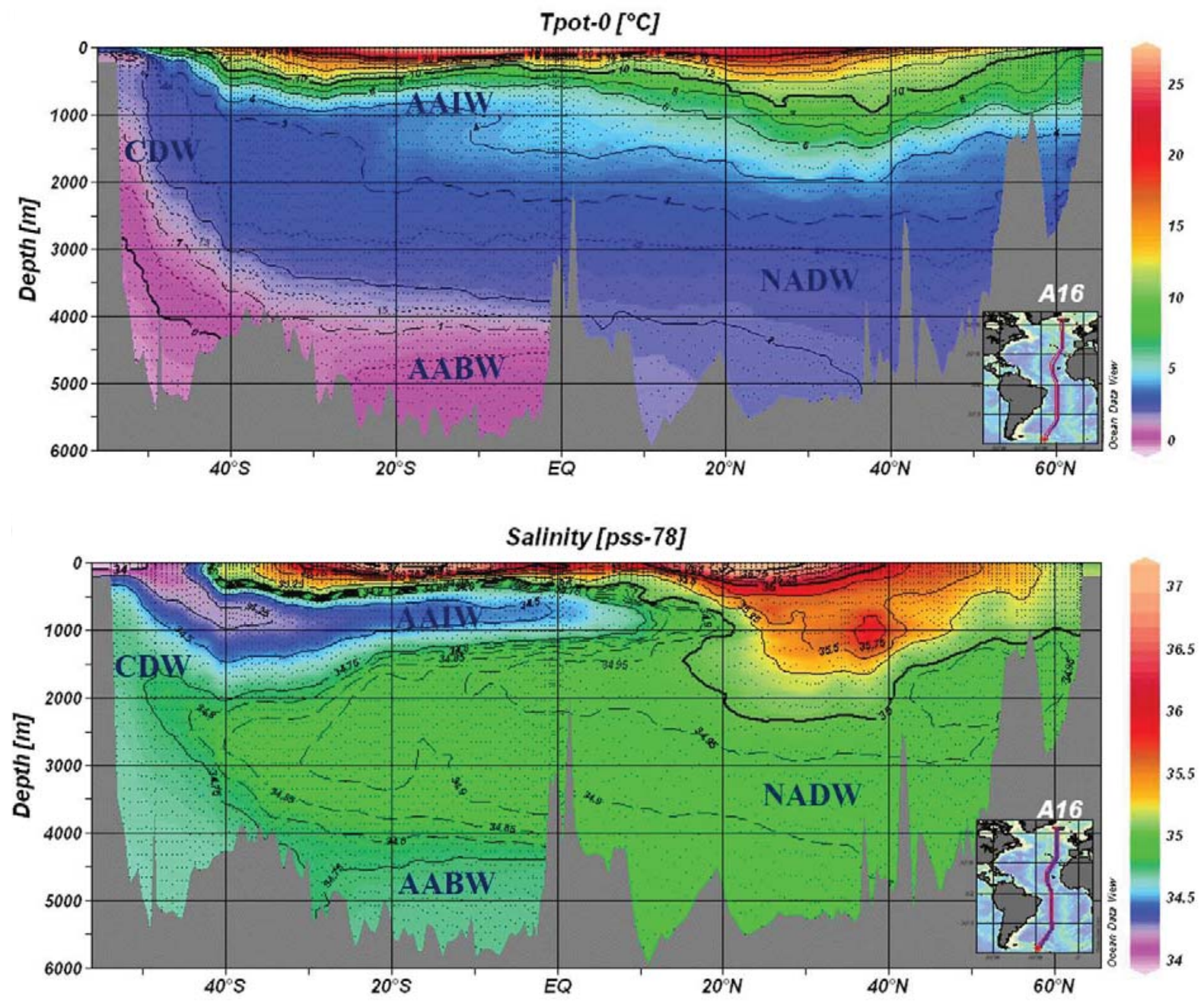

Figure 6: Cross sections of the temperature and salinity through the Atlantic. 
the increased fresh water in the North Atlantic has currently not had a significant impact on the AMOC. However, continued freshening of the surface waters may begin to effect the formation of the NADW and hence the AMOC before clear signals are observed.

The peak in glacial discharge from Greenland occurs during the summer melt season, which coincides with the post-spring depletion of bloom nutrients. It is thought that Greenland's meltwater could be significant source of bioavailable iron and inorganic nutrients to the ocean through sediment at the base of glaciers. These would then form buoyant freshwater plumes allowing the maximum potential of primary productivity in the North Atlantic Ocean [2]. Increased aeolian Fe could explain recent evidence of a correlation between peak phytoplankton blooms and increased meltwater runoff from Greenland [5].

Freshwater anomalies have occurred previously. The Great Salinity Anomaly (GSA) in the 1970s caused deep convection to cease for three mild winters in a row and affected the Labrador Sea by freshening the surface layer. This increased ocean stratification and confined convective mixing to the top fresh layer. Very cold winters during 1971/1972 allowed convection to begin again to normal depths of around 1500m [6]. Currently the freshwater anomaly in the North Atlantic is about a third of the magnitude of the GSA with a cumulative freshwater output of $3200 \pm 358 \mathrm{~km}^{3}$ since 1995 [1]. However, if the accelerating trend of increased fresh water discharge continues, it is estimated to exceed that of the GSA by 2025.

Ice sheet mass balance couples together the surface mass balance (SMB) due to accumulation, surface melt and sublimation and ice discharge (D),

where

$$
\frac{D \mathrm{M}}{D t}=\mathrm{SMB}-\mathrm{D}
$$

$\mathrm{SMB}=$ precipitation $-($ surface melt + sublimation $), \quad \mathrm{D}=\mathrm{ice}$ bergs + ocean melt

GRACE satellite data has recently shown that surface mass balance and ice discharge contributed roughly equally to the cumulative mass loss during the period from 1996-2015, with SMB accounting for between $40-60 \%$ loss and ice discharge the remaining $40 \%$ of loss [17]. In recent years, Greenland has had extreme melt years, for example in 2012 where the Greenland ice sheet was peppered all the way to the center with surface melt ponds. There has also been increased ice discharge moving ice from basins to the ocean. However, thus far models have struggled to constrain the contribution ice discharge makes.

During the last decade Greenland's large outlet glaciers have accelerated. Helheim glacier doubled its flow rate from $6 \mathrm{~km} / \mathrm{yr}$ to $11 \mathrm{~km} / \mathrm{yr}$ between $2002-2005$ whilst Kangerlussuaq glacier almost tripled its flow rate from $5 \mathrm{~km} / \mathrm{yr}$ to $14 \mathrm{~km} / \mathrm{yr}$ between $2002-2005$, with similar changes seen across the south of Greenland. These two glaciers had a combined mass loss of $208 \pm 15 \mathrm{~km}^{3}$ during the period $2001-2005$, or equivalently $51 \pm 8 \mathrm{~km}^{3} / \mathrm{yr}$. The increased flow rate increases longitudinal stresses in the ice and hence results in thinning rates of around $90 \mathrm{~m} / \mathrm{yr}$ contributing $0.31 \pm 0.07 \mathrm{~mm} / \mathrm{yr}$ to global mean sea level which is a significant portion of the $0.57 \mathrm{~mm} / \mathrm{yr}$ from Greenland as a whole [11]. This suggests that mass loss from glaciers in southeast Greenland is significant and hence understanding of the forcing that causes these rapid acceleration is important.

The Greenland ice sheet is influenced by waters originating in the North Atlantic (figure 7; red-yellow) and the Arctic (figure 1; blue). On the Eastern side of the continent the North 


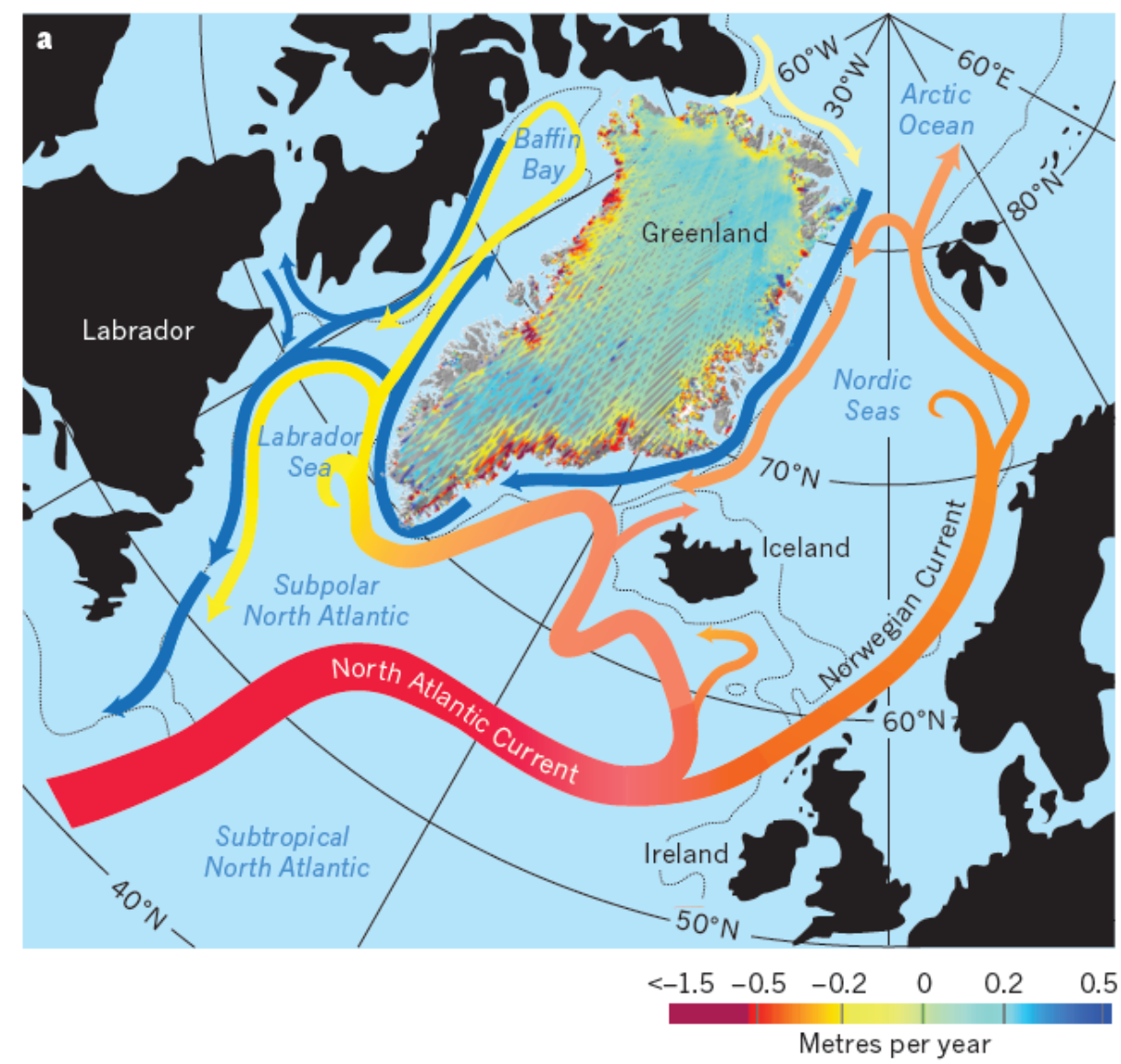

Figure 7: A schematic diagram showing the Ocean flow around Greenland overlain with the ice sheet elevation changes of the Greenland ice sheet. From [15]

Atlantic waters are much warmer than the Arctic water, but are separated from the land by a thin current of cold Arctic water. Without this layer of cold water the warm water would be able to melt the ice sheet.

Even with this layer however it is still possible for the warm Atlantic waters to reach ice shelves. This process occurs across many different scales. The large scale circulation determines the background temperature gradient and can be resolved by current models; mesoscale eddies that transport heat to the coast are significantly smaller; dynamics inside the fjords themselves are even smaller; heat transport between the water and the shelf occurs across a boundary layer that might be only millimeters to centimeters thick. Resolving the process across many scales is a major challenge for models.

Recent changes in the heat content of the North Atlantic appear to be driving changes in the glaciers surrounding Greenland. Observations of the subpolar Atlantic ocean show that there is a decadal variation in heat content, possibly related to the Atlantic Multidecadel Oscillation. Recently, the subpolar Atlantic ocean has seen the largest increase in temperature on current record. These changes are likely related to an inflow of warm subtropical 


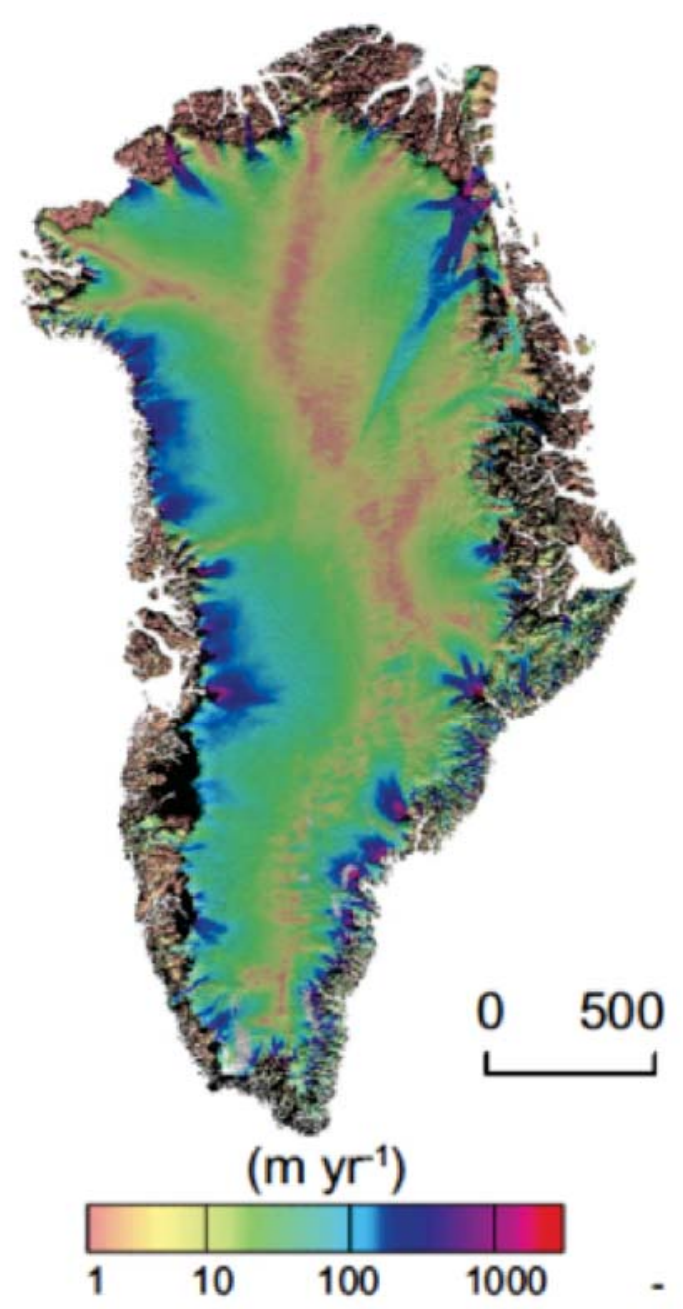

Figure 8: Ice velocity of the Greenland ice sheet. From [12]

waters. These waters have been warmed by increased atmospheric temperatures, but have not been able to propagate poleward into the subpolar Atlantic until the recent phase shift of the North Atlantic Oscillation.

Observations of ice velocity of the Greenland ice sheet show many ice streams carrying ice to the coast, especially on the southeast side (figure 8). Recent reconstructions of the surface elevation have shown that the Greenland ice sheet is not only losing mass, but that the ice rate itself is accelerating. The reconstructions are derived by differencing the observed elevation changes with the observed surface mass balance budget; the residual can be attributed to ice dynamics. The reconstructed data shows an acceleration in the ice, particularly on the southwest side of the continent. Although reconstructions and models have been able to provide us with important information, they have to be constrained by observations in order to provide reliable information since so many of the processes controlling the retreat of the ice sheet are small scale and parameterized in models. 

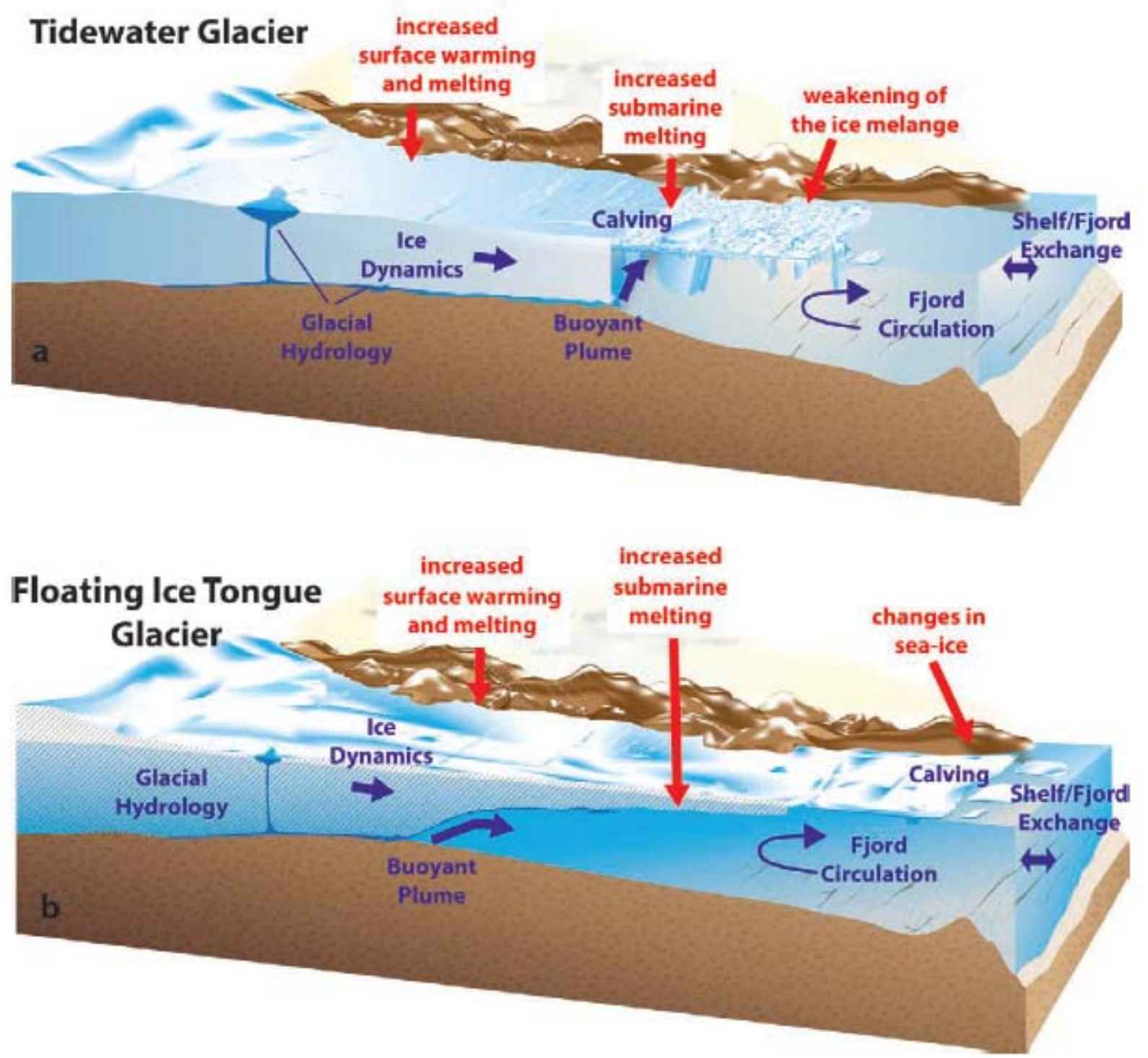

Figure 9: A: A schematic diagram showing the mechanisms by which a tidewater glacier is able to lose mass into the ocean. B: A schematic diagram showing the mechanisms by which a floating ice tongue glacier can lose mass. From [16]

In order to improve our understanding of the mass changes in the Greenland ice sheet we need to understand the different mechanisms for ice loss (figure 9) and their relative magnitudes. For both Tidewater glaciers and Floating Ice Tongue glaciers, increased surface warming from the atmosphere and increased submarine melting driven by warm Atlantic waters circulating in the fjord can cause an increase in ice speed. This could affect the two types of glaciers in different ways since the ice dynamics transporting ice to the ocean and the contact with the ocean look very different between the two cases. Since the problem of 
melting Greenland glaciers touches many different subjects, including glaciology, oceanography, hydrology and geology, a multi-disciplinary approach is needed.

\section{References}

[1] J. Bamber, M. Van Den Broeke, J. Ettema, J. Lenaerts, and E. Rignot, Recent large increases in freshwater fluxes from Greenland into the North Atlantic, Geophys. Res. Lett., 39 (2012), pp. 8-11.

[2] M. P. Bhatia, E. B. Kujawinski, S. B. Das, C. F. Breier, P. B. HenderSON, AND M. A. CharetTe, Greenland meltwater as a significant and potentially bioavailable source of iron to the ocean, Nat. Geosci., 6 (2013), pp. 274-278.

[3] C. W. Böning, E. Behrens, A. Biastoch, And K. Getzla, Emerging impact of Greenland meltwater on deepwater formation in the North Atlantic Ocean, 9 (2016), pp. 523-528.

[4] R. M. DeConto And D. Pollard, Contribution of Antarctica to past and future sea-level rise, Nature, 531 (2016), pp. 591-597.

[5] E. Frajka-Williams And P. B. Rhines, Physical controls and interannual variability of the Labrador Sea spring phytoplankton bloom in distinct regions, Deep Sea Res. Part I Oceanogr. Res. Pap., 57 (2010), pp. 541-552.

[6] R. Gelderloos, F. Straneo, and C. A. Katsman, Mechanisms behind the temporary shutdown of deep convection in the labrador sea: Lessons from the great salinity anomaly years 1968-71, J. Clim., 25 (2012), pp. 6743-6755.

[7] M. McMillan, A. Shepherd, A. Sundal, K. Briggs, A. Muir, A. Ridout, A. Hogg, and D. Wingham, Increased ice losses from Antarctica detected by CryoSat2, Geophys. Res. Lett., 41 (2014), pp. 3899-3905.

[8] F. S. Paolo, H. A. Fricker, and L. Padman, Volume loss from Antarctic ice shelves is accelerating, Science (80-. ) , 348 (2015), pp. 327-331.

[9] S. Rahmstorf, J. E. Box, G. Feulner, M. E. Mann, A. Robinson, S. RutherFORD, AND E. J. SCHAFFERnicht, Exceptional twentieth-century slowdown in Atlantic Ocean overturning circulation, 5 (2015).

[10] E. Rignot, S. Jacobs, J. Mouginot, And B. Scheuchl, Ice-Shelf Melting Around Antarctica, Science (80-. )., 341 (2013), pp. 266-270.

[11] E. Rignot and P. Kanagaratnam, Changes in the Velocity Structure of the Greenland Ice Sheet, Science (80-. ) , 311 (2012), pp. 986-990.

[12] E. Rignot And J. Mouginot, Ice flow in greenland for the international polar year 2008-2009, Geophysical Research Letters, 39 (2012). 
[13] E. Rignot, J. Mouginot, And B. Scheuchl, Ice flow of the antarctic ice sheet, Science, 333 (2011), pp. 1427-1430.

[14] A. Shepherd, E. R. Ivins, G. A, And V. R. Barletta, A Reconciled Estimate of Ice-Sheet Mass Balance, 1183 (2013).

[15] F. Straneo and P. Heimbach, North atlantic warming and the retreat of greenland's outlet glaciers, Nature, 504 (2013), pp. 36-43.

[16] F. Straneo, P. Heimbach, O. Sergienko, G. Hamilton, G. Catania, S. Griffies, R. Hallberg, A. Jenkins, I. Joughin, R. Motyka, et Al., Challenges to understanding the dynamic response of greenland's marine terminating glaciers to oceanic and atmospheric forcing, Bulletin of the American Meteorological Society, 94 (2013), pp. 1131-1144.

[17] M. van den Broeke, J. Bamber, J. Ettema, E. Rignot, E. Schrama, W. J. van de Berg, E. van Meijgaard, I. Velicogna, and B. Wouters, Partitioning Recent Greenland Mass Loss, Science (80-. ), 326 (2009), pp. 984-986. 


\section{GFD 2017 Lecture 2: Ice Dynamics}

Andrew Fowler; notes by Federico Fuentes and Madelaine Gamble Rosevear

June 20, 2017

This document comprises the first full lecture given by Andrew Fowler during the 2017 Geophysical Fluid Dynamics program at the Woods Hole Oceanographic Institution (WHOI). It is about ice dynamics, and is divided in two parts: ice sheet flow, and sliding and subglacial hydrology. Most of the details were taken from Dr. Fowler's book [Fowler, 2011], which the reader is invited to consult if more information is required.

\section{Ice sheet flow}

\subsection{Governing equations}

Over sufficiently long periods, ice behaves as a viscous fluid, deforming under applied stress. The strain rate $\dot{\varepsilon}_{i j}$ is given by

$$
\dot{\varepsilon}_{i j}=\frac{1}{2}\left(\frac{\partial u_{i}}{\partial x_{j}}+\frac{\partial u_{j}}{\partial x_{j} i}\right),
$$

and is commonly modeled using Glen's flow law

$$
\dot{\varepsilon}_{i j}=A(T) \tau^{n-1} \tau_{i j}
$$

where $\tau_{i j}$ is the deviatoric stress tensor in index notation, $\tau$ is the second stress invariant, defined by $2 \tau^{2}=\tau_{i j} \tau_{i j}$, and $A(T)$ is a temperature dependent term. The Glen exponent $n$ is typically taken to be 3 , although values $1 \leq n \leq 4$ have been proposed in the literature.

For the flow of a glacier the Reynolds number is approximately $10^{-13}$, so inertial terms are small with respect to viscous terms (Stokes flow) and mass and momentum conservation may be expressed as

$$
\begin{aligned}
\boldsymbol{\nabla} \cdot \boldsymbol{u} & =0 \\
0 & =\boldsymbol{\nabla} p+\boldsymbol{\nabla} \cdot \boldsymbol{\tau}+\rho \boldsymbol{g} .
\end{aligned}
$$

where $p$ is the pressure, $\boldsymbol{g}$ is the gravity vector and $\boldsymbol{\tau}$ is the deviatoric part of the stress tensor. The assumption of incompressibility in (3) holds within the ice. This is a reasonable assumption as the surface layer in which snow and firn are compacted into ice is very thin. 


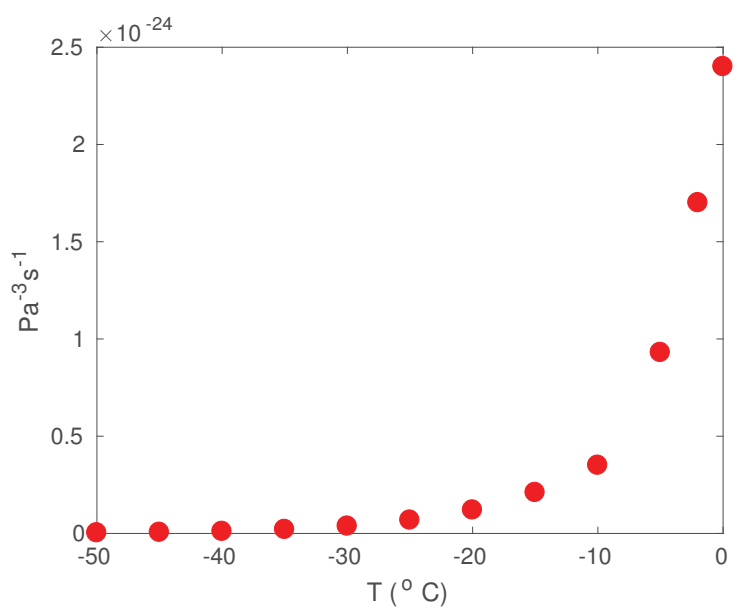

Figure 1: Controls on Creep Parameter A. Data from [Cuffey and Paterson, 2010].

Finally we have the energy equation:

$$
\rho c_{p}(T_{t}+\underbrace{\boldsymbol{u} \cdot \nabla T}_{\text {advection }})=\underbrace{k \nabla^{2} T}_{\begin{array}{c}
\text { heat } \\
\text { conduction }
\end{array}}+\underbrace{\tau_{i j} \dot{\varepsilon}_{i j}}_{\begin{array}{c}
\text { viscous } \\
\text { dissipation }
\end{array}}
$$

where $\rho$ is the ice density, $c_{p}$ is the specific heat, and $k$ is the thermal conductivity. The final term in (5) is the viscous heating term describing the conversion of mechanical energy to heat. Whilst this term is often neglected in other geophysical flows, it significant for ice sheet flow.

Stress and strain are related by $\tau_{i j}=2 \eta \dot{\varepsilon}_{i j}$ where $\eta$ is the effective viscosity. Using (2) we can write

$$
\eta=\frac{1}{2 A(T) \tau^{n-1}} .
$$

The term $A(T)$ is strongly dependent on temperature, increasing over three orders of magnitude for a temperature change of $50 \mathrm{~K}$ (Figure 1), and thus viscosity is inversely related to temperature.

\subsection{Bi-stability and thermal runaway}

It is the strong temperature dependence of the viscous heating term that provides the mechanism for "thermal runaway". If heat is supplied to the ice, the temperature increases and the viscosity decreases. This allows the ice to flow faster, increasing stresses at the bed and warming the ice through the viscous heating term in (5). This in turn lowers viscosity, creating a positive feedback loop.

\subsection{Boundary conditions}

A complicating factor for the modeling of ice flows is that one cannot assume a no-slip boundary condition at the bedrock. Water present beneath the ice allows sliding, and thus 


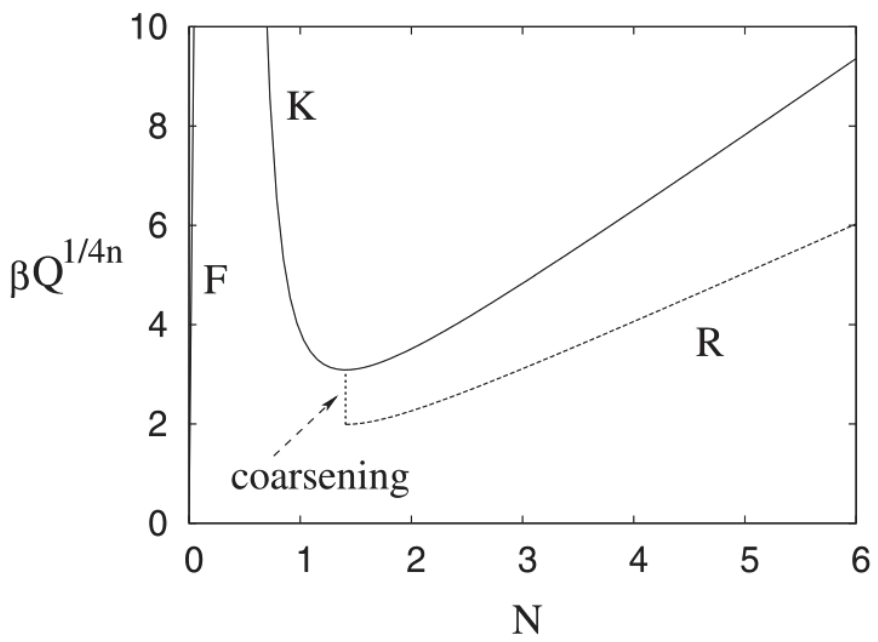

Figure 2: Illustrative relation between the effective pressure $N$ and the water flow $Q$ through a field of linked cavities [Fowler, 2011] where $F$ represents film flow, $K$ is linked cavities and $R$ is Röthlisberger channels. To the right of the minimum, distributed drainage is unstable; the channels coarsen resulting in a single Röthlisberger channel. To the left, distributed drainage in the form of linked cavities is stable. As $N$ goes to zero a thin film flow is permitted.

a sliding law is required. The basal stress,

$$
\tau_{b}=N f\left(\frac{u_{b}}{N^{n}}\right),
$$

is modeled as an increasing function $f$ of the velocity at the base $u_{b}$ and the effective pressure $N=p_{i}-p_{w}$, where $p_{i}$ is the overburden pressure and $p_{w}$ is the water pressure. The effective pressure, $N$, is analogous to that used in soil mechanics, and is typically positive. In order to relate $N$ to the subglacial water flow rate $Q$, subglacial hydraulic theory is required. Three of the prevailing theories, are:

- Röthlisberger channels, where

$$
N \approx \beta Q^{1 /(4 n)}
$$

- A linked system of canals, where

$$
N \approx \frac{\gamma}{Q^{1 / n}} .
$$

- A Creyts-Schoof film, where

$$
N \approx \frac{\delta}{Q^{\mu}} .
$$



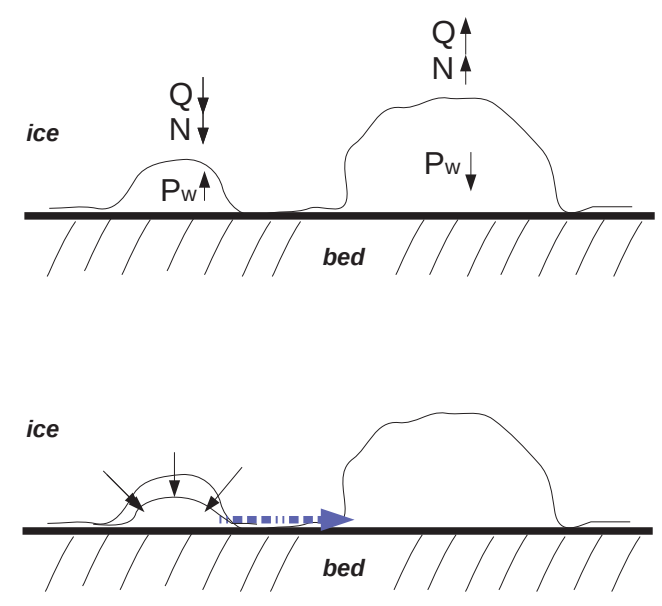

Figure 3: Schematic showing the draining and subsequent closure of a smaller channel due to the presence of a neighboring larger channel.

\subsubsection{Röthlisberger channels}

Subglacial water is present due to both basal melt and, where a conduit to the base is present, surface melt or rainfall. One theory of subglacial drainage involves the formation of semicircular channels within the ice. The fact that the effective pressure $N$ is typically positive means that these channels would close through the deformation of the ice in the absence of a mechanism to keep them open. This mechanism is melting due to frictional heating from the water flow.

The expression for the effective pressure of one of these channels is

$$
N \sim \beta Q^{1 / 4 n}
$$

where $Q$ is the flow rate of the water and $\beta$ is a material parameter that depends inversely on roughness.

An interesting feature of this system is that a decrease in water flux decreases $N$ and therefore increases the water pressure $p_{w}$. If we consider a small channel next to a large channel (Figure 3), then $Q$ is small within the small channel, and thus the water pressure $p_{w}$ must be large. In the larger channel the opposite is true, so the water pressure is low. The bed separating the two is rough, and water is able to leak from high to low pressure. As a result of this, smaller channels drain towards larger ones and close down, creating an arterial system of channels.

\subsection{Thermal boundary conditions}

The pressure melting point of ice decreases with increasing pressure, meaning that even very cold ice may be above the in-situ melting temperature at the base of an ice sheet. This 
means we need to consider whether the ice is above or below the melting point in our thermal boundary conditions. When the temperature at $z=b$ is less than the freezing temperature $T_{m}$ the ice is frozen to the bed and we have a no slip boundary,

$$
-k \frac{\partial T}{\partial n}=G, \quad T<T_{m}, \quad u=0
$$

where $G$ is the geothermal heat flux and $n$ is direction normal to the bed. Once the base reaches the melting temperature a layer of water is present, lubricating the base of the ice. This allows some sliding, however less than the full sliding velocity $u_{b}$ as there is not yet a net production of water,

$$
-k \frac{\partial T}{\partial n}=G+\tau_{b} u, \quad T=T_{m}, \quad 0<u<u_{b} .
$$

This introduces a frictional heating term $\tau_{b} u$ due to the sliding. When there is net production of water, the ice attains its full sliding velocity. In this regime,

$$
0<-k \frac{\partial T}{\partial n}<G+\tau_{b} u, \quad T=T_{m}, \quad u=u_{b} .
$$

Note that each of these regimes contains an inequality, adding another layer of complexity to the model.

\subsection{Shallow ice approximation}

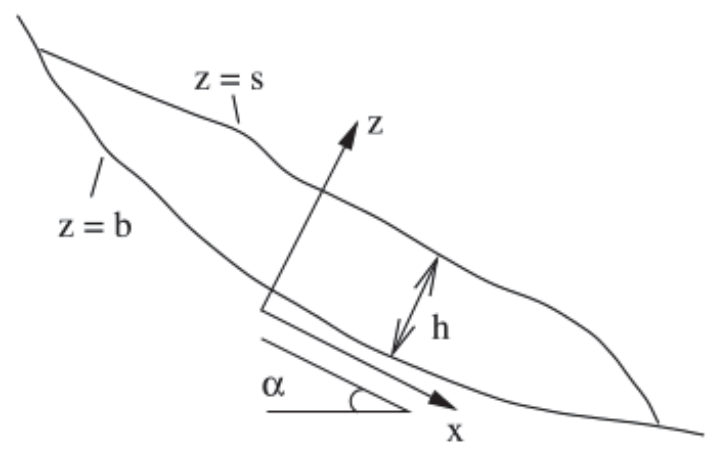

Figure 4: Schematic of a valley glacier showing thickness $h$ (elsewhere $H$ ), bed elevation $b$ and surface height s. Figure from [Fowler, 2011].

Ice sheets may be thousands of kilometers in extent but are only kilometers deep (Figure 5), allowing the use of the shallow ice approximation. For an ice sheet of thickness $d$ and extent $l$ the aspect ratio is given by $\varepsilon=d / l$. For the Antarctic Ice Sheet $d \sim 3 \times 10^{3} \mathrm{~m}$, $l \sim 3 \times 10^{6} \mathrm{~m}$ giving $\varepsilon \sim 10^{-3}$. As a result of this, longitudinal derivatives of stress, velocity and temperature are small compared to vertical derivatives and may be neglected, reducing 


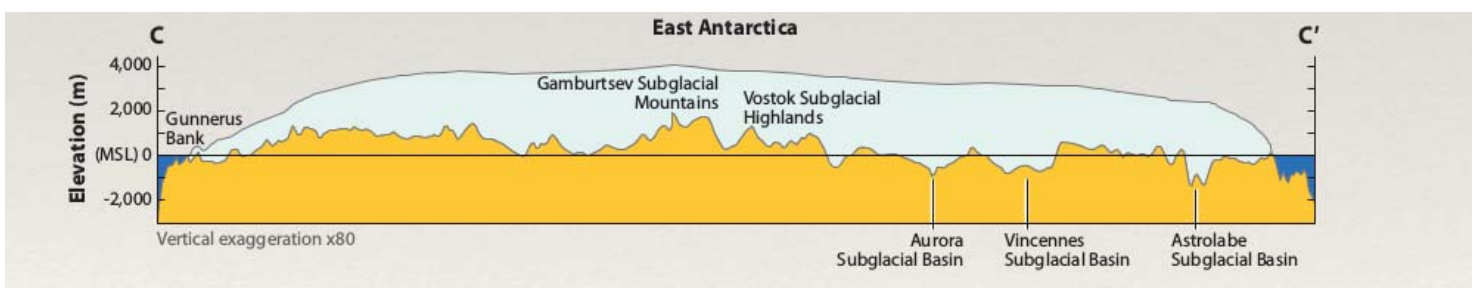

Figure 5: Cross section of the Antarctic Ice Sheet with exaggerated vertical scale. Figure from lecture slides.

the problem to a balance between the driving stress due to surface slope and resistive forces at the boundaries [Huybrechts, 2007].

This allows us to write a diffusion equation governing the evolution of the ice sheet thickness, $H$,

$$
H_{t}=\boldsymbol{\nabla} \cdot(\underbrace{\left(\frac{|\boldsymbol{\nabla} s|^{n-1} H^{n+2}}{n+2} \nabla s\right)}_{\text {nonlinear diffusion }}-H \boldsymbol{u}_{b})+a
$$

where $H$ is the thickness of the ice sheet, $b$ is the bed elevation, $\boldsymbol{u}_{b}$ is the basal velocity, $s=H+b$ is the surface elevation and $a$ is the accumulation from snowfall (or, where negative, ablation). The nonlinear diffusion causes degeneracy at the boundaries and singularities may be involved. Whilst the term $-H \boldsymbol{u}_{b}$ looks like an advective term, $\boldsymbol{u}_{b}$ is typically in the direction of the shear stress and so is proportional to the surface slope $\left(\boldsymbol{u}_{b} \sim \tau_{b} \sim-\nabla s\right)$, meaning that this term is also diffusive.

\subsection{Accumulation and hysteresis}

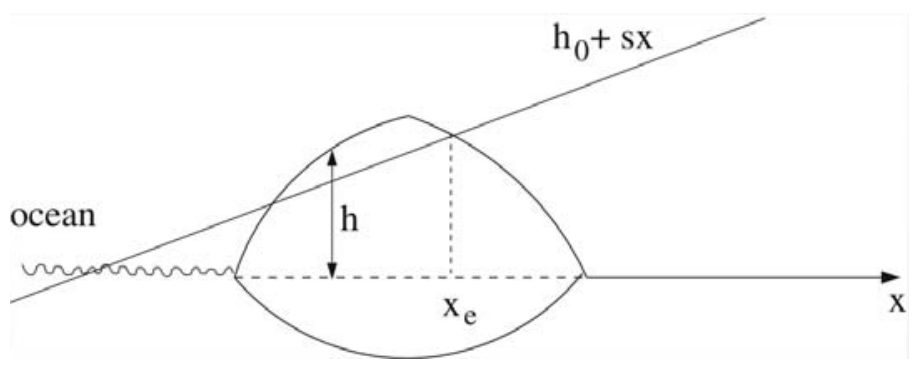

Figure 6: Schematic of an ice sheet with extent $x_{e}$ and height $H$.

Ice sheet mass is determined by the balance between accumulation of snow above the snow line and ablation at the margins, where the snow line is given by $h_{0}+s x$, as shown in Figure 6. As the ice sheet extent decreases the height $h$ decreases, meaning less of the ice sheet is above the snow line, and therefore accumulation is less and ice sheet extent decreases. When the height falls beneath the snow line accumulation goes to zero. In the absence of any gain terms, the ice sheet collapses. 


\section{Sliding and Subglacial Hydrology}

\subsection{Weertman's sliding law}

Consider ice over a set of obstacles as illustrated in Figure 7. The obstacles are separated on average by a distance $l$ and have heights roughly of size $a$, so that the aspect ratio is defined as

$$
\nu=\frac{a}{l} .
$$

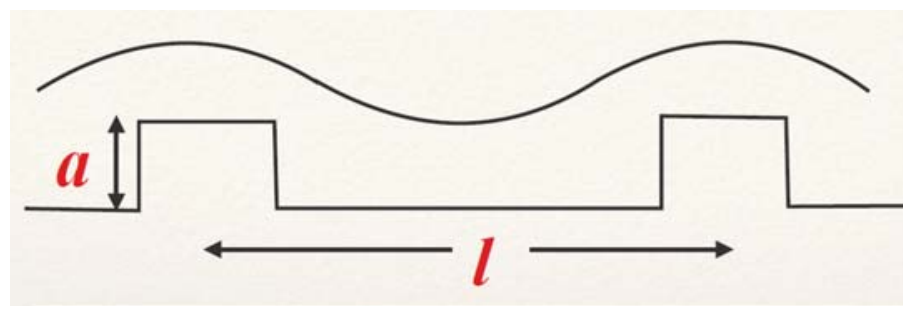

Figure 7: Weertman's sliding law.

The ice is assumed to slide at a particular velocity. Weertman's law is derived by assuming that a "regelation" velocity is roughly the same as a "viscous" velocity associated to Glen's flow law.

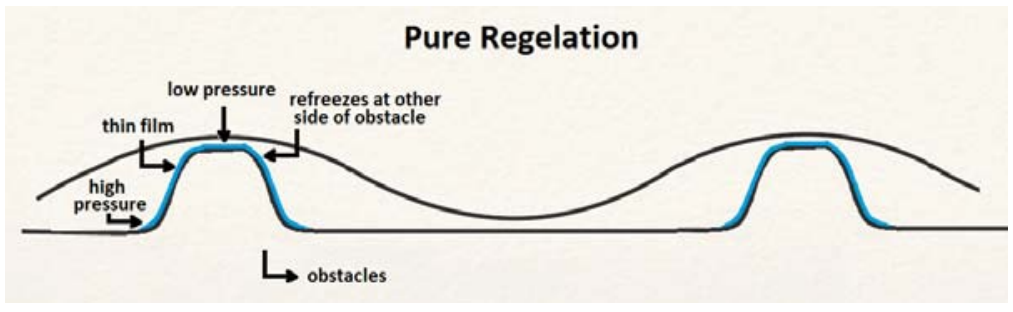

Figure 8: Pure regelation.

Regelation occurs when ice at high pressure melts and then refreezes at areas of low pressure. This creates a very thin film, with a thickness of the order of $1 \mu \mathrm{m}$ over which the ice flows. Figure 8 shows the case of pure regelation. Under regelation, the pressure difference across the obstacle is roughly

$$
\delta p \approx-\frac{\tau}{\nu^{2}}
$$

where $\tau$ is the average shear stress at the bed, and $p$ is the pressure. Hence, using that

$$
-\frac{d T_{m}}{d p}=C,
$$

where $T_{m}$ is the melting temperature, it follows that there is a temperature difference of

$$
\delta T \approx C \frac{\tau}{\nu^{2}}
$$


where $T$ is the temperature. The regelative water flux is $u_{R} a^{2}$, where $u_{R}$ is the regelative ice velocity, meaning that to melt the ice a latent heat of $\rho_{i} L u_{R} a^{2}$ is necessary, where $\rho_{i}$ is the ice density and $L$ is the specific latent heat. This must be equal to the heat conducted through the obstacle, so that

$$
\left(k \frac{\delta T}{a}\right) a^{2}=\rho_{i} L u_{R} a^{2} \Rightarrow u_{R}=\left(\frac{k C}{\rho_{i} L a}\right) \frac{\tau}{\nu^{2}},
$$

where $k$ is the thermal conductivity of the bedrock. It follows regelation is important for small obstacle sizes of size $a$.

Meanwhile, the velocity due to viscous shearing is related to Glen's flow law. It is

$$
u_{V} \approx 2 a A\left(\frac{\tau}{\nu^{2}}\right)^{n}
$$

where $n$ is the exponent in Glen's flow law. Thus, this velocity dominates for large obstacles of size $a$.

There is a controlling obstacle size $a$ for which both effects are important. Selecting $a$ so that both velocities are equal, means that $u=u_{R}=u_{V}$, so that multiplying both equations yields,

$$
\tau=\nu^{2}\left(\frac{\rho_{i} L}{2 k C A}\right)^{\frac{1}{n+1}} u^{\frac{2}{n+1}} .
$$

This is known as Weertman's sliding law.

\subsection{Lliboutry cavitation}

For large obstacles, cavities are formed due to the fact that the film pressure after the obstacle is lower than the water pressure in the local subglacial drainage system. In practice, it is common to find these cavities. Figure 9 illustrates this cavitation.

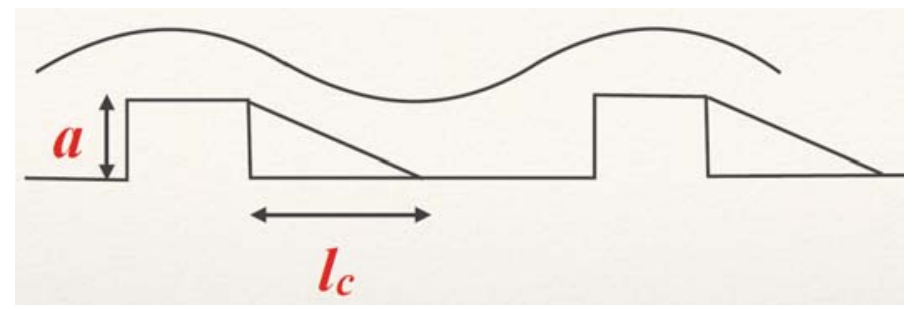

Figure 9: Lliboutry cavitation.

In this case, the velocity due to viscous shearing, which is assumed to dominate, takes the form

$$
u \approx 2\left(a+l_{c}\right) A\left(\frac{\tau}{\nu^{2}}\right)^{n}
$$

where $l_{c}$ is the length of the cavity. Additionally, the pressure difference between the ice and water relates to the velocity by

$$
\frac{u}{l_{c}}=A N^{n}, \quad N=p_{i}-p_{w},
$$


where $N$ is the effective pressure, $p_{i}$ is the ice pressure (or overburden pressure) and $p_{w}$ is the water pressure. Substituting $l_{c}$ then yields

$$
\frac{\tau}{N}=\nu^{2}\left(\frac{\Lambda}{2(1+\Lambda)}\right)^{\frac{1}{n}}, \quad \Lambda=\frac{u}{A N^{n} a} .
$$

\subsection{Drainage and the Nye-Röthlisberger model}

Weertman films have a tendency to become unstable. In these cases, Röthlisberger channels form, where water flows from regions of higher pressure to regions of lower pressure. The channels are maintained open by melting in the channel walls. The melting is due to the frictional heat resulting from the flow of the water itself. The channels are schematically shown in Figure 10.

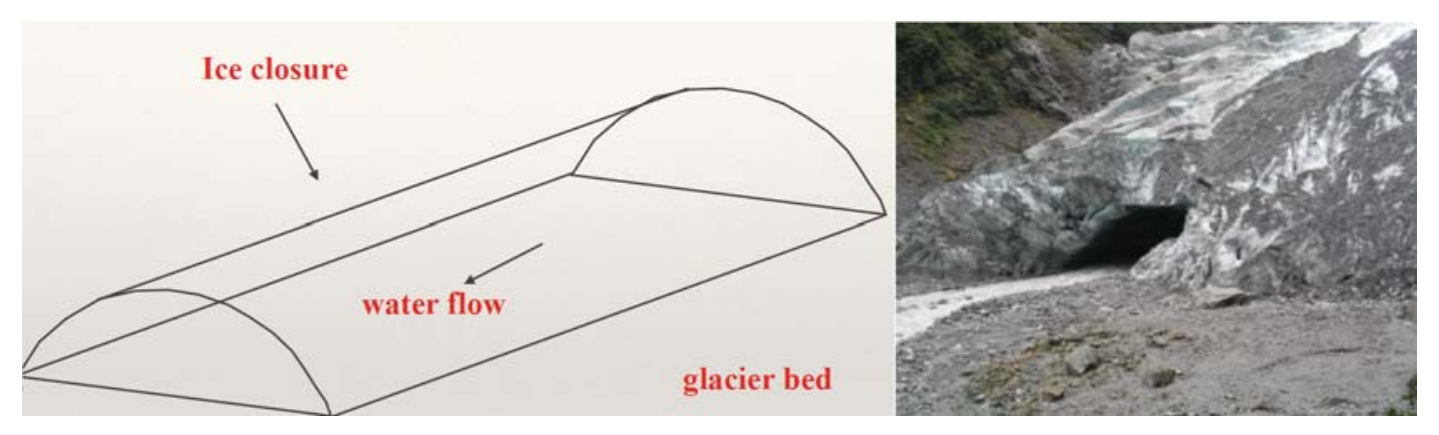

Figure 10: Röthlisberger channels.

The Nye-Röthlisberger model assumes that a channel of cross-sectional semi-circular area $S$ is governed by the closure equation

$$
\frac{\partial S}{\partial t}=\underbrace{\frac{m}{\rho_{i}}}_{\text {melt }}-\underbrace{K S N^{n}}_{\begin{array}{c}
\text { viscous closure } \\
\text { due to ice creep }
\end{array}}
$$

where $m$ is the melt rate, $K$ is a constant (proportional to $A$ ) derived from the ice creep problem, and $N$ is the effective pressure (see $(24)$ ).

Conservation of mass in the slowly varying channel can be written as

$$
\frac{\partial S}{\partial t}+\frac{\partial Q}{\partial x}=\underbrace{\frac{m}{\rho_{w}}}_{\begin{array}{c}
\text { volume source due } \\
\text { to side-wall melt }
\end{array}}+M,
$$

where $x$ is the downstream spatial coordinate, $Q$ is the volume flux, $\rho_{w}$ is the water density, and $M$ is a prescribed source accounting for tributary flow, surface melt-water supply, etc.

Ignoring inertial terms and using a Manning correlation to account for turbulent friction, the conservation of momentum can be written as

$$
\underbrace{\rho_{w} g \sin \alpha-\frac{\partial p_{w}}{\partial x}}_{\text {hydraulic gradient }}=f \rho_{w} g \frac{Q|Q|}{S^{8 / 3}},
$$


where $g$ is gravity, $f$ is a friction coefficient related to the Manning roughness factor and $\alpha$ is the mean bedrock slope.

Meanwhile, the energy equation is given by

$$
\underbrace{\rho_{w} c_{w}\left(S \frac{\partial \theta_{w}}{\partial t}+Q \frac{\partial \theta_{w}}{\partial x}\right)}_{\begin{array}{c}
\text { material rate of change } \\
\text { of water temperature }
\end{array}}=\underbrace{Q\left(\rho_{w} g \sin \alpha-\frac{\partial p_{w}}{\partial x}\right)}_{\text {frictional heat source }}-\underbrace{m\left(L+c_{w}\left(\theta_{w}-\theta_{i}\right)\right)}_{\begin{array}{c}
\text { enthalpy change } \\
\text { on melting }
\end{array}},
$$

where $\theta_{w}$ is the water temperature, $\theta_{i}$ is the ice temperature, $c_{w}$ is the specific heat capacity of water, and $L$ is the specific latent heat.

Lastly, a local heat transfer condition at the ice wall for a cylindrical tube is given by

$$
a_{D B}\left(\frac{\rho_{w}|Q|}{\eta_{w} S^{1 / 2}}\right)^{0.8} k\left(\theta_{w}-\theta_{i}\right)=m\left(L+c_{w}\left(\theta_{w}-\theta_{i}\right)\right),
$$

where $a_{D B}$ is a constant, $\eta_{w}$ is the viscosity of water and $k$ is the thermal conductivity of water.

The five equations, (26)-(30), constitute the Nye-Röthlisberger model which solves for the five unknowns $S, Q, m, p_{w}$ and $\theta_{w}$.

The effective pressure can be estimated under the assumption of steady state conditions. In this case, the equations reduce to

$$
\begin{aligned}
\frac{m}{\rho_{i}} & =K S N^{n} \\
\rho_{w} g \sin \alpha-\frac{\partial p_{w}}{\partial x} & =f \rho_{w} g \frac{Q^{2}}{S^{8 / 3}}, \\
m L & =Q\left(\rho_{w} g \sin \alpha-\frac{\partial p_{w}}{\partial x}\right) .
\end{aligned}
$$

These equations can be solved numerically, but in general it is found that $\frac{\partial p_{w}}{\partial x} \ll \rho_{w} g \sin \alpha$, and neglecting $\frac{\partial p_{w}}{\partial x}$ yields a boundary layer, so that away from the snout it follows that

$$
N=\left(\frac{m}{K S \rho_{i}}\right)^{\frac{1}{n}}, \quad S \approx\left(\frac{f Q^{2}}{\sin \alpha}\right)^{\frac{3}{8}}, \quad m \approx \frac{Q}{L} \rho_{w} g \sin \alpha .
$$

Lastly, substituting the latter two in the former yields that the effective pressure is

$$
N \approx \beta Q^{\frac{1}{4 n}}, \quad \beta=\left(\frac{\rho_{w} g \sin ^{11 / 8} \alpha}{\rho_{i} L K f^{3 / 8}}\right)^{\frac{1}{n}},
$$

where sometimes $f$ is taken as $f=\left(n^{\prime}\right)^{2} G$, where $n^{\prime}$ is the Manning roughness factor, and $G=\left(\frac{\ell^{2}}{S}\right)^{2 / 3}$ is a geometric factor with $\ell$ being the wetted perimeter.

\subsection{Linked cavities}

Next, one might consider linked cavities such as those shown in Figure 11. Let $s$ be the shadowing function which represents the fraction of the bed that is cavity-free. It is a decreasing function of

$$
\Lambda=\frac{u}{N^{n}},
$$


where $u$ is the sliding velocity. Then using the theory yields that

$$
\frac{n_{K}^{1 / 4 n} N}{s(\Lambda)} \approx \beta Q^{\frac{1}{4 n}},
$$

where $n_{K}$ is the number of cavities across the width of the glacier. Therefore, linked cavities within a glacier operate at a higher pressure than a channel-based system.

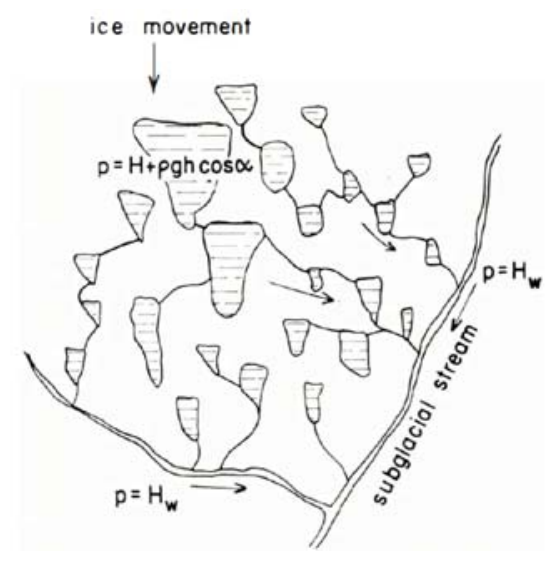

Figure 11: Linked cavities.

\subsection{Creyts-Schoof water film}

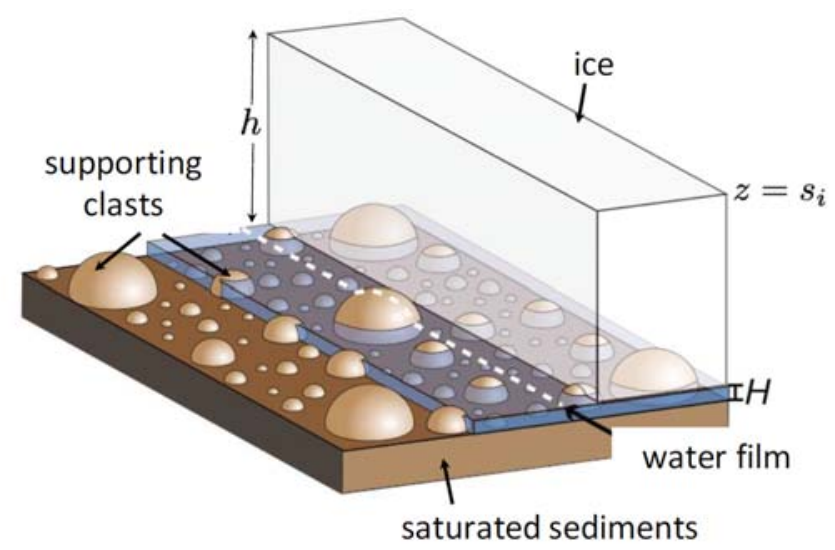

Figure 12: Creyts-Schoof water film [Creyts and Schoof, 2009].

There are other models for the films of water that develop between the bedrock and the ice. One of the most recent is the Creyts-Schoof water film [Creyts and Schoof, 2009]. Under this model, the "obstacles" actually become supporting clasts for the ice, as shown in Figure 12. In this case, there is a different scaling for the effective pressure,

$$
Q \sim h^{3}, \quad N \sim \frac{1}{h^{3 \mu}}, \quad \Rightarrow \quad N \sim \frac{1}{Q^{\mu}} .
$$




\section{References}

Creyts, T. T. and Schoof, C. G. (2009). Drainage through sub-glacial water sheets. Journal of Geophysical Research: Earth Surface, 114(F4).

Cuffey, K. M. and Paterson, W. S. B. (2010). The Physics of Glaciers. Academic Press.

Fowler, A. (2011). Mathematical Geoscience, volume 36. Springer Science \& Business Media.

Huybrechts, P. (2007). Ice sheet modelling. In: Riffenburgh, B., editor, Encyclopedia of the Antarctic, pages 514-517. Taylor \& Francis. 


\title{
GFD 2017 Lecture 3: Subglacial Control of Ice Flow
}

\author{
Andrew Fowler; notes by Eric Hester and Jessica Kenigson
}

June, 21, 2017

\section{Subglacial Floods: Grímsvötn}

Jökulhlaups or "glacier-bursts" are flooding events that are associated with glaciers; these events may be quasi-periodic or periodic. In 1996, a massive Jökulhlaups occurred at the Skeið̄arárjökull glacier at Iceland, which partially overlays a lake within a geothermally heated caldera. The ice overburden pressure at the caldera rim forms a "seal" which prevents the lake from emptying. Figure 1 shows a simplified geometric profile of the region.

Flooding events at Grímsvötn occur regularly, indeed quasi-periodically ( $\sim 5-10$ years) (Figure 2). A plausible mechanism for flooding from Grímsvötn would involve the water pressure in the lake growing to exceed the ice overburden pressure and causing flotation of the glacier, releasing a burst of water through the broken "seal." During observed flooding conditions, however, the water level within the lake has not been observed to reach the necessary height to achieve glacier flotation. A simplified physical model will be developed to shed light on the flow of water beneath the glacier during flood events as well as the periodicity of these events. Much of the following theory follows the exposition by [4].

\section{$1.1 \quad$ Model}

Water flows through a semi-circular conduit (i.e., a Röthlisberger Channel) of cross-sectional area $S$ at the glacier base. In the Röthlisberger model, melting of the channel walls occurs through frictional heating via contact with the flowing water, and creep closure occurs because the ice overburden pressure exceeds the water pressure in the channel. That is, in general, $N=p_{i}-p_{w}>0$. Equation 2 models the change in the cross-sectional area of the conduit under the competing effects of melting of the sidewalls $m$ and creep closure $S N^{n}$. Therefore

$$
\frac{\partial S}{\partial t}=\frac{m}{\rho_{i}}-K S\left(p_{i}-p_{w}\right)^{n}
$$

where subscripts $i$ and $w$ indicate ice and water, respectively, and $K$ is a constant which depends upon the geometry of the conduit. The second term arises from the nonlinear flow law for plastic deformation. Two separate sources are assumed for volumetric flux $Q$ to the channel: melt of the channel walls and other sources such as surface meltwater and outflow 


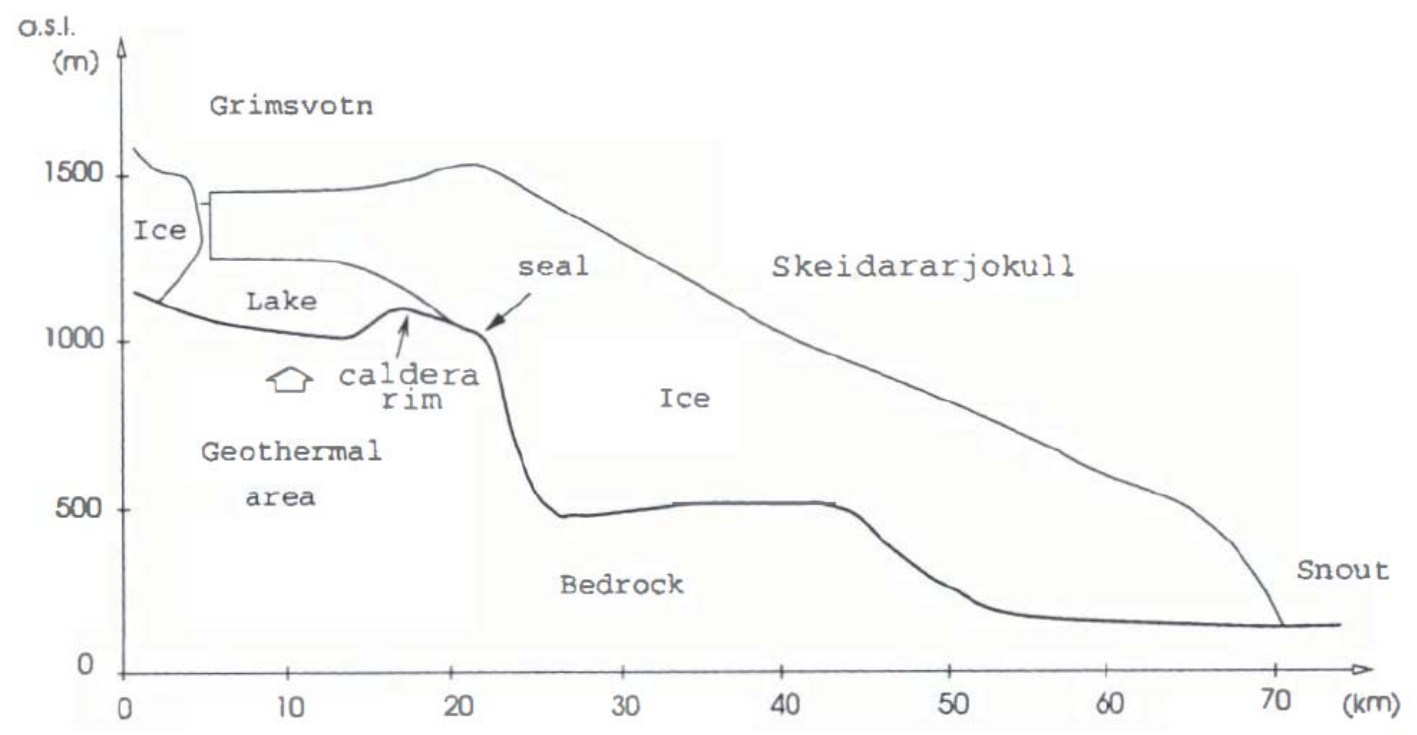

Figure 1: Simplified profile of the landscape near Grímsvötn. In the Röthlisberger-Nye model $x$ is increasing to the right and is measured from the seal (which is assumed to be fixed in space). Figure from [1].

from the lake, which are subsumed into a single term $M$. Therefore, continuity of mass within the conduit implies

$$
\frac{\partial S}{\partial t}+\frac{\partial Q}{\partial x}=\frac{m}{\rho_{w}}+M
$$

A momentum balance arises from rearranging the Gauckler-Manning formula for a mean (turbulent) flow $\bar{u}=Q / S$ :

$$
\bar{u}=\frac{R^{2 / 3}}{n^{\prime}}\left[\frac{1}{\rho_{w} g}\left(\rho_{w} g_{s}-\frac{\partial p}{\partial s}\right)\right]
$$

where $R$ is the hydraulic radius, $g$ is the gravitational constant, $g_{s}$ is the component of the gravitational constant in the $s$-direction (here $x$-direction), $n^{\prime}$ is a Manning roughness factor, and $\rho_{w}$ is the density of water [7]. It follows that

$$
\rho_{w} g \sin \alpha-\frac{\partial p}{\partial x}=f \rho_{w} g \frac{Q|Q|}{S^{8 / 3}}
$$

where the first term represents the gravitational driving force, the second term represents the water pressure gradient, and the third term represents bed friction. Here $f$ is a friction factor and $\alpha$ is the bed inclination. Finally, the energy equation is 

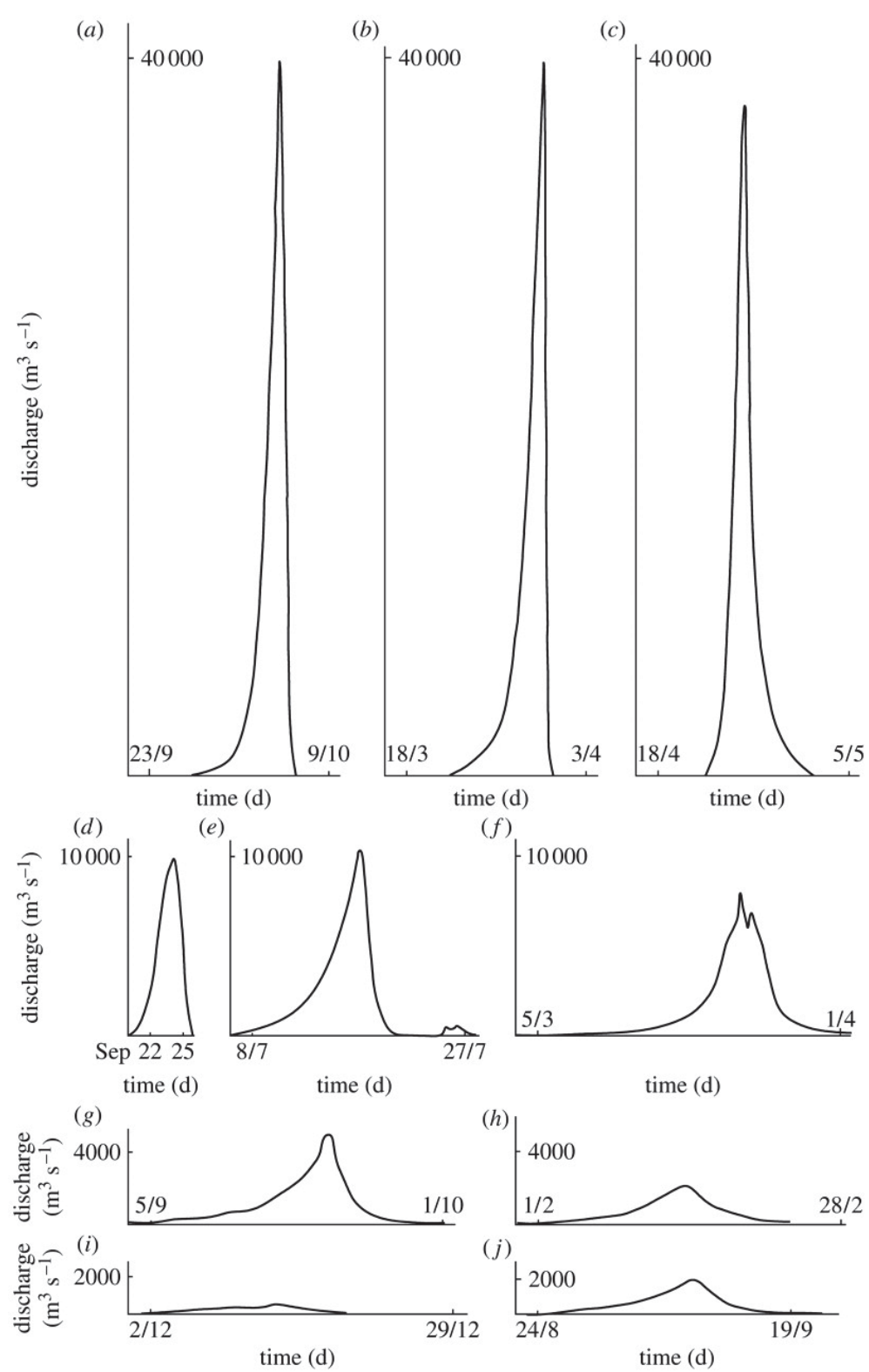

Figure 2: Hydrograph showing extreme flooding events at Grímsvötn in 1922, 1934, 1938, 1945, 1954, 1972, 1976, 1982, 1983, and 1986. Figure from [3]. 


$$
\rho_{w} c_{w}\left[S \frac{\partial \theta_{w}}{\partial t}+Q \frac{\partial \theta_{w}}{\partial x}\right]=Q\left(\rho_{w} g \sin \alpha-\frac{\partial p}{\partial x}\right)-m\left[L+c_{w}\left(\theta_{w}-\theta_{i}\right)\right]
$$

where the rate of change of internal energy is given by the sum of two terms: the energy needed to change the temperature of water already in the conduit (term related to the total derivative of $\theta_{w}$ on the LHS), and the energy needed to melt ice from the conduit walls (second term on the RHS), which consists of a sum of the energy required to raise the ice temperature to the water temperature and the latent heat needed for the phase change. The first term on the RHS is the frictional heating due to viscous dissipation (see Equation 4). Finally, a heat transfer equation is given by

$$
a_{D B}\left(\frac{\rho_{w}|Q|}{\eta_{w} S^{1 / 2}}\right)^{4 / 5} k\left(\theta_{w}-\theta_{i}\right)=m\left[L+c_{w}\left(\theta_{w}-\theta_{i}\right)\right]
$$

where the term on the LHS is an empirical expression for heat transfer at the ice conduit walls given a turbulent flow; this is obtained from an empirical relation among the Nusselt number, the Reynolds number, and the Prandtl number $([7])$. Here $a_{D B} \sim 2$, $\eta_{w}$ is the viscosity of water, and $k$ is the thermal conductivity.

At the lake inlet, the "refilling" condition is given by

$$
-\frac{A_{L}}{\rho_{w} g} \frac{\partial N}{\partial t}=m_{L}-Q \text { at } x=0
$$

where $A_{L}$ is the (fixed) surface area of the lake and $m_{L}$ is the geothermal melt rate in the caldera. That is, changes in the effective pressure at the seal are driven by the meltwater flux in the lake due to geothermal heating and the volumetric flux of water into the subglacial conduit. Here

$$
\Phi=\rho_{w} g \sin \alpha-\frac{\partial p_{i}}{\partial x}
$$

is the hydraulic gradient. These equations are nondimensionalized with

$Q=Q_{0} Q^{*}, \quad S=S_{0} S^{*}, \quad p_{i}-p=N_{0} N^{*}, \quad m=m_{0} m^{*}, \quad x=l x^{*}, t=t_{0} t^{*}, \quad \theta_{w}=\theta_{i}+\theta_{0} \theta^{*}$, which gives 


$$
\begin{aligned}
\frac{\partial S}{\partial t} & =m-S N^{n} \\
\epsilon \frac{\partial S}{\partial t}+\frac{\partial Q}{\partial x} & =\epsilon r m+\Omega \\
\Phi+\delta \frac{\partial N}{\partial x} & =\frac{Q|Q|}{S^{8 / 3}} \\
\epsilon S \frac{\partial \theta}{\partial t}+Q \frac{\partial \theta}{\partial x} & =Q\left[\Phi+\delta \frac{\partial N}{\partial x}\right]-m(1+\epsilon r \theta) \\
\theta\left(\frac{|Q|}{S^{1 / 2}}\right)^{0.8} & =\gamma m(1+\epsilon r \theta)
\end{aligned}
$$

The nondimensional parameters are given by

$$
\begin{aligned}
\epsilon & =\frac{\Phi_{0} l}{\rho_{i} L} \\
\delta & =\frac{1}{\Phi_{0} l}\left[\frac{Q_{0}^{1 / 4} \Phi_{0}^{11 / 8}}{\rho_{i} K L\left(f \rho_{w} g\right)^{3 / 8}}\right]^{\frac{1}{n}} \\
\gamma & =\frac{\rho_{w} c_{w}}{k a_{D B} l}\left(\frac{\eta_{w}}{\rho_{w}}\right)^{4 / 5} Q_{0}^{1 / 2}\left(\frac{f \rho_{w} g}{\Phi_{0}}\right)^{3 / 20} \\
r & =\frac{\rho_{i}}{\rho_{w}} \\
\Omega & =\frac{M l}{Q_{0}}
\end{aligned}
$$

with the boundary condition

$$
\frac{\partial N}{\partial t}=Q-\nu \text { at } x=0
$$

Reference values for these parameters are

$$
\gamma \sim 2.5, \quad \epsilon \sim 0.05, \quad r \sim 0.9, \quad \delta \sim 0.22, \quad \Omega \sim 0.6 \cdot 10^{-3}
$$

(A table of physical parameter values used in the scaling is available in [4]). Ordinarily, flooding is initiated in the presence of the "seal" at the margin of the caldera. Now re-scale $x=\delta X$ to investigate near the boundary of the caldera, let $\omega=\delta \Omega$ and allow $\Phi<0$ near the lake. Assume that $\epsilon$ and $\gamma$ are small, which implies via Equation 9e that $\theta=0$. The equation set then reduces to 


$$
\begin{aligned}
\frac{\partial S}{\partial t} & =\frac{|Q|^{3}}{S^{8 / 3}}-S N^{n} \\
\frac{\partial Q}{\partial X} & =\omega \\
\Phi+\frac{\partial N}{\partial X} & =\frac{Q|Q|}{S^{8 / 3}}
\end{aligned}
$$

with the boundary conditions

$$
\begin{array}{r}
\frac{\partial N}{\partial t}(0, t)=Q(0, t)-\nu \text { at } x=0 \\
\frac{\partial N}{\partial X} \rightarrow 0 \text { as } X \rightarrow \infty
\end{array}
$$

where

$$
\nu=\frac{m_{L}}{Q_{0}}
$$

Assume the following form for $\Phi$,

$$
\Phi=1-a e^{-b X}
$$

for some parameters $a$ and $b$ (which are related to the strength of the seal). Figure 3 shows the numerical solution of Equations 13a-13c and 14, which agrees quite reasonably with observations.

Note that in a steady state $(\partial S / \partial t=0)$ with $\Phi \sim 1$, Equations 13a-13c reduce to the Röthlisberger relation for $N$ and $Q$.

\subsection{Distributed drainage system}

Massive flooding events have likely occurred beneath ice sheets such as Antarctica, with Lake Vostok potentially implicated. Note that flooding events beneath ice sheets are physically very different from events beneath glaciers, as drainage beneath ice sheets is not typically through Röthlisberger channels.

In Röthlisberger channels, $N$ and $Q$ are related via

$$
N \sim \beta Q^{1 / 4 n}
$$

for a material parameter $\beta$. Under these circumstances, it is typical for a single conduit to develop, owing to the relationship between $Q$ and $N$. If two Röthlisberger channels of differing radii are close together (such that water can escape from one channel to another through the bed), the channel with relatively small $Q$ ( small $N$, large $p_{w}$ ) will experience 


\section{Periodic floods}

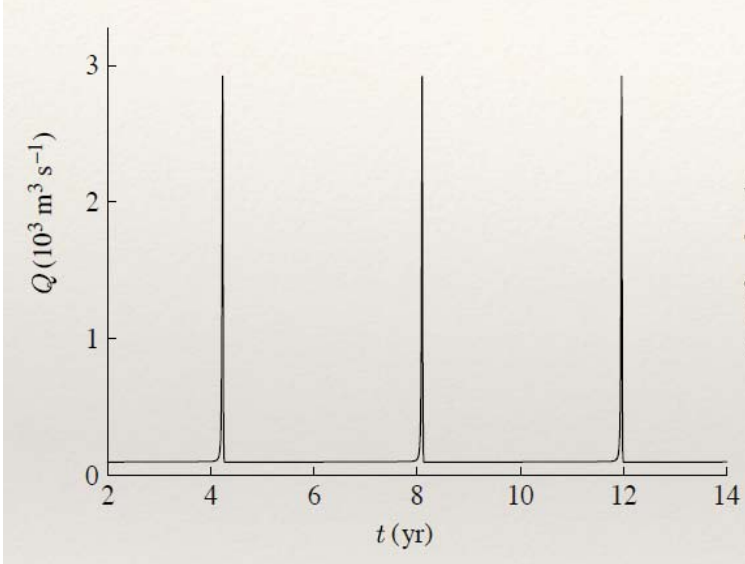

Hydrograph fit

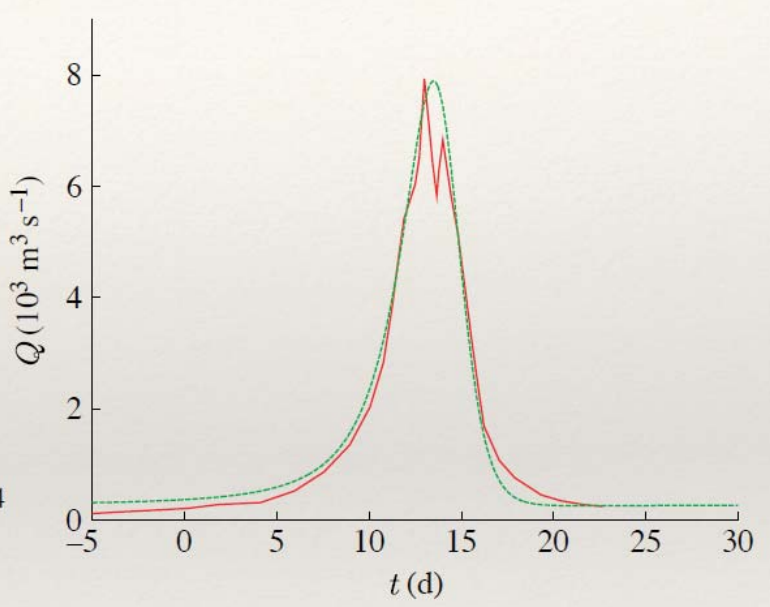

Figure 3: (a) Model showing periodic flooding at Grímsvötn. (b) Hydrograph of the observed (red) and modeled (green) discharge based upon the solution of Equations 13a-13c and 14 under the assumption that $a=2.8, b=4.316$. Figure adapted from [3].

leakage into the nearby channel of large $Q$, and the small channel will gradually close. If the subglacial sediment is relatively stiff, then it is possible for Röthlisberger channels to develop beneath a glacier. However, if sediment is significantly erodible, then it is likelier for a distributed drainage network to develop (rather than a single channel).

In prior derivations, Röthlisberger channels were assumed to be semi-circular with $h \sim w$, where $w$ is the mean width and $h$ is the mean depth. Now relax the assumption (as in the Röthlisberger theory) that $h \sim w$. Instead $R=S / l$, where $R$, the hydraulic radius, is a fraction of the cross section $S=w h$ and $l$, the wetted perimeter, and

$$
\begin{aligned}
w^{2} & =\frac{2^{4 / 3} \rho_{w} n^{2} Q^{2}}{\rho_{i} S_{i} h^{10 / 3}} \\
K w^{2} N^{n} & =\frac{g S_{i} Q}{2 L}
\end{aligned}
$$

where $n^{\prime}$ a Manning roughness coefficient, $K$ is related to the closure rate and is dependent upon the geometry, and $S_{i}$ is the ice surface slope. This is a generalization of the Röthlisberger theory and reduces to it in the case $w \approx h$. It is derived from a Manning law and the assumption that the closure rate due to melting balances channel closure due to the ice overburden pressure. Assume that the channel depth is close to the critical depth at which sediment transport occurs. It then follows that 


$$
N=\frac{\gamma}{Q^{1 / n}}
$$

with

$$
\gamma=\left[\frac{\rho_{i} g S_{i}^{2} h_{c}^{10 / 3}}{2^{7 / 3} K L \rho_{w} n^{\prime 2}}\right]^{1 / n}
$$

where $h_{c}$ is a critical depth fixed by the critical stress for sediment transport and $L$ is a function of the sliding velocity and effective pressure $N$. This suggests an inverse relationship between $N$ and $Q$, unlike for Röthlisberger channels. Therefore, the closure mechanism

discussed previously for Röthlisberger channels is avoided, which permits the existence of a distributed drainage network [7].

\section{Subglacial Bedforms: Drumlins, Ribbed Moraine, and Mega- scale Glacial Lineations (MSGL)}

The action of ice sheets during the last ice age has had profound effects on topography throughout the world. Ribbed moraines, drumlins, and Mega-Scale Glacial Lineations (MSGLs) (seen in Figures 4, 5, and 6, respectively) are prime examples of such effects. They are always seen in large clusters in areas of past glaciation (Sweden, Ireland, Canada), with the prime differences being the main direction of variation. Ribbed moraines, much like dunes, form transverse to the flow, while MSGLs are instead directed longitudinally. Drumlins, however, are fully three dimensional, being between these two extremes.

The glacial dynamics responsible for these features have not been settled. However, [6] has developed a model which couples ice sheet and deformable sediment dynamics, with a thin intermediate water film. This model exhibits all three of these formations, and we outline its development below.

\section{$2.1 \quad$ Ice}

We model the ice as a Newtonian fluid of viscosity $\eta_{i}$. The finite depth ice lies above a thin water layer, which in turn rests on a deformable bed of till (Figure 7). The inertial terms are negligible in the Navier-Stokes equations, giving Stokes flow

$$
\begin{aligned}
\nabla \cdot \boldsymbol{u} & =0 \\
0 & =-\nabla P-\rho_{i} g \nabla z_{i}+\eta_{i} \nabla^{2} \boldsymbol{u}
\end{aligned}
$$

where $\boldsymbol{u}$ is the ice velocity, $\rho_{i}$ is the ice density, $\eta_{i}$ is the dynamic viscosity, and $P$ is the deviation from the cryostatic pressure. 


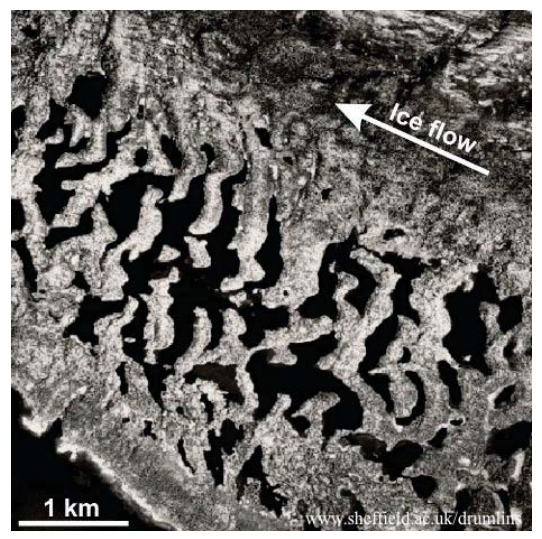

Figure 4: Ribbed moraines in lake Rogen, Sweden. Ridges form transverse to ice flow [6]. Figure adapted from https://www.sheffield.ac.uk /drumlins/rogen

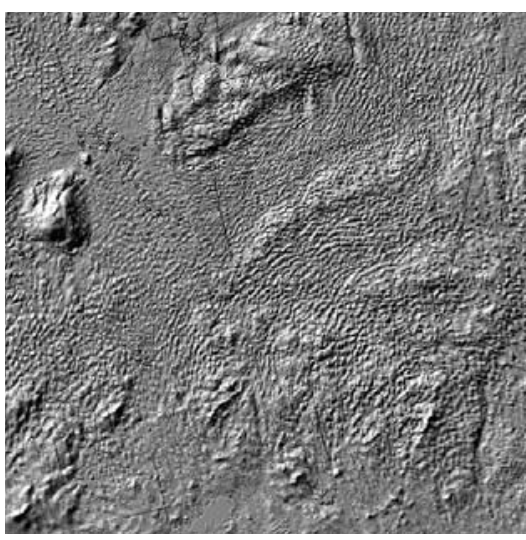

Figure 5: Digital elevation map of part of north central Ireland. The small bumps are drumlins, which are roughly $10 \mathrm{~m}$ high, and several hundred metres in length [6]. Figure adapted from [6].

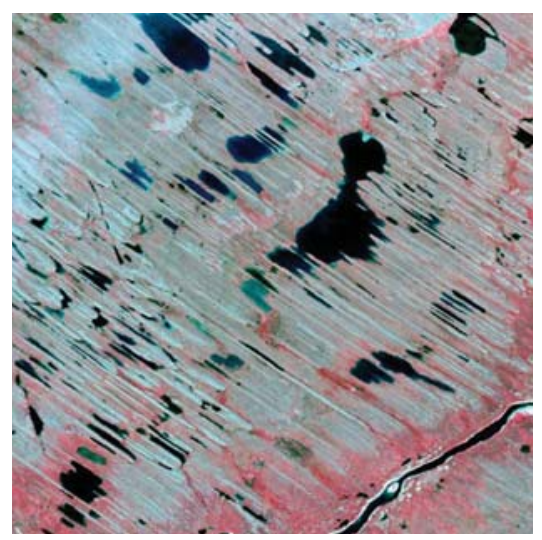

Figure 6: MSGLs in Canada, which are instead parallel to sheet flow [5]. Figure adapted from https://www.sheffield.ac.uk/ drumlins/msgl.

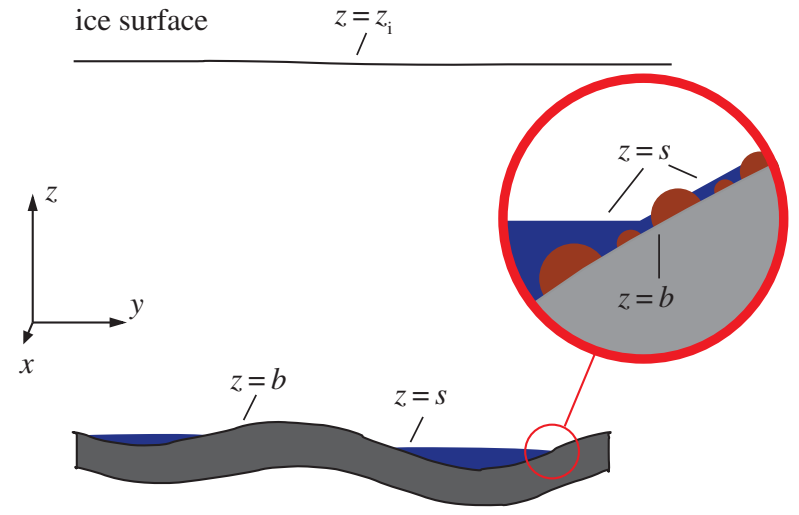

Figure 7: A view upstream of the model. The ice sheet, with upper surface $z=z_{i}$, rests on a water layer extending from $z=s$ to the sediment bed at $z=b[6]$. Figure from [6].

At $z=z_{i}$ we specify matching normal stress $\tau_{n n}$, with zero horizontal shear stress $\boldsymbol{\tau}=$ $\left(\tau_{13}, \tau_{23}\right)$

$$
\begin{aligned}
P-\tau_{n n} & =0, \\
\tau & =0,
\end{aligned}
$$

and additionally require a kinematic equation for $w$, which prescribes its value at the top boundary in terms of the ice sheet elevation $z_{i}$ and the accumulation rate $a$

$$
w=z_{i, t}+u z_{i, x}+v z_{i, y}-a .
$$

At the bottom of the ice $(z=s)$, we now require some relation for the shear stress. We assume it depends on the basal ice velocity $u_{b}$ and the effective pressure at the interface 
$N=p_{i}-p_{w}$,

$$
\boldsymbol{\tau}=f\left(u_{b}, N\right) \frac{\boldsymbol{u}_{b}}{u_{b}}
$$

We specify a generalised Weertman sliding law for $f$

$$
f\left(u_{b}, N\right)=R N^{b} u_{b}^{c}
$$

where $R$ is the roughness coefficient, and $b$ and $c$ are the respective powers of the effective

pressure $N$ and basal velocity $u_{b}$. Finally, we have our second kinematic equation for $w$ at the bottom boundary

$$
w=s_{t}+u s_{x}+v s_{y} .
$$

\section{$2.2 \quad$ Water}

As mentioned, the water exists between the two interfaces, giving the layer thickness $h$ as

$$
h=s-b .
$$

The hydraulic potential in the water is then

$$
=\rho_{i} g\left(z_{i}-d_{i}\right)+\Delta \rho_{w i} g s-N+P-\tau_{n n},
$$

where $\Delta \rho_{w i}=\rho_{w}-\rho_{i}$ is the density difference between water and ice, and $d_{i}$ is the ice depth, which is assumed to be constant over the smaller scale of the deformations we will observe.

The water between the ice and sediment is then modelled as a thin film. Its evolution is governed by Poiseuille-type flow

$$
h_{t}=\nabla \cdot\left[\frac{h^{3}}{12 \eta_{w}} \nabla \psi\right]+\Gamma .
$$

Here, $\Gamma$ represents sources due to ice melt (from geothermal heating, frictional heating, and heat flux into the ice).

\section{$2.3 \quad$ Sediment}

The sediment is also a deformable medium. However, unlike the water or ice, it will not deform until the basal stress applied to it by the ice $\tau=f(\bar{u}, N)$ exceeds the yield stress $\mu N$, where $\mu$ is the coefficient of friction. Hence, it will have a finite deformation depth $h_{A}$, below which the sediment is unperturbed, given by

$$
h_{A}=\frac{[\tau / \mu-N]_{+}}{\Delta \rho_{s w}(1-\phi) g},
$$

(where $\left.[y]_{+}=\max \{y, 0\}\right)$. 
The governing conservation equation of the sediment is called the Exner equation, and models the change in the bed elevation $b$ in terms of the sediment deformation depth $h_{A}$, an effective sediment viscosity $\eta_{s}$, and

$$
b_{t}+\nabla \cdot\left[\frac{1}{2} u_{0} \bar{u} h_{A} i-\frac{h_{A}^{3}}{12 \eta_{s}} \nabla N+Q\left(\tau_{e}\right) \frac{\boldsymbol{\tau}_{e}}{\tau_{e}}\right]=0 .
$$

The first advective flux term represents shearing from the average ice flow $u_{0} \bar{u}$, where $\bar{u}$ is a spatial average of $u$ at the base, defined so that $\overline{f(\bar{u}, N)}=\overline{f(u, N)}$. The second diffusive term then represents squeezing of the till in a thin layer $h_{A}$ due to the effective pressure $N$. The final flux term represents sediment transport due to the effective stress $\boldsymbol{\tau}_{e}$ transmitted by the water to the bed, given by

$$
\tau_{e}=-\frac{1}{2} h \nabla \psi-\Delta \rho_{s w} g D_{s} \nabla b .
$$

This is the actual viscous stress in the water plus a term related to the tendency of sediment to roll downhill, which depends on the difference in sediment and water density $\Delta \rho_{s w}=\rho_{s}-\rho_{w}$, and the average grain size of the sediment, $D_{s}$.

\subsection{Reduced model}

We can then non-dimensionalise this system, and significantly simplify the model. For more details on each step, please refer to [6].

Our upper ice equations are completely solvable in terms of the stress at the boundaries. By linearising the boundaries as constant, we can solve the upper system using the Fourier transform, defined to be

$$
\hat{f}\left(k_{x}, k_{y}\right)=\int_{-\infty}^{\infty} \int_{-\infty}^{\infty} f(x, y) e^{i k_{x} x} e^{i k_{y} y} d x d y .
$$

This leaves only the kinematic equation for $w$, which is now given by a Fourier convolution

$$
w=\alpha s_{t}+\bar{u} s_{x}=J * \Phi,
$$

where $\Phi$ represents a perturbation to the pressure, and $J$ is given by the inverse transform of

$$
\hat{J}=\frac{\sinh ^{2} j}{2 k(j+\cosh j \sinh j)}, \quad j=\frac{k}{\sigma}, \quad k=\sqrt{k_{x}^{2}+k_{y}^{2}} .
$$

Here $\sigma=l_{D} / d_{i}$ is the ratio of the bedform length scale $l_{D}$ to the ice depth scale $d_{i}$, and $\alpha=d_{T} / d_{D}$ is the ratio of the deformable till depth scale $d_{T}$ to the bedform depth scale $d_{D}$. Finally, $\bar{u}(t)$ represents the $x$-averaged basal velocity, defined to give the average shear stress at the bed, which gives (when non-dimensionalised)

$$
\overline{f(\bar{u}, N)}=1, \quad \text { implying } \quad f(\bar{u}, N)=\frac{N^{b}}{\overline{N^{b}}} .
$$


Our thin film evolution equation for the water depth simplifies considerably (throwing out small terms), to become

$$
\nabla \cdot\left[h^{3} \nabla \Psi\right]=\sigma h^{3},
$$

where

$$
\Psi=s-N+\Phi,
$$

can be thought of as akin to pressure in the water.

Our water thickness equation is given by

$$
b=s-\delta h,
$$

where $\delta=h_{0} / d_{D}$ is the ratio of the average film thickness to bedform depth scale.

Finally, our Exner equation simplifies to

$$
b_{t}+\bar{u} A_{x}=\nabla \cdot\left[\beta A^{3} \nabla N-\gamma B\left(\tau_{e}\right) \boldsymbol{\tau}_{e}\right] .
$$

Here, $A$ is a non-dimensional deformable till depth, given as

$$
A=\frac{1}{2}\left[\frac{f(\bar{u}, N)}{\mu}-N\right]_{+} .
$$



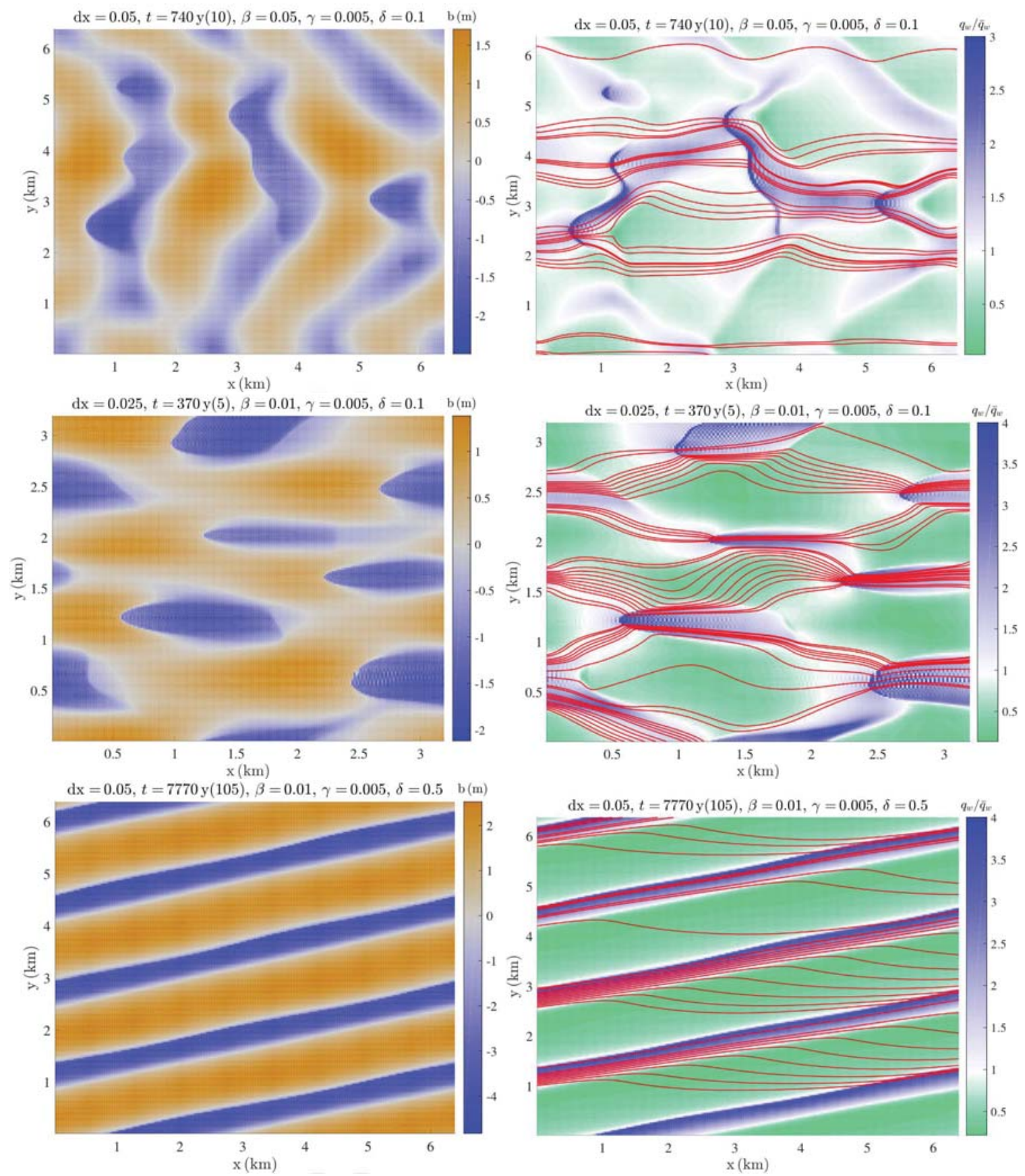

Figure 8: Simulations of the system on a periodic domain [2]. The left column shows topography and the right shows water flux, with red streamlines. Depending on the parameter choice, we can generate ribbed moraines, drumlins, or MSGLs. The incline of the MSGL stems from the periodicity of the domain, which does not enforce a direction of outflow. The chief numerical limit on the model is the smallness of $\delta$. Figure adapted from [2]. 
The parameters $\beta$ and $\gamma$ are given by

$$
\beta=\frac{2 d_{T}}{3 l_{D}}, \quad \gamma=\frac{q_{b}}{d_{T} u_{0}}
$$

where $d_{T}$ is the scale of the deformable till depth, $q_{b}$ is the scale of the sediment flux, and $u_{0}$ is the scale of the basal velocity. While small, both of these terms are necessary. The $\beta$ term is required to stabilise the growth of the bedform, while the sediment transport $\gamma$ term is required to generate the rilling instability responsible for MSGL formations. The advective $\bar{u} A$ term is responsible for the ribbing instability that generates the moraine formations. Finally, the stress $\tau_{e}$ represents the effective stress of the water on the sediment, given by

$$
\tau_{e}=\sigma h i-h \nabla \Psi
$$

Combined, we now have 8 unknowns, $\Psi, b, s, h, A, N, \boldsymbol{\tau}_{e}, \Phi$, but only 7 equations (numbered above). We therefore require an additional equation to close the system. We achieve this by specifying a relation between the height $h$ and effective pressure $N$ of the till.

The water film can be thought of as a porous layer. This porosity will decrease with the effective pressure, and increase with the film thickness. We then reason that the film thickness will be a decreasing function of effective pressure - if we squeeze harder, the layer thins.

To infer the scale of this process, consider some critical clast size $h_{c}$. If the film is thicker than this, the ice no longer rests on rocks jutting from the till, and the effective pressure drops to zero. If $N$ changes by $O\left(N_{0}\right)$ when $h$ changes by $O\left(h_{c}\right)$, then

$$
-\frac{\partial N}{\partial h} \sim \frac{N_{0} h_{0}}{\tau_{b} h_{c}} \equiv \frac{1}{\Lambda}
$$

where $\tau_{b}$ represents the basal stress scale. The simplest such relation satisfying these requirements is given by

$$
\Lambda h N=1 .
$$

Unsurprisingly, this is the least secure aspect of the model. However, when simulated, the model is able to recreate all three types of bedforms (Figure 8) by varying only three parameters.

\section{References}

[1] H. BuöRnsson, Explanations of jökulhlaups from Grímsvötn, Vatnajökull, Iceland., 1974.

[2] J. S. Fannon, A. C. Fowler, And I. R. Moyles, Numerical simulations of drumlin formation, Proceedings of the Royal Society of London A: Mathematical, Physical and Engineering Sciences, 473 (2017).

[3] A. Fowlen, Dynamics of subglacial floods, in Proceedings of the Royal Society of London A: Mathematical, Physical and Engineering Sciences, vol. 465, The Royal Society, 2009, pp. 1809-1828. 
[4] A. Fowler, Mathematical geoscience, vol. 36, Springer Science \& Business Media, 2011.

[5] A. C. Fowler, The formation of subglacial streams and mega-scale glacial lineations, Proceedings of the Royal Society of London A: Mathematical, Physical and Engineering Sciences, 466 (2010), pp. 3181-3201.

[6] A. C. Fowler And M. Chapwanya, An instability theory for the formation of ribbed moraine, drumlins and mega-scale glacial lineations, Proceedings of the Royal Society of London A: Mathematical, Physical and Engineering Sciences, 470 (2014).

[7] J. NyE, Water flow in glaciers: jökulhlaups, tunnels and veins, Journal of Glaciology, 17 (1976), pp. 181-207. 


\title{
GFD 2017 Lecture 4: Processes at the Ice-Ocean Interface
}

\author{
Andrew Fowler; notes by Margaret Lindeman, Agostino Meroni, and Earle Wilson
}

June 22,2017

\section{Ice Streams and Ice Shelves}

\subsection{Ice Streams}

Ice streams are channels of fast flow within an ice sheet. These frozen rivers of ice provide the main drainage pathways for the large masses of ice that accumulate on Greenland and Antarctica. Since ice streams typically flow orders of magnitude faster than the surrounding ice sheet, they are usually delineated by elongated crevasses. Notable examples of ice streams are the Jakobshavn glacier in West Greenland, which flows at a mean rate of 15 kilometers per year, and the Siple Coast ice streams in Antarctica, which have undergone rapid retreat over the past century.

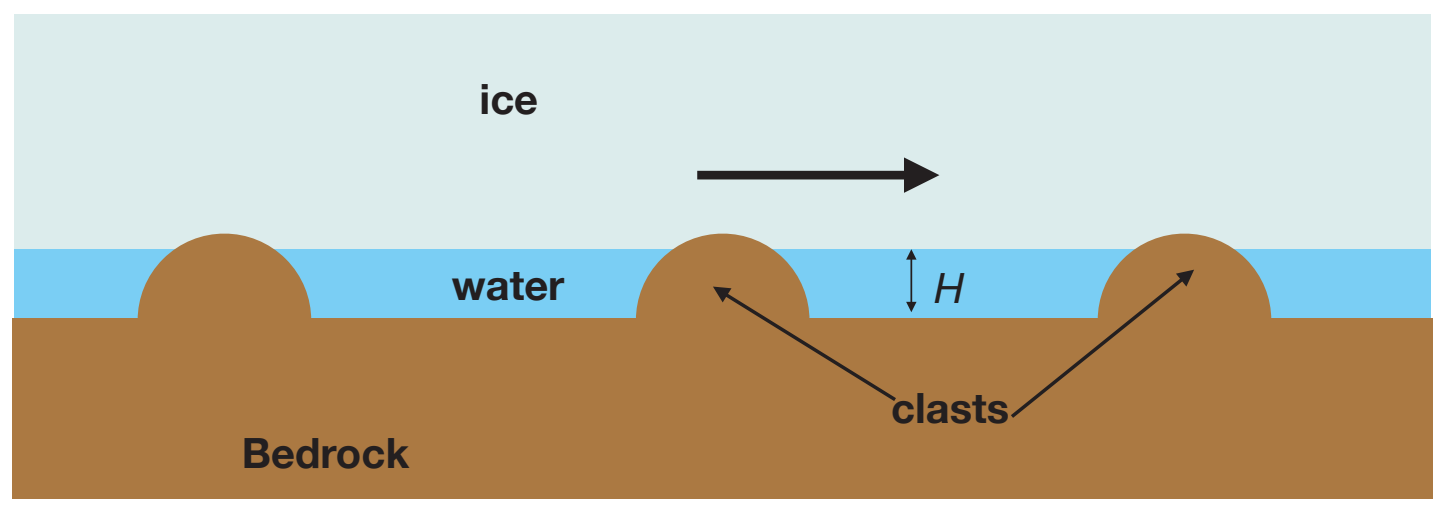

Figure 1: A schematic showing a cross-sectional view of an ice-sheet flowing over bedrock. This schematic is an adaption of Figure 1 in Kyrke-Smith et al. (2013).

Figure 1 provides a simplified cross-sectional view of an ice sheet sliding over bedrock. Here, the ice sheet slides on top of rigid clasts that provide an opposing frictional drag. Between the ice sheet and bedrock is a thin layer of meltwater that has thickness $H$. The 
shear stress at the base of this ice-stream is modeled using the sliding law

$$
\tau_{b}=c\left|u_{b}\right|^{p} N^{q} \frac{u_{b}}{\left|u_{b}\right|}
$$

where $\tau_{b}$ is the basal stress, $c$ is a measure of effective roughness, $u_{b}$ is the basal velocity, $N$ is the effective pressure, and $p$ and $q$ are positive. The effective pressure is defined as $N=p_{i}-p_{w}$, where $p_{i}$ is the ice pressure and $p_{w}$ is the basal water pressure. As the water level increases, the points of contact between the ice and the bedrock decreases. Thus, $N$ should decrease with $H$. Once the ice is lifted above the highest clasts, it will experience much less resistance from the bedrock and flow much more freely. Further increases to the meltwater thickness will have a relatively small effect on the effective pressure. We can therefore identify two distinct states of ice sheet flow: one where the ice is in full contact with bed and $N$ is strongly dependent on water film thickness, and another where the ice is essentially floating on top of meltwater and experiences very little frictional drag.

Mass conservation for the meltwater layer takes the form

$$
\frac{\partial H}{\partial t}+\nabla \cdot \mathbf{q}=\Gamma
$$

where $\mathbf{q}$ is the water flux and $\Gamma$ is the water source due to basal melting. Assuming a local Poiseuille flow, the meltwater flux $\mathbf{q}$ is given by

$$
\mathbf{q}=-\frac{h^{3}}{12 \eta_{w}} \nabla \psi=\frac{h^{3}}{12 \eta_{w}}\left(-\rho_{i} g \nabla s_{i}-\Delta \rho_{w i} g \nabla s_{w}+\nabla N\right),
$$

where $\psi$ is the hydraulic potential of the water film, $\eta_{w}$ is the viscosity of water, $\rho_{i}$ is the density of ice and $\Delta \rho_{w i}=\rho_{w}-\rho_{i}$ is the difference between water density and ice density. The melt rate is given by

$$
\Gamma=\frac{G+\mathbf{u}_{\mathbf{b}} \cdot \tau_{\mathbf{b}}-q_{T}+|\mathbf{q} \cdot \nabla \psi|}{\rho_{w} L}
$$

where $G$ is the geothermal heat flux, $\mathbf{u}_{\mathbf{b}} \cdot \tau_{\mathbf{b}}$ is the work done by the ice on its bed, $q_{T}$ is the sensible heat loss to the overlying ice and $|\mathbf{q} \cdot \nabla \psi|$ is the heating due to viscous dissipation. Equations (1)-(4) reveal the potential for a positive feedback between ice-velocity and basal heating whereby a positive perturbation in the flow field leads to an increase in basal heating and a decrease in effective pressure. With lower effective pressure the ice is able to slide faster, which then leads to more basal heating and meltwater production. This cycle of amplification is known as hydraulic runaway.

For a more thorough discussion on the dynamics of ice streams and hydraulic runaway, the reader is referred to Kyrke-Smith et al. (2013). For more details on the drainage of subglacial water sheets, the reader is referred to Creyts and Schoof (2009). 


\section{$1.2 \quad$ Ice shelves}

When an ice sheet reaches the ocean, it may begin to float as it continues to flow outward, forming an ice shelf. The scales and dynamics of an ice shelf are distinct from those of the ice sheet.

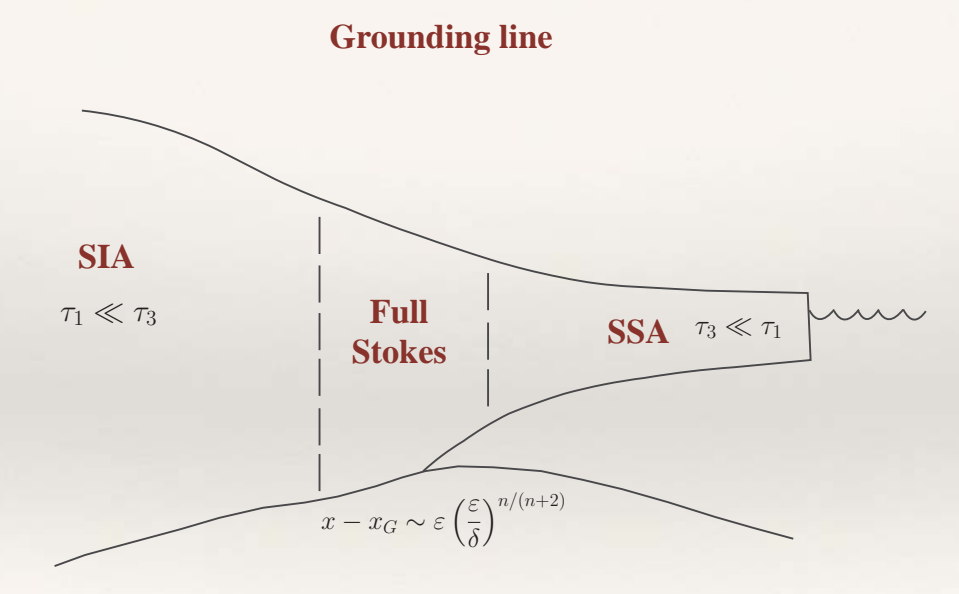

Figure 2: Schematic of grounding line dynamics. The shallow ice approximation (SIA) is applicable to the ice sheet upstream of the grounding line, while the shallow shelf approximation (SSA) is applied to the ice shelf. The grounding line region width is given by (15).

To model the ice sheet, we previously referred to the shallow ice approximation (SIA), with aspect ratio

$$
\epsilon=\frac{d}{l} \ll 1 .
$$

The dominant balance in this approximation was between shear stress $\tau_{3}$ and horizontal pressure gradient, taking longitudinal stress $\tau_{1}$ to be negligible (e.g. Meur et al. (2004); Kirchner et al. (2016); figure 2).

Now we will use the shallow shelf approximation (SSA). As the name suggests, the aspect ratio is the same, but now the dominant balance is between the horizontal pressure gradient and longitudinal stress $\tau_{1}$ (figure 2). This requires a rescaling of the equations in order to apply them to the ice shelf. To this end, we introduce a parameter,

$$
\delta=\frac{\rho_{w}-\rho_{i}}{\rho_{i}},
$$

such that we can define a scaling for the ice shelf depth,

$$
\nu=\frac{\epsilon}{\delta}\left(\frac{\delta}{\lambda}\right)^{1 /(n+1)},
$$


where the ice shelf length scale is given by $\lambda$.

Making the assumption that velocity is purely horizontal and varies only in the along-flow direction $x$, we get the following equations for ice velocity in the SSA:

$$
\begin{aligned}
u & =\left\{\frac{1}{2}(n+1)\left(\frac{1}{4} q_{I}\right)\right\}^{1 /(n+1)}\left(x-x_{G}\right)^{1 /(n+1)}, \\
u_{x} & =\frac{1}{2}\left(\frac{1}{4} H\right)^{n} .
\end{aligned}
$$

Defining the ice height at the grounding line $x_{G}$ as $H$, we also have an expression for ice flux to the ice shelf at the grounding line,

$$
q_{I}=H u \text {. }
$$

\section{Grounding Line Dynamics, Calving and Tidewater Glaciers}

\subsection{The grounding line}

To determine $q_{I}$ and the location of the grounding line $x_{G}$, we need to solve a boundary layer problem where we match the SIA and the SSA through a transitional region where neither approximation is applicable.

First, recall these governing equations for inland ice sheet flow:

$$
\begin{aligned}
H & =s-b, \\
H_{t}+q_{x} & =a, \\
q & =\frac{H^{n+2}\left|s_{x}\right|^{n+1}\left(-s_{x}\right)}{n+2},
\end{aligned}
$$

where $H$ is the depth of the ice, $q$ is the ice flux, and (12) is an expression of mass conservation. To match the two sides of the grounding line, we take the boundary conditions as $x \rightarrow x_{G}$ to be (at leading order)

$$
H \rightarrow 0, \quad q \rightarrow q_{G},
$$

where $q_{G}$ is the ice flux from the ice sheet. These conditions describe a point-sink at the grounding line. (Note that in steady state, $q_{I}=q_{G}$. However, in an unsteady state, the grounding line can move, such that $q_{I}$ and $q_{G}$ are related by (36).)

The full Stokes equations can be used to describe the dynamics of the transition region surrounding the grounding line, the width of which scales as

$$
x-x_{G} \sim \epsilon\left(\frac{\epsilon}{\delta}\right)^{n /(n+2)} .
$$


Rescaling the variables for the transition zone, we redefine a coordinate system where the grounding line is at $X=0$, and derive the following matching condition for the ice surface as $X \rightarrow-\infty$ :

$$
\begin{aligned}
& S \sim-\lambda X, \\
& \lambda=\frac{\left\{(n+2) q_{G}\right\}^{1 / n}}{\left(-B_{G}\right)^{(n+2) / n}} .
\end{aligned}
$$

The rescaled model for incompressible Stokes flow in the transition zone where $\Pi=P+S$ is

$$
\begin{aligned}
U_{X}+W_{Z} & =0 . \\
\Pi_{X} & =T_{3 Z}+T_{1 X}, \\
\Pi_{Z} & =-T_{1 Z}+T_{3 X}, \\
U_{Z}+W_{X} & =T^{n-1} T_{3}, \\
2 U_{X} & =T^{n-1} T_{1}, \\
T^{2} & =T_{3}^{2}+T_{1}^{2} .
\end{aligned}
$$

The boundary conditions on the surface $(Z=0$ in the scaled coordinates) are

$$
T_{3}=W=0 .
$$

The base of the floating ice is a free boundary $(Z=B, X>0)$, with boundary conditions

$$
\begin{aligned}
B & =-\left(\Pi+T_{1}+T_{3} B_{X}\right), \\
T_{3}\left(1-B_{X}^{2}\right) & =2 T_{1} B_{X}, \\
W & =\left(-\dot{x}_{G}+U\right) B_{X},
\end{aligned}
$$

where $\dot{x}_{G}=\frac{\mathrm{d}}{\mathrm{d} t^{*}} x_{G}$ (and $t^{*}$ is rescaled time). The boundary conditions on the grounded base $\left(Z=B_{G}, X<0\right)$ are

$$
\begin{aligned}
& W=0, \\
& T_{3}=\beta U,
\end{aligned}
$$

where (29) is a sliding law with basal sliding parameter $\beta$.

Now, we will define the necessary conditions to match between the three regions. For the ice sheet (SIA), the matching conditions as $X \rightarrow-\infty$ are

$$
\begin{aligned}
\Pi_{X} & \rightarrow-\lambda, \\
W & \rightarrow 0, \\
T_{3} & \rightarrow-\lambda Z .
\end{aligned}
$$

For the ice shelf (SSA), the matching conditions as $X \rightarrow \infty$ are

$$
\begin{aligned}
T_{1} & \sim-\frac{1}{4} B, \\
B & \sim-\frac{q_{I}}{U}, \\
U & \sim\left[\frac{1}{2}(n+1)\left(\frac{1}{4} q_{I}\right)^{n} X\right]^{1 /(n+1)} .
\end{aligned}
$$


Mass flux to the ice shelf, $q_{I}$, is defined by

$$
q_{I}=q_{G}+\dot{x}_{G} B_{G}
$$

The surface is defined as

$$
S=\left.\left(\Pi+T_{1}\right)\right|_{Z=0} .
$$

It should be possible to determine $B$ from (27), but $\dot{x}_{G}$ is still unknown.

To address this, we introduce physically straightforward contact conditions. We require a downward normal stress upstream of the grounding line $(X<0)$ :

$$
B+\Pi+T_{1}>0
$$

Downstream of the grounding line $(X>0)$, we require that the base of the ice be floating:

$$
B>B_{G}
$$

Finally, at the grounding line $(X=0)$, the effective normal stress is zero, allowing the ice to lift off of the bed:

$$
B+\Pi+T_{1}=0 .
$$

Numerical solutions suggest that there is a unique value of ice flux from the ice sheet at the grounding line, $q_{G}$, (proportional to $\lambda^{n}$ ) that satisfies the contact conditions:

$$
q_{G}=\frac{\lambda^{n} H_{G}^{n+2}}{n+2},
$$

where $H_{G}$ is the grounding line ice thickness.

\subsubsection{Marine ice sheet instability}

A marine ice sheet, which is grounded below sea level, may become unstable if its bed slopes downwards inland (Fowler, 2011). The dynamics of this instability, which is known as the marine ice sheet instability (MISI), is described in Figure 3. Here, the delivered ice flux, $q_{0}$, is assumed to be a linear function of distance and proportional to snow accumulation. The grounding line ice flux, $q_{G}$, is an increasing function of ice depth, as in (41).

In the example shown in figure 3 , the slope of the bed depth below sea level, $H_{G}$, changes sign twice, so there are three intersections between $q_{0}$ and $q_{G}$. Such an equilibrium is unstable where $\frac{\partial q_{0}}{\partial x}$ is greater than $\frac{\partial q_{G}}{\partial x}$, so A and

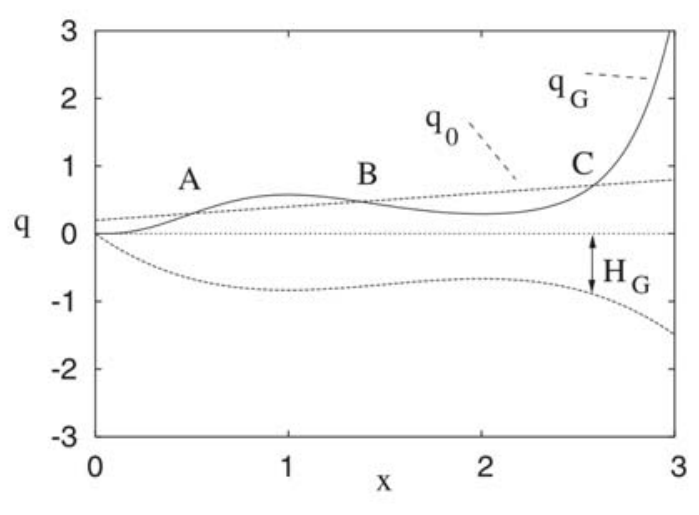

Figure 3: Schematic of MISI. $q_{0}$ is the delivered ice flux, $q_{G}$ is the grounding line flux, $H_{G}$ is the depth of the bed below sea level, and $\mathrm{A}, \mathrm{B}$, and $\mathrm{C}$ are the equilibrium positions. 
$\mathrm{C}$ are stable, while $\mathrm{B}$ is unstable. Intuitively, this makes sense, because if the grounding line $x_{G}$ advances from B, the delivered flux $q_{0}$ exceeds the flux through the grounding line $q G$, causing the $x_{G}$ to advance until it reaches the next stable equilibrium, C. Conversely, if $x_{G}$ is perturbed in the opposite direction, retreating from $\mathrm{B}, q_{G}$ exceeds $q_{0}$, resulting in unabated retreat until $\mathrm{A}$ is reached. In contrast, the ice sheet recovers from small perturbations in either direction at A or C.

\subsection{Calving}

The process of calving refers to the fracture of an ice-shelf or a glacier terminus in the sea. Iceberg calving is a sink in the overall ice sheet mass balance and it has been observed to be especially important in Antarctica. In particular, there are circumstances in which glaciers can undergo rapid ice loss through iceberg calving, and these events can have a significant impact on global sea level. Due to the complexity of the phenomena involved and the danger inherent in making observations near a calving face, there is still not a complete and thorough theory describing calving.

\subsubsection{Calving mechanisms}

Consider a tidewater glacier and let $h_{i}$ be the thickness of the ice and $h_{w}$ be the depth of the water. By scaling the momentum equation along $x$ on an ice shelf, one finds that the net balance is between the longitudinal shear stress term and the pressure gradient term, namely

$$
\frac{\partial \tau_{11}}{\partial x}=\frac{\partial p}{\partial x}
$$

By integrating the above equation along $x$ across the interface

$$
\tau_{11}=p_{i}-p_{w}
$$

and integrating over the depth, one gets

$$
h_{i} \overline{\tau_{11}}=\frac{g}{2}\left(\rho_{i} h_{i}^{2}-\rho_{w} h_{w}^{2}\right),
$$

where the overbar denotes vertical average, $g$ is the acceleration due to gravity and $\rho_{i}$ and $\rho_{w}$ are ice and water density, respectively. This leads to

$$
\overline{\tau_{11}}=\frac{\rho_{i} g h_{i}}{2}\left(1-\frac{\rho_{w} h_{w}^{2}}{\rho_{i} h_{i}^{2}}\right),
$$

indicating that if the pressure jump at the interface balances the depth integrated longitudinal stress, the calving front is in equilibrium. Whether the ice is grounded $\left(\rho_{i} h_{i}>\rho_{w} h_{w}\right)$ or floating $\left(\rho_{i} h_{i}=\rho_{w} h_{w}\right), \overline{\tau_{11}}$ is greater than 0 . In the case that $\overline{\tau_{11}}$ exceeds the yield stress, the ice may fracture. 
In particular, calving occurs when fractures propagate to a suffucient depth to isolate blocks from the main glacier mass. Nye (1957) suggested that the crevasses penetrate to a depth $d$ where a balance between the tensile strain rate and the creep closure rate due to the hydrostatic pressure is reached, namely

$$
d=\frac{2}{\rho_{i} g}\left(\frac{\dot{\epsilon}}{A}\right)^{1 / n},
$$

where $A$ and $n$ come from the Glen's flow law, which relates the strain rate $\dot{\epsilon}_{i j}$ to the stress tensor $\tau_{i j}$ as $\dot{\epsilon}_{i j}=A \tau^{n-1} \tau_{i j}$. The presence of meltwater in the crevasses can help to deepen them because of the additional hydrostatic water pressure. In Nye's model this is accounted for as

$$
d=\frac{2}{\rho_{i} g}\left[\left(\frac{\dot{\epsilon}}{A}\right)^{1 / n}+\rho_{w} g d_{w}\right],
$$

where $d_{w}$ is the water column depth in the crevasse.

Benn et al. (2007) affirm that the four major mechanisms that control calving are, in order of importance:

1. stretching in response to large-scale velocity gradients: the velocity distribution at the ice surface is a primary control on the crevasse depth, which is enhanced by meltwater, and the calving margin, which is also influenced by the ice cliff height;

2. force imbalances at an unsupported ice cliff;

3. undercutting of the ice cliff by melting at the submerged ice interface; and

4. torque arising from buoyant forces.

\subsubsection{Calving laws}

In the literature there have been multiple attempts to quantify the calving rate as a function of other ice or ocean parameters. While no calving laws have yet been established for ice shelves, for tidewater glaciers the rate of change of the ice front position $\dot{x}_{s}$ has been related to the calving rate $u_{c}$ through

$$
\dot{x}_{s}=\bar{u}-u_{c},
$$

where $\bar{u}$ is the vertically averaged glacier velocity at the terminus (Benn et al., 2007). Multiple works, such as Haresign (2004); Benn et al. (2007), have shown that an empirical linear law links the calving rate to the height of the water column at the terminus as

$$
u_{c}=a+b h_{w} .
$$

Although the behavior seems to be quite universal, the coefficients $a$ and $b$ have been found to be glacier- and time-dependent (seasonally). In addition, calving rates of freshwaterterminating glaciers are around one order of magnitude lower than tidewater glacier calving 
rates, which seems to be due to differences in water densities, upwelling turbulent heat transfers and underwater melting rates, among others.

Not all the calving laws have been written as a function of the water depth. Other works, such as Sikonia (1982), have attempted to write down calving laws in terms of the height of the terminal ice cliff above buoyancy $h_{0}$. This represents the excess height with respect to a free floating ice body in the same water and is defined as

$$
h_{0}=h_{i}-\frac{\rho_{w}}{\rho_{i}} h_{w}
$$

\section{$2.3 \quad$ Tidewater glacier cycles}

The dependence of the calving rate on the water column depth is thought to play a crucial role in tidewater glacier cycles. Observations and paleoclimate proxies suggest that in warmer climates tidewater glaciers tend to undergo catastrophic retreats. For example, the Columbia glacier was observed to retreat about $12 \mathrm{~km}$ between 1982 and 2002. To explain that, the following simple reasoning has been developed. Let us start from a condition in which the calving front is in a fixed position set by balance between the inflow mass and the mass loss due to calving and melting. If at some point the up-glacier dynamics start pushing the front further, a moraine shoal develops at the base of the front and the calving rate reduces because of the decrease of the effective water depth at the glacier front. In this way, the glacier is able to advance because the height of the moraine increases and keeps the calving rate low. This is hypothesized to be sustainable for up to $1000 \mathrm{y}$ at a rate of order $30 \mathrm{~m}$ $\mathrm{y}^{-1}$ (Meier and Post, 1987). If then, perhaps due to inherent instability of the steady state, the glacier starts retreating, it finds itself in contact with the full water column depth, with no moraine shielding it, and the calving rate suddenly increases, causing the front to retreat further. The retreat stops when the water column is shallow enough to return to a quasistable equilibrium between inflow and mass loss at the front. This kind of retreat is thought to happen over scales of $100 \mathrm{y}$ at a rate of order $1 \mathrm{~km} \mathrm{y}^{-1}$ and figure 4 shows a schematic of it.

Another feedback loop that might explain the initiation and the sudden retreat of the tidewater glaciers described above has been has been hypothesized for the relationship between thinning, acceleration and calving retreat by Benn et al. (2007). In particular, this study affirms that an increase in surface melting drives the thinning of the glacier, which is responsible for a reduced effective pressure and a consequent increase in velocity and longitudinal strain rate. First, this generates dynamic thinning, leading to a further decrease in

effective pressure. Second, this process leads to deeper crevasses, which causes the calving margin to retreat more quickly.

\section{Conclusion: A Cautionary Tale}

As a conclusion, Dr. Fowler warned the theorists among us not to get too enamoured of theory, to the exclusion of its application: 


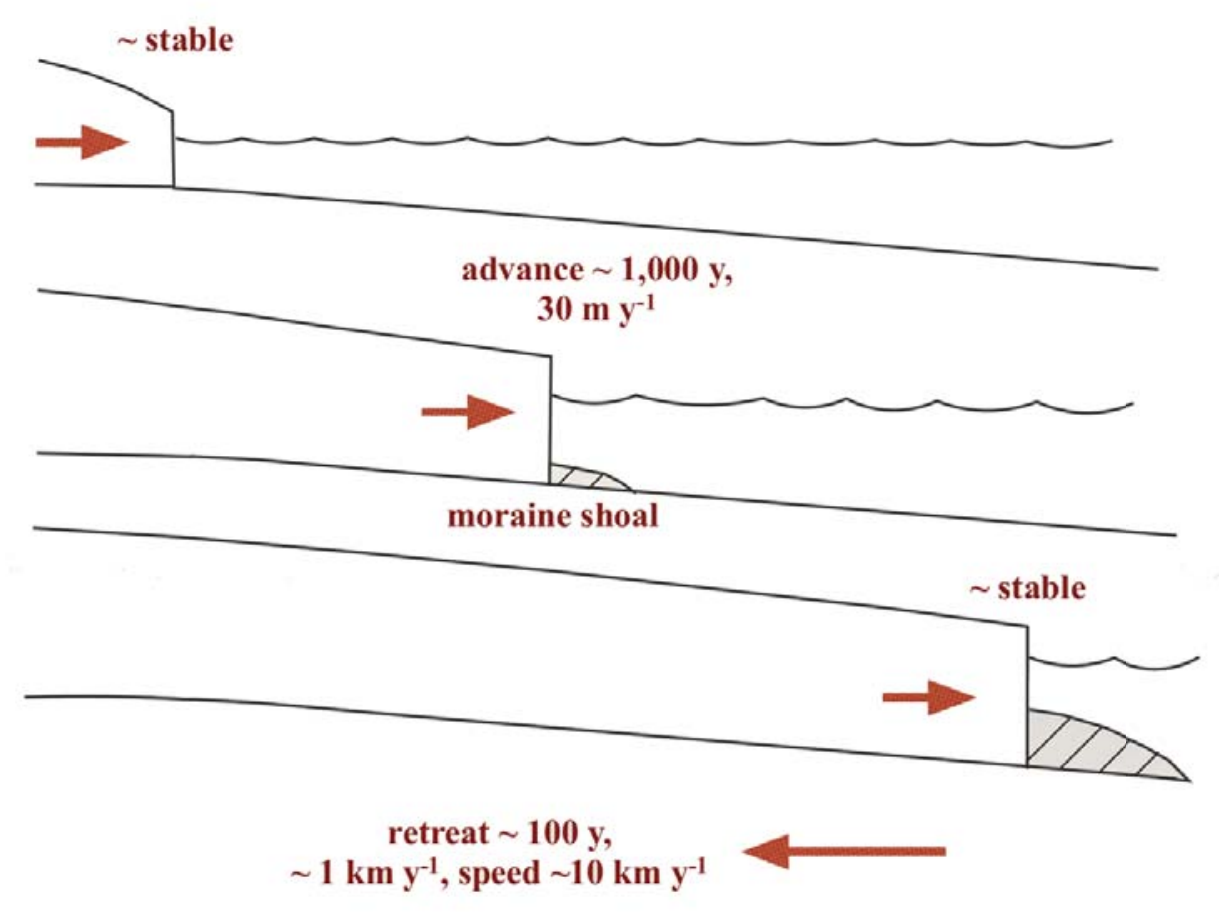

Figure 4: Schematic of the tidewater glacier cycle.

"Pfuel was one of those theorists who so love their theory that they forget the purpose of the theory - its application in practice; in his love for theory, he hated everything practical and did not want to know about it. He was even glad of failure, because failure, proceeding from departures from theory in practice, only proved to him the correctness of his theory." Tolstoy, War and Peace, III, I, X.

\section{References}

Benn, D. I., Warren, C. R., and Mottram, R. H. (2007). Calving processes and the dynamics of calving glaciers. Earth-Science Reviews.

Creyts, T. T. and Schoof, C. G. (2009). Drainage through subglacial water sheets. Journal of Geophysical Research, 114(F4):F04008.

Fowler, A. (2011). Mathematical Geoscience, volume 36 of Interdisciplinary Applied Mathematics. Springer London, London.

Haresign, E. C. (2004). Glacio-limnological interactions at lake-calving glaciers. PhD thesis, University of St Andrews.

Kirchner, N., Ahlkrona, J., Gowan, E., Lötstedt, P., Lea, J., Noormets, R., von Sydow, L., Dowdeswell, J., and Benham, T. (2016). Shallow ice approximation, second order shallow 
ice approximation, and full stokes models: A discussion of their roles in palaeo-ice sheet modelling and development. Quaternary Science Reviews, 147(Supplement C):136 - 147. Special Issue: PAST Gateways (Palaeo-Arctic Spatial and Temporal Gateways).

Kyrke-Smith, T. M., Katz, R. F., and Fowler, A. C. (2013). Subglacial hydrology and the formation of ice streams. Proceedings of the Royal Society A: Mathematical, Physical and Engineering Sciences, 470(2161):20130494-20130494.

Meier, M. F. and Post, A. (1987). Fast tidewater glaciers. Journal of Geophysical Research, 92(B9):9051-9058.

Meur, E. L., Gagliardini, O., Zwinger, T., and Ruokolainen, J. (2004). Glacier flow modelling: a comparison of the shallow ice approximation and the full-stokes solution. Comptes Rendus Physique, 5(7):709 - 722. Ice: from dislocations to icy satellites.

Nye, J. F. (1957). The Distribution of Stress and Velocity in Glaciers and Ice-Sheets. Proceedings of the Royal Society A: Mathematical, Physical and Engineering Sciences, 239(1216):113-133.

Sikonia, W. G. (1982). Finite-element glacier dynamics model applied to Columbia glacier, Alaska. U. S. Geol. Surv. Prof. Pap., 1258-B. 


\title{
GFD 2017 Lecture 5: Basic Theory of Ice-Ocean Interaction
}

\author{
Adrian Jenkins; notes by Madeleine Youngs and Guillaume Michel
}

June 23, 2017

In this lecture, we detail the structures of the ice-ocean boundary layers and of the water motion beneath an ice shelf. These flows strongly depend on the thermodynamic properties of water (e.g. latent heat, phase diagram, equation of state) and we therefore first review these properties of fresh and salty water before turning to fluid mechanics.

\section{The Ice-ocean Interface and the Boundary Layer}

\subsection{Impact of the melting of ice on the ocean}

\subsubsection{Phase diagram of water}

For a mixture of ice and fresh water to be at equilibrium, the temperature has to be equal to the freezing temperature $T_{\mathrm{f}}$, which only depends on the pressure. At one atmosphere, this temperature is very close to $0^{\circ} \mathrm{C}$, and (unlike most other pure substances) it decreases as the pressure increases, approximately as $-1 \times 10^{6} \mathrm{~Pa} \cdot \mathrm{K}^{-1}$.

Because seawater contains ions, its thermodynamic properties also depend on the salinity. Once the pressure is fixed, the dependence of the freezing point on salinity can be seen on the phase diagram Fig. 1. For simplicity, we describe in the following a mixture of pure water and salt $(\mathrm{NaCl})$.

In this figure, the grey zones are delimited by the liquidus (boundary with the liquid solution) and the solidus (boundary with the solid solution). If the temperature and salinity are set such that the system falls into one of these two grey zones, the equilibrium state is a coexistence between a liquid solution and a pure solid (either ice or salt). The point $E$ is called an eutectic point, and corresponds, at one atmosphere, to a temperature of $-21.2^{\circ} \mathrm{C}$ and a salinity per mass of $23.3 \%$.

These coexistence zones are of importance for ice-ocean interfaces: the melting of pure ice in seawater tends to drive the liquid toward the liquidus. For instance, consider the total melting of a mass of ice $\Delta M$ (temperature $T_{\mathrm{i}}$, salinity $S_{\mathrm{i}}=0$ ) into a mass of seawater $M$ (temperature $T_{\mathrm{w}}$, salinity $S_{\mathrm{w}}$ ). The final properties of the liquid $\left(T_{\text {mix }}, S_{\text {mix }}\right)$ follow from the conservation of energy and mass. The first principle of thermodynamics reads

$$
M c_{\mathrm{w}}\left(T_{\text {mix }}-T_{\mathrm{w}}\right)+\Delta M\left[c_{\mathrm{w}}\left(T_{\text {mix }}-T_{\mathrm{f}}\right)+\ell+c_{\mathrm{i}}\left(T_{\mathrm{f}}-T_{\mathrm{i}}\right)\right]=0,
$$




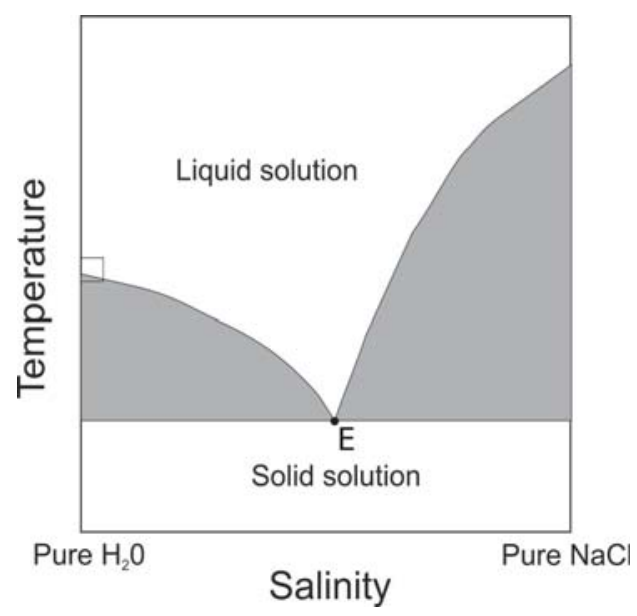

Figure 1: Phase diagram of a mixture of water and salt, at a fixed pressure.

where $c_{\mathrm{w}}$ and $c_{\mathrm{i}}$ are the specific heat capacities of water and ice, and $\ell$ is the specific latent heat at the freezing temperature $T_{\mathrm{f}}$. The conservation of salt mass prescribes

$$
M\left(S_{\text {mix }}-S_{\mathrm{w}}\right)+\Delta M\left(S_{\text {mix }}-S_{\mathrm{i}}\right)=0 .
$$

The changes in the seawater properties result from (1) and (2),

$$
\begin{gathered}
T_{\text {mix }}=T_{\mathrm{w}}-\left(\frac{\Delta M}{M+\Delta M}\right)\left[\left(T_{\mathrm{w}}-T_{\mathrm{f}}\right)+\frac{\ell}{c_{\mathrm{w}}}+\frac{c_{\mathrm{i}}}{c_{\mathrm{w}}}\left(T_{\mathrm{f}}-T_{\mathrm{i}}\right)\right]<T_{\mathrm{w}}, \\
S_{\text {mix }}=S_{\mathrm{w}}-\left(\frac{\Delta M}{M+\Delta M}\right) S_{\mathrm{w}}<S_{\mathrm{w}} .
\end{gathered}
$$

As can be seen in Fig. 1, a decrease of the temperature and salinity of a liquid solution favors the coexistence of pure ice and liquid in a liquidus state. Moreover, until two phases coexist at equilibrium, the liquid properties evolve according to

$$
\left(T_{\text {mix }}-T_{\mathrm{w}}\right)=\left(\frac{\Delta M}{M+\Delta M}\right)\left(T_{\mathrm{eff}}-T_{\mathrm{w}}\right), \quad\left(S_{\mathrm{mix}}-S_{\mathrm{w}}\right)=\left(\frac{\Delta M}{M+\Delta M}\right)\left(S_{\mathrm{eff}}-S_{\mathrm{w}}\right),
$$

with $S_{\text {eff }}=0$ and $T_{\text {eff }}=T_{\mathrm{f}}-\ell / c_{\mathrm{w}}-\left(c_{\mathrm{i}} / c_{\mathrm{w}}\right)\left(T_{\mathrm{f}}-T_{\mathrm{i}}\right)$, that ranges between $-85^{\circ} \mathrm{C}$ to $-100^{\circ} \mathrm{C}$ depending on the ice temperature. Equation (5) has a graphical interpretation on the phase diagram, and shows that during the melting, the liquid evolves along a straight line toward the point $\left(S_{\text {eff }}, T_{\text {eff }}\right)$.

\subsubsection{A closer look at the low salinity zone}

Since the salinity of the eutectic points is by far larger than the actual salinity observed in the ocean, the actual zone of the phase diagram that is being used for the study of ice-ocean interaction is reduced. In the following, we focus on the framed zone of Fig. 1.

The phase diagram in this zone is sketched in Fig. 2. As previously explained, the evolution of seawater during the melting of ice corresponds on the phase diagram to evolutions 


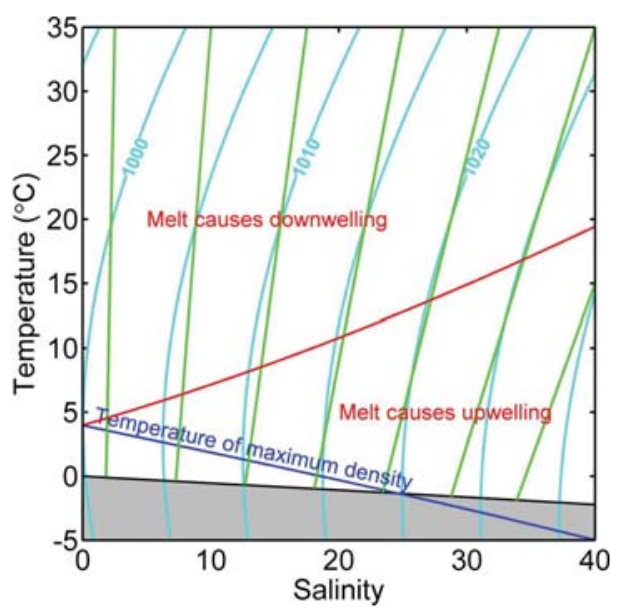

Figure 2: Phase diagram of a mixture of water and salt, at a fixed pressure and low salinities. Salinity is expressed in PSU (practical salinity unit).

along straight lines, that are plotted in green. The lines of constant density are isopycnals (in light blue) and the curve of maximal density is in dark blue).

From this information, we deduce that the melting of ice may either increase or decrease the liquid density, that is either cause downwelling or upwelling. The boundary between these two regimes is plotted in red in Fig. 2 and can be deduced from the other curves. Note that all these phase diagrams evolve with the pressure, i.e. with the depth.

\subsubsection{Application: the "ice pump" effect}

As an application of these thermodynamics properties, we describe the "ice pump" effect, sketched in Fig. 3.

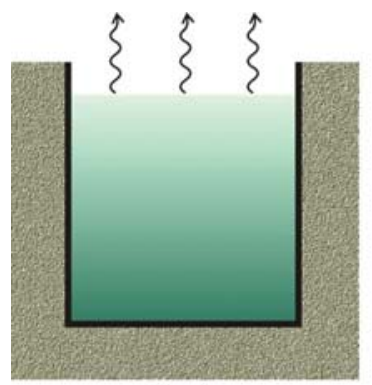

(a)

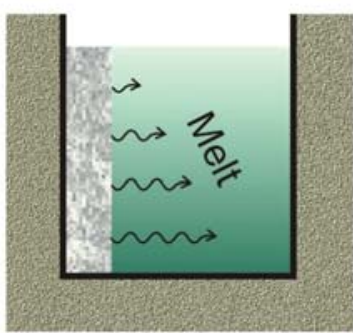

(b)

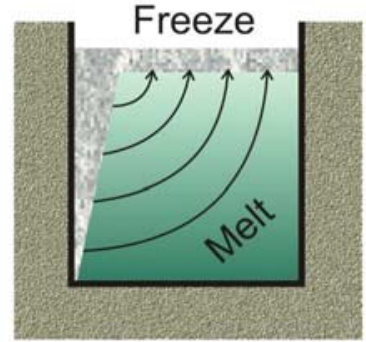

(c)

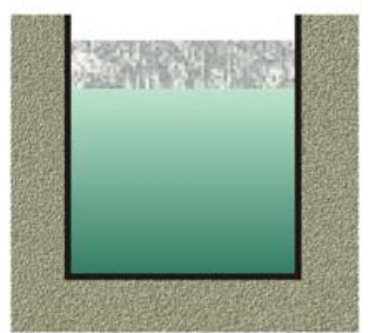

(d)

Figure 3: Sketch of the ice pump effect.

(a) We consider an insulated tank filled with water of uniform salinity and temperature, the latter being the freezing temperature at room pressure. 
(b) We introduce a vertical slab of ice. Although the water at the surface is at the freezing temperature, the water at depth is not, because of the pressure dependence of the freezing temperature. This causes the ice at depth to melt, freshen, and cool the adjacent water.

(c) As seen in Fig. 2, the melting creates less dense water because of the freshening, leading to the cooled water rising. When close to the surface, the reduced freezing temperature leads the cooled water to freeze and form ice.

(d) At equilibrium, all the ice is at the surface.

\subsection{The ice-ocean boundary layer}

Considering the conditions required for an equilibrium between ice and seawater, we discuss the boundary layers in the ocean, in particular the ones below ice shelves. We first disregard the flow motion, then take it into account in turbulent boundary layer models.

\subsubsection{Laminar boundary layers}

If the water in the ocean is at rest, the situation below ice shelves could be represented by one of the sketches of Fig. 4. To connect the ocean temperature and salinity to the ones at the iceocean interface, where they are prescribed by the phase equilibrium, a boundary layer develops. As heat and molecular diffusion take place, this boundary layer thickens. This process may lead to convective instabilities.

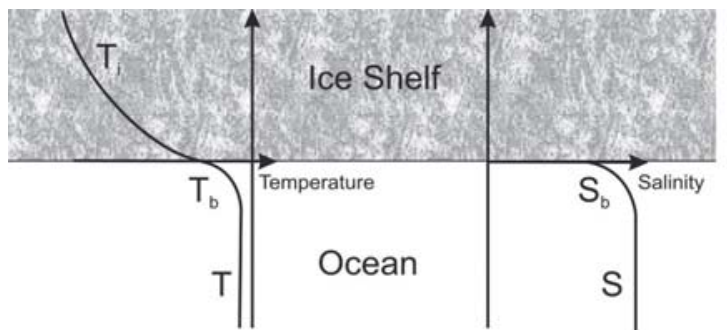

(a)

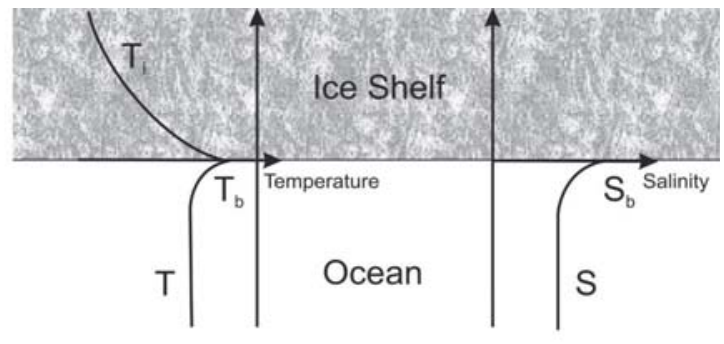

(b)

Figure 4: Temperature and salinity close to the interface.

(a) If the temperature in the ocean is larger than the one at the interface, heat is transfered to the ice shelf, that causes melting. That ablation process is unstable because of the dynamics of double diffusion: whereas the salt diffusion stabilizes the stratification, the faster heat diffusion triggers a convective instability ${ }^{1}$. This leads to thermohaline staircases, that are well-mixed layers separated by sharp interfaces [1]. Note that in the presence of enough shear, this differential diffusive pattern vanishes, which stabilizes the boundary (melting causes upwelling, see Fig. 2).

\footnotetext{
${ }^{1}$ As can be seen in Fig. 2, for ocean salinity of $\sim 34.5 \mathrm{psu}$, the density evolution of water with temperature no longer presents an anomaly at low temperatures.
} 
(b) If the temperature in the ocean is smaller than that at the interface, heat is transfered from the ice shelf to the ocean, which causes freezing. This process is unstable, because in this range of parameters freezing causes downwelling (see Fig. 2).

\subsubsection{Turbulent boundary layers}

Even though laminar boundary layers can be observed where the currents are weak, turbulent boundary layers are more common. They occur when there is enough shear to observe mixing in the boundary layers. They can be modeled as shown in Fig. 5, where we identify

- A surface layer, where turbulent mixing is influenced by the boundary, and an outer layer, where is it not.

- An interfacial sublayer, where the turbulence is greatly damped by viscosity. Its width, of the order of a millimeter or less, is determined by the turbulence in the outer layer. The rapid evolution of the temperature and salinity needed to match the bulk flow ones to the surface ones occur within this thin layer.

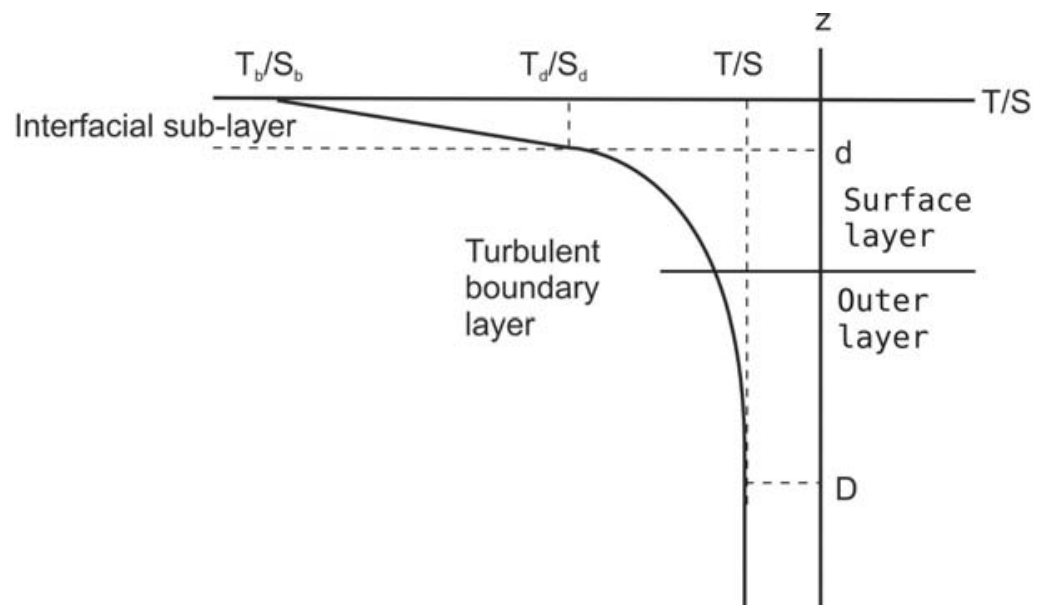

Figure 5: Turbulent boundary layer.

Whereas heat and salt fluxes, crucial to predict the dynamics of the ice shelf, can be easily worked out for a laminar boundary layer, they are much more difficult to predict in the case of a turbulent boundary layer. We present one model that describes the ice shelf evolution from these fluxes. We start by making the assumption that the freezing point at the ice-ocean interface $T_{\mathrm{b}}$ can be expressed as a linear function of salinity $S_{\mathrm{b}}$ and pressure (i.e. depth $z_{\mathrm{b}}$ ) at this same place which we call the liquidus relationship:

$$
T_{\mathrm{b}}=a S_{\mathrm{b}}+b+c z_{\mathrm{b}} .
$$

We then write the energy flux balance at the ice-ocean interface,

$$
\rho_{\mathrm{i}} a_{\mathrm{b}} \ell_{\mathrm{i}}=\rho_{\mathrm{i}} c_{\mathrm{i}} \kappa_{\mathrm{i}}\left(\frac{\partial T_{\mathrm{i}}}{\partial z}\right)_{\mathrm{b}}-q_{\mathrm{b}}^{T}
$$

where: 
- $\rho_{\mathrm{i}} a_{\mathrm{b}} \ell_{\mathrm{i}}$ is the heat flux resulting from ice melting $\left(a_{b}\right.$ is the ablation rate, and $\ell_{\mathrm{i}}$ is the specific latent heat).

- $\rho_{\mathrm{i}} c_{\mathrm{i}} \kappa_{\mathrm{i}}\left(\frac{\partial T_{\mathrm{i}}}{\partial z}\right)_{\mathrm{b}}$ is the heat flux from the ice shelf ( $\rho_{\mathrm{i}}$ is the ice shelf reference density, $c_{\mathrm{i}}$ its specific heat capacity, and $\kappa_{\mathrm{i}}$ its thermal diffusivity).

- $q_{\mathrm{b}}^{T}$ is the heat flux from the turbulent boundary layer that we wish to model.

We also balance the salt flux at the ice-ocean interface $q_{\mathrm{b}}^{S}$ with the height variation of seawater,

$$
\rho_{\mathrm{i}} a_{\mathrm{b}} S_{\mathrm{b}}=-q_{\mathrm{b}}^{S}
$$

where $S_{\mathrm{b}}$ is the salinity at the ice-ocean interface. For given heat and salt fluxes, we can therefore predict the evolution of the ice shelf from this set of equations.

Theoretical predictions for these fluxes in turbulent layers can be carried out by matching solutions for an inner laminar and a turbulent logarithmic layer. The roughness of the surface can also be modeled (see, e.g., [2]). In situ experiments suggest that simple laws apply [3], that read for the heat flux,

$$
q_{\mathrm{b}}^{T}=\rho_{\mathrm{w}} c_{\mathrm{w}}\left(\frac{\sqrt{C_{\mathrm{d}}}}{0.006}\right) U\left(T_{\mathrm{f}}-T\right),
$$

where $w$ refers to the seawater, $C_{\mathrm{d}}$ is the momentum exchange coefficient, $U$ is the velocity of the mixed layer, $T$ is the temperature of the far-field water and $T_{\mathrm{f}}$ the freezing temperature.

\subsubsection{Observations and open questions}

Ice-shelf evolution As mentioned, in situ measurements of the heat and salt fluxes can be done based on oceanic observations of correlations between vertical velocities and temperature/salinity. In this section, we describe how direct observations of the melt rate can be performed.

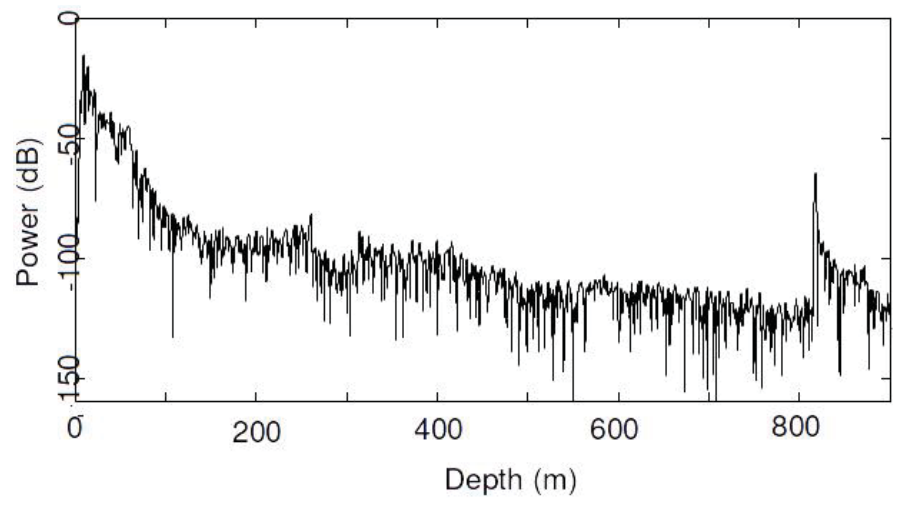

Figure 6: Power spectrum of a radar signal.

To investigate the dynamics of the basal melting, high precision radars can be used. The echo is recorded at the same place and at different times. For a given acquisition, a typical 
power spectra is reported in Fig. 6; it consists of a dominant component at approximately $800 \mathrm{~m}$ (the bottom of the ice shelf), and a multitude of other peaks, resulting from internal reflectors. The precise positions of these reflectors, randomly distributed among the ice, evolve between each acquisition because the ice shelf thickens. By looking at the displacement of the peaks in the power spectrum, it is therefore possible to track these reflectors, i.e. to acquire the vertical deformation field in the ice shelf, see Fig. 7.

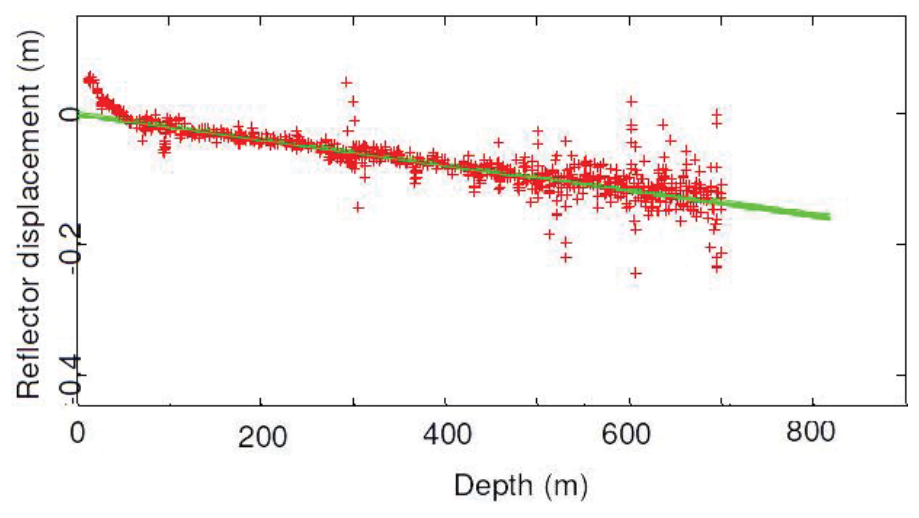

Figure 7: Evolutions of the reflectors positions.

Another possibility is to record the temperature, salinity and current below the ice shelf. This provides the information necessary to calculate the ice-ocean heat flux and the melting rate. The comparison of these two experimental methods can be used, for instance, to constrain the ice-shelf evolution models.

Vertical natural convection boundary layers The flow generated by a heated wall has been theoretically studied by Wells and Worster [4], who discuss three regimes:

1. At the smallest scales, molecular diffusion controls the heat transfer.

2. A turbulent flow develops, but initially heat transfer is controlled by the buoyancy generated at the wall.

3. As the turbulent flow grows, heat transfer is eventually controlled by the shear generated by the flow.

This problem shares similar features as the one met in the ice-ocean interactions, and may provide a model for the melting rate of vertical ice surfaces. The first regime has been sampled by early lab experiments (see, e.g., [5]), the second one has been recently described [6], but the third regime has so far not been fully characterized. It remains also unclear how the processes and scales change when the ice-ocean interface becomes near-horizontal (the bottom of the ice shelf). Finally, the effect of the roughness of the interface would also need to be taken into account. 


\section{Buoyancy-driven Flow on Geophysical Scales}

In this section, we study buoyancy-driven flows outside the boundary layer.

\subsection{Scales of motion beneath an ice shelf}

In the earth-fixed reference frame, the motion of seawater beneath an ice shelf obeys the Navier-Stokes equation,

$$
\rho\left(\frac{\mathrm{d} \vec{v}}{\mathrm{~d} t}\right)+2 \rho \vec{\Omega} \times \vec{v}=\vec{\nabla} \cdot \stackrel{\leftrightarrow}{\mathcal{T}}+\rho \vec{g}
$$

where $\vec{\Omega}$ stands for the rotation of the earth, and $\stackrel{\leftrightarrow}{\mathcal{T}}$ is the stress tensor (the centrifugal force has been incorporated in the pressure field). For water, the stress tensor is given by

$$
\mathcal{T}_{i, j}=-P \delta_{i, j}+\rho \nu\left(\partial_{i} v_{j}+\partial_{j} v_{i}\right)
$$

Compared to the dynamics of ice described in Lecture 1, we have retained the left-hand side of (10) and assumed water to be a Newtonian fluid.

As we shall see, depending on the part of the flow described (boundary layer, large scales, ... ), some of the terms in (10) can be neglected. Typical values of the parameters for an ice shelf are given in Tab. 1.

\begin{tabular}{|c|c|c|c|}
\hline Horizontal length & Depth & Horizontal velocity & Rotation \\
\hline$L \sim 10^{5} \mathrm{~m}$ & $H \sim 10^{2} \mathrm{~m}$ & $U \sim 10^{-1} \mathrm{~m} \cdot \mathrm{s}^{-1}$ & $\Omega \sim 10^{-4} \mathrm{~s}^{-1}$ \\
\hline
\end{tabular}

Table 1: Scales of motion beneath an ice shelf.

Horizontal flow beneath an ice shelf For the horizontal large scales, the Rossby number (ratio of the inertial force to the Coriolis force) scales as

$$
\operatorname{Ro}_{L} \sim \frac{U}{L \Omega} \sim 10^{-2} \ll 1
$$

and the inertial term can therefore be dropped. Similarly, the Reynolds number scales as

$$
\operatorname{Re} \sim \frac{L U}{\nu} \sim 10^{2} \gg 1
$$

and viscous terms remain small. Therefore, the dynamics of these large scales results from a balance between the pressure gradient and Coriolis force, which is called geostrophic balance. This approximation may not always be valid, for instance if the velocity is high and/or the relevant length scale is small (for instance, in or close to the boundary layer). 
Structure of the Ekman layers Velocity must vanish at the solid boundaries (ice shelf base or seabed), which leads to Ekman layers. Their thickness $\delta$ can be evaluated by balancing the Coriolis force with the viscous term,

$$
\rho \Omega U \sim \rho \nu\left(\frac{U}{\delta^{2}}\right) \Rightarrow \delta \sim \overline{\bar{\nu}}
$$

For a typical eddy viscosity of $10^{-2}$, the depth scale is $\delta \sim 10 \mathrm{~m}$. Within the Ekman layer, the velocity reduces (vanishes at the solid boundary) and its direction changes (becomes perpendicular to the geostrophic current close to the solid boundary).

Vertical motion beneath an ice shelf Since the ratio $H / L$ is small, the flow can be described in hydrostatic balance. It turns out that gravity is balanced by the vertical pressure gradient, i.e. that

$$
\frac{\partial P}{\partial z} \simeq-\rho g .
$$

Therefore, we can diagnose the pressure within the ocean directly from the density distribution, as if the water were at rest.

\subsection{Buoyancy-driven flow on a slope}

\subsubsection{Frame of reference and driving pressure gradient}

We apply these approximations to the description of a flow generated by the buoyancy forcing associated with melting ice. The ice-ocean interface is assumed to be planar, but not horizontal: we note the angle $\alpha$ with the horizontal. This tilt allows light water to upwell along the ice shelf base.

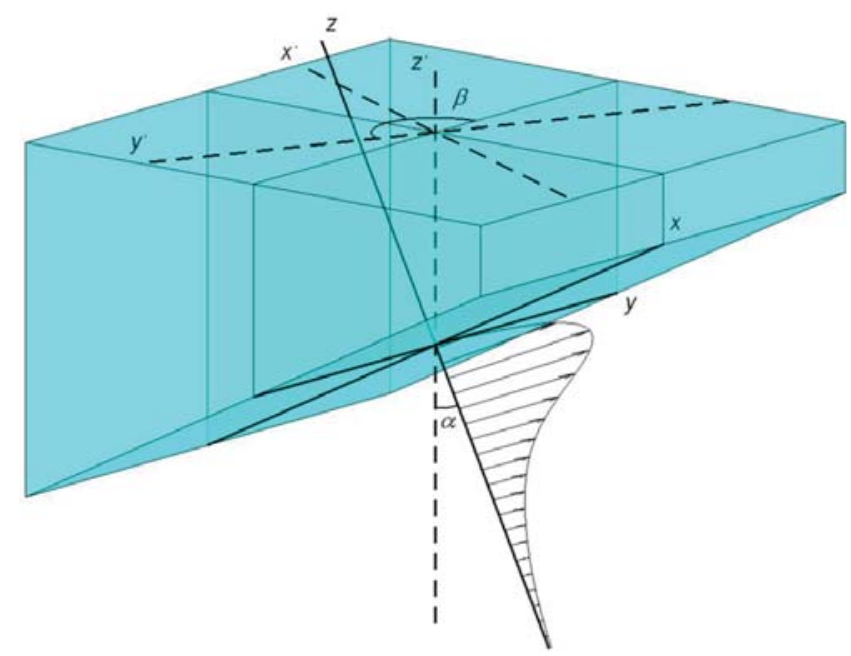

Figure 8: Coordinate system used to model the problem.

To use the same shallow water approximation as before, we consider the reference frame aligned with the boundary, see Fig. 8. We then write the Navier-Stokes equation with the 
Boussinesq approximation,

$$
\left(\frac{\mathrm{d} \vec{v}}{\mathrm{~d} t}\right)+2 \vec{\Omega} \times \vec{v}=-\frac{1}{\rho_{0}} \vec{\nabla} P-\frac{\rho}{\rho_{0}} g \vec{k}+\vec{\nabla} \cdot \nu \vec{\nabla} \cdot \vec{v}
$$

where $\vec{k}$ is a unit vector in the local vertical direction ( $z^{\prime}$ in Fig. 8) and $\rho_{0}$ is the reference density. Similarly to (15), we can apply the hydrostatic approximation along the transformed $z$ coordinate axis, that is

$$
\left(\frac{\partial P}{\partial z}\right)+\rho g \cos \alpha=0 \Longrightarrow P(x, y, z, t)=P(x, y, \eta, t)+g \cos \alpha \int_{z}^{\eta} \rho \mathrm{d} z
$$

where $\eta(x, y, t)$ is the instantaneous deviation of the ice-ocean interface from its equilibrium position. We wish to compute the horizontal pressure gradient, that as mentioned before is essential for the dynamics of the large scales. For this use, we define the gradient parallel to the ice-ocean interface $\vec{\nabla}_{\mathrm{H}}$, and apply it to (17):

$$
\vec{\nabla}_{\mathrm{H}} P(x, y, z, t)=\vec{\nabla}_{\mathrm{H}} P(x, y, \eta, t)+g \cos \alpha\left(\rho \vec{\nabla}_{\mathrm{H}} \eta+\int_{z}^{\eta} \vec{\nabla}_{\mathrm{H}} \rho \mathrm{d} z\right)
$$

We further remove the pressure field associated with a stationary state of the ambient fluid, and assume that the ice sheet float in equilibrium with this fluid $(P(x, y, \eta, t)=0)$ : this leads to the following expression for the reduced pressure gradient in the $x$ and $y$ direction,

$$
\vec{\nabla}_{\mathrm{H}} P^{\prime}=g \cos \alpha\left(\rho_{0} \vec{\nabla}_{\mathrm{H}} \eta+\int_{z}^{\eta} \vec{\nabla}_{\mathrm{H}} \rho^{\prime} \mathrm{d} z\right)
$$

\subsubsection{Evolution for the layer thickness}

If we consider a single active layer, depth-averaged equations sufficiently describe its dynamics. For instance, the incompressibility condition depth-averaged becomes an equation for the layer thickness $D(x, y, t)$ :

$$
\int_{-D}^{0} \vec{\nabla} \cdot \vec{v} \mathrm{~d} z=\int_{-D}^{0} \frac{\partial}{\partial x} v_{x} \mathrm{~d} z+\int_{-D}^{0} \frac{\partial}{\partial y} v_{y} \mathrm{~d} z+v_{z}(z=0)-v_{z}(z=-D)=0 .
$$

The vertical velocity $v_{z}(0)$ describes how the layer develops upward, i.e. is related to the melt rate $\dot{m}(\dot{m}>0$ if ice melts),

$$
v_{z}(z=0)=\dot{m} .
$$

Moreover, the kinematic evolution of the layer thickness is, with $\dot{e}$ the rate at which ambient water is entrained into the active layer, ${ }^{2}$

$$
\left(\frac{\partial D}{\partial t}\right)+\vec{v}(z=-D) \cdot \vec{\nabla}_{\mathrm{H}} \quad D=v_{z}(-D)+\dot{e} .
$$

\footnotetext{
${ }^{2}$ This kinematic condition is similar to the one describing the evolution of surface elevation in the surface wave theory, where $\dot{e}=0$.
} 
Combining equations (20), (21) and (22), we obtain

$$
\left(\frac{\partial D}{\partial t}\right)+\vec{\nabla}_{\mathrm{H}} \cdot \quad \vec{D}=\dot{m}+\dot{e}
$$

where $\vec{U}$ is the depth-averaged velocity, defined as

$$
\vec{U}=\frac{1}{D} \int_{-D}^{0} \vec{v} \mathrm{~d} z=U \vec{e}_{x}+V \vec{e}_{y}
$$

\subsubsection{Depth integration of the momentum equation}

The equations of motion can also be projected on the frame of reference, then depthintegrated. Note that the subscript $a$ represents the ambient fluid and $b$ represents the fluid at the ice-plume interface. This gives (see [7] for some details):

$$
\begin{aligned}
& \left(\frac{\partial(D U)}{\partial t}\right)+\vec{\nabla}_{\mathrm{H}} \cdot D v_{x} \vec{U}-\dot{e} v_{x, a}-\dot{m} v_{x, b}-\phi D V= \\
& -\frac{D \bar{\rho}}{\rho} g \sin \alpha+g \cos \alpha\left[D \frac{\partial}{\partial x}(\eta+D \bar{\rho})\right]+\vec{\nabla}_{\mathrm{H}} \cdot\left(D v_{y} \vec{\nabla}_{\mathrm{H}} U\right)+\left[\left(\nu \frac{\partial v_{x}}{\partial z}\right)_{0}-\left(\nu \frac{\partial v_{x}}{\partial z}\right)_{-D}\right]
\end{aligned}
$$

and

$$
\begin{aligned}
& \left(\frac{\partial(D V)}{\partial t}\right)+\vec{\nabla}_{\mathrm{H}} \cdot \quad D v_{y} \vec{U}-\dot{e} v_{y, a}-\dot{m} v_{y, b}+\phi D U= \\
& g \cos \alpha\left[D \frac{\partial}{\partial y}(\eta+D \bar{\rho})\right]+\vec{\nabla}_{\mathrm{H}} \cdot\left(D v_{x} \vec{\nabla}_{\mathrm{H}} V\right)+\left[\left(\nu \frac{\partial v_{y}}{\partial z}\right)_{0}-\left(\nu \frac{\partial v_{y}}{\partial z}\right)_{-D}\right]
\end{aligned}
$$

where $\bar{\rho}$ is the depth-averaged density, subscript $a$ refers to the ambient fluid, subscript $b$ to the base of the ice shelf, and $\phi$ is the Coriolis parameter, defined as

$$
\phi=2 \Omega(\cos \theta \sin \beta \sin \alpha+\sin \theta \cos \alpha) .
$$

The surface stress term can be modeled by a quadratic drag law:

$$
\left(\nu \frac{\partial v_{x}}{\partial z}\right)_{0}=-C_{d}|\vec{U}| U, \quad\left(\nu \frac{\partial v_{y}}{\partial z}\right)_{0}=-C_{d}|\vec{U}| V
$$

\subsubsection{Depth integration of conservation equations}

Similarly, conservation equations of temperature and salinity can be derived and integrated over the depth. We get for the temperature

$$
\frac{\partial(D \bar{T})}{\partial t}+\vec{\nabla}_{\mathrm{H}} \cdot \quad D \vec{U} \bar{T}-\dot{m} T_{b}-\dot{e} T_{a}=\vec{\nabla}_{\mathrm{H}} \cdot D \kappa_{T} \vec{\nabla}_{\mathrm{H}} \bar{T}+\left[\left(\kappa_{T} \frac{\partial T}{\partial z}\right)_{0}-\left(\kappa_{T} \frac{\partial T}{\partial z}\right)_{-D}\right]
$$

where $\kappa_{T}$ is the thermal diffusivity, and for the salinity

$$
\frac{\partial(D \bar{S})}{\partial t}+\vec{\nabla}_{\mathrm{H}} \cdot \quad D \vec{U} \bar{S}-\dot{m} S_{b}-\dot{e} S_{a}=\vec{\nabla}_{\mathrm{H}} \cdot \quad D \kappa_{S} \vec{\nabla}_{\mathrm{H}} \bar{S}+\left[\left(\kappa_{S} \frac{\partial S}{\partial z}\right)_{0}-\left(\kappa_{S} \frac{\partial S}{\partial z}\right)_{-D}\right]
$$


where $\kappa_{S}$ is the diffusion coefficient. Models can also be used to describe the fluxes at the ice-ocean interface, for instance

$$
\left(\kappa_{T} \frac{\partial T}{\partial z}\right)_{0}=\overline{C_{d}} \Gamma_{T} U\left(T_{b}-T\right), \quad\left(\kappa_{S} \frac{\partial S}{\partial z}\right)_{0}=\overline{C_{d}} \Gamma_{S} U\left(S_{b}-S\right)
$$

where $\Gamma_{T}$ and $\Gamma_{S}$ are thermal and salinity transfer parameters.

\subsubsection{Simplifications}

Although restricted to a single layer, this model is relatively complete and complex to solve. Some assumptions are needed to obtain an equation that describes, at a first approximation, the flow of an inclined plume. We make the following assumptions:

- the flow is in steady state

- the gradients in the cross-slope direction are negligible

- the layer is thin

- the flow is primarily baroclinic and the barotropic forcing term is unimportant

- the flow is supercritical (i.e. sufficiently fast compared to the speed of waves)

This leads to a simple differential equation for momentum conservation,

$$
\frac{\partial\left(D U^{2}\right)}{\partial x}=-\frac{D \Delta \rho}{\rho_{0}} g \sin \alpha-C_{d} U^{2}
$$

\subsection{A simple plume model of ice-ocean interaction}

We use these simplifications to consider an even simpler problem of a buoyant plume driven by melting ice but with no outflow from underneath the glacier. In this model, ambient water melts the ice shelf at depth. It then refreezes as the plume travels upwards, like the "ice pump" example (Fig. 9). Entrainment of ambient water supplies the heat that drives melting, which modifies the buoyancy through cooling and freshening. These plumes are turbulent and entrain fluid from the surroundings, so they grow in volume as they rise. The entrainment rate is also a function of the plume velocity. Since the flow is driven by the component of gravity along the ice base, circulation and melting are sensitive to the interface slope. This process is just like a dense overflow turned upside-down.

The geometry of this problem is now simplified into one dimension where $D$ is the depth of the plume layer, $X$ is the along slope direction, $U$ is the velocity of the plume in the along-slope direction, $\alpha$ is the angle of the slope, and $T$ and $S$ are the temperatures and salinities in the plume, $T_{i}, S_{i}$ are in the ice, $T_{b}, S_{b}$ are at the ice-ocean boundary layer, $T_{a}, S_{a}$

are the ambient properties of the reservoir of the ocean (Fig. 10). We consider the reservoir of the ocean to be infinite in depth and to have no flow. 


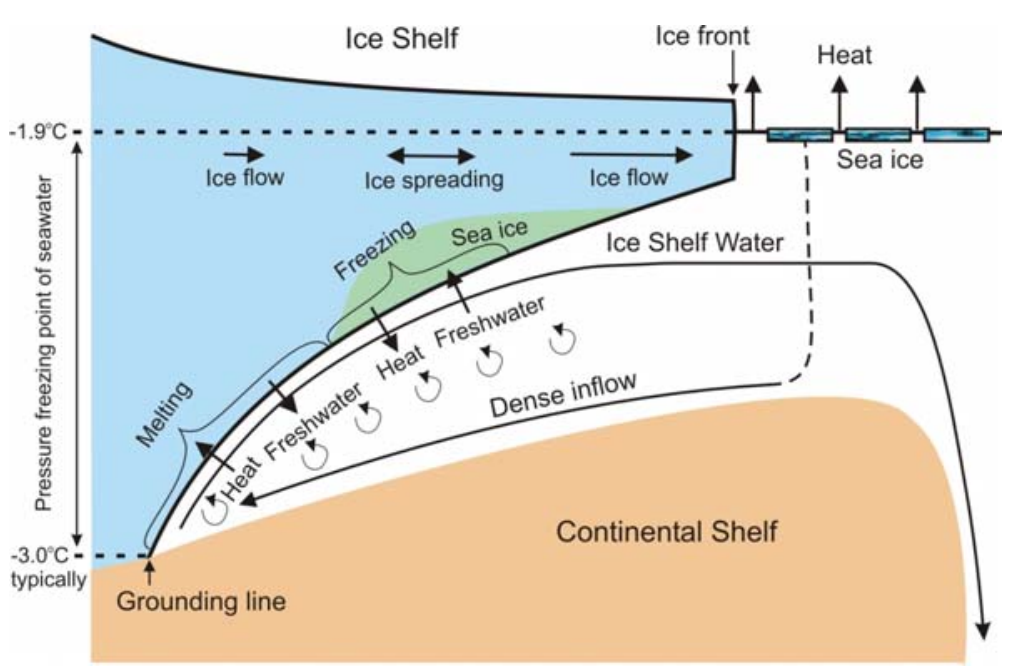

Figure 9: A schematic of a simple plume model of ice-ocean interaction. Dense water inflows at depth and melts the ice shelf, it then refreezes as the plume travels upwards.

\subsubsection{Equations}

We simplify the across-slope integrated equations from the previous section into 1 dimension as discussed in the previous section [8]. Conservation of mass becomes:

$$
\frac{d}{d X}(D U)=\dot{e}+\dot{m}
$$

where $\dot{e}$ is the entrainment rate and $\dot{m}$ is the melt rate. This tells us that the mass flux upward is equal to the entrainment rate plus the melt rate, because we have no other sources of mass in the system. Conservation of momentum is as derived above (Eq. 32)

$$
\frac{d}{d X}\left(D U^{2}\right)=D \frac{\Delta \rho}{\rho_{0}} g \sin (\alpha)-C_{d} U^{2}
$$

where $C_{d}$ is the drag coefficient. This tells us that the momentum imparted by the buoyancy of the plume is balanced by the drag. Conservation of heat is then written as:

$$
\frac{d}{d X}(D U T)=\dot{e} T_{a}+\dot{m} T_{b}-C_{d}^{1 / 2} \Gamma_{T} U\left(T-T_{b}\right)
$$

where $\Gamma_{T}$ is the turbulent transfer coefficient for heat. The equation tells us that the convergence of the heat flux is equal to the amount of heat fluxed in by entrainment of ambient seawater at temperature $T_{a}$ and the heat fluxed by the entrainment of boundary water, minus the turbulent transfer of heat out of the boundary layer into the plume. Conservation of salinity gives a very similar equation:

$$
\frac{d}{d X}(D U S)=\dot{e} S_{a}+\dot{m} S_{b}-C_{d}^{1 / 2} \Gamma_{S} U\left(S-S_{b}\right)
$$

where $\Gamma_{S}$ is the turbulent transfer coefficient for salt. We take

$$
\dot{e}=E_{0} U \sin (\alpha)
$$




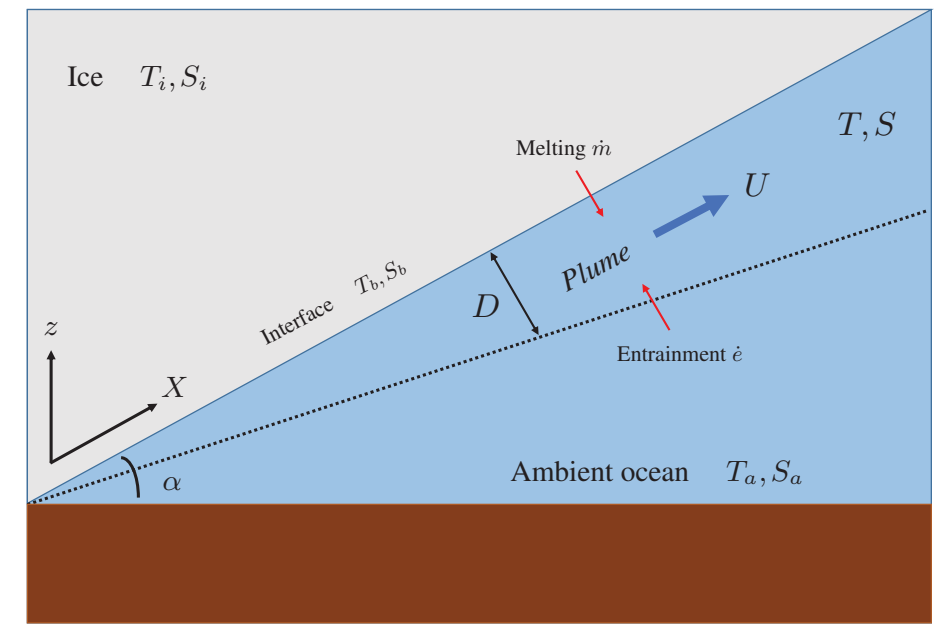

Figure 10: Geometry of the 1-dimensional problem.

where $E_{0}$ is a constant. This parameterization is related to the Richardson number of the flow, i.e. when $\alpha=0$, then the flow cannot entrain any fluid nor travel up the slope. The equation of state is given by

$$
\frac{\Delta \rho}{\rho_{0}}=\beta_{S}\left(S_{a}-S\right)-\beta_{T}\left(T_{a}-T\right)
$$

The boundary conditions on the interface are given by:

$$
C_{d}^{1 / 2} \Gamma_{T} U\left(T-T_{b}\right)=\dot{m}\left[\frac{L}{c}+\frac{c_{i}}{c}\left(T_{b}-T_{i}\right)\right]
$$

This states that the turbulent transfer of heat at the boundary is equal to the amount of heat required to bring up the ice to its melting point and melt the ice with melting flux $\dot{m}$. The boundary condition for the salinity at the interface is:

$$
C_{d}^{1 / 2} \Gamma_{S} U\left(S-S_{b}\right)=\dot{m}\left(S_{b}-S_{i}\right)
$$

which says that the turbulent flux of salt through the boundary is balanced by a flux of salt generated by the entrainment of melt water. The final equation in our set is the liquidus relationship Eq. (6). These equations are a complete set that can be solved to understand the system.

\subsubsection{Results from simplified model}

First we show that the slope of the ice shelf determines how effective the buoyancy forcing is at driving the plume. Figure 11 shows the dependence of the buoyancy and velocities on the slope of the ice shelf. The plume buoyancy changes down the length of the ice shelf. The buoyancy initially increases because of the input of meltwater from the ice shelf, then decreases as freezing transfers freshwater back to the ice shelf. The steeper slopes experience 

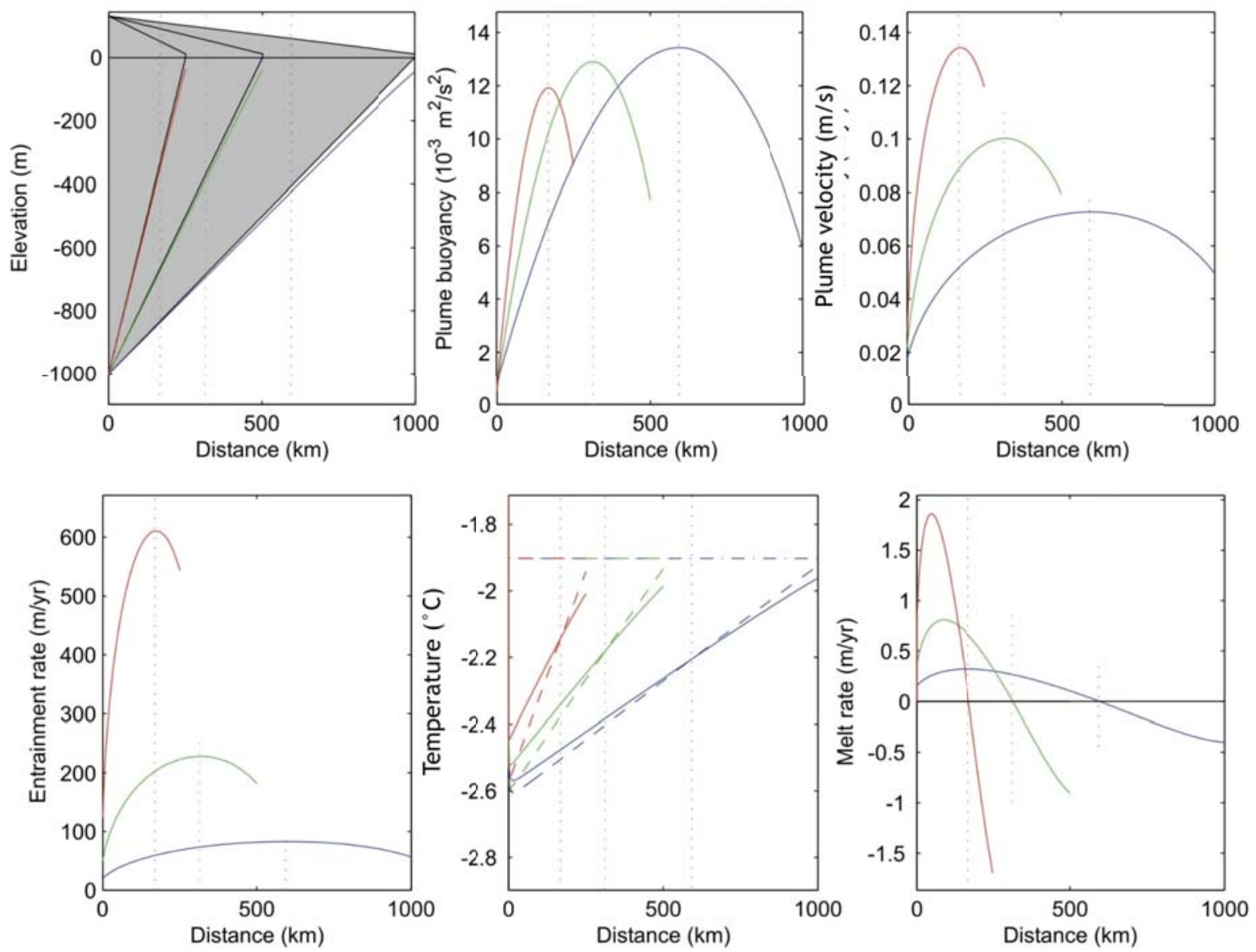

Figure 11: A figure showing the properties of plumes with different slopes. Red describes the steepest slope, green moderate, and blue shallowest. The top left panel shows the slope of the shelf, the top middle panel shows the plume buoyancy, and the plume velocity in the top right panel versus distance along the shelf (should be in $\mathrm{m} / \mathrm{s}$ ). The bottom left shows the entrainment rate, the bottom middle shows the temperature of the plume (solid) and the freezing temperature (dashed) and the melt rate is shown in the bottom right panel.

the maximum buoyancy at shorter distances along the shelf. The other thing to notice is that with a steeper shelf, the plume velocity is larger. The velocity also has a maximum corresponding to the location of maximum buoyancy in the plume. The plume grows in thickness as it entrains ambient seawater, which supplies the heat for melting. The buoyancy imparted by the melting drives the plume up the sloping ice shelf base. The entrainment rate is larger for a steeper slope, both because the velocity is larger, but also because $\alpha$ is larger (Eq. 37). We also see that the temperature begins above the freezing temperature, but depth decreases along the slope (raising the freezing temperature) and ice is melted (lowering the plume temperature), so the temperature in the plume falls below the freezing point, which leads to freezing. For a steeper slope, the melt rate is large but it quickly transitions to freezing as we move along the slope. For a shallower slope, the melt rate is lower and freezing starts much further along the slope. The heat supplied by entrainment and 

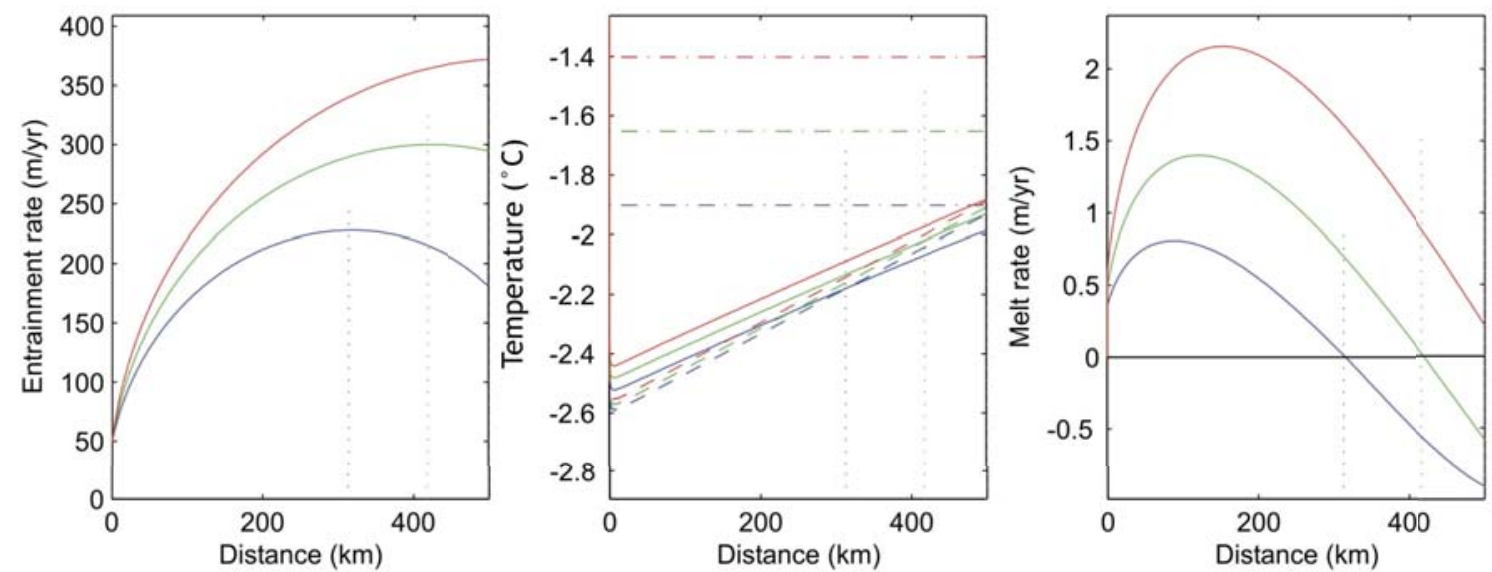

Figure 12: Entrainment rate (left), temperature (middle) and melt rate (right) for different ambient ocean temperatures. The warmest ambient ocean temperature is given in red and the coolest is blue. The dashed line gives the freezing temperature in the middle panel and the solid line gives the plume temperature.

heat lost to the ice shelf in the production of melt water are both proportional to velocity. Melting is also proportional to the temperature difference across the ice-ocean boundary layer. Entrainment warms the plume towards the ambient temperature but melting cools it towards the freezing point. As the plume grows in volume and rises towards the surface, warming by entrainment becomes less effective, leading to freezing.

Warming the ambient ocean increases the effectiveness of entrainment, so the temperature difference across the boundary layer also increases. More rapid melting implies greater buoyancy and a faster plume. Entrainment and melting both rise in response. Eventually, the zone of freezing is eliminated. In Fig. 12 we see that it takes longer for the entrainment rate to decrease in a warmer ocean and it takes longer for the freezing to begin. This begins to quantify how changing ambient ocean temperatures will affect plumes and the melting of ice shelves.

\subsubsection{Melt rate scaling}

In this section we will derive the temperature dependence of the melt rate. The melt rate has a non-linear dependence on the thermal driving, the difference between the ambient ocean temperature and the freezing point or $T_{a}-T_{b}$. From the thermal boundary condition in the boundary layer (Eq. 39), since the latent heat of fusion is generally much larger than the heat required to bring ice up to the melting point, this equation scales like

$$
C_{d}^{1 / 2} \Gamma_{T} U\left(T-T_{b}\right) \sim \dot{m} \frac{L}{c}
$$

or that

$$
\dot{m} \sim U\left(T-T_{b}\right)
$$

where $T$ is the temperature of the plume. 


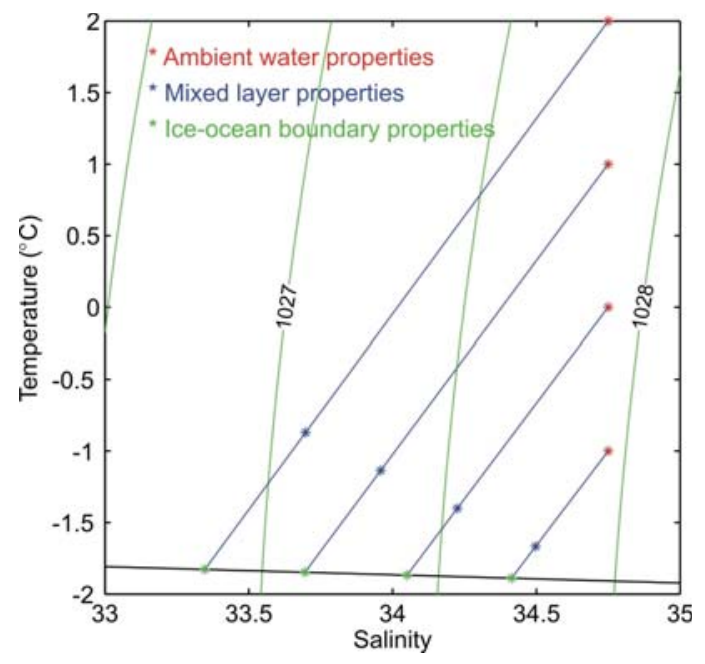

Figure 13: Mixing of the water masses.

The plume is ambient water that is modified by the addition of meltwater. Right at the ice shelf base, the water is at the freezing point. The properties of the plume water lie in between the properties of the boundary and the ambient (Fig. 13). This is evident when considering the case in steady state, where the heat entrained by mixing is equal to the heat used to melt, or

$$
U E_{0} \sin \theta\left(T_{a}-T\right) \approx U C_{d}^{1 / 2} \Gamma_{T}\left(T-T_{b}\right)
$$

so that

$$
\frac{\left(T-T_{b}\right)}{\left(T_{a}-T\right)} \approx \text { constant }
$$

or a function of slope. Thus,

$$
\left(T-T_{b}\right) \sim\left(T_{a}-T_{b}\right)
$$

or this temperature difference scales linearly with the thermal driving. Our scaling then becomes

$$
\dot{m} \sim U\left(T_{a}-T_{b}\right)
$$

Now we consider the scaling for the velocity. From the momentum equation, assuming along slope changes are small, we have the scaling that

$$
U^{2} \sim \Delta \rho \sim\left(T_{a}-T\right)
$$

Using the linear relationship with temperature again, we write that

$$
U \sim \sqrt{T_{a}-T_{b}}
$$

Finally this gives us the melt rate dependence on the temperature difference,

$$
\dot{m} \sim\left(T_{a}-T_{b}\right)^{3 / 2}
$$

That scaling is confirmed by solving the entire set of equations where we see that that the melt rate depends on $\left(T_{a}-T_{b}\right)^{3 / 2}$ (Fig. 14). While the relationship is always of the form given in (41), the proportionality depends on the slope of the ice-ocean interface. Steeper slopes increase the sensitivity to temperature change. 


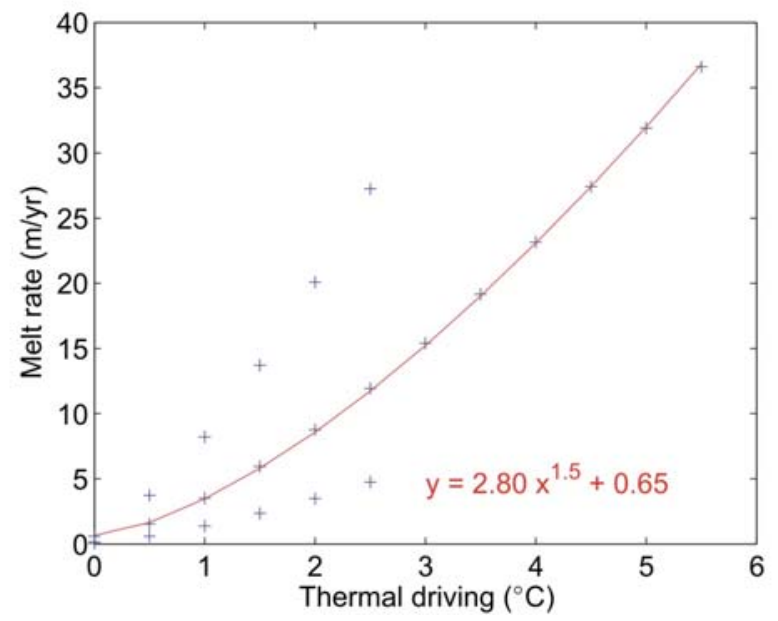

Figure 14: A figure showing the relationship between melt rate and the driving temperature.

\section{References}

[1] S. Kimura, Nicholls K. W., and Venables, E. (2015) Estimation of ice shelf melt rate in the presence of a thermohaline staircase. J. Phys. Oceanogr. 45, 133.

[2] A. M. Yaglom and Kader, B.A. (1974 ) Heat and mass transfer between a rough wall and turbulent fluid flow at high Reynolds and Péclet numbers. J. Fluid Mech. 62, 601.

[3] M. G. McPhee, Kottmeier, C. and Morison, J.H. (1999) Ocean heat flux in the Central Weddell Sea during winter. J. Phys. Oceanogr. 29, 1166.

[4] A. J. Wells and Worster, M.G. (2008) A geophysical-scale model of vertical natural convection boundary layers. J. Fluid Mech. 609, 111.

[5] H. E. Huppert and Turner, J.S. (1980) Ice blocks melting into a salinity gradient J. Fluid Mech. 100, 367.

[6] R. C. Kerr and McConnochie, C.D. (2015) Dissolution of a vertical solid surface by turbulent compositional convection. J. Fluid Mech. 765, 211.

[7] A. Jenkins (2016) A simple model of the ice shelf-ocean boundary layer and current. J. Phys. Oceanogr. 46, 1785.

[8] W. M. J. Lazeroms, Jenkins, A., Gundmundsson, G.H., and van de Wal, R.S.W. (2018) Modelling present-day basal melt rates for Antarctic ice shelves using a parametrization of buoyant meltwater plumes. The Cryosphere 12, 49. 


\section{GFD 2017 Lecture 6: Ocean Circulation beneath Ice Shelves}

Adrian Jenkins; notes by Thomasina Ball and Robert Fajber

May 17, 2018

\section{Insights from Plume Theory}

\subsection{Impact of meltwater outflow at the grounding line}

Near the grounding lines of ice shelves and tidewater glaciers there is often an outflow of freshwater. The buoyancy flux from this meltwater will initially dominate over buoyancy due to melting to form a simple plume. As shown in figure1, the plume rises up the ice face entraining the ambient more saline water. This supplies heat which drives melting of the ice face adding further buoyancy to the plume and in turn driving the flow. The generation of meltwater at the grounding line can be due to a combination of factors. For glaciers in polar regions, geothermal heating and frictional heating causes melting at the base of the glacier, which drains through to the grounding line. In contrast, for more temperate glaciers, the freshwater is generated by surface melting and rain which drains to the base of glacier and then flows along to the grounding line.

We consider the full model derived in the previous lecture [3] that describes the conservation of mass, momentum, heat, and salt, respectively,

$$
\begin{gathered}
\frac{d}{d X}(D U)=\dot{e}+\dot{m}, \\
\frac{d}{d X}\left(D U^{2}\right)=D \Delta \rho g \sin \alpha-C_{d} U^{2}, \\
\frac{d}{d X}(D U T)=\dot{e} T_{a}+\dot{m}\left[T_{b}-\frac{L}{c}\right], \\
\frac{d}{d X}(D U S)=\dot{e} S_{a}+\dot{m} S_{i},
\end{gathered}
$$

where subscripts $i, a$ and $b$ are for water properties evaluated in the ice, ambient and at the ice-ocean boundary, respectively; $\alpha$ is the slope angle, and $C_{d}$ is the drag coefficient. The entrainment is assumed proportional to the speed of the plume and written as $\dot{e}=E_{0} U \sin \alpha$. By defining the density contrast $\Delta \rho$ and thermal driving $\Delta T$ of the plume as

$$
\Delta \rho=\beta_{S}\left(S_{a}-S\right)-\beta_{T}\left(T_{a}-T\right),
$$




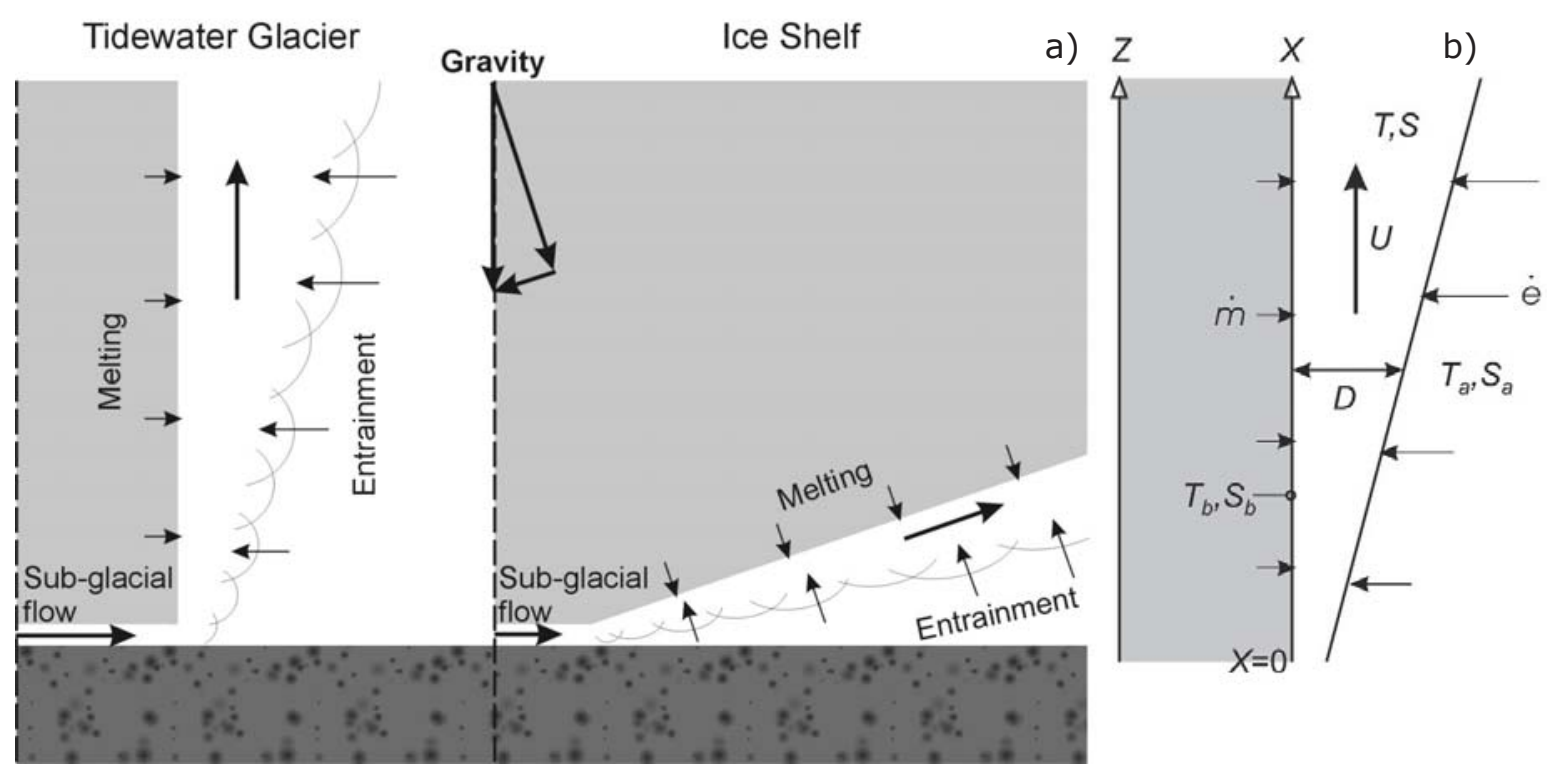

Figure 1: (a) Diagram of a plume originating from a flow of freshwater at the grounding line. (b) Schematic of the plume model with key variables indicated. From [3].

$$
\Delta T=T-T_{f} \quad \text { where } \quad T_{f}=\lambda_{1} S+\lambda_{2}+\lambda_{3} Z_{b},
$$

and using equations (1-4), evolution equations for the buoyancy and sensible heat flux can be found,

$$
\begin{gathered}
\frac{d}{d X}(D U \Delta T)=\left(T_{a}-T_{a f}\right) \dot{e}+\left(T_{i}^{e f}-T_{i f}\right) \dot{m}-\left(\lambda_{3} \sin \alpha\right) D U \\
\frac{d}{d X}(D U \Delta \rho)=\left(\frac{d \Delta \rho_{a}}{d Z} \sin \alpha\right) D U+\Delta \rho_{i}^{e f} \dot{m},
\end{gathered}
$$

where $T_{i}^{e f}=T_{f}-\frac{L}{c}-\frac{c_{i}}{c}\left(T_{f}-T_{i}\right)$ is the effective meltwater temperature and $\Delta \rho_{i}^{e f}=$ $\beta_{S}\left(S_{a}-S_{i}\right)-\beta_{T}\left(T_{a}-T_{i}^{e f}\right)$ is the effective meltwater density contrast.

When the flow of freshwater at the grounding line is large, the initial buoyancy flux dominates the flow, and hence, terms involving feedback due to melting can be neglected (e.g. setting $\dot{m}=0$ in equations $(1,2,7,8)$ ). In an unstratified ambient ocean, $d \Delta \rho_{a} / d Z=0$, and neglecting the pressure dependence on the freezing point, $\lambda_{3}=0$, there is a simple solution where the plume increases linearly in thickness and all other parameters remain constant. Substituting in ansatz $D=A X^{d}, U=B X^{u}, \Delta \rho=C X^{p}$ and $\Delta T=D X^{t}$ results in $d=1, u=0, p=-1$ and $t=0$. Hence, the solution is given by

$$
\begin{gathered}
U_{0}=\left(\frac{B_{0} \sin \alpha}{E_{0} \sin \alpha+C_{d}}\right)^{1 / 3}, \quad D=E_{0} \sin \alpha X, \quad B_{0}=g D_{0} U_{0} \Delta \rho_{0} \\
\left(T-T_{f}\right)_{0}=\left(\frac{E_{0} \sin \alpha}{E_{0} \sin \alpha+C_{d}^{1 / 2} \Gamma}\right)\left(T_{a}-T_{a f}\right) .
\end{gathered}
$$


a)

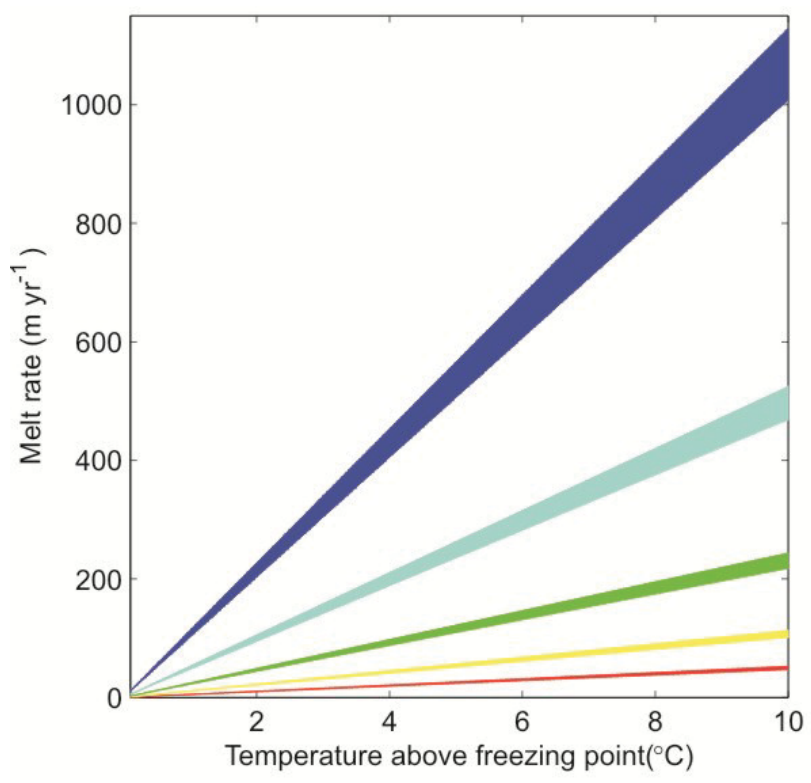

b)

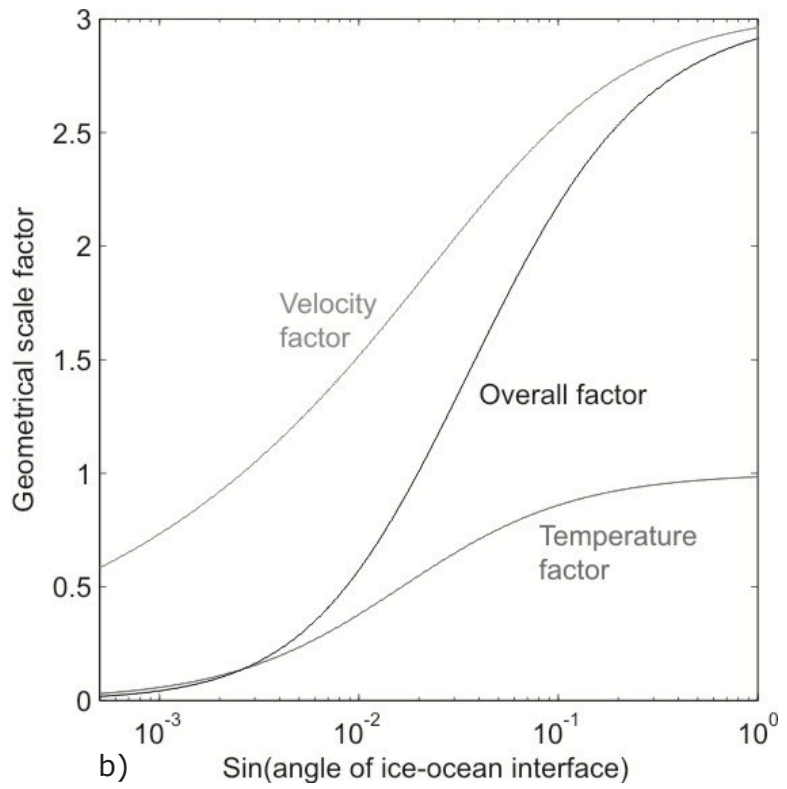

Figure 2: (a) Melt rate $\dot{m}$ plotted against thermal driving showing the linear relationship. The line width represent the spread of gradients for a the range of salinities from 25-35. (b) The geometrical factors, the second and third factors in melt rate in equation 11, plotted against the slope of the ice-ocean interface demonstrating the strong dependence on geometry. From [3].

The melt rate is then derived from the heat balance at the ice-ocean interface where the sensible heat is balanced by the heat flux from the plume mixture,

$$
\dot{m}_{0} L+\dot{m}_{0} c_{i}\left(T_{f}-T_{i}\right)=c C_{d}^{1 / 2} U_{0} \Gamma_{T}\left(T-T_{f}\right)_{0}
$$

Rearranging and substituting in $U_{0}$ and $\left(T-T_{f}\right)_{0}$ from equation (9) then gives the constant melt rate as

$$
\dot{m}_{0}=\left(\frac{c C_{d}^{1 / 2} \Gamma}{L+c_{i}\left(T_{f}-T_{i}\right)}\right)\left(\frac{\sin \alpha}{E_{0} \sin \alpha+C_{d}}\right)^{1 / 3}\left(\frac{E_{0} \sin \alpha}{E_{0} \sin \alpha+C_{d}^{1 / 2} \Gamma}\right) B_{0}^{1 / 3}\left(T_{a}-T_{a f}\right) .
$$

The melt rate in equation 11 is made up of several factors. The first factor is made up of physical constants such as the drag coefficient, heat capacity, transfer coefficients and the latent heat of fusion for ice. The second and third factors come from the dependence of the velocity and temperature gradient on the slope of the interface. The fourth and fifth factors identify the linear dependence on the thermal driving from the ambient ocean and the cube root dependence on the buoyancy flux, see figure 2.

The approximations made thus far allow progress to be made analytically but are unrealistic in terms of modeling plume dynamics at the ice-ocean interface. Therefore, the ambient stratification, increasing freezing point with depth and feedback from melting need to be included. The full system given by equations $(1,2,7,8)$ can be normalised by the scales 
found in equation (9). A scale for the along slope distance $X$ can be found by considering the lengthscale over which melting balances the initial amount of buoyancy from the grounding line,

$$
L_{0}=\frac{B_{0}}{\dot{m}_{0} \Delta \rho_{i}^{e f} g} .
$$

\subsection{Impact of ambient properties}

Ambient conditions can also be important in the plume dynamics. Figure 3 shows dimensionless solutions for the melt rate, volume flux, momentum flux, thermal driving, buoyancy flux, and sensible heat flux against the distance along slope for increasing stratifications. Consider first the unstratified case (red line in figure 3). As the plume rises, entrainment of the ambient ocean provides heat to keep the plume above the freezing point, with more heat required as the volume flux increases. However, as the plume rises the freezing point increases and in turn decreases the sensible heat flux; the ambient thermal driving can be seen to fall almost linearly as a result. There is a transition from melting to freezing when the thermal driving equals zero. As thermal driving continues to decrease all of the meltwater is subsequently refrozen. As the ambient stratification is increased, towards the purple line, the buoyancy flux reaches its maximum further downslope and hence loses momentum before all of the meltwater has frozen out. By increasing the stratification further, the section of freezing can be reduced to zero before the plume runs out of momentum.

The importance of the ambient conditions can be summarised in two key lengthscales. The first is the lengthscale over which the plumes' buoyancy changes and can be written as

$$
L_{\rho a}=\frac{\Delta \rho_{0}}{(d \Delta \rho / d Z) \sin \alpha} .
$$

The second is the lengthscale over which thermal driving changes,

$$
L_{t f}=\frac{\left(T-T_{f}\right)_{0}}{\lambda_{3} \sin \alpha}
$$

initially recognised by [6] to be an important lengthscale characterizing the distance from the source to the ambient freezing point.

Slater et al [10] looked at the importance of lengthscale $L_{\rho a}$ when the plume buoyancy is dominated by subglacial discharge, and hence feedback from submarine melting on the plume can be neglected, and $L_{T F}=\infty$. Initially they considered a uniform stratification, $L_{\rho a}=\infty$, and found that melt rate scaled with $B_{0}^{1 / 3}$ regardless of plume geometry providing discharge was below a critical value. This is consistent with the results derived above in equation (11). The addition of temperature stratification increased the sensitivity of the plume temperature to subglacial discharge. However, when the initial buoyancy at the grounding line is taken to be a point source the temperature in the plume becomes independent of discharge and so they found the exponent to be only slightly different from $1 / 3$. Finally, if the salinity or temperature and salinity set the stratification, the melt rate exponent can vary from $1 / 3$ to as large as $2 / 3$ depending on other plume conditions. These higher exponents suggest that melt rates may depend more on subglacial discharge than previously thought. 

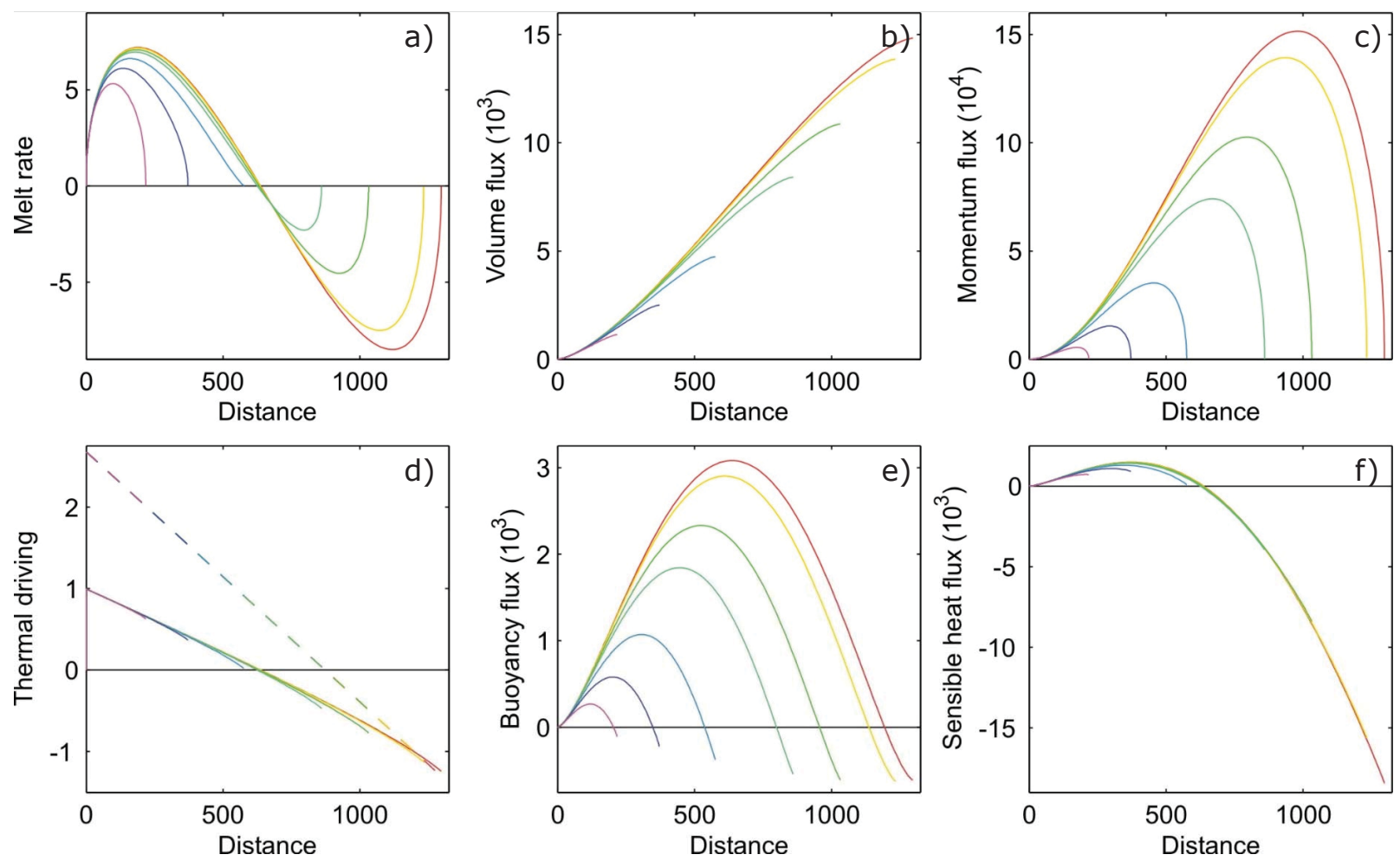

Figure 3: Change in plume dynamics depending on the ambient stratification. Dimensionless solutions for (a) melt rate, (b) volume flux, (c) momentum flux, (d) thermal driving, (e) buoyancy flux and (f) sensible heat flux plotted against distance along slope from the grounding line for an initial fresh water flux of $5 \times 10^{-5} \mathrm{~m}^{2} \mathrm{~s}^{-1}$ with slope $\sin \alpha=0.01$. Colored lines indicate varying ambient stratification with zero stratification given by the red line and ambient stratification of $-1 \times 10^{-6} \mathrm{~m}^{-1}$ given by the purple line. From [3]. 

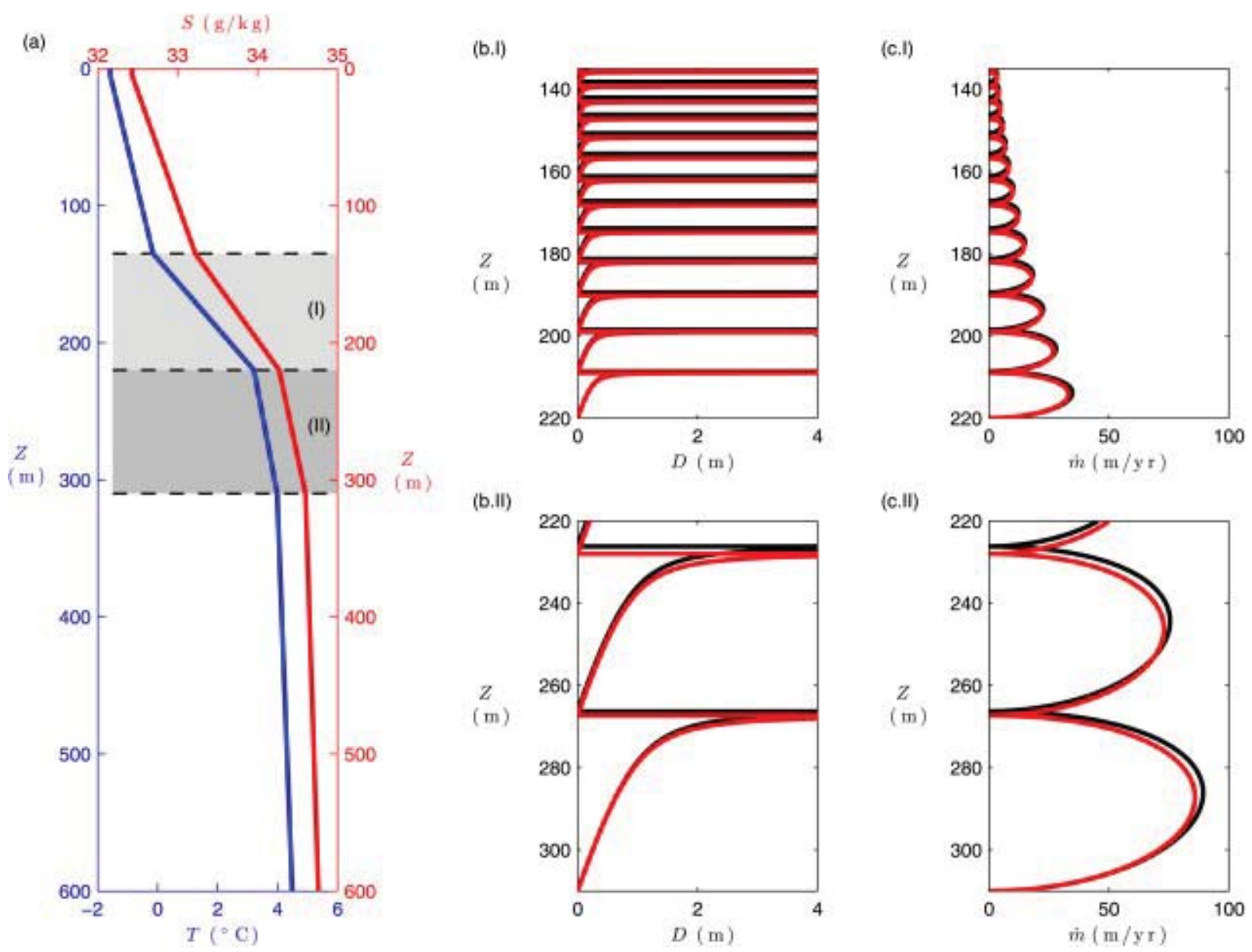

Figure 4: Case study of Helheim glacier in Sermelik fjord. (a) Linear approximation to change in temperature (blue line) and salinity (red line) with depth motivated by observations. Variation of width (bI and bII) and melt rate (cI and cII) with depth for the two regions shaded in grey in (a). From [7].

Conversely, Magorrian and Wells [7] studied the case when the initial discharge at the grounding line is zero and the buoyancy is dominated by meltwater from the ice-ocean interface, again with $L_{T F}=\infty$. They applied their theoretical and numerical results to a case study of melting of Helheim glacier in Sermelik fjord, Greenland, in winter, see figure 4. The numerical solution showed a repeated layered intrusion pattern as the plume reached its neutral buoyancy and the width $d$ diverged. Further melting at the ice-ocean interface then starts the next intrusion. They argue that the layered melting pattern would lead to the formation of notches on the ice-ocean interface. As the depth decreases, the temperature and salinity decreases reducing thermal driving and melt rate causing the layered scaling to decrease, as seen in figure 4.

To identify when the governing lengthscales become important, the size of the lengthscale can be plotted against the thermal driving, temperature above the freezing point. Figure (5) compares the three lengthscales given in equations 12, (13) and (14) for an ice shelf and a 

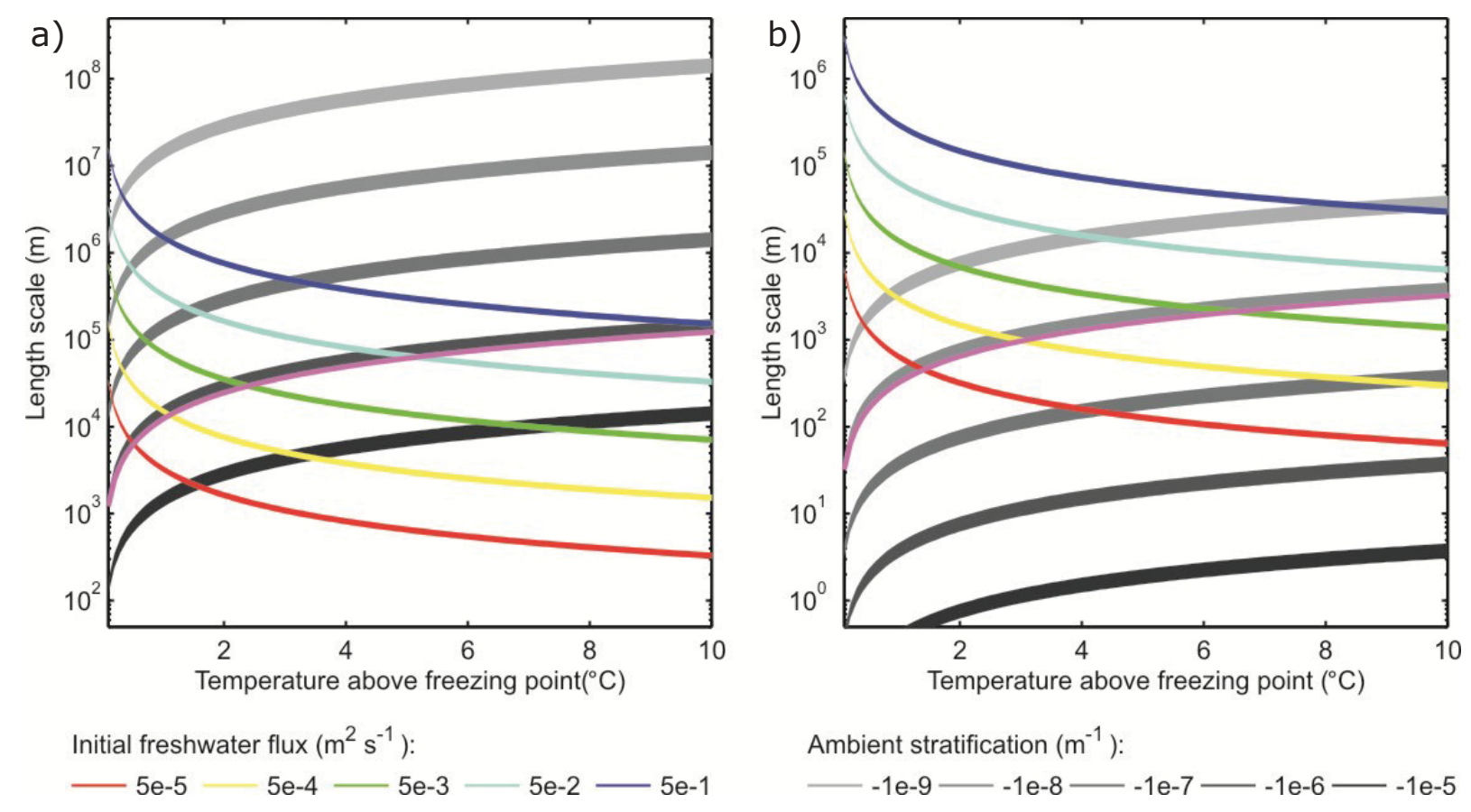

Figure 5: Governing lengthscales for a plume flowing upslope along an ice-ocean interface of slope (a) $\sin \alpha=0.01$ (ice shelf grounding line) and slope (b) $\sin \alpha=1$ (tidewater glacier). The coloured lines are plots of $L_{0}$ for different initial freshwater fluxes, the grey lines are $L_{\rho a}$ for a range of ambient stratifications, and the magnenta line is $L_{T F}$. From [3].

tidewater glacier. For the ice shelf, 5(a), other than the strongest stratification (black line), $L_{T F}$ is the first lengthscale that becomes important for scales of hundreds of metres to tens of kilometres. Below this lengthscale the approximation of a plume in an unstratified environment, with freezing temperature independent of depth and no feedback from melting on the buoyancy of the plume is valid; see section 1.1. For a tidewater glacier, 5(b), entrainment is more important with the strongest stratification limiting the approximation in section 1.1 to tens of metres.

Slater et al [10] and Magorrian and Wells [7] both looked at the role of $L_{\rho a}$ in plume evolution. To consider the impacts of $L_{T F}$ we need to return to the full model. We can run the model for a range of basal slopes from $10^{-3}$ to $10^{-2}$ with a grounding line depth of $500 \mathrm{~m}$ and ambient water temperatures from 0 to $7 \mathrm{C}$ above the surface freezing point to get a series of melt rate curves. Figure $6(\mathrm{~b})$ shows these melt rates where the evolution of the plume is stopped by the plume reaching the surface (termination of the ice shelf).

From the lengthscale recognised by Lane-Serff [6], see equation (14), one can see that the plume dynamics are unchanged by a linear transformation of the ambient temperature profile. Thus, we can construct equivalent ambient temperature profiles with a surface temperature fixed at $T_{f}$ by varying grounding line from depths of $500 \mathrm{~m}$ to $10000 \mathrm{~m}$. Running the model for these new profiles produces a series of melt rates given in figure $6(\mathrm{c})$, where again the solution is stopped by reaching the ice shelf edge.

In order to understand the role of lengthscales in the problem we would like to collapse 

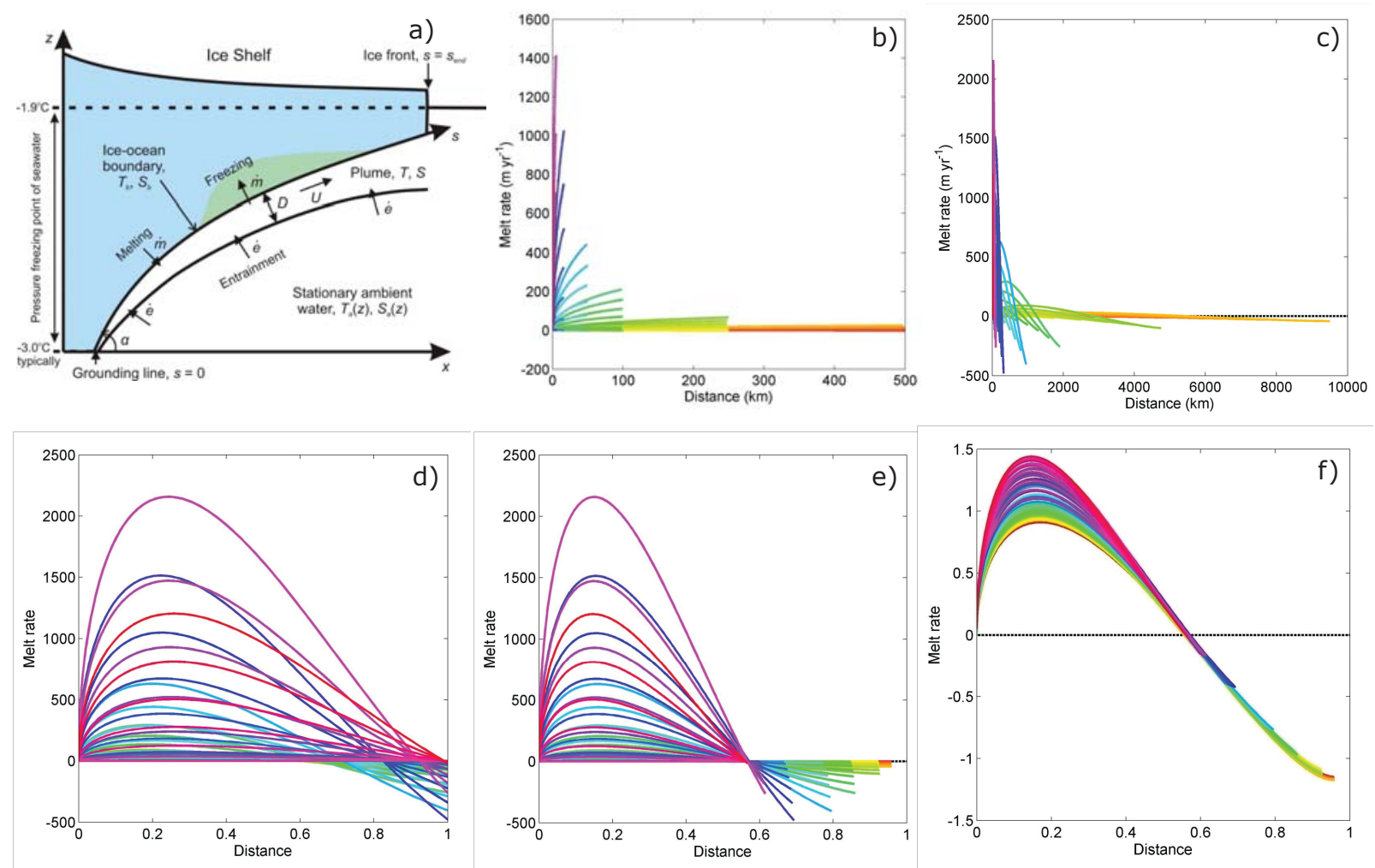

Figure 6: (a) Schematic of full solution. (b) Melt rate against (m/yr) against horizontal distance away from the grounding line for a range of ice shelves with a grounding line $(\mathrm{km})$ depth of $500 \mathrm{~m}$, basal slopes from $10^{-3}$ to $10^{-2}$ and ambient ocean temperatures from 0 to $7 \mathrm{C}$. (c) Melt rate $(\mathrm{m} / \mathrm{yr})$ against distance away from the grounding line $(\mathrm{km})$ for a fixed surface freezing point temperature and grounding line depths from $500 \mathrm{~m}$ to $10000 \mathrm{~m}$. (d) Melt rate (m/yr) against dimensionless distance away from the grounding line. (e) Melt rate (m/yr) for a rescaled dimensionless distance away from the grounding line. (f) Dimensionless melt rate against dimensionless distance away from the grounding line. See section 1.2. 
all of the lines onto a universal curve by scaling the melt rate and distance from the origin suitably. By scaling the distance by $L_{T F}$ the graph transforms to 6(d). Here the transition between melting and refreezing doesn't coincide exactly for all curves because the balance between entrainment and melting is a function of the basal slope. Rearranging equation (3) we have

$$
D U \frac{d T}{d X}=\dot{e} T_{a}+\dot{m}\left[T_{b}-\frac{L}{c}\right]-(\dot{e}+\dot{m}) T .
$$

By balancing the heat due to entrainment and latent heat that goes into melting we can get a scaling for the thermal driving,

$$
\begin{aligned}
& E_{0} U \sin \alpha\left(T_{a}-T\right)-C_{d}^{1 / 2} U \Gamma_{T}\left(T-T_{b}\right) \simeq 0, \\
& \Rightarrow\left(T-T_{b}\right) \simeq \frac{E_{0} \sin \alpha}{C_{d}^{1 / 2} \Gamma_{T}+E_{0} \sin \alpha}\left(T_{a}-T_{b}\right) .
\end{aligned}
$$

The prefactor here allows us to rescale $L_{T F}$ such that the transition between melting and freezing is the same for each run, see figure $6(\mathrm{e})$.

Finally, the melt rate scaling comes from the plume speed and thermal driving. From equation (2) we have

$$
D U \frac{d U}{d X}=D \Delta \rho g \sin \alpha-C_{d} U^{2}-(\dot{e}+\dot{m}) U
$$

By balancing the momentum due to plume buoyancy with entrainment and friction, we can get a scaling for plume velocity,

$$
U^{2} \simeq \frac{\sin \alpha}{C_{d}+E_{0} \sin \alpha} D \Delta \rho g
$$

By considering the remainder of the thermal driving budget we have

$$
\left(T_{a}-T\right) \simeq \frac{C_{d}^{1 / 2} \Gamma_{T}}{C_{d}^{1 / 2} \Gamma_{T}+E_{0} \sin \alpha}\left(T_{a}-T_{b}\right),
$$

which can be used to scale the buoyancy $\Delta \rho$. Hence, this finally allows the solutions to collapse on to one universal curve, see figure $6(\mathrm{f})$.

\subsection{Adding further processes}

The model investigated thus far has only considered the refreezing of meltwater at the iceocean interface. In reality, freshwater can freeze in the plume in the form of suspended disc-shaped frazil ice crystals [5]. This increases the buoyancy and causes the plume to accelerate which in turn promotes rapid crystal growth creating a positive feedback. If the ice crystals are able to deposit out in a manner opposite to sedimentation this reduces the bulk density causing the plume to decelerate and hence allowing crystals to settle out more easily. The formation of frazil ice comes in intense bursts that settle out in discrete intervals. These high rates of accumulation on the order of $1 \mathrm{~m} / \mathrm{yr}$ then give a mechanism for creation of thick layers of marine ice beneath ice shelves [1]. 
The coupling between ice shelf geometry and plume flow can also provide a mechanism for positive feedback. Le Brocq et al [2] used satellite imagery to show that channelisation often forms on the base of ice shelves. They found that these channels coincide with the predictions of outflow locations of freshwater at the grounding line. This suggests that meltwater plumes create ice-shelf channels which in turn focus plume flow promoting further melting in the channels. These features have been explored numerically in the form of 2D fully coupled ice-shelf/sub-ice shelf ocean models [9], which has shed light on the dynamics involved in the formation of these channels. However, plume models have yet to be able to simulate realistic circulation and melt rates beneath ice shelves.

\section{Models of the 3D Circulation within a Cavity}

\subsection{Structure normal to the ice-ocean interface}

To study the structure of the circulation normal to the ice-ocean interface, a simplified version of the rotated viscous Boussinesq equations are used. Since the focus is on the vertical structure, the gradients along the shelf are assumed to vanish, which eliminates the horizontal advection and diffusion terms. This leaves

$$
\begin{aligned}
\frac{\partial u}{\partial t}-\phi v & =\Delta \rho g \sin \alpha-g \cos \alpha \frac{\partial \eta}{\partial x}+\frac{\partial}{\partial z}\left(\nu \frac{\partial u}{\partial z}\right) \\
\frac{\partial v}{\partial t}+\phi u & =-g \cos \alpha \frac{\partial \eta}{\partial y}+\frac{\partial}{\partial z}\left(\nu \frac{\partial u}{\partial z}\right)
\end{aligned}
$$

where $\eta$ is the deviation of the ice-ocean interface from its equilibrium position, and $\phi$ is the Coriolis parameter in the rotated system. Under the same assumptions the equations for conservation of energy and salinity become

$$
\begin{aligned}
& \frac{\partial T}{\partial t}=\frac{\partial}{\partial z}\left(\kappa_{T} \frac{\partial T}{\partial z}\right), \\
& \frac{\partial S}{\partial t}=\frac{\partial}{\partial z}\left(\kappa_{S} \frac{\partial S}{\partial z}\right) .
\end{aligned}
$$

By linearizing the dependence of the freezing temperature on salinity and pressure, the thermal driving can be expressed as

$$
T_{*}=T-\left(\lambda_{1} S+\lambda_{2}+\lambda_{3} P(\eta)\right),
$$

and applying equation (24) with equations (23) allows us to write a conservation equation for thermal driving as

$$
\frac{\partial T_{*}}{\partial t}=\frac{\partial}{\partial z}\left(\kappa \frac{\partial T_{*}}{\partial z}\right) .
$$


In order to simplify the analysis throughout this section, we will take $\nu=\kappa$ and make $\kappa$ a fixed constant. To relate $T_{*}$ to buoyancy, we will use equation (7).

Equations (7) and (24) can be used to write a simple expression for the ratio of the density difference to the difference in thermal driving due to melting of ice into the plume

$$
\frac{\Delta \rho}{\Delta T_{*}}=\frac{S_{a} \beta_{S}-\beta_{T}\left[T_{* a}+\left(L_{i}-c_{i} T_{* i}\right) c_{w}^{-1}\right]}{T_{* a}+\left(L_{i}-c_{i} T_{*} i\right) c_{w}^{-1}-S_{a} \lambda_{1}} .
$$

Note that the expression includes both the enthalpy required to reduce the ice to the freezing point temperature and the latent heat.

Requiring that the solutions are in equilibrium with the ice-ocean interface (no slip momentum condition and at the freezing point) implies an upper boundary condition of

$$
\mathbf{u}=0, \mathrm{~T}_{*}=0 \text { at } z=0
$$

- Since we are interested in studying the boundary layer near to the ice-ocean interface we will consider solutions that decay to the ambient conditions in the far field. Taking the ambient flows to be in geostrophic balance, this gives the lower boundary of

$$
\mathbf{u}=\frac{i g \nabla \eta}{\phi}, T_{*}=T_{* a} \text { at } z=-\infty .
$$

Similar equations have been used to describe the flow of dense currents down a continental slope. Here, however, the top condition is to fix the temperature to the freezing point, a Dirichlet boundary condition, instead of the zero flux Neumann boundary condition that would be applied at the seabed.

In order to get a lengthscale to normalize the solutions, we start by calculating the Ekman depth for the system. By looking for stationary solutions at the ambient density with the ice-ocean interface at its equilibrium position equations (21) become

$$
\begin{aligned}
-\phi v & =\kappa \frac{\partial^{2} u}{\partial z^{2}}, \\
\phi u & =\kappa \frac{\partial^{2} v}{\partial z^{2}} .
\end{aligned}
$$

These have the well known (bottom layer) Ekman solution:

$$
\begin{aligned}
& u=v_{g}^{i} \exp \left(-\frac{z}{d_{E}}\right) \sin \left(\frac{z}{d_{E}}\right), \\
& v=v_{g}^{i} \exp \left(-\frac{z}{d_{E}}\right) \cos \left(\frac{z}{d_{E}}\right),
\end{aligned}
$$

with relevant scales

$$
\begin{aligned}
v_{g}^{i} & =\frac{g}{\phi} \sin \alpha \frac{\Delta \rho}{\Delta T_{*}}, \\
d_{E} & =\sqrt{\frac{2 \kappa}{|\phi|}} .
\end{aligned}
$$


The depth scale is the e-folding distance of the boundary layer. The velocity scale is the geostrophic current that would occur in the absence of friction along the ice-ocean interface. This is why it is $T_{*} a$ and not $T_{*}$ that appears in $u_{g}^{i}$. When the slope is small, the Coriolis parameter $\phi \approx 2 \Omega \sin \theta \cos \alpha$ and the velocity scale is, incidentally, the same as the Nof speed, which describes the translation of cold eddies along a sloped bottom. The temperature scale is simply chosen to be the thermal driving of the ambient system, $T_{* a}$.

The solution to this system for some simple cases is given in figure 7 . The thermal driving is shown in panels (a) and (d); the solution shows the diffusion of cold water into the far field. This produces a gradually weakening stratification. If a finite domain was used, equation (24) with a constant diffusivity would imply that the steady state solution is just a linear profile joining the thermal driving at the ice-ocean interface and the ambient thermal driving. This is why the transient solutions are studied, since the steady state solution (or the asymptotic solution in the case where the boundary condition is applied in the far field) does not permit a boundary layer.

Panels (b) and (e) show the velocity components of the system when a background pressure gradient is applied with a flat ice-ocean interface. A relatively shallow boundary layer is formed at the surface, and quickly converges to the Ekman solution. This is to be expected, since the Ekman solution is calculated without the influence of the sloped iceocean interface. When the ice interface slope is sloped, as in panels (c) and (f), the boundary layer thickens initially, and the cross slope currents do not appear to converge to the Ekman solution. When the ice interface is sloped, it introduces baroclinicity and links the thermal and current profiles. Thus, the impact of thermal diffusion will be felt on the currents in this case.

We can further explore impacts of the slope of the ice-ocean interface on the response of the boundary layer current by decomposing the long term response of the boundary layer with and without a sloped interface (figure 7, panels (e) and (f), respectively) into geostrophic and ageostrophic components (figure 8). This is done by assuming that the geostrophic current is time dependent and in the cross shelf direction:

$$
\begin{aligned}
\phi v_{g} & =\Delta \rho g \sin \alpha \\
\frac{\partial v_{g}}{\partial t} & =\nu \frac{\partial^{2} v_{g}}{\partial z^{2}} .
\end{aligned}
$$

The time dependence results from the diffusion of less buoyant water away from the ice-ocean interface. This is shown in panels (b) and (e) of figure 8. In the case without a slope in the ice-ocean interface, the geostrophic component has no vertical shear, since the applied forcing is barotropic. In the case with a sloped ice interface condition the geostrophic component shows a vertical structure, in thermal wind balance with the applied baroclinic forcing. The ageostrophic components are assumed to be time independent, and can be shown to the same as the Ekman solution (27). Thus the frictional boundary layer response is unaffected by whether a barotropic or baroclinic forcing is applied to the system (panels (e) and (f) in figure 8).

In general the total response will be a combination of the frictional boundary layer, the applied barotropic and baroclinic forcings, and the ice shelf geometry. For instance, if the baroclinic forcing is chosen to oppose the barotropic forcing, the results can stop or even reverse the upslope current $(u)$ near the ice-ocean interface. For a finite cavity, curvature in 

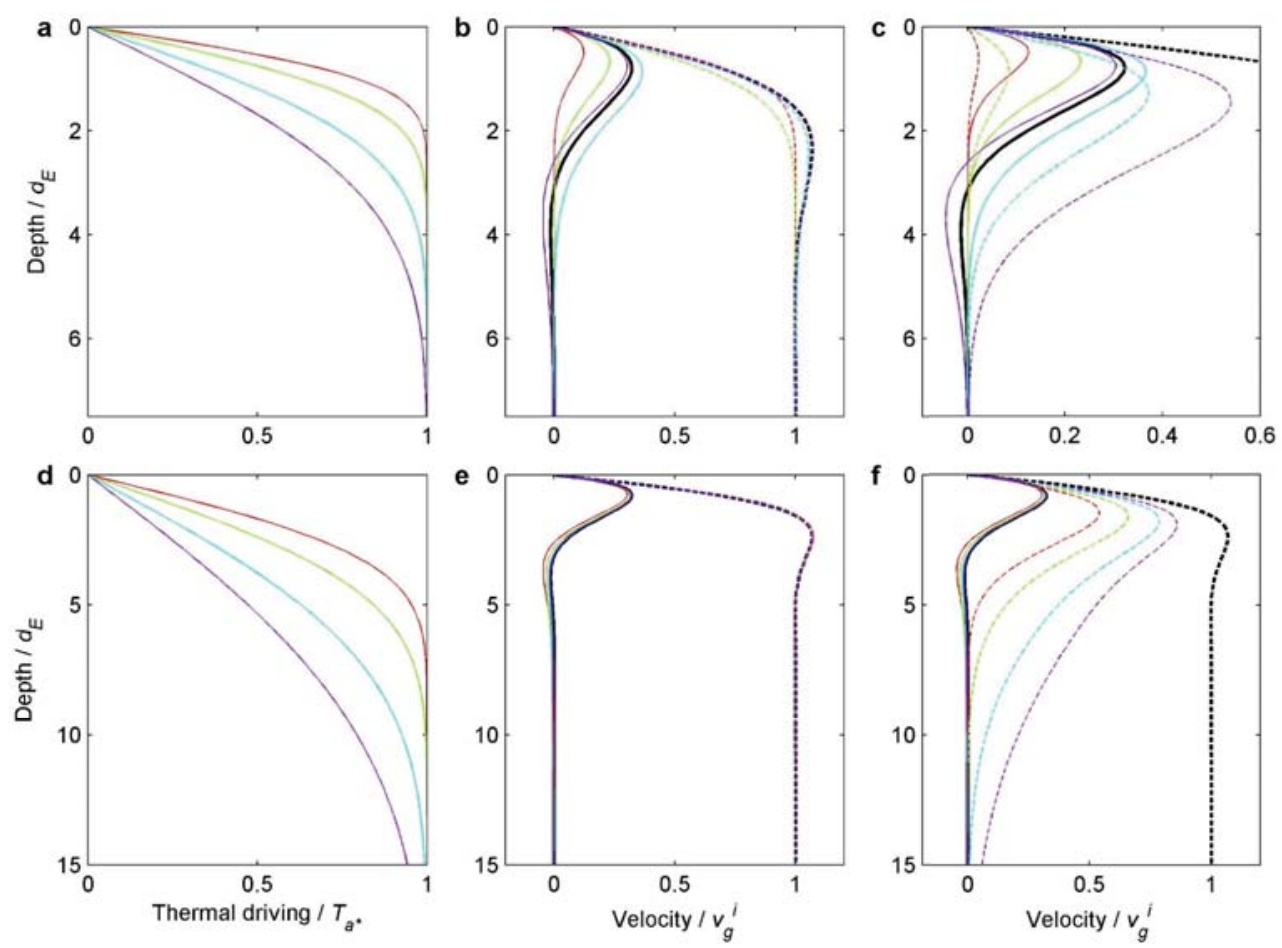

Figure 7: The thermal driving, (a) and (d), and boundary layer currents, (b),(c),(e) and (f), for two simple cases described in the text. The solid lines in (b),(c),(e) and (f) correspond to the up slow flow $(\mathrm{u})$ and the dashed lines correspond to across slope flow. In the top row is the transient solution after 0.1 (red), 0.2(green), 0.5(cyan) and 1.0(magenta) inertial periods, and the bottom row is the transient solution after 1.0 (red), 2.0(green), 5.0(cyan) and 10.0 (magenta) inertial periods, $T=\frac{2 \pi}{f}$. The black lines show the Ekman solution obtained from (29). From [4]

the sea floor bottom, $h$, can also create a current that can oppose the barotropic forcing and oppose the upslope flow, since the planetary vorticity is $\frac{\phi}{h}$.

Another interesting regime occurs when it is assumed that the upslope density gradient balances the turbulent diffusion of upslope momentum instead of the Coriolis term that balances it in the Ekman regime. The density gradient is assumed to be replenished by advection from a steady state upslope current. This results in a solution similar to the Prandtl model of the Katabatic wind

$$
\begin{aligned}
-g \sin \alpha \Delta \rho & =\kappa \frac{\partial^{2} u}{\partial z^{2}} \\
\frac{\partial \Delta \rho}{\partial x} & =\kappa \frac{\partial^{2} \Delta \rho}{\partial z^{2}} .
\end{aligned}
$$

which has solutions 

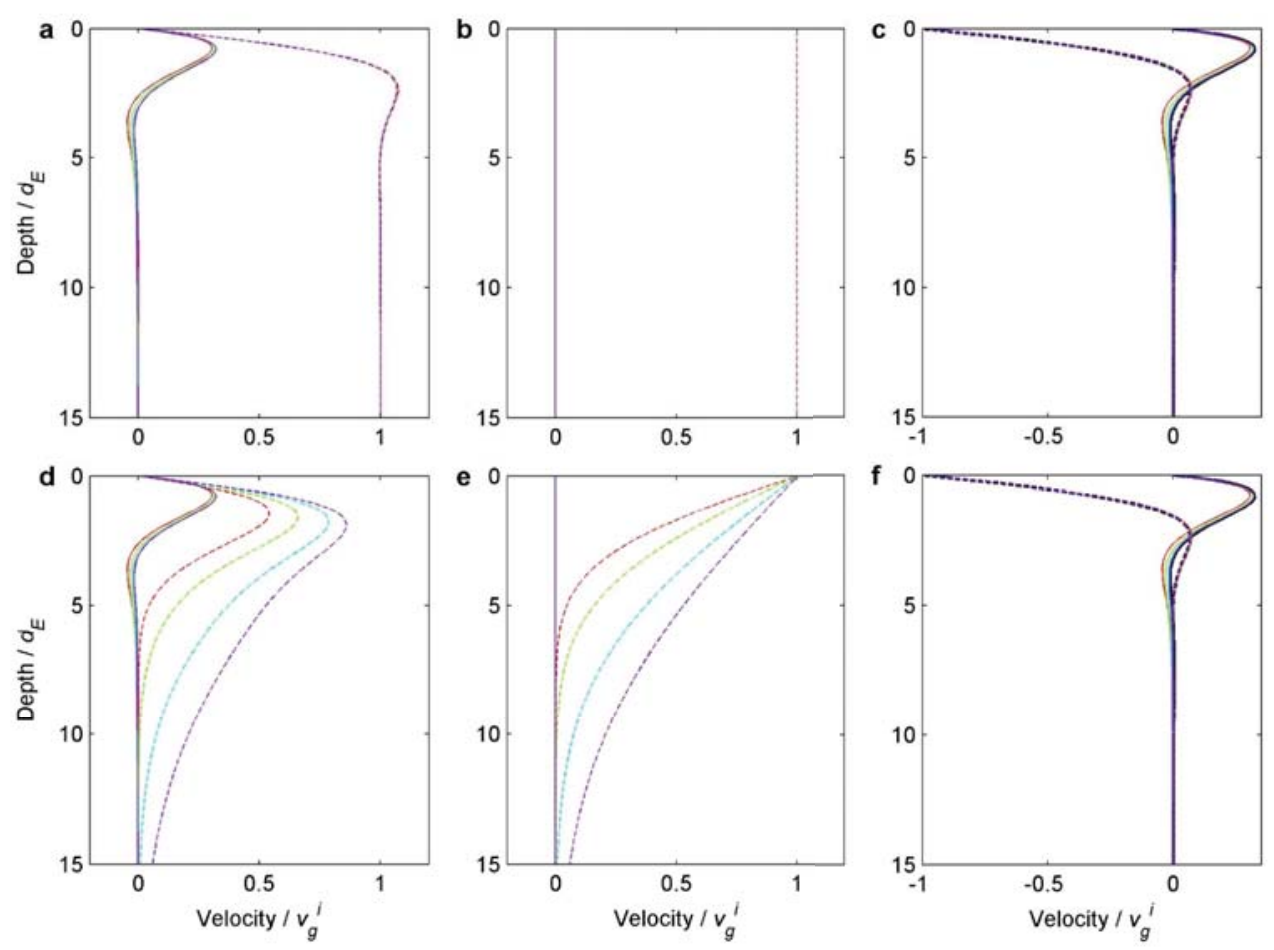

Figure 8: The decomposition of panels (e) (top row) and (f) (bottom row) into their geostrophic (middle column) and ageostrophic (right column) components. The times and current directions indicated by the lines are the same as in panels (e) and (f). From [4].

$$
\begin{aligned}
u & =u_{p} \exp \left(-\frac{z}{d_{P}}\right) \sin \left(\frac{z}{d_{P}}\right) \\
\Delta \rho & =\frac{\Delta \rho}{\Delta T_{*}} T_{* a} \exp \left(-\frac{z}{d_{P}}\right) \cos \left(\frac{z}{d_{P}}\right)
\end{aligned}
$$

where the scales are

$$
\begin{aligned}
& u_{P}=\left(\frac{g \sin \alpha}{\frac{\partial \Delta \rho}{\partial x}}\right)^{\frac{1}{2}} \frac{\Delta \rho}{\Delta T_{*}} T_{* a} \\
& d_{P}=\left(\frac{4 K^{2}}{g \sin \alpha \frac{\partial \Delta \rho}{\partial x}}\right)^{\frac{1}{4}}
\end{aligned}
$$

These solutions look similar to the Ekman solutions, but with a different scaling. Notably, $\phi$ no longer appears in the equations, but instead the horizontal buoyancy gradient, $\partial \Delta \rho / \partial x$ appears.

To determine whether the boundary layer will better resemble the Prandlt or Ekman solution, we note that $u_{P} \exp (-z / d P)$ and $u_{E} \exp \left(-z / d_{E}\right)$ so that $u_{P} \geq_{E}$ when $d_{P} \leq d_{E}$. This is true when 

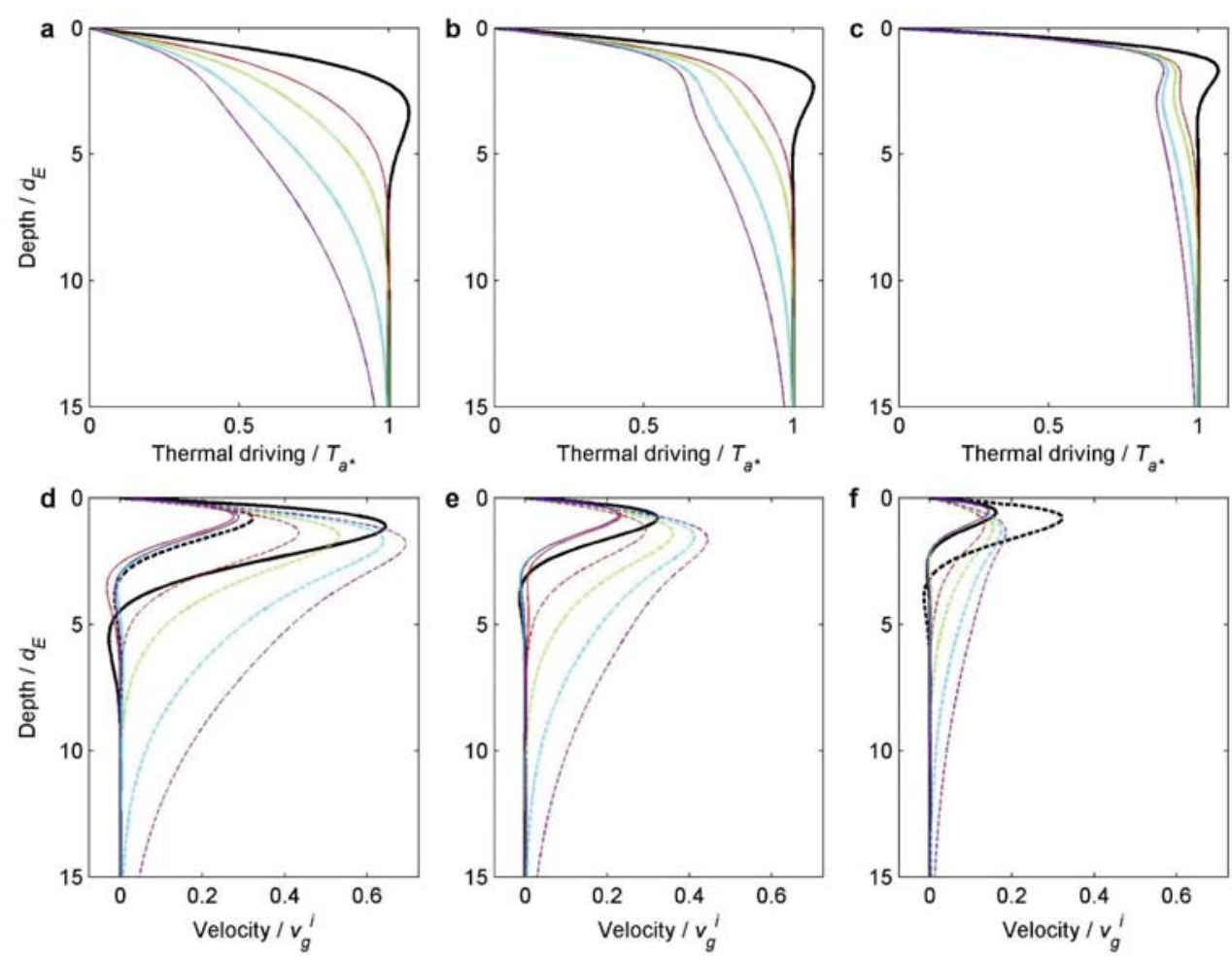

Figure 9: Top Row: the thermal driving for the case with $d_{P} / d_{E}=\sqrt{(2)}$, (left column), $d_{P} / d_{E}=1$ (middle column), and $d_{P} / d_{E}=\sqrt{\frac{1}{2}}$ (right column). The black line is the theoretical Prandtl solution given by (35) The colors indicate the same time periods as in figure 8. Bottom Row: as for the top row, but now for the velocity components. The dashed black line is the theoretical Ekman solution, and the solid black line is the theoretical Prandtl solution. In the middle column they overlie each other. The colors indicate the same as in figure 8. from [4]

$$
\frac{\partial T_{*}}{\partial x} \geq \frac{\Delta T_{*}}{\Delta \rho} \frac{\phi^{2}}{g \sin \alpha}
$$

or equivalently when

$$
\left(g \frac{\partial \Delta \rho}{\partial z}\right)\left(\frac{\sin \alpha}{\phi}\right)^{2} \geq 1
$$

This last equation has the form of a Boundary Layer Burger number. Solutions to the full for a case with upslope temperature advection are shown in figure 9 for differing values of $d_{P} / d_{E}$. For low values of $d_{P} / d_{E}$ the solution has a thick and strong boundary layer near the ice-ocean interface. When the values of $d_{P} / d_{E}$ are higher, the boundary layer becomes thinner and sharper. The addition of the along slope temperature gradient allows for a maintained stratification in a thin layer near the surface. Since, typically, we expect that $\left(g \frac{\partial \Delta \rho}{\partial z}\right)>\phi^{2}$, and for an ice shelf with $\sin \alpha \approx 0$ we would expect the Ekman solution to be more appropriate. For $\sin \alpha \approx 1$ however the Prandtl solution might become appropriate. 
The solutions shown in figure 7 panels (c) and (f) can also be compared with a model with a more realistic diffusivity from a parametrized turbulence closure scheme. We take

$$
\begin{aligned}
\nu & =\lambda u_{*}, \\
u_{*} & =\left(\nu\left|\frac{d u}{d z}\right|\right)^{\frac{1}{2}}, \\
\lambda & =\min \left(\kappa_{v} u_{*}, \lambda_{\max }\right) .
\end{aligned}
$$

This allows us to include the production of TKE from shear layers. Inspection of the solution with the scheme included (figure 10) reveals that the boundary layer becomes trapped in a layer near the surface.

\subsection{From a $1 \mathrm{~d}$ column to the $3 \mathrm{~d}$ circulation}

Now we consider what affects the finite geometry of a real cavity would have on the $1 \mathrm{~d}$ model of the previous section. The pressure at any point in the fluid can be written as

$$
P(z)=g\left[\int_{z_{i o}+\eta}^{z_{s}+\eta} \rho_{i} d z+\int_{z}^{z_{i o}+\eta} \rho d z\right],
$$

and assuming that density of the ice is constant, we find that

$$
\nabla P(z)=g\left[\rho_{i} \nabla H_{i}+\rho_{i o} \nabla\left(z_{i o}+\eta\right)+\int_{z}^{z_{i o}+\eta} \nabla \rho d z\right] .
$$

The first term is the contribution to pressure from the weight of the ice, the second term is the gradient in the ice-ocean interface (both the equilibrium position and the deviation), and the third is the is baroclinic contribution of the (assumed) density profile. Assuming that the weight of the ice shelf is balanced by the water in its equilibrium position, we can write the flotation condition as

$$
\rho_{i} \nabla H_{i}+\rho \nabla z_{i o}=0 .
$$

Applying this equation to the model and solving it over a finite depth reveals a deep cross slope geostrophic flow and a second Ekman layer in the cavity along the seabed. This second Ekman layer creates a mass flux convergence along the grounding line. By conservation of mass, this would raise the ice shelf there and generate an opposing barotropic flow. For a semi-infinite ice sheet we can rationalize this by supposing that this flow is in the across shelf direction. However for an actual $2 \mathrm{~d}$ dimensional cavity this paradox needs to be resolved differently.

In order to understand the structure of the circulation in the cavity we start by constructing a $2 \mathrm{~d}$ idealized model of a steady state current in balance with the pressure gradients applied at the ice-ocean interface. This will allow us to understand the two-dimensional structure imposed by finite cavity geometry. We start by assuming that the momentum equations are in approximate geostrophic balance, with only the vertical momentum diffusion equation term retained: 

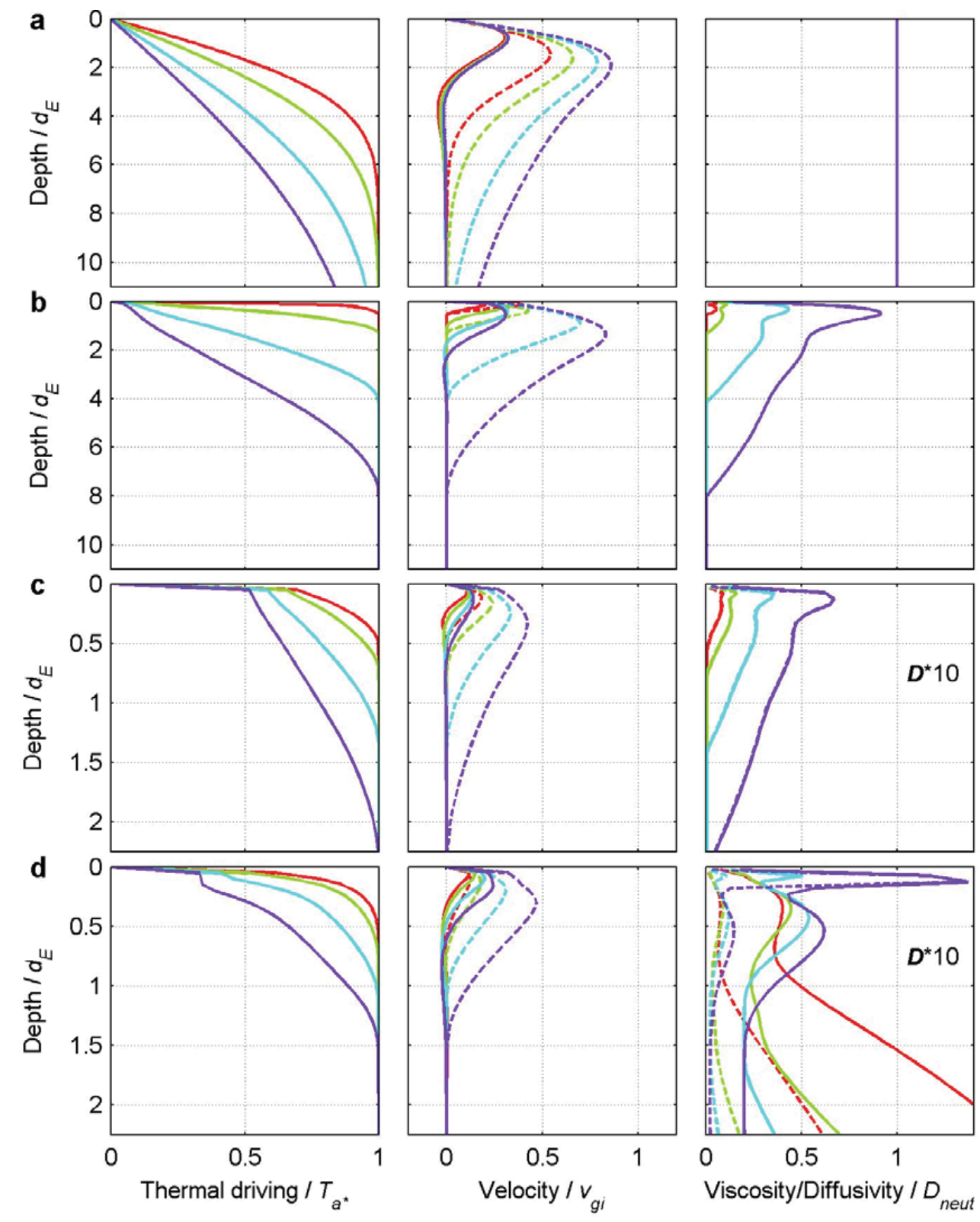

Figure 10: The solutions from a model using a parametrized turbulence closure scheme. The situation is the same as shown for figure 7, panels (c) and (f). The first row uses the scheme described in the text; going down rows the turbulence closure scheme increases in complexity.

$$
\begin{aligned}
-f v & =-\frac{1}{\rho_{0}} \frac{\partial P}{\partial x}+\frac{\partial}{\partial z} \nu \frac{\partial u}{\partial z} \\
f u & =-\frac{1}{\rho_{0}} \frac{\partial P}{\partial y}+\frac{\partial}{\partial z} \nu \frac{\partial v}{\partial z} .
\end{aligned}
$$

The inclusion of the vertical mixing terms allows us to retain the features of Ekman 
solution that were studied in the previous section. The pressure is as given in (42) but with the flotation condition applied and the baroclinic term approximated by the equilibrium height of the ice-ocean interface:

$$
\nabla P=g\left[\rho_{0} \nabla \eta+\int_{z}^{z_{i o}} \nabla \rho d z\right]
$$

The depth integrated geostrophic flow is given by

$$
V_{g}=\frac{g}{f} \mathbf{k} \times\left(H_{w} \nabla \eta+\int_{z}^{z_{i o}} \nabla \rho\left(z-z_{b}\right) d z\right)
$$

where $H_{w}$ is the depth of the water column. The depth integrated ageostrophic velocity comes from the top and bottom Ekman layers and is given by

$$
V_{a}=\frac{d_{E}}{2} \mathbf{k} \times\left(v_{\text {gio }}+v_{g b}\right)+\frac{d_{E}}{2}\left(v_{\text {gio }}+v_{g b}\right),
$$

where the top and bottom geostrophic currents are given by

$$
v_{\text {gio }}=\frac{g}{f} \mathbf{k} \times \nabla \eta
$$

and

$$
v_{g b}=\frac{g}{f} \mathbf{k} \times \nabla \eta+\int_{z_{b}}^{z_{i o}} \nabla \rho d z
$$

respectively. The first term in (49) is a transport normal to the geostrophic flow, and the second term is a transport in the along geostrophic flow created by a reduction in the geostrophic flow speed in the boundary layer.

Mass conservation implies that the divergence of these two currents has to vanish in steady state:

$$
\nabla \cdot\left(\mathbf{V}_{\mathbf{g}}+\mathbf{V}_{\mathbf{a}}\right)=0
$$

Substituting equations (48) and (49) into (52) and assuming a constant linear stratification (constant $N^{2}$ ) profile parallel to the ice shelf gives a second order hyperbolic equation for the deviation of the ice-ocean interface from its equilibrium position:

$$
\begin{array}{cl}
\frac{g}{f}\left[\frac{\partial H_{w}}{\partial y} \frac{\partial \eta}{\partial x}-\frac{\partial H_{w}}{\partial x} \frac{\partial \eta}{\partial y}\right]+\frac{N^{2}}{f} H_{w}\left(\frac{\partial H_{w}}{\partial y} \frac{\partial z_{i}}{\partial x}-\frac{\partial H_{w}}{\partial x} \frac{\partial z_{i}}{\partial y}\right) & + \\
\frac{g}{f} d_{E}\left[\frac{\partial^{2} \eta}{\partial x^{2}}+\frac{\partial^{2} \eta}{\partial y^{2}}\right]+\frac{N^{2}}{f} H_{w} \frac{d_{E}}{2}\left[\frac{\partial^{2} z_{i}}{\partial x^{2}}+\frac{\partial^{2} z_{i}}{\partial y^{2}}\right] & + \\
\frac{N^{2}}{f} \frac{d_{E}}{2}\left[\frac{\partial H_{w}}{\partial x} \frac{\partial z_{i}}{\partial x}+\frac{\partial H_{w}}{\partial y} \frac{\partial z_{i}}{\partial y}-\frac{\partial H_{w}}{\partial y} \frac{\partial z_{i}}{\partial x}+\frac{\partial H_{w}}{\partial x} \frac{\partial z_{i}}{\partial y}\right] & =0
\end{array}
$$

The first term represents the barotropic geostrophic flow caused by the ice ocean interface forced through depth contours. The second term is similar, it is the geostrophic flow being forced by the tilt of the sea level. The second two terms are the Ekman transports that result from the curvature of the ice ocean interface and the sea level, respectively. These terms are similar to the windstress forcing that would occur in an Ekman layer exposed to the atmosphere. The last term is a correction to the second term that results from the depth 

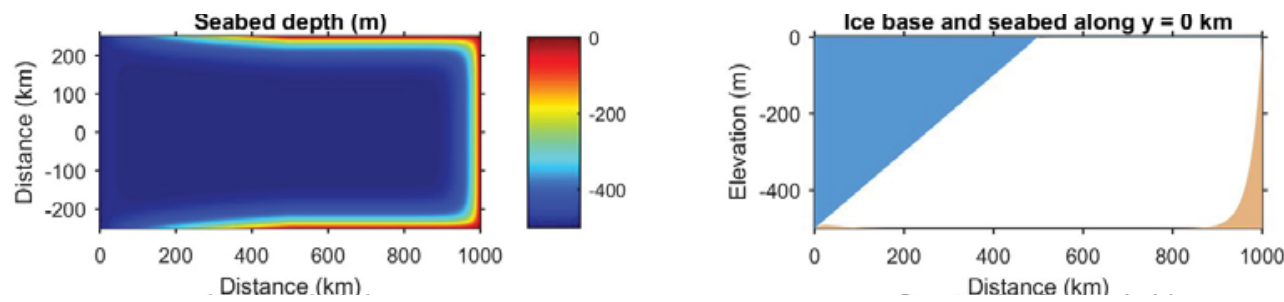

Figure 11: The simplified geometry used to test the circulation models. Left: the seabed depth and Right: a cross section showing the geometry of the ice shelf. Note that the bed has been tapered in order to avoid numerical artifacts.
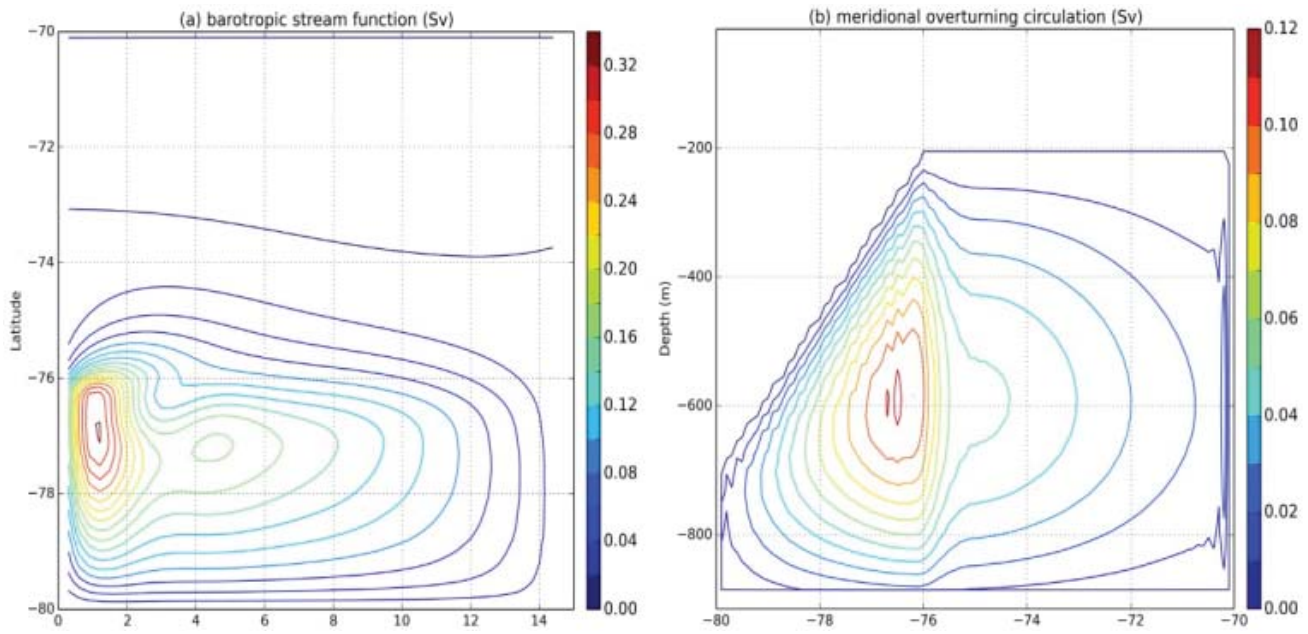

Figure 12: The barotropic streamfunction (left) and the meridional overturning circulation (right) from the primitive equation model using the same geometry show in figure 11. From [8].

of the Ekman depth. Over this depth part of the current in the second term will be canceled by the Ekman divergence, and this is accounted for in the last term.

If boundary conditions are given, this model can be solved for $\eta$. Using a simple test geometry (figure 11) this gives an asymmetric circulation, with increased sea heights on the bottom left side of the domain and decreased sea heights on the upper right side of the domain. It should be noted that since this calculation is done with an f-plane, this intensification is not related to the usual western boundary current intensification, and is instead related to the meridional gradient in water column thickness. These results compare favorably with a primitive equation model (NEMO) run with full physical parametrization (figure 12, left panel). The primitive equation model also shows the full three dimensional circulation, which shows a meridional overturning cell (figure 12, right panel) as well as a melt freeze pattern similar to an ice pump (figure 13). The gyre circulation is imprinted on the melt freeze pattern, and shifts the horizontal structure so that the melting is in the west, and the freezing in the east.

The full primitive equation model can be used to simulate the full circulation beneath all of the antarctic ice shelves, including observed bathymetry. The model simulation shows that 


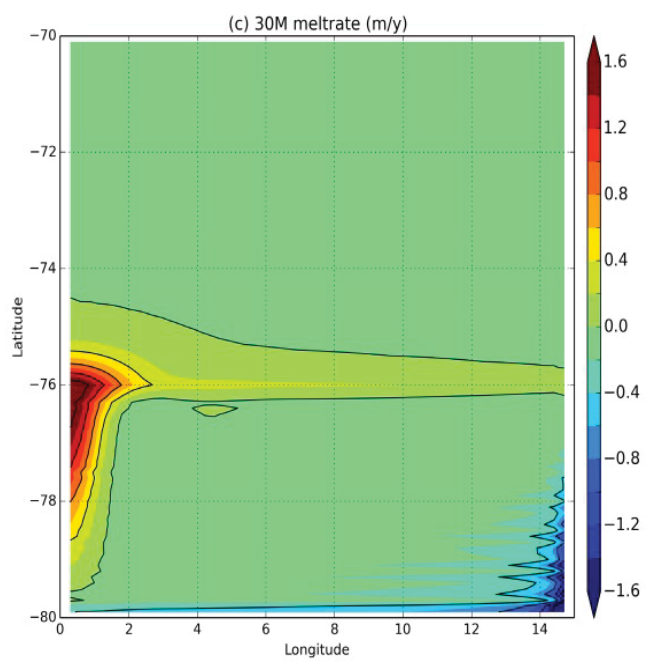

Figure 13: The freezing patterns from the primitive equation model, from [8].

the ice shelves in the warm regions of West Antarctica are rapidly melting (figure 14, panel (a)). In the cold water regions of the Ross and Weddell seas and also East Antarctica the melting occurs more slowly, and there are also extensive areas of refreezing (figure 14, panels (b), (c), and (d)). This model compares much better to observations than the plume model does in the area of the Ronne ice shelf. This is because the fully $3 \mathrm{~d}$ model can simulate the buoyancy driven circulation that carries water away from melt zone, which is not included in the depth integrated plume model.

Sensitivity tests which involve removing the ice shelves show that there is a large influence of the ice shelves on sea ice formation. When the shelves are removed, large buildups of sea ice occur on West Antartica. This occurs because the melting of the land ice is introduced into the ocean at the surface in the grid cell nearest to the coast. This introduces a layer of fresh water at the surface that produces an unrealistically strong stratification, which prevents heat fluxes from the ocean from reaching the ice and an unrealistically thick layer of sea ice can form. The impact of the ice shelves is to input a similar amount of water as the land ice, but by inputing the water at depth, the ice shelves change the stratification and so do not allow such large regions of ice to grow. Similarly, melting of the shelves drives gyres within troughs in the continental shelf. These gyres can connect otherwise separate troughs, and also introduce cold fresh water fluxes at depth, which is critical to the formation of Antartic Bottom Water.

A caveat to these simulations is that the bathymetry is unknown in many circumstances. The areas where the bathymetry are the least well known are also the areas where the disagreement between the model and the observations are the largest. Improving the knowledge of the bathymetry could be a key step in our ability to simulate the circulation under the Antarctic ice shelves. 
(a) West Antarctica ice shelves

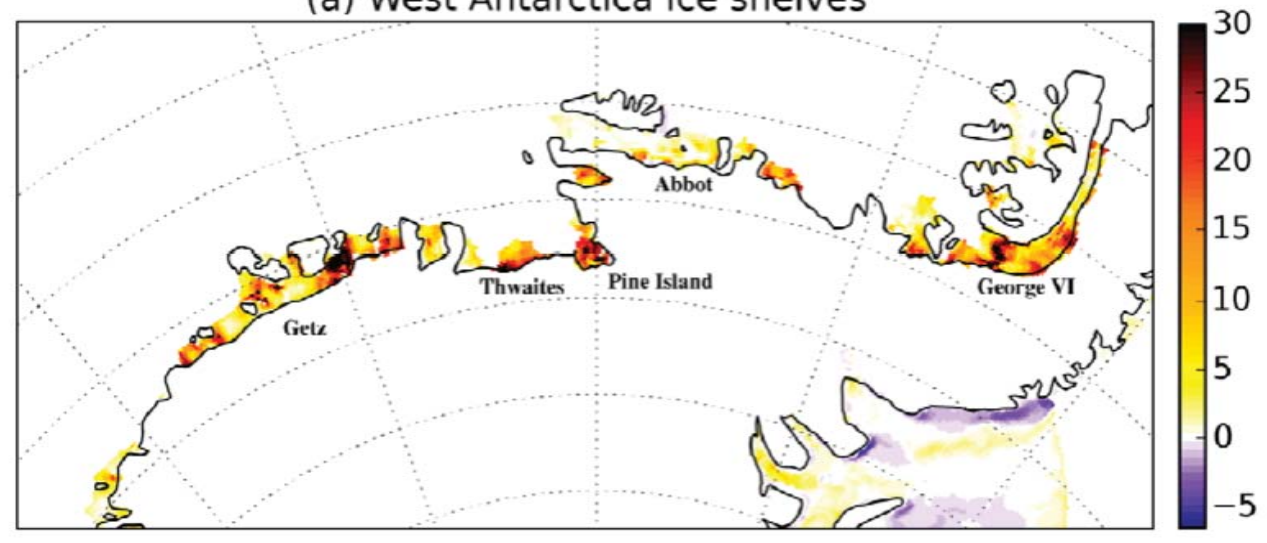

(b) Ross ice shelf
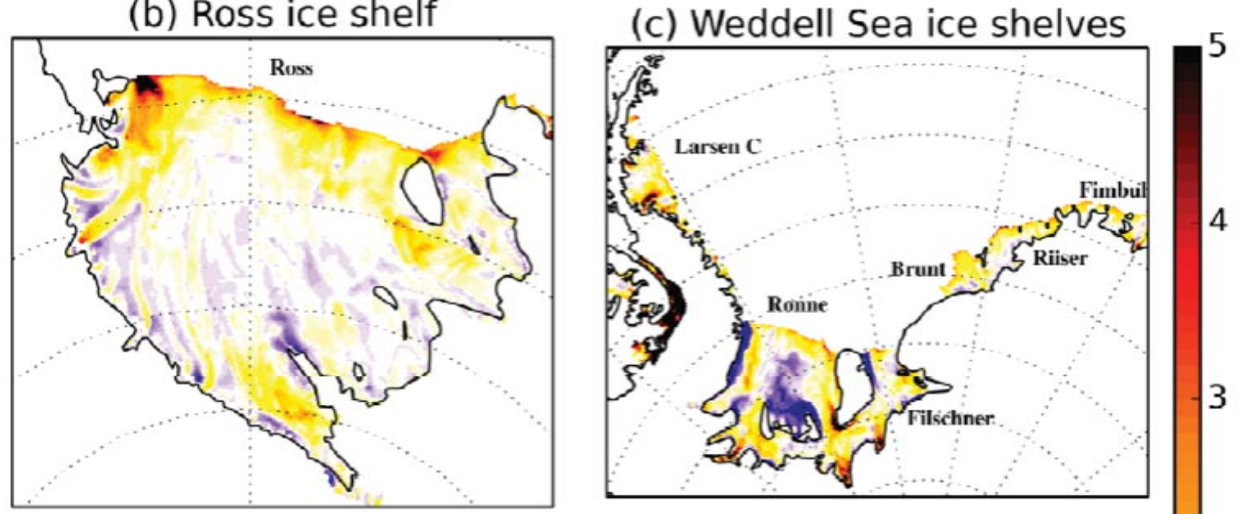

(d) East Antarctica ice shelves

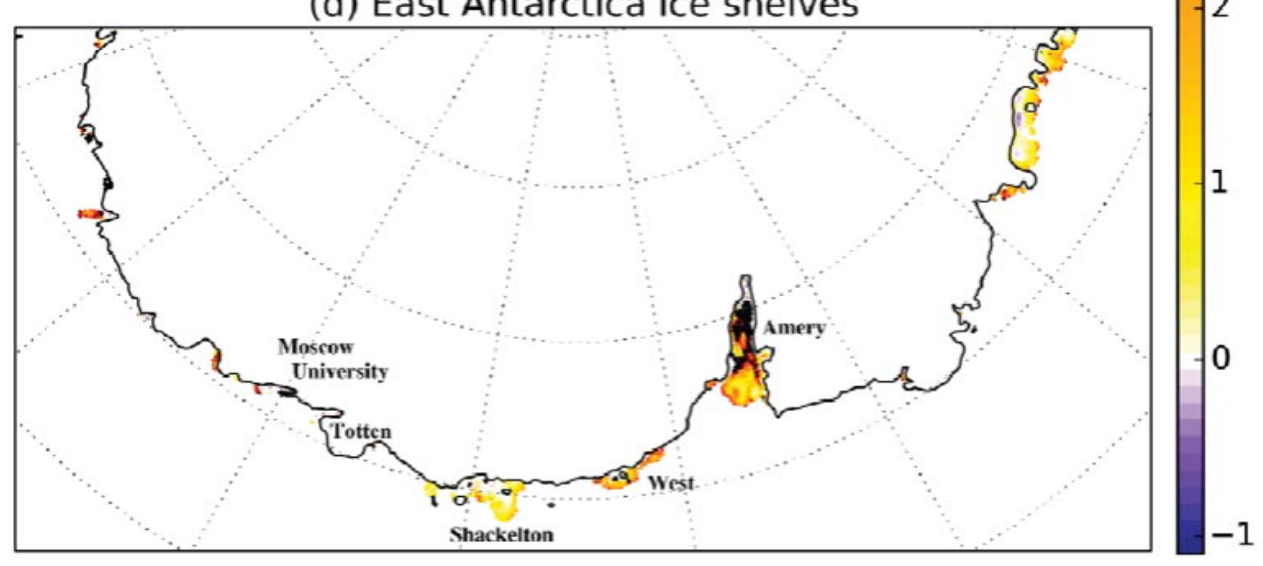

Figure 14: The melting patterns from the primitive equation model, run using observed bathemetry. From [8]. 


\section{References}

[1] A. Bombosch And A. Jenkins, Modeling the formation and deposition of frazil ice beneath filchner-ronne ice shelf, Journal of Geophysical Research: Oceans, 100 (1995), pp. 6983-6992.

[2] A. M. L. Brocq, N. Ross, J. A. Griggs, R. G. Bingham, H. F. J. Corr, F. Ferraccioli, A. Jenkins, T. A. Jordan, A. J. Payne, D. M. Rippin, And M. J. SiEgERT, Evidence from ice shelves for channelized meltwater flow beneath the Antarctic Ice Sheet, Nat. Geosci., 6 (2013), pp. 1-4.

[3] A. Jenkins, Convection-Driven Melting near the Grounding Lines of Ice Shelves and Tidewater Glaciers, Am. Meteorol. Soc., (2011).

[4] —, A simple model of the ice shelf-ocean boundary layer and current, Journal of Physical Oceanography, 46 (2016), pp. 1785-1803.

[5] A. Jenkins And A. Bombosch, Modeling the effects of frazil ice crystals on the dynamics and thermodynamics of ice shelf water plumes, Journal of Geophysical Research: Oceans, 100 (1995), pp. 6967-6981.

[6] F. Lane-Serff, G, On meltwater under shelves, J. Geophys. Res., 100 (1995), pp. 6961-6965.

[7] S. J. MagorRian And A. J. Wells, Turbulent plumes from a glacier terminus melting in a stratified ocean, J. Geophys. Res. Ocean., 121 (2016), pp. 4670-4696.

[8] P. Mathiot, A. Jenkins, C. Harris, And G. Madec, Explicit and parametrised representation of under ice shelf seas in a $z^{*}$ coordinate ocean model, Geosci. Model Dev. Discuss, (in review).

[9] O. V. Sergienko, Basal channels on ice shelves, Journal of Geophysical Research: Earth Surface, 118 (2013), pp. 1342-1355.

[10] D. A. Slater, D. N. Goldberg, P. W. Nienow, and T. R. Cowton, Scalings for Submarine Melting at Tidewater Glaciers from Buoyant Plume Theory, Am. Meteorol. Soc., 46 (2016), pp. 1839-1855. 


\section{GFD 2017 Lecture 7: Ice Ocean Interactions around Antarctica}

Adrian Jenkins; notes by Federico Fuentes and Madelaine Gamble Rosevear

June 27, 2017

This document comprises the third review lecture given by Adrian Jenkins during the 2017 Geophysical Fluid Dynamics program at the Woods Hole Oceanographic Institution (WHOI). It is about the ice-ocean interaction in the continent of Antarctica, and is divided in two parts: cold water regimes and warm water regimes. Several figures and figure captions were copied literally or almost literally from their original sources to facilitate comprehension. In those cases, the relevant references are given.

\section{Cold Water Regimes}

\subsection{Surface properties of Antarctica}

For ease of reference, a map of Antarctica is included (Figure 1).

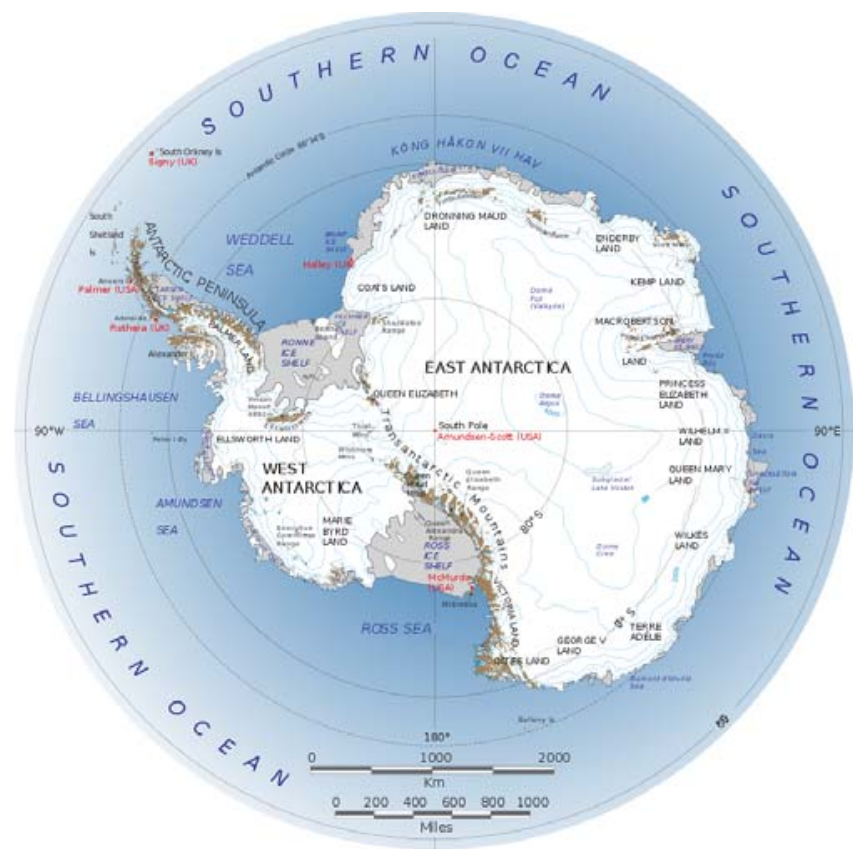

Figure 1: Map of Antarctica (image from NASA is in the public domain). 
The continent of Antarctica is uniformly surrounded by very cold near-freezing water close to the surface. However, the distribution of near-surface (0-100 m) salinity is not as simple. Indeed, it can vary depending on precipitation and redistribution by sea ice, which adds salt during formation and fresh water when it melts. This can be observed in Figure 2 , where the ocean tends to be fresher towards the north. Wherever the salinity is higher, it is easier to deepen the cold layer of water.
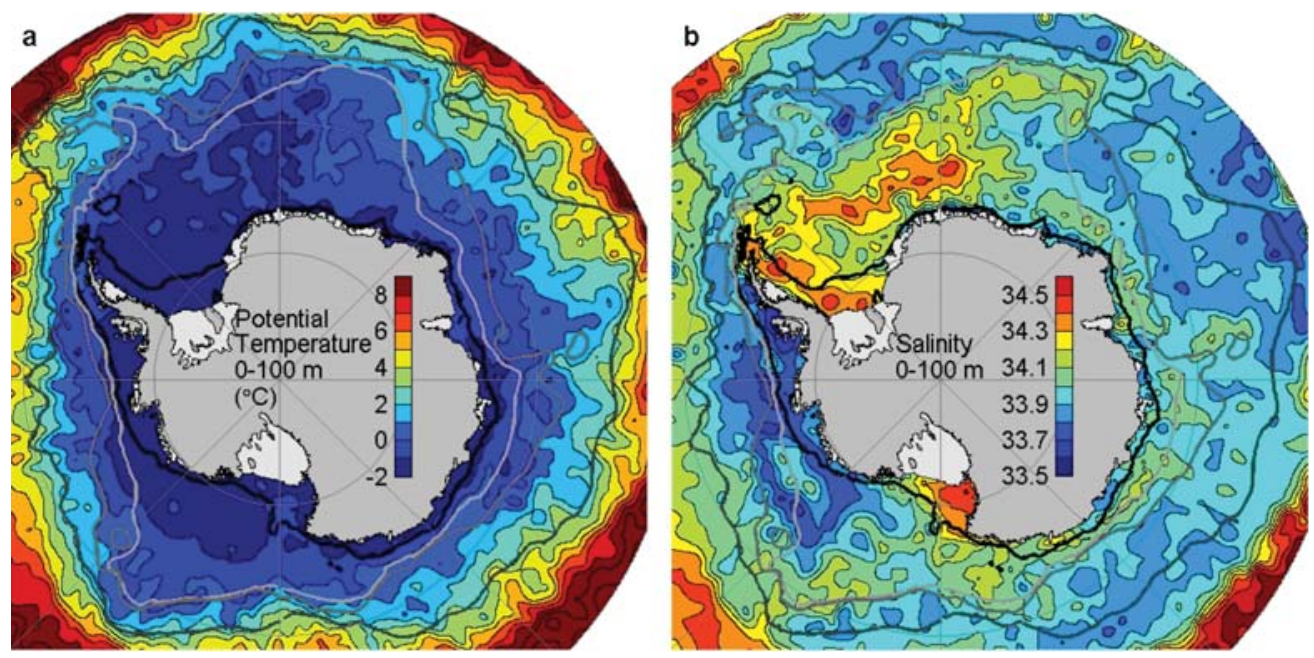

Figure 2: Near-surface temperature and near-surface salinity in the continent of Antarctica.

Around the Antarctic peninsula precipitation is high because the westerlies (winds blowing from west towards east, i.e., clockwise in Antarctic maps) encounter steep topography on the Antarctic Peninsula and are forced to rise, losing moisture as snowfall. This orographic effect can be observed in Figure 3, which shows the surface mass balance (effectively the snowfall) on the continent. The precipitation is then much lower in the Ross and Weddell seas due to the cold dry air which flows from the interior of the continent. Freezing (ice production) occurs pretty uniformly around the continent.

Strong katabatic winds (from higher elevations to lower elevations) are carried by the high and steep topography, especially in East Antarctica, where winds with speeds up to 320 $\mathrm{km} / \mathrm{hr}$ have been reported in winter. These katabatic winds feed the near-coastal easterly winds. Meanwhile, in the Ross and Weddell seas winds are deviated north by topographic barriers. All this can be observed in Figure 4.

\subsection{Shelf properties of Antarctica}

In [23] an idealised model was used to study the processes setting the shelf water properties. The transport of relatively warm and salty circumpolar deep water (CDW) across the Antarctic slope front (ASF) (which almost completely surrounds the Antarctic continental shelf) was of particular interest, as the steep isopycnals (surfaces of constant density) associated with the ASF provide a barrier to on-shelf transport. The model was idealized by being essentially 2D, with no variations in the along-shore direction and periodic boundary conditions assumed. This is shown in Figure 5. 

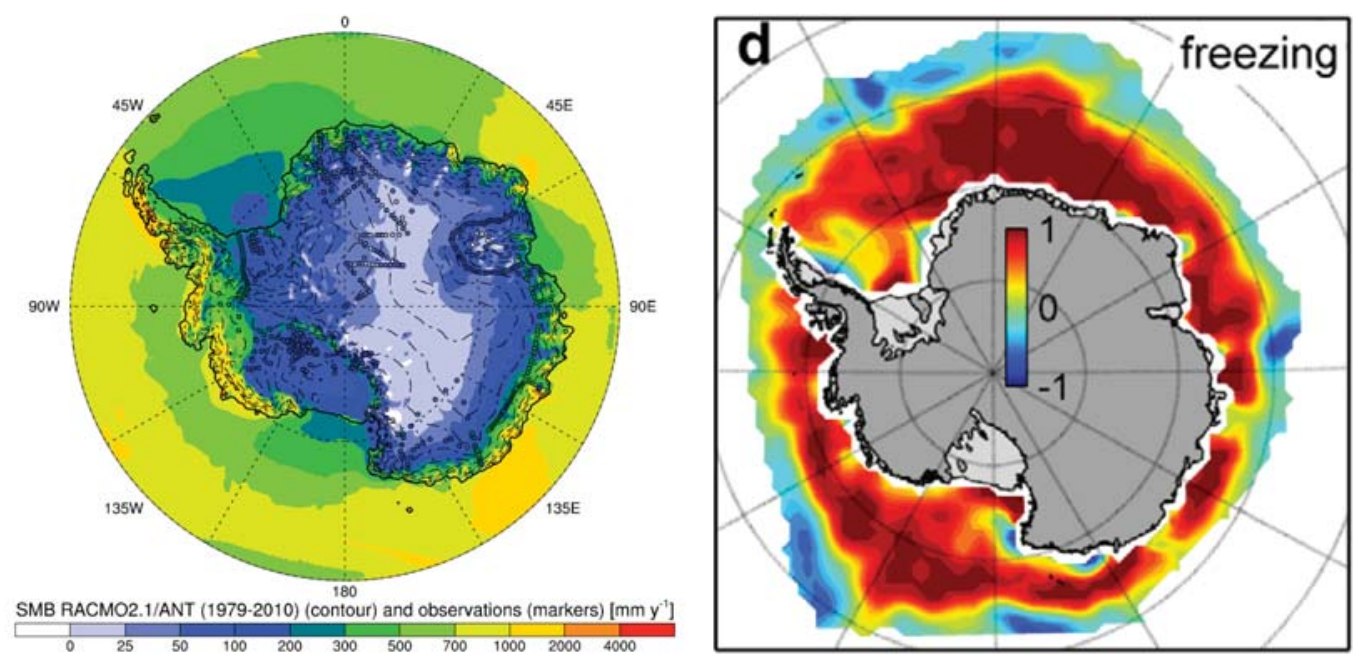

Figure 3: Left: Mean (1979-2010) surface mass balance $(S M B)$ in mm w.e./yr (where w.e. means water equivalent) using simulations and observations. Note that on the seasonal sea-ice, $S M B$ equals precipitation $(P)$ minus surface sublimation $\left(S U_{s}\right)$ and on open ocean $S M B=P$ [13]. Right: Mean concentration difference from residual freezing (does not represent ice thickness!) [5].

The investigation showed that shelf-water properties were mainly defined by wind-driven transport of Antarctic surface water (AASW) and by mesoscale eddies carrying CDW along isopycnals. Weaker winds allowed more warm CDW to flow onto the shelf, heating up the shelf waters, as did broader and deeper shelves. A higher surface salt flux (representative of higher sea ice formation) had the same effect, as it increased the production and outflow of cold, salty Antarctic bottom water (AABW). It is the presence of AABW that creates a connection between the shelf waters and the offshore CDW and allows mesoscale eddies to transport CDW onto the shelf. All these results are shown in Figure 6.

Another process that affects the shelf water properties is precipitation. When precipitation is high, the upper ocean is stabilised and the thermocline is shallower, thus in regions with high precipitation and weak coastal easterly winds (i.e. the Antarctic peninsula), warm CDW intrudes onto the shelf. Meanwhile, in regions where precipitation is lower and coastal easterlies are stronger, downwelling (accumulation and sinking of higher density water below lower density water) is sufficient to exclude CDW from the shelf. In the case where precipitation is low and the sea ice production is high, cold, salty shelf water is present.

This picture is confirmed by comparing temperature and salinity (Figure 7), precipitation (Figure 3) and winds (Figure 4) with shelf properties observed around Antarctica (Figure 8). At depth, the shelves are dominated by cold and salty shelf water off the coasts of the Filchner and Ross ice shelves; by cold and fresh AASW off the coasts of Dronning Maud Land and Wilkes Land; and by warm and salty CDW off the coast of Ellsworth Land and the Antarctic peninsula (see Figure 1 for geographic references). 

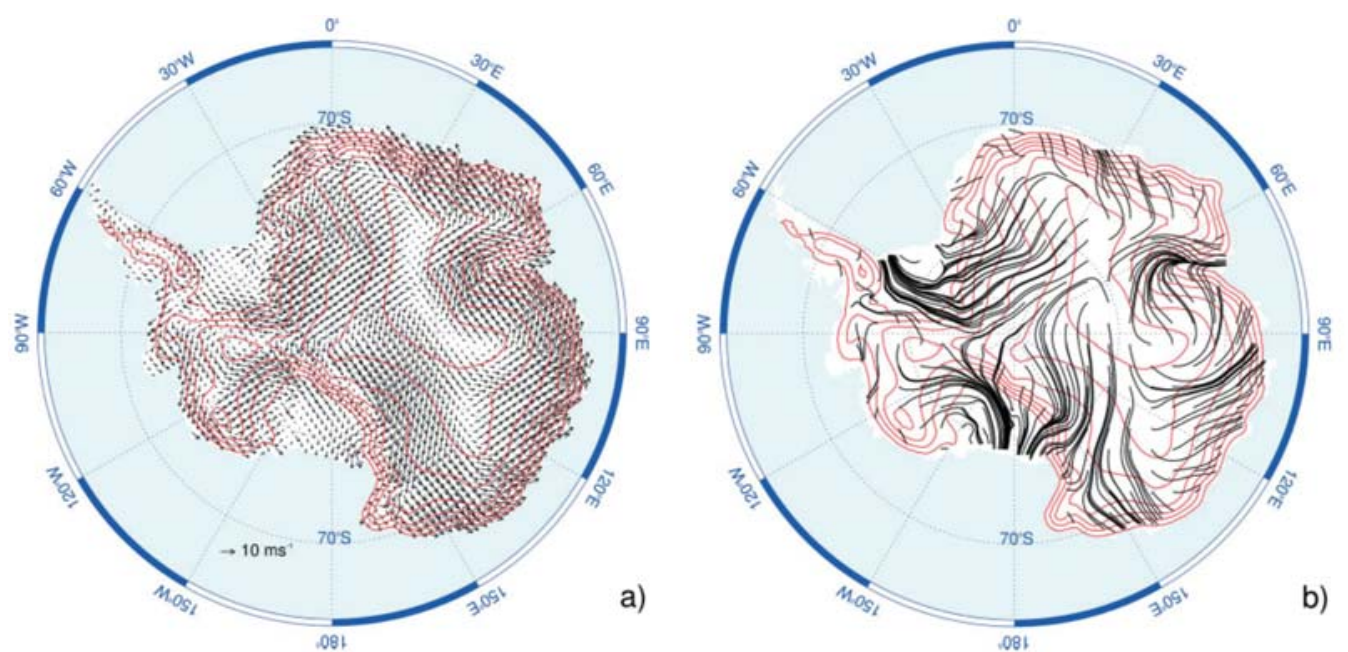

Figure 4: Left: Mean winter wind vector. Right: Mean winter stream lines. Data is from 1980-93. The elevation of the surface is shown by contour lines in red [24].

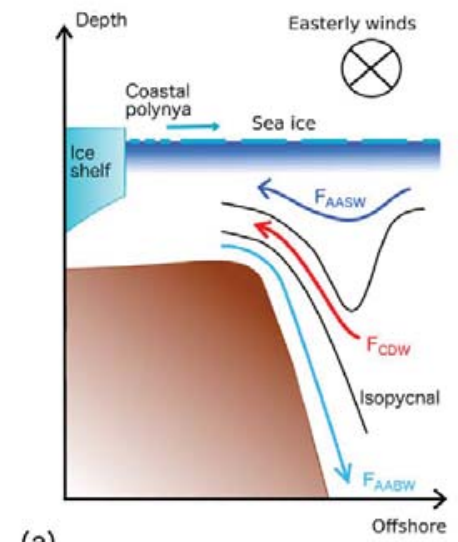

(a)

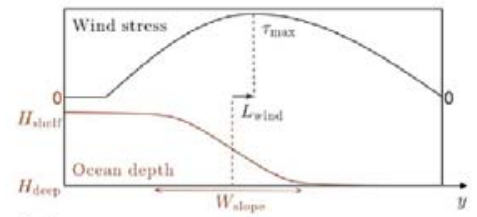

(b)

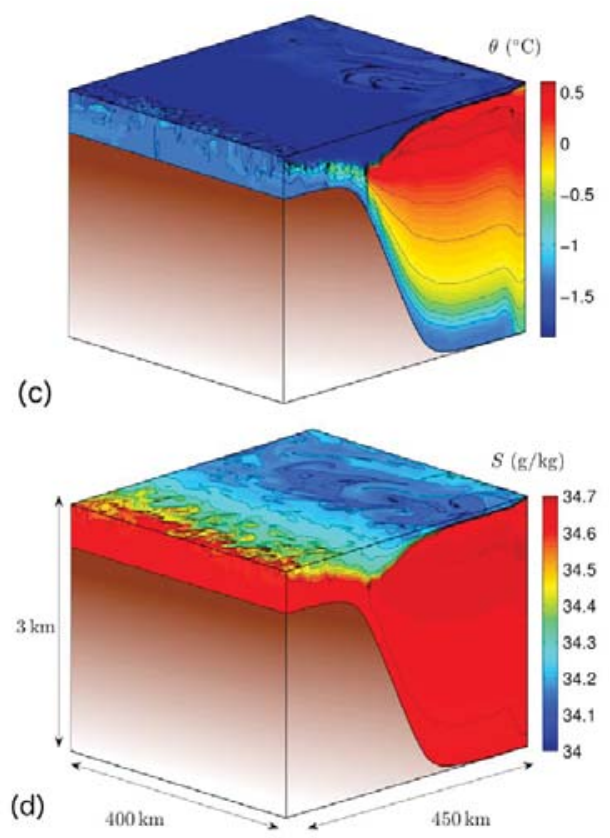

Figure 5: Taken from [23]. (a) Schematic cross-section of the Antarctic slope front (ASF), which separates the continental shelf waters from the warm circumpolar deep water (CDW) at mid-depth offshore. In regions of Antarctic bottom water (AABW) outflow, such as the western Weddell and Ross Seas, isopycnal (i.e. constant density) surfaces connecting the shelf waters to CDW may facilitate onshore heat transport and AABW export via the action of mesoscale eddies. (b) Schematic profiles of ocean depth, along-shore surface wind stress, and associated parameters. (c) potential temperature profile used as reference, where eddy boluses of warm CDW are visible crossing the shelf break. (d) Salinity profile used as reference. 

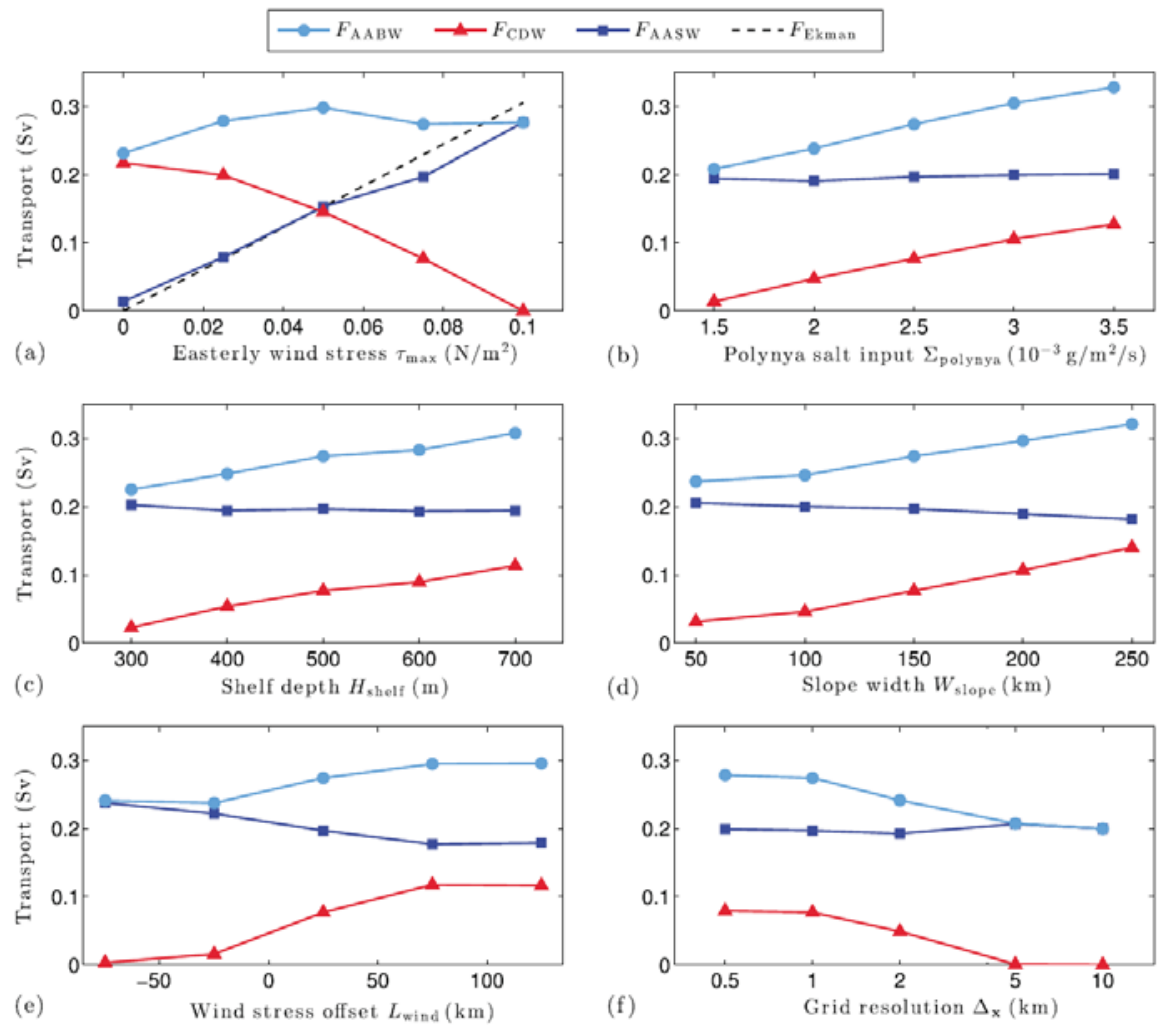

Figure 6: Taken from [23]. Left: Sensitivity of the transports crossing the Antarctic slope front (ASF) of Antarctic surface water $\left(F_{A A S W}\right)$, circumpolar deep water $\left(F_{C D W}\right)$, and Antarctic bottom water $\left(F_{A A B W}\right)$. The sensitivity is to (a) the wind stress maximum amplitude $\tau_{\max }$, (b) the brine rejection rate on the continental shelf $\Sigma_{\text {polynya }}$, (c) the depth of the continental shelf $H_{\text {shelf }}$, (d) the width of the continental slope $W_{\text {slope }}$, (e) the offset of the wind stress maximum from the center of the continental slope ( $\left.L_{\text {wind }}\right)$, and (f) the model's horizontal grid spacing $\Delta_{x}$. In (a) the theoretical wind-driven southward surface Ekman transport is also shown, and it agrees closely with the shoreward transport of Antarctic surface water (AASW).
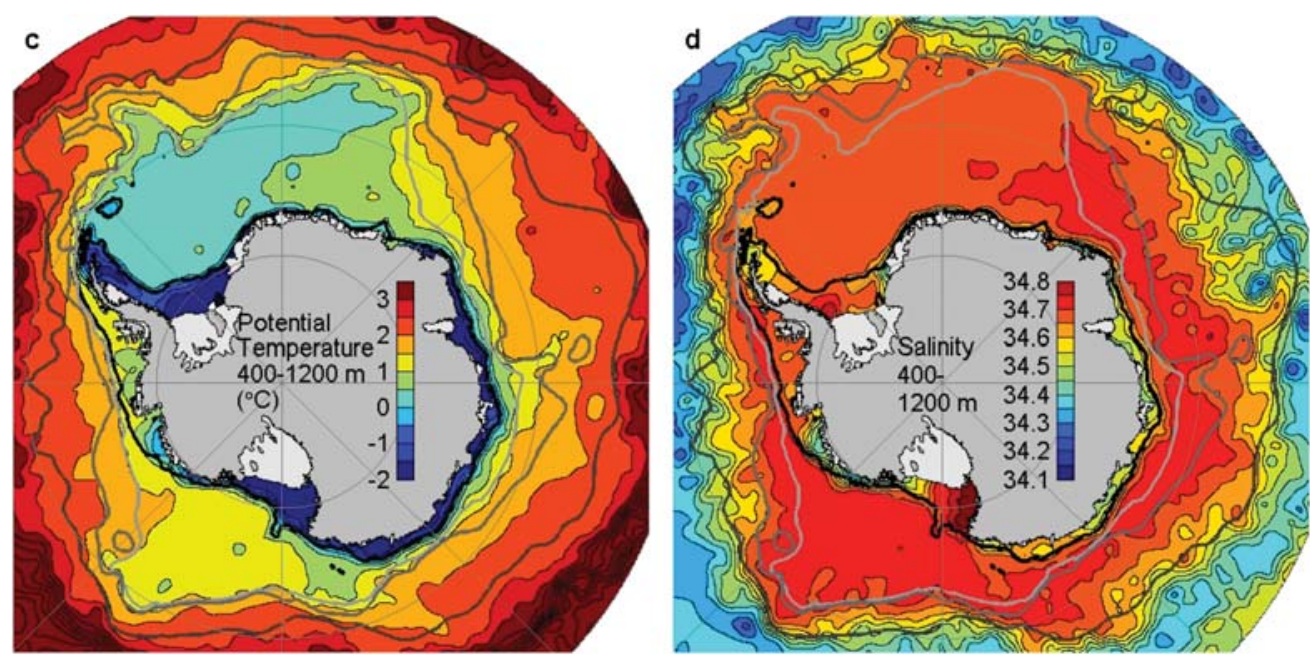

Figure 7: Deep water temperature and salinity in the continent of Antarctica. 

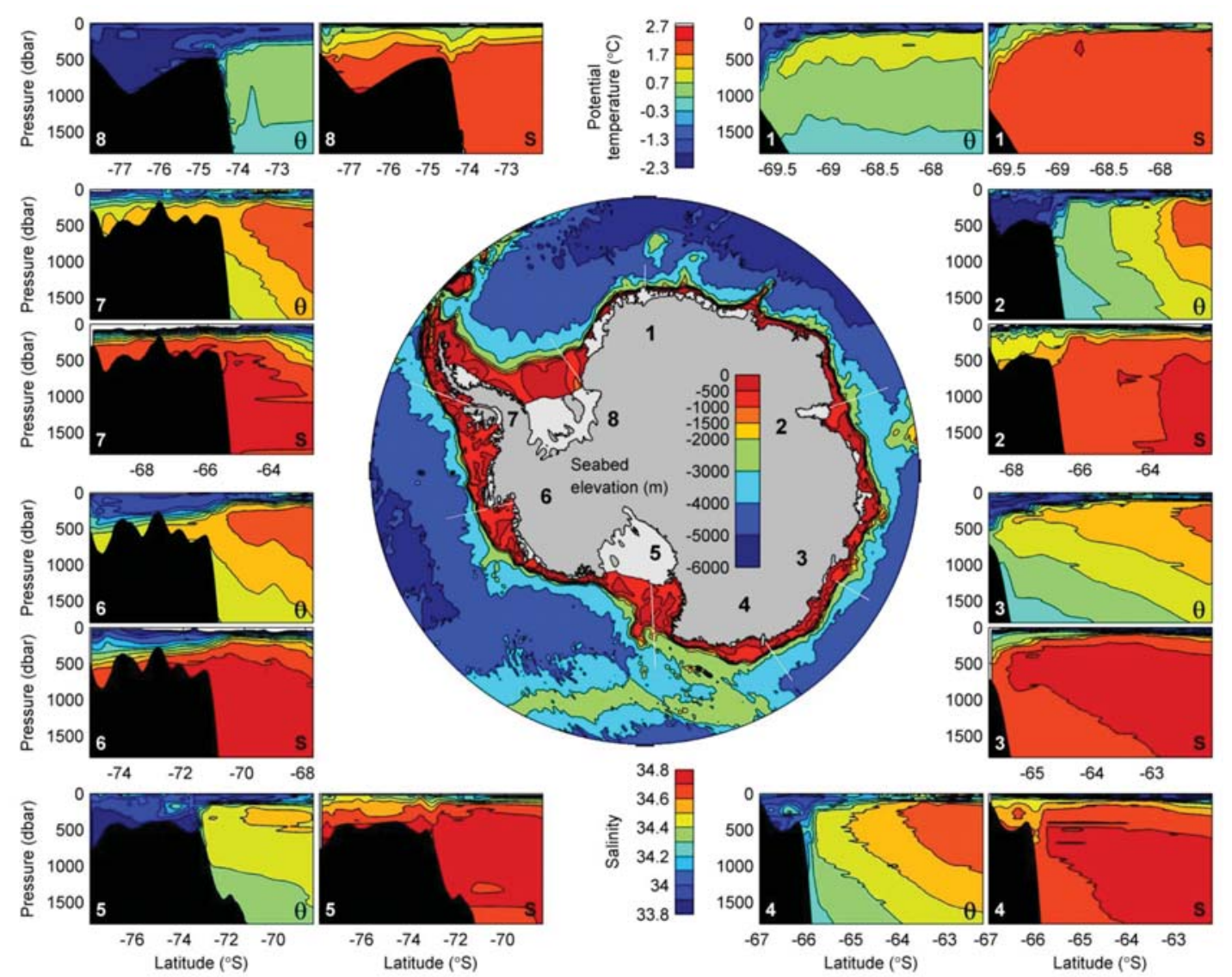

Figure 8: Temperature $(\theta)$ and salinity $(S)$ profiles at different parts of Antarctica. At depth the shelves are dominated by either: cold and salty shelf water $(8,5$, and to a lesser extent 2 and 4); cold and fresh Antarctic surface water (AASW) (1, 3, and much of the area around 2 and 4); or warm and salty circumpolar deep water (CDW) (7 and 6). 


\subsection{Melting modes}

The different melting modes are illustrated in Figure 9, and are briefly described next:

- Mode 1: This form of melting occurs when shelf water (SW) dominates. There are extensive areas of refreezing and melt rates on the order of $0.1 \mathrm{~m} / \mathrm{yr}$. Both SW and ice shelf water (ISW) are sufficiently dense to form Antarctic bottom water (AABW).

- Mode 2: Here, the warmer circumpolar deep water (CDW) dominates. There is no refreezing and much higher melt rates on the order of 1-10 m/yr are observed.

- Mode 3: This occurs when Antarctic surface water (AASW) dominates and CDW intrusions only occur at the seabed. Some refreezing does occur and due to AASW being seasonally warmer than SW, higher melt rates than Mode 1 are observed; on the order of $0.1-1 \mathrm{~m} / \mathrm{yr}$.

\subsection{Melting mode 1}

In the southern Weddell Sea, where the Filchner-Ronne ice shelf (FRIS) lies, the cold SW is dominant and denser than CDW. The complicated seabed and ice shelf geometry results in a modified (with respect to the usual) pattern of melting and freezing, as shown in Figure 10, where the basic "ice pump" mechanism is visible from the satellite data. From the figure it can be observed that relatively little warm water enters the cavity, and that the highest melting occurs at depth, near the grounding line.

Figure 11 presents results from models, which show an overturning circulation, but also strong horizontal flows guided by ice-base and seabed topography. The presence of tides induces much stronger circulation, where increased melting results in higher buoyancy forcing. The model also reproduces the observed pattern of melting where the warm water enters the cavity and reaches the deep grounding lines, while freezing is present along the outflow paths (right in Figure 11). The freezing and melting are low when the effects of the tides are not considered, but increase significantly with tidal forcing. Even though tides generate only weak time-averaged currents, tidal currents can dominate the instantaneous flow if the buoyancy forcing is weak. In that case, the tides control the turbulent transport of heat to the ice shelf base.

The outflows of ice shelf water (ISW) are possibly supercooled, due to the fact that inflowing waters have a temperature close to the surface freezing point. The addition of meltwater at depth, where the in situ freezing temperature is even lower due to the effect of pressure, means that the buoyant outflow may become supercooled as it rises. In that case, platelet ice may form and can generate regions of very thick (about $10 \mathrm{~m}$ ) land-fast sea ice. These insights can be appreciated in Figure 10 (bottom).

Most ISW exits the cavity at depth and contributes to the Antarctic bottom water (AABW) formation as it spills off the continental shelf. Similar processes occur in the Ross Sea, but melting and production of ISW appear to be slightly lower, probably because the ice shelf is thinner on average.

Ice shelves of the Ross and Weddell seas are probably relatively insensitive to climate change. The shelf water will be fixed at the surface freezing point as long as enough sea ice 

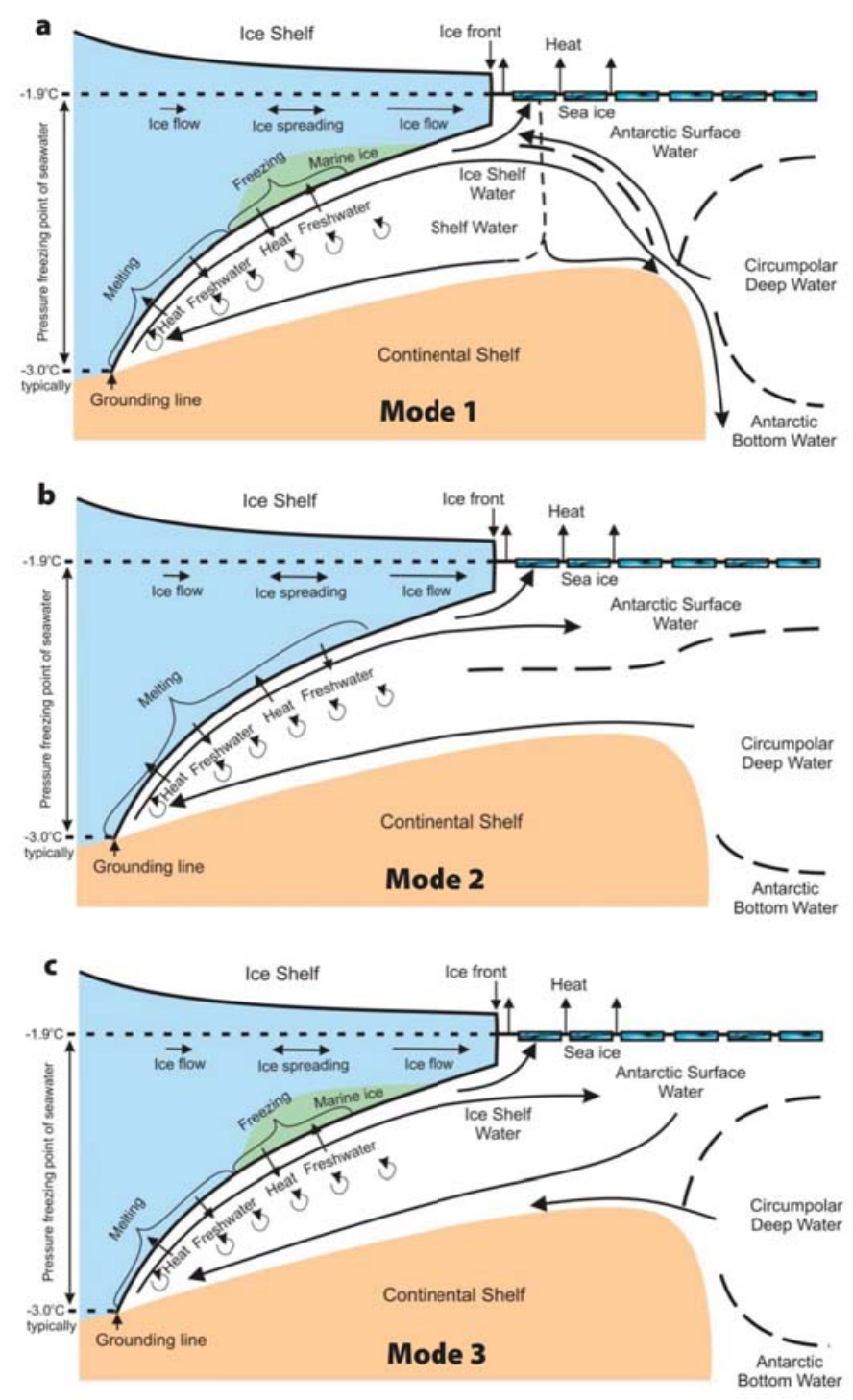

Figure 9: Taken from [8]: Three modes of sub-ice-shelf circulation and associated stratification on the continental shelf. (a) In Mode 1, dense shelf water (SW), formed by brine rejection beneath growing sea ice, dominates the sub-ice cavity. SW has a temperature at or close to the surface freezing point, and can melt ice at depth only because of the pressure dependence of the freezing point. Some refreezing occurs in the cavity because the water produced by melting (ice shelf water (ISW)) becomes supercooled as it rises along the shoaling ice shelf base. (b) Mode 2 dominates if SW is absent and circumpolar deep water (CDW) is the densest water on the shelf. CDW temperatures are typically around $3^{\circ} \mathrm{C}$ above the surface freezing point so melting is rapid, no ISW forms, and there is no refreezing. (c) Mode 3 dominates where both SW and CDW are absent, leaving Antarctic surface water (AASW) as the densest water on the shelf. Only the upper layer of AASW is seasonally warmer than the surface freezing point, so melt rates are low and ISW formation and refreezing can result. Although the CDW is denser, its access to the shelf is limited by the deepening of the AASW layer at the coast, where the southward Ekman transport driven by the easterly wind is blocked. Note that in (a), Modes 2 and 3 may influence the outer cavity because AASW and modified CDW are present in the upper water column, while in (b), Mode 3 melting may occur above the permanent thermocline separating AASW and CDW. 


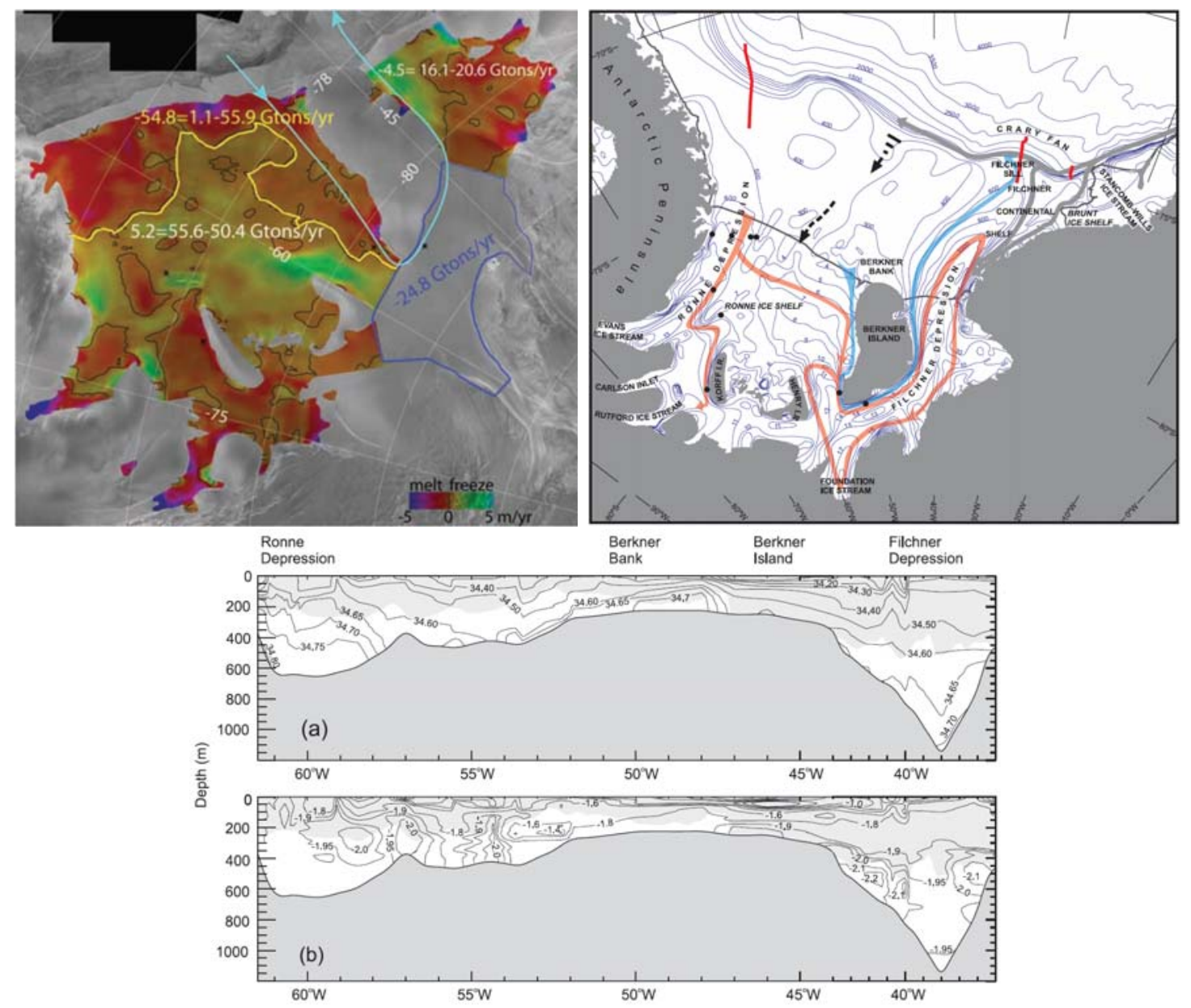

Figure 10: Top left: Basal melt rates under assumptions of steady-state ice shelf and conservation of mass. Positive values represent freezing and negative values melting (with color saturation for magnitudes over $5 \mathrm{~m} / \mathrm{yr}$ ). Yellow line shows separation of region with strong melt at the Ronne shelf, while light blue lines illustrate inferred ocean circulation paths [10]. Top right: Southwestern Weddell Sea. Bathymetric contours are labeled in hundreds of meters beneath the ice shelves. Dotted black arrows indicate inflow of modified warm deep water (MWDW) [18]. Bottom: (a) Salinity and (b) potential temperature at the Filchner and Ronne ice fronts, where the light gray represents the draft of the ice shelf in the ice front [18].

is produced. One model suggests the possibility of a future regime change [4]. It argues that thinning of sea ice may increase the wind stress and strengthen the inflow of modified CDW. However, as this happens, there must also be a decrease in shelf water density.

\section{$1.5 \quad$ Melting mode 3}

The Fimbul ice shelf (FIS) is located in the eastern Weddell Sea, where wind-forced downwelling of Antarctic surface water (AASW) dominates and keeps the shelf cold. Note that the topography in this region is very steep, as can be observed in Figure 12. It is thought that the wind-forced overturning is opposed by eddy overturning of the front, which brings warm water on-shelf at depth (see Figure 12). This has a subtle difference to the case 

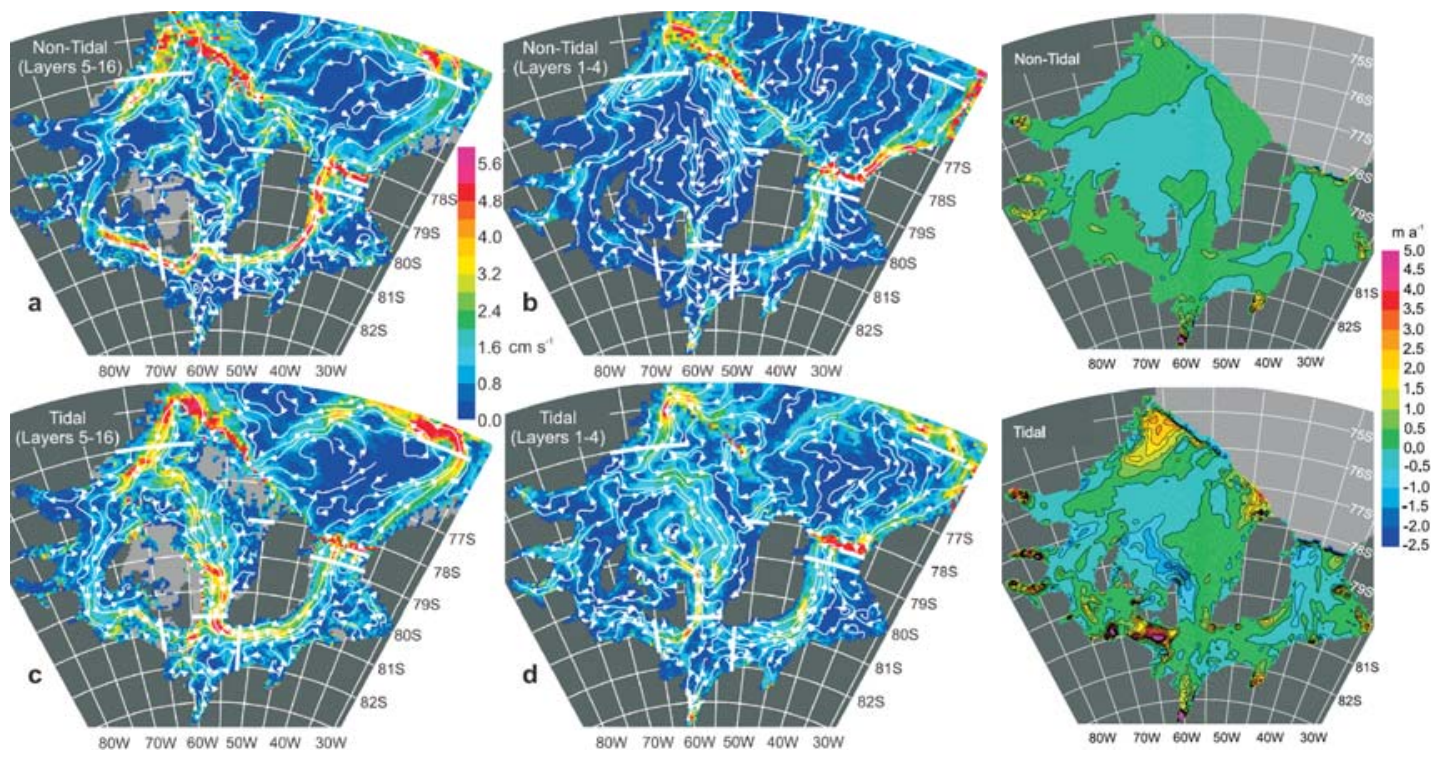

Figure 11: From [14]. Left-center: Flow lines of the modeled mean annual circulation in (a) the lower twelve layers without tides, (b) the upper four layers (including the mixed layer) without tides, (c) the lower twelve layers with tides, and (d) the upper four layers with tides. The color scale shows the mean current speed. Right: Mean basal ice melt rates beneath the Filchner-Ronne ice shelf (FRIS) in cm/yr from the model for non-tidal (top) and tidal (bottom) simulations. Negative values indicate freezing.

where shelf water (SW) formation creates an isopycnal connection to the deep waters offshelf. That being said, an analogous model with the ice shelf included and without any SW formation, shows a similar result. As observed in Figure 12 (right), a weaker easterly wind allows warmer CDW to intrude along the seabed (note the higher temperatures at the seabed when the wind is $3 \mathrm{~m} / \mathrm{s}$ as compared to when the wind is $9 \mathrm{~m} / \mathrm{s}$ ). Therefore, the wind is a fundamental factor of the heat transfer.

In the summer, the seasonally warmer upper layer of Antarctic surface water (AASW) drives melting in the outer cavity (see left of Figure 13). This layer is relatively fresh and typically too thin to get beneath the ice, but again, wind-forced coastal downwelling is what allows it to access the Fimbul cavity. However, in this case, stronger easterly winds lead to a greater flux of warm water into the cavity.

Before observations were made in the Fimbul cavity, a modeling study suggested that more circumpolar deep water (CDW) could be present at depth (see right of Figure 13). This ended up being wrong, but for an interesting reason. An eastward-flowing undercurrent beneath the westward-flowing AASW carried CDW along the upper slope. It turned onshelf within a seabed trough that cut the shelf edge and carried CDW beneath the ice shelf. Stronger easterly winds could exclude the inflow by pushing the front and the undercurrent deeper. This undercurrent has been identified in observations, but always deep down on the continental slope [1]. 

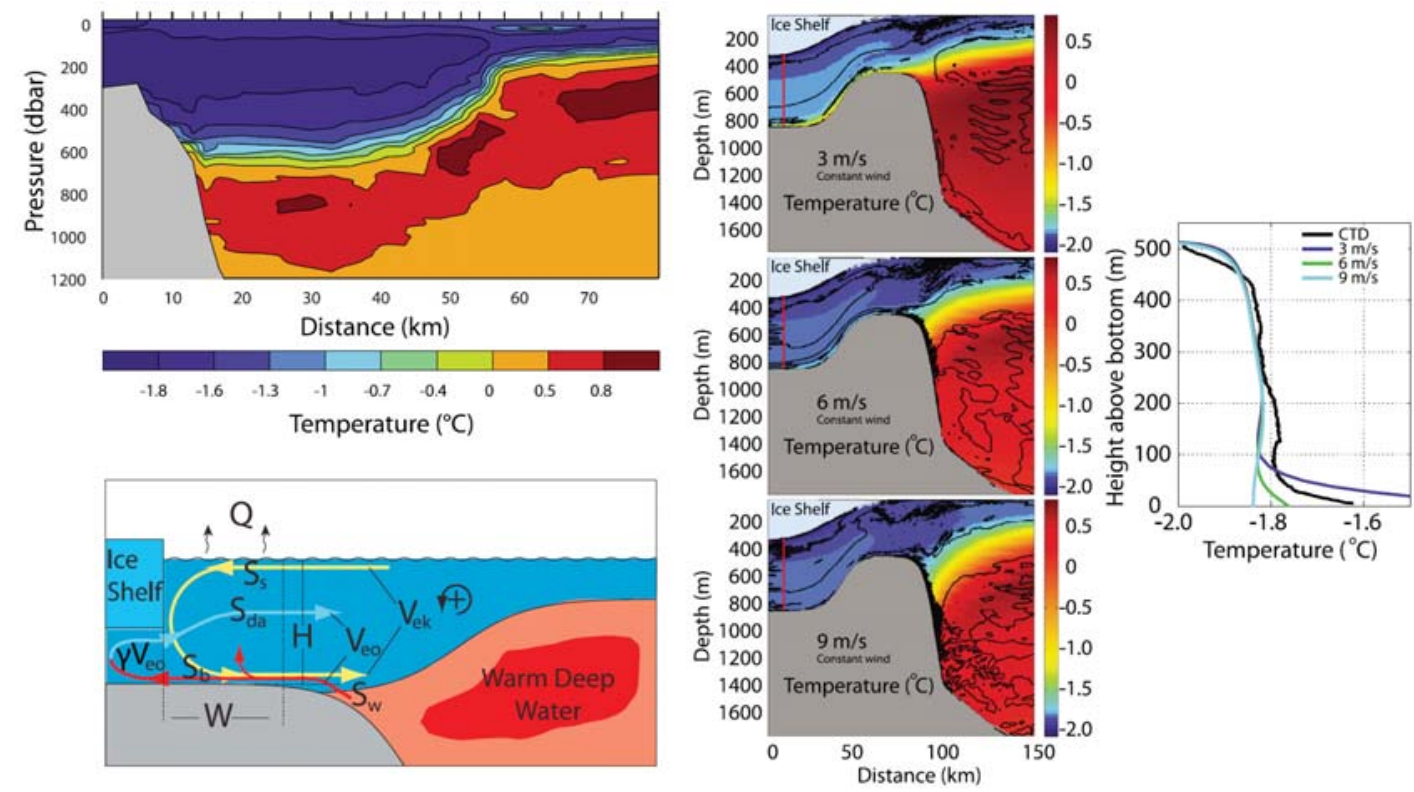

Figure 12: From [19]. Top left: Temperature section across the Antarctic slope front (ASF) at $17^{\circ} \mathrm{W}$ showing the typical structure of the ASF. Bottom left: Sketch illustrating the hypothesized exchange processes across the ASF. Yellow arrows illustrate Ekman overturning $\left(V_{e k}\right)$, while red and blue arrows illustrate the overturning of the slope front $\left(V_{e o}\right)$ and the sub-ice shelf overturning $\left(\gamma V_{e o}\right)$ respectively. Right: The along-slope-averaged temperature estimated from daily snapshots. Negative velocities are to left. The black lines are contours of zero residual velocity. Further right is the observed temperature profile beneath the Fimbul ice shelf (FIS) (in black) along with the modeled temperature profiles (in colors) taken from the vertical red line in the previous plots.

\subsection{A note on the warm water regimes}

Warm derivatives of warm circumpolar deep water (CDW) are found at depth on the shelf from $55-155^{\circ} \mathrm{W}$. Often, it is assumed that the dominant processes are common and connected with the proximity of the Antarctic circumpolar current (ACC). Nevertheless, the ACC does not reach the continental slope until about $90^{\circ} \mathrm{W}$ (see left of Figure 14). In the Amundsen Sea it flows north of the Marie-Byrd Seamounts.

In the Bellingshausen Sea, upper CDW comes on the shelf as eddies that shed from the ACC (see right of Figure 14). Thus, upper CDW is not confined to troughs. On the other hand, lower CDW intrusions follow the trough topography and appear to be steadier. Meanwhile, in the Amundsen Sea, the deeper thermocline seems to exclude most upper CDW, while lower CDW intrudes along the troughs. The presence of CDW on the shelf is not a novelty, since it was observed as early as the Belgian Antarctic expedition from 1897-1899. 

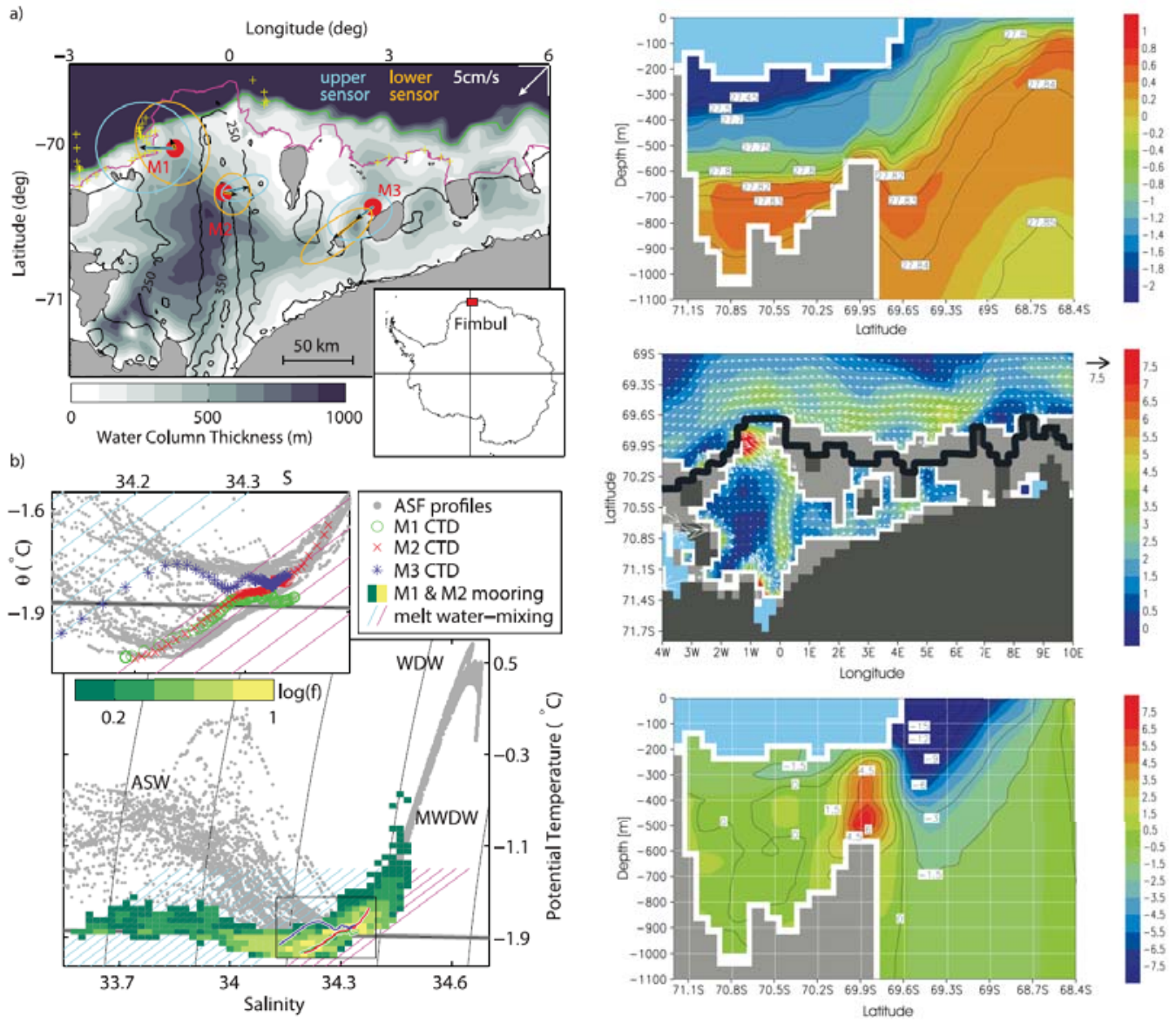

Figure 13: Left: From [3]. (a) Map of Fimbul ice shelf (FIS), with mooring locations M1, M2, and M3 indicated by red dots, together with water column thickness (gray shading). Black contours show the ice draft in $100 \mathrm{~m}$ intervals. The ice front is shown in magenta, and the continental shelf break in green (1500 $\mathrm{m}$ isobath). Vectors originating at each site show the annual mean value of the currents, surrounded by their associated variance ellipses (the white arrow in the upper right corner indicates the velocity scale). (b) Potential temperature-salinity diagram comparing observations below the FIS with coastal hydrography. The color shading shows the relative occurrence of different water masses at the mooring sensors, binned in T-S space, with yellow indicating many observations on a logarithmic scale. Two arrays of melt water mixing lines, as described in the text, highlight the melting regimes associated with Antarctic surface water (AASW or ASW) (blue) and modified warm deep water (MWDW) (magenta). Right: From [21]. (Top) Annual mean potential temperature $\left({ }^{\circ} \mathrm{C}\right)$ along $1^{\circ} \mathrm{W}$ in the steady state solution. FIS is shown in light blue, and the bedrock in gray. Potential density contours are shown as black lines. (Middle) Annual mean flow at $450 \mathrm{~m}$ depth in the steady state solution. The color scale shows current magnitude $(\mathrm{cm} / \mathrm{s})$ and the arrows are current vectors with a $7.5 \mathrm{~cm} / \mathrm{s}$ arrow to scale in the upper right corner. The thick black line is the ice front of FIS. (Bottom) Annual mean eastward current speed $(\mathrm{cm} / \mathrm{s})$ at $1^{\circ} \mathrm{W}$ in the steady state solution. 

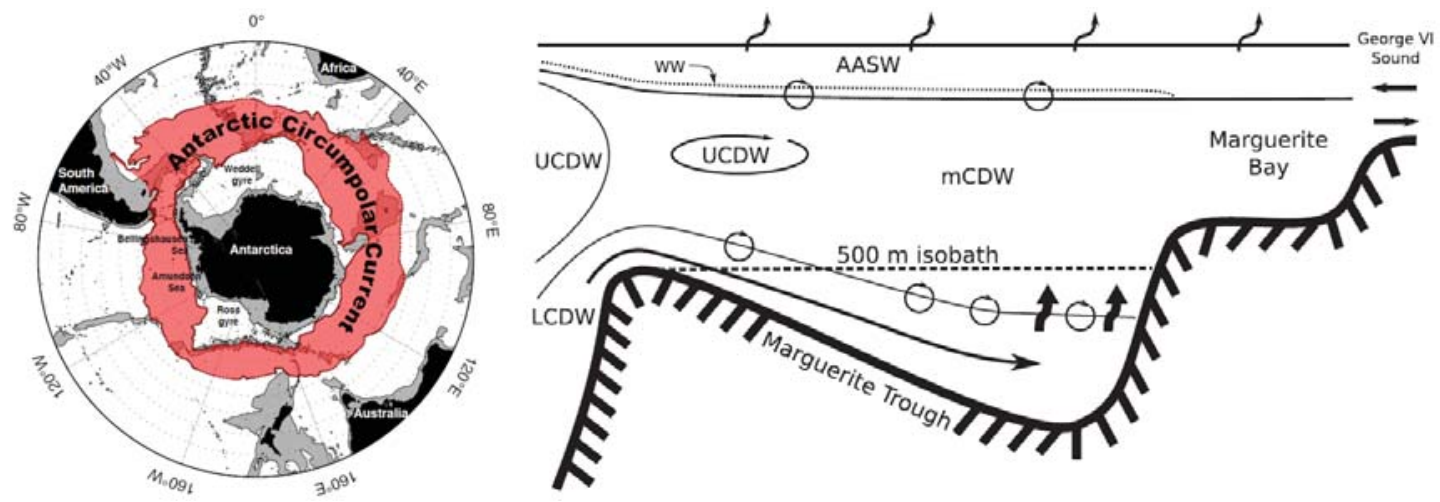

Figure 14: Left: From [15]. Location of climatological Antarctic circumpolar current (ACC), transporting warm upper circumpolar deep water (UCDW). The ACC flows along the slope-shelf break for the entire western Antarctic. Bathymetry shallower than $3 \mathrm{~km}$ is shaded. Right: From [16]. Conceptual diagram showing the characteristics of circumpolar deep water (CDW) intrusions to the western Antarctic Peninsula (wAP) shelf. UCDW intrudes on the shelf in the form of relatively small and frequent middepth features. Lower circumpolar deep water (LCDW) is found at the bottom of deep depressions. The thick black arrows represent upwelling of LCDW water to the overlying water, and the rounded arrows represent mixing across layers.

\section{Warm Water Regimes}

Here, we turn our attention the high melt regime driven by the transport of warm circumpolar deep water (CDW) onto the continental shelf, or mode 2 melting. Whilst CDW is present all around Antarctica, there are only a few regions in which it can penetrate beneath ice shelves. The Amundsen sea sector is one such region.

\subsection{Mean state}

In the eastern Amundsen Sea, the broad continental shelf is interspersed with a series of deeper troughs (Figure 15). These troughs, which were carved out by glaciers during previous advances of the Antarctic ice sheet, now provide a pathway for CDW to access the ice shelves in the region.

Moving east to west along the shelf, the thermocline deepens (Figure 16) and the CDW present below becomes cooler. In the west, the combination of a shallower shelf and a deeper thermocline means that CDW is almost completely blocked from coming on-shelf. As a result, the ice shelves in the western part of the Amundsen sector are close to a transition to the cold water regimes discussed in Part I, where the shelf is effectively isolated from CDW.

\subsubsection{Observations of heat transport to Pine Island Glacier}

Access for CDW to Pine Island Glacier occurs through two troughs, Pine Island Trough East (PITE) and Pine Island Trough West (PITW). Observations of PITW show an inflow of CDW of about $0.2 \mathrm{~Sv}$ which delivers an on-shelf heat flux of around 2.8 TW [25]. 


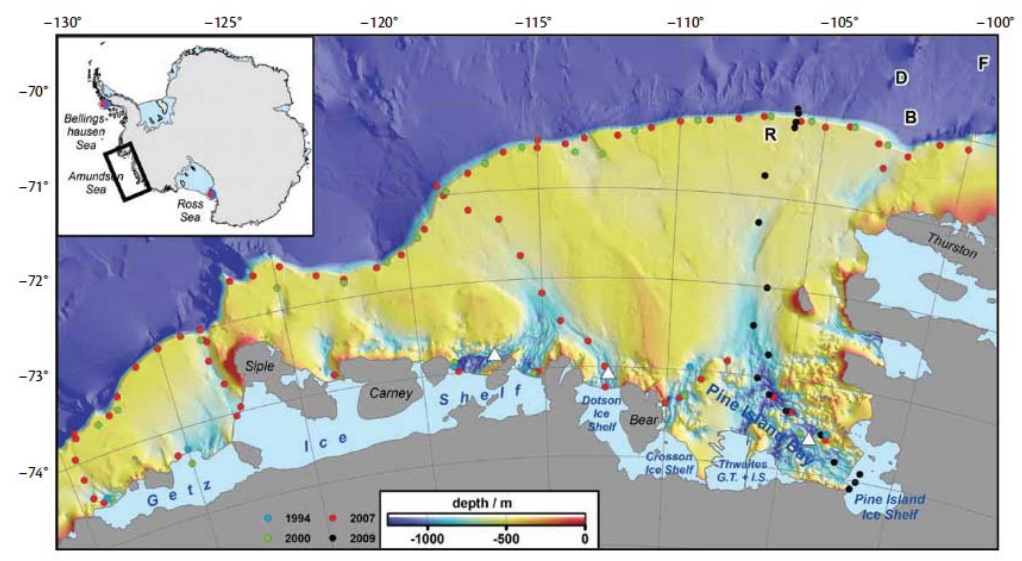

Figure 15: The Amundsen Sea continental shelf and floating ice shelves of the region. Figure taken from $[6]$.

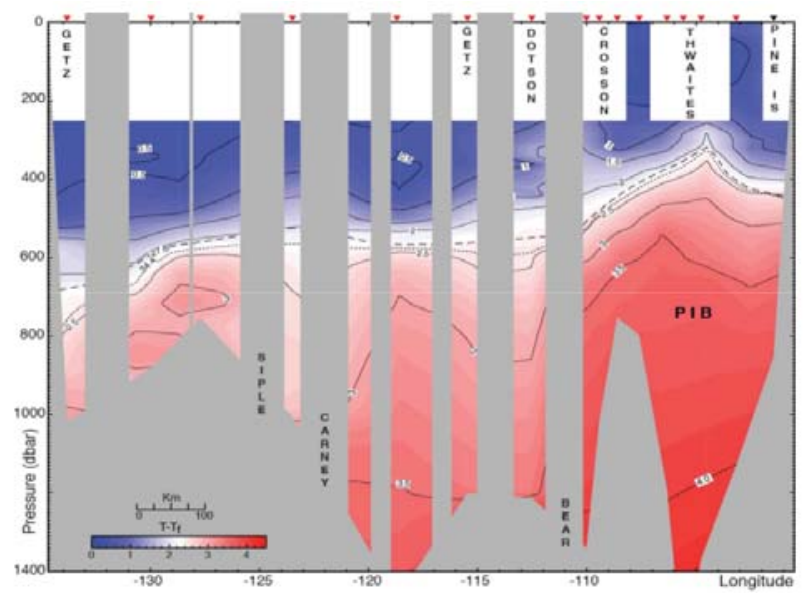

Figure 16: Temperature $\left({ }^{\circ} \mathrm{C}\right)$ above pressure freezing point $\left(T-T_{f}\right)$ moving along the shelf, with nominal ice shelf draft (white). Figure from [6]

Along shelf flow is dominated by the Antarctic Slope Current, an eastward flow driven by winds off the Antarctic continent. Intriguingly, observations on the western side of PITW show a strong, eastward flowing undercurrent carrying CDW along the shelf edge (see upper two panels of Figure 17). The troughs are approximately $30 \mathrm{~km}$ wide, considerably larger in scale than the Rossby radius of deformation which is on the order of $3 \mathrm{~km}$, so when the current encounters the trough it is steered south onto the shelf (see lower two panels of Figure 17).

Kimura et al. (in review) find that the inflows combined supply about 6 TW of heat to the shelf. Of this heat, approximately one third is lost to the atmosphere, one third is used to melt ice shelves and one third is carried westward by the on-shelf circulation. 


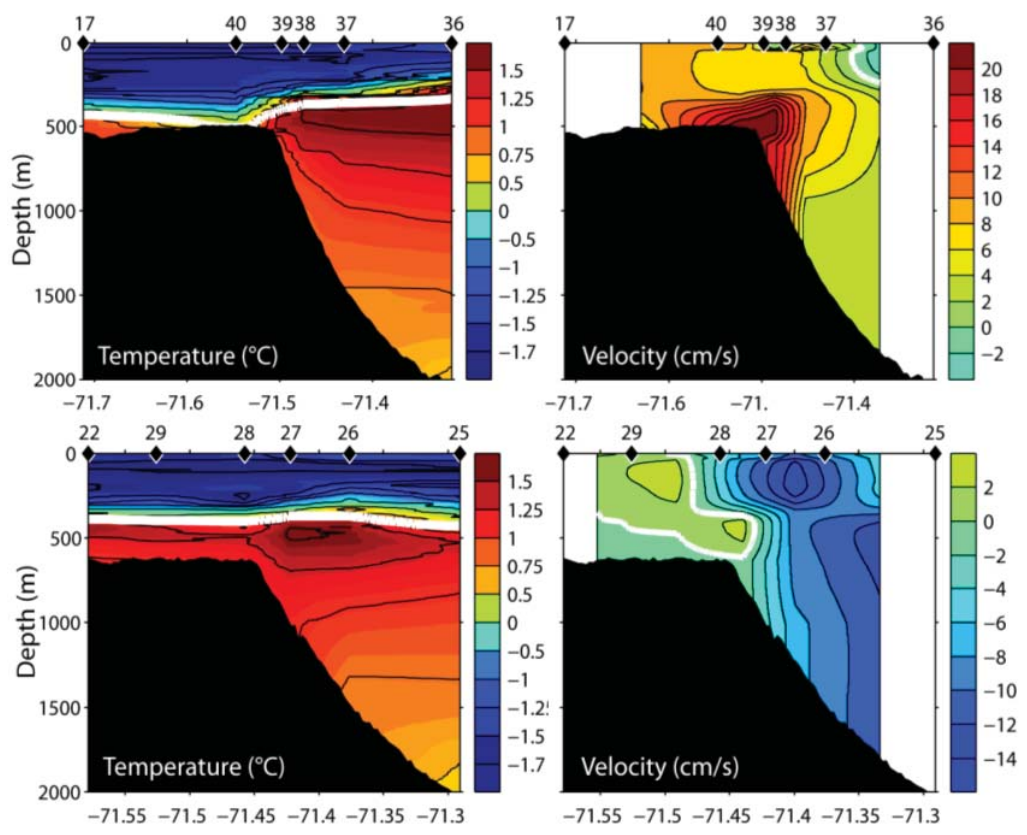

Figure 17: Potential temperature and geostrophic velocity sections for two separate transects; one slightly westward of Pine Island Trough West (PITW) and the other at the entrance to PITW. Positive values are eastward flowing currents. Figure from [26].

\section{$2.2 \quad$ Variability}

\subsubsection{Instrumental record}

Conditions in the Amundsen Sea lie somewhere in between the strongly stratified conditions to the east (e.g. Marguerite Bay) and the weakly stratified conditions in the Ross Sea to the west. In Marguerite Bay the thermocline is extremely shallow and warm CDW occupies most of the water column (Figure 18), however the Ross Sea is almost uniformly cold with some freshening at the surface.

The thermocline depth in the Amundsen sea is highly variable and is sensitive to both wind and buoyancy forcing. Furthermore, variability in thermocline depth drives variability in ice shelf melt. Observations from in front of the Pine Island Glacier (PIG) calving front show that changes in the depth of the thermocline are accompanied by changes in meltwater fraction and thus ice shelf melt (Figure 19). At the PIG calving front, meltwater is identified as a warm, salty anomaly. This result, which is somewhat counter-intuitive as melting is associated with cooling and freshening, can be understood in terms of the watermasses in Figure 20. If the cavity beneath an ice shelf has the temperature-salinity (TS) properties of Ambient 1, warm, salty CDW $\left(x_{1}\right)$ may drive melt and follow the meltwater mixing line to $x_{2}$. The mix of meltwater and ambient fluid will then rise in the water column to its level of neutral buoyancy. Tracing the isopycnal between $x_{2}$ and the open circle on Ambient 1 , we can see that the meltwater mix will be warmer and saltier than the ambient fluid of the same density, and will therefore appear as a warm and salty anomaly. This effect will only occur if the slope of the ambient in TS space is steeper than the meltwater mixing lines; if 


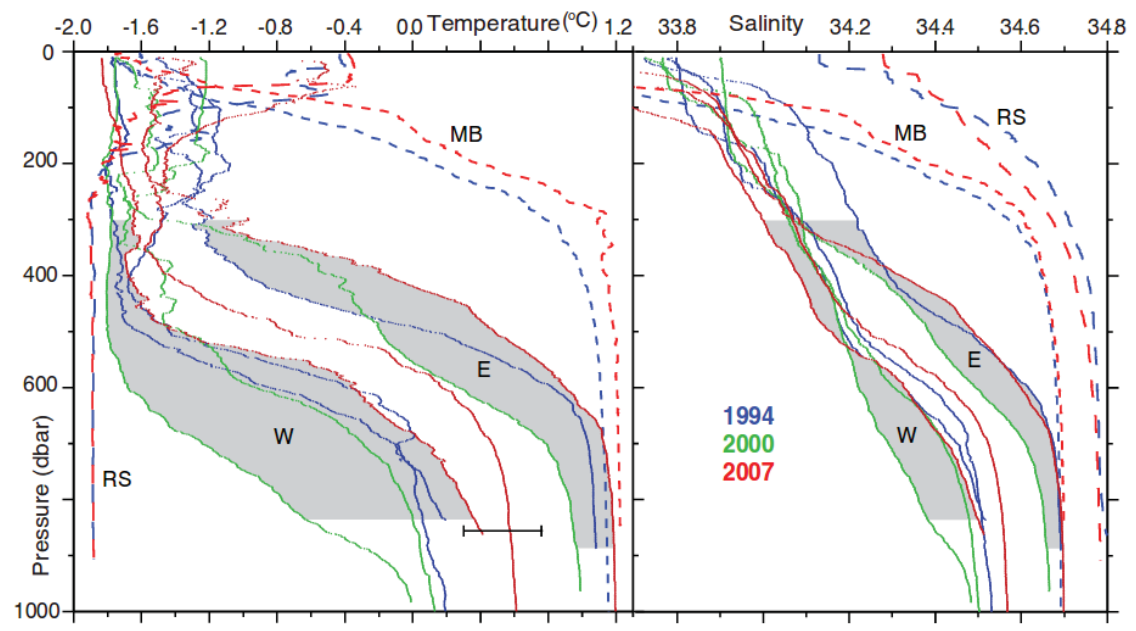

Figure 18: In situ temperature and salinity averages showing thermohaline properties in the Amundsen Sea for 1994, 2000 and 2007. Labels indicate profiles from east of Bear Island (E) and west of Siple Islands (W). The dashed profiles, which are provided for comparison, are from Marguerite Bay (MB) and near Ross Island (RS). Figure from [6].

the slope is shallower (e.g. Ambient 2) the water mass will be cooled and freshened through the addition of meltwater.

In the Amundsen sector, the ambient stratification is set by the mixing of Antarctic surface water (AASW) and CDW. Provided that the AASW salinity is above 33.6 psu, the CDW/meltwater mix will be warmer and saltier at any given density. In winter, the meltwater outflows contain enough heat to melt sea ice.

How different water masses interact with ice shelves is also determined by the geometry of the ice shelf cavity. Beneath Pine Island Glacier, a $300 \mathrm{~m}$ high seafloor ridge provides a partial barrier to inflowing CDW. Much like the relative depths of the thermocline and continental shelf control the flow of CDW onto the shelf, the relative positions of the thermocline depth to the ridge determine how much heat reaches the grounding zone. However, here a positive feedback may be present; the more the ice thins, the wider the gap over the ridge becomes, allowing more CDW to access the grounding line.

West of Pine Island Glacier, at the Dotson Ice Shelf, a time series of temperature sections across the calving front shows that variability in the depth of the thermocline is the primary influence on the average temperature of inflowing water, and that this drives extreme variability in melt fluxes. The melt fluxes calculated from oceanic properties are consistent with satellite-derived measurements of ice shelf melt rate.

Results from the Dotson suggest that melt rate is a non-linear function of temperature. Whilst it is difficult to ascertain this from the Dotson data alone, Figure 22 also includes an estimate of melt in cold water ice shelves, as we know that the pressure dependence of the freezing point allows melting to occur beneath an ice shelf even when the water masses driving it are at surface freezing point. Thus, the melt flux is positive definite even at zero degrees on Figure 22. As a result of this non-linearity, the Amundsen ice shelves are more sensitive to ocean variability because the mean state is warm. 

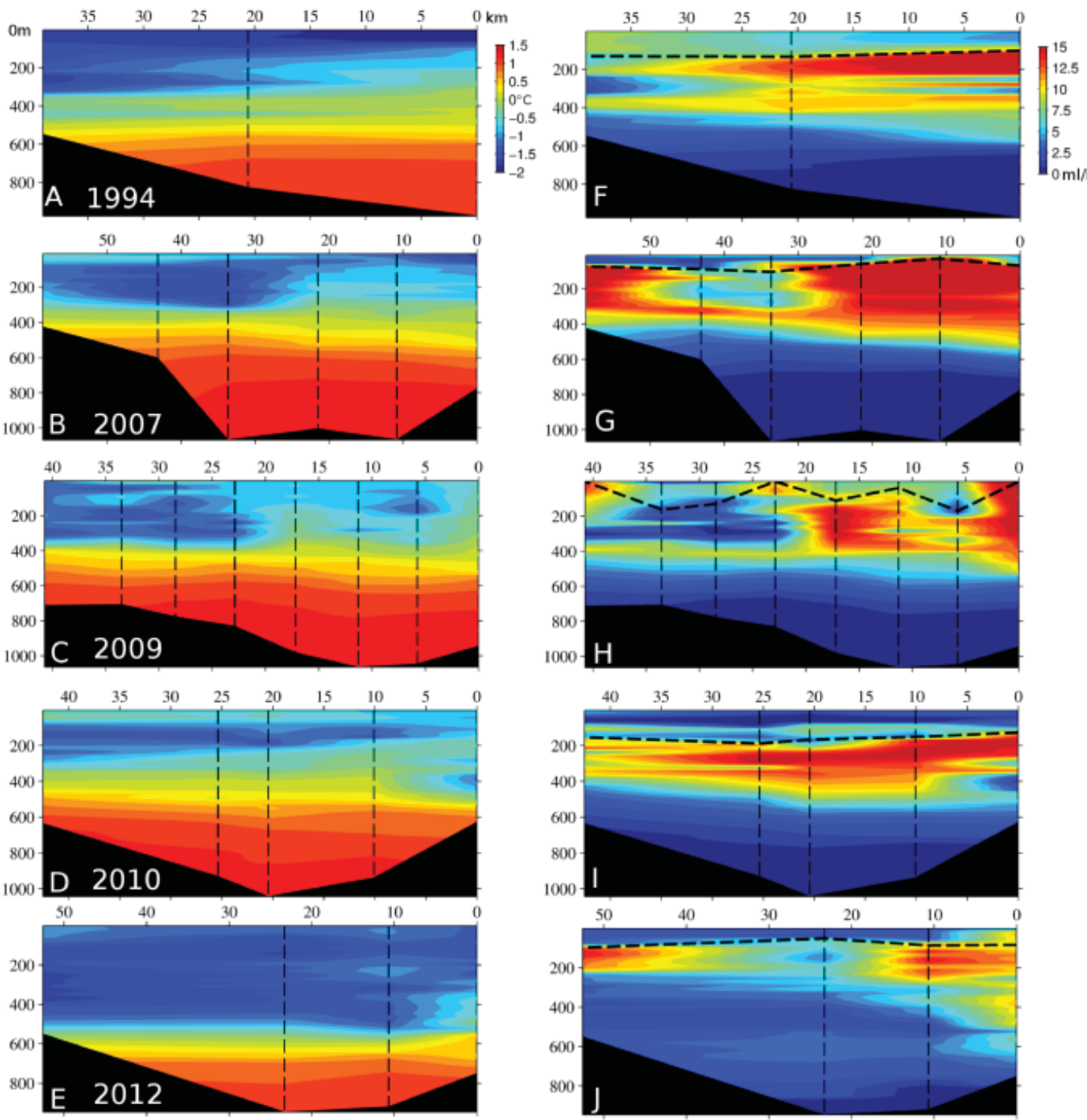

Figure 19: Potential temperature $(\mathrm{A}-\mathrm{E})$ and meltwater fraction $(\mathrm{F}-\mathrm{J})$ sections at the Pine Island Glacier ice shelf calving front for individual years of observation (labeled). Sections are facing into the cavity beneath the ice shelf. Figure from supplementary material of [2].

\subsection{What's driving the variability?}

Model results from Kimura et al. (in review) suggest that variability in shelf edge inflows rather than surface fluxes is the primary control on themocline depth, and hence on melt rates. Whilst surface fluxes affect the AASW layer, they have little impact at the depths that matter to the ice shelf.

The variability in the shelf edge currents themselves is driven by wind, although the mechanism for this is not yet clear. Periods of strong easterlies (Figure 23a) enhance downwelling on the shelf and buoyancy forcing in polynyas and suppress the slope front undercurrent, decreasing transport of CDW onto the shelf and making it more likely to mix with the overlying waters as it passes over the ridge. This results in an overall cooling and decrease in 


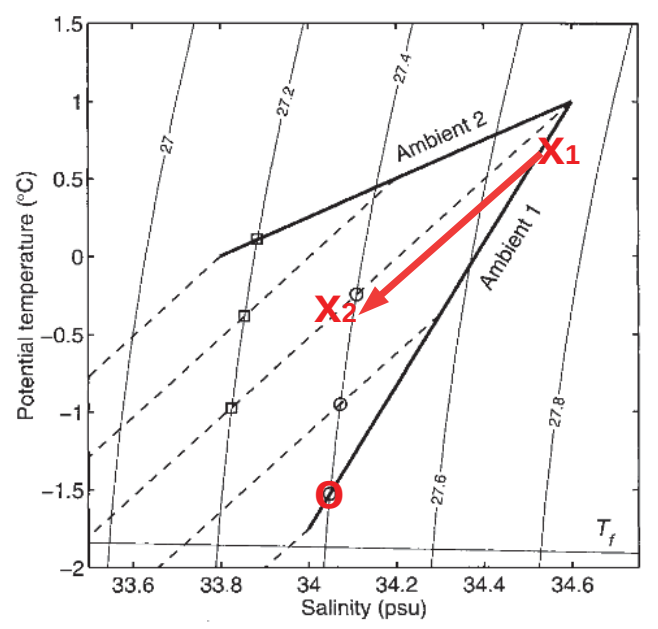

Figure 20: Potential temperature vs salinity diagram illustrating properties of two idealized ambient water columns (bold lines), with melt-water mixing lines (dashed lines) extending from the top, the bottom, and the midpoint of each. Open circles indicate possible properties produced by melting into Ambient 1, while open squares indicate the same for Ambient 2. Figure adapted from [7].

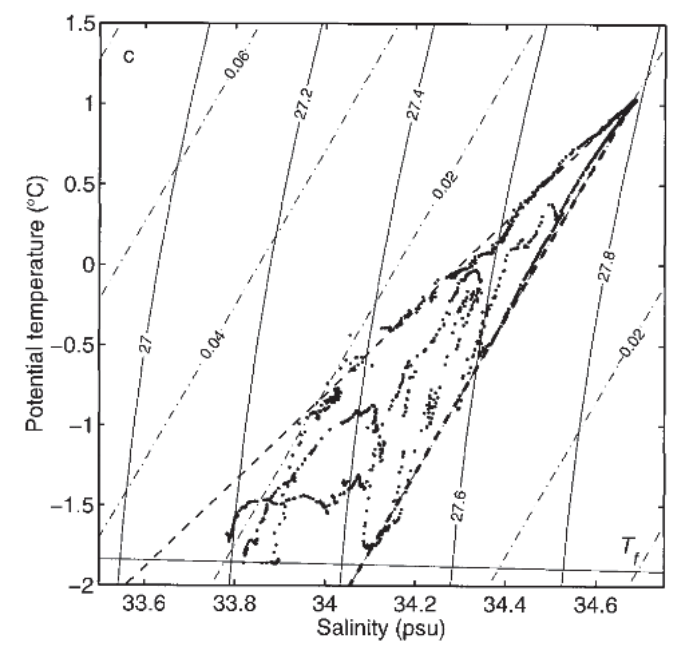

Figure 21: Potential temperature vs salinity plot at the calving front of Pine Island Glacier. Numbered solid lines are isopycnals, and the solid line near the bottom of the diagram indicates the surface freezing point. In (c) dashed lines represent approximations to the ambient trend in the main thermocline (bold) and a melt-water mixing line. The dash-dotted lines are contours of melt-water fraction. Figure from [7]. 


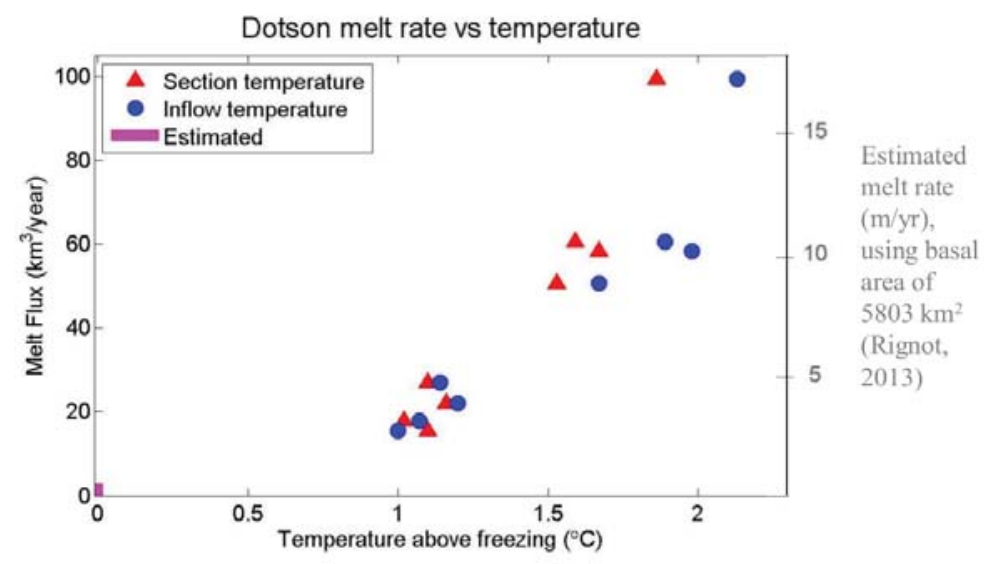

Figure 22: Average temperatures and melt fluxes from the Dotson Ice Shelf. Figure from Jenkins et al. (in preparation).

melt. Conversely, weaker easterly winds are often accompanied by weak westerlies at the shelf edge (Figure 23b). This enhances inflow of CDW which raises the thermocline, allowing more transport over the ridge and increased melting.
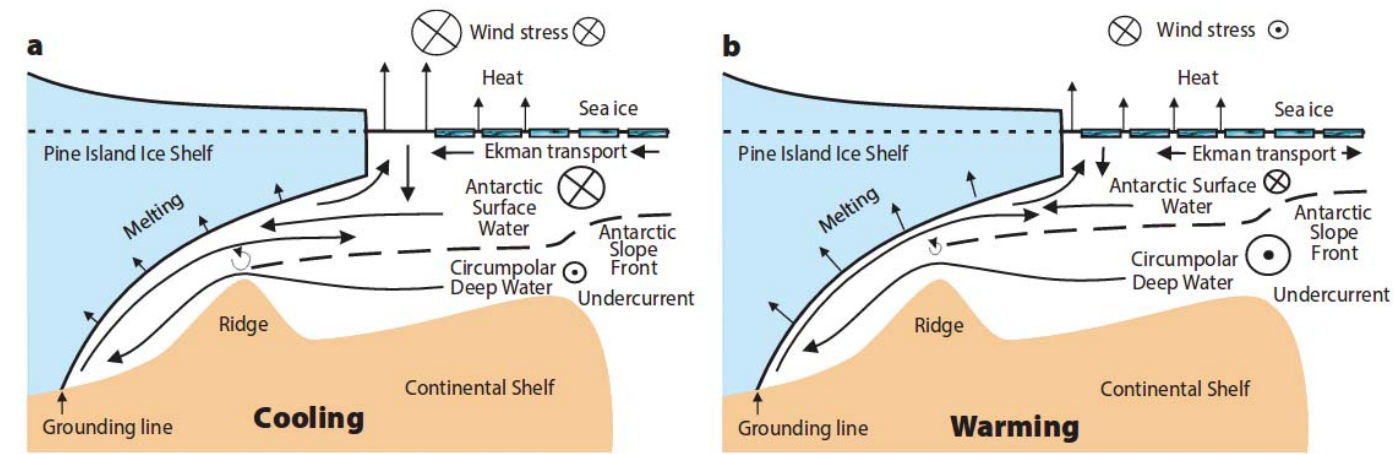

Figure 23: Schematic of processes that lead to (a) cooling and (b) warming of the eastern Amundsen Sea continental shelf. Figure from [9].

\subsubsection{A mechanism for changing the winds}

The weak easterlies in the Amundsen sector are themselves associated with El Niño Southern Oscillation (ENSO). Anomalous heating in the central equatorial Pacific triggers a standing Rossby wave that which results in sea level pressure anomalies in the Amundsen Sea. These anomalies weaken the easterly winds over the Amundsen Sea shelf and can result in westerly winds over the shelf edge.

Over the instrumental record, mooring and CTD (sonde used to measure conductivity, temperature and pressure) observations of inflow properties show a good agreement with zonal wind anomalies and central Pacific Sea Surface Temperature (SST) anomalies (Figure 


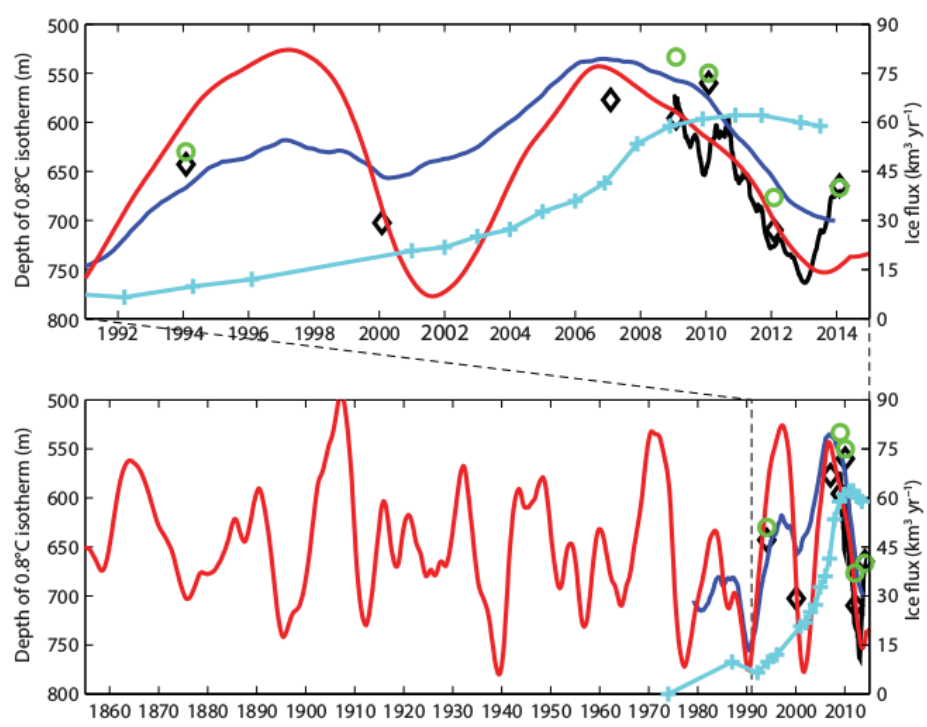

Figure 24: Proxies for thermocline depth on the inner shelf of the eastern Amundsen Sea (the upper panel is an expanded version over the observational period). The depth of the $0.8^{\circ} \mathrm{C}$ isotherm (lefthand axis) is extracted from mooring data (black line) and averages of summer CTD stations (black diamonds). Less direct proxies come from the cumulative zonal wind anomaly (dark blue line) and cumulative central tropical Pacific sea surface temperature anomaly (red line). Figure from [9].

24, upper panel), suggesting that the link between these may explain the dominant mode of variability.

\subsection{Implications for driving ice sheet change}

The Last Glacial Maximum (LGM) was the period during the last glacial cycle during which Earth's ice sheets were at their maximum extent. As such, the retreat of ice between the LGM and present day extent is used to understand the mechanisms for ice loss from Antarctica, and the speed at which they occur.

In the Amundsen sector, the retreat of ice from LGM to present day extent occurred mainly between 10 and 20 thousand years before present. In the following period, the margin appears to have been stable [12].

\subsubsection{The retreat of Pine Island Glacier}

Sediment cores from the ridge beneath Pine Island Glacier reveal that there has been recent change. Pre-1940s sediment records show only coarse grained sediments, transported by the glacier itself, to either side of the ridge (Figure 25). Behind the ridge is a small cavity of water, but this cavity has no connection to the ocean. Post 1945, the presence of fine grained sediments transported by plumes suggest the there was an oceanic cavity behind the ridge with a connection to the outer cavity, either through a bathymetric feature or due to tides. However, the coarse sediments on the front of the ridge demonstrate that the ice shelf is still pinned to the ridge. Post 1970, the presence of fine sediments on both sides of the ridge 
suggest that this is when the cavity took on its current geometry. This assertion is confirmed by early satellite imagery.

Satellite radar interferometry since the early 1990s shows the glacier is still retreating, as are many others along the eastern Amundsen coastline.

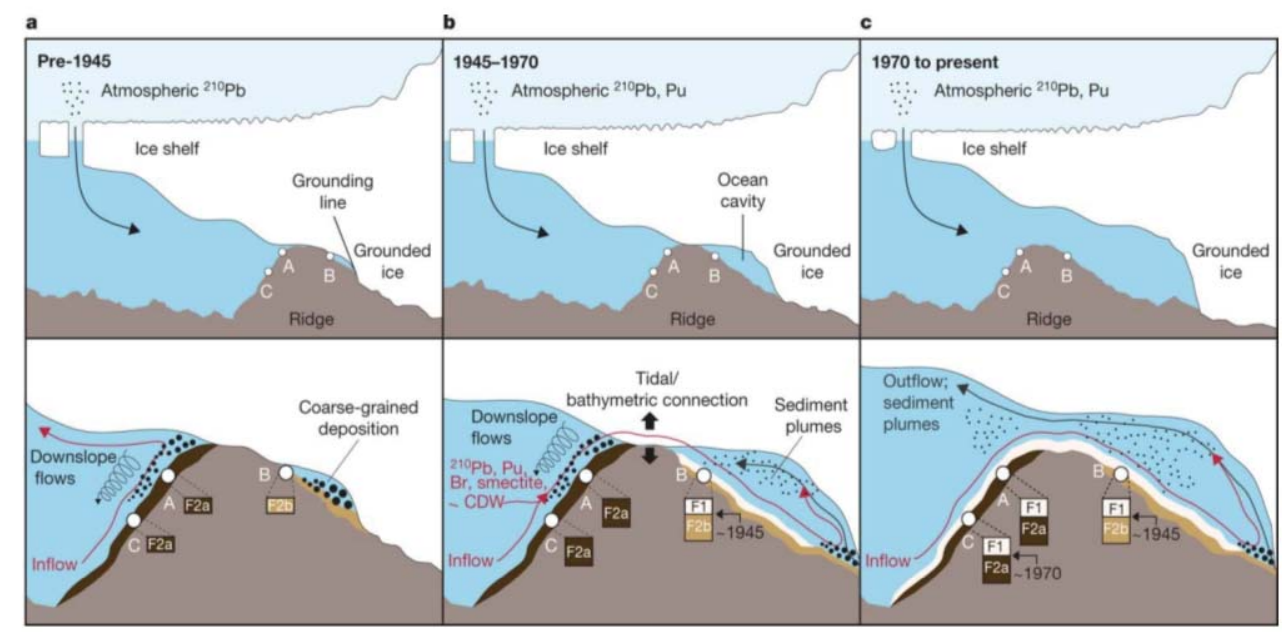

Figure 25: Sedimentation and processes beneath Pine Island Glacier. Figure from [22].

The retreat has been explained as a result of warming of the waters on the Amundsen Sea Shelf [20]. However, the study that published this result uses data up to early 2010, and thus does not include the subsequent cooling of shelf waters. Instead, the recent record of grounding line movement is more consistent with the ocean variability. Grounding line retreat has slowed during the cool phase and the Kohler Glacier has even re-advanced.

\subsubsection{Glacier thinning}

Analysis from satellite altimetry shows that the thinning of glaciers is not a continuous process. During warm periods, enhanced thinning at the grounding line triggers a wave of thinning that propagates inland (Figure 26). Periods of reduced thinning are similarly followed by reduced thinning, or even thickening, inland. For example, the Kohler glacier (Figure 26c) shows a thinning signature initiated pre 1996, however the stability of the current grounding line has prevented any more recent thinning, in contrast to Pine Island and Thwaites glaciers (Figures 26a and 26b).

Using the relationships established in Section 2.3.1 between shelf edge winds (and thus thermocline depth and melt flux) and Pacific SSTs, we can extend our proxy record back in time. Two prominent anomalies occur in the 1940s and 1970s, coinciding with the grounding line events seen in the sediments beneath the Pine Island Glacier. The anomalous period in the 1940s is well documented in ice core records from the West Antarctica, and is the most anomalous period in the 20th century, with the exception of the 1990s.

Records of ice flux across the grounding line of glaciers in the Amundsen region start in the 1970s, when satellite imagery became available. Since the 1970s, each glacier has experienced periods of rapid acceleration and periods of relatively steady flow or slight deceleration. Whilst responses vary from glacier to glacier, it is clear that accelerations correspond with 


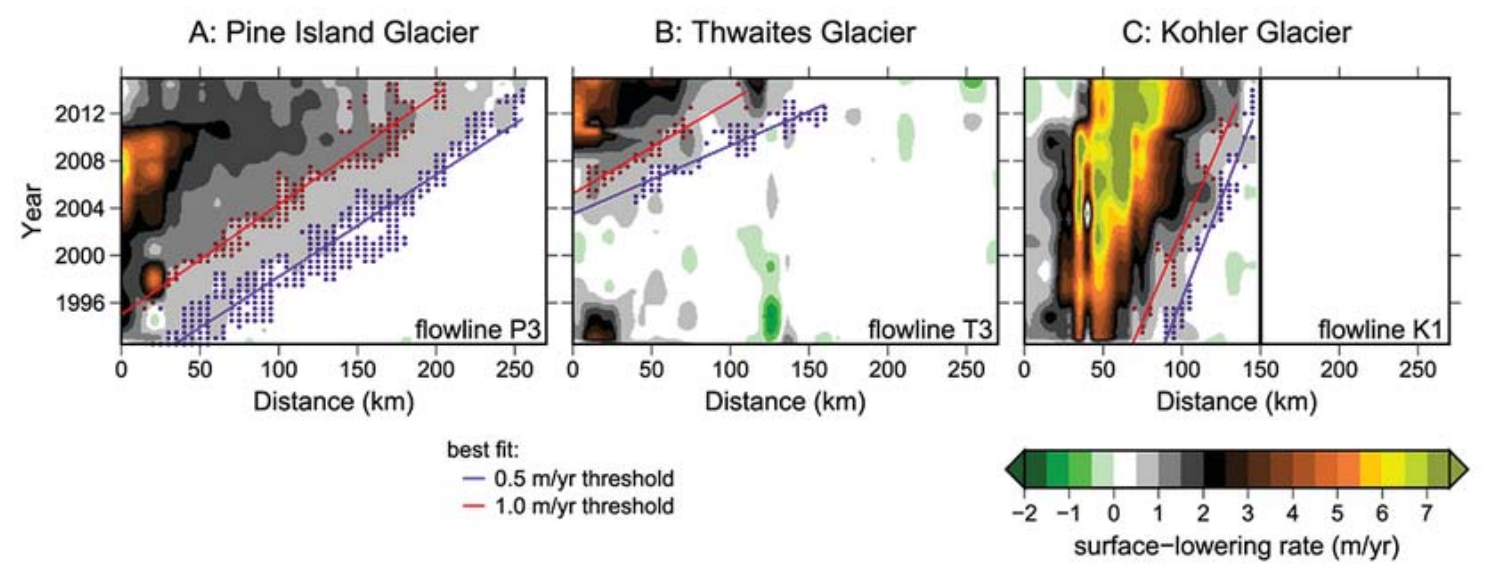

Figure 26: Ice surface-lowering rates along flowlines in the three basins. Figure from [11].
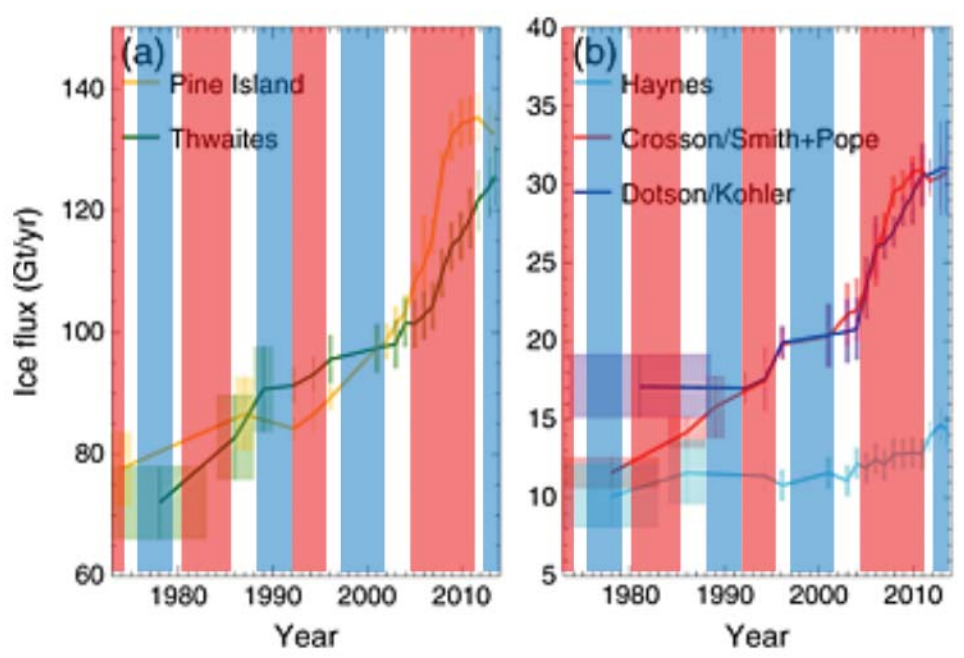

Figure 27: Evolution of ice discharge at the grounding line, where the red shading indicates a warm period and the blue shading is a cool period. Figure adapted from [17].

warm periods, while steady flow coincides with cool periods (Figure 27). The varied responses of individual glaciers is determined by the geometry and properties.

The mechanism for increased melting of an ice shelf driving thinning of a glacier upstream is illustrated in Figure 28. Increased transport of CDW on shelf increases melt rates and thins the glacier (top panel). This decreases the buttressing effect the ice shelf can provide and moves the grounding line back (middle panel), and the glacier accelerates. This changes the surface slope of the glacier, accelerating flow upstream and allowing the thinning signal to propagate. In the lower panel, the ice shelf thickens due to decreased melt, itself a result of a deeper thermocline, which isolates the shelf from CDW.

The propagation of the thinning signal upstream operates on far longer timescales than the adjustment which occurs at the glacier front. Thus, at any one time we may be looking 

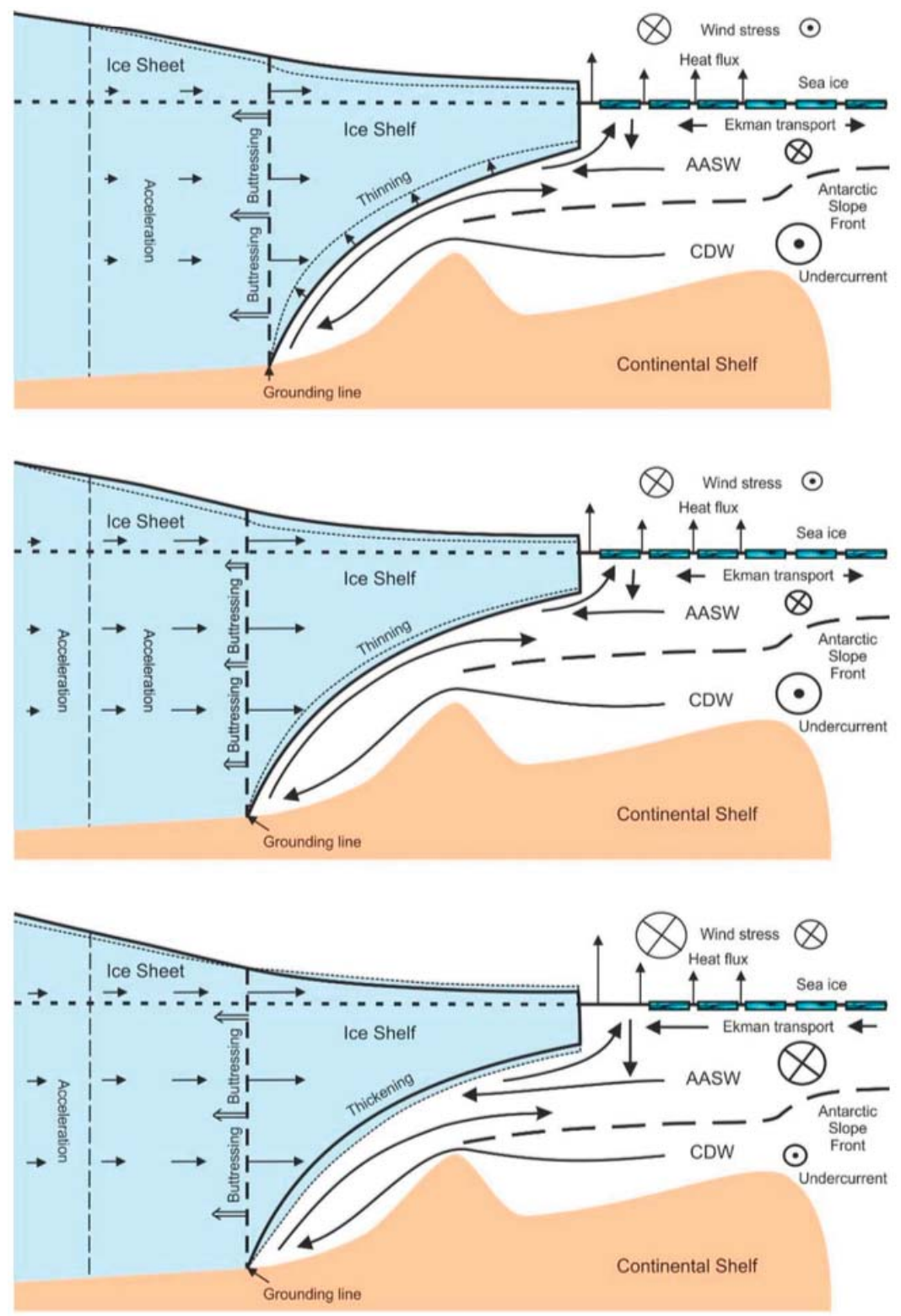

Figure 28: Schematic linking atmospheric and oceanic forcing to ice sheet flow via the thinning/thickening of an ice shelf. Upper: CDW accesses the ice shelf cavity, driving high melt. Middle: The ice shelf thins and the grounding line retreats. The surface slope increases, as does the grounded ice flow. Lower: Increased westerlies lower the thermocline and restrict CDW access, thickening and flattening the ice shelf. 
at a superposition of cooling (thickening, advancing) and warming (thinning, retreating) signals. To further complicate the matter, a key factor in the stability of the grounding line is the geometry of the bed, meaning that a given forcing may influence different glaciers in different ways.

\subsection{Summary}

The Amundsen sea sector is an especially interesting region in that wind variability has the capacity to drive a large response in ice shelves, and consequently in the upstream flow of grounded ice. The sensitivity of the ice to the winds is a result of the ocean state and bathymetry of the region; variability in the thermocline depth and the strength of the slope front undercurrent modulate the on-shelf flow of warm CDW.

Since the 1940s, the ice sheet has been experiencing episodic retreat. Decadal ocean variability can trigger retreat, and once the grounding line is forced from a seabed high, it will continue until the grounding line stabilises long enough for the inland flow to equilibrate. A key question to address is whether, were the shelf to thicken, the previous grounding line could be re-established. In other words, is this a cycle, or is it irreversible?

\section{References}

[1] Chavanne, C. P., Heywood, K. J., Nicholls, K. W., and Fer, I. (2010). Observations of the Antarctic slope undercurrent in the southeastern Weddell sea. Geophysical Research Letters, 37(13).

[2] Dutrieux, P., De Rydt, J., Jenkins, A., Holland, P. R., Ha, H. K., Lee, S. H., Steig, E. J., Ding, Q., Abrahamsen, E. P., and Schröder, M. (2014). Strong sensitivity of Pine Island ice-shelf melting to climatic variability. Science, 343(6167):174-178.

[3] Hattermann, T., Nøst, O. A., Lilly, J. M., and Smedsrud, L. H. (2012). Two years of oceanic observations below the Fimbul Ice Shelf, Antarctica. Geophysical Research Letters, $39(12)$.

[4] Hellmer, H. H., Kauker, F., Timmermann, R., Determann, J., and Rae, J. (2012). Twenty-first-century warming of a large Antarctic ice-shelf cavity by a redirected coastal current. Nature, 485(7397):225-228.

[5] Holland, P. R. and Kwok, R. (2012). Wind-driven trends in antarctic sea-ice drift. Nature Geoscience, 5(12):872-875.

[6] JacoBS, S., Jenkins, A., Hellmer, H., Giulivi, C., Nitsche, F., Huber, B., and Guerrero, R. (2012). The Amundsen Sea and the Antarctic ice sheet. Oceanography, 25(3):154-163.

[7] Jenkins, A. (1999). The impact of melting ice on ocean waters. Journal of Physical Oceanography, 29(9):2370-2381. 
[8] Jenkins, A., Dutrieux, P., Jacobs, S., Steig, E. J., Gudmundsson, G. H., Smith, J., and Heywood, K. J. (2016a). Decadal ocean forcing and Antarctic ice sheet response: Lessons from the Amundsen Sea. Oceanography, 29(4):106-117.

[9] Jenkins, A., Dutrieux, P., Jacobs, S., Steig, E. J., Gudmundsson, G. H., Smith, J., and Heywood, K. J. (2016b). Decadal ocean forcing and Antarctic ice sheet response: Lessons from the Amundsen Sea. Oceanography, 29(4):106-117.

[10] Joughin, I. and Padman, L. (2003). Melting and freezing beneath Filchner-Ronne ice shelf, Antarctica. Geophysical Research Letters, 30(9). 1477.

[11] Konrad, H., Gilbert, L., Cornford, S. L., Payne, A., Hogg, A., Muir, A., and Shepherd, A. (2017). Uneven onset and pace of ice-dynamical imbalance in the Amundsen Sea Embayment, West Antarctica. Geophysical Research Letters, 44(2):910-918.

[12] Larter, R. D., Anderson, J. B., Graham, A. G., Gohl, K., Hillenbrand, C.-D., Jakobsson, M., Johnson, J. S., Kuhn, G., Nitsche, F. O., Smith, J. A., et al. (2014). Reconstruction of changes in the Amundsen Sea and Bellingshausen sea sector of the West Antarctic ice sheet since the last glacial maximum. Quaternary Science Reviews, 100:55-86.

[13] Lenaerts, J. T. M., van den Broeke, M. R., van de Berg, W. J., van Meijgaard, E., and Kuipers Munneke, P. (2012). A new, high-resolution surface mass balance map of Antarctica (1979-2010) based on regional atmospheric climate modeling. Geophysical Research Letters, 39(4). L04501.

[14] Makinson, K., Holland, P. R., Jenkins, A., Nicholls, K. W., and Holland, D. M. (2011). Influence of tides on melting and freezing beneath Filchner-Ronne Ice Shelf, Antarctica. Geophysical Research Letters, 38(6).

[15] Martinson, D. and McKee, D. (2012). Transport of warm Upper Circumpolar Deep Water onto the western Antarctic Peninsula continental shelf. Ocean Science, 8(4):433.

[16] Moffat, C., Owens, B., and Beardsley, R. C. (2009). On the characteristics of Circumpolar Deep Water intrusions to the west Antarctic Peninsula continental shelf. Journal of Geophysical Research: Oceans, 114(C5).

[17] Mouginot, J., Rignot, E., and Scheuchl, B. (2014). Sustained increase in ice discharge from the Amundsen Sea Embayment, West Antarctica, from 1973 to 2013. Geophysical Research Letters, 41(5):1576-1584.

[18] Nicholls, K. W., Østerhus, S., Makinson, K., Gammelsrød, T., and Fahrbach, E. (2009). Ice-ocean processes over the continental shelf of the southern Weddell Sea, Antarctica: A review. Reviews of Geophysics, 47(3).

[19] Nøst, O., Biuw, M., Tverberg, V., Lydersen, C., Hattermann, T., Zhou, Q., Smedsrud, L., and Kovacs, K. (2011). Eddy overturning of the Antarctic Slope Front controls glacial melting in the Eastern Weddell Sea. Journal of Geophysical Research: Oceans, 116(C11). 
[20] Schmidtko, S., Heywood, K. J., Thompson, A. F., and Aoki, S. (2014). Multidecadal warming of Antarctic waters. Science, 346(6214):1227-1231.

[21] Smedsrud, L. H., Jenkins, A., Holland, D. M., and Nøst, O. A. (2006). Modeling ocean processes below Fimbulisen, Antarctica. Journal of Geophysical Research: Oceans, 111(C1).

[22] Smith, J., Andersen, T. J., Shortt, M., Gaffney, A., Truffer, M., Stanton, T., Bindschadler, R., Dutrieux, P., Jenkins, A., Hillenbrand, C.-D., et al. (2016). Sub-ice-shelf sediments record history of twentieth-century retreat of Pine Island Glacier. Nature.

[23] Stewart, A. L. and Thompson, A. F. (2015). Eddy-mediated transport of warm Circumpolar Deep Water across the Antarctic Shelf Break. Geophysical Research Letters, 42(2):432-440.

[24] van Lipzig, N. P. M., Turner, J., Colwell, S. R., and van Den Broeke, M. R. (2004). The near-surface wind field over the Antarctic continent. International Journal of Climatology, 24(15):1973-1982.

[25] Walker, D. P., Brandon, M. A., Jenkins, A., Allen, J. T., Dowdeswell, J. A., and Evans, J. (2007). Oceanic heat transport onto the Amundsen Sea shelf through a submarine glacial trough. Geophysical Research Letters, 34(2).

[26] Walker, D. P., Jenkins, A., Assmann, K. M., Shoosmith, D. R., and Brandon, M. A. (2013). Oceanographic observations at the shelf break of the Amundsen Sea, Antarctica. Journal of Geophysical Research: Oceans, 118(6):2906-2918. 


\title{
GFD 2017 Lecture 8: Testing the Ocean Trigger Hypothesis for Greenland's Recent Glacier Retreat
}

\author{
Fiamma Straneo; notes by Eric Hester and Jessica Kenigson
}

June 28, 2017

\section{Testing the Ocean Trigger Hypothesis for Greenland's Glaciers}

\subsection{Establishing rates of mass loss in Greenland}

The change in mass $M$ of an ice sheet with respect to time is given by

$$
\frac{d M}{d t}=S M B-D
$$

where $S M B$ represents the surface mass balance and $D$ represents the rate of ice discharge. Here $S M B$ is the difference between the rate of accumulation due to precipitation and the rate of ablation due to surface melt and sublimation, while $D$ represents the rate of loss due to glacial calving and melting at the ice margins by the ocean. In order to accurately monitor and predict the ice sheet mass balance in a changing climate, it is necessary to isolate $S M B$ and $D$. In Antarctica, mass loss occurs primarily though $D$ (as ambient temperatures are too low to permit significant mass loss from surface melting); in Greenland this is not the case.

Since 2002, GRACE satellite observations of Greenland mass balance changes through gravimetry have provided data on an ice-sheet-wide scale. Figure 1 shows the cumulative mass change of the ice sheet since 2002 as resolved by GRACE. The declining mass is superimposed upon a significant seasonal cycle of $S M B$.

Prior to GRACE, ice mass changes were necessarily interpolated from scattered in situ observations. Greenland $S M B$ has been relatively adequately monitored since $\sim 1980$. In order to obtain estimates for earlier periods, Greenland Ice Sheet SMB is reconstructed from (typically) atmospheric and snow-pack models. The Regional Atmospheric Climate Model (RACMO) simulates the spatial distribution of climatological SMB from 1958 - 2007 [3]. Spatially, $S M B$ is $\mathcal{O}\left(1000 \mathrm{~kg} \mathrm{~m}^{-2} \mathrm{yr}^{-1}\right)$ along the coast of southeast Greenland due to significant orographic precipitation. Over much of northern Greenland, $S M B$ is $\mathcal{O}(100 \mathrm{~kg}$ $\mathrm{m}^{-2} \mathrm{yr}^{-1}$ ) due to the relatively low precipitation rates in the interior (and by relatively cold temperatures at high latitudes). Over the coastal margin of southwest Greenland, $S M B$ can reach $\mathcal{O}\left(-1000 \mathrm{~kg} \mathrm{~m}^{-2} \mathrm{yr}^{-1}\right)$, which is attributable to significant surface melting. 


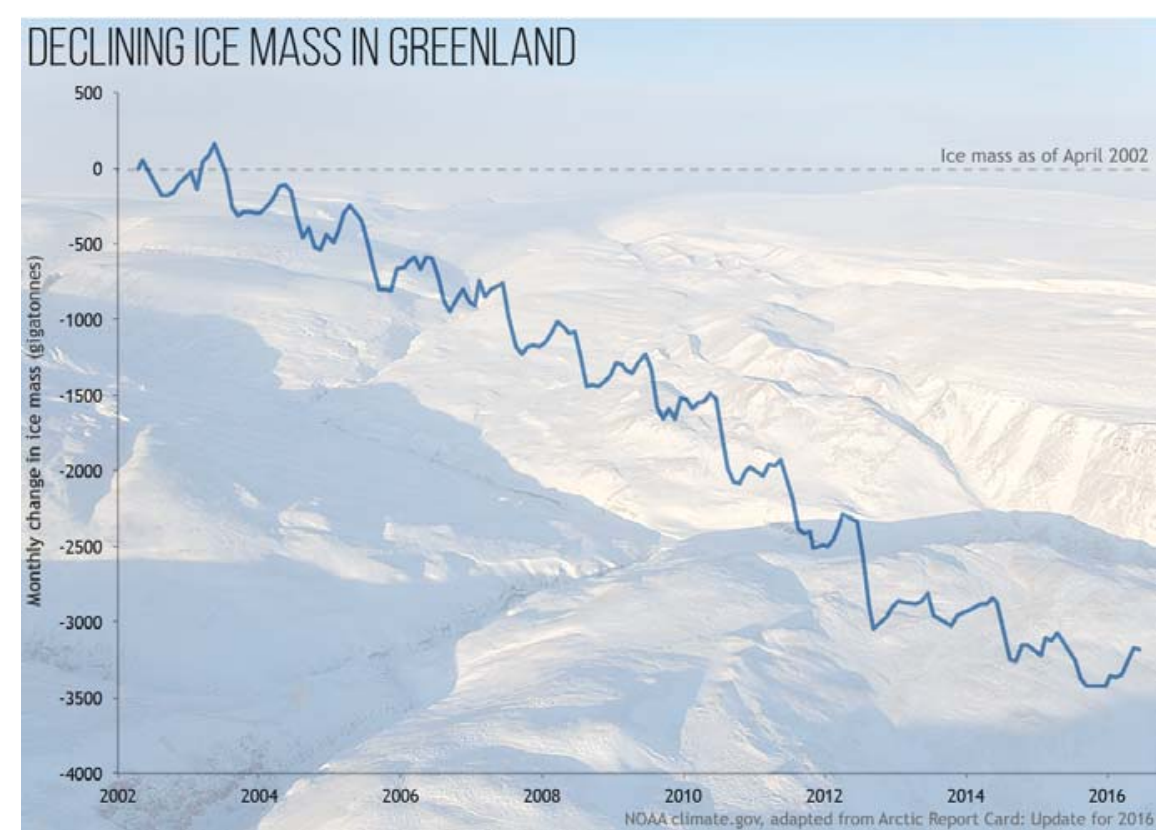

Figure 1: Monthly change in mass of Greenland from April 2002 - April 2016 (cumulative). Figure reproduced from https://www.climate.gov/news-features/featuredimages/greenland-ice-mass-loss-continued-2016.

Measurements of $D$ for a particular glacier are made via remote sensing of the ice velocity across a transect (fluxgate) as near as possible to the grounding line, and $D$ is approximated by

$$
D=V h H
$$

where $V$ is the velocity perpendicular to the transect, $h$ is the width of the fluxgate, and $H$ is the depth of the glacier [5,2]. Typically, $D$ is assumed to be seasonally invariant (due both to a paucity of observations and, when observations have been available, a lack of evidence of a clear seasonal dependence). In one study, $D$ was calculated at 178 outlet glaciers [2]; $\mathrm{V}$ was estimated (where possible) by repeat imaging from the Landsat 7 Enhanced Thematic Mapper Plus and the Advanced Spaceborne Thermal and Reflectance Radiometer (ASTER). In addition, $H$ was obtained from digital elevation models (DEMs) by differencing the bed elevation from the surface elevation (where possible; bed elevation data was not available in the cross-flow direction at all glaciers).

Historical reconstructions of the total mass balance ( $T M B$, defined as $S M B-D)$ require estimates of $D$, which are often based upon correlations between $S M B$ and $D$ over periods in which both quantities have been observed. Figure 2 shows a reconstruction of the Greenland Ice Sheet $S M B, D$, and $T M B$ from 1900 - 2010 [6]. The historical reconstruction is based upon differences between the maximum extent of the ice sheet during the Little Ice Age (as inferred from trimlines and moraines) and aerial photogrammetry from 1978-1987, which allows the change in elevation around the entire perimeter of the ice sheet to be calculated. This is then interpolated to the interior. SMB modeling is used to resolve the mass balance 


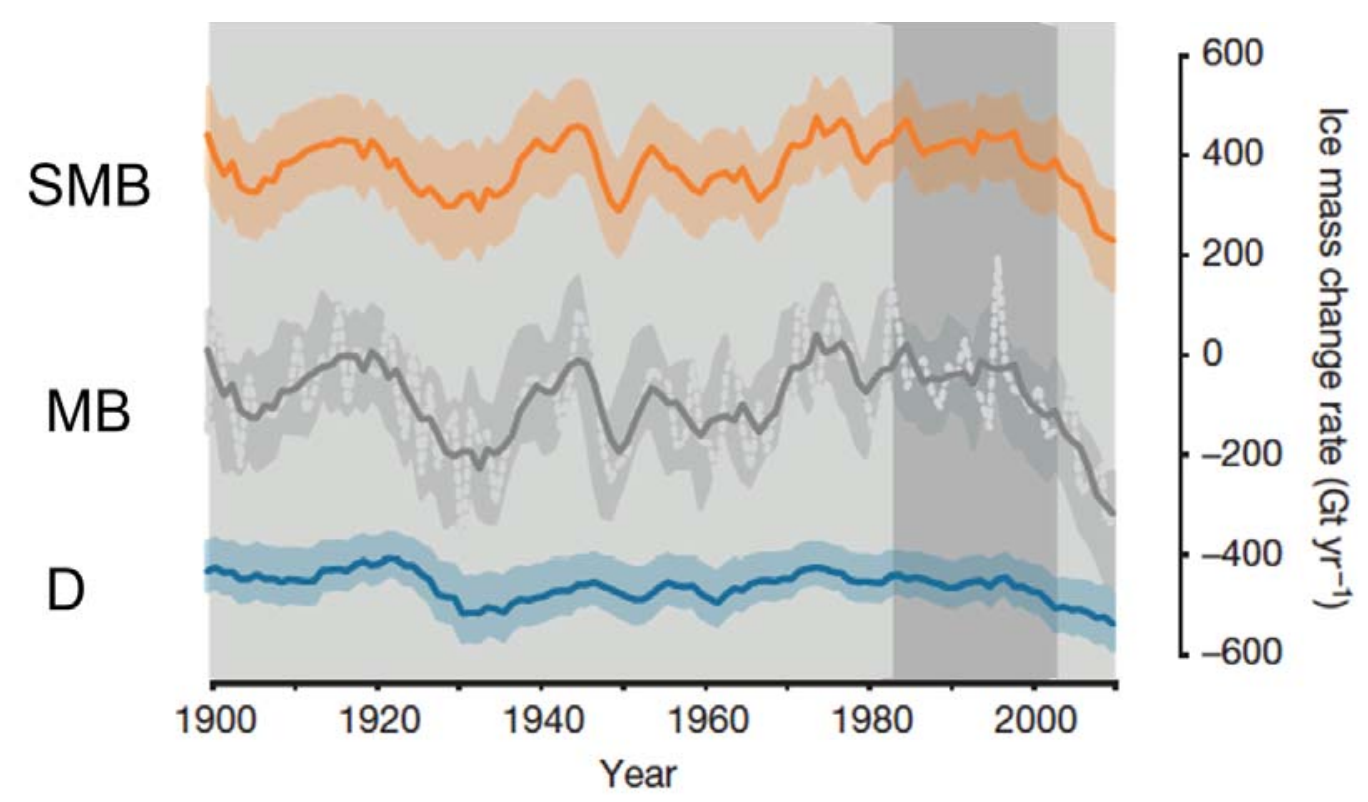

Figure 2: Five-year mean of $S M B$ (orange line), modeled ice discharge (blue line), and 5-year mean of total mass balance (gray) with $1 \sigma$ uncertainty range (shading). Figure subsetted from $[6]$.

into components arising from $S M B$ and $D$ (see their "Methods" section). Mass loss is seen to significantly accelerate around $\sim 1990$, with mass balance deficits increasing at a rate not seen since perhaps $\sim 1920^{-} 1930$. What might account for the accelerated mass loss since $\sim 1990$ ?

\subsection{Ocean trigger hypothesis}

Changes in both $S M B$ and $D$ contribute in approximately equal part to the mass loss from the Greenland Ice Sheet since $\sim 1990$. Moreover, an accelerated retreat of large outlet glaciers beginning around $\sim 2000$ (primarily around the western and southern coast of Greenland) has been documented.

Two major types of glaciers exist along the margins of Greenland: "floating ice tongue" glaciers and tidewater glaciers. Tidewater glaciers are characterized by a relatively shear vertical face and primarily lose mass through glacial calving, while floating ice tongue glaciers are characterized by a long, thin, floating ice protrusion into the ocean from the grounding line and primarily lose mass through melting. Many of Greenland's large tidewater glaciers (including, for example, Jacobskavn Isbrae, Helheim, and Zachariae Isstrom) had floating ice tongues in the recent past.

The ocean trigger hypothesis [13] suggests that the glacier retreat beginning around $\sim 2000$ (Figure 3) and contributing to the relative increase of $D$ (as in [6]) was initiated by oceanic drivers. The intrusion of anomalously warm ocean water onto the shelf causes submarine melting of the floating ice tongue, triggering rapid thinning and ungrounding, which reduces buttressing and causes acceleration and calving. For instance, Jacobshavn Isbrae transitioned from a regime of slow ice accumulation to rapid thinning beginning around 


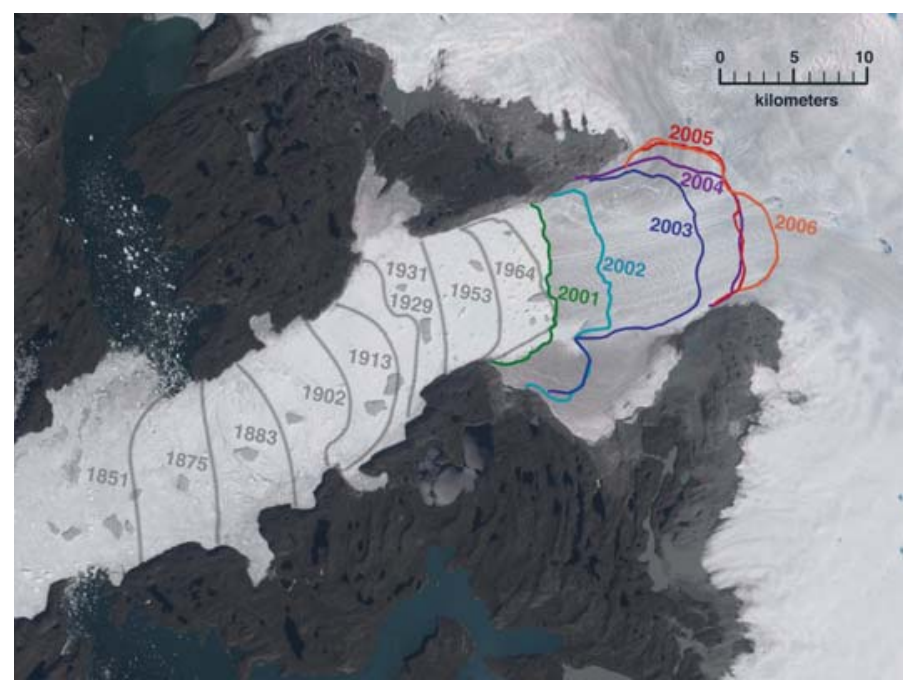

Figure 3: Shift in the calving front at Jacobshavn Isbrae from 1851-2006. Figure reproduced from https://svs.gsfc.nasa.gov/3395.

1997, and this was accompanied by an approximate doubling of velocity [4]. The accelerated mass loss is thought to be associated with warm oceanic inflow from the Irminger Sea. This is in contrast to the hypothesis that atmospheric warming causes enhanced surface melt and bed lubrication, leading to accelerated sliding. Hydrographic data in and around Greenland's fjords is difficult to obtain, particularly at depth. However, this warming signal beginning around 1997 (at depths of 150-600 m) was captured by trawl fishery measurements made from $1991-2006$.

The ocean trigger hypothesis is supported by several independent lines of evidence. Indeed, ocean currents which bifurcate from the North Atlantic Current transport warm equatorial waters close to the southern coastal shelf of Greenland (Figure 5 shows a schematic diagram), suggesting that it is plausible for outlet glaciers to respond sensitively to changes in ocean temperature. However, few direct measurements of ocean temperature at depth along the shelf are available over the period of interest, requiring the use of sparse direct measurements, proxy data, and models. For instance, a numerical ice-flow model with a dynamic calving front has been used to study the reponse of Helheim glacier to various front-stress perturbations, changes in basal lubrication, and changes in the ablation rate [8]. Experiments with front-stress perturbations (which could occur due to rapid thinning of the floating ice tongue) best captured the observed rate of retreat and lend credence to the ocean trigger hypothesis.

Furthermore, paleooceanographic reconstructions fail to refute the ocean trigger hypothesis. For example, at Disko Bugt (West Greenland), a 100-year long (1910 - 2007) record of ocean temperature at approximately $300 \mathrm{~m}$ depth was reconstructed based upon the relative presence of warm and cold water taxa of benthic foraminifera in a series of sediment cores [7]. Indeed, the accelerated retreat of Jacobshavn Isbrae beginning after 1998 coincided with a period of ocean warming locally (and local ocean temperatures were related to the Atlantic Multidecadal Oscillation). 


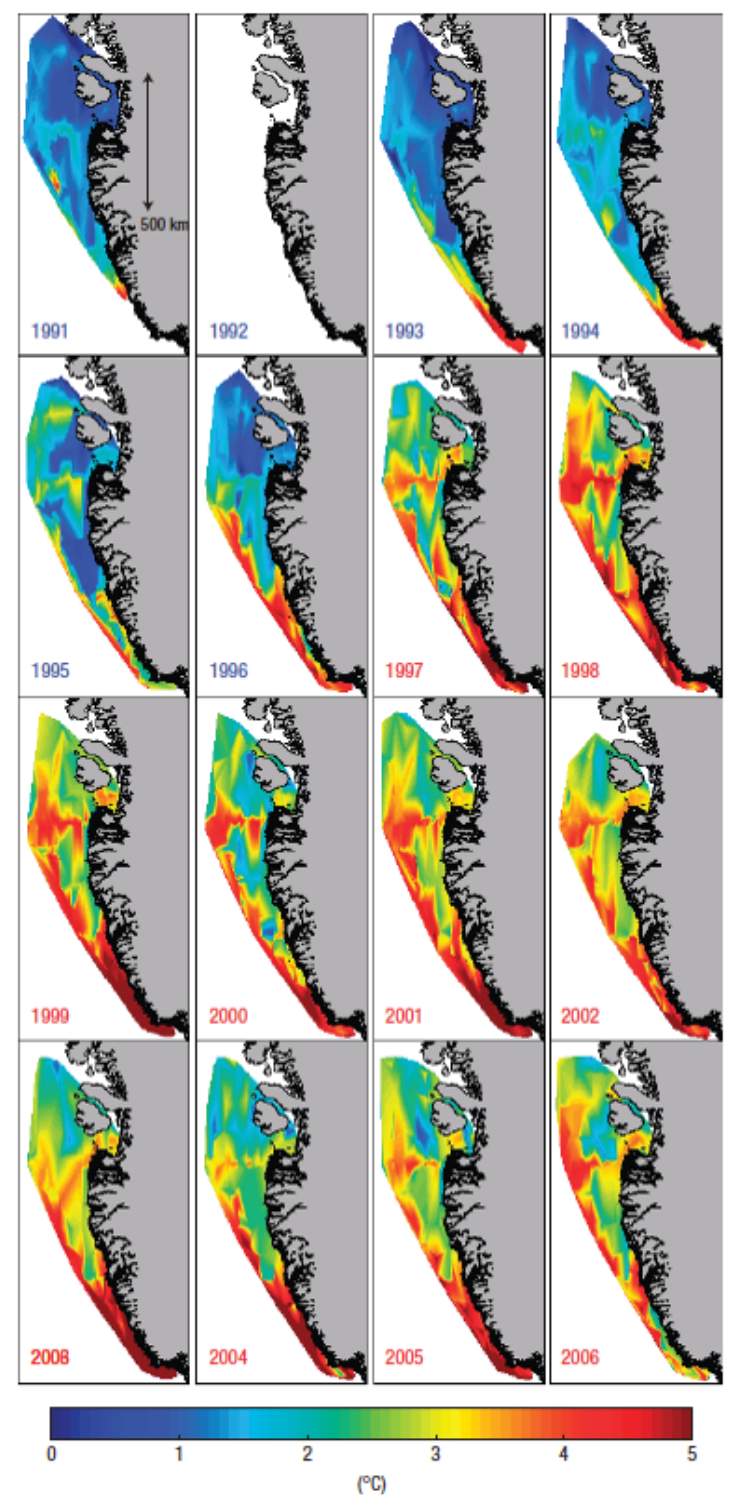

Figure 4: Depth-averaged temperature as obtained from trawl fisheries for1991-2006 (150$600 \mathrm{~m}$ average). Note the increase in temperature near Jacobshavn Isbrae in 1997. Figure reproduced from [4]. 


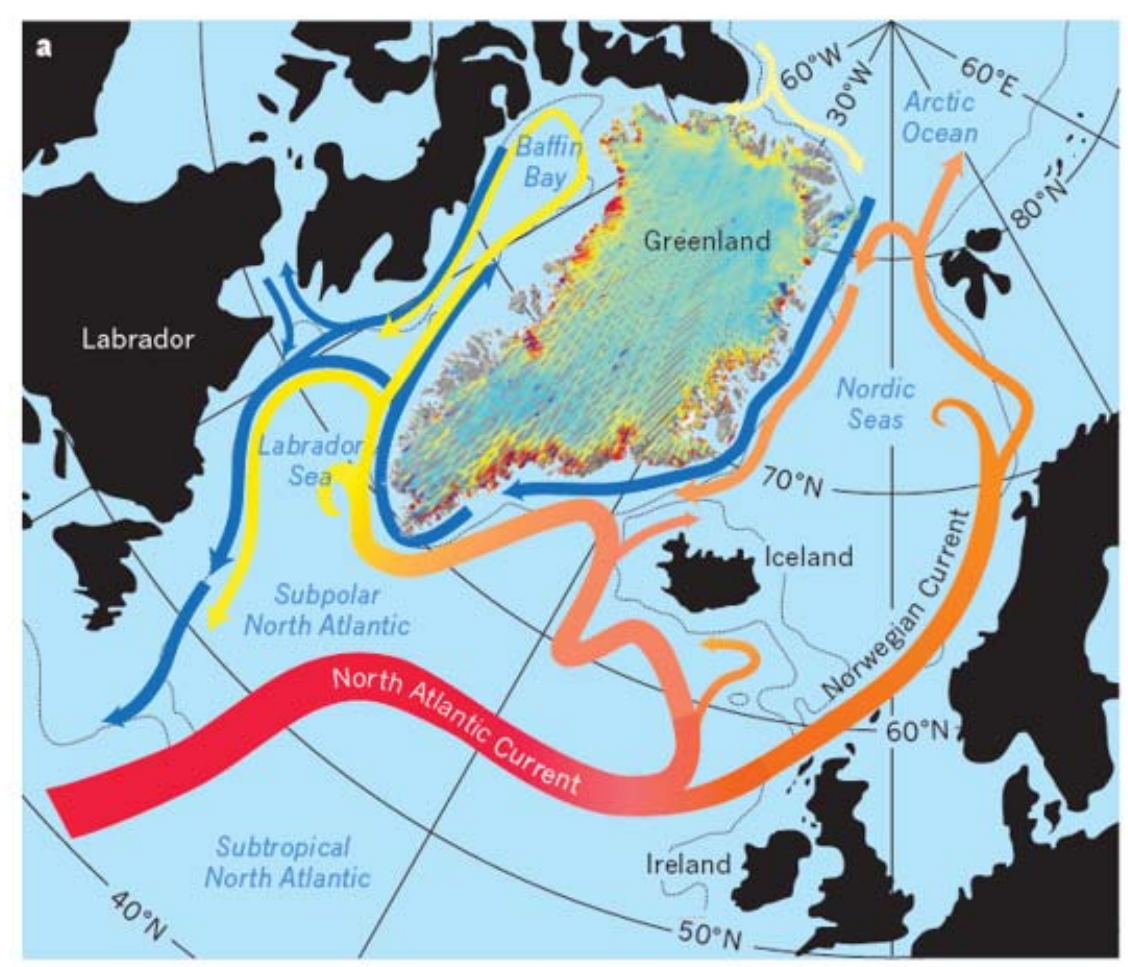

Figure 5: Schematic diagram of the ocean circulation around Greenland. Note the proximity of the warm Irminger Current (warm northward-flowing current branching from the North Atlantic Current to the west) to the coastal shelf of Greenland. Figure adapted from [13]. 
Calving rates may be reconstructed using the observation that icebergs are "dirty." Specifically, Ice-Rafted Debris (IRD) deposited in Semilik Fjord near the Helheim Glacier terminus has been used as a proxy for glacial calving [1]. Based upon measurements from sediment cores, a record of the calving rate has been reconstructed from 1890 to near present. In particular, the sand fraction is used to represent the IRD since sand grains are likely to have been transported by icebergs rather than advected by meltwater plumes due to their large size (which causes them to rain out of suspension). The authors note that the accelerated calving event of the early 2000s (as well as a period during the early $\sim 1930$ s -1940 s) was associated with warm phases of the Atlantic Multidecadal Oscillation (indicating that inflowing Atlantic waters were relatively warm) and with relatively low export of cold Arctic water. This supports the hypothesis that enhanced submarine melt at Helheim was triggered by contact with anomalously warm ocean water.

Indeed, the influx of cold Arctic water through the Fram Strait to the coastal margins of Greenland (as indicated by the Storis index, related to the latitude of the sea ice extent along the coast of southwest Greenland) versus the influx of relatively warm water from the south via the North Atlantic Current/Irminger Current (as given by a temperature transect south of Iceland) likely influences the calving rate [1]. For this reason, a "Shelf Index" is constructed as the sum of these indices, and the Shelf Index is seen to correlate with the calving rate on interannual and longer timescales $(r=0.41$ for 3-year mean, which is statistically significant at the $95 \%$ level). Correlations between the (negative) Storis index and Atlantic water temperatures as measured along the transect are nearly as strong, yet correlations with atmospheric variables such as the wintertime North Atlantic Oscillation index are also significant $(r=-0.45)$.

Thus, we see that there are multiple independent lines of evidence to support the Ocean Trigger hypothesis.

\section{Ice-ocean Interactions in Greenland Glaciers}

The evolution of Greenland glaciers depends on a range of complex phenomena, associated with changes in atmospheric and oceanic conditions on multiple spatial and temporal scales.

This lecture will outline the current understanding of the effect of oceanic forcing on Greenland glaciers, and the techniques used to establish these facts.

\subsection{Greenland glaciers: tidewater vs tongues}

There are two types of outlet glaciers in Greenland, characterised by their structure beyond their grounding line (the furthest point at which they are in contact with the sea bed).

The first and most common, tidewater glaciers, do not extend far beyond their grounding line, and display vigorous calving (iceberg production) at their edge. The other type, floating tongue glaciers, instead extend tens of kilometres beyond their grounding line. Further, floating ice tongues typically balance the incoming ice flux by melt, and do not strongly calve. Floating tongue glaciers are able to balance the incoming ice flux by melt as they have a much larger area in contact with the fjord waters. 


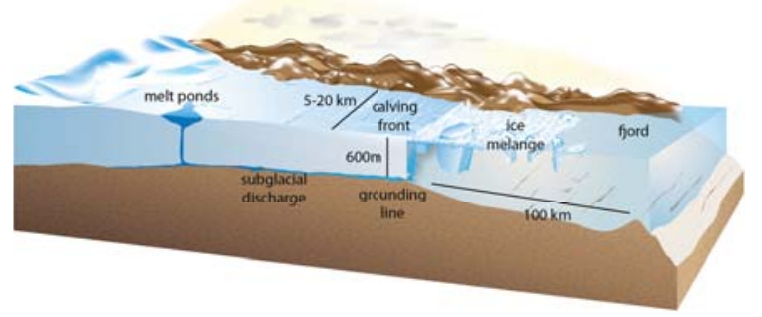

Figure 6: Schematic of typical Greenland tidewater glacier. Figure adapted from $[14]$.

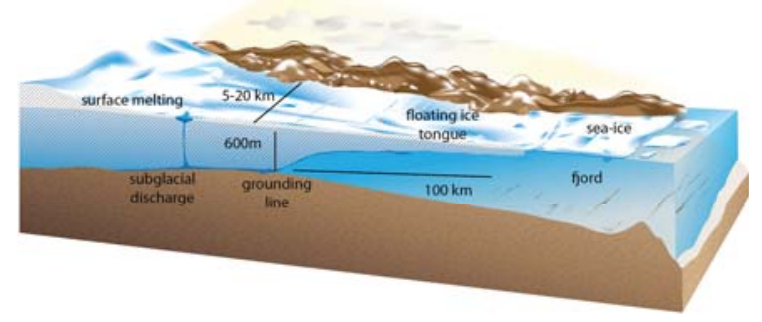

Figure 7: Floating tongue glaciers extend far beyond the grounding line. Figure adapted from [14].

\subsection{Ocean water in fjords}

The waters surrounding East Greenland are divided between two dominant water types - the warmer Atlantic water (AW) supplied by the North Atlantic current, and cooler polar water (PW) from the pole (Figure 8). The location of these waters, and in particular their interaction with glaciers when within the fjord, is believed to control glacial melting.

Straneo et. al. [12] performed ship and mooring based measurements of oceanographic data in Sermilik Fjord during 2008. They found the bottom of the fjord (beyond 200-300 m) was filled with warmer Atlantic water, while cooler polar water resided in the higher layers. These two modes were supplemented by a third water mass of glacial meltwater during the summer.

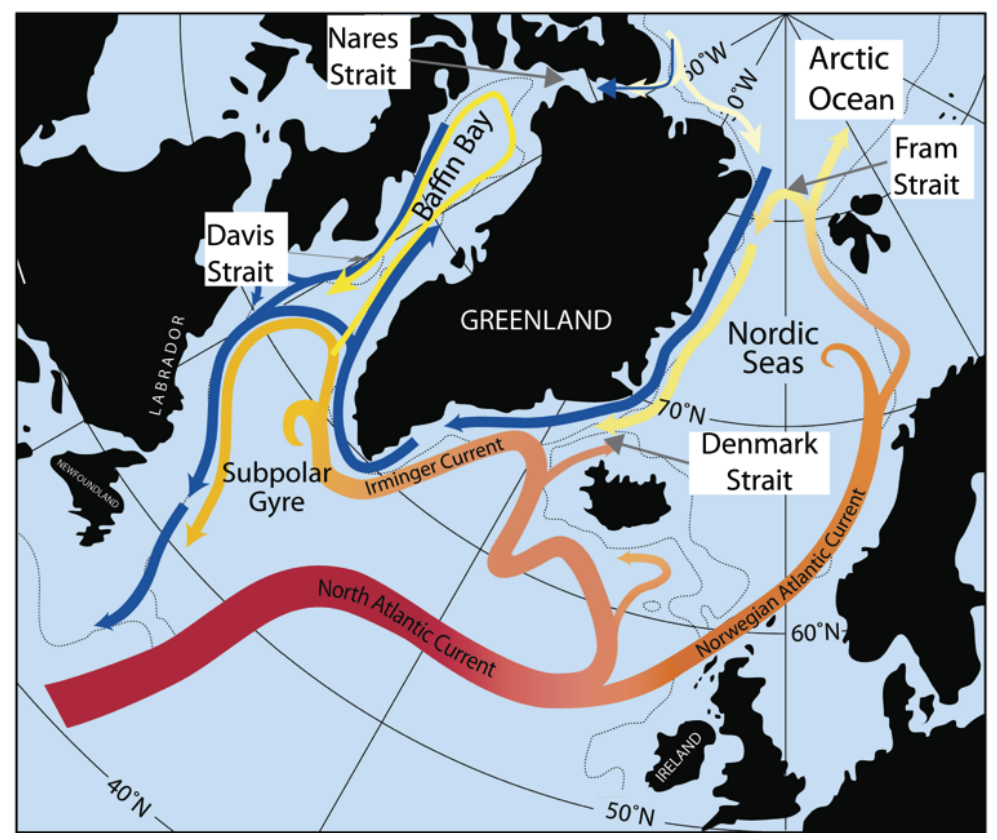

Figure 8: Schematic of ocean currents around Greenland. Figure from [10].

Importantly, they found that these waters were continuously replenished [12]. There are several mechanisms that contribute to this replacement, but one main driver of fjord/shelf 
exchange is variations in the pycnocline on the shelf near the mouth of the fjord.

Figure 9 illustrates this mechanism with the example of Ekman transport by along coast winds. These winds (into the page) force transport of the surface layer toward the right (into the fjord), depressing the shelf pycnocline. The fjord waters then equilibrate to this new stratification by inflow of the top polar water, and outflow of the bottom Atlantic water. When the forcing ceases, the fjord waters will then relax to the original equilibrium, thereby replenishing the waters.
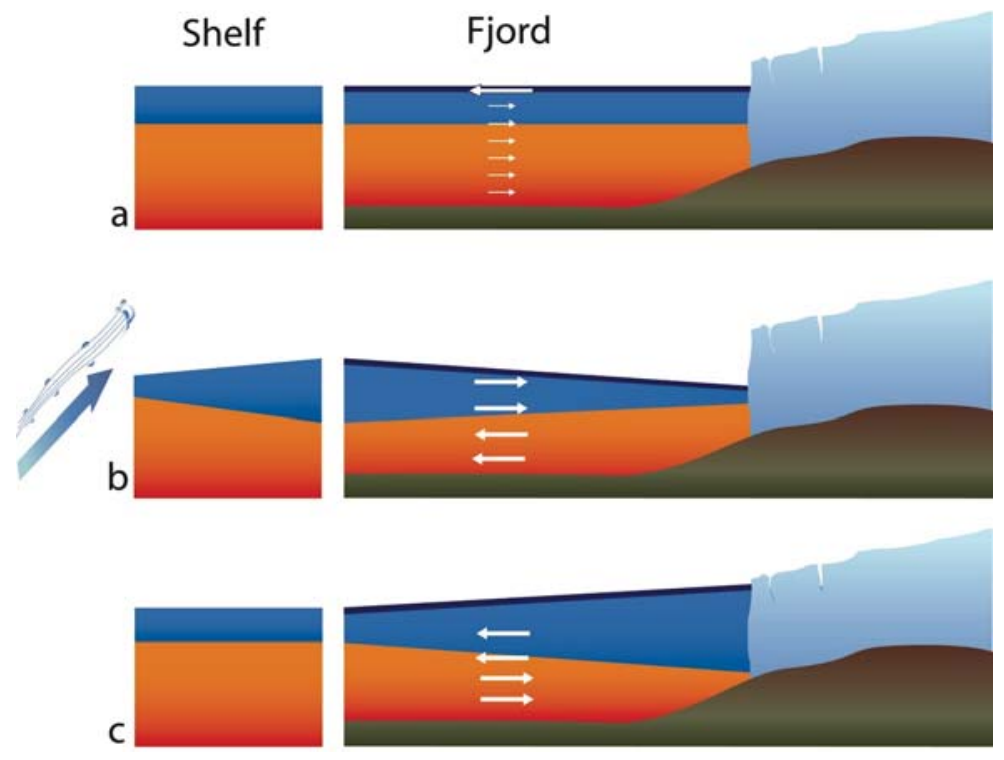

Figure 9: Wind driven forcing of shelf waters will adjust the shelf pycnocline, to which the fjord waters equilibrate. When the forcing ceases, the fjord waters readjust to the previous equilibrium (c). Figure adapted from [12] (Supplementary Information).

The presence of high sills in fjords may be able to block this transfer however $[9,16]$, mitigating the i nfluence of the warmer Atlantic water.

\subsection{Glacial melt from temperature-salinity diagrams}

The distribution of fjord water characteristics is highly revealing when plotted on a (potential) Temperature $(\theta)$ - Salinity $(S)$ diagram. This is because when salt water melts ice, the properties of the water-ice mixture will evolve along a straight line in $\theta-S$ space - as explained in Adrian Jenkins' first lecture. Water measurements close to the line imply the melting of glacial ice; divergence implies some other process is occurring (such as mixing with glacial runoff).

Straneo and others $[11,15]$ found that measurements around Greenland glaciers were consistent with melting of glaciers by Atlantic Water. The red curves in Figure 10 show $\theta-S$ measurements of water near the fjord mouth, while the blue curves are near the glacier. The winter measurements (on the right), show that water near the glacier lies closer to the melting line of Atlantic water, implying that the water is melting the glacier. 
The summer measurements tell a different story however. Now, the near-glacier water diverges from the melting line of the Atlantic water, instead being much fresher than expected. This is due to discharge, including at depth, of surface melt driven by a warm atmosphere above the ice.
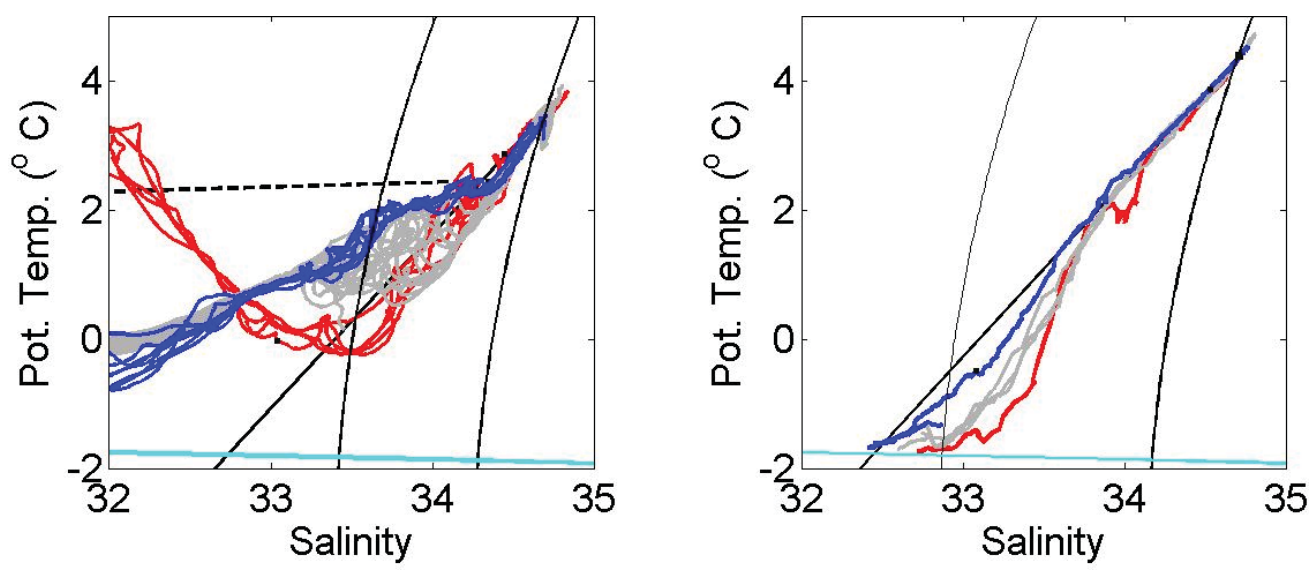

Figure 10: $\theta-S$ measurements near Helheim glacier in summer (left) and winter (right). The red curves are of waters near the mouth of the fjord, and the blue readings are as close as possible to the glacier edge. The influence of the glacier is seen through the differences between the red and blue curves. The solid black line shows the melting line of Atlantic water, while the dashed line shows the line for mixing with glacial runoff. The curved lines are isopycnals, the cyan line shows the freezing temperature at zero pressure for varying salinities. Figure adapted from [11].

\subsection{Lagrangian ice flux divergence measurements}

The melting of ice tongues can be inferred by measuring the divergence of the ice flow. Assuming a vertically uniform velocity $\boldsymbol{u}=(u, v)$ and density $\rho_{i}$, the melt rate $\dot{a}$ of a floating ice tongue can be inferred from the conservation law for ice thickness $h$ :

$$
\frac{\partial h}{\partial t}+\nabla \cdot(h \boldsymbol{u})=\frac{\partial h}{\partial t}+\boldsymbol{u} \cdot \nabla h+h \nabla \cdot \boldsymbol{u}=\dot{a} .
$$

This Eulerian framework suffers from a key drawback however; for sparse sampling times, the calculation of time derivatives will be affected by aliasing - If the sampling time is too sparse and a second peak is in the same location as a past peak, then there is no way to infer a change in thickness of the ice.

A more effective approach is to switch to a Lagrangian framework, in which we track the time derivative of the ice thickness following the ice, Dh/Dt $=\partial h / \partial t+\boldsymbol{u} \cdot \nabla h$. By tracking the ice, we are able to minimize aliasing. This gives our conservation law as

$$
\frac{D h}{D t}+h \nabla \cdot \boldsymbol{u}=\dot{a}
$$




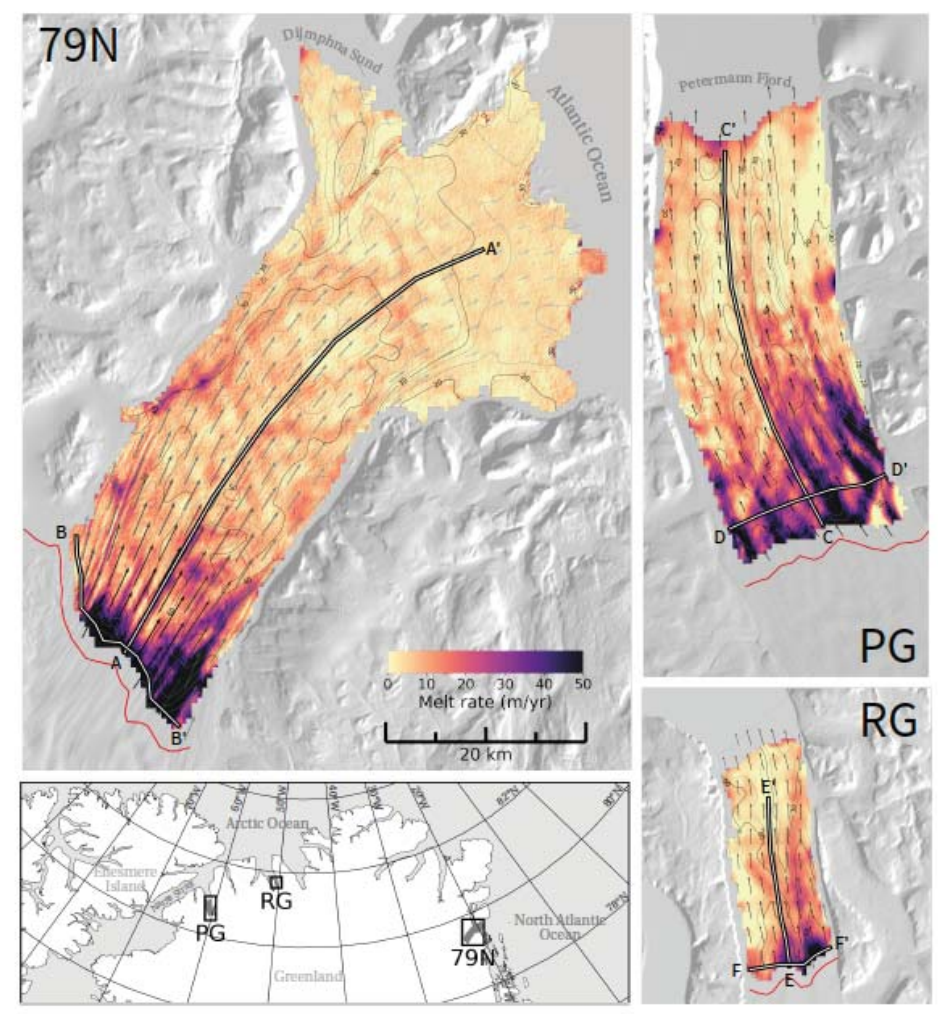

Figure 11: Greenland glacier melt rates determined using Lagrangian ice flux divergence measurements. Figure from [17].

The first step in estimating these quantities is to measure the surface elevation of the ice tongue using Digital Elevation Maps at multiple times.

The thickness of the ice tongue can the be measured given knowledge of the tidal data, and assuming hydrostatic balance of the ice. The hydrostatic approximation becomes invalid within several kilometres of the grounding line, preventing the use of this technique in these areas.

By cross correlating successive elevation maps, the velocity of the ice $\boldsymbol{u}$ can be inferred, and the elevation (thus thickness) change $D h / D t$ can be measured. From these measurements, the total melt rate of the ice tongue can then be inferred. The submarine melting can finally be isolated by subtracting the surface melt using a model of atmospheric melting. Putting all this together, the subglacial melt of glaciers can be calculated, as seen in Figure 11 for three Greenland glaciers [17].

\section{References}

[1] C. S. Andresenn, F. Straneo, M. H. Ribergaard, A. A. Buørk, T. J. Andersen, A. Kuijpers, N. Nørgaard-Pedersen, K. H. Kjær, F. Schjøth, 
K. WeCKStröm, ET AL., Rapid response of Helheim Glacier in Greenland to climate variability over the past century, Nature Geoscience, 5 (2012), pp. 37-41.

[2] E. M. Enderlin, I. M. Howat, S. Jeong, M.-J. Noh, J. H. Angelen, And M. R. Broeke, An improved mass budget for the Greenland ice sheet, Geophysical Research Letters, 41 (2014), pp. 866-872.

[3] J. Ettema, M. R. van den Broeke, E. van Meijgaard, W. J. van de Berg, J. L. Bamber, J. E. Box, And R. C. BAles, Higher surface mass balance of the Greenland ice sheet revealed by high-resolution climate modeling, Geophysical Research Letters, 36 (2009).

[4] D. M. Holland, R. H. Thomas, B. De Young, M. H. Ribergaard, and B. LyBERTH, Acceleration of Jakobshavn Isbrae triggered by warm subsurface ocean waters, Nature geoscience, 1 (2008), pp. 659-664.

[5] I. M. Howat, I. Joughin, And T. A. Scambos, Rapid changes in ice discharge from Greenland outlet glaciers, Science, 315 (2007), pp. 1559-1561.

[6] K. K. Kueldsen, N. J. Korsgaard, A. A. Buørk, S. A. Khan, S. Funder, N. K. Larsen, J. L. Bamber, W. Colgan, M. van den Broeke, M.-L. SiggaARD-Andersen, ET AL., Spatial and temporal distribution of mass loss from the Greenland Ice Sheet since AD 1900, Nature, 528 (2015), pp. 396-400.

[7] J. Lloyd, M. Moros, K. Perner, R. J. Telford, A. Kuijpers, E. Jansen, AND D. MCCARTHY, A 100 yr record of ocean temperature control on the stability of Jakobshavn Isbrae, West Greenland, Geology, 39 (2011), pp. 867-870.

[8] F. M. Nick, A. Vieli, I. M. Howat, And I. Joughin, Large-scale changes in Greenland outlet glacier dynamics triggered at the terminus, Nature Geoscience, 2 (2009), pp. 110-114.

[9] F. Schjoth, C. S. Andresenb, F. Straneo, T. Murray, K. Scharrer, and A. Korablev, Campaign to map the bathymetry of a major Greenland fjord, Eos, Transactions American Geophysical Union, 93 (2012), pp. 141-142.

[10] F. Straneo And C. Cenedese, The dynamics of Greenland's glacial fjords and their role in climate, Annual Review of Marine Science, 7 (2015), pp. 89-112. PMID: 25149564 .

[11] F. Straneo, R. G. Curry, D. A. Sutherland, G. S. Hamilton, C. Cenedese, K. VAGE, AND L. A. SteARns, Impact of fjord dynamics and glacial runoff on the circulation near Helheim Glacier, Nature Geosci, 4 (2011), pp. 322-327.

[12] F. Straneo, G. S. Hamilton, D. A. Sutherland, L. A. Stearns, F. Davidson, M. O. Hammill, G. B. Stenson, And A. Rosing-Asvid, Rapid circulation of warm subtropical waters in a major glacial fjord in East Greenland, Nature Geosci, 3 (2010), pp. $182-186$. 
[13] F. Straneo and P. Heimbach, North Atlantic warming and the retreat of Greenland's outlet glaciers, Nature, 504 (2013), pp. 36-43.

[14] F. Straneo, P. Heimbach, O. Sergienko, G. Hamilton, G. Catania, S. Griffies, R. Hallberg, A. Jenkins, I. Joughin, R. Motyka, W. T. Pfeffer, S. F. Price, E. Rignot, T. Scambos, M. Truffer, and A. Vieli, Challenges to understanding the dynamic response of Greenland's marine terminating glaciers to oceanic and atmospheric forcing, Bulletin of the American Meteorological Society, 94 (2013), pp. 1131-1144.

[15] F. Straneo, D. Sutherland, D. Holland, C. Gladish, G. Hamilton, H. Johnson, E. Rignot, Y. Xu, And M. Koppes, Characteristics of ocean waters reaching Greenland's glaciers, Annals of Glaciology, 53 (2012), pp. 202-210.

[16] D. A. Sutherland, F. Straneo, G. B. Stenson, F. J. Davidson, M. O. HamMill, And A. Rosing-Asvid, Atlantic water variability on the SE Greenland continental shelf and its relationship to sst and bathymetry, Journal of Geophysical Research: Oceans, 118 (2013), pp. 847-855.

[17] N. Wilson, F. Straneo, and P. Heimbach, Submarine melt rates and mass balance for Greenland's remaining ice tongues, The Cryosphere, 2017 (in press, 2017), pp. 1-17. 


\title{
GFD 2017 Lecture 9: Greenland Glacier-Ocean Interaction Part I
}

\author{
Fiamma Straneo; notes by Margaret Lindeman, Agostino Meroni, \\ and Earle Wilson
}

June 29, 2017

\section{The Near Ice Zone}

\subsection{Diagnosing the influence of subglacial discharge}

The Greenland Ice Sheet (GIS) is l osing mass at an accelerated rate and is responsible for approximately $25 \%$ of the current rate of global sea-level rise (Church et al., 2011; Chen et al., 2017). Much of this mass loss occurs via the release of ice and melt water at outlet glaciers, which terminate in deep and narrow fjords (van den Broeke et al., 2009). Here it is argued that the mass loss is in part affected by the release of subglacial melt water into the ocean.

Subglacial discharge is due to large catchments upstream of the glacier's marine interface and has a peak discharge of approximately $30 \times 10^{3} \mathrm{~m}^{3} / \mathrm{s}$ during summer months (Jackson and Straneo, 2016). This seasonal discharge of subglacial meltwater can be diagnosed from glacier-induced changes in water properties in Sermilik Fjord, the fjord adjacent to Helheim Glacier. Figure 1 shows distributions of potential temperature and salinity in the Sermilik Fjord for the summer of 2009 (left) and the winter of 2010 (right).

Ambient waters within the fjord can be modified by two glacial sources of freshwater: subglacial discharge and submarine melt. Subglacial discharge is assumed to be fresh and at its local freezing point at depth. If no other sources of freshwater are present, mixing between the deep ambient waters of the fjord and the cold, fresh subglacial discharge water from beneath glacier results in a modified water mass whose properties lie along the runoff line indicated by the dashed line in Figure 1. Submarine melt modifies the ambient water in a similar fashion but causes additional ocean cooling through the extraction of latent heat. If no other sources of freshwater are present, the melting of the ice and its subsequent mixing with ambient ocean water will result in a new water mass that lies along the melting line indicated by the solid line in Figure 1.

Figure 1 shows that, during the summer months, the waters of the Sermilik Fjord are modified by both runoff/subglacial discharge and submarine melt. The is evidenced by the fact that the near-glacier fjord water has a $\theta-S$ distribution that lies between the runoff 


\section{Summer}

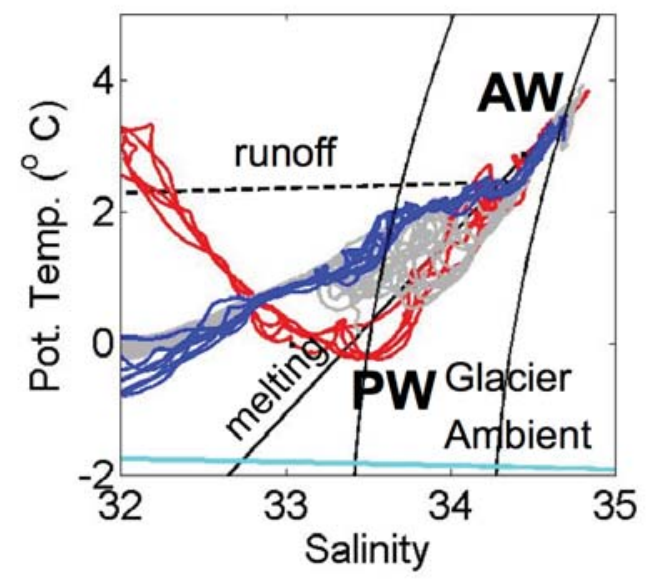

Winter

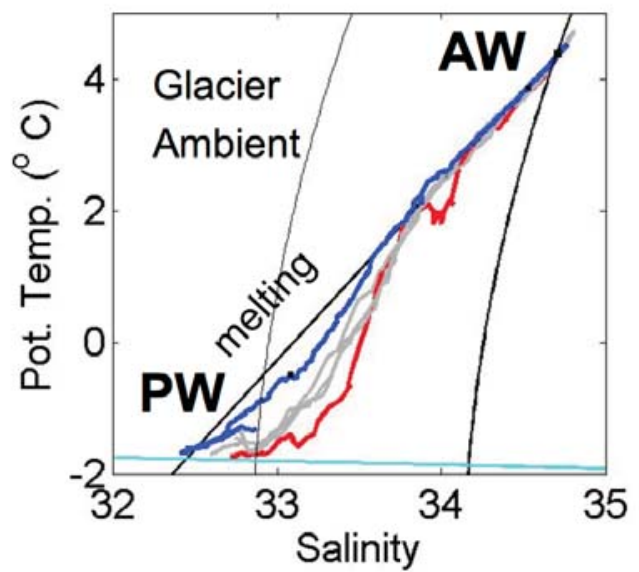

Figure 1: Seasonal distribution of water mass properties in the Sermilik Fjord. Left: Potential temperature $(\theta)$ versus salinity $(S)$ of the ocean at the mouth of the fjord (red) and near the glacier (blue) collected in August 2009. Right: Same, but for March 2010. The dashed and solid line represent the mixing lines for runoff and submarine melt, respectively. The cyan line shows the freezing point of seawater for different salinities. Figure is adapted from Straneo et al. (2011).

and melt lines. During the winter months, the $\theta-S$ distribution of the ocean indicates that submarine melt is the main source of freshwater for the fjord.

If the ambient waters consist of a single water mass, the relative contributions of freshwater from submarine melt and subglacial discharge can be quantified (e.g Mortensen et al., 2011; Jackson and Straneo, 2016).

\subsection{Dynamics at the ice-ocean interface}

When subglacial discharge enters the ocean at the grounding line, it rises as a turbulent buoyant plume. The mixing generated by this turbulent plume enhances the exchange of heat between the ambient ocean and the ice surface, thereby elevating the submarine melt rate. Since submarine melting has a primary control on the mass balance of the entire ice sheet, it is essential that we understand the dynamics of this interaction. 


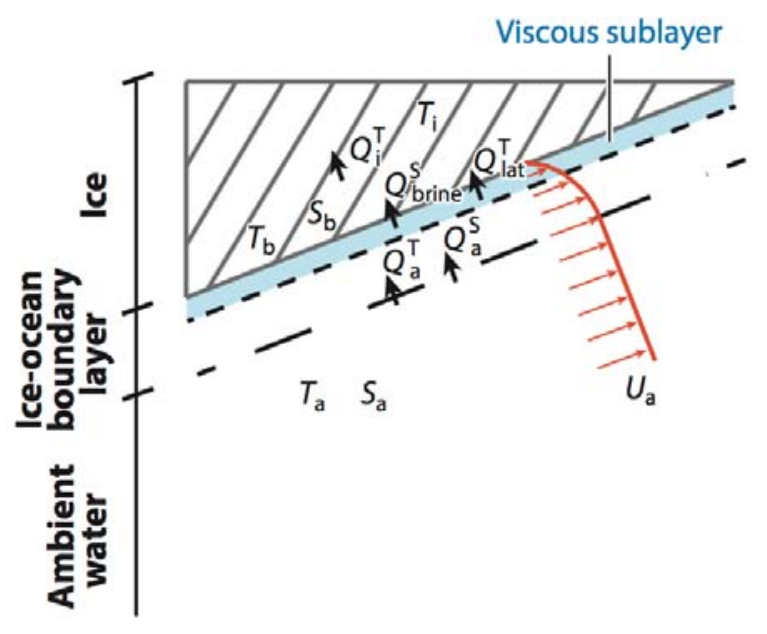

Figure 2: A schematic describing the processes governing the temperature, salinity and melt rate at the ice-ocean interface. $Q_{a}^{T}$ is the heat flux from the ambient fjord water to the glacier, $Q_{i}^{T}$ is the heat flux into the ice, and $Q_{\text {lat }}^{T}$ is the latent heat flux from phase changes. Corresponding freshwater fluxes are denoted by the superscript $\mathrm{S}$ with the addition of $Q_{b r i n e}^{S}$, associated with the melting or freezing of ice. All other variables are defined in the text. Figure is taken from Straneo and Cenedese (2015), which is modified from Holland and Jenkins (1999).

The submarine melt rate is typically determined through the use of a three-equation plume model. This model, which was first developed for the floating ice shelves of Antarctica, solves for the temperature $T_{b}$, salinity $S_{b}$ and melt rate $\dot{m}$ at the ice-ocean interface(Hellmer and Olbers, 1989; Holland and Jenkins, 1999). A schematic of the processes represented by the model is provided in Figure 2.

$T_{b}$ is constrained to be at the in situ freezing point of seawater, which is governed by

$$
T_{b}=\lambda_{1} S_{b}+\lambda_{2}+\lambda_{3} p_{b},
$$

where $\lambda_{1}, \lambda_{2}$ and $\lambda_{3}$ are known constants, and $Z_{b}$ is the pressure at the interface. $T_{b}$ and $S_{b}$ are further constrained by the heat and salt fluxes across the viscous sublayer that separates the ice boundary from the ambient ocean. The heat budget of the viscous sublayer is a balance of the heat flux supplied by the ambient ocean and the sensible and latent heat flux to the ice

$$
C_{p} \gamma_{T}\left(T_{a}-T_{b}\right)=\dot{m} C_{i}\left(T_{b}-T_{i}\right)+\dot{m} L,
$$

where $T_{a}$ is the ambient ocean temperature, $C_{p}$ and $C_{i}$ are the specific heat capacities of seawater and ice, $L$ is the latent heat of fusion, and $\gamma_{T}$ is the thermal exchange velocity. Likewise, the salt budget of the viscous sublayer is a balance of the salt flux supplied by the ambient ocean and the salt flux across the ice boundary. This is given by

$$
\gamma_{S}\left(S_{a}-S_{b}\right)=\dot{m}\left(S_{b}-S_{i}\right),
$$


where $\gamma_{S}$ is the salinity exchange velocity, $S_{a}$ is the salinity of the ambient ocean and $S_{i}$ is the salinity of the ice, which is sometimes assumed to be zero. In the turbulent region outside the viscous sublayer, heat and salt diffuse at the same rate. However, within the viscous sublayer, the exchange of heat and salt are governed by molecular diffusion. In this region, heat is transferred at a faster rate than salt. Additionally, these exchange rates are dependent on the shear generated by the ambient ocean as it drags along the ice. These effects are parameterized as

$$
\gamma_{T, S}=C_{D}^{\frac{1}{2}} \Gamma_{T, S} U_{a}
$$

where $C_{D}^{\frac{1}{2}} \Gamma_{T, S}$ represents the thermal and haline Stanton numbers for a hydraulically smooth surface (Kader and Yaglom, 1972; Steele et al., 1989).

From equations (1)-(4), we see that an increase in near-ice ocean velocity $U_{a}$, will lead to an increase in the submarine melt rate $m$. This velocity can be influenced by either large scale ocean circulation, driven by processes like tidal motions, or by local buoyant plumes supplied by subglacial discharge. For the near-vertical calving fronts typically found in Greenland, the latter mechanism is dominant, especially during summer months (Sciascia et al., 2013).

A major caveat to the three-equation model is that it was developed for the near horizontal floating ice-shelves of Antarctica. The tidewater glaciers of Greenland have a much steeper ocean interface and receive much greater freshwater input from subglacial discharge. These differences 1 ikely affect the t urbulent exchange $\mathrm{r}$ ates parameterized by (4).

\subsection{Plume modeling}

The ultimate goal of plume modeling is to predict the submarine melt rate (SMR) along the front of a glacier. The SMR will depend on the plume's buoyancy forcing, vertical extent and lateral extent. Additionally, the vertical structure and velocity of the nearby ocean will also have an impact. Due to the paucity of in situ data, many of these factors remain largely unconstrained. We therefore rely on models to inform our understanding of these processes.

Plume models currently fall into two broad categories: simple one-dimensional buoyant plume models (e.g. Hellmer and Olbers, 1989; Jenkins, 2011) based on buoyant plume theory originally developed by Morton et al. (1956) and Turner (1973), and fully three-dimensional plume models that utilize physics from high-resolution, non-hydrostatic general circulation models (e.g. Sciascia et al., 2013; Slater et al., 2015). In each case, the subglacial discharge forcing the plume may be funneled through a single point source, a distribution of small point sources or across the full width of the grounding line.

One-dimensional plume models have been used to varying degrees of success to explain observations of water mass properties near tidewater glaciers. For example, Stevens et al. (2016) showed that the line plume model introduced by (Jenkins, 2011) is able to reproduce the measured vertical extent and composition of glacially modified waters near a major subglacial discharge site at the Saqqarliup sermia outlet glacier system in West Greenland. However, the same model was unable represent the properties of glacially modified waters at another nearby subglacial discharge site. This discrepancy was attributed to uncertainties 
in subglacial discharge and missing physics (such as the detachment of the plume after it reaches neutral buoyancy).

Other studies have used three-dimensional plume models to quantify the sensitivity of SMR to certain unknown parameters. For example, Slater et al. (2015) showed that subglacial discharge, when distributed over a wide area, could produce up to five times as much submarine melt as when the amount of discharge is passed through a single localized outlet. Additionally, Sciascia et al. (2013) showed that the intrusion depth of a buoyant plume will depend on the magnitude of the subglacial discharge. These sensitivity studies highlight the great uncertainty surrounding SMR and stresses the need for more observational studies.

\subsection{Summary}

1. The seasonal injection of subglacial discharge affects ice-ocean exchanges by affecting the dynamics at the interface.

2. Plume models work well near the glacier front, but the far-field impacts of plumes is not represented by these models.

3. Melt rates from models are highly uncertain, to a large extent because they have not been validated by data.

\section{Fjord Dynamics}

Many tidewater glaciers, especially in Greenland, do not have terminate in open ocean waters, but in a fjord. The fjord connects the glacier and its catchment, which are influenced by the atmospheric dynamics of the region, with the open ocean circulation, which determines the heat input to the glacier front, all of which influence ice sheet and grounding line dynamics. Due to the inherent difficulties in performing field campaigns in these regions, the fjord dynamics is still a topic of very active research. The fjord circulation is known to be mainly driven by the freshwater input of the subglacial discharge (Motyka et al., 2003; Rignot et al., 2010), the along-fjord katabatic winds that flow downhill from the ice-sheet and the alongshelf winds that drive the so-called intermediary circulation, by imposing density fluctuations at the mouth of the fjord itself (Jackson et al., 2014). Additionally, processes of deep water renewal and transient motions (namely internal waves or internal seiches) characterize the fjord circulation.

\section{$2.1 \quad$ Iceberg trajectories}

Using GPS trackers such as the one in figure 3, icebergs can be tracked until they completely melt or capsize. The sensors are deployed from a helicopter, on large icebergs with a waterline length longer than $100 \mathrm{~m}$. The motion of large icebergs is primarily driven by the ocean currents, rather than wind. 


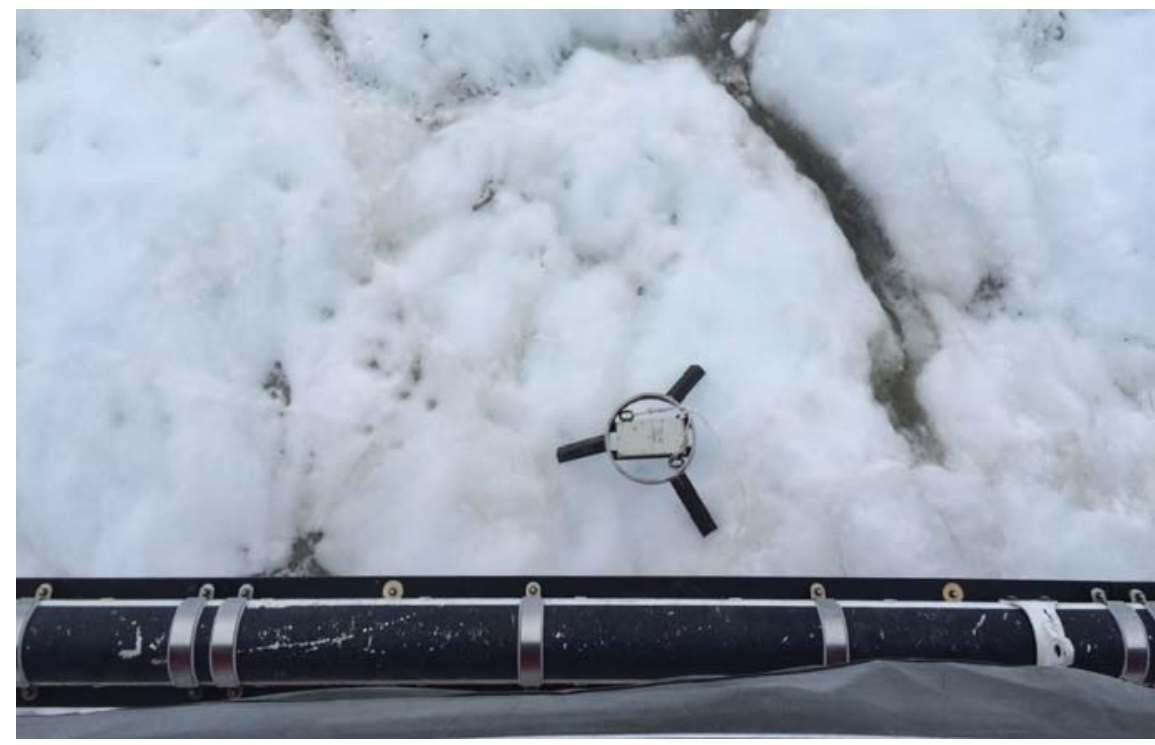

Figure 3: Picture of a GPS tracker placed on the surface of a large iceberg in the Sermilik Fjord (South-East Greenland) from a helicopter. (Photo by F. Straneo)

Observations of iceberg trajectories through GPS tracking show a net mean displacement of the ice mélange, a mixture of icebergs and sea ice extending $O(10 \mathrm{~km})$ beyond the glacier front, out of the fjord (Sutherland et al., 2014). In particular, multiple icebergs in the mélange are observed to undergo sudden simultaneous motions. These are caused either by strong calving events at the glacier front or by the action of intense katabatic winds that can flush the whole ice mélange out of the fjord in few days. Figure 4, from Sutherland et al. (2014), shows the daily average distance from the glacier front of three icebergs in the ice mélange as a function of time deployed. The average velocities marked on the intervals of constant slope shows that the mélange is a compact system that moves at a roughly constant speed for its whole extension. The sudden changes in position that happens in two or three days (around day 120) indicates a strong calving event that pushed the entire mélange out of the fjord. After this first phase of motion, the icebergs, if they do not capsize or become trapped by bottom topography, are observed to move on average out of the fjord until they reach the open ocean, where they are driven south-westward by the East Greenland Coastal Current. This mean displacement is indicative of the buoyancy-driven estuarine-like circulation due to the subglacial runoff at the glacier front (Motyka et al., 2003; Rignot et al., 2010).

\subsection{Buoyancy-driven circulation}

As mentioned earlier, the freshwater released at the base of the glacier front has been observed to form plumes that rise buoyantly near the glacier front, entraining ambient water until they reach the surface or a neutral buoyancy depth. This gives rise to a buoyancy-driven circulation, with the relatively cold, fresh plume detaching from the glacier front and flowing toward the fjord mouth, while the entrainment drives flow of warmer, saltier Atlantic Water 


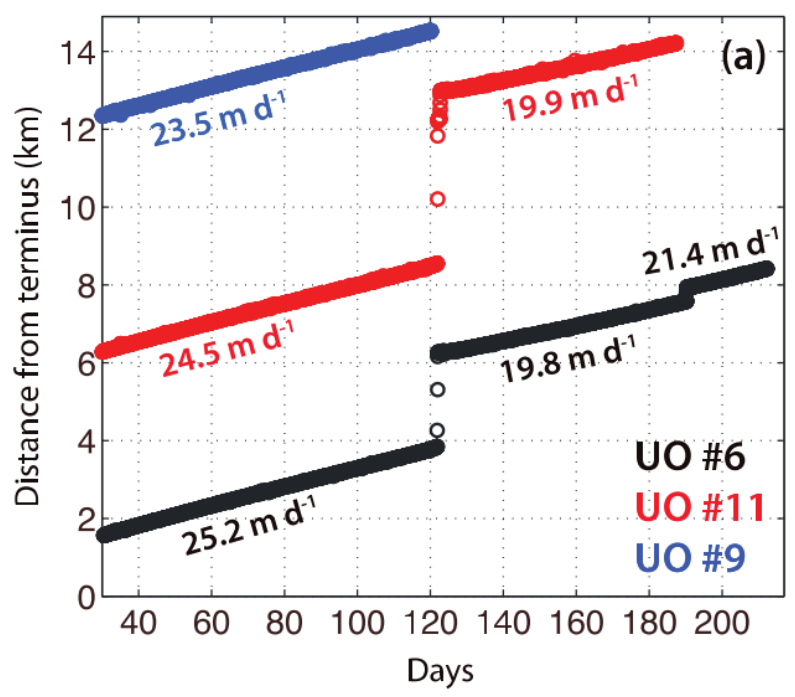

Figure 4: Displacement from the glacier terminus of three icebergs in the ice mélange in the Sermilik Fjord in South-East Greenland as a function of the time deployed (Sutherland et al., 2014).

toward the glacier (Straneo and Cenedese, 2015). Figure 5 shows a scheme of this kind of circulation, highlighting the salty water input on the bottom of the fjord and the relatively fresher water export at the surface (Rignot et al., 2010).

The flux of subglacial discharge and resulting entrainment of ambient water thus have a strong influence on the heat flux to the glacier front from warm Atlantic Water. Observations of enhanced submarine melting corresponding to the buoyant plumes have shown a strong seasonal variability, corresponding to the seasonality of subglacial discharge, which has a maximum in summer or after intense rainfalls (Motyka et al., 2003). Moreover, it was found that submarine melting can contribute to the ice-sheet mass balance as significantly as the calving, making it an important factor in grounding-line and ice-flow dynamics (Rignot et al., 2010). 


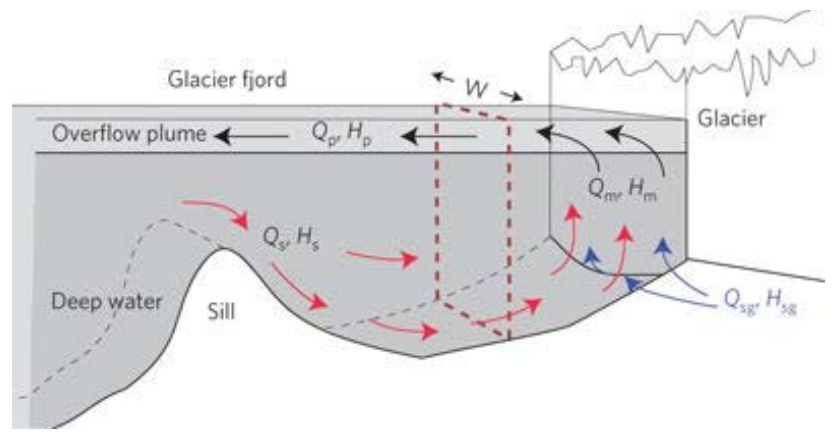

Figure 5: Scheme of the estuarine-like circulation observed in the fjords driven by the positively buoyant freshwater input of the subglacial runoff. In most fjords, the dynamics is almost two-dimensional and small across-fjord variations are generally observed (Rignot et al., 2010).

Exercise: The importance of heat transport from outside a fjord can be shown with a simple calculation using typical characteristics of each fjord and glacier, as given in table 1. What would the change in mean fjord temperature be if the entire ice flux from each glacier was melted by water in the fjord?

The necessary input of heat is calculated as the heat needed to warm the ice to its freezing point plus the heat needed for the phase change:

$$
Q_{H}=\rho_{i} Q_{i}\left[c_{i}\left(T_{\mathrm{f}}-T_{i}\right)+L\right] * 1 \text { year. }
$$

The change in fjord temperature is calculated as

$$
\Delta T=\frac{Q_{H}}{c_{w} \rho_{w} V_{\text {fjord }}},
$$

where $V_{\text {fjord }} \equiv l * w * d$. Inputting the values from table 1 gives a temperature decrease of approximately $8^{\circ} \mathrm{C}$ for Sermilik Fjord and $1^{\circ} \mathrm{C}$ for 79 North Fjord. With no renewal of water from outside the fjord, this would bring the temperature of both fjords below freezing. This exercise is indicative of the importance of heat transport from outside the fjords to maintain a steady state balance between the fjord and glacier.

\subsection{Observing seasonal variability}

Moored observations of current velocities are crucial to understanding fjord dynamics during the non-summer months, when the subglacial runoff forcing is weak, but icebergs pose a significant challenge to collecting long timeseries of observations. Figure 6 shows how iceberg impacts may affect a mooring (Jackson, 2016). Panel (A) shows how the pressure measurements at three different levels all collapse to the bottom value simultaneously, indicating that the impact with an iceberg has pushed the buoy below a critical depth at which the water pressure has compressed it, so that is it no longer able to float. Panel (B) shows the 


\begin{tabular}{|c|c|c|}
\hline & $\begin{array}{l}79 \text { North Glacier } \\
+ \text { Fjord }\end{array}$ & $\begin{array}{l}\text { Helheim Glacier } \\
+ \text { Sermilik Fjord }\end{array}$ \\
\hline Ice flux $Q_{i}\left(\mathrm{~km}^{3} \mathrm{yr}^{-1}\right)$ & 15 & 30 \\
\hline Grounding depth $d(\mathrm{~m})$ & 600 & 600 \\
\hline Width $w(\mathrm{~km})$ & 20 & 6 \\
\hline Length $l(\mathrm{~km})$ & 80 & 80 \\
\hline Ice temperature $T_{i}\left({ }^{\circ} \mathrm{C}\right)$ & -10 & -10 \\
\hline $\begin{array}{l}\text { Upper } 100 \mathrm{~m} \text { water temp. } \\
\left({ }^{\circ} \mathrm{C}\right)\end{array}$ & $\mathrm{T}_{\mathrm{f}}$ & $\mathrm{T}_{\mathrm{f}}$ \\
\hline $\begin{array}{l}\text { Lower } 500 \mathrm{~m} \text { water temp. } \\
\left({ }^{\circ} \mathrm{C}\right)\end{array}$ & 1 & 4 \\
\hline \multicolumn{3}{|c|}{$\begin{array}{l}\text { Heat capacity of ice } c_{i}: 2 \mathrm{~kJ} \mathrm{~kg}^{-1}{ }^{o} \mathrm{C}^{-1} \\
\text { Heat capacity of seawater } c_{w}: 4 \mathrm{~kJ} \mathrm{~kg}^{-1}{ }^{o} \mathrm{C}^{-1} \\
\text { Latent heat of fusion } L: 334 \mathrm{~kJ} \mathrm{~kg}^{-1} \\
\quad \text { Ice density } \rho_{i}: \sim 917 \mathrm{~kg} \mathrm{~km}^{-3} \\
\text { Seawater density } \rho_{w}: \sim 1025 \mathrm{~kg} \mathrm{~km}^{-3}\end{array}$} \\
\hline
\end{tabular}

Table 1: Estimates of typical characteristics of the 79 North Glacier and Fjord, Helheim Glacier, and Sermilik Fjord and pertinent physical constants to be used in the exercise.

track of the iceberg that hit the mooring and panel $(\mathrm{C})$ contains a scheme for the two kinds of impact with an iceberg. In the type 1 hit, the buoy is not compressed and thus it still floats after the iceberg has passed, while in the type 2 hit, the buoy sinks after the pressure has squeezed it, as shown in the picture of panel (D).

Using timeseries of moored observations in Sermilik Fjord, a new decomposition of the mass, salt and heat budgets that include mechanisms that have been neglected in the past literature is introduced (Jackson and Straneo, 2016). Two major circulation regimes are identified: shelf variability via barrier winds (dominant in nonsummer months) and freshwater discharge f rom $r$ unoff (dominant in s ummer).

\subsection{Other drivers of fjord circulation}

Figure 7 shows the timeseries of along-fjord velocity (a,b) and potential temperature (c,d) at two different locations in the Sermilik fjord.(Jackson et al., 2014). The moorings that survived the season show that the currents have a strong variability on the $O(2-3$ days $)$ scale, associated with the periodic tilting of the halocline at the mouth of the fjord due to the atmospheric mesoscale forcing. When cyclonic winds blow along the continental shelf in front of the south-eastern coasts of Greenland (see figure 8), the Ekman transport induces an increase in pressure in the upper layer at the mouth of the fjord. This generates an overturning circulation that opposes the buoyancy-driven one. This explains the short scales $O$ (days) variability in the direction of the fjord circulation, as opposed to the monthly variations induced by the subglacial runoff forcing that controls the estuarine-like circulation 

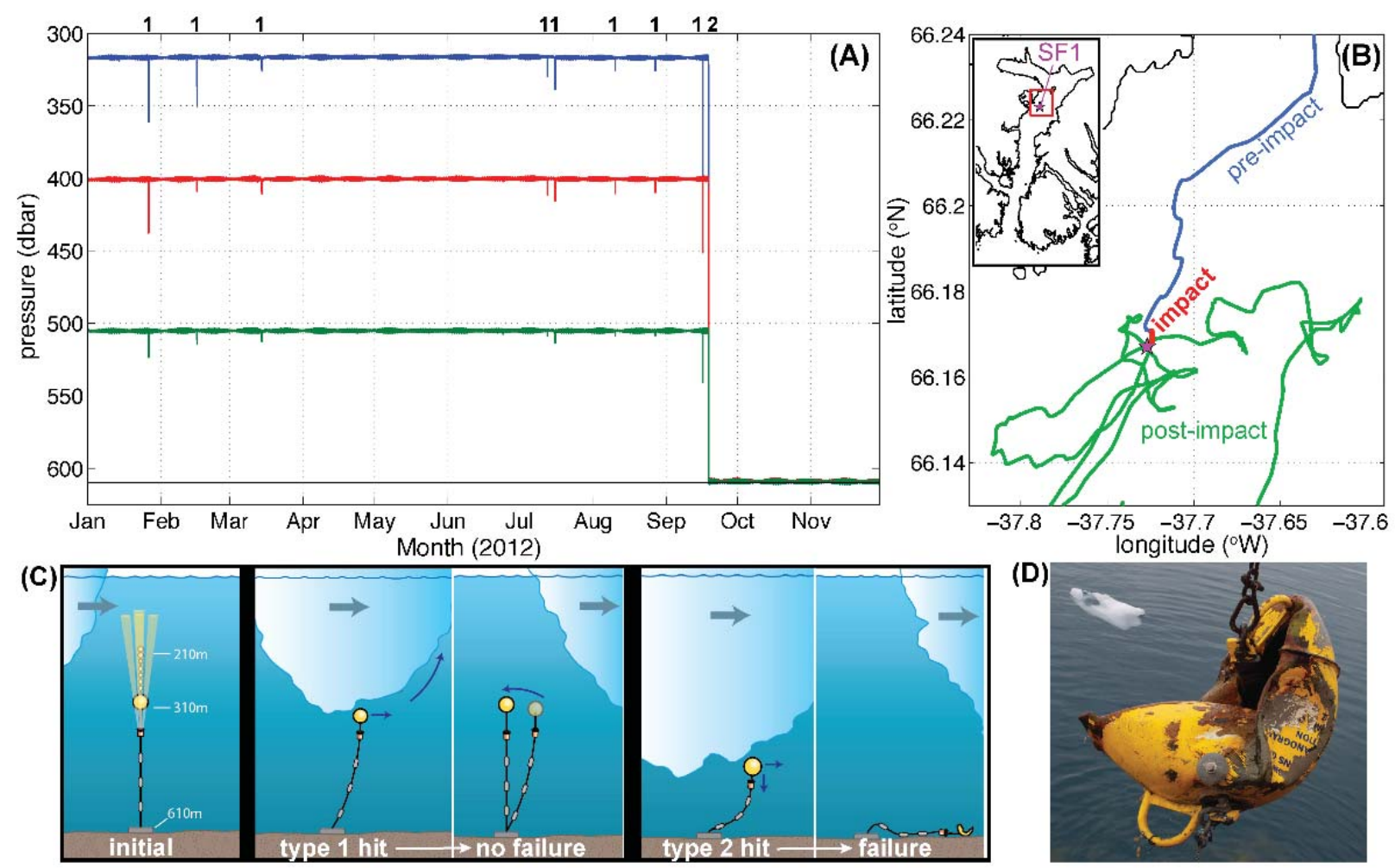

Figure 6: (A) pressure measurements before and after the impact with the iceberg. (B) Track of the iceberg. (C) Schemes of the two types of impact, in the former the buoy is still able to float because the pressure has not deformed it, while in the latter the buoy has been pushed at such a depth that the it cannot sustain the water pressure and it sinks. (D) Picture of a buoy recovered after a type 2 hit (Jackson, 2016). 

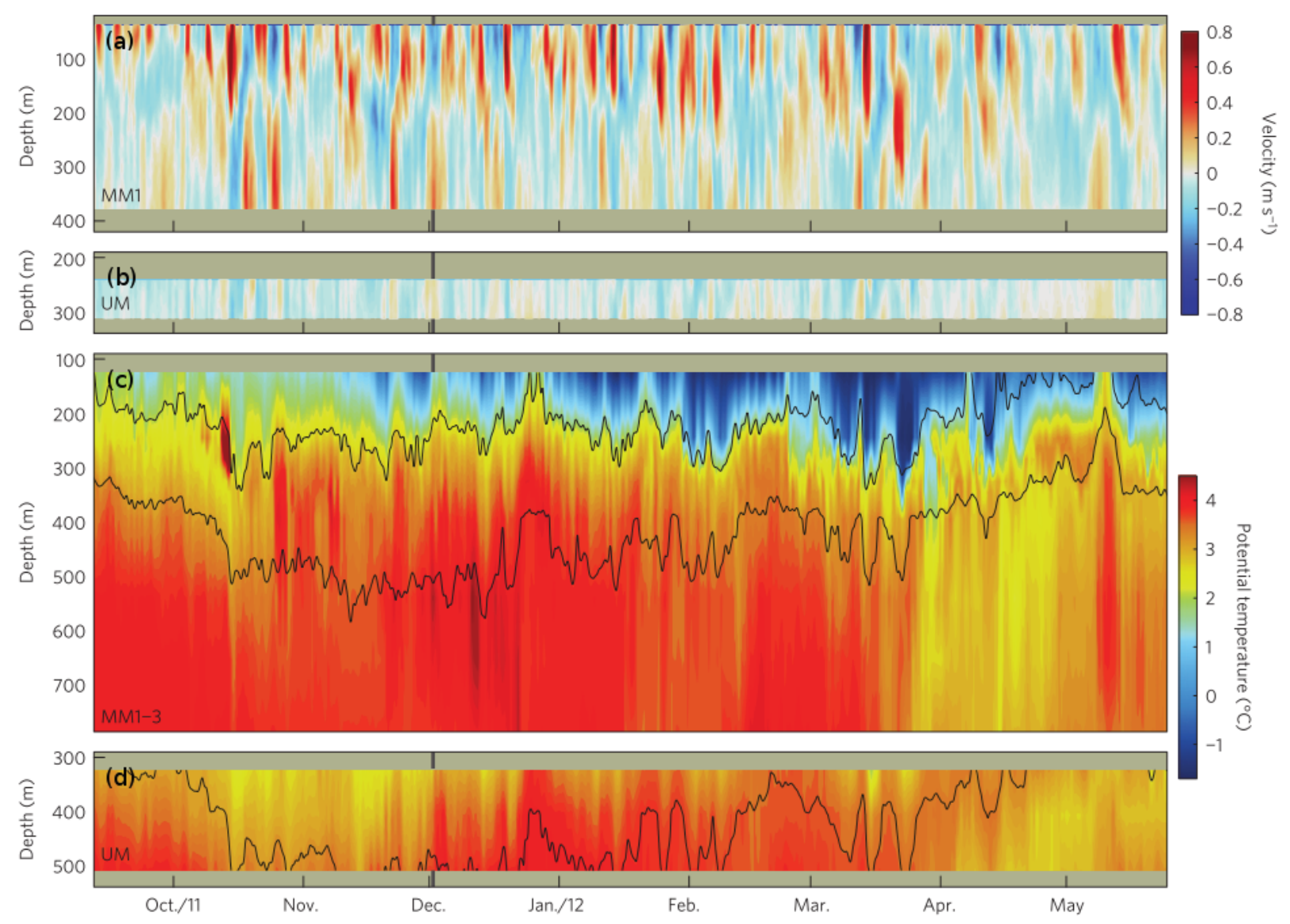

Figure 7: (a), (b) Along-fjord velocity (positive is towards the glacier) in two locations in the Sermilik fjord. (c), (d) Potential temperature timeseries for the same two locations with the contours of potential density anomaly $\sigma_{\theta}=[27.0,27.5] \mathrm{kg} \mathrm{m}^{-3}$ overlaid. (b), (d) are closer to the glacier front than (a) and (c)." Adapted from (Jackson et al., 2014).

described above.

The fjord circulation is also driven by along-fjord katabatic winds, which have been observed to flush out the entire ice mélange of a fjord on a $O(1$ day) scale. These winds, which blow from the ice sheet to the open ocean and can reach hurricane velocities, have a significant influence on the fjord circulation in the same direction as the buoyancy-driven one (Oltmanns et al., 2014, 2015). An example of this kind of event is shown in figure 9, where a series of three satellite images (Moderate Resolution Imaging Spectroradiometer, MODIS) shows how a strong wind event removes almost completely the ice mélange of the Ammassalik fjord in roughly one day (Oltmanns et al., 2014). Numerical simulations of a typical katabatic wind event in Greenland fjords show that $O(10 \%)$ of the upper layer is flushed out in a single event, in agreement with observations (Spall et al., 2017). 


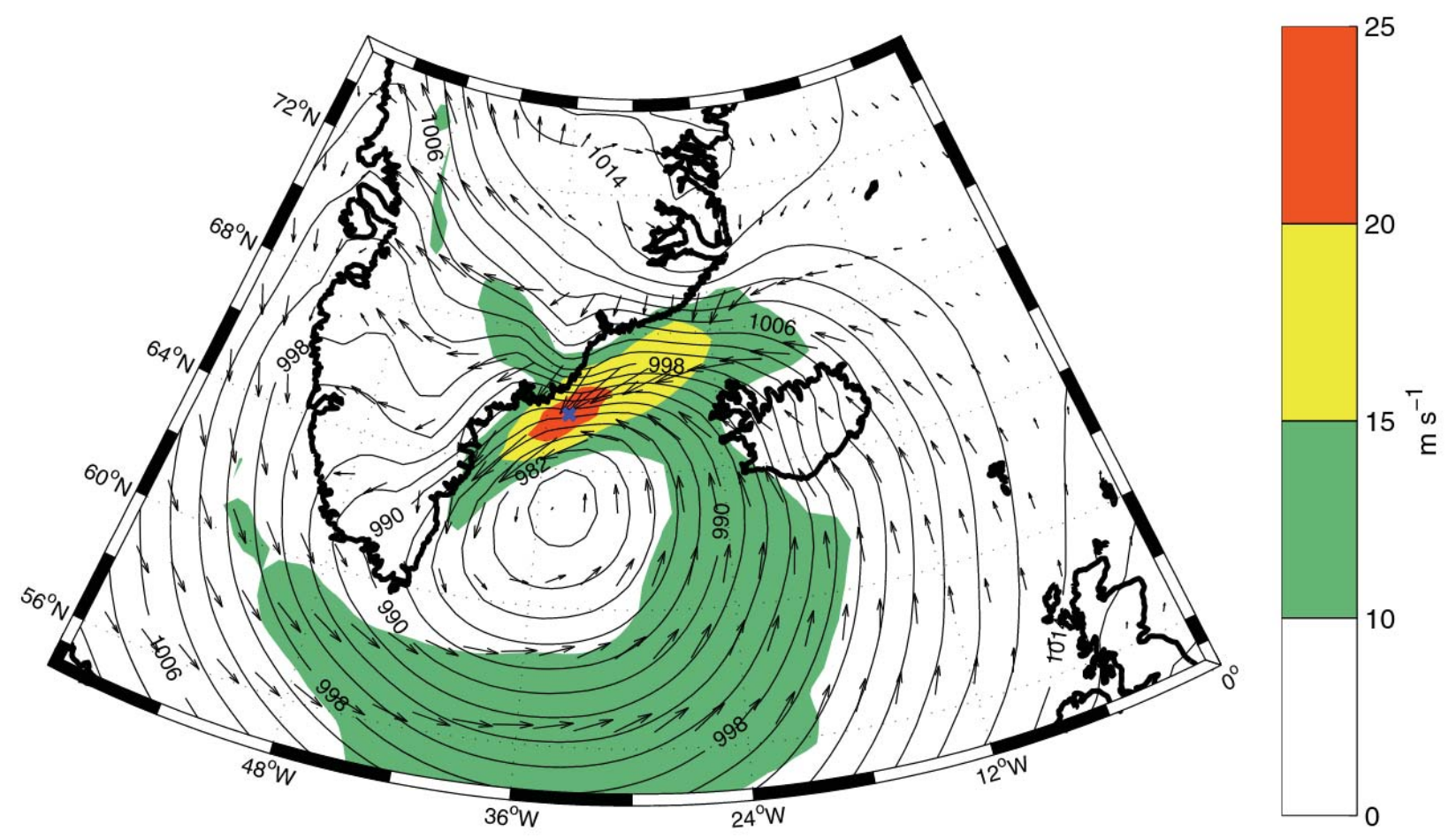

Figure 8: Composite analysis of the 10 meters winds (shading) and mean sea level pressure (contours) for the times of wind events over $15 \mathrm{~m} \mathrm{~s}^{-1}$ in the location indicated by the blue cross roughly between August 2009 and August 2013 (Harden et al., 2014).
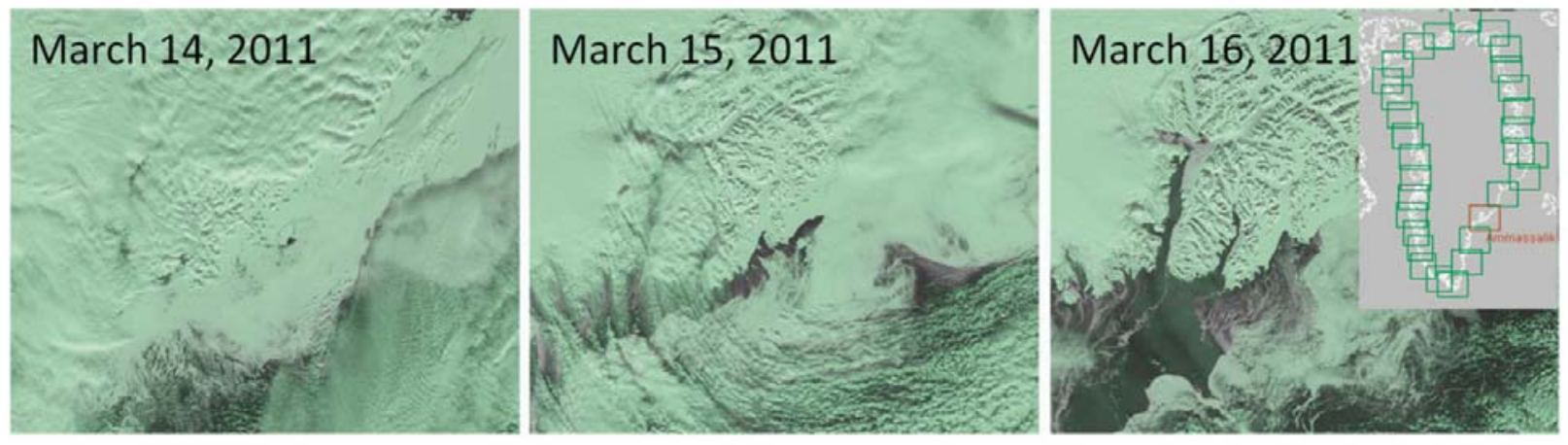

Figure 9: MODIS satellite images showing in the visible range the flushing of the ice mélange out of the Ammassalik fjord by the action of an intense katabatic wind event in roughly one day. Adapted from (Oltmanns et al., 2014). 


\subsection{Impact of topographic sills}

With the same numerical setup it is possible to simulate the role of a sill in the fjord bottom topography, a feature present at the mouth of some glacial fjords. Numerical simulations by Gladish et al. (2015), focused on the water renewal in Illulisat Fjord, West Greenland, show that the position of the pycnocline with respect to the height of the sill is crucial in determining the circulation. In particular, a sill shallower than the pycnocline will block the inflow of warmer deep waters, reducing submarine melt at the glacier front. Moreover, numerical simulations show that while the subglacial runoff circulation can drive the water renewal in the fjord in a single summer, the external baroclinic forcing cannot, because of the presence of the sill blocking the flow at depth. The effect of a sill deeper than the pycnocline on the fjord water renewal mechanism is small.

\subsection{Modeling the full fjord circulation}

From the above discussion, it is evident that phenomena on multiple scales control the complex interaction between the ice-sheet and ocean components that characterize the Greenland coastal system. Examples of recent numerical efforts in modeling this broad range of processes, encompassing buoyant plumes due to the subglacial runoff at the glacier front, fjord buoyancy and wind circulation, iceberg displacement and open ocean dynamics include Carroll et al. (2015) and Cowton et al. (2015). The sensitivity of the glacier melting to the subsurface runoff, through the oceanic warmer water entrainment in the buoyant plume, is studied by means of numerical simulations by modifying the runoff flux and geometrical configuration (line plume versus point source plume, for example). Despite the lack of knowledge of some feedback mechanisms, for example between submarine melting and iceberg calving at the front, numerical results show that the submarine melt rates increase with subglacial runoff, but they appear to be insensitive to the annual runoff variability (Cowton et al., 2015). Instead, there is both numerical (Carroll et al., 2015) and observational (Beaird et al., 2015) evidence that the stratification at the glacier front influences the terminal level of a buoyant plume. In fact, depending on the depth profile of density, the subglacial runoff, and the turbulent entrainment, the plume can reach a neutral buoyancy level before surfacing. Other works are trying to model case studies of fjord circulation and to link the fjord variability to the large scale ocean variability.

\subsection{Summary}

1. Drivers of the circulation in the fjord include buoyancy due to meltwater release, regional winds, shelf-forced exchanges, tides.

2. The geometry of the fjord (sills, width, ice tongue extent) affects the circulation.

3. Theories of the fjord circulation typically do not cover the parameter space of the glacial fjords. 
4. Future studies will need to explore the coupling between near-ice dynamics and fjord scale circulation, which are characterized by different length scales.

\section{References}

Beaird, N., Straneo, F., and Jenkins, W. (2015). Spreading of greenland meltwaters in the ocean revealed by noble gases. Geophysical Research Letters, 42(18):7705-7713. 2015GL065003.

Carroll, D., Sutherland, D. A., Shroyer, E. L., Nash, J. D., Catania, G. A., and Stearns, L. A. (2015). Modeling Turbulent Subglacial Meltwater Plumes: Implications for Fjord-Scale Buoyancy-Driven Circulation. Journal of Physical Oceanography, 45(8):2169-2185.

Chen, X., Zhang, X., Church, J. A., Watson, C. S., King, M. A., Monselesan, D., Legresy, B., and Harig, C. (2017). The increasing rate of global mean sea-level rise during 1993-2014. Nature Climate Change, 7(7):492-495.

Church, J. A., White, N. J., Konikow, L. F., Domingues, C. M., Cogley, J. G., Rignot, E., Gregory, J. M., van den Broeke, M. R., Monaghan, A. J., and Velicogna, I. (2011). Revisiting the Earth's sea-level and energy budgets from 1961 to 2008. Geophysical Research Letters, 38(18):n/a-n/a.

Cowton, T., Slater, D., Sole, A., Goldberg, D., and Nienow, P. (2015). Modeling the impact of glacial runoff on fjord circulation and submarine melt rate using a new subgrid-scale parameterization for glacial plumes. Journal of Geophysical Research: Oceans, 120(2):796812.

Gladish, C. V., Holland, D. M., Rosing-Asvid, A., Behrens, J. W., and Boje, J. (2015). Oceanic Boundary Conditions for Jakobshavn Glacier. Part I: Variability and Renewal of Ilulissat Icefjord Waters, 2001-14*. Journal of Physical Oceanography, 45(1):3-32.

Harden, B. E., Straneo, F., and Sutherland, D. A. (2014). Moored observations of synoptic and seasonal variability in the East Greenland Coastal Current. Journal of Geophysical Research: Oceans, 119(12):8838-8857.

Hellmer, H. and Olbers, D. (1989). A two-dimensional model for the thermohaline circulation under an ice shelf. Antarctic Science, 1(04).

Holland, D. M. and Jenkins, A. (1999). Modeling Thermodynamic Ice-Ocean Interactions at the Base of an Ice Shelf. Journal of Physical Oceanography, 29(8):1787-1800.

Jackson, R. H. (2016). A mooring in iceberg alley. Oceanus, 51(2):16-19.

Jackson, R. H. and Straneo, F. (2016). Heat, Salt, and Freshwater Budgets for a Glacial Fjord in Greenland. Journal of Physical Oceanography, 46(9):2735-2768. 
Jackson, R. H., Straneo, F., and Sutherland, D. A. (2014). Externally forced fluctuations in ocean temperature at Greenland glaciers in non-summer months. Nature Geoscience.

Jenkins, A. (2011). Convection-Driven Melting near the Grounding Lines of Ice Shelves and Tidewater Glaciers. Journal of Physical Oceanography, 41(12):2279-2294.

Kader, B. and Yaglom, A. (1972). Heat and mass transfer laws for fully turbulent wall flows. International Journal of Heat and Mass Transfer, 15(12):2329-2351.

Mortensen, J., Lennert, K., Bendtsen, J., and Rysgaard, S. (2011). Heat sources for glacial melt in a sub-Arctic fjord (Godthåbsfjord) in contact with the Greenland Ice Sheet. Journal of Geophysical Research, 116(C1):C01013.

Morton, B. R., Taylor, G., and Turner, J. S. (1956). Turbulent Gravitational Convection from Maintained and Instantaneous Sources. Proceedings of the Royal Society A: Mathematical, Physical and Engineering Sciences, 234(1196):1-23.

Motyka, R. J., Hunter, L., Echelmeyer, K. A., and Connor, C. (2003). Submarine melting at the terminus of a temperate tidewater glacier, LeConte Glacier, Alaska, U.S.A. Annals of Glaciology, 36:57-65.

Oltmanns, M., Straneo, F., Moore, G. W. K., and Mernild, S. H. (2014). Strong Downslope Wind Events in Ammassalik, Southeast Greenland. Journal of Climate, 27(3):977-993.

Oltmanns, M., Straneo, F., Seo, H., and Moore, G. W. K. (2015). The Role of Wave Dynamics and Small-Scale Topography for Downslope Wind Events in Southeast Greenland. Journal of the Atmospheric Sciences, 72(7):2786-2805.

Rignot, E., Koppes, M., and Velicogna, I. (2010). Rapid submarine melting of the calving faces of West Greenland glaciers. Nature Geoscience, 3(3):187-191.

Sciascia, R., Straneo, F., Cenedese, C., and Heimbach, P. (2013). Seasonal variability of submarine melt rate and circulation in an East Greenland fjord. Journal of Geophysical Research: Oceans, 118(5):2492-2506.

Slater, D. A., Nienow, P. W., Cowton, T. R., Goldberg, D. N., and Sole, A. J. (2015). Effect of near-terminus subglacial hydrology on tidewater glacier submarine melt rates. Geophysical Research Letters, 42(8):2861-2868.

Spall, M. A., Jackson, R. H., and Straneo, F. (2017). Katabatic Wind-Driven Exchange in Fjords. Journal of Geophysical Research: Oceans.

Steele, M., Mellor, G. L., and Mcphee, M. G. (1989). Role of the Molecular Sublayer in the Melting or Freezing of Sea Ice. Journal of Physical Oceanography, 19(1):139-147.

Stevens, L. A., Straneo, F., Das, S. B., Plueddemann, A. J., Kukulya, A. L., and Morlighem, M. (2016). Linking glacially modified waters to catchment-scale subglacial discharge using autonomous underwater vehicle observations. The Cryosphere, 10(1):417-432. 
Straneo, F. and Cenedese, C. (2015). The dynamics of Greenland's glacial fjords and their role in climate. Annu. Rev. Mar. Sci., 7:89-112.

Straneo, F., Curry, R. G., Sutherland, D. A., Hamilton, G. S., Cenedese, C., Våge, K., and Stearns, L. A. (2011). Impact of fjord dynamics and glacial runoff on the circulation near helheim glacier. Nature Geoscience, 4:322 EP -. Article.

Sutherland, D. A., Roth, G. E., Hamilton, G. S., Mernild, S. H., Stearns, L. A., and Straneo, F. (2014). Quantifying flow regimes in a Greenland glacial fjord using iceberg drifters. Geophysical Research Letters, 41(23):8411-8420.

Turner, J. S. (1973). Buoyancy Effects in Fluids. Cambridge University Press, Cambridge.

van den Broeke, M., Bamber, J., Ettema, J., Rignot, E., Schrama, E., van de Berg, W. J., van Meijgaard, E., Velicogna, I., and Wouters, B. (2009). Partitioning Recent Greenland Mass Loss. Science, 326(5955):984-986. 


\title{
GFD 2017 Lecture 10: Greenland Glacier-Ocean Interaction Part II
}

\author{
Fiamma Straneo; notes by Guillaume Michel and Madeleine Youngs
}

June 30, 2017

In this lecture, we detail the two-way coupling between the dynamics of glaciers and of the ocean: the melting of glaciers is caused by an inflow of warm seawater, that in return is cooled and freshened. This interaction affects the large-scale ocean dynamics, and is usually only considered as a boundary condition in numerical simulations.

In the first $\mathrm{p}$ art of this lecture, we show how the melt $\mathrm{r}$ ate $\mathrm{c}$ an $\mathrm{b}$ e e valuated. We then present how it is connected to the ocean dynamics.

\section{How to estimate the melt rate?}

For a given glacier, the melt rate depends on the characteristics of the water flow (e.g. temperature, salinity, vertical stratification and $\mathrm{v}$ elocity). These p roperties set by the farfield o cean properties, but it remains challenging to infer a variation of the melt $\mathrm{r}$ ate based on records in the ocean. This is a consequence of the large range of length-scales lying between an ice edge, a fjord, the nearby ocean, and the large-scale ocean currents, see Tab. 1.

\begin{tabular}{|c|c|c|c|}
\hline Ice edge & Fjord & Nearby ocean & Far-field ocean \\
\hline$L \sim 10-100 \mathrm{~m}, H \sim \mathrm{km}$ & $W \sim 10 \mathrm{~km}, L \sim 100 \mathrm{~km}$ & $\sim 100 \mathrm{~km}$ & $\sim 1000 \mathrm{~km}$ \\
\hline
\end{tabular}

Table 1: Typical length-scales involved in the glacier-ocean coupling. $L$ stands for length, $H$ for height, and $W$ for width.

To estimate the melt rate, one can either:

1. For floating ice tongues, use the ice-flux divergence method, presented in the previous lectures. This method is recent and there is so far not enough data to investigate seasonal variability.

2. Perform a numerical simulation, and/or use a theoretical model. The thermal and salinity fluxes must then be characterized by transfer parameters (see e.g. equation (31) of lecture 5), whose values are not universal.

Since we lack data, we therefore lack an experimental way to estimate these fluxes. To fill this gap, we propose the gate flux method. 


\subsection{The gate flux method}

\subsubsection{General overview}

Given that the width of a fjord is much smaller than its length, the large-scale dynamics of the water in this domain can be supposed to be uniform over the width. Therefore, local measurements of the water temperature, salinity and velocity with depth combined with conservation equations can be used to estimate the melt rate, see e.g. [1, 2, 3, 4].

For instance, the measurements of Inall et al. [4] performed in the calving front of Kangerdlugssuaq have been used to deduce that heat delivered by the warm water coming from the ocean is equivalent to a melt rate of $\sim 10 \mathrm{~m} \cdot$ day $^{-1}$ (between $30 \%$ and $60 \%$ of the ice flux). The measurements, some of them being reported in F ig. 1, show a general feature of these exchanges:

1. Relatively hot water heads for the glacier below a few hundred meters.

2. Melting causes cooling, freshening and eventually upwelling (see Fig. 2 of Lecture 5).

3. Cold water leaves the Fjord near the surface.
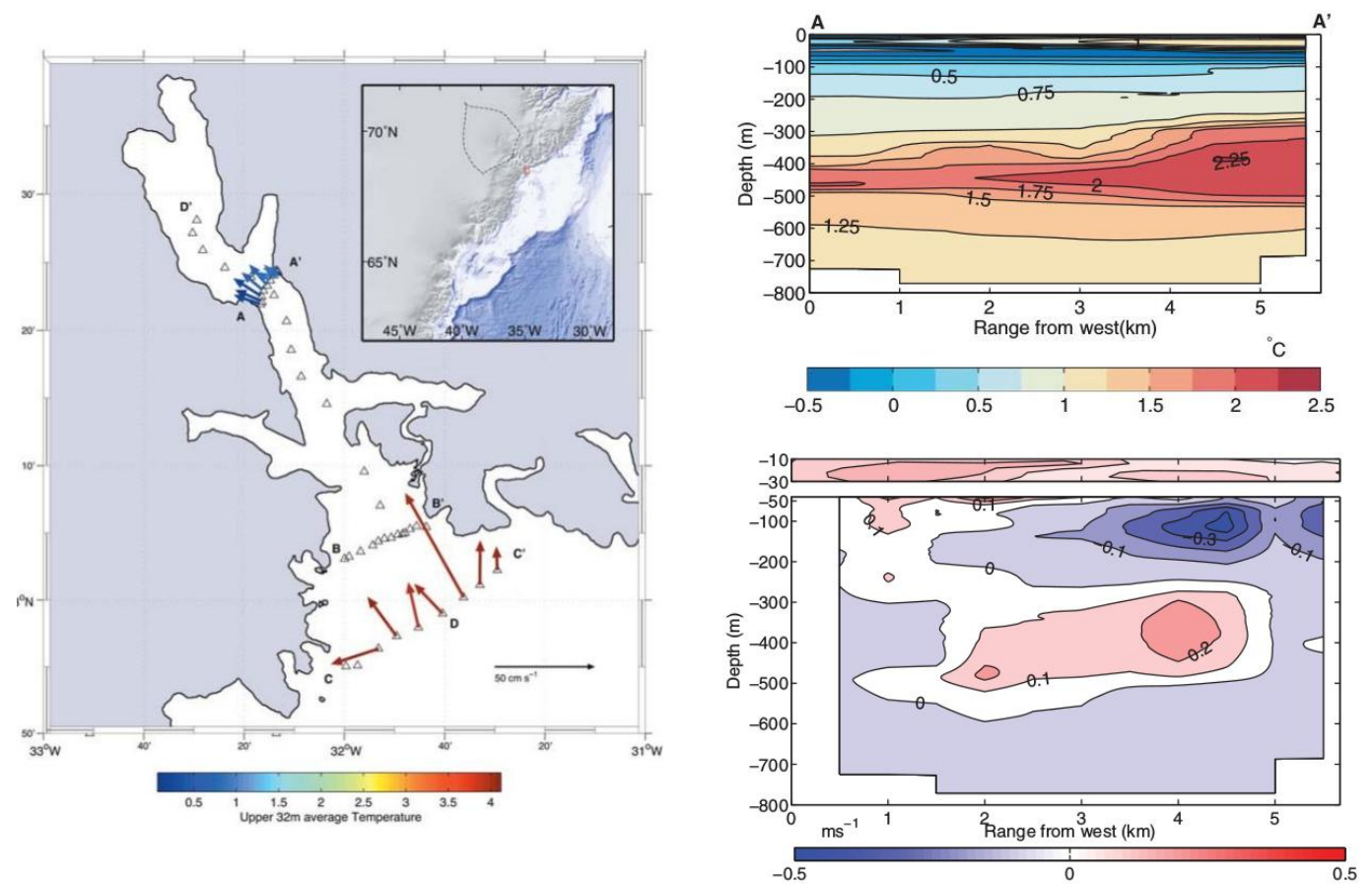

Figure 1: Left: schematic of the Kangerdlugssuaq Fjord, vector arrows represent upper velocity. Right: temperature and along-axis velocity across the measurement section AA'. Figures extracted from [4]. 


\subsubsection{The underlying equations}

In the gate flux framework, the complete heat, salt and mass budgets have been worked out by Jackson and Straneo [5]. Given the processes sketched in Fig. 2, they are as follows.

- Mass budget:

$$
\int_{\mathcal{A}_{x}} u \mathrm{~d} A+Q_{\mathrm{R}}+Q_{\mathrm{MW}}=0
$$

where $u$ is the velocity perpendicular to the cross-section $\mathcal{A}_{x}$ (vertical left boundary of the control volume), $Q_{\mathrm{R}}$ is the runoff volume flux and $Q_{\mathrm{MW}}$ is the meltwater volume flux.

- Heat budget:

$$
\rho c_{\mathrm{p}} \int_{\mathcal{A}_{x}} u \theta \mathrm{d} A+\rho c_{\mathrm{p}} Q_{\mathrm{R}} \theta_{\mathrm{R}}+\rho c_{\mathrm{p}} Q_{\mathrm{MW}} \theta_{\mathrm{MW}}+\rho c_{\mathrm{p}} Q_{\text {surf }} \theta_{\text {surf }}=\rho c_{\mathrm{p}} \frac{\partial}{\partial t} \int_{\mathrm{V}} \theta \mathrm{d} V+H_{\mathrm{m}}+H_{\mathrm{s}},
$$

where the left-hand side terms stand for the advective heat flux through the control volume's boundaries (respectively : the cross-section $\mathcal{A}_{x}$, runoff, meltwater and surface). In the right-hand side, we identify the evolution of the properties of the control volume, the heat required to melt ice and the heat flux through the surface.

- Salt budget:

$$
\int_{\mathcal{A}_{x}} u S \mathrm{~d} A=\int_{V} \frac{\partial S}{\partial t} \mathrm{~d} V
$$

that balances advective transport through the section $\mathcal{A}_{x}$ with changes in the amount of salt stored in the control volume.

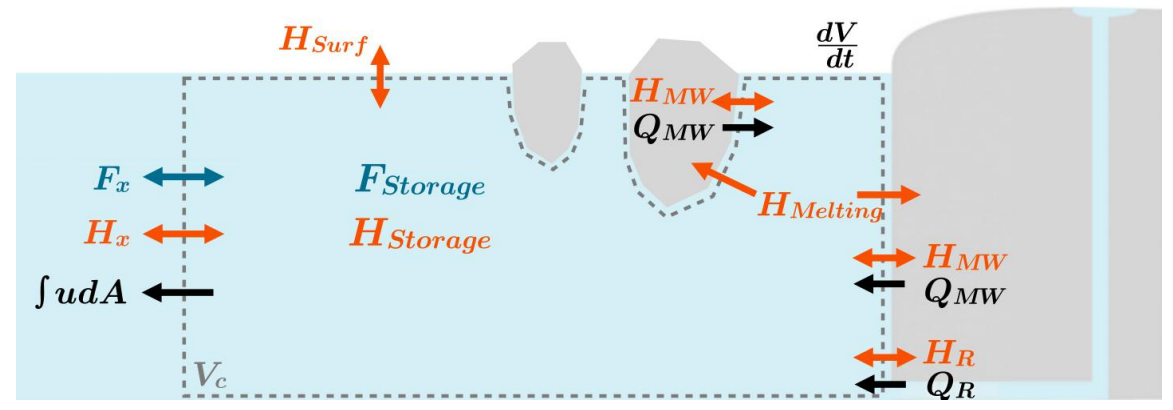

Figure 2: Control volume (grey dashed volume) and different processes taken into account. Figure extracted from [5].

For a practical use, time averaging as well as a differentiation between barotropic and baroclinic flow are useful. This leads to a new set of equations, see [5] for more details. Eventually, the mean meltwater volume flux, as well as other dominant fluxes, can be deduced from measurements. 


\subsubsection{Application to the Helheim glacier}

The resulting set of equations is used to analyze measurements performed in the Sermilik Fjord, that connects to the Helheim Glacier. A satellite image is shown in Fig. 3, together with records of the water properties with time and depth. They strongly fluctuate, hence the need to distinguish between fast and slow time-scales.
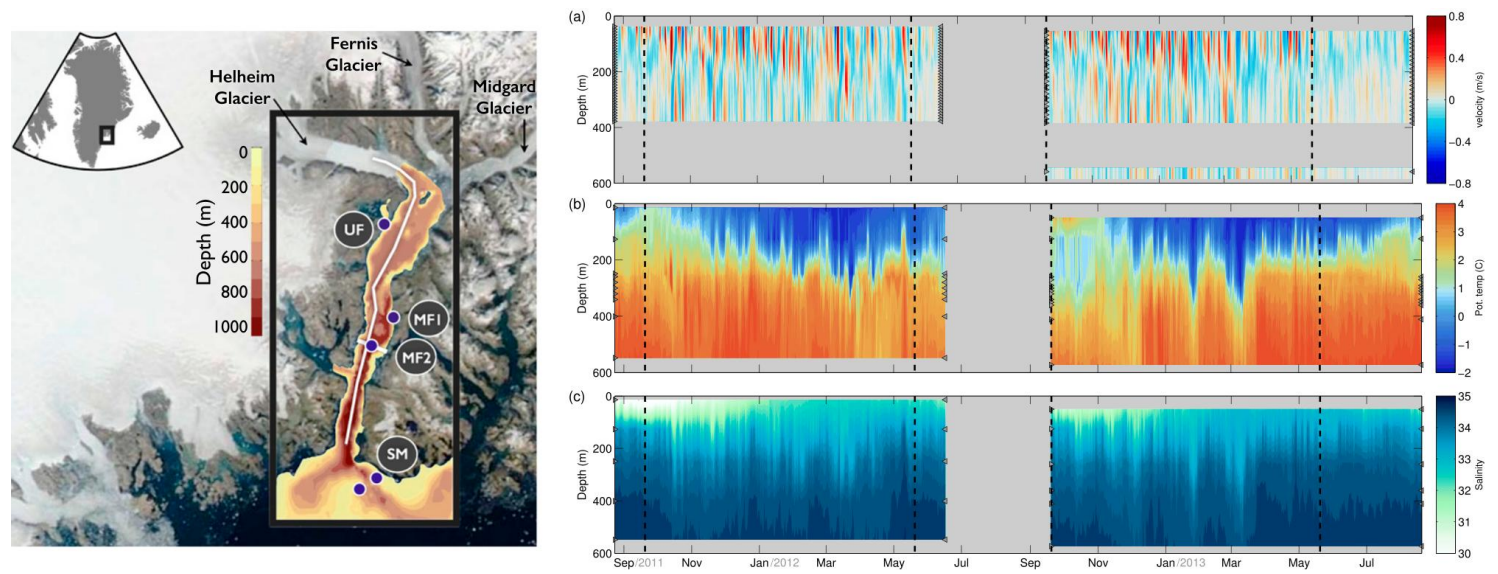

Figure 3: Left: sketch of the Sermilik Fjord. Right: velocity, potential temperature and salinity records at positions MF1 and MF2. Figure extracted from [5].

In winter, signal to noise ratio is low and freshwater fluxes do not appear c learly. On the other hand, in summer, the energy balance can be deduced and involves both the transported heat toward the glacier, storage in the water close to the glacier and melting.

Note that the melt rate deduced from these measurements includes icebergs melt, and thus differs from the melt rate of the glacier.

\subsubsection{Application to an ice tongue}

Exercise Consider the situation within the 79 North Cavity, sketched in Fig. 4, where $Q_{\text {in }}, Q_{\text {out }}$ and $S_{\mathrm{m}}$ are the incoming, outgoing and melting volume fluxes, and $T_{\text {in }}$ and $T_{\text {out }}$ are the associated temperatures.

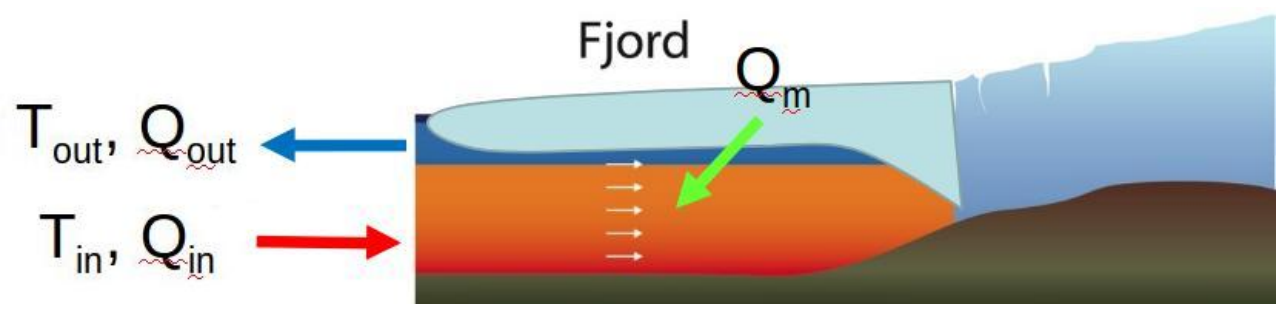

Figure 4: Exchanges with the 79 North Cavity. 
Question: in a steady-state, give numerical estimates for $Q_{\text {in }}$ and $Q_{\text {out }}$. Below are some numerical values that may be useful:

- The incoming temperature is $T_{\mathrm{in}}=1^{\circ} \mathrm{C}$.

- The outgoing temperature is $T_{\text {out }}=0.2^{\circ} \mathrm{C}$.

- The temperature of ice is $T_{\mathrm{i}}=-10^{\circ} \mathrm{C}$.

- The flux of meltwater is $Q_{\mathrm{m}}=0.36 \mathrm{mSv} \cdot\left(1 \mathrm{~Sv}=10^{6} \mathrm{~m} \cdot \mathrm{s}^{-1}\right)$

- The heat capacity of ice is $c_{\mathrm{i}}=2 \mathrm{~kJ} \cdot \mathrm{kg}^{-1} \cdot \mathrm{K}^{-1}$.

- The heat capacity of seawater is $c_{\mathrm{w}}=4 \mathrm{~kJ} \cdot \mathrm{kg}^{-1} \cdot \mathrm{K}^{-1}$.

- The latent heat of fusion is $L_{\mathrm{f}}=334 \mathrm{~kJ} \cdot \mathrm{kg}^{-1}$.

- The density of ice is $\rho=917 \mathrm{~kg} \cdot \mathrm{m}^{-3}$.

Solution: In a steady-state, the mass budget reads

$$
Q_{\text {in }}=Q_{\text {out }}=Q_{\mathrm{e}}
$$

with $Q_{\mathrm{e}}$ defined as the overturning exchange flow. The incoming heat balances the outgoing one and sustains the melting process,

$$
Q_{\mathrm{e}}\left(T_{\mathrm{in}}-T_{\text {out }}\right) c_{\mathrm{w}}=L_{\mathrm{f}} Q_{\mathrm{m}} \Longrightarrow Q_{\mathrm{e}}=\frac{L_{\mathrm{f}}}{c_{\mathrm{w}}}\left(\frac{Q_{\mathrm{m}}}{T_{\mathrm{in}}-T_{\text {out }}}\right)=38 \mathrm{mSv}
$$

See [6] for a detailed discussion of these water exchanges. Note that we ignore the heat required to raise the ice temperature from $-10{ }^{\circ} \mathrm{C}$ to $0^{\circ} \mathrm{C}$ because the latent heat is much higher than the heat capacity of ice.

\subsection{Diagnosing meltwater and runoff concentrations}

We thereafter present three methods that can be used to estimate both the meltwater and the runoff fluxes.

\subsubsection{Using $T-S$ diagrams}

As seen in a previous lecture, the melting of ice results, on a temperature-salinity diagram, in an evolution along a straight line toward the point $\left(T_{\text {eff }}, S_{\text {eff }}\right)$ given in equation $(5)$ of lecture 5 .

Experimental measurements of the water properties at different depths may lie on this line (see the winter profiles in F ig. 5) or not (e.g. the summer measurements in F ig. 5 ). This deviation from the theoretical melting evolution indicates that another process is involved, namely the addition of run-off to ambient water. In this case, the addition of fresh run-off water at freezing temperature in the heat and salt budgets (equations (1) and (2) of lecture $5)$ can be used to deduce the run-off concentration from measurements.

For applications of this method, see e.g. $[7,8,9]$. 


\section{Winter}

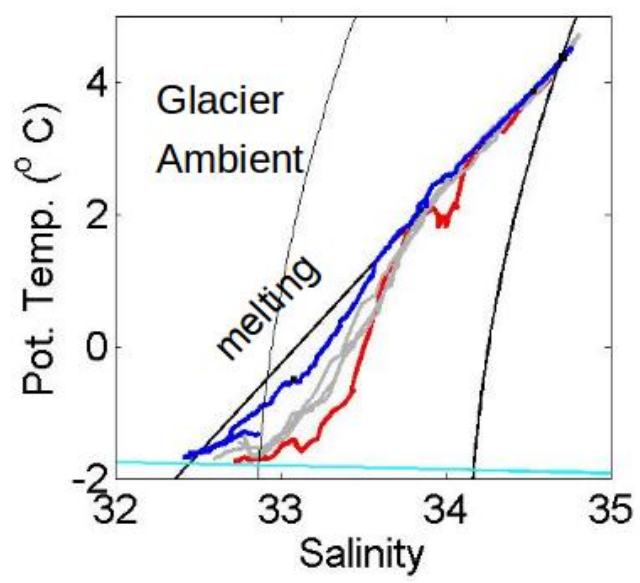

Summer

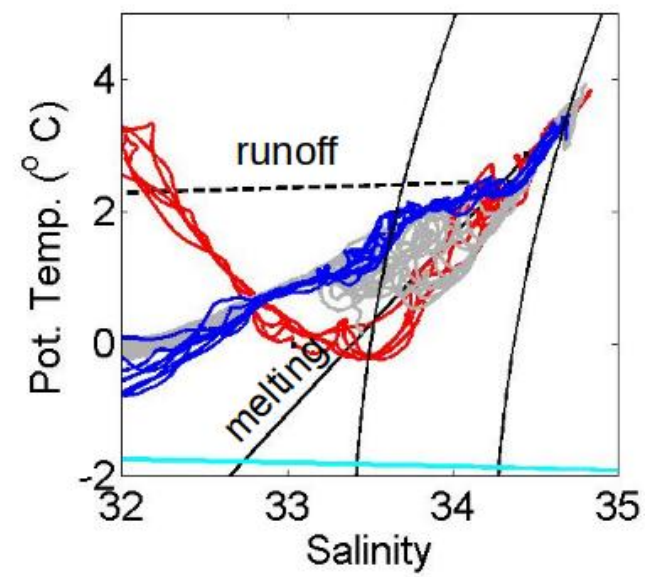

Figure 5: $(T, S)$ diagram measurement in winter (left) and in summer (right) [7].

\subsubsection{Using noble gases}

Another method is based upon the non-uniform distribution of noble gases in seawater. Noble gases are inert and do not undergo chemical reactions: their concentrations only depend on the diffusive processes they have been through. Depending on the quantity of noble gas measured, it is therefore possible to distinguish between the different water origins:

- Surface melt runoff is formed at the surface of an ice sheet, is isolated and eventually melts into fresh water. The fraction of noble gas is then the one associated with solubility in fresh water at zero degree and one atmosphere.

- Submarine melt water, on the other hand, comes from melting under pressure, that results in anomalous noble gas concentration signals.

Examples of results than can be deduced from these measurements are reported in Fig. 6. We recognize the general structure of water fluxes close to a glacier, described in section 1.1.1, see [10] for an extensive discussion. This method has recently been used in the Sermilik fjord [11], to disentangle the subglacial discharge from the submarine melt, that turned out to be of similar amplitude.

\subsubsection{Using nutrients}

The upwelling of subglacial discharge and the entrained waters also results in an upwelling of nutrients, including nitrate, silicate and phosphate. They have been recently evidenced [12]. This provides an interesting information to discuss the biological life in a fjord. $A$ posteriori, nutrients could also be used instead of noble gases. 


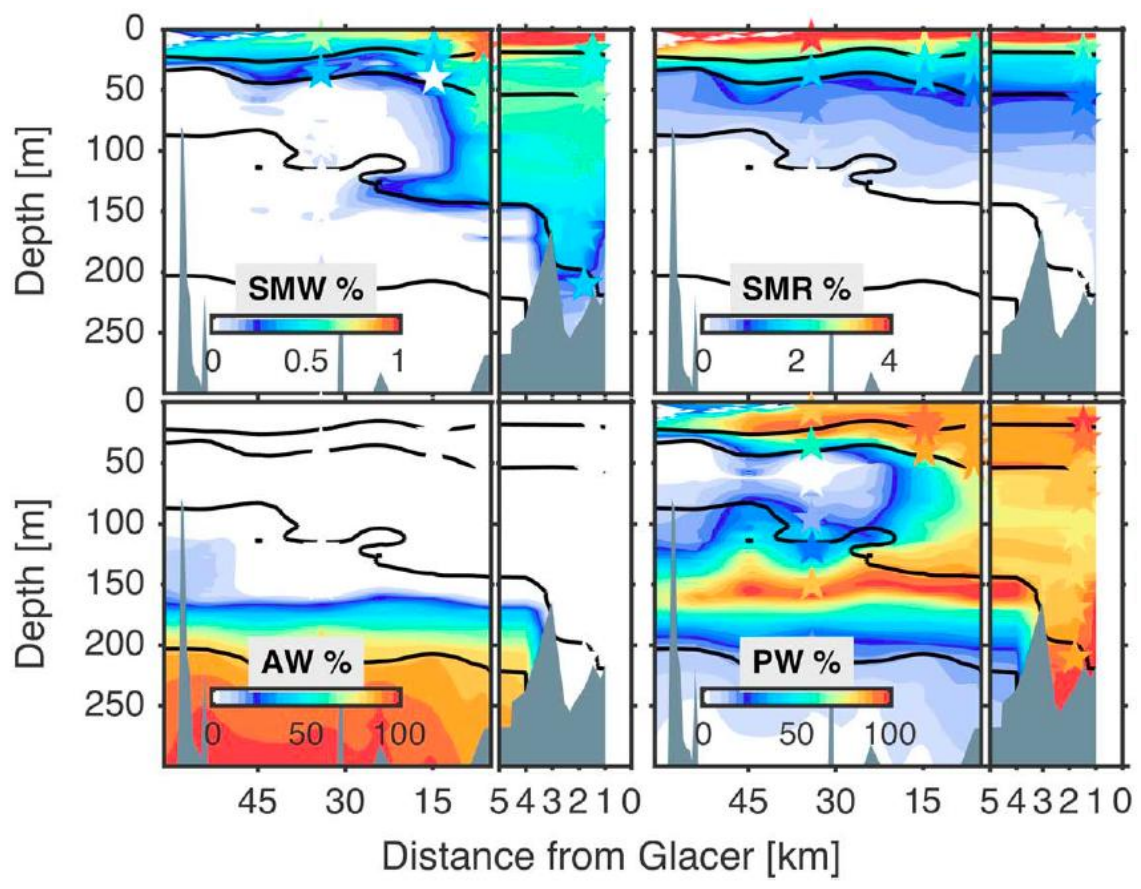

Figure 6: Extrapolation of the data to infer the distributions of submarine melt water (SMW), Antartic water (AW), surface melt runoff (SMR) and polar water (PW). Figure extracted from [10].

\section{Ocean forcing by Greenland}

There is clear evidence that freshwater fluxes from Greenland into the North Atlantic increase $[13,14,15]$, which may have an impact on the ocean dynamics $[16,17,18]$. So far, ocean forcing by Greenland is taken into account through freshwater discharge conditions (see, e.g., [15]), that are rough estimates and are not consistent with progress in ice/ocean exchanges. These difficulties in obtaining the freshwater flux partly come from icebergs.

\subsection{Shortcomings in the current estimates of the freshwater flux}

Consider the Sermilik Fjord, that consists of three glaciers and a significant catchment basin. A first approach to estimate the freshwater flux (FWF) consists of adding the discharge and the runoff fluxes, where:

- The discharge is obtained by adding the discharge of each of the three glaciers.

- The runoff is obtained by defining a catchment basin then by estimating the net melt from a regional climate models.

However, the freshwater flux evaluated this way differs from the freshwater flux at the fjord mouth, because of the transformation (my mixing) or delay (by storing) inside the fjord. Therefore, it is necessary either to resolve the dynamics at these small scales, which 
is unrealistic for the current oceanic models, or to model this dynamics. This has been reviewed in [19], and we thereafter focus on the effect of icebergs. The calving of icebergs is necessary to balance the mass flux of a glacier when the melt rate from the glacier cannot remove enough mass.

\section{$2.2 \quad$ Icebergs in fjords}

\subsubsection{Measurements from satellite images}

Icebergs affect the FWF because they can either leave the fjord or melt in the fjord (some of this meltwater could then be trapped subsurface and/or affect the fjord circulation).

This solid ice flux has recently been estimated from satellite images and showed to dominate the freshwater budget in iceberg-congested glacial fjord [20]. Many properties of the icebergs can be deduced from satellite images (combined with models), such as the icebergs aspect ratio, size distribution and melt rates [21]. As can been seen in Fig. 7, the aspect ratio of iceberg is roughly constant $(W \simeq 2 H$, where $W$ is the width and $H$ is the thickness), and the size distribution is self-similar. Similarly, the meltwater flux c an be characterized as a function of the draft or as a function of the size of the dense matrix of floating i ce [21].
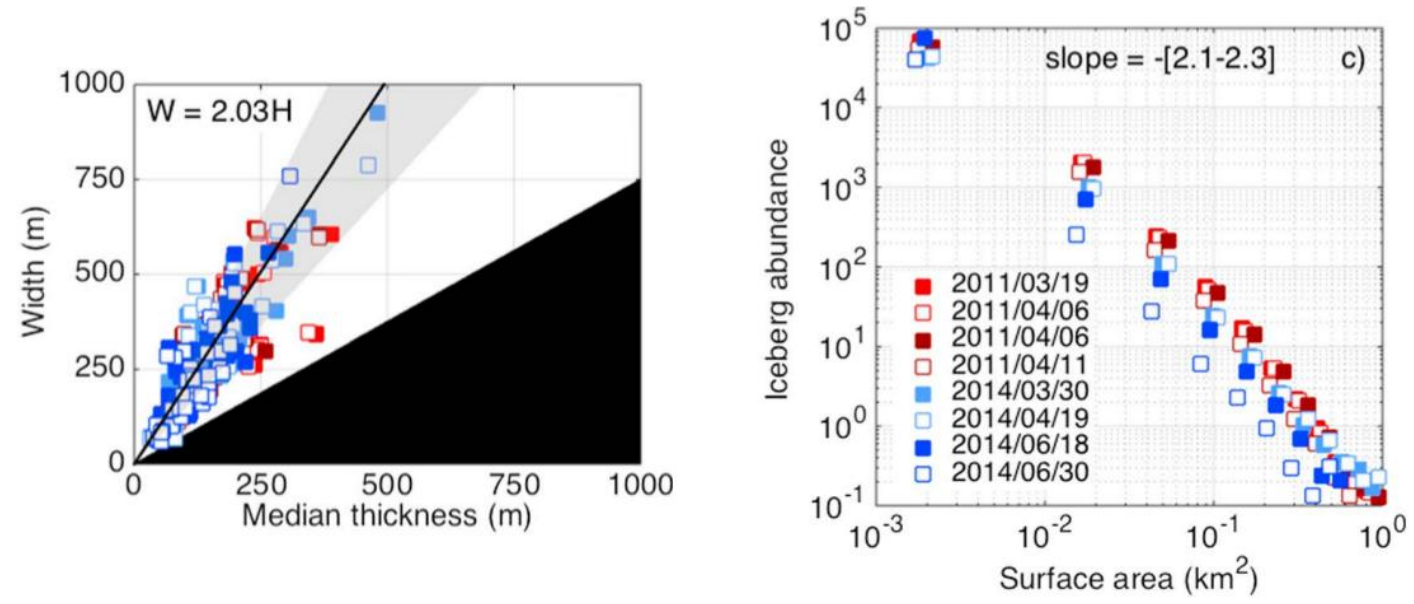

Figure 7: Icebergs ratio and size distribution for Ilulissat fjord. Figure extracted from [20].

\subsubsection{In-situ measurements}

Icebergs properties including draft and speed can also be measured using a inverted echo sounder at the bottom of the ocean [22]. This information gives us the mass flux of ice out of the domain and information about the freshwater budget. The current parameterizations of freshwater flux due to icebergs only use the surface velocities, but an iceberg actually travels using the vertical average of speeds along its draft, so we need better iceberg parameterizations to appropriately model the freshwater fluxes from glaciers [23]. 


\subsubsection{Models of the iceberg melt flux}

Models of iceberg melting are also used to examine the contribution of meltwater flux from icebergs [24]. This model includes a seasonality of the freshwater fluxes from icebergs due to changes in temperature distribution where the icebergs are melting (Fig. 8). Overall, there is more melting in the summer as expected, but the vertical distribution of the melting changes with the season and temperature distributions in the fjords.
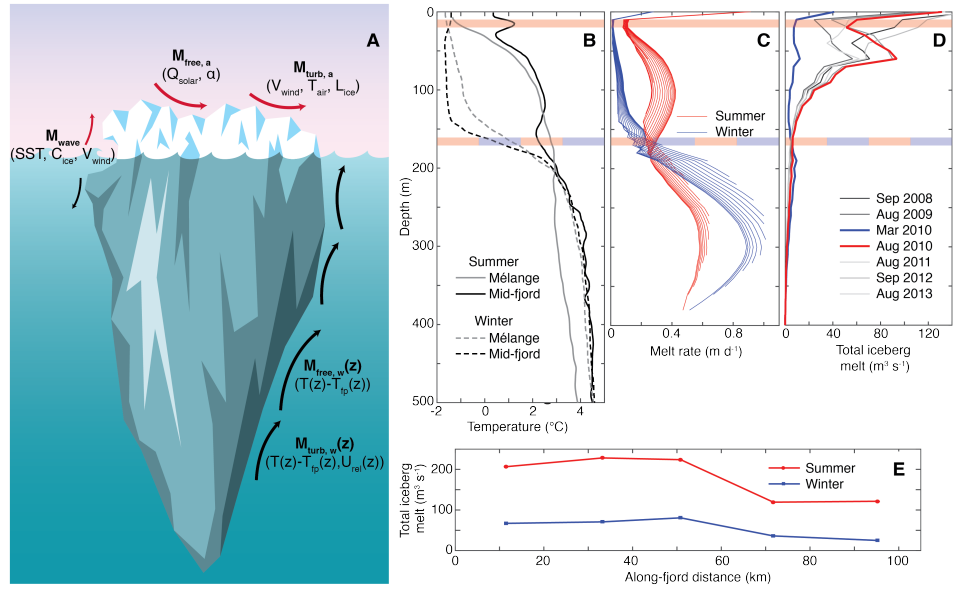

Figure 8: A figure showing the model of freshwater flux into ocean from icebergs. Figure extracted from [24].

This iceberg model is coupled with data to estimate the total freshwater flux into the ocean from the glacial system (Fig. 9). There is a large peak in the freshwater fluxes due to subglacial discharge in the summer months as the largest contribution. The second largest contribution is from iceberg melting which is lagged behind the subglacial discharge with a minimum in April and a maximum in September. The observations of iceberg melt agree well with the model where observations exist.

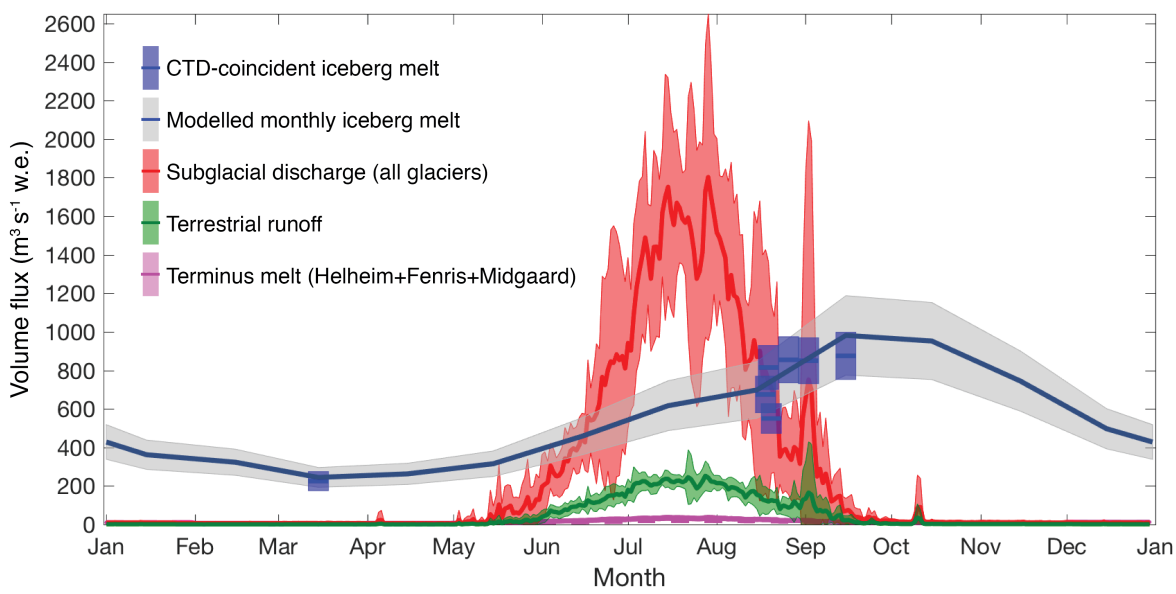

Figure 9: A figure showing the total freshwater flux into ocean throughout a year. Figure extracted from [24]. 


\section{References}

[1] R. Motyka et al. (2003) Submarine melting at the terminus of a temperate tidewater glacier, LeConte Glacier, Alaska, U.S.A., Ann. Glaciol. 36, 57.

[2] E. Rignot et al. (2010) Rapid submarine melting of the calving faces of West Greenland glaciers. Nat. Geosci. 3, 187.

[3] D. Sutherland and F. Straneo (2012) Estimating ocean heat transports and sumarine melt rates in Sermilik Fjord, Greenland, using lowered acoustic Doppler current profiler (ADCP) velocity profiles. Ann. Glaciol. 53, 50.

[4] M. E. Inall et al., (2014) Oceanic heat delivery via Kangerdlugssuaq Fjord to the southeast Greenland ice sheet. J. Geophys. Res. Oceans 119, 631.

[5] R. H. Jackson and F. Straneo (2016) Heat, salt and freshwater budgets for a glacial fjord in Greenland. J. Phys. Oceanogr. 46, 2735.

[6] N. J. Wilson and F. Straneo (2015) Water exchange between the continental shelf and the cavity of Nioghalvfjerdsbrae (79 North). Geophys. Res. Lett. 42, 7648 (2015)

[7] F. Straneo (2011) Impact of fjord dynamics and glacial runoff on the circulation near Helheim Glacier. et al., Nat. Geosci. 4, 322.

[8] A. Jenkins (1999) The impact of melting ice on ocean waters. J. Phys. Oceanogr. 29, $2370(1999)$

[9] H. G. Gade (1979) Melting of ice in sea water: A primitive model with application to the Antarctic ice shelf and icebergs. J. Phys. Oceanogr. 9, 189.

[10] N. Beaird et al. (2015) Spreading of Greenland meltwaters in the ocean revealed by noble gases. Geophys. Res. Lett. 42, 7705.

[11] N. Beaird et al., in prep.

[12] Cape, M. et al., in prep.

[13] T. W. N. Haine et al. (2015) Arctic freshwater export: Status, mechanisms, and prospects. Global Planet. Change 125, 13.

[14] E. C. Carmack et al. (2016) Freshwater and its role i the Arctic marine system: Sources, disposition, storage, export, and physical and biogeochemical consequences in the Arctic and global oceans. Geophys. Res. Bio. 121, 675.

[15] J. Bamber et al. (2012) Recent large increases in freshwater fluxes from Greenland into the North Atlantic. Geophys. Res. Lett. 39, L19501. 
[16] C. W. Böning et al., (2016) Emerging impact of Greenland meltwater on deepwater formation in the North Atlantic Ocean. Nat. Geosci. 9, 523.

[17] H. Luo et al. (2016) Oceanic transport of surface meltwater from the southern Greenland ice sheet. Nat. Geosci. 9, 528.

[18] S. Rahmstorf et al., (2015) Exceptional twentieth-century slowdown in Atlantic Ocean overturning circulation. Nat. Clim. Change 5, 475.

[19] F. Straneo and C. Cenedese (2015) . The dynamics of Greenland's glacial fjords and their role in climate. Ann. Rev. of Marine Sci. 7, 89.

[20] E. M. Enderlin et al. (2016) Iceberg meltwater fluxes dominate the freshwater budget in Greenland's iceberg-congested glacial fjords. Geophys. Res. Lett. 43, 287.

[21] E. M. Enderlin and G. S. Hamilton (2014) Estimates of iceberg submarine melting from high-resolution digital elevation models: application to Sermilik Fjord, East Greenland. J. Glaciol. 60, 1084.

[22] M. Andres et al. (2015) Icebergs and sea ice detected with inverted echo sounders. J. Atmos. Oce. Tech. 32, 1042.

[23] A. FitzMaurice et al. (2016) Effect of a sheared flow on iceberg motion and melting. Geophys. Res. Letters 43 12,520 (2016)

[24] Moon et al., Submitted 


\title{
Seeing the Ocean through Sea Ice
}

\author{
Robert A Fajber
}

November 15, 2017

\begin{abstract}
Signatures of submesoscale ocean filaments are commonly observed in satellite images of thin, sparse sea ice. However, sea ice is not a passive tracer, and its distribution over regions of surface ocean convergence depends on a balance between the external ocean drag and the internal stresses due to ice-floe interactions. Therefore, the distribution of sea ice thus depends on ice-ocean interactions at the submesoscale.

This study looks at two idealized cases. The first is a convergent filament with surface flow that is independent from the sea-ice. Sea ice accumulates over the center of the filament, and shows multi-timescale behavior. The second case is a filament where the surface flow is driven by ocean dynamics through Ekman transport. It is shown that the coupled ice-ocean system is able to accumulate sea-ice for certain rheological regimes.

The results of these two idealized test cases show that concentrations of sea ice over filaments are able to develop on timescales of 1-2 days, and that the peak of the concentration profile can be several times the background. This gives an indication that snapshots of sea ice could be used to constrain surface ocean currents.
\end{abstract}

\section{Motivation}

Upper ocean dynamics contains a rich array of submesoscale eddies and filaments with high Rossby numbers and scales below the Rossby deformation radius, resulting from mixed layer and submesoscale instabilities [9]. Submesoscale currents can frequently develop energetic ageostrophic vertical velocities. This provides a mechanism of vertical tracer transport across the mixed layer that is critical for biogeochemical processes in the ocean [6] and also creates strong vertical heat fluxes. In addition, submesoscale eddies provide a mechanism for the forward energy cascade towards dissipation (contrary to mesoscale dynamics, which leads to energy cascade to large scales). Understanding submesoscale dynamics as it relates to upper ocean tracer transport, modification of surface buoyancy fluxes, and energy cycles remains a longstanding problem in oceanography. 


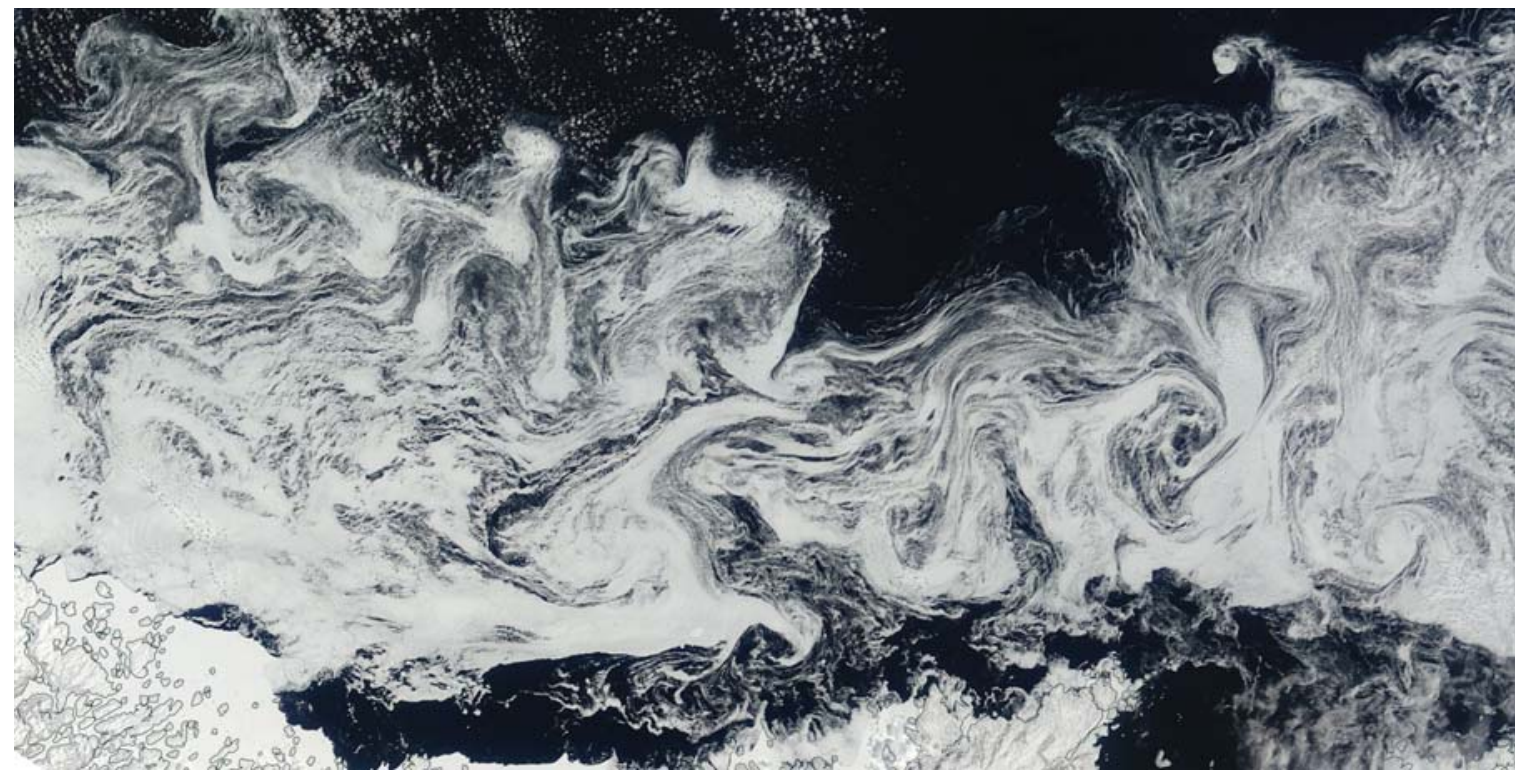

Figure 1.1: Satellite reflectance image (Aqua/MODIS) of sea ice in the marginal ice zone in the Labrador sea, taken from NASA Worldview: https://worldview.earthdata.nasa.gov/. Image resolution is $250 \mathrm{~m}$ and total width is about $400 \mathrm{~km}$ with eddy sizes $\mathrm{O}(20 \mathrm{~km})$ and filament widths $\mathrm{O}(5 \mathrm{~km})$. The Labrador coastline is visible in the bottom of the photograph, and small clouds are visible at the top of the photograph. 
Satellite observations of ocean altimetry and sea ice are limited to the larger, geostrophically constrained scales [2]. Making in situ observations in the polar oceans is particularly complicated due to the presence of sea ice and icebergs, which can destroy instruments [1]. As a result, studies of submesoscale currents in the polar oceans are limited to relatively few observations [13]. Under the heavily packed multi-year sea ice, the submesoscale variability is significantly damped since ice acts as a momentum and energy sink for upper ocean flows [10]. However, submesoscale variability is energetic in marginal ice zones and can substantially enhance the oceanice heat source by bringing warm sub-mixed layer waters in contact with the ice [8].

At sufficiently low concentrations, the sea ice can accumulate in convergent surface ocean currents creating submesoscale concentration patterns visible from satellite images [8], see figure 1.1 for example. The sea ice accumulation is opposed by internal ice stresses, leading to a quasi-steady balance with elevated sea ice concentrations over converging cyclonic eddies and filaments. Identifying conditions favorable for pattern formation in sea ice is complicated by the fact that the sea ice rheology is still largely unknown [3]. Yet, satellite images of marginal ice zones suggest that ice concentrations are indeed tightly related to the divergence patterns of the underlying ocean currents.

Here, we present a theoretical framework to explain the development of sea ice concentration patterns over idealized ocean eddies and filaments. Throughout this work we prioritize analytical tractability over rheological complexity by retaining only the very critical aspects of sea ice rheology, with the goal of elucidating the key physical balances. The report is organized as follows: In Section 2 we derive an idealized model of sea ice evolution driven by converging upper ocean flow. In Section 3 we apply the model to a cyclonic filament that has a strong ageostrophic surface flow resulting in sea ice accumulation. In Section 4 we explore sea ice accumulation over near-geostrophic flows where it is the presence of sea ice (and a corresponding iceocean stress) that generates the Ekman convergence. In Section 5 we discuss potential applications of our theoretical framework to estimating the statistical properties of upper-ocean divergence field from still images of sea ice concentration.

\section{Equations of Sea Ice Motion}

\subsection{Development of equations}

The model that we use treats sea ice as a continuous media, similar to $[4,11]$. The development of the model follows a standard approach for the fluid dynamics of a continuum mixture, which we review below to introduce the key assumptions made.

Although sea ice is composed of individual floes that strongly interact with each other, we assume that the the dynamics can be averaged over a sufficiently large scale that the properties of the floes (e.g. floe-floe stresses) can be represented using 

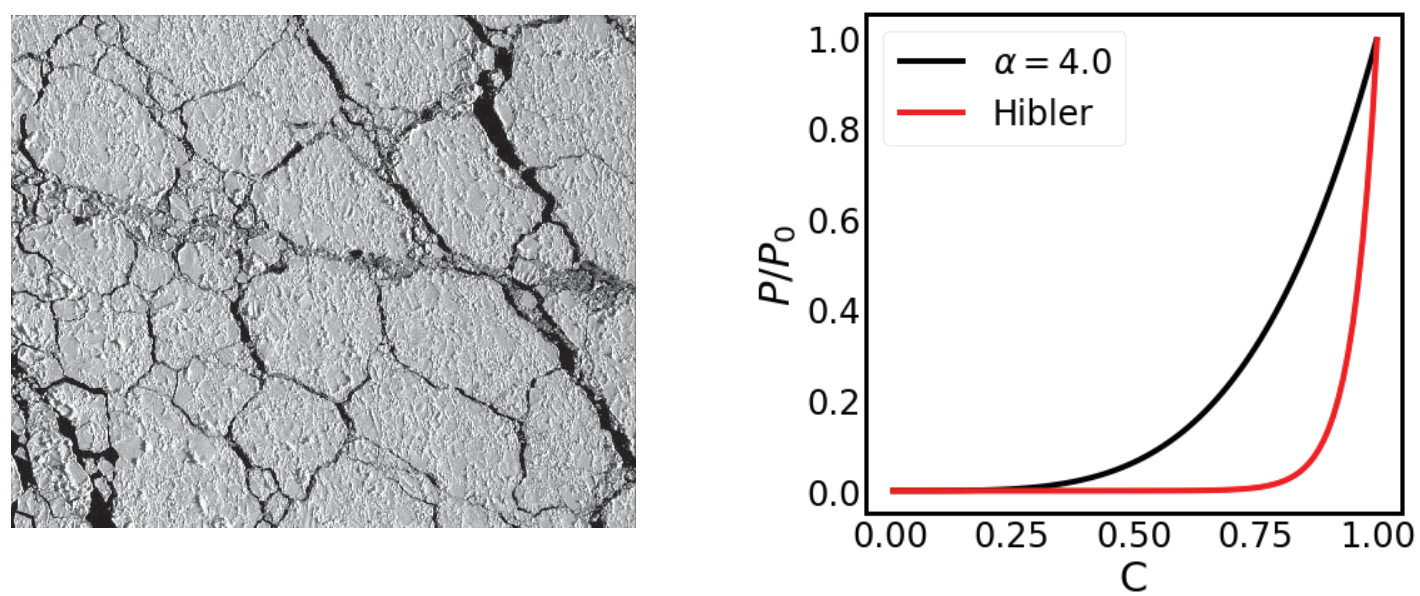

Figure 2.1: (a) Distribution of sea ice (with dark areas representing open ocean) emphasizing strong interactions between individual ice floes (with characteristic diameters of about $100 \mathrm{~m}$ ). The ice occupies an area $A_{I}$ within a total image area $A$, over which the sea ice is considered as a continuous media (see Eq. 2.4 and discussion in the text). Taken from [7]. (b) The continuous power-law 'equation of state' showing the critical dependence of sea ice pressure on concentrations (black). The Hibler rheology [4] is shown in red for comparison.

average properties (e.g. the concentration and average velocity). Our study will focus only on the dynamic equations of sea ice motion, since on submesoscale timescales (order of days to a week), much shorter than the sea ice thermodynamic timescale of several months [11], justifying the neglect of the sea ice.

We assume that the ice within some region $A$ has a uniform thickness $h$ and covers an area $A_{I}$, a typical sea ice state is shown in Fig. 2.1a. The resulting concentration $C(\mathbf{r}, t)=A_{I} A^{-1}$ can evolve in time and space. We consider purely two-dimensional ice motion along the ocean surface with sea ice velocities $\underline{u}=(u, v)$ that may be divergent. Dividing the mass conservation equation by $A$ yields

$$
0=\partial_{t} \int_{V} \rho C d V+\int_{V} \rho \nabla \cdot(C \underline{u}) d V
$$

where $\rho$ is the density of the ice. Doing this integral and noting that the vertical component vanishes, and dividing by $\rho h$ gives the concentration evolution equation

$$
0=\partial_{t}(C)+\nabla \cdot(\underline{u} C),
$$

with $\nabla=\left(\partial_{x}, \partial_{y}\right)$. We note that $C \in[0,1]$, but this constraint is not enforced by equation 2.1. If a system produces $C>1$, then this corresponds to a breakdown of model physics, implying that the assumption of constant height is broken.

Assuming that the ice has negligible inertia, the conservation of momentum equation 
can be written as a balance between the internal, $\underline{\underline{\tau}}_{\text {int }}$, and external stresses $\underline{\underline{\tau}}_{\text {ext }}$,

$$
0=\oint_{S}\left(\underline{\underline{\tau}}_{e x t}+\underline{\tau}_{i n t}\right) \cdot d \underline{S}
$$

where $S$ is the surface of $V$. To simplify the external stress we assume

- $\underline{\underline{\tau}}_{\text {ext }}=0$ except on the top and bottom surfaces.

- $\underline{\tau}_{\text {ext }}$ acts only on the sea ice .

- $\underline{\underline{\tau}}_{\text {ext }}$ is constant on the lower surface of $S$.

Taken together these assumptions give

$$
A^{-1} \int_{S} \underline{\tau}_{e x t} \cdot d \underline{S}=C \underline{\tau}_{w},
$$

where $\underline{\tau}_{w}$ is the water stress on the bottom surface of the ice. We note that there is also an atmospheric force acting on the top of the ice in principle, but we will ignore it here since the focus of the project is on the interaction of the ice and the ocean. To simplify the internal stress we assume that:

- $\underline{\tau}_{\text {int }}$ parameterizes collisions between sea ice on the boundary of $V$.

- $\underline{\underline{\tau}}_{\text {int }}$ is independent of $\mathrm{z}$,

so that

$$
\begin{aligned}
\oint_{\underline{\tau}_{i n t}} \cdot d \underline{S} & =\int_{V} \nabla \cdot \underline{\underline{\tau}}_{i n t} d V \\
& =h \int_{A} \nabla \cdot \underline{\underline{\tau}}_{\text {int }} d A .
\end{aligned}
$$

To understand why the integral involving $\tau_{\text {int }}$ appears as a boundary term, consider an ensemble of interacting floes in an area A. If there are no other forces other than the collisions between floes, then the total force acting on all the floes must be 0 , since by Newton's third law every collisional force will have an equal and opposite force. If the area $\mathrm{A}$ is subdivided into two areas, $A_{1}$ and $A_{2}$, then the total force on the sea ice inside area $A_{1}$ will be due to collisions between floes in $A_{1}$ and $A_{2}$, since collisions between floes only in $A_{1}$ will cancel. Thus, the interactions which can exert a force on an ensemble of floes are interactions that involve floes from another ensemble. If we consider the ensembles of floes to be in neighboring areas, then the only place for these floes to interact is at the boundary of the area that we are averaging over. 
Since this internal stress represents the average statistical properties of the collisions within the area A, then they have to be evaluated over the entire boundary. After applying the divergence theorem, this is equivalent to saying that they have to be integrated over the entire volume. This is different than the external stress, which only acts on the surface of the sea ice, hence the factor of $\mathrm{C}$ difference between the two terms.

The internal stress can be further separated into an isotropic component inlcuding a pressure $P$ that we assume only depends on the concentration of ice, and a deviatoric stress $\underline{\underline{\sigma}}$ :

$$
\underline{\underline{\tau}}_{\text {int }}=-P \underline{\underline{I}}+\underline{\underline{\sigma}} \text {. }
$$

By assuming that the area $A$ is arbitrary and combining equations (2.2)-(2.5), we find

$$
0=C \underline{\tau}_{w}-h[\nabla P+\nabla \cdot \underline{\underline{\sigma}}]
$$

\subsection{Simplified sea ice rheology}

To complete the system we have to specify a drag law for $\tau_{w}$, an equation of state for $P$ and an equation for $\underline{\sigma}$. Our choices for this are motivated by the physics governing the system, but we have also made additional simplifications to allow for a more tractable mathematical anlaysis. Our final system differs therefore from other more complicated models (for example the Hibler model [4]) that include more terms in the momentum equation in order to have simulations that are realistic enough to compare to large scale observations of thick, high concentration sea ice.

We assume that the drag law can be linearized so that

$$
\underline{\tau}=-\rho_{w} \Gamma_{d}\left(\underline{u}-\underline{u}_{w}\right)
$$

where $\rho_{w}$ is the density of water, and $\Gamma_{d}$ is a drag coefficient with units $\mathrm{ms}^{-1}$, and $\underline{u}_{w}$ is the water velocity.

The pressure term includes the large scale effect of collisions between individual ice floes. To include this we use an 'equation of state' which describes the pressure as a function of the concentration. This means that a concentration gradient in the ice will create a force on the ice due to internal stresses. Physically, this corresponds to there being more collisions in a region with high ice concentration and fewer collisions in a region with low ice concentration, with the result of an average force towards the region with lower concentration (the intuition here is similar to an ideal gas, hence the comparison with the equation of state). In principle, the equation of state should be determined by either detailed field measurements or by consideration of a model of sea ice floe collisions. In this study however, we will pick a form that matches our physical intuition, namely that at low concentrations the pressure should become 
very small, and at concentrations approaching 1 the pressure should become very large, with a sharp transition in between. We also choose a form that is convenient for analytical analysis. One form that accomplishes these goals is

$$
P=P_{0} C^{\alpha},
$$

In this study we choose $\alpha=4$; note that the choice $\alpha=4$ corresponds to the dynamics of a thin viscous film (see section 3 in [14]). The choice of the rheology is essentially arbitrary. The rheology of [4] is chosen on the basis of observational comparison with wintertime ice distributions. The function is somewhat arbitrary the only real constraints are the ones mentioned in the previous section. Our choice of a power law gives a good qualitative comparison to the rheology of [4], but is more more analytically tractable, allowing us to advance our analysis further.

We specify the deviatoric stress by assuming that the fluid behaves like a Newtownian fluid so that

$$
\underline{\underline{\sigma}}=\eta\left(\nabla \underline{u}+(\nabla \underline{u})^{T}\right)
$$

where the viscosity $\eta$ is a constant. This approximation is a significant simplification compared to the physical nature of sea ice, which is thought to behave as a shear thinning fluid [3]. Other models have assumed that ice can be treated either as a visco-plastic material [4], or an elastic-visco-plastic material [5] where the elasticity is introduced for numerical convenience. However, in these models it is found that the ice usually goes between the two extremes, either as a plastic fluid at high concentrations or as a viscous fluid at low concentrations. Since we are primarily interested in the low concentration limit with high shear, the Newtownian approximation should be valid in this region. In section 4 we will also take both the $\eta \rightarrow 0$ and the $\eta \rightarrow \infty$ limits of the system to further understand the implications of this choice.

Equations (2.1), (2.6), (2.7), (2.8), and (2.9) are a closed set of equations that can be solved to find the time varying ice concentration field. In the geometry of our problems, we will only be interested in motion that varies in one direction, which we will take to be $y$. We can substitute equations (2.7), (2.8), and (2.9) into equation (2.6) and simplify all the equations by eliminating terms involving $\partial_{x}$ to get

$$
\begin{aligned}
\partial_{t} C & =-\partial_{y}(v C), \\
0 & =-C \rho_{w} \Gamma_{d}\left(v-v_{w}\right)-h P_{0} \partial_{y} C^{\alpha}+\eta \partial_{y}^{2} v, \\
0 & =-C \rho_{w} \Gamma_{d}\left(u-u_{w}\right)+\eta \partial_{y}^{2} u .
\end{aligned}
$$

\subsection{Comment on assumptions}

Throughout the development of the model we have made several assumptions to simplify the theory, some of which have been made for mathematical expediency. In deriving equations (2.1) and (2.6), it was assumed that the distribution of floe sizes was irrelevant. If the distribution of floe thicknesses and sizes is important, an additional equation that constrains the distribution (like the thickness equation) 
would have to be added. This would also add a meaningful constrain on $C$, as well to constrain it to be $C \in[0,1]$.

Atmospheric drag is removed primarily for the sake of simplicity. At the scales below $1 \mathrm{~km}$, atmospheric drag is highly variable. At the larger scale, the atmospheric drag can be very important for the sea ice distribution, but the scales are much larger than the ones here. During strong atmospheric storms, the atmospheric drag destroys all of the structure described in this study. For instance, atmospheric arctic mesocyclones referred to as "polar lows" can reach hurricane force winds, but typically have horizontal scales of 100-500km [12]. Atmospheric drag at much smaller scales could also create deviations from a purely ocean-driven sea ice distribution while not completely removing all of the structures inherited from the ocean currents. Ignoring the atmospheric drag thus restricts us to using the model on relatively calm days, with weak atmospheric drag.

The choices of the sea ice rheology are not expected to make a large difference compared to Hibler's model [4] and are primarily done for the sake of mathematical tractability. This is because we are interested in low-concentration sea ice with strong shear, and so we are less interested in the plastic part of the rheology. The choice of pressure function could be made the same as [4], however it would not change much, since at low concentrations it is similar to our pressure function.

The boundary condition in $C$ used for both the uncoupled and coupled filament model is to impose an outer boundary condition $C(L)=C_{\infty}$. As will be seen in sections 3 and 4, this choice of boundary condition allows a flux of mass through the domain. Outside the filament there are other processes acting to redistribute sea ice. If these processes act to produce a concentration of $C_{\infty}$ then it is reasonable to impose this as a condition at the outside of the domain. Additionally, this situation allows us to approximate the case where there is a small filament in a large domain, i.e. a case where $v_{w}=0$ for $|y|>l$ and $\partial_{y} C(L)=0$. In such a case, the flux through

$y=l$ is supplied by the diffusion in the outer boundary. Numerically simulating such a setup can be computationally challenging however, due to the range of concentrations involved. Analysis of this case is currently ongoing.

\subsection{Non-dimensionalization}

These equations can be non-dimensionalized with:

$$
y=\hat{y} Y, v=V \hat{v}, v_{w}=V \hat{v}_{w}, u=U \hat{u}, u_{w}=U \hat{u}_{w}, \eta=\lambda \hat{\eta} .
$$

The values for these scales are given in table 2.4, except for $\lambda$, which is investigated in section 4 . The length scale of $1 \mathrm{~km}$ is characteristic of motion in the submesoscale [9]. If we use the constraint that the Rossby number is 1 , and we assume a latitude of $60 \mathrm{~N}$, then we get a velocity scale $V \sim 0.05 \mathrm{~ms}^{-1}$. The other scales are chosen either from [8] or [4]. 


\begin{tabular}{cccccccc}
\hline Parameter & $P_{0}$ & $V$ & $U$ & $L$ & $\Gamma_{d}$ & $h$ & $\rho_{w}$ \\
Value & $5 \times 10^{3} \mathrm{Nm}^{-1}$ & $10^{-2} \mathrm{~ms}^{-1}$ & $1 \mathrm{~ms}^{-1}$ & $10^{3} \mathrm{~m}$ & $5 \times 10^{-3} \mathrm{~ms}^{-1}$ & $1 \mathrm{~m}$ & $10^{3} \mathrm{kgm}^{-3}$ \\
\hline
\end{tabular}

Table 1: Parameters for the Uncoupled Filament problem (e.g. without Ekman divergence): sea ice pressure scale $P_{0}$, across- and along-filament ocean velocities $U, V$, filament width $L$, linear drag coefficient $\Gamma_{d}$, characteristic sea ice thickness $h$, and water density $\rho_{w}$.

We can find scalings for the variables $t$ and $C$ by using the equations 2.10. In order for the concentration equation for $\mathrm{C}$ to have leading order time dependence, then time time must scale advectively with $v$, e.g.

$$
t=\hat{t} Y V^{-1} .
$$

In steady state, it must be the case that $v=0$. This means that any steady state momentum balance in the $\mathrm{y}$ direction must be between the pressure term and the drag term, e.g.

$$
C\left(v^{w}-v\right)=\frac{h P_{0}}{\rho_{w} \Gamma_{d}} \partial_{y} C^{\alpha} .
$$

This yields an intrinsic scale for $C$;

$$
\begin{aligned}
& \mathcal{C} \sim\left(V Y \frac{\rho_{w} \Gamma_{d}}{h P_{0}}\right)^{\frac{1}{\alpha-1}} \\
& \mathcal{C} \sim\left(10^{-4}\right)^{\frac{1}{\alpha-1}} .
\end{aligned}
$$

This is an intrinsic concentration scale that we use to scale the concentration $C=\mathcal{C} \hat{C}$. If the concentration scale departs greatly from this scale then no steady state balance will be possible. In the limit of low concentration (free ice drift), this corresponds to insufficient ice to provide collisions or a resistive force against the underlying ocean currents. In the limit of high concentrations (packed ice) this represents ice that is unable to be significantly influenced by the ocean currents. For convenience, we include the non-dimensionalized equations here, ignoring the hat symbol hereafter:

$$
\begin{aligned}
\partial_{t} C & =-\partial_{y}(v C,) \\
0 & =-C\left(v-v_{w}\right)-\partial_{y} C^{\alpha}+\lambda \partial_{y}^{2} v \\
0 & =-C\left(u-u_{w}\right)+\lambda \partial_{y}^{2} u .
\end{aligned}
$$

\section{Sea Ice Dynamics Over Strongly Converging Cyclonic Filaments}

\subsection{Model formulation}

The geometry of the problem is summarized in figure 3.1. If we assume that there is 


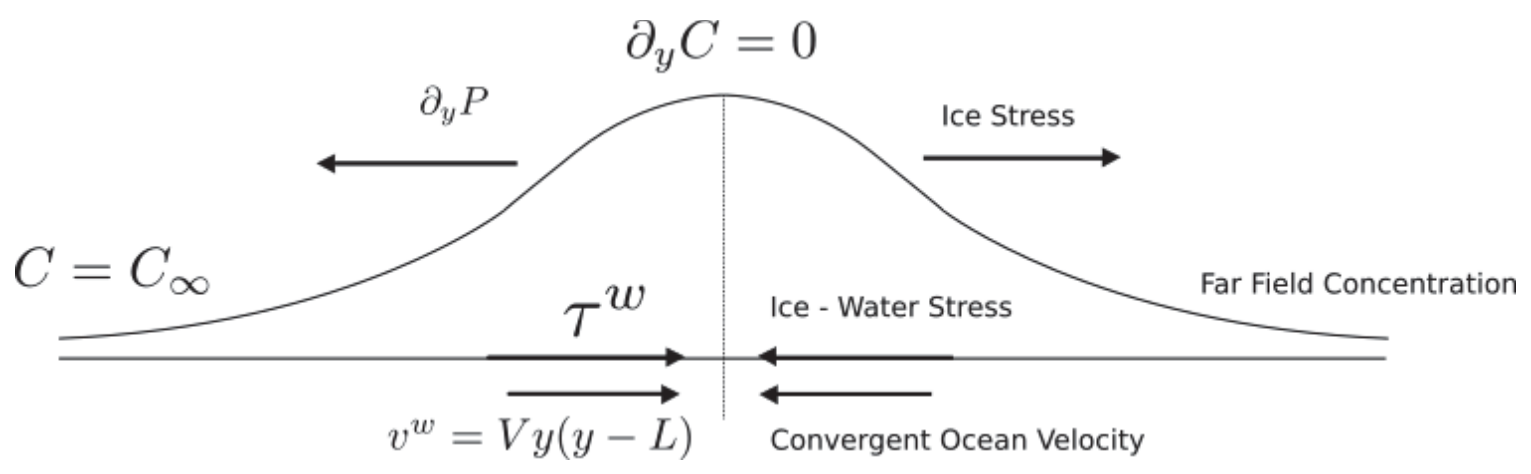

Figure 3.1: Schematic of the of the various forces on acting on the sea ice, the concentration profile that is produced (top line), and the boundary conditions.

no Ekman velocity, and further ignore the viscous term in the $y$ momentum equation (see appendix), we find that the equations become

$$
\begin{aligned}
\partial_{t} C+\partial_{y}(v C) & =0 \\
0 & =C\left(v^{w}-v\right)+\partial_{y} C^{\alpha} .
\end{aligned}
$$

Doing this allows us to write a single PDE for the concentration (in terms of the dimensionless variables):

$$
\partial_{t} C=-\partial_{y}\left(v_{w} C\right)+\partial_{y}^{2} C^{\alpha}
$$

We assume that the filament is symmetric on the domain $y \in[-1,1]$, so that we don't expect there to be any diffusive flux through the center of the domain. Hence we simulate over $0 \leq y \leq 1$ with a symmetry condition

$$
\partial_{y} C(y=0)=0
$$

Outside of the domain we assume that there are processes unrelated to the filament that maintain the concentrations at some far-field value $C_{\infty}$. This leads us to impose the condition

$$
C(y=1)=C_{\infty}
$$

In ongoing work we have also done some analysis using a no flux condition at the outer boundary, although these results will be described elsewhere. We also assume that initially the entire domain is at the far field concentration before the eddy begins applying a stress to the ice, so that

$$
C(t=0)=C_{\infty} .
$$

The filament is assumed to have a parabolic structure in the ageostrophic direction, so that in dimensionless form the velocity is

$$
v^{w}=y(y-1)
$$




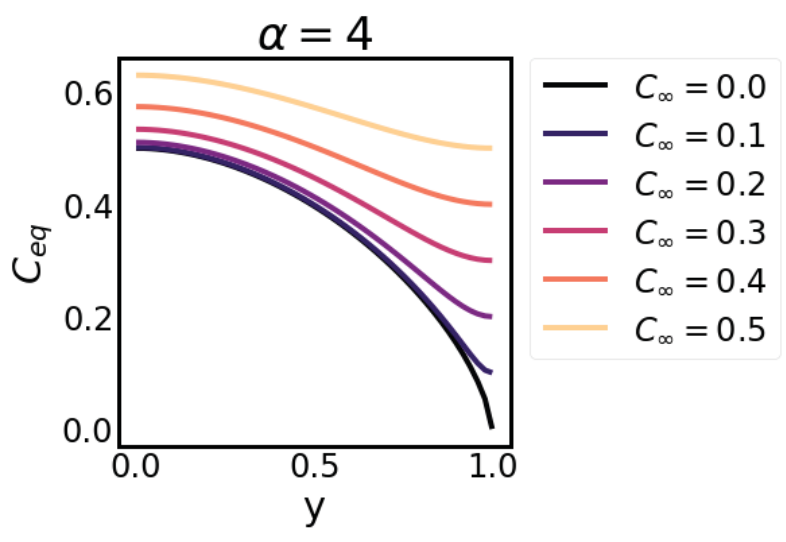

Figure 3.2: Solutions to equation (3.7), for different values of $\alpha$ and $C_{\infty}$.

This form is chosen because it satisfies $v(0)=v(1)=0$ and permits simple analytical steady state solutions.

Equations (3.2)-(3.6) define the system describing the evolution of the sea ice field over the ocean filament.

\subsection{Steady state solutions}

We can find the steady state solution by integrating (3.1)

$$
\begin{aligned}
0 & =-\frac{d}{d y}\left(C v^{w}-\frac{d}{d y} C^{\alpha}\right) \\
\int_{1}^{y} v^{w} d y & =\int_{1}^{y} \alpha C^{\alpha-1} d C \\
C & =\left[\frac{\alpha-1}{\alpha} \int_{1}^{y} v^{w} d y+C(1)^{\alpha-1}\right]^{\frac{1}{\alpha-1}} \\
& =\left[\frac{\alpha-1}{\alpha}\left(\frac{y^{3}-1}{3}-\frac{y^{2}-1}{2}\right)+C(1)^{\alpha-1}\right]^{\frac{1}{\alpha-1}}
\end{aligned}
$$

These solutions are plotted in figure (3.2) for various choices of $\alpha$ and $C(1)=C_{\infty}$. The results show that the solution tends to flatten when $C_{\infty}$ is increased. This is consistent with our physical intuition that when the far-field concentration is increased, the diffusivity is increased, and so the solution becomes flatter.

\subsection{Transient solutions}

We use a simple numerical scheme to simulate the transient evolution to equation 3.2. The advective term is discretized with an upwind advection scheme, and the diffusive term is solved using a second order difference:

$$
\partial_{t} C^{i}=\frac{C^{i-1} v_{w}^{i-1}-C^{i} v_{w}^{i}}{\Delta y}+\frac{\left(C^{i+1}\right)^{\alpha}-2\left(C^{i}\right)^{\alpha}+\left(C^{i-1}\right)^{\alpha}}{(\Delta y)^{2}} .
$$



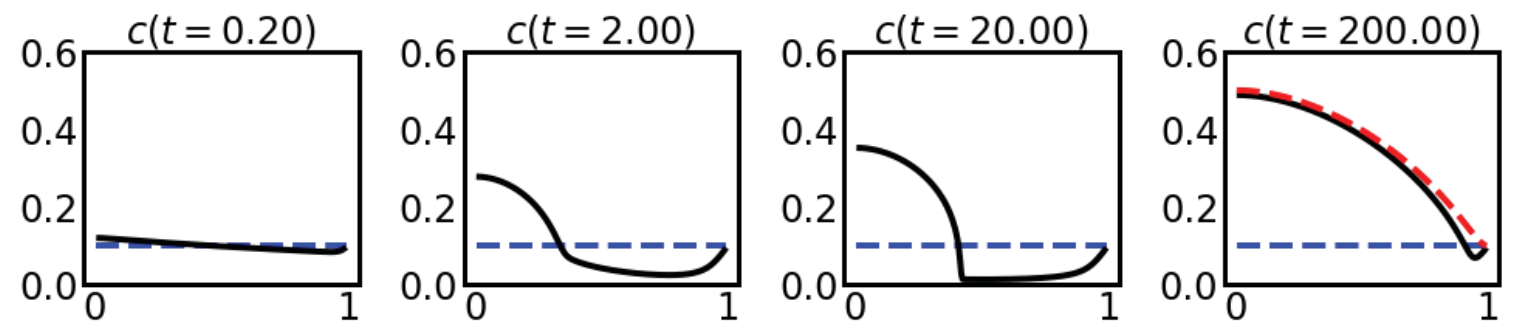

Figure 3.3: Transient evolution of the sea ice concentrations over the uncoupled filament for several exponentially sampled times showing the different stages of evolution. The blue dashed line is the initial condition. The last panel also shows the steady state solution (red dashed line).

The time stepping is done using the integrate.odeint routine which is part of the SciPy package (http://www.scipy.org/). The exact details of the scheme are unimportant, but it includes both implicit and explicit methods; deciding between an implicit or explicit method with substepping depending on the stiffness of the problem.

The transient solution to equations $(2.10) C_{\infty}=0.1$ is shown in figure 3.3. The solution shows that initially the system responds advectively until close to $t=2$. This is because initially $\partial_{y} C=0$, and so there is no diffusion in the system. The result of this early time evolution is shown in the $C(t=2)$ panel; there is a buildup of concentration in the center of the domain (near $y=0$ ), a region of near zero concentrations (between $y=0.5$ and $y=1.0$ in the $C(t=2)$ panel), and a region near $\mathrm{y}=1$ which connects the low-concentration region to the boundary condition $C(1)=C_{\infty}$. This boundary condition has a profound consequence for solutions of our equations. Since there is a non-zero flux through the right hand boundary, the total concentration

$$
M=\int_{0}^{1} C d y
$$

is not constant in time.

We can think of the evolution of the system as having three distinct stages, an initially advective state where the concentration is redistributed in the system, then an adjustment to equilibrium as $M$ grows larger, and then an approach to the final equilibrium stage (shown in the panel of figure 3.3 corresponding to $C(t=200)$ ). These different stages can be visualized by looking at $C(y=0)$ and $M$ as functions of time, shown in figure 3.4. The initial advective stage happens in the first few time units, and is shown by a rapid change in $C(y=0)$. This is shown in the $t=2$ panel. Following this, there is a quasistatic adjustment to the final state, whereby the the central built-up region slowly grows to the edge of the domain due to the flux through the boundary. This happens between approximately times $t=5$ and $t=200$. The final adjustment to equilibrium occurs with very little change in either $C(y=0)$ or $M$. 

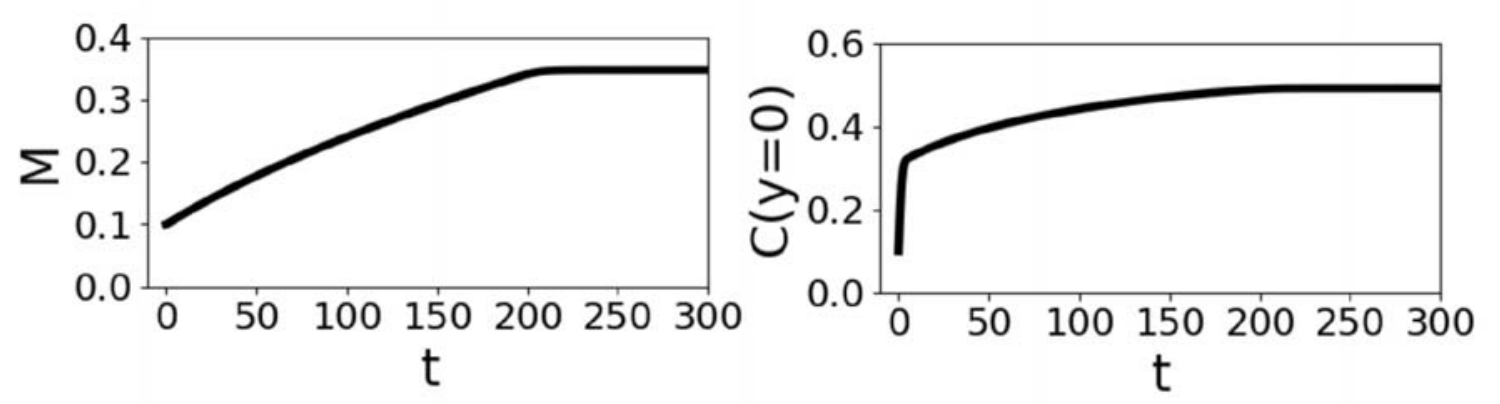

Figure 3.4: The integrated concentration over the domain $M=\int_{0}^{1} C d y$ and the concentration in the center of the filament over the entire simulation, $C(y=0)$.

\subsection{Asymptotic structure}

We can understand the slow evolution towards equilibrium by analyzing the concentration equation

$$
\partial_{t} C=-\partial_{y} q=\partial_{y} A-\partial_{y} D
$$

where $A=-C v_{w}$ is the advective component ; $D=-\partial_{y} C^{\alpha}$ is the diffusive component; and $q=A-D$ is the total flux of concentration. This is shown in figure 3.5 for a single time in the experiment shown in figure 3.3. We define two lengths: $y=L$ is defined as the point where there is a minimum in $\partial_{y} C$, and $y=\delta$ is defined as the point in the outer region where $A(\delta)=D(\delta)$. These lines are shown in figure 3.5, and can be used to split the domain into three regions.

The inner region, $0 \leq y \leq L$ contains high concentrations. Since the adjective and diffusive flux are close to balancing, the concentrations are changing slowly, and so the profile inside this region can be approximated with the steady state solution. By symmetry, $\partial_{y} C(0)=0$, and if we make the approximation that $C(L)=0$ (e.g. so that the flux at $y=L$ vanishes) we can solve the steady state profile in the region $y \in[0, L]$ up to a choice of constant, equivalent to a choice of $C(0)$. We can choose this constant by requiring the total mass $\mathrm{M}$ to match the $\mathrm{M}$ from the numerical simulation. This solution is shown as a dashed redline in the upper left panel of 3.5. This is the quasi-steady approximation: at any given time the concentration profile inside this region can be approximated by knowing only the total mass. In this region the fluxes are large, and diffusion can balance advection since the concentration is large. The small residual balance between $\mathrm{A}$ and $\mathrm{D}$ is what allows the built-up region to accrue mass in time.

In the outer region, $\delta \leq y \leq 1$, the diffusive flux dominates (by definition). The reason for this is $v_{w} \rightarrow 0$ as $y \rightarrow 0$, but there is no constraint on the diffusive flux. Since $C(1)=C_{\infty}$, and $\partial_{y} C(1) \neq 0$, there is a diffusive flux into the domain and a diffusive boundary layer. Immediately away from the outer boundary $v_{w}$ increases and removes sea ice from the diffusive boundary layer. Since $D \sim C^{\alpha}$, this decreases the diffusivity and limits the size of the boundary layer. 

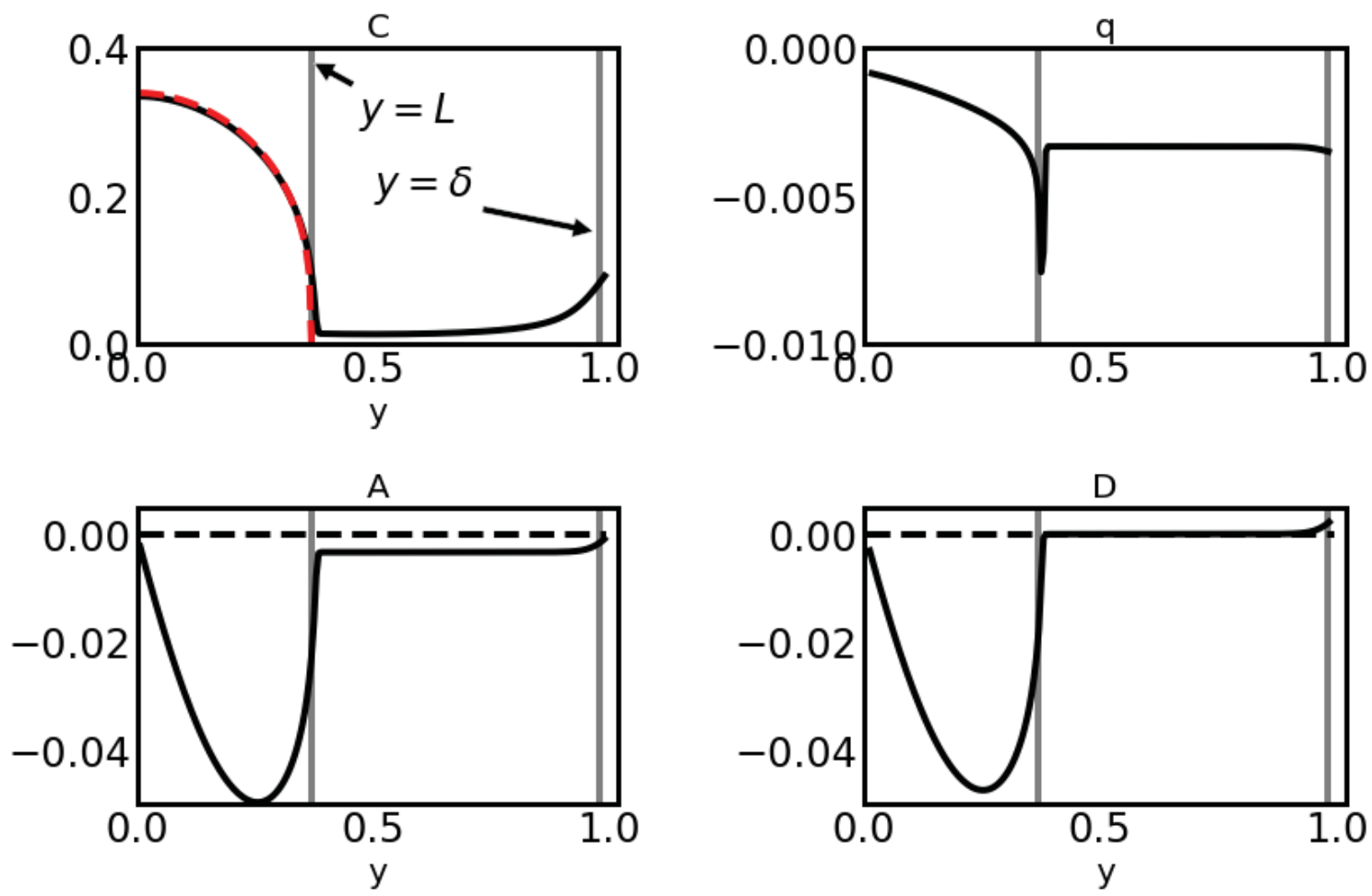

Figure 3.5: The concentration (top left), concentration flux $q$ (top right), advective flux A (bottom left) and the diffusive flux D (bottom right) taken at $\mathrm{t}=10$ from the experiment shown in figures 3.3 and 3.4. The red dashed line in the top plot is the steady state solution described in the text. The two grey lines show $y=L$ and $y=\delta$

In the middle region, $L \leq y \leq \delta$, the concentrations are very small, and since $D / A \sim C^{\alpha-1}$, the flux of concentration $q$ is dominated by advection. The advection effectively transfers sea ice from the outer region to inner region without diffusive losses, since the concentrations are low. There is a sharp discontinuity at the left boundary of this region, $y=L$. This is where the regions join, and there is a large growth right at the boundary of the built-up region, which indicates the expansion of the built-up region towards the outer boundary.

Taken together, these observations give an explanation for how the outer boundary fluxes drive the expansion of the central built-up region towards the outer boundary. In the outer region the diffusive flux puts sea ice into the domain, but the advection removes sea ice from this region and advects it through the middle region into the edge, $y=L$ of the central built-up region. 


\subsection{A heuristic theory for $L$}

Based on the observations in the previous section we can develop a theory for the position of $L$. We start by assuming that the total mass $M=\int_{0}^{L} C(y) d y$ is given. If we approximate $C(L) \approx 0$, then we can calculate $L$ from $M$, assuming that the system is in a quasi steady state. This gives

$$
\frac{d L}{d t}=\frac{\partial L}{\partial M} \frac{d M}{d t}(L)=\frac{\partial L}{\partial M} q(\delta) .
$$

The last line comes from the observation that $d M / d t(L) \approx q(\delta)$ since $q$ does not vary over the interior region. The term $\partial L / \partial M$ can be determined numerically from the steady state solutions. To complete a theory for $d L / d t$, all we need is a theory for $q(\delta)$.

To determine $q(\delta)$, we start by assuming that $\delta$ is quite small, so we can write $v^{w} \sim \beta \delta$ where $\beta$ is a shear scale. The advective and diffusive fluxes scale like

$$
\begin{aligned}
& A=v^{w} C \sim \beta \delta C_{\infty}, \\
& D=\frac{\partial}{\partial y} C^{\alpha} \sim \delta^{-1} C_{\infty}^{\alpha},
\end{aligned}
$$

and since this is the length scale where $A=D$, we can solve for $\delta$, and so find

$$
q \sim \beta^{\frac{1}{2}} C_{\infty}^{\frac{\alpha+1}{2}} .
$$

We can plot this scaling by doing simulations over a wide range of $V$ and $C_{\infty}$, as shown in figure 3.6(a). There is generally good agreement, but for the larger values of $C_{\infty}$ the values deviate from the prediction. This is not unexpected since for larger $C_{\infty}$ the inner advective region is not able to develop since the concentrations never become small enough.

We can also use the scaling of (3.11) and (3.9) to propagate $L$ in time. At later times the estimate for $L$ diverges from the value determined by simulating the full system. This is because as the edge of the buildup region approaches the edge of the domain, the scaling theory ceases to apply since there is no longer a clear advective region with small $C$. The numerical estimate of $\mathrm{L}$ also shows some step-like behaviour. This is due to the finite size used for the computational grid.

\subsection{Summary}

When a parabolic velocity is used, the uncoupled filament equations can be solved for both the steady state as well as for the transient case. The steady state solutions show a strong dependence on the outer boundary concentration condition. The transient solution shows two different timescales. The first is a short advective timescale, which ends when the system is split into three regions; an inner region of high concentrations, a middle region with very low concentrations and an outer diffusive boundary layer. The approach to equilibrium from this state takes a long time because the fluxes from the diffusive boundary layer are limited through the middle region. 


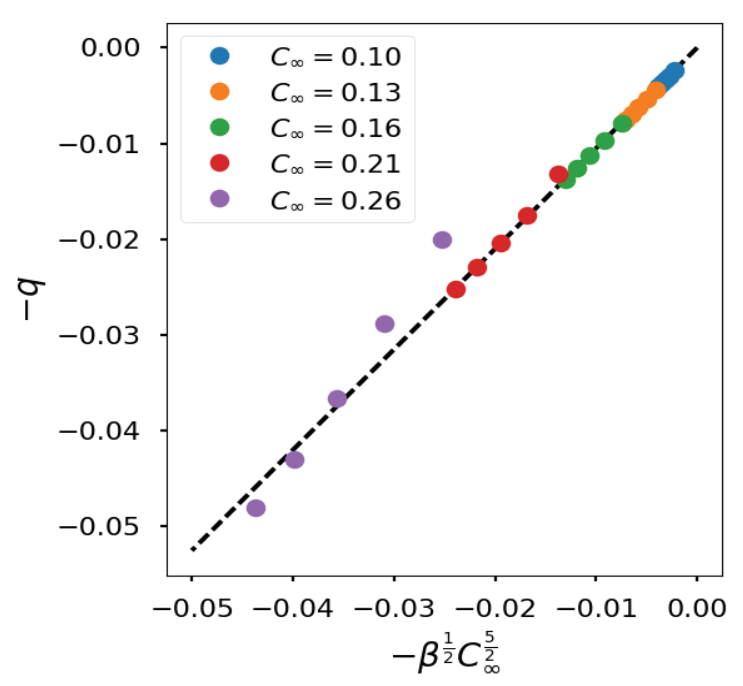

Figure 3.6: (a) Scaling of q given in equation (3.11). (b) $L(t)$ calculated from (3.9) and the numerical solution.

\section{Coupled Filament}

For a balanced filament or eddy, there will, in general, be no secondary circulation supplied by the ocean that can create convergent motions in the sea ice. The presence of sea ice introduces an additional stress into the system that couples the ice and ocean together by creating an Ekman flow in the surface layer of the ocean. In this section we ask whether this added flow can induce convergent motion of the sea ice, and, if so, what properties does the convergence have that are different from the uncoupled filament? In section 4.1 we introduce the equations, and in section 4.2 we consider some transient solutions. The geometry of the problem is summarized in figure 4.1.

\subsection{Equations}

For convenience we rewrite equation (2.10) with $v_{w}=0$ but with an Ekman velocity $v_{E}$ (in the non-dimensionalized variables):

$$
\begin{array}{ccc}
\partial_{t} C & = & -\partial_{y}(v C) \\
0 & = & -C\left(v-v_{E}\right)-\partial_{y} C^{\alpha} \\
0 & = & -C\left(u-u_{w}\right)+\lambda \partial_{y}^{2} u
\end{array}
$$

where once again we ignore the viscous stress in the equation for $v$. For the purposes of this work, we treat the case of a filament using cartesian coordinates (rather than working in polar coordinates as would be required for a circular eddy). We assume that $v_{E}$ can be taken as an average over the depth $D$ of the Ekman layer. Assuming that in this layer the surface stress from the ice balances a Coriolis force, the stress 

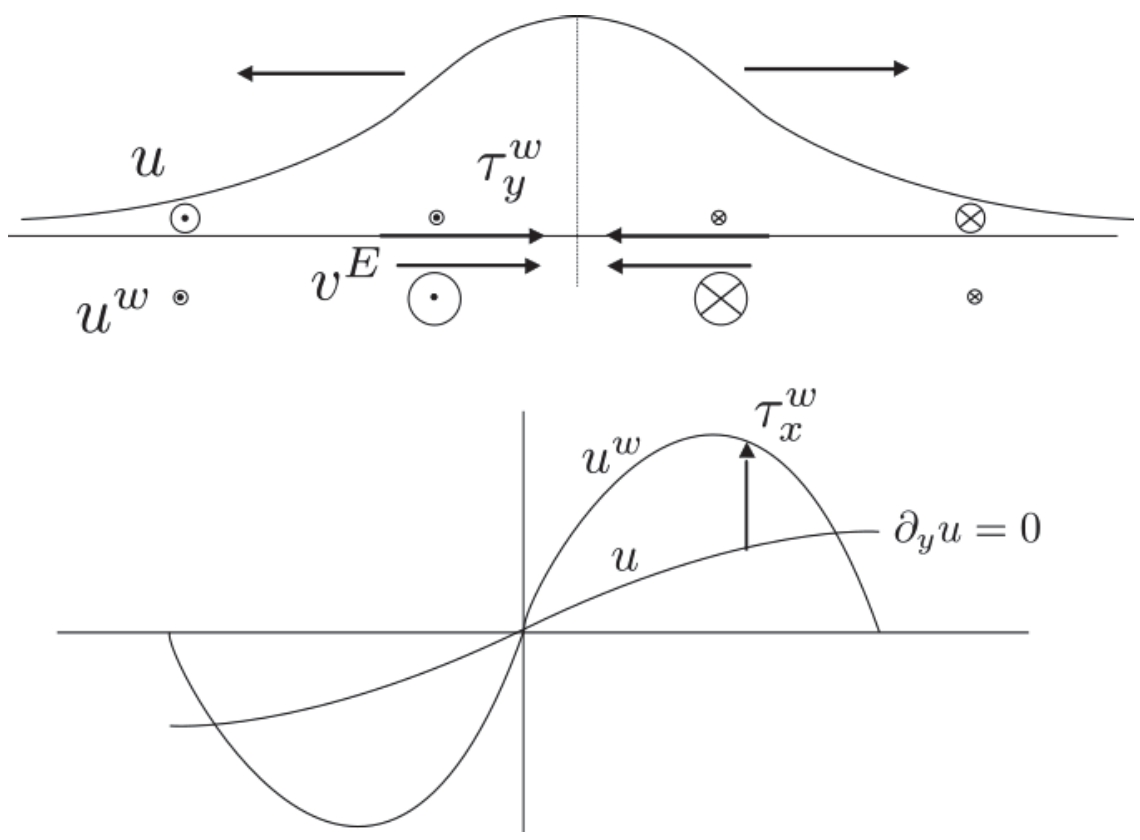

Figure 4.1: Schematic of the of the various forces on acting on the sea ice and the concentration profile (similar to figure 3.1, in a plane (top) and bird's eye (bottom) views. The bottom figure also contains the along filament velocity profile.

in the across frontal direction is

$$
\tau_{w, x}=-\rho_{w} f D v_{E}
$$

Since the drag in the across frontal direction is

$$
\tau_{w, x}=C\left(u-\underline{u}_{w}\right)
$$

we can write the Ekman velocity in non-dimensional variables as

$$
v_{E}=C\left(u-u_{w}\right)
$$

where we have rescaled $V$ to be $V=U C \Gamma / f D$. Note that this velocity scale is very different than in the previous section where we assumed that this velocity scale was supplied by the imposed ocean velocity. We model the geostrophic water velocity $u_{w}$ in a similar way to the ageostrophic component $v_{w}$ in the previous section and choose

$$
u_{w}=y(y-1),
$$

in non-dimensional variables.

The addition of equation (4.2) couples the momentum balances in the $x$ and $y$ directions, and hence changes the nature of solving the system in equation (4.1). Before, the equation for the geostrophic velocity $u$ could be ignored in solving the 

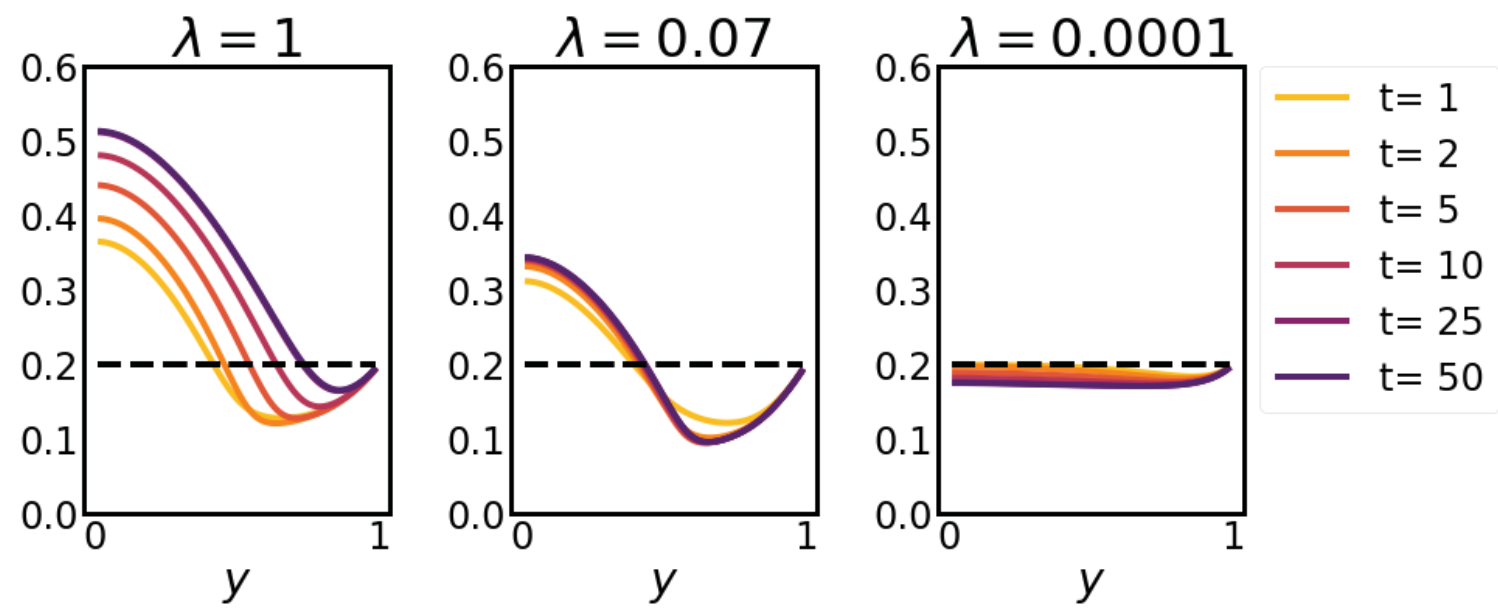

Figure 4.2: $C$ with 3 different values of $\lambda$, sampled at various times. The dashed lines show the value of $C_{\infty}$ for that experiment, 0.2 .

rest of the system. We now instead have to solve a boundary value problem to find $u$, which in turn can be used with $C$ to find $v_{E}$. Once $v_{E}$ is found, this allows $v$ to be determined and used to advance $C$ to a new time step, using the procedure described in the previous section.

Since we now have to solve for $u$, we have to introduce boundary conditions. We choose

$$
u(0)=0
$$

on the basis of symmetry, and

$$
\partial_{y} u(1)=0,
$$

equivalent to a no-stress boundary at the edge of the domain. If we did not impose the second condition, then there would be a viscous stress at the edge of the domain that could change the sea ice field outside the domain. Similar to the previous section we choose the boundary condition so that the motion of sea ice inside the domain does not impact outside of the domain.

\subsection{Transient solutions}

We solve the transient solutions to the system posed in the previous section using a method similar to that in section 3. Now, however, we have to solve the boundary problem associated with $u$ in order to determine $v_{E}$ before we can timestep $C$. We do this by using the solve_BC package in the scipy library (http://www.scipy.org/). Briefly, the package uses a collocation method with a cubic spline interpolation of $C$.

Figure 4.2 shows $C$ for several times and 3 different parameter values of $\lambda$. All of the simulations were run for 50 time units. For the smallest value of $\lambda$, the total mass

of sea ice in the domain decreases by approximately $25 \%$ over the simulation and the 

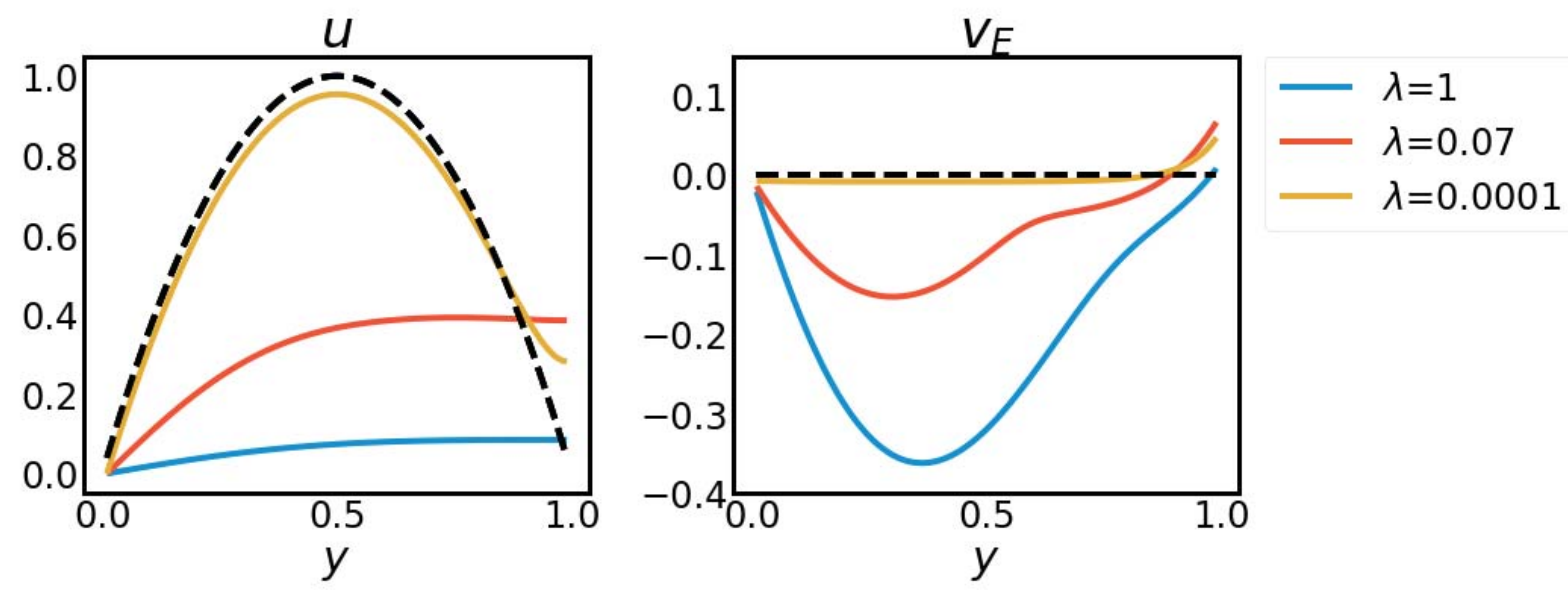

Figure 4.3: $u$ left and $v_{E}$ right sampled at the end of the experiment show in figure 4.2. In the left figure the dashed black line is the ocean velocity $u_{W}$, and in the right figure it is $v_{E}$.

profile becomes almost flat. For the largest value of $\lambda$, the total mass increases by approximately $60 \%$ and the built-up region is able to extend over almost the entire domain. The intermediate value of $\lambda$ keeps the total mass of ice almost constant over the domain, and has a structure similar to the uncoupled case at short times.

To understand why $\lambda$ has this control on the total mass and concentration profile inside the domain, we examine profiles of $u$ and $v_{E}$ taken from the end of the simulation (figure 4.3). In all cases we see that the ice velocity is faster than the ocean velocity near the outer boundary of the domain. The viscous stress is able to spread momentum throughout the domain, and since the outer boundary condition is $\partial_{y} u=0$, the momentum is not completely removed from the ice and the ice moves faster than the ocean. By contrast, the inner boundary condition is $u=0$, and so there is a momentum sink.

For the largest value of $\lambda$ the ice velocity is very close to 0 , and for the smallest $\lambda$ the ice velocity is very close to the ocean velocity. This matches our intuition, since for a very small viscosity the ice has little viscous resistance and should be moving with the ocean, and for a very large viscosity all of the momentum is transferred to the momentum sink at $y=0$.

Since $v_{E} \propto\left(u-u_{W}\right)$, the point where $u$ and $u_{W}$ cross separates $v_{E}$ into a positive and negative region. Near the outer boundary $u>u_{W}$, and so the Ekman transport is actually exporting ice from the eddy into the far field. In the interior of the domain the ice transport is towards the center, and the velocity vanishes at the inner boundary since $u=u_{W}=0$. This means that for the largest viscosity there is only a small export of ice near the outer boundary and a strong Ekman transport in the interior. For the smallest viscosity there is a strong export of ice near the outer boundary and a weak convergence in the interior of the domain (since $u \approx u_{w}$ there). For 
the intermediate case the structure of the Ekman transport is not unlike $v_{w}$ in the uncoupled case.

These differences explain why some parameter values of $\lambda$ accrue ice in the domain, and others reduce the amount of ice in the domain. For small values of $\lambda$, the convergence of $v_{E}$ is not very strong, and so the system loses some ice mass. This mass loss is eventually stopped because of the diffusive flux across the boundary. For large values of $\lambda$, the convergence into the center of the domain is quite strong, and the mass is able to accrue in the center of the domain.

\subsection{Summary}

The viscous stresses in the ice redistribute momentum in the along-filament direction in such a way that ice near the inner boundary moves slower than the ocean underneath, while the ice near the outer boundary moves faster than the ocean. This creates an Ekman transport that exports sea ice out of the filament near the outer boundary, and pushes sea ice towards the center in the rest of the domain. Strongly viscous sea ice is able to create large Ekman transports that accrue ice over top of the filament because of the large difference between the ice and water velocities. Weakly viscous sea ice is only able to create a weakly convergent Ekman transport and exports ice into the far field. This implies that understanding sea ice rheology in this regime will be important for constraining the ability of eddies and filaments to self-accrue ice.

\section{$5 \quad$ Ramifications for Estimating Surface Ocean Conver- gences from Sea Ice Concentrations}

The previous two sections have discussed two one-dimensional models of sea ice and ocean interaction. In this section we discuss the implications of these models for doing inversions to determine upper ocean velocity from sea ice concentration fields. The main challenges of doing inversions from single sea ice images are:

1. Is there a large difference between $C(0)$ and $C_{\infty}$ ? If not, the concentration buildup will not be detectable from the background.

2. Does the system reach an equilibrium on a timescale similar to or shorter than the persistence timescale of ocean flows (approximately 5 days)? If not, then we need time dependent information to properly constrain the flow and so will not be able to do so from a single image.

Here we try to recast the results from previous sections in terms of these questions and try to provide some heuristic criteria about which conditions will be suitable for inversions. 

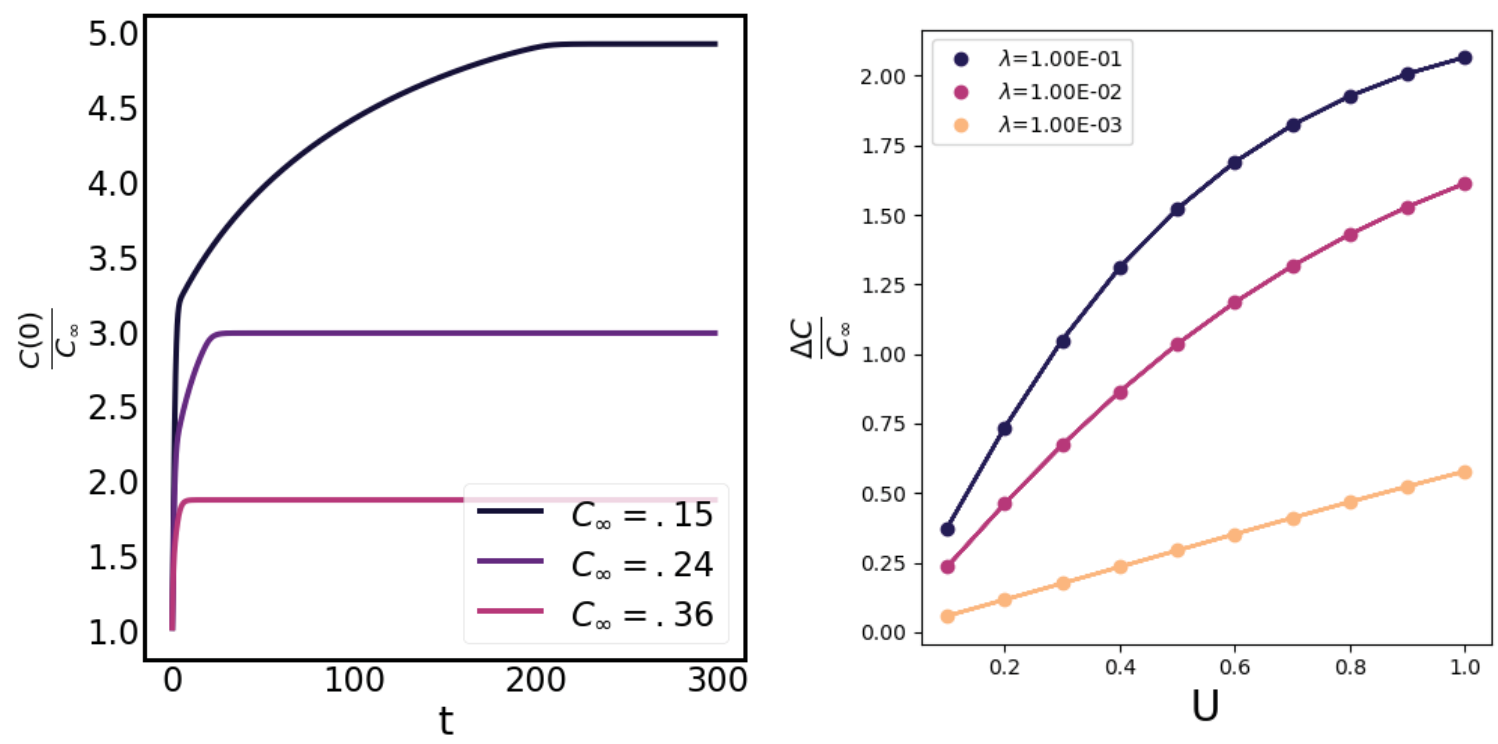

Figure 5.1: (a) Time series of the signal-to-background ratio $C(0) / C_{\infty}$ for different values of $C_{\infty}$, from the uncoupled model. (b) $\left[C(0)-C_{\infty}\right] / C_{\infty}$ for different values of $\lambda$ and the forcing velocity scale $(U)$ after 5 time units $(\sim 5.8$ days). for the coupled model

\subsection{Timescales for uncoupled inversions}

To answer the above questions we compute the signal-to-background ratio $C(0) / C_{\infty}$, which quantifies the concentration buildup in the center of the domain compared with the outer boundary. This is shown for several boundary conditions in figure 5.1. Two timescales are clearly visible: there is a sharp increase for $t<1$, and then a second longer timescale with a smaller increase. For large values of $C_{\infty}$ there is a small signal-to-background ratio over the entire time. This means that the steady state solution will be a good approximation to the time-dependent solution, since the period of transience is relatively small and the solution quickly converges to the steady state.

These results yield two rules of thumb:

1. Low background concentrations give higher signal-to-background ratios, which means that inversions will be able to be computed more accurately. This also means that packed ice ( $100 \%$ concentration) are unlikely to be invertible, even without taking account of any plastic part of the rheology [4].

2. The fast time scale of the sea ice adjustment means that the ice concentration will be approximately in balance with the ocean forcing on short (5-10 day time scales). 


\subsection{Timescales for coupled inversions}

For the coupled system, the solutions evolve in a much more complicated manner compared with the uncoupled system. If there were a non-monotonic relationship between the strength of the forcing velocity $U$ and the other system parameters, then the system will no longer be invertible. We test whether or not this in the case in figure 5.1 by calculating the enhancement of the sea ice concentration over the center of the filament for various $U$ and $\lambda$. We find a monotonic relationship between the enhancement and the scale of the forcing velocity when $\lambda$ is fixed, although the relationship is strongest for the stronger sea ice.

This suggest the following simple observations:

1. Higher viscosity allows a stronger coupling between the ocean currents and the sea ice concentrations.

2. There is an approximately monotonic relationship between the scaling of the ocean velocity and the enhancement of sea ice above.

Together, these results suggest that it may be possible to invert submesoscale currents from observations of ice concentration. Note that this result was not guaranteed, nor obvious a priori. There are many ways that this model could have failed to validate the hypothesis that inversions would be possible. The adjustment timescales between the ice and the ocean could have been too different, or the enhancement of sea ice in the center of the domain could have been too low to be meaningful. However our results suggest that the fast adjustment timescale associated with advection of low concentration sea ice allows ocean forcing to enhance sea ice concentration over convergent ocean currents. Future studies could analyze models with more realistic rheologies, or attempt simple inversion methods on data from realistic ice-ocean circulation models.

\section{Conclusions}

In the marginal ice zone, ocean filaments are able to accrue significant sea ice. In this study we have considered two idealized test cases. We first considered an uncoupled filament, where the surface ocean currents create a convergence of ice over the filament. We find that there are two timescales. The first is a short advective timescale that is able to accumulate sea ice into a built-up region over the filament in a few days. The second timescale can take several hundred days, and is related to a diffusive boundary layer (where the diffusion is controlled by internal ice stresses providing a driving force to redistribute ice mass). We have developed a simple theory to calculate the scaling of the diffusive flux and shown how it can be used to propagate the boundary of the built-up region near the center where the sea ice concentration is in quasi-steady equilibrium. 
We also considered a coupled filament, where we assume that the surface currents are non-divergent, but the ocean boundary layer is coupled to the ice stress through Ekman transport. The viscous stress spreads ice velocity in the along-filament direction so that the ice near the outer boundary of the filament is moving faster than the ocean underneath. This results in an Ekman transport that exports sea ice through the outer boundary but also has a convergent section pushing sea ice towards the inner section. We show that in this case the ability of the filament to accumulate sea ice is strongly dependent on the strength of the ice viscosity. When the ice viscosity parameter is very large, there are strong convergent velocities at the ocean surface. When the ice viscosity parameter is very small, the convergent velocity is small. This idealized study shows that both the uncoupled and coupled filaments are able to accumulate significant amounts of sea ice on oceanographically relevant timescales. These results suggest that ocean turbulence statistics can be inferred from still satellite images of sea ice in marginal ice zones.

\section{Appendix: justification for ignoring the viscous term in the uncoupled filament}

The Hibler model [4] assumes that the viscosity is

$$
\nu=\max ,\left(\frac{1}{E^{2}} \frac{P}{\max \left(e_{\min },|\underline{e}|\right)}, \nu_{\min }\right)
$$

where

$$
|\underline{e}|=\frac{1}{\alpha} \sqrt{2 \operatorname{tr}(\underline{e} \cdot \underline{e})+\left(E^{2}-1\right)[\operatorname{tr}(\underline{e})]^{2}}
$$

is the strain invariant associated with an elliptical yield curve of eccentricity $E=4$, with the strain rate tensor $e_{i j}=\frac{1}{2}\left(\partial_{i} u_{j}+\partial_{j} u_{i}\right)$.

If we assume that the geostrophic velocity is larger than the ageostrophic velocity, but that both vary over the same spatial scale, then we find that

$$
|\underline{e}| \sim \frac{U}{L}
$$

For the purposes of this scaling, we will be interested in only the part of the viscosity that does not involve $e_{\min }$ and $\nu_{\min }$. We ignore $\nu_{\min }$ because we are interested in the largest values of $\nu$ and ignore $e_{\text {min }}$ because this value is much smaller (by a factor of $10^{6}$ ) than $U / L$ (from the parameters listed in table 1 ).

The viscosity then scales like

$$
\nu \sim \frac{P L}{U}
$$

so the ratio of the pressure gradient force to the viscous force in the along-filament direction is 


$$
\frac{\partial_{y}\left(\nu \partial_{y} v\right)}{\partial_{y} P} \sim \frac{V}{U}
$$

which we assume to be small.

\section{Acknowledgments}

I would sincerely like to thank Georgy Manucharyan, Andrew Wells, and Sam Pegler for their time and guidance through this project. I would also like to thank MaryLouise Timmermans and Claudia Cenedese for organizing the summer school, and my fellow fellows for their companionship.

\section{References}

[1] E. Abrahamsen, Sustaining observations in the polar oceans, Philosophical Transactions of the Royal Society A, 372 (2014), p. 20130337.

[2] T. W. Armitage, S. Bacon, A. L. Ridout, S. F. Thomas, Y. Aksenov, AND D. J. Wingham, Arctic sea surface height variability and change from satellite radar altimetry and grace, 2003-2014, Journal of Geophysical Research: Oceans, 121 (2016), pp. 4303-4322.

[3] D. L. Feltham, Sea ice rheology, Annual Review of Fluid Mechanics, 40 (2008), pp. $91-112$.

[4] W. Hibler III, A dynamic thermodynamic sea ice model, Journal of Physical Oceanography, 9 (1979), pp. 815-846.

[5] E. Hunke And J. Dukowicz, An elastic-viscous-plastic model for sea ice dynamics, Journal of Physical Oceanography, 27 (1997), pp. 1849-1867.

[6] P. Klein and G. LAPeyre, The oceanic vertical pump induced by mesoscale and submesoscale turbulence, Annual Review of Marine Science, 1 (2009), pp. 351-375.

[7] V. Lytle, R. Massom, A. Worby, And I. Allison, Floe sizes in the east antarctic sea ice zone estimated using combined sar and field data, in Space at the service of our Environment, 1997, pp. 931-936.

[8] G. E. Manucharyan And A. F. Thompson, Submesoscale sea iceocean interactions in marginal ice zones, Journal of Geophysical Research: Oceans, (2017).

[9] J. C. MCWilliams, Submesoscale currents in the ocean, 2016. 
[10] J. A. Mensa And M.-L. Timmermans, Characterizing the seasonal cycle of upper-ocean flows under multi-year sea ice, Ocean Modelling, 113 (2017), pp. $115-130$.

[11] B. Rallabandi, Z. Zheng, M. Winton, and H. A. Stone, Formation of sea ice bridges in narrow straits in response to wind and water stresses, Journal of Geophysical Research: Oceans, (2017).

[12] M. C. Serreze And R. G. Barry, The Arctic climate system, Cambridge University Press, 2014.

[13] M.-L. Timmermans And P. Winsor, Scales of horizontal density structure in the chukchi sea surface layer, Continental Shelf Research, 52 (2013), pp. 39-45.

[14] J. A. WhiteheAd, Dimensions of continents and oceans-water has carved a perfect cistern, Earth and Planetary Science Letters, 467 (2017), pp. 18-29. 


\title{
A Mechanism for Secondary Sea Ice Formation Driven by Double-diffusive Supercooling
}

\author{
Margaret R. Lindeman
}

November 16, 2017

\begin{abstract}
Sea ice forms rapidly in gaps in ice cover, driven by large heat fluxes from the ocean to the atmosphere. The resulting brine rejection forms a cold, salty water mass that sinks and flows away from its source. Here, we present a mechanism for secondary ice formation due to supercooling at the interface between this cold, salty water mass and the relatively fresh mixed layer overlying it. Motivated by Ice-Tethered Profiler (ITP) measurements from the Canada Basin that show the onset and persistence of these conditions, we develop a model for supercooling-driven frazil ice formation that can be generalized to the Arctic or Antarctic. We quantify the contribution of the frazil ice formed through this mechanism to the thickness of the overlying sea ice and the resulting evolution of the mixed layer temperature and salinity fields.
\end{abstract}

\section{Introduction}

\subsection{Motivation}

Sea ice has a key impact on the planetary energy budget, and impacts air-sea exchanges near the poles $[1,16]$. In a changing climate, the fate of sea ice is of critical importance, but complicated dynamics make prediction challenging. Sea ice has long posed an impediment to observing ocean properties in the Arctic. Since the mid-2000s, Ice Tethered Profilers (ITPs) have been deployed to mitigate this challenge [15]. ITPs are anchored in sea ice and periodically measure conductivity, temperature, and depth (CTD) profiles of the underlying water column, producing unprecedented year-round hydrographic observations of the ice-covered Arctic Ocean. These measurements provide insight into ocean circulation under sea ice as well as thermodynamic interactions between the sea ice and ocean [14].

Most sea ice growth is driven directly by local surface cooling where the ocean is 

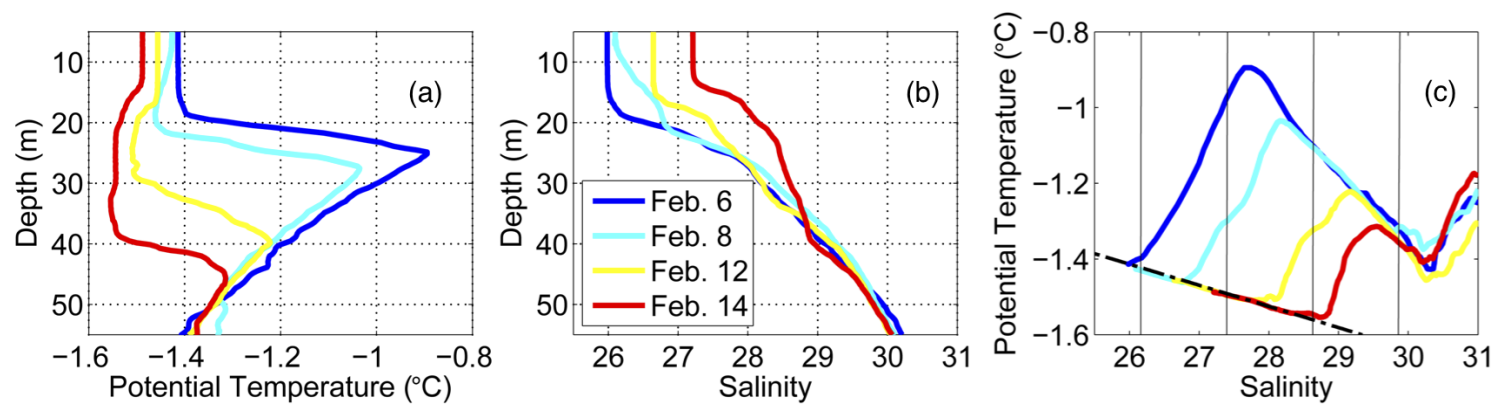

Figure 1: Ice Tethered Profiler observations from the Canada Basin (figure from [13]).

exposed to the atmosphere, such as in polynyas and leads, which are gaps in sea ice cover [8]. As sea ice forms, it excludes salt from its crystal structure, leaving behind very cold, saline water known as brine. The dense brine then sinks until it is neutrally buoyant, and may flow outward from where it formed, intruding beneath the relatively warm and fresh mixed layer insulated by the surrounding sea ice. Some ITP profiles show the arrival of cold, salty water mass at a depth of around $20 \mathrm{~m}$ (e.g. the transition from the dark blue to red lines in figure 1a-b), which is speculated to be brine generated by this primary ice formation process. Due to the salinity dependence of the freezing point, each of these water masses is at its local freezing temperature (figure 1c). Because heat diffuses faster than salt, as the mixed layer loses heat to the underlying brine, it may become supercooled (figure 2). This could result in the nucleation of ice crystals called frazil ice. However, measuring supercooling directly is difficult, because the instrument itself can act as a nucleus for ice formation. It is desirable to develop a model for this mechanism to identify an observable signature of the process and to quantify its potential for secondary frazil ice production.

\subsection{Double-diffusive supercooling}

A number of earlier studies utilized theory and laboratory experiments to investigate similar processes related to double-diffusive supercooling. Notably, Martin and Kaufmann's 1974 experimental setup allowed them to describe a three-phase ice growth process that occurs in under-ice melt ponds [5]. In this situation, the meltwater temperature is $0^{\circ} \mathrm{C}$ and salinity is $0 \mathrm{~g} / \mathrm{kg}$, causing the upper layer to become less dense as it cools (freshwater has a maximum density at $4^{\circ} \mathrm{C}$ ), which drives convective instability. Stigebrandt (1981) similarly developed a theory where convective instability arises in both layers [11]. Voropayev et al. (1995) build on these earlier results, still considering an overlying layer of purely fresh meltwater but adding turbulence to their model to better reproduce observed quantities of frazil production [17]. Observational results are presented in McPhee et al. (2013), who propose a similar supercooling mechanism. Instead of an intrusion of cold brine on a timescale of days to weeks, they observe a tidally-advected salinity front that is speculated to induce double-diffusive supercooling in a fully turbulent water column [7]. 


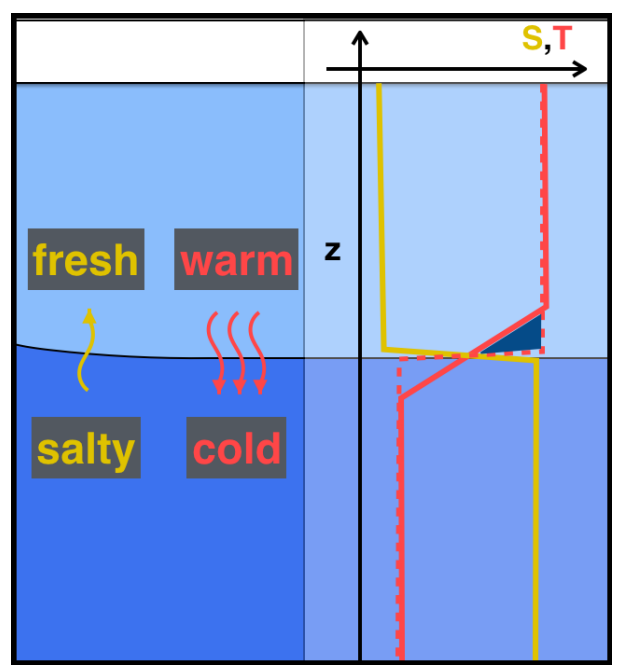

Figure 2: Schematic of double-diffusive supercooling. The initial temperature indicated by the red dashed profile is the salinity- dependent freezing point (right). Because heat diffuses faster than salt (left), after a short time, the temperature in the upper layer has decreased (solid red profile), while the salinity remains at its initial value (yellow profile). The blue triangle represents supercooling of the boundary layer, where the temperature is below the freezing point.

\subsection{Frazil ice}

Jeffries et al. (1995) measured sea ice cores from the Beaufort Sea and found that 9\% of ice thickness was formed from incorporated frazil ice, including layers ranging from $5 \mathrm{~cm}$ to $60 \mathrm{~cm}$ thick [2]. They speculate that most of that ice may be attributed to under-ice melt pond growth (see section 1.2), with a plausible alternative being "ice pumps," analogous to those observed in the Antarctic by Langhorne et al. (2015). Ice pumps arise due to the pressure dependence of the freezing point. In the Ross Sea, this happens where deep ice shelves melt and the buoyant meltwater becomes supercooled as it ascends, producing a platelet ice layer up to about $1 \mathrm{~m}$ thick [4]. Building on these observational studies, modeling studies including Svensson and Omstedt (1994) and Rees Jones and Wells (2017) make significant contributions to understanding the conditions that generate frazil ice and facilitating generalization of supercoolingdriven frazil ice growth and the subsequent dynamics to other situations $[12,10]$.

The aim of this project was to use an idealized model to quantify the parameters affecting the magnitude and persistence of supercooling driven by double-diffusion at the interface between a mixed layer and underlying cold brine water mass, and ultimately quantify the contribution of the resulting frazil ice formation to the thickness of the overlying sea ice. 


\section{$2 \quad$ Model}

\subsection{Model setup}

We use an idealized model of supercooling at the base of a well-mixed under-ice layer to simulate a situation similar to the one seen in ITP observations (e.g. figure 1). See figure 3 for a schematic representation of the model setup. The model simulates mixed layer temperature $T$ and salinity $S$, as well as the number density $N$ and mean radius $\bar{R}$ of ice crystals suspended in the layer. The layer is assumed to be well-mixed, with the temperature and salinity both uniform with depth. The frazil ice crystals are assumed to be disc shaped, with a constant thickness $H$ and variable radius $R$ [6]. The crystals rise through the layer at a velocity proportional to the mean radius, but otherwise there is no flow in the mixed layer.

The initial salinity $S_{0}$ of the mixed layer is chosen based on observations, and its initial temperature $T_{0}$ is the freezing point determined by its salinity. The underlying cold brine layer will be referred to as the reservoir. The reservoir salinity is assumed to be constant and greater than the mixed layer salinity, and its temperature (also constant) is also at the salinity-dependent freezing point, making the reservoir colder than the mixed layer. The freezing point $T_{f}$ is assumed to be approximately independent of pressure (i.e. a function of salinity $S$ ) and calculated as

$$
T_{f}(S)=T_{f}\left(S_{0}\right)+\Gamma\left(S-S_{0}\right)
$$

where $T_{f}(S)$ is the freezing temperature at salinity $S_{0}$ and $\Gamma=-0.06^{\circ} C$ [3]. Mixed layer temperature and salinity are subject to diffusion of heat and salt across the bottom boundary, with diffusivities of heat $\kappa_{T}$ and salt $\kappa_{S}$ acting across a boundary layer of thickness $\delta$. Under molecular diffusion, $\kappa_{T} \gg \kappa_{S}$, but the ratio $\tau=\kappa_{T} / \kappa_{S}$ may vary with the level of turbulence.

\subsection{Model formulation}

To model the ice crystal suspension, we assume a crystal size distribution such that $n(R)$ is the number density of crystals of any given radius $R$, as illustrated in figure 4. The total number density $N$ is defined as

$$
\bar{N}=\int_{0}^{\infty} n \mathrm{~d} R
$$

measured in units of crystal number per unit volume, and the mean radius $\bar{R}$ as

$$
\bar{R}=\frac{1}{N} \int_{0}^{\infty} R n \mathrm{~d} R
$$




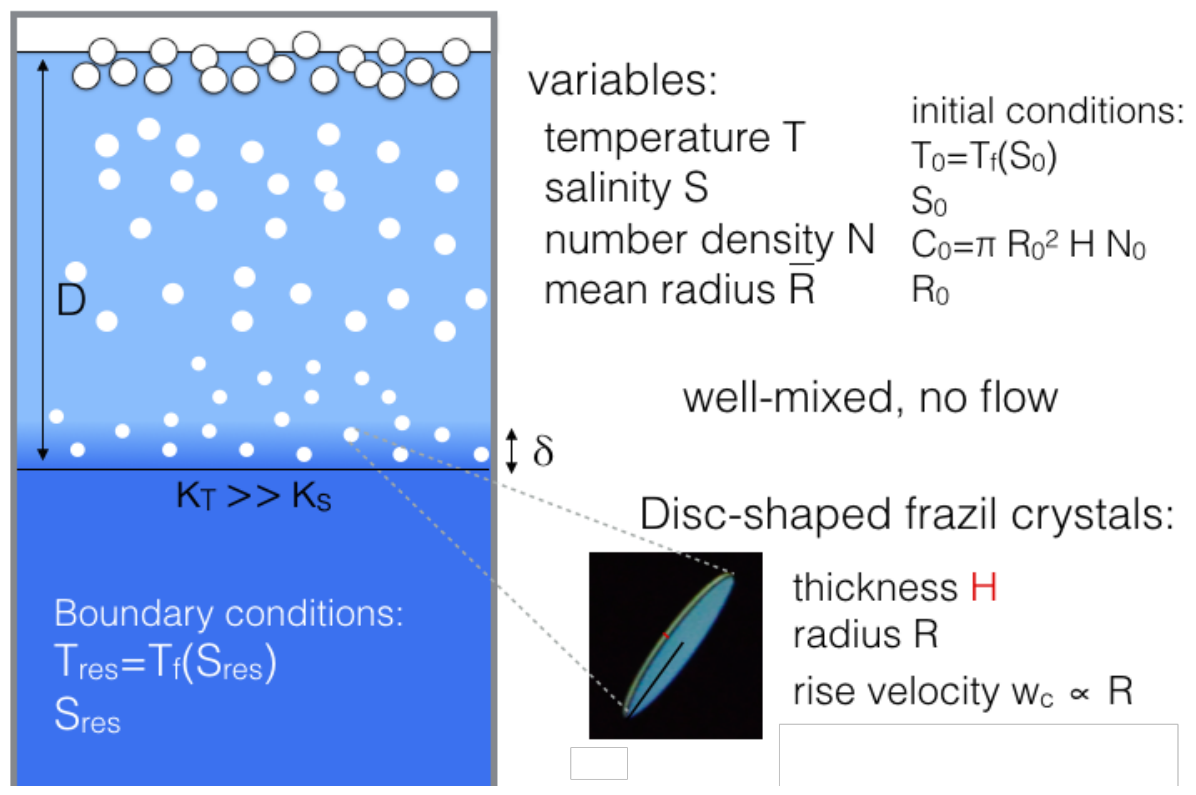

Figure 3: Model schematic and list of variables. Subscript 0 indicates the initial value of a variable. $T_{f}(S)$ is the salinity-dependent freezing point as defined in (1). $C_{0}$ is an initial ice crystal concentration as defined in (10). Ice crystal image from [6].

The rise velocity of disc shaped ice crystals may be parameterized simply with the linear relationship

$$
w_{c}=\gamma R
$$

where the constant $\gamma=16 \mathrm{~s}^{-1}$ is estimated from experimental data $[6,10]$.

The time derivative of $n(R)$ can be formulated relatively simply following [10] as

$$
\frac{\partial n}{\partial t}=-\frac{\partial}{\partial R}(G n)-w_{c} \frac{\partial n}{\partial z}
$$

where $G=G_{0}\left[T_{f}(S)-T\right]$ and the growth constant $G_{0}$ is defined as

$$
G_{0}=\frac{\kappa_{T m} \rho_{w} c_{w}}{\rho_{i} L H}
$$

The first term on the right hand side of (5) is due to crystal growth, which shifts the crystal size distribution $n(R)$ to the right. The second term is the settling of crystals as they rise. In order to model the suspension of ice crystals in a well-mixed layer, we integrate (5) with respect to $R$ and $z$. 


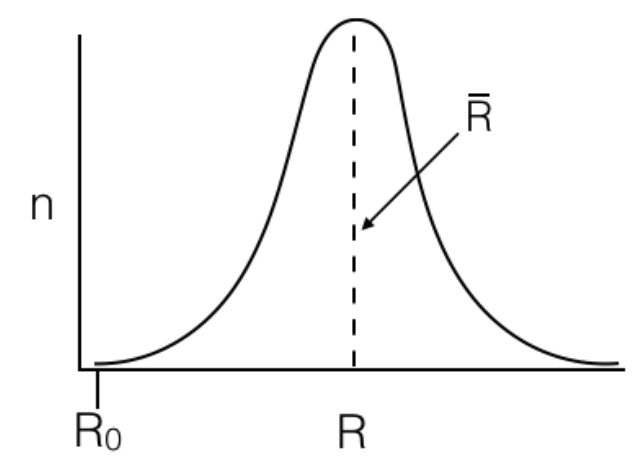

Figure 4: Schematic of crystal size distribution. $R_{0}$ is the initial radius and $\bar{R}$ is the mean radius.

This leads to the equation for evolution of the depth-averaged number density $N$ :

$$
D \frac{\partial N}{\partial t}=\gamma R_{0} N_{0}-\gamma \bar{R} N
$$

which is a balance between influx of crystals at the initial radius $R_{0}$ and number density $N_{0}$ at the base of the mixed layer, and the settling of crystals at the mean radius and number density at the top. This is independent of the growth term because, while crystal growth shifts the size distribution, it has no effect on the total number of crystals.

Because the mixed layer temperature and freezing temperature are assumed to be independent of depth, the supercooling is distributed through the mixed layer and crystals continue to grow radially as they rise. By multiplying (5) by $R$ before integrating, we arrive at an equation for the change in mean radius $\bar{R}$ :

$$
D \frac{\partial(N \bar{R})}{\partial t}=D G_{0}\left[T_{f}(S)-T\right] N+(\alpha+1)\left[\gamma R_{0}^{2} N_{0}-\gamma \bar{R}^{2} N\right]
$$

with mixed layer depth $D$, latent heat of solidification $L$, ice density $\rho_{i}$, seawater density $\rho_{w}$, and molecular diffusivity of heat $\kappa_{T m} . \alpha$ is a parameter that accounts for the shape of the crystal size distribution, defined as

$$
\alpha \equiv \frac{\int_{0}^{\infty} R^{2} n \mathrm{~d} R}{\bar{R} \int_{0}^{\infty} R n \mathrm{~d} R} .
$$

The distribution shape determines the relationship between $\bar{R}^{2}$ and $\overline{R^{2}}$. If all crystals are the same size (corresponding to a delta function distribution), $\alpha=0$. Otherwise, $\alpha>0$. For example, for a Gaussian distribution with standard deviation $\sigma$, 


$$
\alpha \approx \frac{\sigma}{\bar{R}}
$$

For the purposes of this report, we found the impact of $\alpha$ to be negligible, so henceforth we consider (8) with $\alpha=0$.

The ice concentration can thus be expressed as the characteristic volume of a crystal times the number of crystals per unit volume:

$$
C=\pi \bar{R}^{2} H N
$$

The evolution of mixed layer temperature $T$ and salinity $S$ are governed by diffusion of heat and salt across the interface and the addition of salt and latent heat due to the growth of ice crystals:

$$
\begin{gathered}
\frac{\partial T}{\partial t}=\frac{L \rho_{i}}{c_{w} \rho_{w}} G_{0}\left[T_{f}(S)-T\right] N \bar{R} 2 \pi H-\kappa_{T}\left[\frac{T-T_{\mathrm{res}}}{D \delta}\right], \\
\frac{\partial S}{\partial t}=\frac{\rho_{i}}{\rho_{w}} G_{0}\left[T_{f}(S)-T\right] N \bar{R} 2 \pi H S-\kappa_{S}\left[\frac{S-S_{\mathrm{res}}}{D \delta}\right],
\end{gathered}
$$

with diffusivities of heat and salt $\kappa_{S}$ and $\kappa_{T}$ reservoir temperature $T_{\text {res }}$ and salinity $S_{\text {res }}$.

It is useful to define supercooling $\theta$ as

$$
\theta=T-T_{f}(S)
$$

choosing a sign convention where negative $\theta$ indicates supercooling (i.e. the mixed layer temperature is below the freezing point determined by its current salinity). We can directly compute the evolution of $\theta$ as

$$
\frac{\partial \theta}{\partial t}=\frac{\partial T}{\partial t}-\Gamma \frac{\partial S}{\partial t}
$$

as we do in our definition of the dimensionless system below, or it can be computed post hoc given the evolution of $T$ and $S$. 


\subsection{Dimensionless parameters}

We nondimensionalize the variables as

$$
\hat{\theta}=\frac{T-T_{f}(S)}{\Delta T}, \quad \hat{S}=\frac{S}{S_{0}}, \quad \hat{R}=\frac{\bar{R}}{R_{0}}, \quad \hat{N}=\frac{D G_{0} \Delta T}{\gamma R_{0}^{2} N_{0}} N, \quad \hat{t}=\frac{\kappa_{T}}{D \delta} t,
$$

with $\Delta T$ defined as

$$
\Delta T=T_{f}\left(S_{0}\right)-T_{\text {res }}
$$

The timescale we have chosen is the adjustment timescale for cooling of the whole mixed layer depth $D$ by diffusion of heat across the boundary layer $\delta$.

The resulting nondimensional governing equations corresponding to (14), (12), (7), and (8), respectively, can be written as

$$
\begin{gathered}
\frac{\partial \hat{\theta}}{\partial \hat{t}}=-2 \operatorname{Pe} C_{0} \hat{N} \hat{R} \hat{\theta}\left[\operatorname{St}-\mathcal{L} \frac{\rho_{i}}{\rho_{w}} \hat{S}\right]-[\hat{\theta}+\mathcal{L}(\hat{S}-1)+1]+\mathcal{L} \tau\left[\hat{S}-\hat{S}_{\mathrm{res}}\right], \\
\frac{\partial \hat{S}}{\partial \hat{t}}=-2 \operatorname{Pe} C_{0} \frac{\rho_{i}}{\rho_{w}} \hat{N} \hat{R} \hat{\theta} \hat{S}-\tau\left(\hat{S}-\hat{S}_{\mathrm{res}}\right), \\
\frac{\partial \hat{N}}{\partial \hat{t}}=-\operatorname{Pe} \hat{R} \hat{N}+\mathcal{G} r \\
\frac{\partial(\hat{R} \hat{N})}{\partial \hat{t}}=\mathcal{G} r \hat{N} \hat{\theta}-\operatorname{Pe} \hat{R}^{2} \hat{N}+\mathcal{G} r,
\end{gathered}
$$

where the six dimensionless parameters are defined as follows:

$$
\begin{gathered}
\tau \equiv \frac{\kappa_{S}}{\kappa_{T}}, \\
\mathrm{Pe} \equiv \frac{\gamma R_{0} \delta}{\kappa_{T}}, \\
\mathrm{St} \equiv \frac{\rho_{i} L}{\rho_{w} c_{w} \Delta T}, \\
\mathcal{G} r \equiv \frac{G_{0} \Delta T \delta D}{\kappa_{T} R_{0}}, \\
\mathcal{L} \equiv \frac{\Gamma S_{0}}{\Delta T}, \\
\hat{S}_{\mathrm{res}} \equiv \frac{S_{\mathrm{res}}}{S_{0}} .
\end{gathered}
$$


It is worth briefly explaining the significance of these parameters and their approximate values for the parameter ranges that will be explored in section 3 . It is critical to this problem that $\tau$ (18a), the ratio of salt diffusivity to heat diffusivity, have a value much less than 1 , because that is the origin of the supercooling. The Péclet number $\mathrm{Pe}(18 \mathrm{~b})$ is defined as a ratio of crystal rise velocity to heat diffusion across the basal boundary layer, which has a value on the order of 50 , indicating that heat diffuses slowly relative to the timescale on which crystals remain in the suspension. The Stefan number $\mathcal{S}$ t (18c) describes the ratio of latent to sensible heat, an indicator of the efficiency of ice production, and is on the order of $10^{3}$. The growth parameter $\mathcal{G} r(18 \mathrm{~d})$ increases as $\mathcal{S}$ t decreases, and describes the relative change in size by crystal growth over the cooling timescale, compared to the initial radius. It is on the order of $10^{4}$, indicating that the timescale of crystal growth is much faster than the timescale of heat diffusion, and crystals will grow substantially. The liquidus number $\mathcal{L}(18 \mathrm{e})$ is the ratio of the initial mixed layer freezing temperature to temperature gradient, and is on the order of 20 . The scaled reservoir salinity $\hat{S}_{\text {res }}$ is order 1 .

\section{$3 \quad$ Results}

\subsection{Timescales of system evolution}

The system of equations (17) is solved numerically to simulate the evolution of the system over 30 days, providing some insight into the basic behavior and important timescales of this system (figure 5). Henceforth, dimensional quantities will be used in the figures and discussion. The initial conditions and parameter values used for this simulation are initial mixed layer salinity $S_{0}=28 \mathrm{~g} / \mathrm{kg}$, reservoir salinity $S_{\text {res }}=29$ $\mathrm{g} / \mathrm{kg}$, temperature gradient $\Delta T=0.06^{\circ} \mathrm{C}$, initial concentration $C_{0}=10^{-7}$, initial radius $R_{0}=0.2 \mathrm{~mm}$, and mixed layer depth $D=10 \mathrm{~m}$. Consistent with previous laboratory and modeling experiments of supercooling and frazil ice formation, the system first cools rapidly, which is accompanied by an explosion in ice concentration (e.g. $[9,10])$.

After the time of peak supercooling, which scales as the cooling adjustment timescale discussed earlier,

$$
t \sim \frac{D \delta}{\kappa_{T}}
$$

the magnitude of supercooling shows a small and gradual decrease, along with the ice concentration and mean radius. This change coincides with the salinity increasing approximately linearly with time.

By running the simulation over 500 days (which is unrealistic physically because of the

relative ephemerality of these conditions), we can see the full theoretical evolution of 

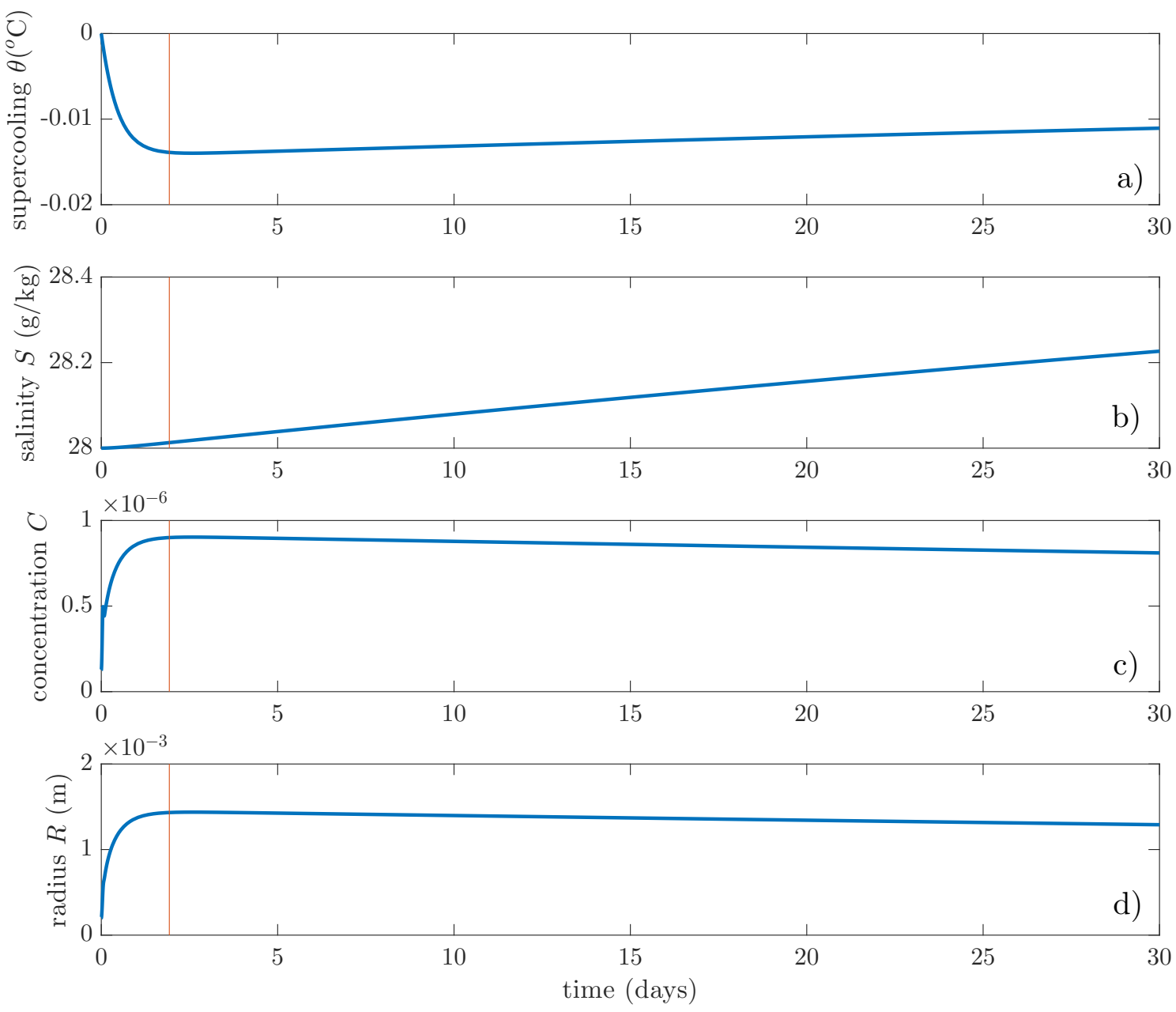

Figure 5: 30-day evolution of mixed layer (a) supercooling $\theta$, (b) salinity $S$, (c) ice concentration $C(10)$, and (d) mean crystal radius $\bar{R}$. The red line indicates the timescale of peak supercooling (19). The initial conditions used for this simulation are $S_{0}=28 \mathrm{~g} / \mathrm{kg}, S_{\text {res }}=29 \mathrm{~g} / \mathrm{kg}, \Delta T=0.06^{\circ} \mathrm{C}, C_{0}=10^{-7}, R_{0}=0.2 \mathrm{~mm}$, and $D=10 \mathrm{~m}$. 

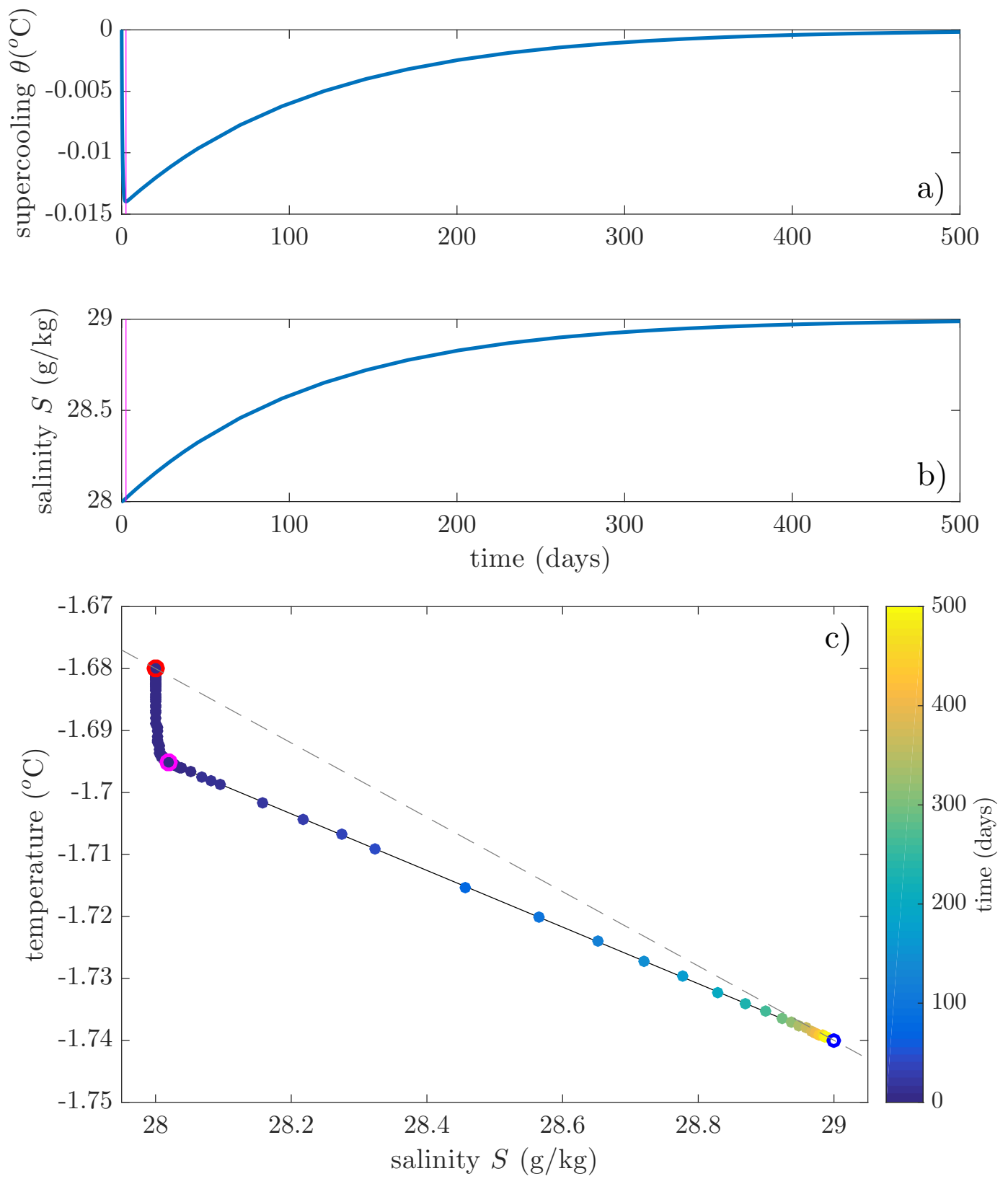

Figure 6: 500-day simulation of mixed layer (a) temperature and (b) salinity. The magenta lines indicate the time of peak supercooling. (c) Points on the T-S plot are colored by day and indicate the mixed layer conditions. The red circle in the T-S plot indicates the initial temperature and salinity of the mixed layer; magenta indicates the magnitude of peak supercooling; blue is the reservoir temperature and salinity. The dashed grey line is the freezing line. Parameter values as in figure 5 . 
this system in temperature-salinity space (figure 6c). The magnitude of supercooling decreases monotonically after the peak, qualitatively following the salinity (figure 6ab), although the T-S plot shows that the temperature of the mixed layer continues to decrease, indicating that the slow evolution of the system is driven by the increasing salinity lowering the freezing temperature. The steady decrease in temperature and increase in salinity are qualitatively consistent with the changing temperature and salinity profiles in figure 1.

\subsection{Fast evolution}

Plotting the time evolution of each variable's time derivative gives some more insight into the initial transient period (figure 7e-h). Prior to the peak supercooling, the supercooling is dominated by the heat flux out of the mixed layer. However, as the ice concentration increases, more latent heat is released into the mixed layer as those crystals grow, and the peak supercooling is reached when those tendencies balance (figure 7e):

$$
\frac{L \rho_{i}}{c_{p} \rho_{w}} G_{0}\left(T_{f}-T\right) N \bar{R} 2 \pi H \sim \kappa_{T}\left[\frac{T-T_{\mathrm{res}}}{D \delta}\right] .
$$

The crystal number density $N$ and mean radius $\bar{R}$ also peak at this point, with the crystals settling out at the top balancing the influx of crystals, and radial crystal growth balancing reduction of the mean radius by precipitation of larger crystals (figure $7 \mathrm{~g}-\mathrm{h}$ ), respectively:

$$
\begin{gathered}
\gamma \bar{R} N \sim \gamma R_{0} N_{0} \\
G_{0}\left(T_{f}-T\right) D N \sim \gamma \bar{R} N
\end{gathered}
$$

These balances lead to scalings for the peak supercooling,

$$
\frac{\theta_{\text {peak }}}{\Delta T} \sim \frac{\beta}{2} \frac{\kappa_{T}}{D \delta G_{0}} \frac{\rho_{w} c_{p} \Delta T}{\rho_{i} L} \frac{1}{N_{0} R_{0} \pi H},
$$

mean ice crystal radius,

$$
\frac{\bar{R}_{\mathrm{peak}}^{2}}{R_{0}^{2}} \sim \frac{\beta}{2} \frac{\kappa_{T}}{\gamma \delta} \frac{c_{p} \rho_{w} \Delta T}{\rho_{i} L} \frac{1}{N_{0} R_{0} \pi H},
$$

and ice concentration,

$$
C_{\text {peak }} \sim \frac{C_{0}}{R_{0}} \bar{R}_{\text {peak }}
$$



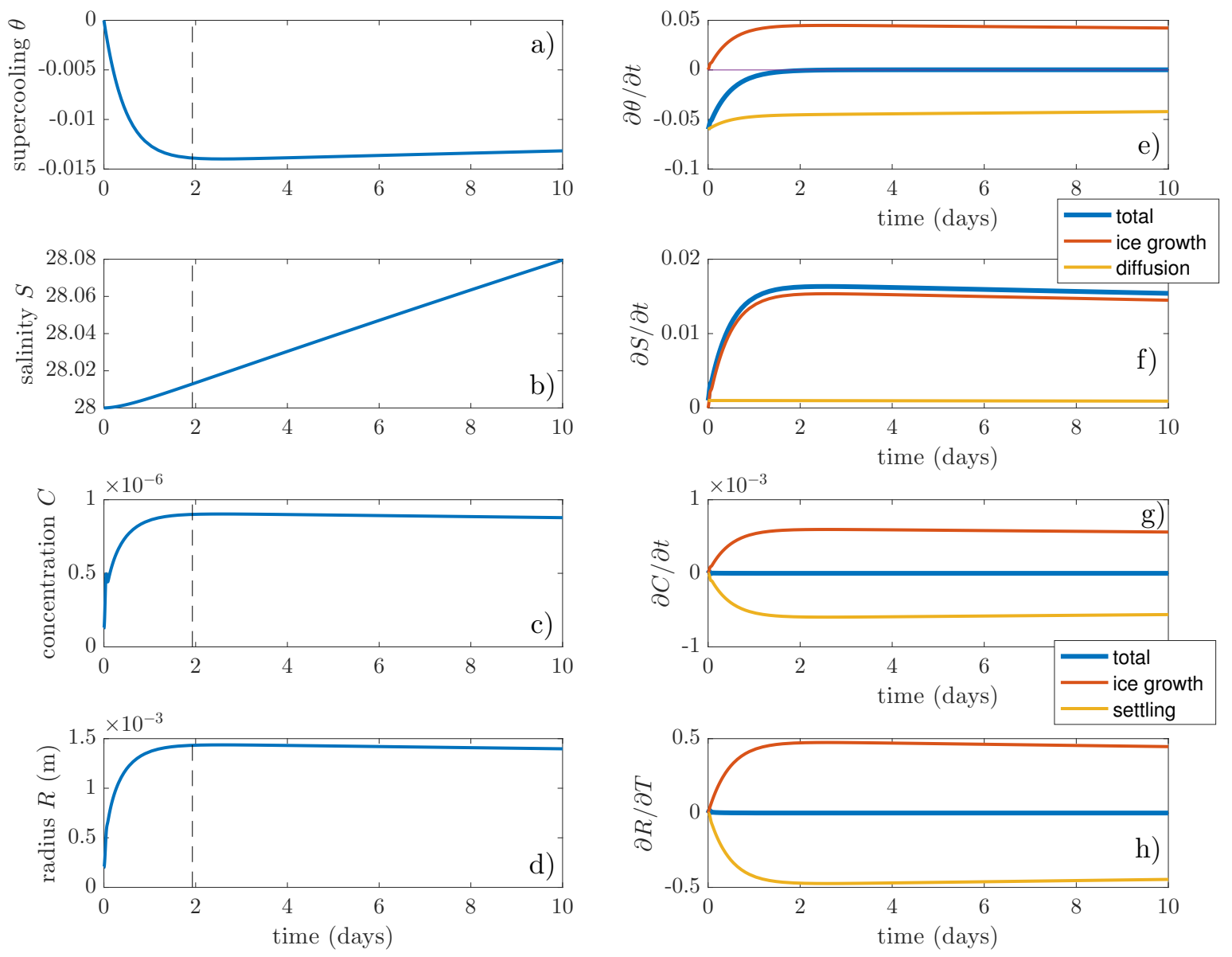

Figure 7: (a-d) Evolution for the first 10 days of the simulation with parameter values as in figure 5 are plotted with the time of peak supercooling indicated by the grey dashed line. (e-h) The associated tendencies are plotted (blue) along with their major constituents (red and yellow). The top legend applies to $\theta$ and $S$; the bottom legend applies to $C$ and $\bar{R}$. 

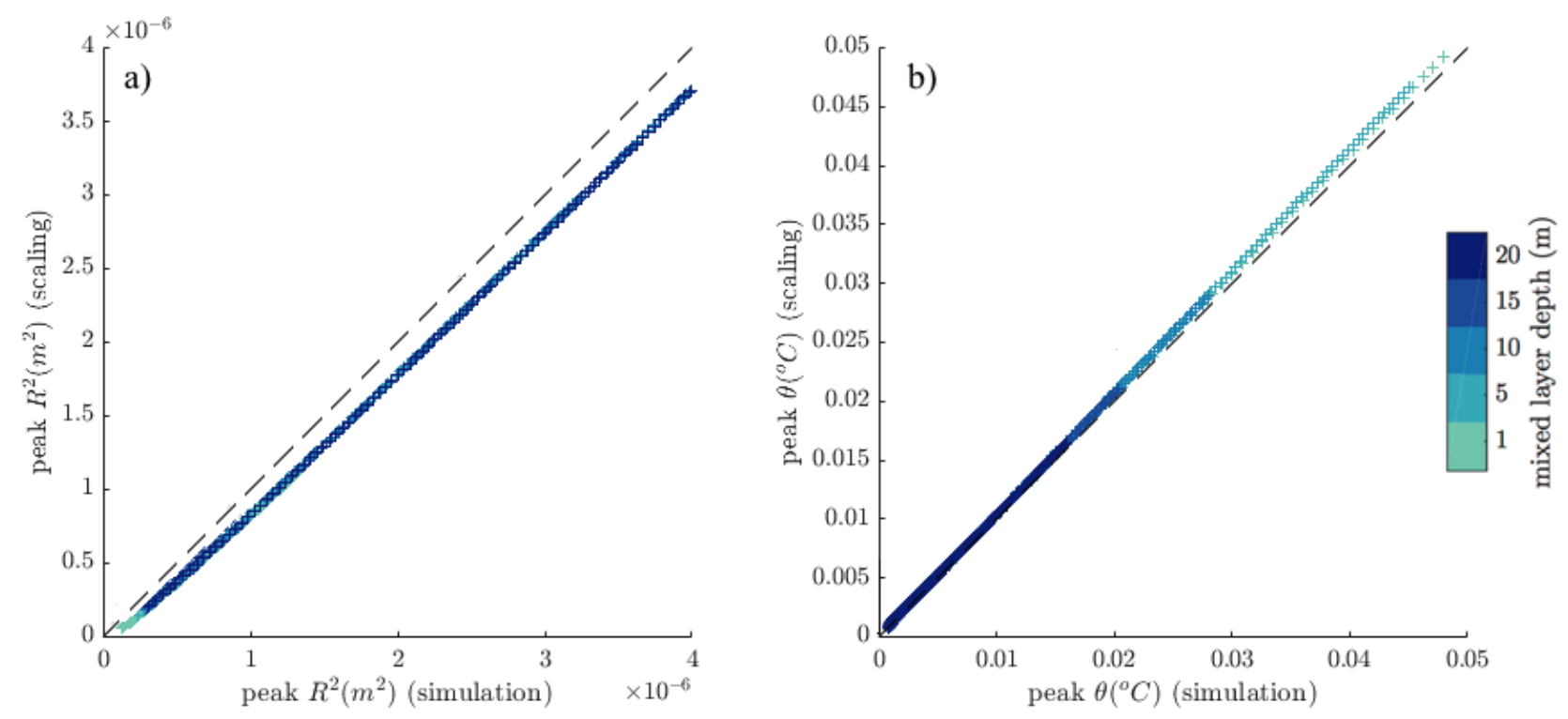

Figure 8: (a) The squared maximum radius $\bar{R}^{2}$ calculated using (21b) is plotted against the simulated value for a range of values of initial heat flux $Q_{0}$ and mixed layer depth $D$. (b) As in (a), but for peak supercooling $\theta$ (21a).

with dimensionless factor $\beta$ arising from the assumption that $T_{f}(S)-T_{\text {res }} \approx \Delta T$ :

$$
\beta \equiv \frac{1}{1+\frac{\kappa_{T}}{2 \pi R_{0} H N_{0} G_{0} D \delta} \frac{c_{w} \rho_{w}}{L \rho_{i}}}=\frac{1}{1+\frac{1}{\operatorname{St} \mathcal{G} r C_{0}}} .
$$

These can also be written in terms of the dimensionless parameters (18):

$$
\begin{aligned}
& \frac{\theta_{\text {peak }}}{\Delta T} \sim \frac{\beta}{2 C_{0} \mathcal{G} r \mathcal{S} t}, \\
& \frac{\bar{R}_{\text {peak }}^{2}}{R_{0}^{2}} \sim \frac{\beta}{2 P e \mathcal{S} t C_{0}}
\end{aligned}
$$

The scaling $\bar{R}_{\text {peak }}^{2}$ slightly underestimates the peak radius relative to the simulation (figure $8 \mathrm{a}$ ), while the $\theta_{\text {peak }}$ scaling slightly overestimates the magnitude of supercooling (figure $8 \mathrm{~b}$ ), but both are linear with respect to initial heat flux $Q_{0}$ and mixed layer depth $D$. 


\subsection{Slow evolution}

The slow evolution of the system after the time of peak supercooling is driven by increasing salinity due to salt rejection as the ice crystals grow (figure $7 \mathrm{f}$ ). This depresses the freezing point, reducing the magnitude of supercooling. As a result, the mean ice crystal radius and concentration also decline very gradually.

To get some more insight into this timescale, we can arrive at a scaling estimate using a few simplifications. First, a balance between latent heat release due to ice freezing and cooling due to the heat flux across the boundary is assumed (20a). Second, mean radius and number density are approximated to be constant, with

$$
\bar{R} N \approx R_{0} N_{0}
$$

We also require $\tau \ll 1$.

This gives us a leading order balance for $\frac{\partial S}{\partial t}$ :

$$
\frac{\partial S}{\partial t} \sim \frac{\rho_{i}}{\rho_{w}} G_{0}\left[T_{f}(S)-T\right] N \bar{R} 2 \pi H S .
$$

Defining $T_{m}=0^{\circ} \mathrm{C}$ and

$$
T_{f}(S)=T_{m}+\Gamma S,
$$

we can substitute for $T_{f}-T$ in (25) to get

$$
\frac{\partial S}{\partial t} \approx\left(\frac{\lambda \Gamma}{T_{r e s}-T_{m}}\right) S\left[S+\frac{T_{m}-T_{r e s}}{\Gamma}\right] .
$$

Here we have defined a parameter $\lambda$, which has units $s^{-1}$ :

$$
\lambda \equiv\left(T_{r e s}-T_{m}\right) \frac{c_{p}}{L} \frac{\kappa_{T}}{D \delta}\left[\frac{1}{1-\frac{1}{N_{0} R_{0} 2 \pi H} \frac{\kappa_{T} c_{p}}{D \delta G_{0} L}}\right] .
$$

The solution of (27) yields a scaling for the slow evolution of salinity $S(t)$ :

$$
S(t) \sim \frac{-T_{\text {res }}}{\Gamma}\left(1+\frac{\Delta T}{\Gamma S_{0}} e^{-\lambda t}\right)^{-1},
$$

and $\frac{1}{\lambda}$ is an approximate timescale for relaxation of the mixed layer salinity $S$ to the reservoir salinity $S_{\text {res }}$ (i.e. the timescale on which supercooling would be depleted if the appropriate conditions persisted). 

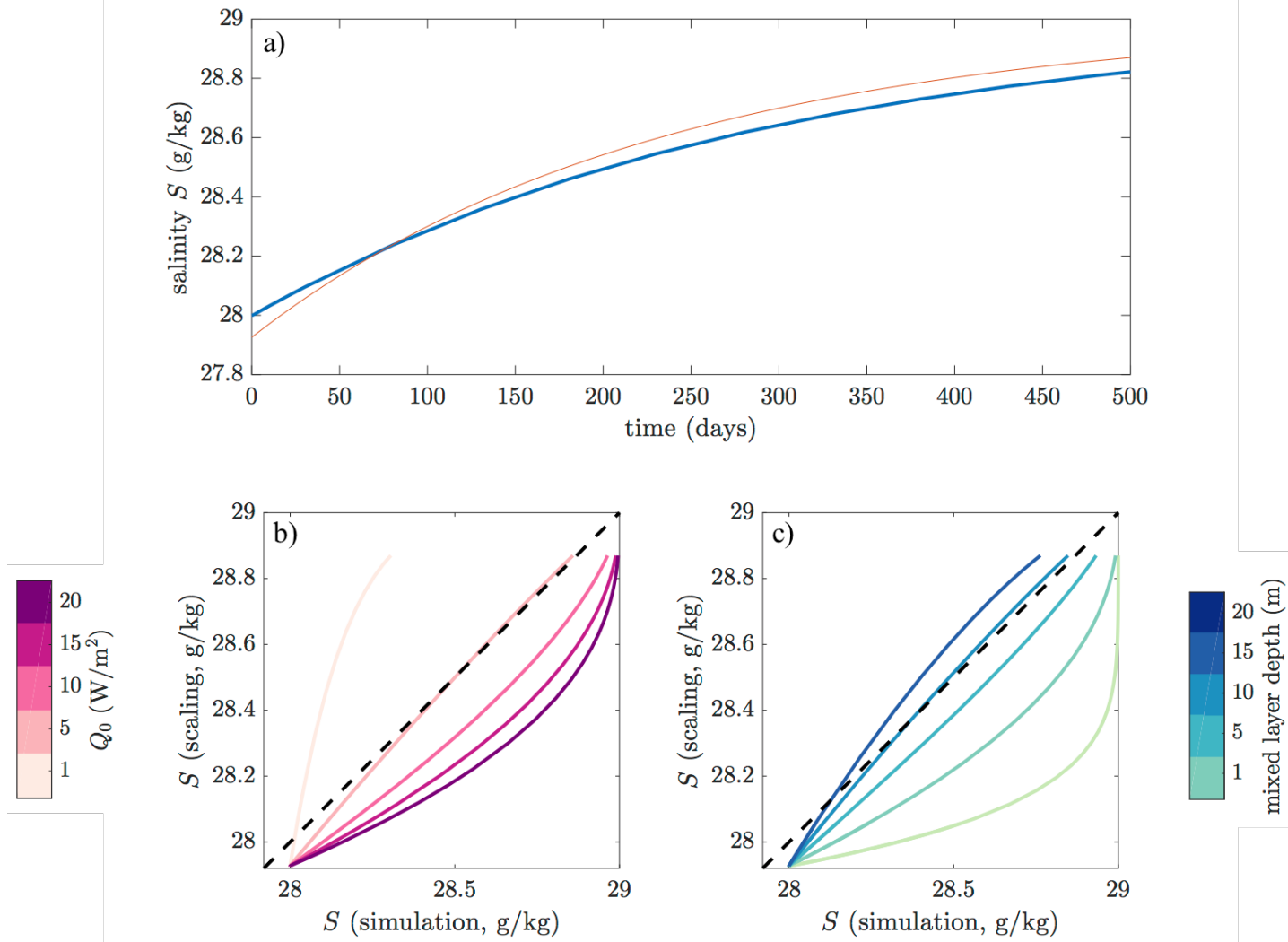

Figure 9: (a) The simulated slow evolution of $S(t)$ over 500 days (blue) is plotted with the scaling estimate from (29) (red) for the baseline initial conditions. (b) The scaling value of the salinity is plotted against the simulation value of the salinity over the 500-day run for a range of values of initial heat flux $Q_{0}$. (c) As in (b), but for a range of values of mixed layer depth $D$.

For the parameter values used in these simulations (see section 3.1), this scaling qualitatively captures the timing and magnitude of mixed layer salinity evolution on long timescales (figure 9a). However, it doesn't apply to all parameter ranges, overestimating salinity for very small heat fluxes and deep mixed layers, and underestimating salinity for large heat fluxes and shallow mixed layers (figure 9b-c).

\subsection{Quantifying ice accumulation}

We are interested in quantifying the potential contribution to ice thickness resulting from this mechanism. We can define accumulation at each timestep as 

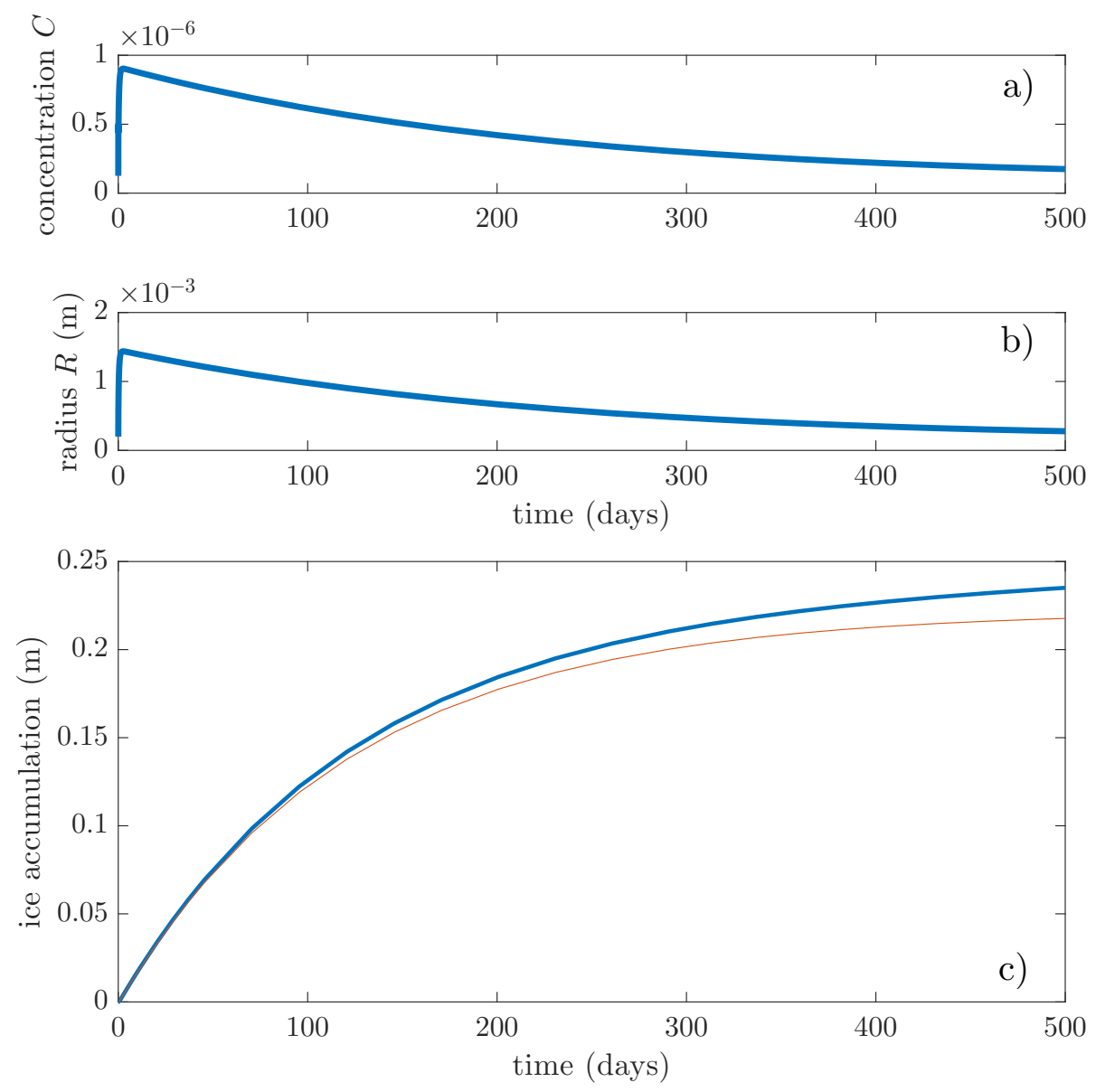

Figure 10: (a-b) Ice concentration $C$ and mean radius $\bar{R}$ are plotted over 500 days. (c) Ice accumulation as calculated in (30) (red) and assuming $\left(\gamma R_{0}\right) \pi R_{0}^{2} N_{0} \ll$ $(\gamma \bar{R}) \pi \bar{R}^{2} H N$ (blue). 


$$
\frac{\partial h}{\partial t}=(\gamma \bar{R}) \pi \bar{R}^{2} H N-\left(\gamma R_{0}\right) \pi R_{0}^{2} N_{0},
$$

where the first term represents settling of ice crystals at the mean radius and the second term removes the contribution of the crystals seeded at the bottom of the mixed layer in the model. We see that for about 100 days of the simulation, the contribution of the second term is negligible (figure 10c). In theory, the system would saturate where these terms were equal, i.e.

$$
\frac{\bar{R}^{3} N}{R_{0}^{3} N_{0}} \sim 1
$$

which is where the red curve levels out in figure 10c. However, as discussed previously, these conditions are unlikely to persist for more than a few weeks. On more realistic timescales, we can approximate that $N$ and $R$ are equal to their peak values $N_{\text {peak }}$ and $R_{\text {peak }}$, leading us to the scaling

$$
\frac{\partial h}{\partial t} \sim \frac{1}{2} \frac{\kappa_{T}}{\delta} \frac{1}{\mathcal{S} t}
$$

with $\mathcal{S}$ t, defined in (18c), representing the efficiency of ice production relative to cooling at the mixed layer boundary. This is easily solved (and the constants rearranged) to find

$$
h(t) \sim \frac{1}{2} \frac{Q_{0}}{L \rho_{i}} t
$$

We find that this is one half of the theoretical upper bound, assuming perfect conversion of cooling to ice production:

$$
h_{\max }(t)=\frac{Q}{L \rho_{i}} t
$$

We test this scaling using a model simulation over 30 days (figure 11a). We find that for small values of $Q_{0}$ up to about $10 \mathrm{~W} / \mathrm{m}^{2}$ and large mixed layer depths of $10-20 \mathrm{~m}$, or relatively slow adjustment timescales (19), the scaling estimate (dashed line) agrees qualitatively with the simulated ice production. In general, larger heat fluxes generate more ice production, which is captured by the scaling, but it does not account for the impact of mixed layer depth $D$. The colors overlaid on the curve for each mixed layer depth show the mean radius $\bar{R}$ at the end of the simulation. Deeper mixed layers have larger $\bar{R}$, indicating that one explanation for the dependence on depth is that ice 
crystals in deep layers remain in the supercooled mixed layer longer before settling at the top, facilitating more radial growth and thus greater total accumulation.

The depth dependence is further explained in figure 11b, which shows the peak and final supercooling $\theta$ of the mixed layer at various values of $D$. The greatest magnitude of supercooling is much greater for the $1 \mathrm{~m}$ mixed layer than for the $20 \mathrm{~m}$ mixed layer. However, the final magnitude of $\theta$ is substantially smaller than the peak for the shallower layer, because the latent heat and salt released by ice freezing in the shallow mixed layer are relatively concentrated and erode the supercooling rapidly. In contrast, the final value of $\theta$ in the $20 \mathrm{~m}$ layer is very close to its peak, and nearly indistinguishable for lower values of $Q_{0}$. This is supported by the depletion timescale $\frac{1}{\lambda}(28)$, which is directly related to $D$ and inversely related to $\frac{\kappa_{T}}{\delta}$. Finally, ice accumulation is also well-correlated with maximum ice concentration $C$ (figure 11c).

In order to compare the values of ice accumulation to frazil ice layer thickness in observational studies, it is necessary to estimate an ice volume fraction. Based on a combination of observational and modeling estimates, this factor is approximated as $\mathcal{T}=0.25 \pm 0.09$ [4], resulting in a layer thickness around 4 times greater than the accumulation values shown in figures $10 \mathrm{c}$ and $11 \mathrm{a}$, or between $1 \mathrm{~cm}$ and $15 \mathrm{~cm}$ over 30 days as calculated using (32b) and $Q_{0}$ between 1 and $10 \mathrm{~W} / \mathrm{m}^{2}$.

\section{Conclusions and Future Work}

Our model reaffirms that differing rates of heat and salt diffusion can generate supercooling at the interface between water masses. We have described the evolution of the mixed layer as a result of this process and derived scalings to quantify the effects of system parameters on key quantities, including the peak supercooling, which defines the overall phase-space trajectory of mixed layer properties, and ice crystal radius. We have shown that under realistic Arctic conditions, this mechanism could contribute non-negligible secondary sea ice growth, and identified key parameters that may determine the quantity of ice production.

In order to rigorously quantify the uncertainties, more observations are necessary to constrain appropriate parameter ranges and evaluate the model predictions. We will continue to improve the model, notably by adding depth variation. We also intend to evaluate the potential for convective mixing by the rising ice crystals. 

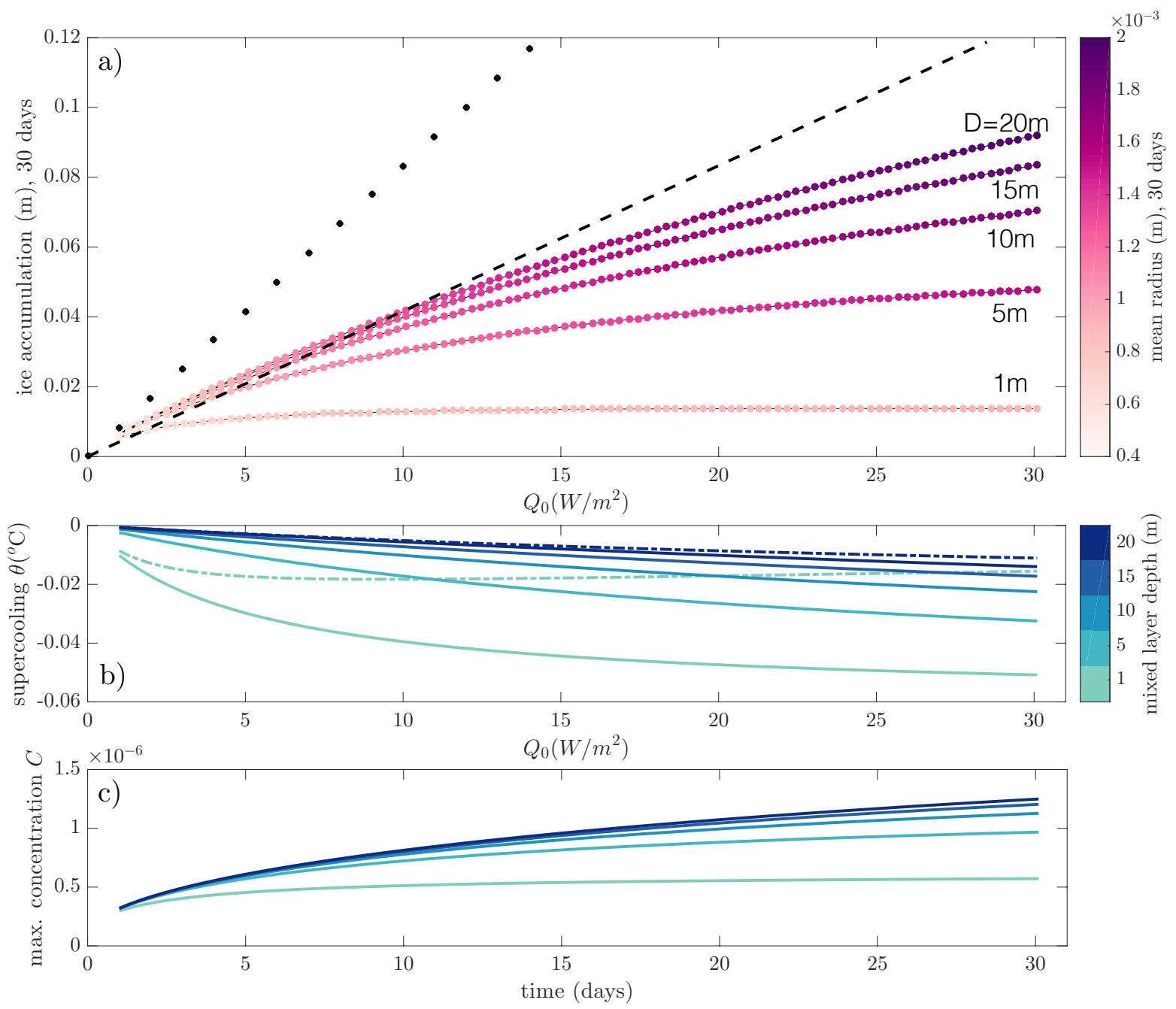

Figure 11: (a) Ice accumulation after 30 days is plotted against initial heat flux $Q_{0}$ for different values of the mixed layer depth $D$ (labeled). The color of the dots indicates the mean ice crystal radius $\bar{R}$ after 30 days. The growth scaling law given in (32b) (dashed) and theoretical upper bound for ice accumulation (33) (dotted) are also plotted. (b) Peak supercooling is plotted against initial heat flux $Q_{0}$ for different values of the mixed layer depth $D$ (solid lines). The remaining supercooling after 30 days is plotted for the $1 \mathrm{~m}$ and $20 \mathrm{~m}$ mixed layer (dashed lines). (c) Maximum concentration $C$ is plotted against initial heat flux $Q_{0}$ for different values of the mixed layer depth $D$. 


\section{Acknowledgments}

I would like to thank Mary-Louise Timmermans and Andrew Wells for their peerless expertise, generous patience, and boundless good humor in guiding me through this project! I am also grateful to everyone in and around Walsh Cottage who helped me throughout the summer, especially Claudia Cenedese, Craig McConnochie, Renske Gelderloos, Ian Hewitt, Sam Pegler, Bruce Sutherland, Colm-cille Caulfield, Janet Fields, Julie Hildebrandt, Joe Pedlosky, Fiamma Straneo, and the many wonderful visitors. Finally, thanks to the Fellows for sharing many "ups" and a few "downs" but food always.

\section{References}

[1] M. I. Budyko, The effect of solar radiation variations on the climate of the Earth, Tellus, 21 (1969), pp. 611-619.

[2] M. O. Jeffries, K. Schwartz, K. Morris, A. D. Veazey, H. R. Krouse, And S. Gushing, Evidence for platelet ice accretion in Arctic sea ice development, Journal of Geophysical Research: Oceans, 100 (1995), pp. 10905-10914.

[3] A. Jenkins, Convection-driven melting near the grounding lines of ice shelves and tidewater glaciers, Journal of Physical Oceanography, 41 (2011), pp. 2279 2294.

[4] P. J. Langhorne, K. G. Hughes, A. J. Gough, I. J. Smith, M. J. M. Williams, N. J. Robinson, C. L. Stevens, W. Rack, D. Price, G. H. Leonard, A. R. Mahoney, C. HaAs, And T. G. Haskell, Observed platelet ice distributions in Antarctic sea ice: An index for ocean-ice shelf heat flux, Geophysical Research Letters, 42 (2015), pp. 5442-5451. 2015GL064508.

[5] S. Martin And P. Kauffman, The evolution of under-ice melt ponds, or double diffusion at the freezing point, Journal of Fluid Mechanics, 64 (1974), pp. 507-528.

[6] V. McFarlane, M. Loewen, And F. Hicks, Laboratory measurements of the rise velocity of frazil ice particles, Cold Regions Science and Technology, 106-107 (2014), pp. $120-130$.

[7] M. G. McPhee, R. Skogseth, F. Nilsen, And L. H. Smedsrud, Creation and tidal advection of a cold salinity front in Storfjorden: 2. supercooling induced by turbulent mixing of cold water, Journal of Geophysical Research: Oceans, 118 (2013), pp. 3737-3751. 
[8] M. A. Morales Maqueda, A. J. Willmott, and N. R. T. Biggs, Polynya dynamics: a review of observations and modeling, Reviews of Geophysics, 42 (2004), pp. 1-37. RG1004.

[9] A. Omstedt And U. Svensson, Modeling supercooling and ice formation in a turbulent Ekman layer, Journal of Geophysical Research: Oceans, 89 (1984), pp. $735-744$.

[10] D. W. Rees Jones And A. J. Wells, Frazil-ice growth rate and dynamics in mixed layers and sub-ice-shelf plumes, The Cryosphere Discussions, 2017 (2017), pp. 1-22.

[11] A. STIGEBRANDT, On the rate of ice formation in water cooled by a more saline sublayer, Tellus, 33 (1981), pp. 604-609.

[12] U. Svensson And A. Omstedt, Simulation of supercooling and size distribution in frazil ice dynamics, Cold Regions Science and Technology, 22 (1994), pp. 221-233.

[13] M.-L. Timmermans, The impact of stored solar heat on Arctic sea ice growth, Geophysical Research Letters, 42 (2015), pp. 6399-6406. 2015GL064541.

[14] M.-L. Timmermans, J. Toole, A. Proshutinsky, R. Krishfield, and A. Plueddemann, Eddies in the Canada Basin, Arctic Ocean, observed from ice-tethered profilers, Journal of Physical Oceanography, 38 (2008), pp. 133-145.

[15] J. Toole, R. Krishfield, A. Proshutinsky, C. Ashuian, K. Doherty, D. Frye, T. Hammar, J. Kemp, D. Peters, M.-L. Timmermans, K. von Der Heydt, G. PaCkARd, and T. Shanahan, Ice-tethered profilers sample the upper Arctic Ocean, Eos, 87 (2006), pp. 434, 438.

[16] T. Vinma, Effects of Arctic sea ice decline on weather and climate: A review, Surveys in Geophysics, 35 (2014), pp. 1175-1214.

[17] S. I. Voropayev, H. J. S. Fernando, and L. A. Mitchell, On the rate of frazil ice formation in polar regions in the presence of turbulence, Journal of Physical Oceanography, 25 (1995), pp. 1441-1450. 


\title{
Eddy Energy Fluxes in Mixed Barotropic-Baroclinic Instability: Upgradient or Downgradient?
}

\author{
Madeleine K. Youngs
}

November 15, 2017

\section{Introduction}

Wave-type instabilities, such as barotropic and baroclinic instability, are important types of variability in the oceanic and atmospheric system. These instabilities generate storms in the atmosphere and eddies in the ocean, set the stratification of the global ocean [Marshall and Speer, 2012], generate surface westerlies in the atmosphere (see Vallis [2006] and references therein) and more. They are generally important for setting the mean state of the system. In the ocean, these instabilities are parameterized as a diffusion of buoyancy along isopycnals, which decreases the baroclinic shear, but does nothing to account for barotropic instability or the momentum fluxes associated with both instabilities and their effects on the mean flow, because they are too complicated [Gent and McWilliams, 1990]. In effect, the mean state of ocean models is likely to be inaccurate at best in some regions. I examine these momentum fluxes to determine the effects of eddies on the mean flow.

The necessary conditions for barotropic and baroclinic instability state that there must be a sign change of the potential vorticity (PV) gradient somewhere in the domain for instability to occur [Charney and Stern, 1962]. Purely barotropic flow and thus instability would have a sign change of the PV gradient within a layer, which would indicate a downgradient momentum flux and extraction of energy from the mean flow [Rayleigh, 1880; Drazin and Howard, 1966]. For a purely baroclinic flow, there must be a sign change in the PV gradient between the layers and this results in a downgradient flux of buoyancy and extraction of energy from available potential energy [Eady, 1949; Charney, 1947]. In the real world, however, flows have both vertical and horizontal shear and thus have two mean energy reservoirs, kinetic and potential energy. The necessary conditions for instability in this case don't indicate which energy reserves are being tapped, but just that energy is being extracted from the mean. Thus, it would be useful to be able to determine a priori the energy pathways in an unstable system, but so far this goal has been elusive. For example, it is sometimes assumed that a change in sign of the PV gradient in a layer implies that there is a down-gradient momentum flux [Pedlosky, 1964]. This assumption is false. I show that the dynamical interaction between layers is responsible for setting the direction of the momentum fluxes in a linearly unstable system.

In a baroclinically unstable fluid with a horizontal shear, interesting interactions occur between the instability and the horizontal shear. There can be upgradient momentum fluxes associated with the baroclinic instability [Pedlosky, 1964], but also downgradient momentum fluxes. Held [1975] found that when a two-layer flow has the same sign PV gradient in both layers, the direction of the momentum fluxes can be determined. Killworth [1980] examines the parameter space of instabilities with horizontal and vertical shear and found that when the horizontal length scale of the shear was larger than the internal deformation radius, then baroclinic conversion dominated, but when the 
horizontal length scale was much smaller than the deformation radius, then barotropic conversion dominated (see also Holland and Haidvogel [1980]).

In this report I look at the linear stability of a zonal channel flow to understand the direction of the momentum and buoyancy fluxes of a wide parameter space and answer the question: what sets the direction of the eddy energy fluxes in mixed instability? In section 2, I set up the model and diagnostics used to analyze the momentum fluxes. In sections 3 and 4, I describe the Gaussian and cosine jet configurations, respectively. I conclude in section 5 .

\section{Model}

\subsection{Linear stability problem}

The derivation that follows parallels Pedlosky [1987]. I consider the two-layer quasi-geostrophic potential vorticity (QGPV) non-dimensionalized equations on a $\beta$-plane:

$$
\left[\frac{\partial}{\partial t}+\frac{\partial \psi_{n}}{\partial x} \frac{\partial}{\partial y}-\frac{\partial \psi_{n}}{\partial y} \frac{\partial}{\partial x}\right]\left[\beta y+\nabla^{2} \psi_{n}-F_{n}(-1)^{n}\left(\psi_{2}-\psi_{1}\right)+\eta_{b} \delta_{n 2}\right]=0 \quad n=1,2
$$

where $\psi_{n}$ is the stream function, $\delta_{i j}$ is the Kronecker delta function and

$$
\nabla^{2}=\frac{\partial^{2}}{\partial x^{2}}+\frac{\partial^{2}}{\partial y^{2}}
$$

I have

$$
\beta=\beta_{0} \frac{L^{2}}{U}
$$

where $\beta_{0}$ is the meridional gradient in planetary vorticity, $L$ is a length scale given by the width of the channel, and $U$ is the velocity scale of the jet and

$$
F_{n}=\frac{f_{0}^{2} L^{2}}{g\left(\rho_{2}-\rho_{1}\right) / \rho_{0} D_{n}}
$$

where $f_{0}$ is the planetary vorticity, $\rho_{n}$ is the density of the $n$-th layer, $\rho_{0}$ is a reference density and $D_{n}$ is the depth of the layer and

$$
F=\frac{f_{0}^{2} L^{2}}{g\left(\rho_{2}-\rho_{1}\right) / \rho_{0} D}
$$

where $D$ is the total depth. I relate $F_{n}$ to $F$ by a ratio of depth of top layer to lower layer $\Delta=D_{1} / D_{2}: F_{1}=F(1+\Delta) / \Delta$ and $F_{2}=F(1+\Delta)$. The topographic forcing is given by:

$$
\eta_{b}=\frac{f_{0} h_{b}}{D_{2}} \frac{L}{U}
$$

and is only included in the lowest layer.

I consider a zonal basic flow

$$
U_{n}(y)=-\frac{\partial \Psi_{n}}{\partial y}
$$

that has a horizontal shear as a source of kinetic energy and a vertical shear (through thermal wind) as a source of available potential energy. Let $\phi_{n}$ be the eddy stream function such that

$$
\psi_{n}=\Psi_{n}(y)+\phi_{n}(x, y, t)
$$


with eddy velocities

$$
u_{n}=-\frac{\partial \phi_{n}}{\partial y}, \quad v_{n}=\frac{\partial \phi_{n}}{\partial x}
$$

I substitute Eq. 7 into Eq. 1 to get:

$$
\left[\frac{\partial}{\partial t}+U_{n} \frac{\partial}{\partial x}\right] q_{n}+\frac{\partial \phi_{n}}{\partial x} \frac{\partial Q_{n}}{\partial y}+\left[\frac{\partial \phi_{n}}{\partial x} \frac{\partial q_{n}}{\partial y}-\frac{\partial \phi_{n}}{\partial y} \frac{\partial q_{n}}{\partial x}\right]=0
$$

where the potential vorticity gradient of the basic state is

$$
\frac{\partial Q_{n}}{\partial y}=\beta-\frac{\partial^{2} U_{n}}{\partial y^{2}}-F_{n}(-1)^{n}\left(U_{1}-U_{2}\right)+\frac{\partial \eta_{b}}{\partial y} \delta_{n 2}
$$

and the perturbation potential vorticity is given by

$$
q_{n}=\nabla^{2} \phi_{n}-F_{n}(-1)^{n}\left(\phi_{2}-\phi_{1}\right)
$$

We then consider the linear stability problem by neglecting the terms of order $\mathcal{O}\left(\phi_{n}^{2}\right)$ and higher where I get the linearized QGPV equation.

$$
\left[\frac{\partial}{\partial t}+U_{n} \frac{\partial}{\partial x}\right] q_{n}+\frac{\partial \phi_{n}}{\partial x} \frac{\partial Q_{n}}{\partial y}=0
$$

with boundary conditions

$$
\frac{\partial \phi_{n}}{\partial x}=0, \quad y= \pm 1
$$

which says that there is no flow into or out of the walls at the boundary.

The necessary conditions for instability in this case are given by:

$$
\int_{-1}^{1} d y \sum_{n} \frac{\partial}{\partial t} \frac{\left|\phi_{n}\right|^{2}}{\left|U_{n}-c\right|^{2}} \frac{\partial Q_{n}}{\partial y}=0
$$

where $c$ is the phase speed of the wave as developed in section 2.2. This equation is derived from the zonally averaged momentum equation. If the flow is unstable, $\frac{\partial}{\partial t} \frac{\left|\phi_{n}\right|^{2}}{\left|U_{n}-c\right|^{2}}$ must be positive such that the eddy energy is growing, so in order for this equation to be satisfied, $\frac{\partial Q_{n}}{\partial y}$ must change sign somewhere in the domain.

\subsection{Diagnostics}

In order to understand the energetics of the flow, I use the energy equation for the perturbations. I set $D_{n} / D=d_{n}$. I take Eq. 12 and multiply by $-d_{n} \phi_{n}$ and sum the two layers, and integrate in $y$.

$$
\begin{aligned}
\frac{\partial}{\partial t} \int_{-1}^{1} d y\left[\mathrm{EKE}_{1}+\mathrm{EKE}_{2}+\mathrm{EAPE}\right] & =\int_{-1}^{1} d y\left[\Delta \mathrm{EKE}_{1}+\Delta \mathrm{EKE}_{2}+\Delta \mathrm{EAPE}\right] \\
\mathrm{EKE}_{n} & =\frac{d_{n} \overline{\left(\frac{\partial \phi_{n}}{\partial x}\right)^{2}}+\left(\frac{\partial \phi_{n}}{\partial y}\right)^{2}}{\left(\phi_{1}-\phi_{2}\right)^{2}} \\
\mathrm{EAPE} & =\frac{\frac{2}{\partial \phi_{n}}}{\partial \phi_{n}} \frac{\partial U_{n}}{\partial y} \\
\Delta \mathrm{EKE}_{n} & =d_{n} \frac{\partial y}{\partial x} \\
\Delta \mathrm{EAPE} & =F_{0}\left(U_{1}-U_{2}\right) \frac{\partial \phi_{2}}{\partial x} \phi_{1}
\end{aligned}
$$


where the left-hand side represents the time change of total eddy energy and the right-hand side represents the conversion from the mean into the eddy energy. If there is energy converted into eddies through a positive (negative) $\Delta \mathrm{EKE}_{n}$, then the momentum fluxes are said to be downgradient (upgradient) because they act to relax (strengthen) the mean flow and transfer kinetic energy from regions of high (low) energy to regions of low (high) kinetic energy. If there is energy converted into eddies through a positive (negative) $\triangle \mathrm{EAPE}$, then the buoyancy fluxes are said to be downgradient (upgradient) because they act to relax (strengthen) the mean buoyancy gradient and transfer potential energy from regions of high (low) potential energy to regions of low (high) potential energy.

Another way to assess how eddies affect the mean flow is to consider the zonally averaged, zonal momentum equation summed over both layers:

$$
\frac{\partial}{\partial t}\left[\sum_{n=1}^{2} d_{n} \overline{u_{n}}\right]=-\frac{\partial}{\partial y}\left[\sum_{n=1}^{2} d_{n} \overline{u_{n} v_{n}}\right]
$$

so the change of zonal momentum is related to the divergence of the Reynolds stresses. I can take the PV equation and the enstrophy equation to show that

$$
\frac{\partial}{\partial t}\left[\sum_{n=1}^{2} d_{n} \overline{u_{n}}\right]=-\frac{\partial}{\partial y}\left[\sum_{n=1}^{2} d_{n} \frac{\overline{q_{n}^{2}}}{\partial Q_{n} / \partial y}\right]
$$

Ultimately, I want to know if the barotropic flow accelerates or decelerates, and this expression tells us that I know the sign of the acceleration if $\partial Q_{n} / \partial y$ has the same sign in both layers [Held, 1975]. If the PV gradients are not the same sign then the sign of the acceleration also depends on the ratio of the magnitudes of $\overline{q_{n}^{2}}$ as well as $\partial Q_{n} / \partial y$.

\subsection{Eigenvalue solver}

I use an eigenvalue solver to compute the solutions to Eq. 12. First I assume a solution of the form

$$
\phi_{n}=\operatorname{Re} \Phi_{n}(y) e^{i k(x-c t)}
$$

where Eq. 12 becomes two coupled ordinary differential equations

$$
\begin{aligned}
& \left(U_{1}-c\right)\left[\frac{d^{2} \Phi_{1}}{d y^{2}}-k^{2} \Phi_{1}-F_{1}\left(\Phi_{1}-\Phi_{2}\right)\right]+\Phi_{1} \frac{\partial Q_{1}}{\partial y}=0 \\
& \left(U_{2}-c\right)\left[\frac{d^{2} \Phi_{2}}{d y^{2}}-k^{2} \Phi_{2}-F_{2}\left(\Phi_{2}-\Phi_{1}\right)\right]+\Phi_{2} \frac{\partial Q_{2}}{\partial y}=0
\end{aligned}
$$

I discretize the various parameters across my channel and use second order finite differencing to create a differentiation matrix for the operator $\frac{d^{2}}{d y^{2}}$ which gives an equivalent matrix expression for Equations 23 and 24. Then for every $k$, I can use an eigenvalue solver to compute the eigenvalues $c$ and the eigenvectors $\left[\frac{d^{2} \Phi_{n}}{d y^{2}}-k^{2} \Phi_{n}+F_{n}(-1)^{n}\left(\Phi_{1}-\Phi_{2}\right)\right]$ and $\Phi_{n}$. Then I normalize $\Phi_{n}$ so that the total energy in the domain is $1\left(\mathrm{EKE}_{1}+\mathrm{EKE}_{2}+\mathrm{EAPE}=1\right)$. Then growth rates $k c_{i}$ and energy conversions are computed (Eq. 18 and 19). After computing the solution for all $k$ s the solution $\left(\Phi_{n}\right)$ with maximum growth rate $\left(k c_{i}\right)$ is selected for further analysis. In reality, the solution that is selected is not always the one with the maximum growth rate but can be another mode [Pedlosky, 1981]. 


\section{Gaussian Jet}

\subsection{Set up}

I set up the two layers with a Gaussian jet of half-width $\delta$ in the upper layer and no flow in the lower layer (Fig. 1).

$$
\begin{aligned}
U_{1} & =\frac{1}{2}+\frac{1}{2} e^{-\frac{y^{2}}{\delta^{2}}} \\
U_{2} & =0
\end{aligned}
$$

Note that the velocity has been scaled out of the problem and is included in the $\beta$ parameter. $\delta$ ranges from 0 to 1 in our non-dimensionalized domain. In this case the basic state potential vorticity gradient is given by

$$
\begin{aligned}
& \frac{\partial Q_{1}}{\partial y}=\beta-\frac{\partial^{2} U_{1}}{\partial y^{2}}+F_{1} U_{1} \\
& \frac{\partial Q_{2}}{\partial y}=\beta-F_{2} U_{1}
\end{aligned}
$$

We have 4 non-dimensional parameters to vary in this case: $F_{0}, \delta, \Delta$, and $\beta$. For experiments discussed here, I set $\Delta=1$.

\subsection{Results}

I examine the energy conversion over a range of $F$ and $\beta$ and set $\delta=0.05$ as a constant. I scale the constants $F$ and $\beta$ with $\delta^{2}$. This rescales the

$$
F \delta^{2}=F=\frac{f_{0}^{2} \delta_{0}^{2}}{g\left(\rho_{2}-\rho_{1}\right) / \rho_{0} D}
$$

and

$$
\beta \delta^{2}=\beta_{0} \frac{\delta_{0}^{2}}{U}
$$

where $\delta=\delta_{0} / L$ is the length scale of the jet width. First, I notice that when the PV gradient changes sign in the upper layer, the momentum fluxes are not always downgradient (Fig. 2). There are also downgradient momentum fluxes associated with no sign change of the PV gradient in the upper layer. So, it is not the introduction of a sign change in the upper-layer PV gradient that leads to downgradient momentum fluxes. I also notice that as I decrease $F$ or increase the deformation radius, the kinetic energy conversion comes to dominate over the potential energy conversion.

I split up the domain into 5 regions. Region 1 has downgradient momentum fluxes but no sign change in the upper layer PV gradient; this is an unexpected result. Region 2 has downgradient momentum fluxes and a sign change in the upper layer PV gradient. Region 3 has upgradient momentum fluxes and no sign change in the upper layer PV gradient. Region 4 has upgradient momentum fluxes and a sign change in the upper layer PV gradient; this is also an unexpected result. Region 5 is dominated by barotropic conversion and has a sign change in the upper layer PV gradient.

In these 5 different regions, there are defining characteristics in the wavenumber space (Fig. 3). In regions $1-4$, the dominant mode (with largest growth rate) is a mode with primarily baroclinic production (downgradient buoyancy fluxes) and weak barotropic production (kinetic energy fluxes). 
When a mode has primarily kinetic energy conversion, I call it a barotropic mode and when it has primarily baroclinic conversion, I call it a baroclinic mode. Regions 1 and 2 have downgradient kinetic energy fluxes and regions 3 and 4 have upgradient kinetic energy fluxes at the most unstable wavenumber. Comparing region 1 and region 2, the primary difference is that modes with higher wavenumber appear. These new modes have primarily barotropic conversion. Similarly, this mode appears in region 4 but not region 3. So when the sign change is introduced into the upper layer PV gradient in regions 2 and 4, a higher-wavenumber barotropic mode appears as a solution. In these regions, the dominate barotropic modes both have locations where $U_{1}-c$ vanishes. This indicates that the barotropic modes are contiguous with neutral modes, and is a result consistent with previous studies $[K u o, 1949]$. The dominant (baroclinic) mode sets the direction of the momentum fluxes in regions 1-4 and the mode primarily dependent on $F, \beta$, and $\delta$ and not on the upperlayer PV gradient in particular. In region 5, the baroclinic mode's growth rate decreases but the barotropic mode's growth rate increases and becomes dominant.

The direction of the momentum fluxes in the baroclinic mode can be visualized geometrically (Fig. 4). When the stream function is tilted with the flow, then the fluxes are upgradient and energy is being fed back into the mean, but when the stream function is tilted against the flow, then the momentum fluxes are downgradient and energy is being extracted from the mean. In region 2 near the center of the jet, the stream function is tilted against the flow, leading to downgradient momentum fluxes. In region 3 near the center of the jet, the stream function is tilted with the flow, indicating upgradient momentum fluxes. This shows us that the particular arrangement of the stream function in the baroclinic mode is important for setting the direction of the momentum fluxes.

As I increase $\delta$ similar structures appear but the line that separates upgradient and downgradient momentum fluxes moves to the right (larger $\beta \delta^{2}$ ), eventually until there is no region 4 (Fig. 5). This indicates the role of the channel walls in setting the structure of the streamfunction and the direction of the fluxes.

\subsection{Physical interpretation}

As I reduce $F$, I reduce the coupling between the layers or increase the internal deformation radius. Physically this corresponds to a weakening of the baroclinic mode, largely without modifying the barotropic mode. The deformation radius becomes much larger than the length scale defining the horizontal shear $\left(F \delta^{2}<<1\right)$, making PV gradients and growth rates dominated by the horizontal shear. When $F \delta^{2}>>1$ the baroclinic mode dominates. This result is discussed at length in Killworth [1980].

\section{Cosine Jet}

In the previous section, I examined the cases where there was always a sign change between the two layers, which doesn't allow an examination of cases with no sign change between two layers. By examining set-ups where there is no change in the PV gradient between layers but a change within each layer, I examine cases that are analogous to the Gaussian jet set-up where there is no change in the PV gradient within the layer. By comparing these two cases, I expect a super-symmetry in this system because baroclinic and barotropic instability are described by the same eigenvalue problem [Drazin and Reid, 2004] and a just a rotation of the same system. 


\subsection{Set up}

I set up a cosine jet in this case, so that I can set up a flow where the potential vorticity gradient can be the same sign between the two layers but a different sign within the layer (Fig. 6). I introduce topography into my lower layer in order to force the PV gradients to change sign at the same location. My flow is then:

$$
\begin{aligned}
& U_{1}=1 / 2+1 / 2 \cos (\pi y) \\
& U_{2}=0
\end{aligned}
$$

The topography that I construct is

$$
\frac{\partial \eta_{b}}{\partial y}=\gamma \cos (\pi y)+C
$$

The potential vorticity gradient

$$
\begin{aligned}
& \frac{\partial Q_{1}}{\partial y}=\beta+\frac{\pi^{2}}{2} \cos (\pi y)+\frac{F_{1}}{2}(1+\cos (\pi y)) \\
& \frac{\partial Q_{2}}{\partial y}=\beta-\frac{F_{2}}{2}(1+\cos (\pi y))+\gamma \cos (\pi y)+C
\end{aligned}
$$

where $\gamma=8$ is the magnitude of the topography variation and $C$ is the magnitude of the constant slope added to enforce the condition that the PV gradient change sign at the same location in both layers.

\subsection{Results}

I analyze this configuration following section 3 by varying $F$ and $\beta$ and interpreting the eddy energy conversion. There are three distinct regions in the domain (Fig. 7). Region 1 has upgradient buoyancy fluxes and downgradient momentum fluxes and has no sign change between the two layers. Region 2 has downgradient buoyancy fluxes and downgradient momentum fluxes and has no sign change between the two layers (this is counter intuitive). Region 3 has downgradient buoyancy fluxes and has a sign change between the two layers. Notice that unlike the Gaussian jet case, there is no region with upgradient buoyancy fluxes but a sign change between the layers.

In comparing region 1 and region 2, the buoyancy fluxes change from upgradient to downgradient (Fig. 8). This is seen in the barotropic mode of the flow. As the sign change between the two layers is introduced a baroclinic mode is introduced, just as I found in section 3.2 for the barotropic mode. I see a symmetry in the modes that appear.

\subsection{Physical Interpretation}

As I increase $F$, I increase the coupling between the two layers so the baroclinic mode becomes stronger and eventually dominates. With a smaller $\beta$ I see a larger region of down-gradient buoyancy fluxes but no sign change. In the Gaussian jet case the walls act to eliminate region 4 , or the region with upgradient momentum fluxes with a sign change in the upper layer PV gradient, but in this case there is no region with upgradient buoyancy fluxes with a sign change between the layers. The QG approximation involves assuming that the layers are thin, which seems to be preventing a region with upgradient buoyancy fluxes and a sign change between the layers, just like the walls prevent region 4 in the Gaussian jet. 


\section{Conclusion}

In this report, I have shown that the change in sign of the PV gradient within a layer (between layers) is not generally responsible for setting the sign of the eddy momentum (buoyancy) fluxes. I have shown that the relative magnitude of the eddy PV of the two layers is fundamental for determining the direction of the eddy momentum fluxes. The relative magnitude of the eddy PV is set by the PV gradients, the position of the walls, and the structure of the most unstable mode, but there is not a straightforward way to determine the relative magnitude.

\section{References}

Charney, J. G., The Dynamics of Long Waves in a Baroclinic Westerly Current, doi:10.1175/15200469(1947)0040136:TDOLWI>2.0.CO;2, 1947.

Charney, J. G., and M. E. Stern, On the Stability of Internal Baroclinic Jets in a Rotating Atmosphere, Journal of the Atmospheric Sciences, 19(2), 159-172, doi:10.1175/15200469(1962)019<0159:OTSOIB>2.0.CO;2, 1962.

Drazin, P. G., and L. N. Howard, Hydrodynamic Stability of Parallel Flow of Inviscid Fluid, Advances in Applied Mechanics, 9(C), 1-89, doi:10.1016/S0065-2156(08)70006-1, 1966.

Drazin, P. G., and W. H. Reid, Hydrodynamic Stability, Cambridge University Press, p. 605, doi:10.1017/CBO9780511616938, 2004.

Eady, E. T., Long Waves and Cyclone Waves, Tellus, 1(3), 33-52, doi:10.3402/tellusa.v1i3.8507, 1949.

Gent, P. R., and J. C. McWilliams, Isopycnal Mixing in Ocean Circulation Models, Journal of Physical Oceanography, 20(1), 150-155, doi:10.1175/1520-0485(1990)020<0150:IMIOCM $>2.0 . C O ; 2$, 1990.

Held, I. M., Momentum Transport by Quasi-Geostrophic Eddies, Journal of the Atmospheric Sciences. doi.org/10.1175/1520-0469(1975)032<1494:MTBQGE>2.0.CO;2

Holland, W. R., and D. B. Haidvogel, A parameter study of the mixed instability of idealized ocean currents, Dynamics of Atmospheres and Oceans, 4(3), 185-215, doi:10.1016/0377-0265(80)900147,1980 .

Killworth, P. D., Barotropic and baroclinic instability in rotating stratified fluids, Dynamics of Atmospheres and Oceans, 4(3), 143-184, doi:10.1016/0377-0265(80)90013-5, 1980.

Kuo, H.-1., Dynamic Instability of Two-dimensional Nondivergent Flow in a Barotropic Atmosphere, Journal of Meterology, 6(2), 105-122, doi.org/10.1175/1520-0469(1949)006<0105:DIOTDN >2.0.CO;2 1949

Marshall, J., and K. Speer, Closure of the meridional overturning circulation through Southern Ocean upwelling, Nature Geoscience, 5(3), 171-180, doi:10.1038/ngeo1391, 2012.

Pedlosky, J., The Stability of Currents in the Atmosphere and the Ocean: Part II, doi:10.1175/15200469(1964)021๔0342:TSOCIT>2.0.CO;2, 1964. 
Pedlosky, J., The nonlinear dynamics of baroclinic wave ensembles, J. Fluid Mech., 102(-1), 169209, doi:10.1017/S0022112081002590, 1981.

Pedlosky, J., Geophysical Fluid Dynamics, New York and Berlin, Springer-Verlag, 1982. 636 p. 742, doi:10.1007/978-1-4612-4650-3, 1987.

Rayleigh, L., On the Stability, or Instability, of Certain Fluids Motions II., Proceedings of the London Mathematical Society, 1(1), 57-72, doi:10.1112/plms/s1-11.1.57, 1880.

Vallis, G. K., Atmospheric and Oceanic Fluid Dynamics, Cambridge University Press, p. 773, doi:10.1002/qj.186, 2006. 

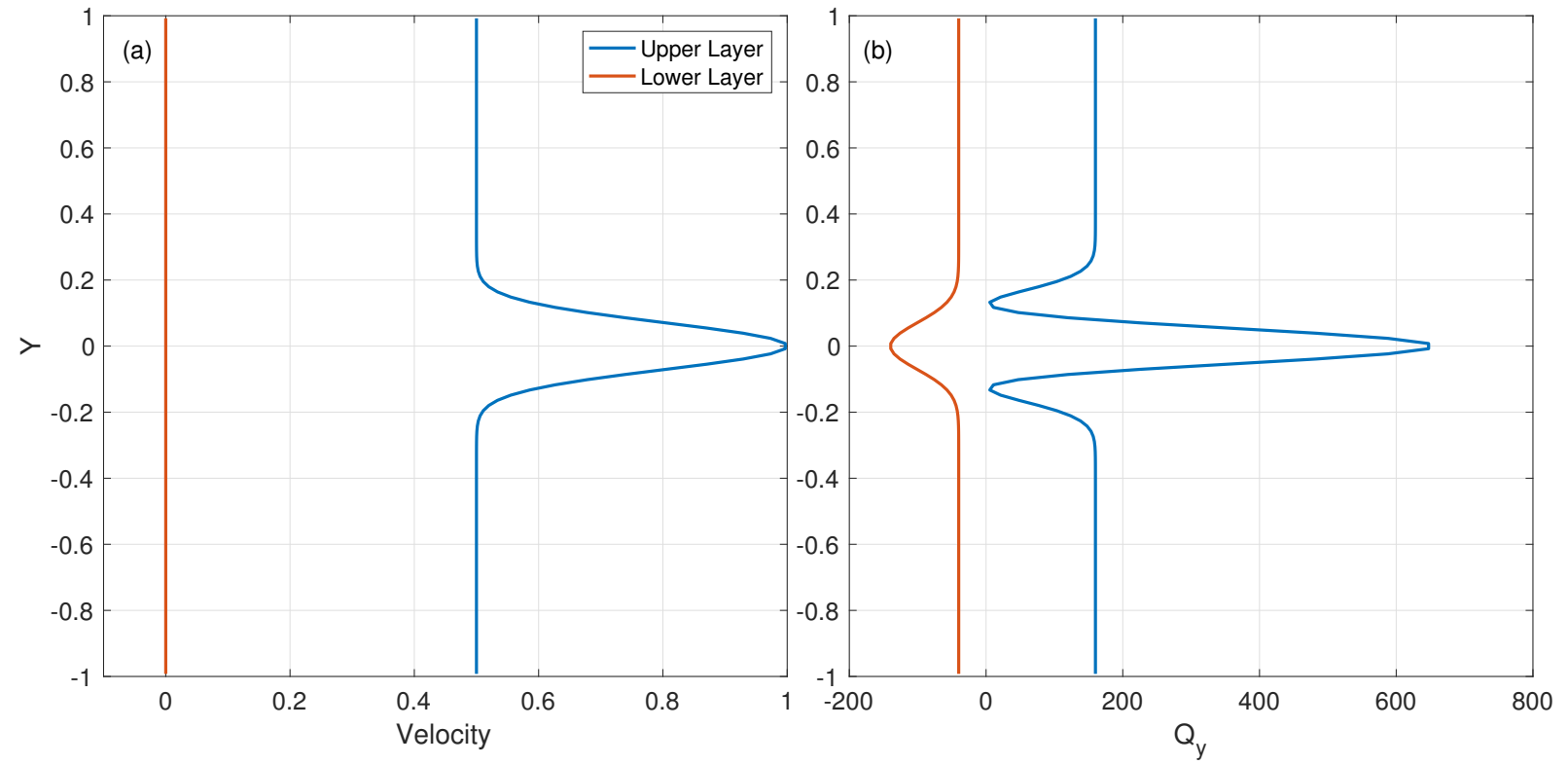

Figure 1: An example velocity profile and PV gradient for $\beta=60, F=100$ and $\delta=0.1$. 


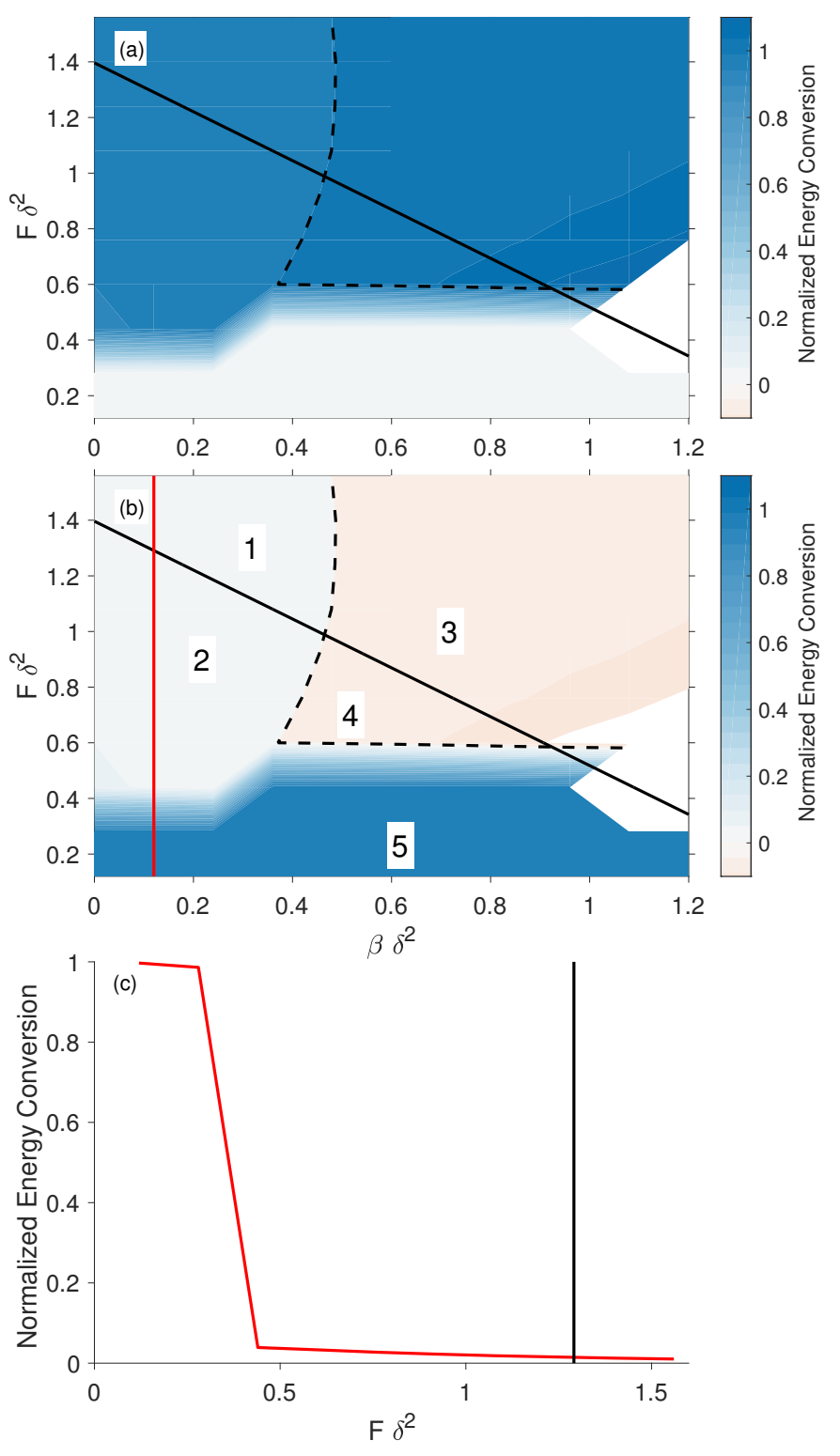

Figure 2: Energy conversion computed for the most unstable eigenfunctions normalized by total (potential plus kinetic) energy conversion at $\delta=0.05$. Potential energy conversion (a) and kinetic energy conversion (b). The domain is divided into 5 regions. The dotted line shows where the momentum fluxes change sign and the solid line shows where the upper layer PV gradient changes sign, with no sign change to the right and a sign change to the left. Blue represents downgradient fluxes and red represents upgradient fluxes. A cross section of kinetic energy conversion (c) as shown in red line of panel (b). 

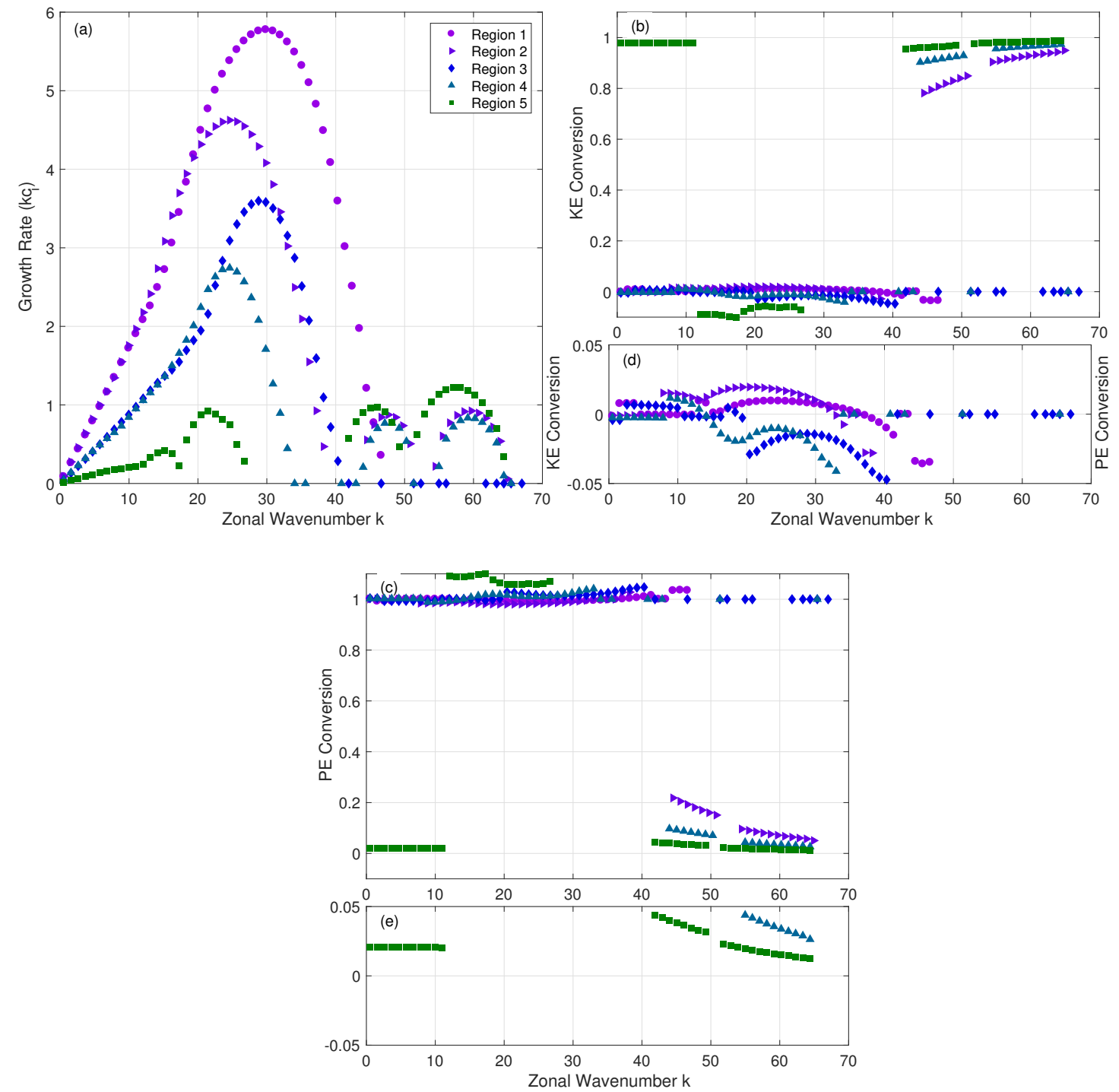

Figure 3: Example growth rates and normalized energy conversions from 5 different regions. Growth rates (a), kinetic energy conversion (b,d) and potential energy conversion (c,e). Region 1: $\beta=120$, $\delta=0.05, F=520$. Region $2: \beta=80, \delta=0.05, F=360$. Region $3: \beta=280, \delta=0.05, F=400$. Region 4: $\beta=200, \delta=0.05, F=280$. Region $5: \beta=240, \delta=0.05, F=200$. 

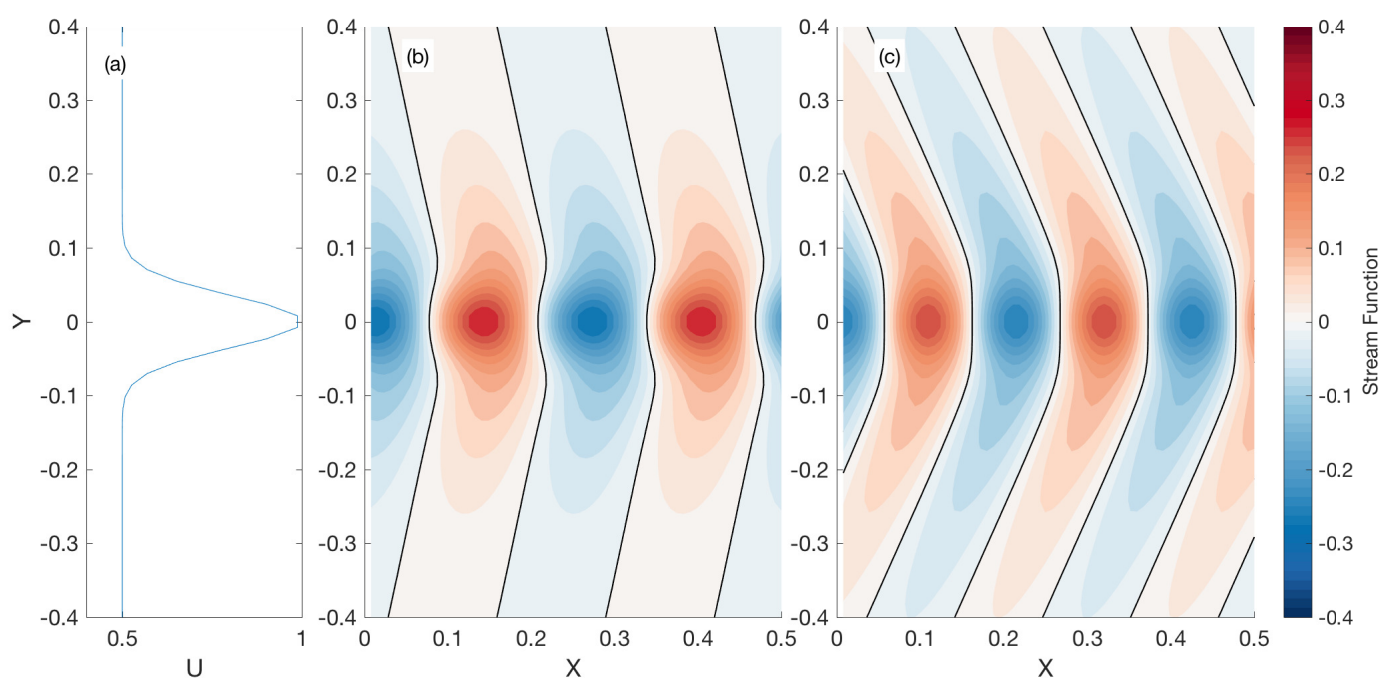

Figure 4: A jet profile (a) with the most unstable eigenfunction plotted in space given at $\beta=20$, $\delta=0.05$ and $F=360$ in region 2 (b) and at $\beta=320, \delta=0.05$ and $F=400$ in region 3 (c). 


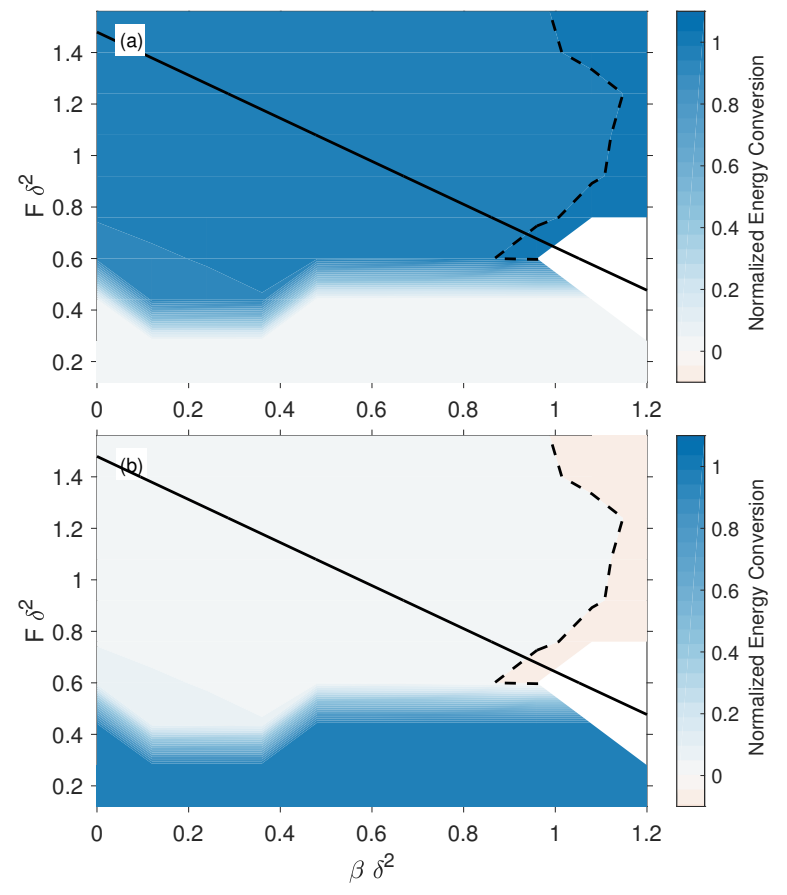

Figure 5: Same as Fig. 2 but for $\delta=0.3$. 

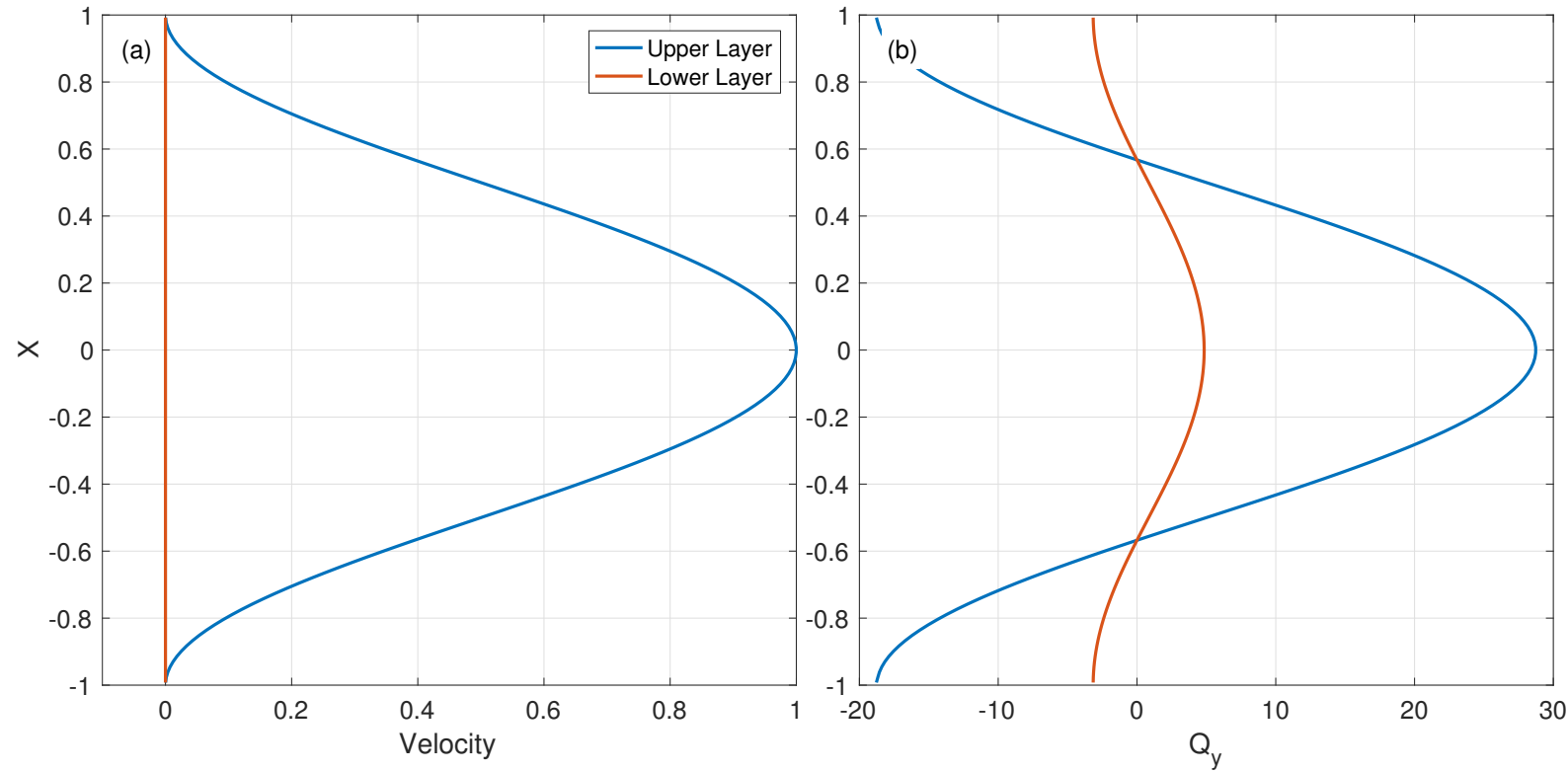

Figure 6: An example velocity profile and PV gradient for $\beta=1, F=4$ for cosine jet case. 

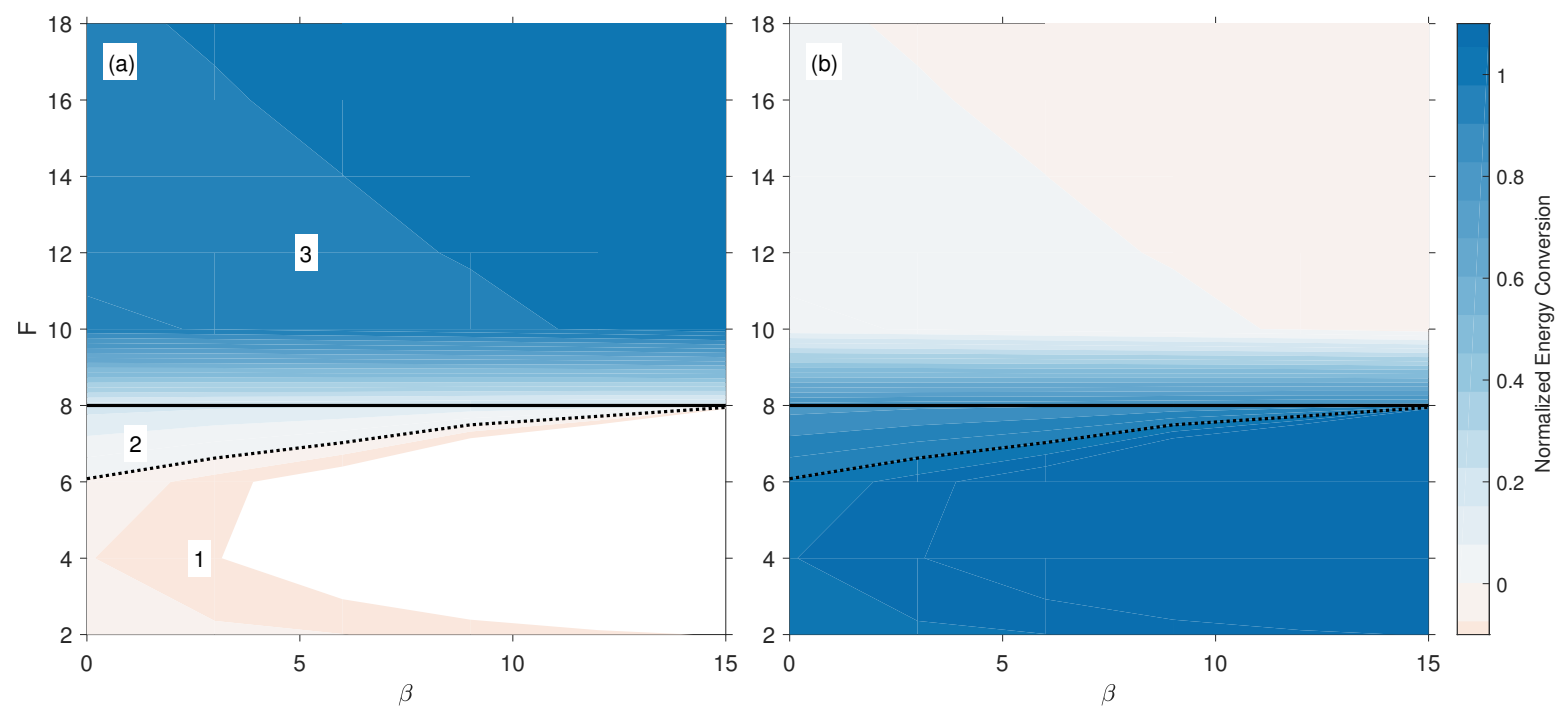

Figure 7: Energy conversion computed for the most unstable eigenfunctions normalized by total (potential plus kinetic) energy conversion for cosine jet. Potential energy conversion (a) and kinetic energy conversion (b). The dotted line shows where the momentum fluxes change sign and the solid line shows where the PV gradient changes sign between layers, with no sign below and a sign change above. Blue represents downgradient fluxes and red represents up-gradient fluxes. 

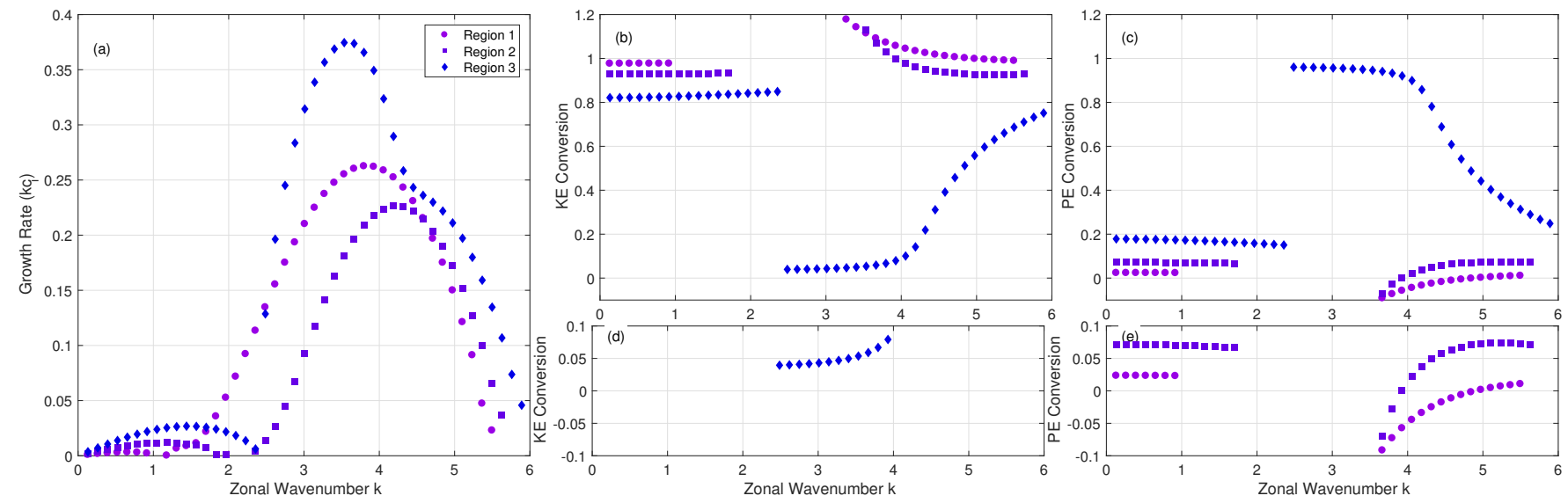

Figure 8: Example growth rates and normalized energy conversions from 3 different regions. Growth rates (a), kinetic energy conversion $(b, d)$ and potential energy conversion (c,e). See text for locations. Region 1: $F=4, \beta=1$. Region 2: $F=7, \beta=1$. Region 3: $F=10, \beta=1$. 


\title{
Global Stability of 2D Plane Couette Flow Beyond the Energy Stability Limit
}

\author{
Federico Fuentes
}

January, 2018

\section{Introduction}

The field of hydrodynamic stability studies the transient responses of an initial perturbation around a known steady flow. Due to being fundamental in the understanding of transition to turbulence, it has garnered the attention of influential scientists over the years, including Reynolds, Orr, and Heisenberg, among others [4].

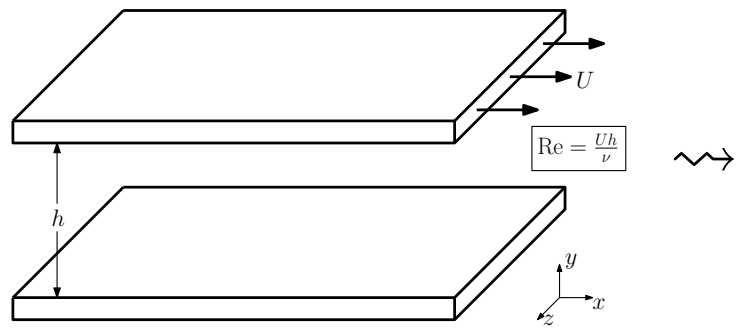

3D Plane Couette Flow

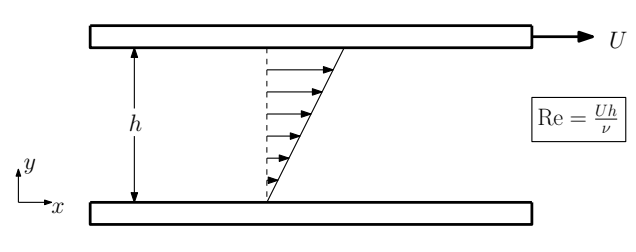

2D Plane Couette Flow

Figure 1.1: Diagram illustrating plane Couette flow (in 3D and 2D) with the corresponding system of coordinates, Reynolds number and steady laminar flow.

Sound theoretical results matching experiments have readily been found in many cases, such as Taylor-Couette flow [17], but others have remained more elusive. One classical example is plane Couette flow, which, as shown in Figure 1.1, is the flow between two infinite parallel plates separated by a distance $h$ with the top plate moving at speed $U$ in a direction parallel to the plates. The Reynolds number is defined as $\operatorname{Re}=\frac{U h}{\nu}>0$, where $\nu$ is the kinematic viscosity, while $x, y$ and $z$ are called the axial, wall-normal and transverse directions respectively. A linear shear flow is well-known to be the steady laminar equilibrium (see Figure 1.1), and it is the evolution of perturbations about this flow that are of interest.

This flow was proved by Romanov [15] to have a linear stability limit of $\operatorname{Re}_{L}=\infty$, meaning that it is linearly stable for any Reynolds number. That is, there always exist initial perturbations, $\mathbf{u}(0)$, under an infinitesimal energy which will decay (i.e., $\mathbf{u}(t) \rightarrow \mathbf{0}$ as $t \rightarrow \infty)$ to the laminar steady flow. Hence, other notions of stability must be studied to explain when and how the flow becomes unstable. With this in mind, it is important to look at global stability, meaning that every initial perturbation $\mathbf{u}(0)$ decays, and conditional 
stability, meaning that a certain subset of initial perturbations $\mathbf{u}(0)$ decay. The global stability limit, denoted $\operatorname{Re}_{G}$, is the largest number under which the flow is globally stable.

The problem of finding the global stability limit of $2 \mathrm{D}$ plane Couette flow dates back to 1907 [13], over 110 years ago, when William McFadden Orr found a lower bound for this flow's global stability limit, the so-called energy stability limit, $\operatorname{Re}_{E}=177.2 \leq \operatorname{Re}_{G}$. He did so using what we now call the energy method, first proposed by Reynolds shortly before, and since then, this has been the only systematic mechanism to rigorously establish lower bounds to the global stability limit of fluid flows. In some rare situations, ad hoc techniques can be developed to find a larger lower bound, but in general this is a difficult task, which, in over a century, has proved to be an unsuccessful endeavor for the particular case of plane Couette flow. The purpose of this work is to find a better lower bound to the $\operatorname{Re}_{G}$ of $2 \mathrm{D}$ plane Couette flow using techniques that can additionally be applied to other flows in the future.

It is worth noting that $2 \mathrm{D}$ plane Couette flow (right of Figure 1.1) is the transverselyindependent $\left(\frac{\partial(\cdot)}{\partial z}=0\right)$ simplification of 3D plane Couette flow (left of Figure 1.1). In 3D plane Couette flow, the energy stability limit was proved to be actually lower than the $2 \mathrm{D}$ counterpart, $\operatorname{Re}_{E}=82.6[1,11,10,16]$, but once again no lower bound to $\operatorname{Re}_{G}$ beyond $\operatorname{Re}_{E}$ has been established. A theoretical upper bound to $\operatorname{Re}_{G}$ was determined for 3D plane Couette flow by the 3D finite-amplitude periodic solutions found by Nagata [12], which occur at about $\operatorname{Re}=500$. Meanwhile, experiments place upper bounds of $\operatorname{Re}_{G}$ on $3 \mathrm{D}$ plane Couette flow to be around $\mathrm{Re}=1300[8,3,18]$. By contrast, no upper bounds of $\operatorname{Re}_{G}$ have been found for $2 \mathrm{D}$ plane Couette flow either through theoretical means or numerical simulations [14]. Thus, it could be true that $\operatorname{Re}_{G}=\infty$ for $2 \mathrm{D}$ plane Couette flow, and finding a set of increasing lower bounds of $\operatorname{Re}_{G}$ could shed some light onto this open question.

This report is organized as follows. In Section 2 a brief review of how to write a fluid system as an uncertain dynamical system will be given. The energy method used to prove global stability will be described, and an alternative based on new techniques coming from sum-of-squares (SOS) polynomials optimization will be introduced. These techniques allow to reduce the problem to a tractable semidefinite program (SDP) which can be solved using a computer. They will produce high-order Lyapunov functions more general than the energy. In Section 3, solving the energy eigenvalue problem for 2D plane Couette flow will be outlined. Section 4 will present the results and discussion, while Section 5 will contain the concluding remarks. Lastly, the family of Appendices A-E will have extensive technical details associated to the computations and mathematical derivations.

\section{Review of Fluid Dynamical Systems}

What follows is a brief review of the uncertain fluid dynamical system first presented in [7].

Assume $\Omega$ is a bounded domain, and boundary conditions for the fluid velocity $\mathbf{v}$ and pressure $p_{0}$ consist of a combination of fixed known velocities and periodicity of the velocity and pressure fields, which additionally satisfy the nondimensional Navier-Stokes equations,

$$
\begin{aligned}
\frac{\partial \mathbf{v}}{\partial t}+\mathbf{v} \cdot \nabla \mathbf{v} & =-\nabla p_{0}+\frac{1}{\operatorname{Re}} \nabla^{2} \mathbf{v}+\mathbf{f}_{g} \\
\nabla \cdot \mathbf{v} & =0
\end{aligned}
$$


where $\mathbf{f}_{g}$ represents the gravity effects. Provided a steady solution, $\mathbf{V}$ and $P$, is known, the Navier-Stokes equations become

$$
\begin{aligned}
\frac{\partial \mathbf{u}}{\partial t}+\mathbf{u} \cdot \nabla \mathbf{u}+\mathbf{u} \cdot \nabla \mathbf{V}+\mathbf{V} \cdot \nabla \mathbf{u} & =-\nabla p+\frac{1}{\operatorname{Re}} \nabla^{2} \mathbf{u}, \\
\nabla \cdot \mathbf{u} & =0,
\end{aligned}
$$

where the unknown perturbations $\mathbf{u}=\mathbf{v}-\mathbf{V}$ and $p=p_{0}-P$ satisfy no-slip boundary conditions $(\mathbf{u}=\mathbf{0})$ wherever $\mathbf{v}$ is fixed, and periodic boundary conditions elsewhere.

Consider the following series expansion for the perturbation velocity field,

$$
\begin{aligned}
\mathbf{u}(\mathbf{x}, t) & =\sum_{i=1}^{m} a_{i}(t) \mathbf{u}_{i}(\mathbf{x})+\mathbf{u}_{\mathbf{s}}(\mathbf{x}, t), \\
\nabla \cdot \mathbf{u}_{i}=0, \quad\left\langle\mathbf{u}_{i}, \mathbf{u}_{j}\right\rangle & =\delta_{i j}, \quad \nabla \cdot \mathbf{u}_{\mathbf{s}}=0, \quad\left\langle\mathbf{u}_{\mathbf{s}}, \mathbf{u}_{i}\right\rangle=0,
\end{aligned}
$$

where $\delta_{i j}$ is the Kronecker delta. Hence, the basis fields, $\mathbf{u}_{i}$, and the residual perturbation velocity, $\mathbf{u}_{\mathbf{s}}$, are solenoidal, meaning their divergence vanishes and implying that the incompressibility of the perturbation velocity is satisfied, $\nabla \cdot \mathbf{u}=0$. Moreover, the $\mathbf{u}_{i}$ are orthonormal in the $L^{2}$ inner product, and $\mathbf{u}_{\mathbf{s}}$ is orthogonal to all the $\mathbf{u}_{i}$. Here, $\langle\cdot, \cdot\rangle$ is the $L^{2}$ inner product, so that

$$
\left\langle\mathbf{w}_{1}, \mathbf{w}_{2}\right\rangle=\int_{\Omega} \mathbf{w}_{1} \cdot \mathbf{w}_{2} \mathrm{~d} \Omega, \quad\|\mathbf{w}\|^{2}=\langle\mathbf{w}, \mathbf{w}\rangle=\int_{\Omega}|\mathbf{w}|^{2} \mathrm{~d} \Omega
$$

where $\|\cdot\|$ is the $L^{2}$ norm, and $|\cdot|$ is the usual Euclidean norm of a vector.

Next, let $\mathbf{a}(t)=\left[a_{1}(t), \ldots, a_{m}(t)\right] \in \mathbb{R}^{m}$ and $q(t)=\left\|\mathbf{u}_{\mathbf{s}}(t)\right\|$, so that the perturbation energy is $\|\mathbf{u}(t)\|^{2}=|\mathbf{a}(t)|^{2}+q^{2}(t)$. Chernyshenko and Goulart [7] show that the dynamical system $\widetilde{\mathbf{a}}(t)=\left(\mathbf{a}(t), q^{2}(t)\right)$ describing the perturbation velocity is

$$
\begin{aligned}
\frac{\mathrm{d} \mathbf{a}}{\mathrm{d} t} & =\mathbf{f}(\mathbf{a})+\boldsymbol{\Theta}_{\mathbf{a}}\left(\mathbf{u}_{\mathbf{s}}\right)+\boldsymbol{\Theta}_{\mathbf{b}}\left(\mathbf{u}_{\mathbf{s}}, \mathbf{a}\right)+\boldsymbol{\Theta}_{\mathbf{c}}\left(\mathbf{u}_{\mathbf{s}}\right), \\
\frac{1}{2} \frac{\mathrm{d} q^{2}}{\mathrm{~d} t} & =-\mathbf{a} \cdot\left(\boldsymbol{\Theta}_{\mathbf{a}}\left(\mathbf{u}_{\mathbf{s}}\right)+\boldsymbol{\Theta}_{\mathbf{b}}\left(\mathbf{u}_{\mathbf{s}}, \mathbf{a}\right)+\boldsymbol{\Theta}_{\mathbf{c}}\left(\mathbf{u}_{\mathbf{s}}\right)\right)+\Gamma\left(\mathbf{u}_{\mathbf{s}}\right)+\chi\left(\mathbf{u}_{\mathbf{s}}, \mathbf{a}\right),
\end{aligned}
$$

where

$$
\begin{array}{rlrl}
f_{i}(\mathbf{a}) & =\overbrace{\left(\frac{1}{\operatorname{Re}}\left\langle\mathbf{u}_{i}, \nabla^{2} \mathbf{u}_{j}\right\rangle-\left\langle\mathbf{u}_{i}, \mathbf{u}_{j} \cdot \nabla \mathbf{V}+\mathbf{V} \cdot \nabla \mathbf{u}_{j}\right\rangle\right) a_{j}}^{(\mathbf{L} \cdot \mathbf{a})_{i}}+\overbrace{\left(-\left\langle\mathbf{u}_{i}, \mathbf{u}_{j} \cdot \nabla \mathbf{u}_{k}\right\rangle\right) a_{j} a_{k}}, \\
\Theta_{\mathbf{a} i}\left(\mathbf{u}_{\mathbf{s}}\right) & =\left\langle\mathbf{u}_{\mathbf{s}}, \mathbf{h}_{i 0}\right\rangle, & \mathbf{h}_{i 0}=\frac{1}{\operatorname{Re}} \nabla^{2} \mathbf{u}_{i}+\mathbf{V} \cdot \nabla \mathbf{u}_{i}-\mathbf{u}_{i} \cdot \nabla^{\mathrm{T}} \mathbf{V}, \\
\Theta_{\mathbf{b} i}\left(\mathbf{u}_{\mathbf{s}}, \mathbf{a}\right) & =\left\langle\mathbf{u}_{\mathbf{s}}, \mathbf{h}_{i j}\right\rangle a_{j}, & & \mathbf{h}_{i j}=\mathbf{u}_{j} \cdot \nabla \mathbf{u}_{i}-\mathbf{u}_{i} \cdot \nabla^{\mathrm{T}} \mathbf{u}_{j}, \\
\Theta_{\mathbf{c} i}\left(\mathbf{u}_{\mathbf{s}}\right) & =\left\langle\mathbf{u}_{\mathbf{s}}, \mathbf{u}_{\mathbf{s}} \cdot \nabla \mathbf{u}_{i}\right\rangle, & \\
\Gamma\left(\mathbf{u}_{\mathbf{s}}\right) & =\frac{1}{\operatorname{Re}}\left\langle\mathbf{u}_{\mathbf{s}}, \nabla^{2} \mathbf{u}_{\mathbf{s}}\right\rangle-\left\langle\mathbf{u}_{\mathbf{s}}, \mathbf{D} \mathbf{u}_{\mathbf{s}}\right\rangle, & \\
\chi\left(\mathbf{u}_{\mathbf{s}}, \mathbf{a}\right) & =2\left\langle\mathbf{u}_{\mathbf{s}}, \mathbf{d}_{j}\right\rangle a_{j}, & \mathbf{d}_{j}=\frac{1}{\operatorname{Re}} \nabla^{2} \mathbf{u}_{j}-\mathbf{D} \mathbf{u}_{j},
\end{array}
$$

with $\mathbf{D}=\frac{1}{2}\left(\nabla \mathbf{V}+\nabla^{\top} \mathbf{V}\right)$ being the rate of strain tensor of the steady flow $\mathbf{V}$, and where $\nabla^{\top} \mathbf{w}=(\nabla \mathbf{w})^{\top}$ for any vector field $\mathbf{w}\left(\right.$ e.g. $\left(\mathbf{h}_{i j}\right)_{k}=\left(\mathbf{u}_{j}\right)_{l}\left(\nabla \mathbf{u}_{i}\right)_{l k}-\left(\mathbf{u}_{i}\right)_{l}\left(\nabla \mathbf{u}_{j}\right)_{k l}$ for $j \geq 1$, where $\left.(\nabla \mathbf{w})_{i j}=\frac{\partial w_{i}}{\partial x_{j}}\right)$. 
The evolution of the fluid dynamical system described through $\frac{\mathrm{d} \widetilde{a}}{\mathrm{~d} t}$ in $(2.5)$, is nonlinear in $\mathbf{a}(\operatorname{via} \mathcal{N}: \mathbf{a} \otimes \mathbf{a}$ in $\boldsymbol{\xi}(\mathbf{a})$ ), and more importantly it is uncertain in $q$ due to the fact that it is multivalued in that variable. Indeed, for a fixed $q$, there are multiple values $\mathbf{u}_{\mathbf{s}}$ such that $q=\left\|\mathbf{u}_{\mathbf{s}}\right\|$ meaning that $\frac{\mathrm{d} \widetilde{\mathbf{a}}}{\mathrm{d} t}$ can take multiple values for a single value of $q$.

\subsection{Lyapunov functionals and the energy method}

Now, consider a real-valued Lyapunov functional $V(\mathbf{u})$, with $V(\mathbf{0})=0$. Then, Lyapunov's theorem says that if $V$ is positive-definite and radially unbounded (i.e. $V(\mathbf{u})>0$ for all $\mathbf{u} \neq \mathbf{0}$ and $V(\mathbf{u}) \rightarrow \infty$ as $\|\mathbf{u}\| \rightarrow \infty$ ), and $\frac{\mathrm{d} V(\mathbf{u}(t))}{\mathrm{d} t}$ is negative definite (i.e. $\frac{\mathrm{d} V(\mathbf{u}(t))}{\mathrm{d} t}<0$ for all $\mathbf{u} \neq \mathbf{0}$ ), it follows that the flow is globally asymptotically stable (meaning that for every initial perturbation $\mathbf{u}(0)$ it follows $\mathbf{u}(t) \rightarrow \mathbf{0}$ as $t \rightarrow \infty)$.

The classical approach to proving global stability is to use the energy method, where the Lyapunov function is chosen as the perturbation energy, $V(\mathbf{u})=E=\frac{1}{2}\|\mathbf{u}\|^{2}$. In the simplest case, where $m=0$ in $(2.3)$, so $\mathbf{u}=\mathbf{u}_{\mathbf{s}}$ and $\widetilde{\mathbf{a}}(t)=q(t)=\|\mathbf{u}(t)\|$, then $V(\mathbf{u}(t))=E(t)=\frac{1}{2} q^{2}(t)$, a does not exist and (2.5) becomes

$$
\frac{\mathrm{d} E}{\mathrm{~d} t}=\frac{1}{2} \frac{\mathrm{d} q^{2}}{\mathrm{~d} t}=\Gamma(\mathbf{u})=\frac{1}{\operatorname{Re}}\left\langle\mathbf{u}, \nabla^{2} \mathbf{u}\right\rangle-\langle\mathbf{u}, \mathbf{D} \mathbf{u}\rangle .
$$

It is clear $V(\mathbf{0})=0, V(\mathbf{u})>0$ for all $\mathbf{u} \neq \mathbf{0}$ and more importantly $\frac{\mathrm{d} V}{\mathrm{~d} t}=\frac{\mathrm{d} E}{\mathrm{~d} t}$. Therefore, if $\frac{\mathrm{d} V}{\mathrm{~d} t} \leq \kappa_{\mathbf{S}} q^{2}$ for some $\kappa_{\mathbf{S}}<0$, the flow will be globally stable.

Thus, solving for the minimum $\kappa_{\mathrm{S}} \in \mathbb{R}$ such that $\frac{\mathrm{d} V}{\mathrm{~d} t} \leq \kappa_{\mathrm{S}} q^{2}$ yields a constrained minimization problem which is equivalent to an eigenvalue problem known as the energy eigenvalue problem,

$$
\begin{aligned}
-\lambda \mathbf{u} & =\mathbf{D} \mathbf{u}-\frac{1}{\operatorname{Re}} \nabla^{2} \mathbf{u}+\nabla \zeta, \\
\nabla \cdot \mathbf{u} & =0,
\end{aligned}
$$

which is solved for $\mathbf{u}$ and $\zeta$ satisfying the same boundary conditions as the perturbation velocity and pressure in (2.2). Its solution is the eigenvalues and eigenfunctions of the Hermitian (symmetric) energy operator, $\mathbf{A}_{E} \mathbf{u}=\frac{1}{\operatorname{Re}} \nabla^{2} \mathbf{u}-\mathbf{D u}-\nabla \zeta$, where $\zeta$ depends on $\mathbf{u}$ through the auxiliary Poisson problem $\nabla^{2} \zeta=-\nabla \cdot$ Du with the boundary conditions $\nabla \zeta \cdot \mathbf{n}=\left(\frac{1}{\mathrm{Re}} \nabla^{2} \mathbf{u}-\mathbf{D u}\right) \cdot \mathbf{n}$ wherever $\mathbf{u}$ has no-slip boundary conditions, and periodic otherwise. Recalling the meaning of $\kappa_{\mathbf{S}}$, yields that the flow is globally stable provided the largest eigenvalue of the energy operator is negative. The energy stability limit, $\mathrm{Re}_{E}$, is obtained by solving for Re in limiting case in which the largest eigenvalue is 0 . This implies any $\operatorname{Re}<\operatorname{Re}_{E}$ is associated to a negative eigenvalue, so that the flow is globally stable, and thus $\operatorname{Re}_{E}$ is a lower bound of the global stability limit, $\operatorname{Re}_{G} \geq \operatorname{Re}_{E}$.

Despite being very practical, it is clear that the energy method is simply a special choice of quadratic Lyapunov functional in the context of a much more general theorem. The ideal scenario would be to find other Lyapunov functionals that hopefully allow to establish that the flow can be globally stable for values of $R e$ above $R_{E}$. It is possible to deduce that if this is desired, then high-order (above quadratic) Lyapunov functionals that are not powers of the energy should be considered. In view of the form of the uncertain system (2.5), look at high-order polynomial functions of the form $V(\mathbf{u})=V\left(\mathbf{a}, q^{2}\right)$ (with $m>0$ ). Additionally, note that the uncertain terms in (2.5) do not impede the use of the Lyapunov theorem as 
long as the terms dependent on $\mathbf{u}_{\mathbf{s}}$ (namely $\boldsymbol{\Theta}_{\mathbf{a}}, \boldsymbol{\Theta}_{\mathbf{b}}, \boldsymbol{\Theta}_{\mathbf{c}}, \Gamma$ and $\chi$ ) are bounded in some sense in terms of a single valued function of $\mathbf{a}$ and $q$.

Before proceeding, it is useful to simplify (2.5) further if possible. In this sense, the energy eigenvalue problem is of practical use. Indeed, from now on, assume that the basis fields $\mathbf{u}_{i}$ for $i=1, \ldots, m$ are a subset of the energy eigenfunctions. That is, for each $i=1, \ldots, m$ assume there exist $\zeta_{i}$ and $\lambda_{i} \in \mathbb{R}$ such that $\left(\mathbf{u}_{i}, \zeta_{i}, \lambda_{i}\right)$ is a solution to the energy eigenvalue problem in (2.8). Under this assumption of the $\mathbf{u}_{i}$, it follows $\chi\left(\mathbf{u}_{\mathbf{s}}, \mathbf{a}\right)=0$ and $\Gamma\left(\mathbf{u}_{\mathbf{s}}\right) \leq \kappa q^{2}$, where $\kappa$ is the largest eigenvalue of (2.8) different from all the $\lambda_{i}$ for $i=1, \ldots, m$.

Then, provided $\frac{\partial V}{\partial q^{2}} \geq 0$, it is obvious that

$$
\begin{aligned}
\frac{\mathrm{d} V}{\mathrm{~d} t} & =\underbrace{\frac{\partial V}{\partial \mathbf{a}} \cdot \mathbf{f}(\mathbf{a})+2 \frac{\partial V}{\partial q^{2}} \Gamma\left(\mathbf{u}_{\mathbf{s}}\right)}_{G\left(\mathbf{a}, q^{2}, \mathbf{u}_{\mathbf{s}}\right)}+\underbrace{\left(\frac{\partial V}{\partial \mathbf{a}}-2 \frac{\partial V}{\partial q^{2}} \mathbf{a}\right)}_{\mathbf{M}\left(\mathbf{a}, q^{2}\right)} \cdot(\underbrace{\boldsymbol{\Theta}_{\mathbf{a}}\left(\mathbf{u}_{\mathbf{s}}\right)+\boldsymbol{\Theta}_{\mathbf{b}}\left(\mathbf{u}_{\mathbf{s}}, \mathbf{a}\right)}_{\boldsymbol{\Theta}_{\mathbf{a b}}\left(\mathbf{u}_{\mathbf{s}}, \mathbf{a}\right)}+\boldsymbol{\Theta}_{\mathbf{c}}\left(\mathbf{u}_{\mathbf{s}}\right)) \\
& \leq \underbrace{\frac{\partial V}{\partial \mathbf{a}} \cdot \mathbf{f}(\mathbf{a})+2 \frac{\partial V}{\partial q^{2}} \kappa q^{2}}_{\tilde{G}\left(\mathbf{a}, q^{2}\right)}+\Xi\left(\mathbf{a}, q^{2}\right),
\end{aligned}
$$

for some $\Xi\left(\mathbf{a}, q^{2}\right)$ satisfying $\mathbf{M}\left(\mathbf{a}, q^{2}\right) \cdot\left(\boldsymbol{\Theta}_{\mathbf{a b}}\left(\mathbf{u}_{\mathbf{s}}, \mathbf{a}\right)+\boldsymbol{\Theta}_{\mathbf{c}}\left(\mathbf{u}_{\mathbf{s}}\right)\right) \leq \Xi\left(\mathbf{a}, q^{2}\right)$, with $\Xi(\mathbf{0}, 0)=0$. Finally, the idea is to use sum-of-squares (SOS) polynomial constraints to setup a semidefinite program (SDP) that ensures that $V(\mathbf{0}, 0)=0$ and $\Xi(\mathbf{0}, 0)=0$, and that $V>0$, $\frac{\partial V}{\partial q^{2}} \geq 0$ and $\tilde{G}+\Xi<0$ whenever $\left(\mathbf{a}, q^{2}\right) \neq(\mathbf{0}, 0)$.

The details to construct a valid $\Xi\left(\mathbf{a}, q^{2}\right)$ and to properly set up the SDP are of tantamount importance (there is no unique way of doing this). Having said that, the details are quite technical and better left for the Appendices, where several techniques were attempted. The only details worth repeating here are the bounds of each component of $\boldsymbol{\Theta}_{\mathbf{a b}}$ and $\boldsymbol{\Theta}_{\mathbf{c}}$, first derived in $[7,9]$. Let $\tilde{\mathbf{a}}=\left[1, a_{1}, \ldots, a_{m}\right]$, indexed from 0 so that $\tilde{a}_{0}=1$ and $\tilde{a}_{i}=a_{i}$ for $i=1, \ldots, m$. Then, $\Theta_{\mathbf{a b} i}\left(\mathbf{u}_{\mathbf{s}}, \mathbf{a}\right)=\left\langle\mathbf{u}_{\mathbf{s}}, \mathbf{h}_{i j}\right\rangle \tilde{a}_{j}$, and the bounds are,

$$
\begin{aligned}
\left|\Theta_{\mathbf{a b} i}\left(\mathbf{u}_{\mathbf{s}}, \mathbf{a}\right)\right| & \leq \sqrt{\tilde{\mathbf{a}}^{\top} \mathbf{R}_{i} \tilde{\mathbf{a}} q^{2}}, & \left(R_{i}\right)_{k l} & =\left\langle\widetilde{\mathbf{h}}_{i k}, \widetilde{\mathbf{h}}_{i l}\right\rangle, \\
\left|\Theta_{\mathbf{c} i}\left(\mathbf{u}_{\mathbf{s}}\right)\right| & \leq C_{i} q^{2}, & C_{i} & =\left\|\rho\left(\mathbf{D}_{i}\right)\right\|_{\infty}=\sup _{\mathbf{x} \in \Omega} \rho\left(\mathbf{D}_{i}(\mathbf{x})\right),
\end{aligned}
$$

where $\mathbf{D}_{i}=\frac{1}{2}\left(\nabla \mathbf{u}_{i}+\nabla^{\top} \mathbf{u}_{i}\right)$, and $\rho\left(\mathbf{D}_{i}(\mathbf{x})\right)$ is the spectral radius of $\mathbf{D}_{i}(\mathbf{x})$. Here, $\widetilde{\mathbf{h}}_{i j}$ is the solenoidal projection (so $\nabla \cdot \widetilde{\mathbf{h}}_{i j}=0$ ) of $\mathbf{h}_{i j}$ such that $\left\langle\widetilde{\mathbf{h}}_{i j}, \mathbf{u}_{k}\right\rangle=0$ for all $k=1, \ldots, m$ and satisfying that $\widetilde{\mathbf{h}}_{i j} \cdot \mathbf{n}=0$ ( $\mathbf{n}$ is the outer normal) wherever the perturbation velocity has no-slip boundary conditions. These bounds are important as they eliminate $\mathbf{u}_{\mathbf{s}}$ and yield expressions only in terms of $a_{1}, \ldots, a_{m}$ and $q^{2}$.

\section{Solving for the Energy Eigenfunctions and Bounds}

Recall the setup and coordinates of 2D plane Couette flow in Figure 1.1 (or Figure 3.1). Assuming all parameters are naturally nondimensionalised, note that the well-known steady solution is $\mathbf{V}=\left[\begin{array}{l}y \\ 0\end{array}\right]$, which satisfies the nondimensional boundary conditions $\mathbf{V}=\mathbf{0}$ at $y=0$ and $\mathbf{V}=\left[\begin{array}{l}1 \\ 0\end{array}\right]$ at $y=1$. 


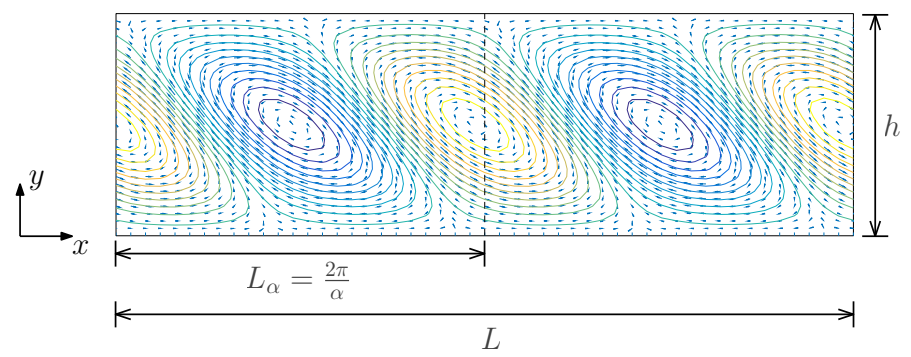

Figure 3.1: Diagram illustrating a periodic 2D plane Couette flow domain with the subperiods.

Assume a periodic $2 \mathrm{D}$ domain $\Omega=\left(0, L_{x}\right) \times(0,1)$ as in Figure $(3.1)$, and consider a perturbed velocity field $\mathbf{v}=\mathbf{V}+\mathbf{u}$ and pressure $p_{0}=P+p$ with respect to the steady solution $\mathbf{V}$ and $P$, such that the perturbation velocity and pressure are $\mathbf{u}=\left[\begin{array}{l}u_{x} \\ u_{y}\end{array}\right]$ and $p$ and subject to no-slip boundary conditions at both plates $\mathbf{u}(x, 0)=\mathbf{u}(x, 1)=\mathbf{0}$.

The energy eigenvalue problem is written in (2.8), and from what is known from the flow, it follows that it can be simplified to

$$
\begin{gathered}
-\lambda\left[\begin{array}{l}
u_{x} \\
u_{y}
\end{array}\right]=\left[\begin{array}{ll}
0 & \frac{1}{2} \\
\frac{1}{2} & 0
\end{array}\right]\left[\begin{array}{l}
u_{x} \\
u_{y}
\end{array}\right]-\frac{1}{\operatorname{Re}}\left[\begin{array}{l}
\frac{\partial^{2} u_{x}}{\partial x^{2}}+\frac{\partial^{2} u_{x}}{\partial y^{2}} \\
\frac{\partial^{2} u_{y}}{\partial x^{2}}+\frac{\partial^{2} u_{y}}{\partial y^{2}}
\end{array}\right]+\left[\begin{array}{l}
\frac{\partial \zeta}{\partial x} \\
\frac{\partial \zeta}{\partial y}
\end{array}\right] \\
\frac{\partial u_{x}}{\partial x}+\frac{\partial u_{y}}{\partial y}=0 .
\end{gathered}
$$

To eliminate $\zeta$ one can take the (2D) curl of (3.1) to get

$$
-\lambda \omega_{z}=(\nabla \times(\mathbf{D u}))_{z}-\frac{1}{\operatorname{Re}} \nabla^{2} \omega_{z}
$$

where $\omega_{z}=\frac{\partial u_{y}}{\partial x}-\frac{\partial u_{x}}{\partial y}$ and $(\nabla \times(\mathbf{D u}))_{z}=\frac{1}{2}\left(\frac{\partial u_{x}}{\partial x}-\frac{\partial u_{y}}{\partial y}\right)$

Moreover, since this is a $2 \mathrm{D}$ problem, there must exist a stream function $\psi$ automatically satisfying the continuity equation (3.2) with

$$
u_{x}=\frac{\partial \psi}{\partial y} \quad \text { and } \quad u_{y}=-\frac{\partial \psi}{\partial x} .
$$

In this case, verification of $\omega_{z}=-\nabla^{2} \psi$ is trivial, while $(\nabla \times(\mathbf{D u}))_{z}=\frac{\partial^{2} \psi}{\partial x \partial y}$, so (3.3) may be rewritten in terms of $\psi$ as

$$
\lambda \nabla^{2} \psi=\frac{\partial^{2} \psi}{\partial x \partial y}+\frac{1}{\operatorname{Re}} \nabla^{2}\left(\nabla^{2} \psi\right)
$$

The equation above is important, because it is sufficient (along with the boundary conditions) to solve the energy problem. The no-slip boundary conditions of the perturbation velocity in terms of the stream function are

$$
\begin{array}{ccc}
u_{x}=\frac{\partial \psi}{\partial y}=0 & \text { at } & y=0 \text { and } y=1 \text { for all } x \in\left(0, L_{x}\right), \\
u_{y}=-\frac{\partial \psi}{\partial x}=0 & \text { at } & y=0 \text { and } y=1 \text { for all } x \in\left(0, L_{x}\right) .
\end{array}
$$


As mentioned before, the perturbation velocity is assumed periodic in the $x$ direction, so $\psi$ must accept a Fourier series expansion. It is written as

$$
\psi(x, y)=\sum_{n \in \mathbb{Z}} \widehat{\psi}_{n}(y) e^{\mathrm{i} \alpha_{n} x}, \quad \text { where } \quad \alpha_{n}=\frac{2 \pi}{L_{x}} n .
$$

Since $\psi: \Omega \rightarrow \mathbb{R}$ is a real function, it easily follows that $\widehat{\psi}_{-n}(y)={\widehat{\psi_{n}}}_{n}(y)$, where the bar denotes complex conjugation. Substitution of (3.7) into equation (3.5) and the boundary conditions in (3.6) yield

$$
\begin{gathered}
\sum_{n \in \mathbb{Z}} \lambda\left(-\alpha_{n}^{2} \widehat{\psi}_{n}+\frac{\mathrm{d}^{2} \widehat{\psi}_{n}}{\mathrm{~d} y^{2}}\right) e^{\mathrm{i} \alpha_{n} x}=\sum_{n \in \mathbb{Z}}\left(\mathrm{i} \alpha_{n} \frac{\mathrm{d} \widehat{\psi}_{n}}{\mathrm{~d} y}+\frac{1}{\operatorname{Re}}\left(\alpha_{n}^{4} \widehat{\psi}_{n}-2 \alpha_{n}^{2} \frac{\mathrm{d}^{2} \widehat{\psi}_{n}}{\mathrm{~d} y^{2}}+\frac{\mathrm{d}^{4} \widehat{\psi}_{n}}{\mathrm{~d} y^{4}}\right)\right) e^{\mathrm{i} \alpha_{n} x}, \\
\frac{\partial \psi}{\partial y}=\sum_{n \in \mathbb{Z}} \frac{\mathrm{d} \widehat{\psi}_{n}}{\mathrm{~d} y} e^{\mathrm{i} \alpha_{n} x}=0 \quad \text { at } \quad y=0 \text { and } y=1 \text { for all } x \in\left(0, L_{x}\right), \\
-\frac{\partial \psi}{\partial x}=-\sum_{n \in \mathbb{Z}} \mathrm{i} \alpha_{n} \widehat{\psi}_{n} e^{\mathrm{i} \alpha_{n} x}=0 \quad \text { at } \quad y=0 \text { and } y=1 \text { for all } x \in\left(0, L_{x}\right) .
\end{gathered}
$$

Now, since the Fourier modes are known to be orthogonal with the $L^{2}\left(0, L_{x}\right)$ inner product, it follows that each Fourier mode can be treated separately. Therefore, dropping the subindex $n$, the equations above become

$$
\begin{aligned}
\lambda\left(-\alpha^{2} \widehat{\psi}+\frac{\mathrm{d}^{2} \widehat{\psi}}{\mathrm{d} y^{2}}\right) & =\left(\mathrm{i} \alpha \frac{\mathrm{d} \widehat{\psi}}{\mathrm{d} y}+\frac{1}{\operatorname{Re}}\left(\alpha^{4} \widehat{\psi}-2 \alpha^{2} \frac{\mathrm{d}^{2} \widehat{\psi}}{\mathrm{d} y^{2}}+\frac{\mathrm{d}^{4} \widehat{\psi}}{\mathrm{d} y^{4}}\right)\right), \\
\widehat{\psi}(0) & =\widehat{\psi}(1)=\frac{\mathrm{d} \widehat{\psi}}{\mathrm{d} y}(0)=\frac{\mathrm{d} \widehat{\psi}}{\mathrm{d} y}(1)=0 .
\end{aligned}
$$

The equation is a fourth order homogeneous ordinary differential equation with constant coefficients and four vanishing boundary conditions. Hence, the solution is known to be a linear combination of exponentials. With this in mind, one first proposes that $\widehat{\psi}(y)=e^{i \beta y}$. Substitution into (3.10) gives

$$
-\lambda\left(\alpha^{2}+\beta^{2}\right)=-\alpha \beta+\frac{1}{\operatorname{Re}}\left(\alpha^{2}+\beta^{2}\right)^{2},
$$

which is the characteristic equation whose solutions are the roots of the characteristic polynomial

$$
p_{\psi}(\lambda, \operatorname{Re}, \alpha, \beta)=\frac{1}{\operatorname{Re}}\left(\alpha^{2}+\beta^{2}\right)^{2}+\lambda\left(\alpha^{2}+\beta^{2}\right)-\alpha \beta,
$$

with discriminant

$$
\begin{aligned}
\Delta_{\psi}(\lambda, \operatorname{Re}, \alpha)=256 \alpha^{8}+ & 384 \alpha^{6} \lambda \operatorname{Re}-27 \alpha^{4} \operatorname{Re}^{2}+120 \alpha^{4} \lambda^{2} \operatorname{Re}^{2} \\
+ & 16 \alpha^{4} \lambda^{4} \operatorname{Re}^{2}-4 \alpha^{2} \lambda^{3} \operatorname{Re}^{3}+16 \alpha^{2} \lambda^{5} \operatorname{Re}^{3} .
\end{aligned}
$$

The characteristic polynomial is symmetric in $\alpha$ and $\beta$. Given a fixed triplet $(\lambda, \operatorname{Re}, \alpha), p_{\psi}$ will have exactly four roots $\beta_{j}(\lambda, \operatorname{Re}, \alpha)$ for $j=1, \ldots, 4$, which are obviously dependent on that triplet. The roots $\beta_{j}$ can even be computed analytically due to $p_{\psi}$ being quartic in $\beta$. Moreover, if only real values of $\lambda$ are considered, which is reasonable due to the operator being symmetric, then the coefficients in $\beta$ of $p_{\psi}$ will be real. This implies the roots $\beta_{j}$ will 
either be real or come in conjugate pairs. Finally, since the coefficient of $\beta^{3}$ is 0 it follows that $\sum_{j=1}^{4} \beta_{j}=0$.

For convenience, assume that all the roots are different, i.e. $\Delta_{\psi}(\lambda, \operatorname{Re}, \alpha) \neq 0$, so that due to linearity, the general solution of (3.10) will be

$$
\widehat{\psi}(y)=C_{1} e^{\mathrm{i} \beta_{1} y}+C_{2} e^{\mathrm{i} \beta_{2} y}+C_{3} e^{\mathrm{i} \beta_{3} y}+C_{4} e^{\mathrm{i} \beta_{4} y},
$$

where the $C_{j}$ are constant coefficients in $\mathbb{C}$.

The constant coefficients $C_{j}$ in (3.15) are chosen to satisfy the boundary conditions (3.11). When $\Delta_{\psi}(\lambda, \operatorname{Re}, \alpha) \neq 0$, substituting (3.15) into (3.11) yields a linear system of equations, which in matrix form is

$$
\underbrace{\left[\begin{array}{cccc}
1 & 1 & 1 & 1 \\
e^{\mathrm{i} \beta_{1}} & e^{\mathrm{i} \beta_{2}} & e^{\mathrm{i} \beta_{3}} & e^{\mathrm{i} \beta_{4}} \\
\mathrm{i} \beta_{1} & \mathrm{i} \beta_{2} & \mathrm{i} \beta_{3} & \mathrm{i} \beta_{4} \\
\mathrm{i} \beta_{1} e^{\mathrm{i} \beta_{1}} & \mathrm{i} \beta_{2} e^{\mathrm{i} \beta_{2}} & \mathrm{i} \beta_{3} e^{\mathrm{i} \beta_{3}} & \mathrm{i} \beta_{4} e^{\mathrm{i} \beta_{4}}
\end{array}\right]}_{\mathbf{M}_{\psi}(\lambda, \operatorname{Re}, \alpha)} \underbrace{\left[\begin{array}{l}
C_{1} \\
C_{2} \\
C_{3} \\
C_{4}
\end{array}\right]}_{\mathbf{C}}=\left[\begin{array}{l}
0 \\
0 \\
0 \\
0
\end{array}\right] .
$$

The complex matrix $\mathbf{M}_{\psi}$ is dependent on the triplet $(\lambda, \operatorname{Re}, \alpha)$ via the distinct roots $\beta_{j}$ of $p_{\psi}(\lambda, \operatorname{Re}, \alpha, \beta)$. To have a nonzero eigenfunction it is then necessary for $\operatorname{det}\left(\mathbf{M}_{\psi}\right)=0$, and $\mathbf{C} \in \operatorname{ker}\left(\mathbf{M}_{\psi}\right) \backslash \mathbf{0}$, which can then be substituted into (3.15) to calculate the complex function $\widehat{\psi}$. By adding the complementary Fourier mode, a real stream function is computed pointwise as

$$
\psi(x, y)=\widehat{\psi}(y) e^{\mathrm{i} \alpha x}+\overline{\widehat{\psi}(y)} e^{-\mathrm{i} \alpha x} \in \mathbb{R} .
$$

Then, a real eigenvelocity field $\left[u_{x}(x, y), u_{y}(x, y)\right]^{\top} \in \mathbb{R}^{2}$ corresponding to that stream function is easily determined via (3.4). In fact, if $\alpha \neq 0$, and $\mathbf{C} \in \operatorname{ker}\left(\mathbf{M}_{\psi}\right) \backslash \mathbf{0}$, then iC is another relevant solution (shift by $\frac{\pi}{2}$ ) which leads to a shifted and linearly independent eigenvelocity field associated to the same eigenvalue. All eigenfunctions can be normalized (to have $\|\mathbf{u}\|=1$ ).

If $\Delta_{\psi}(\lambda, \operatorname{Re}, \alpha)=0$, then the necessary and tedious modifications associated having repeated roots must be done. This is left for the reader to ponder. Additionally, if $\alpha=0$, then $\psi$ is only a function of $y$ and solving (3.1) directly gives $\mathbf{u}_{i}=[\sin (2 \pi k y), 0]^{\top}$ with (unique) eigenvalues $\lambda_{i}=-\frac{(2 \pi k)^{2}}{\operatorname{Re}}$ for $k \in \mathbb{N}$.

Next, assume that the Galerkin basis vector fields $\mathbf{u}_{i}$ for $i=1, \ldots, m$ are chosen as eigenfunctions of the energy eigenvalue problem. The idea is to calculate the matrices $\mathbf{R}_{i}$ in (2.10). Let $\mathbf{b}=\left[b_{x}, b_{y}\right]^{\top}$ be either $\mathbf{h}_{i 0}$ or $\mathbf{h}_{i j}$ for some $i, j=1, \ldots, m$. The first step is to find $\phi_{\mathbf{b}}$ such that $\mathbf{b}=\mathbf{b}+\nabla \phi_{\mathbf{b}}$, with $\nabla \cdot \breve{\mathbf{b}}=0$ and $\breve{\mathbf{b}} \cdot \mathbf{n}=0$ wherever the perturbation velocity has no-slip boundary conditions. This is equivalent to solving the Poisson problem

$$
\nabla^{2} \phi_{\mathbf{b}}=\nabla \cdot \mathbf{b}
$$

subject to the boundary conditions

$$
\nabla \phi_{\mathbf{b}} \cdot \mathbf{n}=\mathbf{b} \cdot \mathbf{n}
$$


wherever the perturbation velocity has no-slip boundary conditions. In this case no-slip boundary conditions occur at the plates, and those are precisely the points $(x, y) \in \partial \Omega$ for which $y=0$ and $y=1$. The outward unit normal $\mathbf{n}$ corresponding to those points are $\mathbf{n}=[0,-1]^{\top}$ when $y=0$ and $\mathbf{n}=[0,1]^{\top}$ when $y=1$.

Proceeding as with the eigenvalue problem, the Fourier series expansions of $\phi_{\mathbf{b}}$ and $\mathbf{b}$ are

$$
\phi_{\mathbf{b}}(x, y)=\sum_{n \in \mathbb{Z}} \widehat{\phi}_{n}(y) e^{\mathrm{i} \alpha_{n} x} \quad \text { and } \quad \mathbf{b}(x, y)=\sum_{n \in \mathbb{Z}} \widehat{\mathbf{b}}_{n}(y) e^{\mathrm{i} \alpha_{n} x} .
$$

One can then substitute these expansions into (3.18) and treat each Fourier mode separately due to their orthogonality with the $L^{2}(\Omega)$ inner product. Therefore, dropping the $n$, the Poisson problem eventually becomes the nonhomogeneous second order ordinary differential equation

$$
\frac{\mathrm{d}^{2} \widehat{\phi}}{\mathrm{d} y^{2}}-\alpha^{2} \widehat{\phi}=\frac{\mathrm{d} \widehat{b}_{y}}{\mathrm{~d} y}+\mathrm{i} \alpha \widehat{b}_{x}
$$

with the boundary conditions

$$
\frac{\mathrm{d} \widehat{\phi}}{\mathrm{d} y}(0)=\widehat{b}_{y}(0) \quad \text { and } \quad \frac{\mathrm{d} \widehat{\phi}}{\mathrm{d} y}(1)=\widehat{b}_{y}(1)
$$

where $\widehat{\mathbf{b}}(y)=\left[\widehat{b}_{x}(y), \widehat{b}_{y}(y)\right]^{\top}$. This equation must be solved for each Fourier mode separately. Due to the form of $\mathbf{h}_{i 0}, \mathbf{h}_{i j}$ and the energy eigenfunctions (only having 1 or 2 Fourier modes), it follows that $\mathbf{b}$ will have at most 4 separate Fourier modes.

The details will be skipped, but as usual, one must find first a general homogeneous solution $\widehat{\phi}_{h}(y)$ such that $\frac{\mathrm{d}^{2} \widehat{\phi}_{h}}{\mathrm{~d} y^{2}}-\alpha^{2} \widehat{\phi}_{h}=0$, followed by a particular solution $\widehat{\phi}_{p}$. This can actually be done analytically for the current problem. In the end, the full solution will be $\widehat{\phi}=\widehat{\phi}_{h}+\widehat{\phi}_{p}$, and $\phi_{\mathbf{b}}$ can be reconstructed via (3.20).

This means $\breve{\mathbf{b}}=\mathbf{b}-\nabla \phi_{\mathbf{b}}$ is known explicitly, and then $\widetilde{\mathbf{b}}$ is easy to compute as

$$
\widetilde{\mathbf{b}}=\mathbf{b}-\nabla \phi_{\mathbf{b}}-\sum_{j=1}^{m}\left\langle\breve{\mathbf{b}}, \mathbf{u}_{j}\right\rangle \mathbf{u}_{j} .
$$

Finally, one can proceed to calculate the integrals to find $\mathbf{R}_{i}$ for each $i=1, \ldots, m$.

As mentioned before, $\kappa_{\mathbf{s}}$ is the $m+1$ largest eigenvalue, provided the Galerkin basis vector fields $\mathbf{u}_{i}$ for $i=1, \ldots, m$ are chosen as eigenfunctions associated to the largest eigenvalues. Their strain rate tensor is $\mathbf{D}_{i}$ for all $i=1, \ldots, m$. Now, given that the flow is two dimensional and using the incompressibility of the eigenfunctions in the form $\operatorname{tr}\left(\mathbf{D}_{i}(\mathbf{x})\right)=0$ for some arbitrary $\mathbf{x} \in \Omega$, it follows that the eigenvalues of $\mathbf{D}_{i}(\mathbf{x})$ can be computed explicitly. They have the same magnitude, which must be the spectral radius. Hence, the spectral radius $\rho\left(\mathbf{D}_{i}\right): \Omega \rightarrow \mathbb{R}$ as a scalar field is

$$
\rho\left(\mathbf{D}_{i}(\mathbf{x})\right)=\sqrt{\left(\frac{\partial^{2} \psi_{i}}{\partial x \partial y}\right)^{2}+\frac{1}{4}\left(\nabla^{2} \psi_{i}\right)^{2}} .
$$

This immediately implies that the problem of finding $\left\|\rho\left(\mathbf{D}_{i}(\mathbf{x})\right)\right\|_{\infty}$ becomes much easier. Nevertheless, it is solved numerically by being formulated as a constrained optimization problem of finding the global maximum of $\rho\left(\mathbf{D}_{i}\right)$ constrained to $\mathbf{x} \in \Omega$. 


\section{Results}

Solving for the energy eigenmodes and bounds for 2D plane Couette flow as described in Section 3 allows to setup an optimization problem with sum-of-squares (SOS) constraints that can be written as a semidefinite program (SDP). This was done for each periodic domain of length $L$. The specific method used to produce the results in this section is described in Section B.1 in Appendix B.

Two families of carefully chosen modes were considered. These can be appreciated in Figure 4.1. One family is composed of six modes (boxed in blue) and the other is comprised of those six modes plus two more for a total of eight modes (boxed in red). Then the SDP was used to attempt to find a quartic Lyapunov function (as opposed to quadratic) for each of the two families.

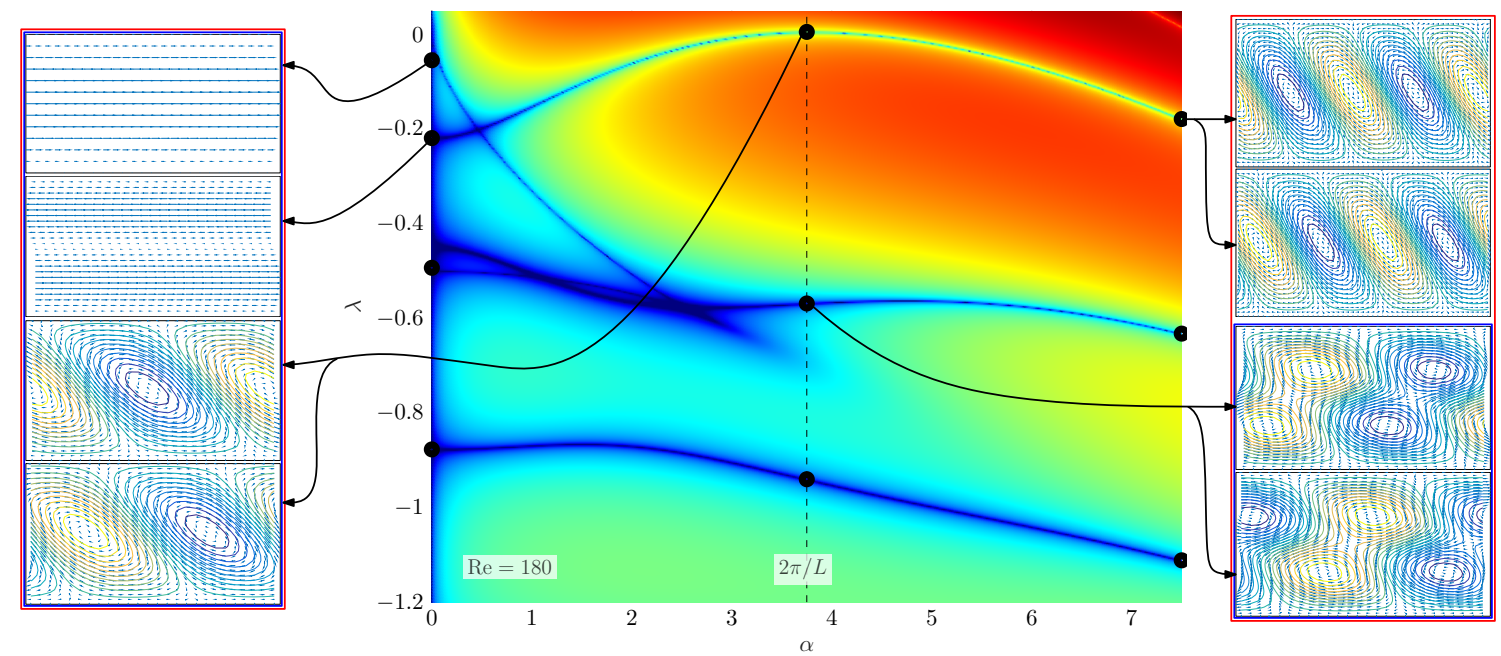

Figure 4.1: Determinant of the matrix associated to the eigenvalue problem. The branches in blue correspond to eigenvalues (where the determinant vanishes) with the exception of a triangle-looking shape in the upper left which corresponds to repeated roots (zero discriminant). The value of the Reynolds number is fixed (at 180), so the eigenvalues are a function of $\alpha$. Each eigenvalue corresponding to $\alpha \neq 0$ has two eigenfunctions associated to it (shifted by a quarter period from each other). Two families of eigenmodes illustrated: six-mode family boxed in blue, and eight-mode family boxed in red.

The results can be observed in Figure 4.2, where the energy stability limit was shown for each periodic length $L$. The two curves above the energy stability limit are new larger lower bounds of the global stability limit, and for every Re under those curves the flow is globally asymptotically stable. For periodic lengths of under $L / h=2.28$ the new lower bounds for $\operatorname{Re}_{G}$ are $\operatorname{Re}_{S O S, 1}=190$ using the six-mode family of eigenmodes and Re $e_{S O S, 2}=200$ using the eight-mode family of eigenmodes, which are both above the energy stability limit of $\operatorname{Re}_{E}=177.2$ found by Orr over a century ago. This is the first improvement to the bound in that time-frame.

It should be noted that being able to find better bounds beyond the "bump" in the energy stability curve that occurs at about $L / h=2.4$ is no easy task. The reason is that it would require using more modes (at least twelve), which make the computation much more expensive. At the current moment, the algorithms and solvers are definitely a limitation 
to getting more and better results. This is why posing the constraints in the more efficient manner possible is an important matter (see the Appendices).

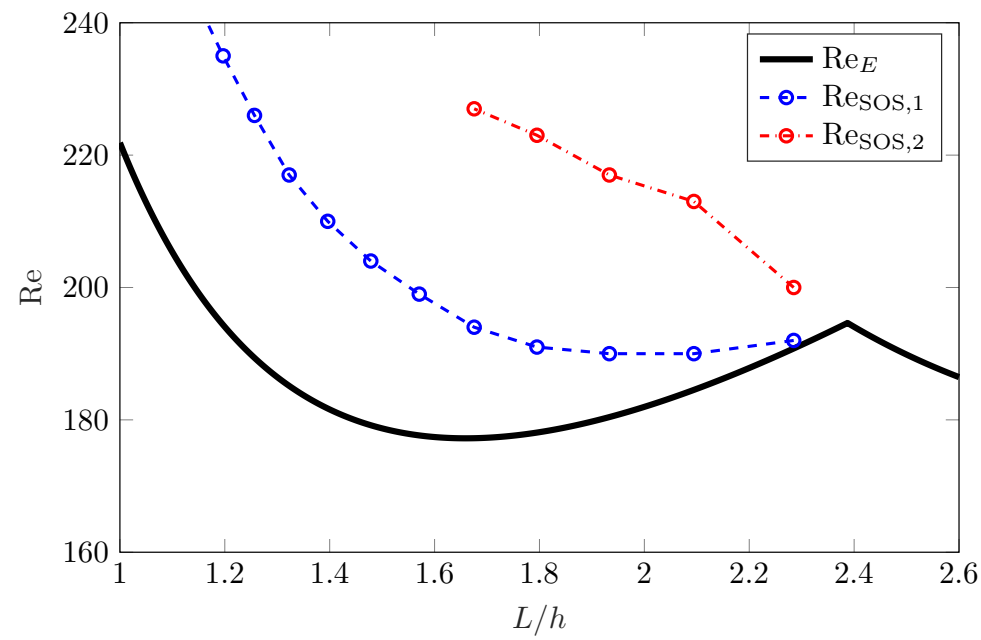

Figure 4.2: Energy stability limit (black) as a function of the periodic length of the domain. Larger lower bounds for the global stability limit resulting from the quartic Lyapunov functions found by the semidefinite program (SDP) with sum-of-squares (SOS) constraints for two families of energy eigenmodes: a six-mode family in blue and an eight-mode family in red.

\section{Conclusions}

Using new techniques from optimization, namely, the tractable imposition of sum-of-squares polynomial constraints in an optimization algorithm, it was possible to construct high-order quartic Lyapunov functions that proved the global stability of 2D plane Couette flow beyond the energy stability limit. This marks the first improvement in such a result in over a century! More importantly, the techniques can be utilized to analyze different flows. This will be left for future work. The current main limitation is computational power or better algorithms, and overcoming this limitation would allow to increase the number of energy eigenmodes to be included in the uncertain dynamical system that describes the fluid.

\section{Acknowledgments}

First, I wish to thank David Goluskin for supervising this project, for his support, and for sharing all his insight with me. I also wish to thank Giovanni Fantuzzi, who contributed some ideas in this work and was always open to bounce opinions back and forth. I also thank Sergei Chernyshenko for telling me about the program and encouraging me to apply. Lastly, I wish to thank all the GFD fellows, Claudia and Mary-Louise for organizing everything, and the whole environment at WHOI for making it a wonderful summer. 


\section{A Original Approach}

First, I will give some general comments on the choice of $V\left(\mathbf{a}, q^{2}\right)$. Then, I will give the bounds derived for $\boldsymbol{\Theta}_{\mathbf{a b}}\left(\mathbf{u}_{\mathbf{s}}, \mathbf{a}\right)$ and $\boldsymbol{\Theta}_{\mathbf{c}}\left(\mathbf{u}_{\mathbf{s}}\right)$, and describe the usual choice of $\Xi\left(\mathbf{a}, q^{2}\right)$, which will be non-polynomial, and how it converts to a valid SDP.

\section{A.1 Choice of Lyapunov function ansatz}

The goal is for $V\left(\mathbf{a}, q^{2}\right)$ to be an SOS, where in this context a polynomial in $\left(\mathbf{a}, q^{2}\right)$ means a linear combination of monomials in the variables $\mathbf{a}=\left[a_{1}, \ldots, a_{m}\right]$ and even powers of $q$ (i.e. monomials like $a_{1}$ of degree $1, a_{1} q^{2}$ of degree $3, a_{1} a_{2} q^{4}$ of degree 6 , etc.). Since $V\left(\mathbf{a}, q^{2}\right)$ will be constrained to be an SOS, it follows that the highest and lowest degree monomials in the linear combination must be of even degree. In particular, $\operatorname{deg}(V)$ must be even.

Additionally, we want $-(\tilde{G}+\Xi)$ to be an SOS too, so it must also have even degree. Here, $\Xi$ is assumed to be positive definite (it is normally a positive bound of $\mathbf{M} \cdot\left(\boldsymbol{\Theta}_{\mathbf{a b}}+\boldsymbol{\Theta}_{\mathbf{c}}\right)$ ). Thus, $\tilde{G}$ must be negative definite such that $\tilde{G}+\Xi$ is still negative definite. If $\operatorname{deg}(\Xi)>$ $\operatorname{deg}(\tilde{G})$, then $\tilde{G}+\Xi$ will be positive for large $\left(\mathbf{a}, q^{2}\right)$ and this is precisely what we do not want. Therefore, assume $\operatorname{deg}(\tilde{G}) \geq \operatorname{deg}(\Xi)$, with $\operatorname{deg}(\tilde{G})$ being even (in order for it to be negative definite). The term $\frac{\partial V}{\partial \mathbf{a}} \cdot \mathbf{f}(\mathbf{a})$ in $\tilde{G}$ seems to be inconveniently of odd degree as $\mathbf{f}(\mathbf{a})$ is quadratic in a and $\operatorname{deg}\left(\frac{\partial V}{\partial \mathbf{a}}\right)$ is odd (as $\operatorname{deg}(V)$ is even). To overcome this hurdle, a viable and elegant approach is to choose the component of highest degree in $V$ to be a power of the kinetic energy $E=\frac{1}{2}\left(|\mathbf{a}|^{2}+q^{2}\right)$, because with this choice, $\frac{\partial V}{\partial \mathbf{a}} \cdot \mathbf{f}(\mathbf{a})$ is of even degree (there are cancellations). Thus, the only component of degree $\operatorname{deg}(V)$ in $V$ is chosen to be $\left(|\mathbf{a}|^{2}+q^{2}\right)^{\operatorname{deg}(V) / 2}$, and this ensures that $\tilde{G}$ and $\mathbf{M}$ are of even degree, with $\operatorname{deg}(V)=\operatorname{deg}(\tilde{G})=\operatorname{deg}(\mathbf{M})+2$.

Next, we know $V(\mathbf{0}, 0)=0$, so there must not be any constants in $V$. Since $V$ will be chosen to be an SOS polynomial, this implies there must not be any linear terms in $a_{1}, \ldots, a_{m}$ either. As a result, in the interest of generality, $V$ is assumed to be a linear combination of monomials in the variables $a_{1}, \ldots, a_{m}$ and $q^{2}$ of degree greater than or equal to 2 and less than or equal to $\operatorname{deg}(V)-1$ along with the polynomial $\left(|\mathbf{a}|^{2}+q^{2}\right)^{\operatorname{deg}(V) / 2}$. That is, it should take the form,

$$
V\left(\mathbf{a}, q^{2}\right)=\sum_{2 \leq \operatorname{deg}\left(\operatorname{mon}_{\iota}\right) \leq \operatorname{deg}(V)-1} c_{\iota} \operatorname{mon}_{\iota}\left(\mathbf{a}, q^{2}\right)+\left(|\mathbf{a}|^{2}+q^{2}\right)^{\operatorname{deg}(V) / 2},
$$

where $\iota$ indexes all monomials in the variables $a_{1}, \ldots, a_{m}$ and even powers of $q, \operatorname{mon}_{\iota}\left(\mathbf{a}, q^{2}\right)$, and the $c_{\iota}$ are unknown real coefficients associated to those monomials. Notice that the last homogeneous polynomial, $\left(|\mathbf{a}|^{2}+q^{2}\right)^{\operatorname{deg}(V) / 2}$, does not have a coefficient. This is because, in principle, $V$ can be scaled by any constant, and this will not change the conclusion that $V>0$ and that $\frac{\mathrm{d} V}{\mathrm{~d} t}<0$, so there is a freedom to fix at least one of the coefficients in $V$. Also, note that with this choice, $\tilde{G}$ does not have any constant or linear terms and $\mathbf{M}(\mathbf{0}, 0)=0$.

Finally, placing the constraint that $V$ should be an SOS polynomial is not sufficient, since this only ensures that $V$ is positive semidefinite. To ensure that it is positive definite, a margin or barrier function must be added. That is, replace the condition $V\left(\mathbf{a}, q^{2}\right)>0$, 
by $V\left(\mathbf{a}, q^{2}\right) \geq \varepsilon\left(|\mathbf{a}|^{2}+q^{2}\right)>0$. Thus, place the constraints

$$
V\left(\mathbf{a}, q^{2}\right)-\varepsilon\left(|\mathbf{a}|^{2}+q^{2}\right) \in \operatorname{SOS}(\mathbf{a}, q), \quad \frac{\partial V\left(\mathbf{a}, q^{2}\right)}{\partial q^{2}} \in \operatorname{SOS}(\mathbf{a}, q),
$$

where $\operatorname{SOS}(\mathbf{a}, q)$ is the set of sum-of-squares polynomials in the variables $a_{1}, \ldots, a_{m}$ and $q$.

\section{A.2 Bounds of $\Theta_{\mathrm{ab}}$ and $\Theta_{\mathrm{c}}$}

In $[7,9]$ the bounds of each component of $\boldsymbol{\Theta}_{\mathbf{a b}}$ and $\boldsymbol{\Theta}_{\mathbf{c}}$ were derived. Let $\tilde{\mathbf{a}}=\left[1, a_{1}, \ldots, a_{m}\right]$, indexed from 0 so that $\tilde{a}_{0}=1$ and $\tilde{a}_{i}=a_{i}$ for $i=1, \ldots, m$. Then, $\Theta_{\mathbf{a b} i}\left(\mathbf{u}_{\mathbf{s}}, \mathbf{a}\right)=\left\langle\mathbf{u}_{\mathbf{s}}, \mathbf{h}_{i j}\right\rangle \tilde{a}_{j}$, and the bounds are,

$$
\begin{aligned}
\left|\Theta_{\mathbf{a b} i}\left(\mathbf{u}_{\mathbf{s}}, \mathbf{a}\right)\right| & \leq \sqrt{\tilde{\mathbf{a}}^{\top} \mathbf{R}_{i} \tilde{\mathbf{a}} q^{2}}, & \left(R_{i}\right)_{k l} & =\left\langle\widetilde{\mathbf{h}}_{i k}, \widetilde{\mathbf{h}}_{i l}\right\rangle, \\
\left|\Theta_{\mathbf{c} i}\left(\mathbf{u}_{\mathbf{s}}\right)\right| & \leq C_{i} q^{2}, & C_{i} & =\left\|\rho\left(\mathbf{D}_{i}\right)\right\|_{\infty}=\sup _{\mathbf{x} \in \Omega} \rho\left(\mathbf{D}_{i}(\mathbf{x})\right),
\end{aligned}
$$

where $\mathbf{D}_{i}=\frac{1}{2}\left(\nabla \mathbf{u}_{i}+\nabla^{\top} \mathbf{u}_{i}\right)$, and $\rho\left(\mathbf{D}_{i}(\mathbf{x})\right)$ is the spectral radius of $\mathbf{D}_{i}(\mathbf{x})$. Here, $\widetilde{\mathbf{h}}_{i j}$ is the solenoidal projection (so $\nabla \cdot \widetilde{\mathbf{h}}_{i j}=0$ ) of $\mathbf{h}_{i j}$ such that $\left\langle\widetilde{\mathbf{h}}_{i j}, \mathbf{u}_{k}\right\rangle=0$ for all $k=1, \ldots, m$ and satisfying that $\widetilde{\mathbf{h}}_{i j} \cdot \mathbf{n}=0$ ( $\mathbf{n}$ is the outer normal) wherever the perturbation velocity has no-slip boundary conditions. These bounds are important as they eliminate $\mathbf{u}_{\mathbf{s}}$ and yield expressions only in terms of $a_{1}, \ldots, a_{m}$ and $q^{2}$.

\section{A.3 A conservative bound of $\mathrm{M} \cdot\left(\Theta_{\mathrm{ab}}+\Theta_{\mathrm{c}}\right)$}

The original approach relies on first bounding with the absolute value, then using the triangle inequality twice, and lastly using the Cauchy-Schwarz inequality in $\mathbb{R}^{m}$,

$$
\begin{aligned}
\mathbf{M}\left(\mathbf{a}, q^{2}\right) \cdot\left(\boldsymbol{\Theta}_{\mathbf{a b}}\left(\mathbf{u}_{\mathbf{s}}, \mathbf{a}\right)+\boldsymbol{\Theta}_{\mathbf{c}}\left(\mathbf{u}_{\mathbf{s}}\right)\right) & \leq\left|\mathbf{M}\left(\mathbf{a}, q^{2}\right) \cdot \boldsymbol{\Theta}_{\mathbf{a b}}\left(\mathbf{u}_{\mathbf{s}}, \mathbf{a}\right)\right|+\left|\mathbf{M}\left(\mathbf{a}, q^{2}\right) \cdot \boldsymbol{\Theta}_{\mathbf{c}}\left(\mathbf{u}_{\mathbf{s}}\right)\right| \\
& \leq \sum_{i=1}^{m}\left|M_{i}\left(\mathbf{a}, q^{2}\right)\right|\left(\left|\Theta_{\mathbf{a b} i}\left(\mathbf{u}_{\mathbf{s}}, \mathbf{a}\right)\right|+\left|\Theta_{\mathbf{c} i}\left(\mathbf{u}_{\mathbf{s}}, \mathbf{a}\right)\right|\right) \\
& \leq\left|\mathbf{M}\left(\mathbf{a}, q^{2}\right)\right| \sqrt{\sum_{i=1}^{m}\left(\left|\Theta_{\mathbf{a b} i}\left(\mathbf{u}_{\mathbf{s}}, \mathbf{a}\right)\right|+\left|\Theta_{\mathbf{c} i}\left(\mathbf{u}_{\mathbf{s}}, \mathbf{a}\right)\right|\right)^{2}} .
\end{aligned}
$$

Next, let $\delta_{i} \in(0, \infty)$ for all $i=1, \ldots, m$, and note that

$$
\begin{aligned}
\sum_{i=1}^{m}\left(\left|\Theta_{\mathbf{a b} i}\left(\mathbf{u}_{\mathbf{s}}, \mathbf{a}\right)\right|+\left|\Theta_{\mathbf{c} i}\left(\mathbf{u}_{\mathbf{s}}\right)\right|\right)^{2} & =\sum_{i=1}^{m} \Theta_{\mathbf{a b} i}^{2}\left(\mathbf{u}_{\mathbf{s}}, \mathbf{a}\right)+2\left|\Theta_{\mathbf{a b} i}\left(\mathbf{u}_{\mathbf{s}}, \mathbf{a}\right)\right|\left|\Theta_{\mathbf{c} i}\left(\mathbf{u}_{\mathbf{s}}\right)\right|+\Theta_{\mathbf{c} i}^{2}\left(\mathbf{u}_{\mathbf{s}}\right) \\
& \leq \sum_{i=1}^{m}\left(\left(1+\delta_{i}\right) \Theta_{\mathbf{a b} i}^{2}\left(\mathbf{u}_{\mathbf{s}}, \mathbf{a}\right)+\left(1+\frac{1}{\delta_{i}}\right) \Theta_{\mathbf{c} i}^{2}\left(\mathbf{u}_{\mathbf{s}}\right)\right) \\
& \leq \sum_{i=1}^{m}\left(\left(1+\delta_{i}\right) \tilde{\mathbf{a}}^{\top} \mathbf{R}_{i} \tilde{\mathbf{a}} q^{2}+\left(1+\frac{1}{\delta_{i}}\right) C_{i}^{2} q^{4}\right)=p_{\Theta}\left(\mathbf{a}, q^{2}\right)
\end{aligned}
$$

where the so-called "Peter-Paul" inequality, $2 w_{1} w_{2} \leq \delta w_{1}^{2}+\frac{1}{\delta} w_{2}^{2}$ for any $\delta \in(0, \infty)$, is used. Hence,

$$
\mathbf{M}\left(\mathbf{a}, q^{2}\right) \cdot\left(\boldsymbol{\Theta}_{\mathbf{a b}}\left(\mathbf{u}_{\mathbf{s}}, \mathbf{a}\right)+\boldsymbol{\Theta}_{\mathbf{c}}\left(\mathbf{u}_{\mathbf{s}}\right)\right) \leq\left|\mathbf{M}\left(\mathbf{a}, q^{2}\right)\right| \sqrt{p_{\boldsymbol{\Theta}}\left(\mathbf{a}, q^{2}\right)}=\Xi\left(\mathbf{a}, q^{2}\right)
$$


Now we need to have that $\tilde{G}+\Xi<0$ for all $\left(\mathbf{a}, q^{2}\right) \neq(\mathbf{0}, 0)$ (it is clear already that $\Xi(\mathbf{0}, 0)=0$ because $p_{\boldsymbol{\Theta}}(\mathbf{0}, 0)=0$ ). Unfortunately, $\Xi$ is not polynomial (but $\Xi^{2}=p_{\boldsymbol{\Theta}}|\mathbf{M}|^{2}=$ $p_{\Theta} \mathbf{M}^{\top} \mathbf{M}$ is), so some manipulations are necessary to obtain a polynomial expression that is linear in the unknown coefficients $c_{\iota}$ of $V$ (note that the $c_{\iota}$ are linearly present in $\tilde{G}$ and $\mathbf{M})$. Using Schur complements, the inequality can be rewritten as,

$$
\tilde{G}+\Xi<0 \Leftrightarrow 0 \leq \Xi<-\tilde{G} \Leftrightarrow\left\{\begin{array}{r}
-\tilde{G}>0 \\
p_{\boldsymbol{\Theta}} \tilde{G}^{2}-p_{\boldsymbol{\Theta}} \Xi^{2}>0
\end{array} \Leftrightarrow\left[\begin{array}{cc}
-p_{\boldsymbol{\Theta}} \tilde{G} & p_{\boldsymbol{\Theta}} \mathbf{M}^{\top} \\
p_{\boldsymbol{\Theta}} \mathbf{M} & -\tilde{G} \mathbf{I}
\end{array}\right] \succ 0 .\right.
$$

Again, use a barrier function to obtain strict positivity, so that you should enforce the constraint

$$
\left[\begin{array}{cc}
-p_{\boldsymbol{\Theta}} \tilde{G} & p_{\boldsymbol{\Theta}} \mathbf{M}^{\top} \\
p_{\boldsymbol{\Theta}} \mathbf{M} & -\tilde{G} \mathbf{I}
\end{array}\right]-\varepsilon\left(|\mathbf{a}|^{2}+q^{2}\right)\left[\begin{array}{cc}
1 & 0 \\
0 & \mathbf{I}
\end{array}\right] \in \operatorname{SOS}_{\mathbb{M}}(\mathbf{a}, q)
$$

where $\operatorname{SOS}_{\mathbb{M}}(\mathbf{a}, q)$ is the set of sum-of-squares polynomial positive semidefinite matrices.

Another slight deviation of this condition is to use the barrier function beforehand, so that $\tilde{G}+\Xi+\varepsilon\left(|\mathbf{a}|^{2}+q^{2}\right) \leq 0$, which results in the constraint

$$
\left[\begin{array}{cc}
-p_{\boldsymbol{\Theta}}\left(\tilde{G}+\varepsilon\left(|\mathbf{a}|^{2}+q^{2}\right)\right) & p_{\boldsymbol{\Theta}} \mathbf{M}^{\top} \\
p_{\boldsymbol{\Theta}} \mathbf{M} & -\left(\tilde{G}+\varepsilon\left(|\mathbf{a}|^{2}+q^{2}\right)\right) \mathbf{I}
\end{array}\right] \in \operatorname{SOS}_{\mathbb{M}}(\mathbf{a}, q) .
$$

Both (A.8) and (A.9) are "matrix" constraints of the form $\mathbf{T} \succeq 0$. These can be quite expensive to enforce. As an example, with only $m=6$ modes and the fastest solver available, the SDP enforcing (A.2) and (A.8) took roughly 2 hours. However, $\mathbf{T}$ is a very sparse matrix so if you are careful, it is natural to expect some savings. The most natural approach is to add variables $\tilde{\mathbf{z}}=\left(z_{0}, z_{1}, \ldots, z_{m}\right)$, where $\mathbf{z}=\left(z_{1}, \ldots, z_{m}\right)$, and consider the equivalent statement $\tilde{\mathbf{z}}^{\top} \mathbf{T} \tilde{\mathbf{z}} \geq 0$. Due to the sparsity, (A.8) is rewritten as

$$
-\left(p_{\Theta} \tilde{G}+\varepsilon\left(|\mathbf{a}|^{2}+q^{2}\right)\right) z_{0}^{2}+2 z_{0} p_{\Theta} \mathbf{M} \cdot \mathbf{z}-\left(\tilde{G}+\varepsilon\left(|\mathbf{a}|^{2}+q^{2}\right)\right) \mathbf{z} \cdot \mathbf{z} \in \operatorname{SOS}(\mathbf{a}, q, \tilde{\mathbf{z}}) .
$$

With this formulation, the computation time was reduced to roughly 1 minute in the same machine. Similarly, (A.8) is rewritten as

$$
-p_{\Theta}\left(\tilde{G}+\varepsilon\left(|\mathbf{a}|^{2}+q^{2}\right)\right) z_{0}^{2}+2 z_{0} p_{\Theta} \mathbf{M} \cdot \mathbf{z}-\left(\tilde{G}+\varepsilon\left(|\mathbf{a}|^{2}+q^{2}\right)\right) \mathbf{z} \cdot \mathbf{z} \in \operatorname{SOS}(\mathbf{a}, q, \tilde{\mathbf{z}}) .
$$

Lastly, an alternative to exploit the sparsity of $\mathbf{T}$ (and does not require extra variables) is to use Agler's theorem as proposed in [5], but we have not implemented it in this note.

The SDP enforcing the constraints (A.2) and (A.10) is called "Original 1", while the SDP enforcing the constraints (A.2) and (A.11) is called "Original 2",

$$
\begin{aligned}
& \max \varepsilon, \quad \text { subject to }(\text { A.2) \& (A.10) } \leftarrow \text { Original 1, } \\
& \max \varepsilon, \quad \text { subject to }(\text { A.2) \& (A.11) } \leftarrow \text { Original } 2 .
\end{aligned}
$$

In both cases $\varepsilon$ is maximized. If $\max \varepsilon$ is ultimately positive, then the problem is feasible, and otherwise it is infeasible. As expected, both methods above seem to behave very similarly and have the same limitations, but Original 2 produces larger values of $\varepsilon$ which are safer to trust. Unfortunately, adding just two more modes $(m=8)$ has huge memory requirements for solvers using interior point methods, so no simulations could be completed. More detailed results are found in Section E. 


\section{B A New Family of Methods}

This family of methods relies on a better bound for $\mathbf{M} \cdot\left(\boldsymbol{\Theta}_{\mathbf{a b}}+\boldsymbol{\Theta}_{\mathbf{c}}\right)$, which still uses (A.4), but considers the bound before the Cauchy-Schwarz inequality is applied,

$$
\mathbf{M}\left(\mathbf{a}, q^{2}\right) \cdot\left(\boldsymbol{\Theta}_{\mathbf{a b}}\left(\mathbf{u}_{\mathbf{s}}, \mathbf{a}\right)+\boldsymbol{\Theta}_{\mathbf{c}}\left(\mathbf{u}_{\mathbf{s}}\right)\right) \leq \sum_{i=1}^{m}\left|M_{i}\left(\mathbf{a}, q^{2}\right)\right|\left(\left|\Theta_{\mathbf{a b} i}\left(\mathbf{u}_{\mathbf{s}}, \mathbf{a}\right)\right|+\left|\Theta_{\mathbf{c} i}\left(\mathbf{u}_{\mathbf{s}}\right)\right|\right) .
$$

The results of the methods about to be described can be found in Section E.

\section{B.1 Method 1}

Consider first

$$
\left|M_{i}\right|\left|\Theta_{\mathbf{a b} i}\right| \leq\left|M_{i}\right| \sqrt{\tilde{\mathbf{a}}^{\top} \mathbf{R}_{i} \tilde{\mathbf{a}} q^{2}} \leq r_{i},
$$

where (A.3) was used and where $r_{i}\left(\mathbf{a}, q^{2}\right)$ is an unknown polynomial of the form,

$$
r_{i}\left(\mathbf{a}, q^{2}\right)=\sum_{2 \leq \operatorname{deg}\left(\operatorname{mon}_{\iota}\right) \leq \operatorname{deg}(V)} c_{\iota}^{r} \operatorname{mon}_{\iota}\left(\mathbf{a}, q^{2}\right),
$$

where the coefficients, $c_{\iota}^{r}$ are unknown. Notice that the constant and linear terms are eliminated because we eventually want $\Xi(\mathbf{0}, 0)=0$. Also, we want that $\operatorname{deg}(V)=\operatorname{deg}(\tilde{G}) \geq$ $\operatorname{deg}(\Xi) \geq \operatorname{deg}\left(r_{i}\right)$, so it follows $\operatorname{deg}\left(r_{i}\right) \leq \operatorname{deg}(V)$. Additionally, $\operatorname{deg}\left(r_{i}\right)$ should be an even power strictly larger than $\operatorname{deg}\left(M_{i}\right)=\operatorname{deg}(V)-2$ (see (B.2)). The only choice is then to set $\operatorname{deg}\left(r_{i}\right)=\operatorname{deg}(V)$ and this explains the expression in (B.3). Proceeding as with (A.7) now yields,

$$
\left|M_{i}\right| \sqrt{\tilde{\mathbf{a}}^{\top} \mathbf{R}_{i} \tilde{\mathbf{a}} q^{2}} \leq r_{i} \quad \Leftrightarrow \quad\left[\begin{array}{cc}
\tilde{\mathbf{a}}^{\top} \mathbf{R}_{i} \tilde{\mathbf{a}} q^{2} r_{i} & \tilde{\mathbf{a}}^{\top} \mathbf{R}_{i} \tilde{\mathbf{a}} q^{2} M_{i} \\
\tilde{\mathbf{a}}^{\top} \mathbf{R}_{i} \tilde{\mathbf{a}} q^{2} M_{i} & r_{i}
\end{array}\right] \succeq 0,
$$

for each $i=1, \ldots, m$. Therefore, add the SOS constraints,

$$
\left[\begin{array}{cc}
\tilde{\mathbf{a}}^{\top} \mathbf{R}_{i} \tilde{\mathbf{a}} q^{2} r_{i} & \tilde{\mathbf{a}}^{\top} \mathbf{R}_{i} \tilde{\mathbf{a}} q^{2} M_{i} \\
\tilde{\mathbf{a}}^{\top} \mathbf{R}_{i} \tilde{\mathbf{a}} q^{2} M_{i} & r_{i}
\end{array}\right] \in \operatorname{SOS}_{\mathbb{M}}(\mathbf{a}, q), \quad \forall i=1, \ldots, m
$$

Next, using (A.3) again, look at

$$
\left|M_{i}\right|\left|\Theta_{\mathbf{c} i}\right| \leq\left|M_{i}\right| C_{i} q^{2} \leq s_{i} C_{i} q^{2},
$$

where $s_{i}\left(\mathbf{a}, q^{2}\right)$ is an unknown polynomial of the form,

$$
s_{i}\left(\mathbf{a}, q^{2}\right)=\sum_{0 \leq \operatorname{deg}\left(\operatorname{mon}_{\iota}\right) \leq \operatorname{deg}(V)-2} c_{\iota}^{s} \operatorname{mon}_{\iota}\left(\mathbf{a}, q^{2}\right),
$$

where the coefficients, $c_{\iota}^{s}$ are unknown. Notice that $\operatorname{deg}\left(s_{i}\right)=\operatorname{deg}(V)-2=\operatorname{deg}(\mathbf{M})$, because we want that $\operatorname{deg}(V)=\operatorname{deg}(\tilde{G}) \geq \operatorname{deg}(\Xi) \geq \operatorname{deg}\left(s_{i} C_{i} q^{2}\right)$ (so $\operatorname{deg}\left(s_{i}\right)$ cannot be any larger than $\operatorname{deg}(V)-2)$. Now, the condition can be rewritten as

$$
\left|M_{i}\right| \leq s_{i} \quad \Leftrightarrow \quad\left\{\begin{array}{c}
M_{i} \leq s_{i} \\
-s_{i} \leq M_{i}
\end{array}\right.
$$


for each $i=1, \ldots, m$. Therefore, add the SOS constraints,

$$
\left\{\begin{array}{l}
s_{i}-M_{i} \in \operatorname{SOS}(\mathbf{a}, q) \\
s_{i}+M_{i} \in \operatorname{SOS}(\mathbf{a}, q)
\end{array}, \quad \forall i=1, \ldots, m .\right.
$$

Lastly, add (B.2) and (B.6) across all $i=1, \ldots, m$ to yield,

$$
\mathbf{M}\left(\mathbf{a}, q^{2}\right) \cdot\left(\boldsymbol{\Theta}_{\mathbf{a b}}\left(\mathbf{u}_{\mathbf{s}}, \mathbf{a}\right)+\boldsymbol{\Theta}_{\mathbf{c}}\left(\mathbf{u}_{\mathbf{s}}\right)\right) \leq \sum_{i=1}^{m}\left(r_{i}\left(\mathbf{a}, q^{2}\right)+s_{i}\left(\mathbf{a}, q^{2}\right) C_{i} q^{2}\right)=\Xi\left(\mathbf{a}, q^{2}\right) .
$$

By design, $\Xi(\mathbf{0}, 0)=0$ and in general $\operatorname{deg}(\Xi)=\operatorname{deg}(\tilde{G})=\operatorname{deg}(V)$. It only remains to enforce the condition that $\tilde{G}+\Xi<0$, which in this case is very simple by imposing the SOS constraint,

$$
-\tilde{G}-\Xi-\varepsilon\left(|\mathbf{a}|^{2}+q^{2}\right) \in \operatorname{SOS}(\mathbf{a}, q) .
$$

The final SDP takes the form,

$$
\max \varepsilon, \quad \text { subject to }(\mathrm{A} .2),(\mathrm{B} .5),(\mathrm{B} .9) \&(\mathrm{~B} .11) \leftarrow \text { Method } 1 .
$$

\section{B.2 Method 2}

This time, let $\delta_{i} \in(0, \infty)$ for all $i=1, \ldots, m$, and proceed as in (A.5) (but with each component separately and taking square roots a posteriori), so that

$$
\left|M_{i}\right|\left(\left|\Theta_{\mathbf{a b} i}\right|+\left|\Theta_{\mathbf{c} i}\right|\right) \leq\left|M_{i}\right|\left(\sqrt{\tilde{\mathbf{a}}^{\top} \mathbf{R}_{i} \tilde{\mathbf{a}} q^{2}}+C_{i} q^{2}\right) \leq\left|M_{i}\right| \underbrace{\sqrt{\left(1+\delta_{i}\right) \tilde{\mathbf{a}}^{\top} \mathbf{R}_{i} \tilde{\mathbf{a}} q^{2}+\left(1+\frac{1}{\delta_{i}}\right) C_{i}^{2} q^{4}}}_{\sqrt{d_{i}\left(\mathbf{a}, q^{2}\right)}} \leq r_{i},
$$

where $r_{i}\left(\mathbf{a}, q^{2}\right)$ is an unknown polynomial with an ansatz as in (B.3). Manipulating as in (B.4), yields the SOS constraints

$$
\left[\begin{array}{cc}
d_{i} r_{i} & d_{i} M_{i} \\
d_{i} M_{i} & r_{i}
\end{array}\right] \in \operatorname{SOS}_{\mathbb{M}}(\mathbf{a}, q), \quad \forall i=1, \ldots, m
$$

Adding (B.13) among all $i=1, \ldots, m$ gives,

$$
\mathbf{M}\left(\mathbf{a}, q^{2}\right) \cdot\left(\boldsymbol{\Theta}_{\mathbf{a b}}\left(\mathbf{u}_{\mathbf{s}}, \mathbf{a}\right)+\boldsymbol{\Theta}_{\mathbf{c}}\left(\mathbf{u}_{\mathbf{s}}\right)\right) \leq \sum_{i=1}^{m} r_{i}\left(\mathbf{a}, q^{2}\right)=\Xi\left(\mathbf{a}, q^{2}\right),
$$

and the remaining SOS constraint is

$$
-\tilde{G}-\Xi-\varepsilon\left(|\mathbf{a}|^{2}+q^{2}\right) \in \operatorname{SOS}(\mathbf{a}, q)
$$

with the final SDP taking the form,

$$
\max \varepsilon, \quad \text { subject to }(\mathrm{A} .2),(\mathrm{B} .14) \&(\mathrm{~B} .16) \leftarrow \text { Method } 2 .
$$




\section{B.3 Method 3}

This method aims to precompute bounds of each $\left|\Theta_{\mathbf{a b} i}\right|$ and then use the bounds of $\left|M_{i}\right|$ via the constraints in (B.9). It is supposed to produce a significant speed up in the computations by avoiding constraints of the form of (B.5) in the global (large) problem, and instead tackling these type of constraints in a previous step consisting of a series of much cheaper and easier small problems that precompute particular bounds. The crux is to develop a viable method to precompute these bounds and to find good criteria to have the most effective bounds possible.

First, consider bounds

$$
0 \leq\left|\Theta_{\mathbf{a b} i}\right| \leq \sqrt{\tilde{\mathbf{a}}^{\top} \mathbf{R}_{i} \tilde{\mathbf{a}} q^{2}} \leq b_{i}
$$

with $b_{i}\left(\mathbf{a}, q^{2}\right)$ being an unknown homogeneous quadratic polynomial of the form,

$$
b_{i}\left(\mathbf{a}, q^{2}\right)=\sum_{\operatorname{deg}\left(\operatorname{mon}_{\iota}\right)=2} c_{\iota}^{b} \operatorname{mon}_{\iota}\left(\mathbf{a}, q^{2}\right)
$$

where the coefficients, $c_{\iota}^{b}$, are unknown. Note the ansatz forces $b_{i}(\mathbf{0}, 0)=0$ (so that later on $\Xi(\mathbf{0}, 0)=0$ as well). This is equivalent to the SOS constraints

$$
\left[\begin{array}{cc}
\tilde{\mathbf{a}}^{\top} \mathbf{R}_{i} \tilde{\mathbf{a}} q^{2} b_{i} & \tilde{\mathbf{a}}^{\top} \mathbf{R}_{i} \tilde{\mathbf{a}} q^{2} \\
\tilde{\mathbf{a}}^{\top} \mathbf{R}_{i} \tilde{\mathbf{a}} q^{2} & b_{i}
\end{array}\right] \in \operatorname{SOS}_{\mathbb{M}}(\mathbf{a}, q), \quad \forall i=1, \ldots, m .
$$

The idea is to precompute the $b_{i}$ beforehand under some optimization criterion (we have to choose what to minimize and maximize). Then, the $c_{\iota}^{b}$ coefficients will be known, and it is valid to use

$$
\mathbf{M}\left(\mathbf{a}, q^{2}\right) \cdot\left(\boldsymbol{\Theta}_{\mathbf{a b}}\left(\mathbf{u}_{\mathbf{s}}, \mathbf{a}\right)+\boldsymbol{\Theta}_{\mathbf{c}}\left(\mathbf{u}_{\mathbf{s}}\right)\right) \leq \sum_{i=1}^{m} s_{i}\left(\mathbf{a}, q^{2}\right)\left(b_{i}\left(\mathbf{a}, q^{2}\right)+C_{i} q^{2}\right)=\Xi\left(\mathbf{a}, q^{2}\right),
$$

where the $s_{i}$ bound $\left|M_{i}\right|$ as in (B.6). This may look viable if one proceeds as in the previous two methods, but it is actually impossible.

To see the problem, simply focus on the assumed bound (B.18), and notice it is impossible to find such a bound. Indeed, assuming $\mathbf{a}=\mathbf{0}$ and $q \neq 0$ (recall $\widetilde{\mathbf{a}}$ has a constant nonzero component) it follows

$$
\sqrt{\tilde{\mathbf{a}}^{\top} \mathbf{R}_{i} \tilde{\mathbf{a}} q^{2}}=\sqrt{D_{i} q^{2}}=\sqrt{D_{i}}|q|, \quad \text { if } \mathbf{a}=\mathbf{0},
$$

where $D_{i}>0$ is a constant. It is impossible to bound this positive function with a positive quadratic polynomial that passes through $q=0$ (see the behaviour near $q=0$ ).

To fix this issue, note that $\frac{\mathrm{d} V}{\mathrm{~d} t}<0$ if and only if $\frac{\mathrm{d} V}{\mathrm{~d} t}\left(|\mathbf{a}|^{2}+q^{2}\right)^{k_{E}}<0$ for any positive integer $k_{E} \in \mathbb{N}$. Thus, let $2 E\left(\mathbf{a}, q^{2}\right)=|\mathbf{a}|^{2}+q^{2}$, choose some $k_{E} \in \mathbb{N}$, and as in (2.9), note that

$$
\frac{\mathrm{d} V}{\mathrm{~d} t}\left(2 E\left(\mathbf{a}, q^{2}\right)\right)^{k_{E}} \leq \tilde{G}\left(\mathbf{a}, q^{2}\right)\left(2 E\left(\mathbf{a}, q^{2}\right)\right)^{k_{E}}+\tilde{\Xi}\left(\mathbf{a}, q^{2}\right),
$$

as long as $\frac{\partial V}{\partial q^{2}} \geq 0$ and that there exists $\tilde{\Xi}\left(\mathbf{a}, q^{2}\right)$ such that $\tilde{\Xi}(\mathbf{0}, 0)=0$ and

$$
\mathbf{M}\left(\mathbf{a}, q^{2}\right) \cdot\left(\boldsymbol{\Theta}_{\mathbf{a b}}\left(\mathbf{u}_{\mathbf{s}}, \mathbf{a}\right)+\boldsymbol{\Theta}_{\mathbf{c}}\left(\mathbf{u}_{\mathbf{s}}\right)\right) \cdot\left(2 E\left(\mathbf{a}, q^{2}\right)\right)^{k_{E}} \leq \tilde{\Xi}\left(\mathbf{a}, q^{2}\right) .
$$


Thus, if such a $\tilde{\Xi}$ exists, $\frac{\partial V}{\partial q^{2}} \geq 0$, and $\tilde{G}(2 E)^{k_{E}}+\tilde{\Xi}<0$ it follows that $\frac{\mathrm{d} V}{\mathrm{~d} t}<0$.

To find such a $\tilde{\Xi}$, instead of (B.18), consider the bounds

$$
\left|\Theta_{\mathbf{a b} i}\right|(2 E)^{k_{E}} \leq \sqrt{\tilde{\mathbf{a}}^{\top} \mathbf{R}_{i} \tilde{\mathbf{a}} q^{2}}(2 E)^{k_{E}} \leq b_{i},
$$

with $b_{i}\left(\mathbf{a}, q^{2}\right)$ being an unknown polynomial of the form,

$$
b_{i}\left(\mathbf{a}, q^{2}\right)=\sum_{2 \leq \operatorname{deg}\left(\operatorname{mon}_{\iota}\right) \leq 2\left(k_{E}+1\right)} c_{\iota}^{b} \operatorname{mon}_{\iota}\left(\mathbf{a}, q^{2}\right),
$$

where the coefficients, $c_{\iota}^{b}$, are unknown. These bounds are now truly viable to find, since the situation in (B.22) no longer holds (i.e., when $\mathbf{a}=\mathbf{0}$ and $k_{E}=1$ the function now grows cubically about the origin and this can be bounded by a positive quartic polynomial passing through $q=0$ ). As before, (B.25) is equivalent to the SOS constraints,

$$
\left[\begin{array}{cc}
\widetilde{\mathbf{a}}^{\top} \mathbf{R}_{i} \tilde{\mathbf{a}} q^{2}(2 E)^{2 k_{E}} b_{i} & \widetilde{\mathbf{a}}^{\top} \mathbf{R}_{i} \widetilde{\mathbf{a}} q^{2}(2 E)^{2 k_{E}} \\
\widetilde{\mathbf{a}}^{\top} \mathbf{R}_{i} \widetilde{\mathbf{a}} q^{2}(2 E)^{2 k_{E}} & b_{i}
\end{array}\right] \in \operatorname{SOS}_{\mathbb{M}}(\mathbf{a}, q), \quad \forall i=1, \ldots, m .
$$

Making $m$ SOS feasibility tests could provide the precomputed bounds $b_{i}$,

$$
\text { Check feasibility of (B.27) } \leftarrow \text { Precompute bound } i, \quad \forall i=1, \ldots, m \text {, }
$$

which yields the coefficients, $c_{\iota}^{b}$ for each $i=1, \ldots, m$.

Then, $\tilde{\Xi}$ is simply,

$$
\mathbf{M} \cdot\left(\mathbf{\Theta}_{\mathbf{a b}}+\boldsymbol{\Theta}_{\mathbf{c}}\right) \cdot(2 E)^{k_{E}} \leq \sum_{i=1}^{m} s_{i}\left(b_{i}+C_{i} q^{2}(2 E)^{k_{E}}\right)=\tilde{\Xi},
$$

and the SOS constraint that implies $\frac{\mathrm{d} V}{\mathrm{~d} t}<0$ is

$$
-\left(\tilde{G}(2 E)^{k_{E}}+\tilde{\Xi}\right)-\varepsilon\left(|\mathbf{a}|^{2}+q^{2}\right) \in \operatorname{SOS}(\mathbf{a}, q) .
$$

Lastly, this method would consist of the SDP

$$
\max \varepsilon, \quad \text { subject to }(\mathrm{A} .2),(\text { B.9) \& (B.30) } \leftarrow \text { Method } 3,
$$

provided the $m$ bounds in (B.25) have been precomputed using (B.28) in a previous step.

The feasibility test in (B.28) is by no means optimal in the sense that one would want the smallest possible upper bound in (B.25), but (B.28) only provides one such upper bound, which could be huge. Ideally, it could be useful to modify the ansatz for $b_{i}$ in (B.26) to include an optimization parameter that somehow ensures that $b_{i}$ is as small as possible. In essence, one should try to change (B.28) from a feasibility problem to an intelligently chosen optimization problem. There are many ways to do this, but no more details are given here for the time being, since the method has not been implemented yet.

From experience, we expect this method to be two or three orders of magnitude faster than the previous two methods. Once we implement this method, more details will be given. 


\section{Improving the Bounds Even More}

Now look at (A.4) once again, but this time stop right after using the triangle inequality for the first time,

$$
\mathbf{M}\left(\mathbf{a}, q^{2}\right) \cdot\left(\boldsymbol{\Theta}_{\mathbf{a b}}\left(\mathbf{u}_{\mathbf{s}}, \mathbf{a}\right)+\boldsymbol{\Theta}_{\mathbf{c}}\left(\mathbf{u}_{\mathbf{s}}\right)\right) \leq\left|\mathbf{M}\left(\mathbf{a}, q^{2}\right) \cdot \boldsymbol{\Theta}_{\mathbf{a b}}\left(\mathbf{u}_{\mathbf{s}}, \mathbf{a}\right)\right|+\left|\mathbf{M}\left(\mathbf{a}, q^{2}\right) \cdot \Theta_{\mathbf{c}}\left(\mathbf{u}_{\mathbf{s}}\right)\right| \cdot
$$

\section{C.1 Bound of $\left|\mathrm{M} \cdot \Theta_{\mathrm{ab}}\right|$}

Proceed as in [2] and using the summation convention note that

$$
\left|\mathbf{M} \cdot \boldsymbol{\Theta}_{\mathbf{a b}}\right|=\left|\left\langle\mathbf{u}_{\mathbf{s}}, M_{i} \widetilde{\mathbf{h}}_{i j} \tilde{a}_{j}\right\rangle\right| \leq\left\|M_{i} \widetilde{\mathbf{h}}_{i j} \tilde{a}_{j}\right\||q| .
$$

In [2], (31) is incorrect (it is even dependent on $\mathbf{x} \in \Omega$ ), because this an $L^{2}$ norm and must be computed explicitly. It can be written as,

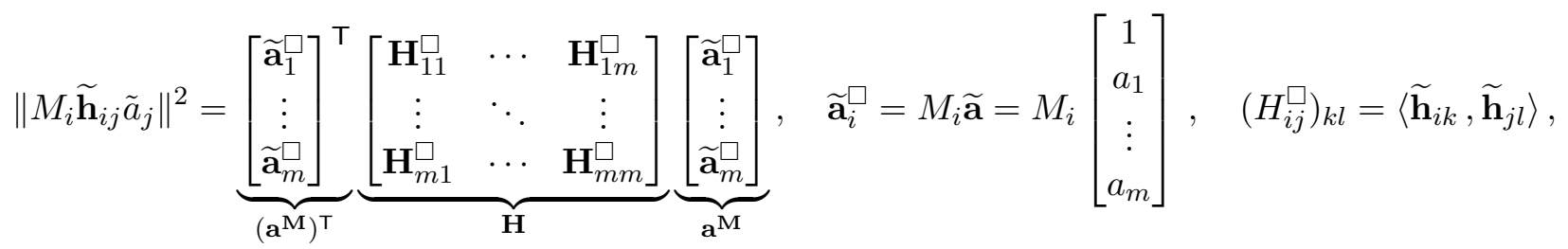

so that $\mathbf{H} \in \mathbb{R}^{m(m+1) \times m(m+1)}$ is a positive semidefinite matrix (compare to the much smaller matrices $\left.\mathbf{R}_{i} \in \mathbb{R}^{(m+1) \times(m+1)}\right)$, and where it is clear that $\mathbf{H}_{i i}^{\square}=\mathbf{R}_{i}$ for every $i=1, \ldots, m$. Thus,

$$
\left|\mathbf{M} \cdot \Theta_{\mathbf{a b} i}\right| \leq \sqrt{\left(\mathbf{a}^{\mathbf{M}}\right)^{\top} \mathbf{H a}^{\mathbf{M}} q^{2}}=\sqrt{\left(\mathbf{H}^{1 / 2} \mathbf{a}^{\mathbf{M}} q\right)^{\top}\left(\mathbf{H}^{1 / 2} \mathbf{a}^{\mathbf{M}} q\right)},
$$

where $\mathbf{H}^{1 / 2}=\left(\mathbf{H}^{1 / 2}\right)^{\top}$ is the unique positive semidefinite square root of $\mathbf{H}$ (computed using the eigenvalue decomposition). Note that a unique Cholesky decomposition also exists and could be used, but the typical algorithm breaks down in the semidefinite case, so it is preferable to use the usual square root of the matrix.

To give a rough idea of how to deal with this bound, assume $\mathbf{M} \cdot \boldsymbol{\Theta}_{\mathbf{c}}$ has been bounded as in (B.6)-(B.9) from Method 1, so that

$$
\mathbf{M}\left(\mathbf{a}, q^{2}\right) \cdot\left(\boldsymbol{\Theta}_{\mathbf{a b}}\left(\mathbf{u}_{\mathbf{s}}, \mathbf{a}\right)+\boldsymbol{\Theta}_{\mathbf{c}}\left(\mathbf{u}_{\mathbf{s}}\right)\right) \leq \sqrt{\left(\mathbf{H}^{1 / 2} \mathbf{a}^{\mathbf{M}} q\right)^{\top}\left(\mathbf{H}^{1 / 2} \mathbf{a}^{\mathbf{M}} q\right)}+\sum_{i=1}^{m} s_{i}\left(\mathbf{a}, q^{2}\right) C_{i} q^{2}=\Xi\left(\mathbf{a}, q^{2}\right) .
$$

Then, to enforce $\tilde{G}+\Xi<0$, note that

$$
\begin{aligned}
\tilde{G}+\Xi<0 & \Leftarrow \underbrace{\tilde{G}+\sum_{0}^{m} s_{i}\left(\mathbf{a}, q^{2}\right) C_{i} q^{2}+\varepsilon\left(|\mathbf{a}|^{2}+q^{2}\right)}_{i=1}+\sqrt{\left(\mathbf{H}^{1 / 2} \mathbf{a}^{\mathbf{M}} q\right)^{\top}\left(\mathbf{H}^{1 / 2} \mathbf{a}^{\mathbf{M}} q\right)} \leq 0 \\
& \Leftrightarrow\left[\begin{array}{cc}
-\tilde{G}_{0} & \left(\mathbf{H}^{1 / 2} \mathbf{a}^{\mathbf{M}} q\right)^{\top} \\
\mathbf{H}^{1 / 2} \mathbf{a}^{\mathbf{M}} q & -\tilde{G}_{0} \mathbf{I}
\end{array}\right] \succeq 0 .
\end{aligned}
$$


This formulation would have been impossible without the decomposition in (C.4) which uses $\mathbf{H}^{1 / 2}$, since $\left(\mathbf{a}^{\mathbf{M}}\right)^{\mathrm{T}} \mathbf{H a}^{\mathbf{M}} q^{2}$ is quadratic in $\mathbf{M}$ and thus quadratic in the unknown coefficients $c_{\iota}$ of $V$. Thankfully, this reformulation avoids any such problems. However, one should note that the matrix in (C.6) is huge. Therefore, creating auxiliary variables $z_{0}, z_{1}, \ldots, z_{m(m+1)}$ to exploit the sparsity might be computationally prohibitive. Perhaps using Agler's theorem as described in [5] is a more viable approach. In any case, this formulation was discovered at the last moment, so it has not been implemented.

\section{C.2 Bound of $\left|\mathrm{M} \cdot \Theta_{\mathrm{c}}\right|$}

The best one could hope for is to proceed as in [9] but with the whole $\mathbf{M} \cdot \boldsymbol{\Theta}_{\mathbf{c}}$ instead. This yields,

$$
\left|\mathbf{M} \cdot \boldsymbol{\Theta}_{\mathbf{c}}\right| \leq\left\|\rho\left(\sum_{i=1}^{m} M_{i} \mathbf{D}_{i}\right)\right\|_{\infty}=\sup _{\mathbf{x} \in \Omega} \rho\left(\sum_{i=1}^{m} M_{i} \mathbf{D}_{i}\right) .
$$

Unfortunately we have not found a way to compute this quantity, even if $M_{i}$ was known, which in principle it is not. The best bounds at this moment are those calculated via (B.6)-(B.9) in Method 1.

\section{Global Stability Using Bounds and Conditional Stability}

This approach was proposed in [2] very briefly, so here I will give some more details. As a heads up, it is more expensive computationally, but, in principle, still worth trying if it allows to prove global stability beyond what the other methods can. It is divided into three steps:

1. Choose a positive definite quantity of interest $\Phi\left(\mathbf{a}, q^{2}\right) \geq 0$, so that $\Phi\left(\mathbf{a}, q^{2}\right)=0$ if and only if $\left(\mathbf{a}, q^{2}\right)=(\mathbf{0}, 0)$, and which you expect to be conditionally stable for the known equilibrium point of the dynamical system, $(\mathbf{0}, 0)$.

2. Compute a time-average bound $U \in \mathbb{R}$ such that $\overline{\Phi\left(\mathbf{a}, q^{2}\right)} \leq U$, where $\overline{\Phi\left(\mathbf{a}, q^{2}\right)}=$ $\lim _{T \rightarrow \infty} \frac{1}{T} \int_{0}^{t} \Phi\left(\mathbf{a}(t), q^{2}(t)\right) \mathrm{d} t$. Obviously this implies that for each initial condition $\left(\mathbf{a}(0), q^{2}(0)\right)$ there exists some instant $t^{*}$, such that $\Phi\left(\mathbf{a}\left(t^{*}\right), q^{2}\left(t^{*}\right)\right)=U$.

3. Show that whenever $0<\Phi<C=U+\bar{\varepsilon}$, then $\frac{\mathrm{d} \Phi}{\mathrm{d} t}<0$. This implies that if $\Phi\left(\mathbf{a}, q^{2}\right)<C$ at some instant $t$, then $\lim _{t \rightarrow \infty} \Phi\left(\mathbf{a}(t), q^{2}(t)\right)=0$ and as a result $\lim _{t \rightarrow \infty}\left(\mathbf{a}(t), q^{2}(t)\right)=(\mathbf{0}, 0)$. Here, $\bar{\varepsilon}>0$ is a fixed positive small quantity. In particular, by step 2 , it follows that regardless of the initial condition $\lim _{t \rightarrow \infty}\left(\mathbf{a}(t), q^{2}(t)\right)=$ $(\mathbf{0}, 0)$, which means that the flow is globally stable (since the velocity satifies $\|\mathbf{u}(t)\|^{2}=$ $\left.|\mathbf{a}(t)|^{2}+q^{2}(t)\right)$.

An important comment on step 3 , is that it is not sufficient to simply prove that $\frac{\mathrm{d} \Phi}{\mathrm{d} t}<0$ in an open set (like any ball of radius $R$ ) containing $\left(\mathbf{a}, q^{2}\right)=(\mathbf{0}, 0)$, as this will only imply that $(\mathbf{0}, 0)$ is locally asymptotically stable, but will not give any information on the critical set (which will be some unknown ball of radius $0<R_{0}<R$ if your initial set was a ball of radius $R$ ) leading to asymptotic convergence. You will only know that such a critical set around $(\mathbf{0}, 0)$ exists and is a subset of the original open set. So it is fundamental to prove that $\frac{\mathrm{d} \Phi}{\mathrm{d} t}<0$ whenever $\Phi<C$, since this actually does imply that the critical set are the points for which $\Phi<C$. 
The combination of the three steps will be referred to as the "Bounding" method for short.

\section{D.1 Step 1}

The most natural choice for a positive-definite $\Phi$, which you expect to be conditionally stable, is precisely the Lyapunov function associated to the linearized system of equations. For a truncated set of $m$ modes, $\mathbf{a}=\left(a_{1}, \ldots, a_{m}\right)$, and forgetting about $\mathbf{u}_{\mathbf{s}}$, the linearized dynamical system (see (2.5) and (2.6)) is simply,

$$
\frac{\mathrm{d} \mathbf{a}}{\mathrm{d} t}=\mathbf{f}(\mathbf{a})=\mathbf{L a}, \quad f_{i}(\mathbf{a})=\left(\frac{1}{\operatorname{Re}}\left\langle\mathbf{u}_{i}, \nabla^{2} \mathbf{u}_{j}\right\rangle-\left\langle\mathbf{u}_{i}, \mathbf{u}_{j} \cdot \nabla \mathbf{V}+\mathbf{V} \cdot \nabla \mathbf{u}_{j}\right\rangle\right) a_{j}=L_{i j} a_{j} .
$$

To find a Lyapunov function simply seek a positive definite matrix $\mathbf{P} \succ 0$ such that $\mathbf{L}^{\top} \mathbf{P}+$ $\mathbf{P L} \prec 0$, and the Lyapunov function will be $\Phi_{T}(\mathbf{a})=\mathbf{a}^{\top} \mathbf{P a}$. As usual to ensure strict positivity or negativity one needs barrier functions, so that one can solve an SDP enforcing,

$$
\max \varepsilon, \quad \text { subject to } \quad \mathbf{P}-\varepsilon \mathbf{I} \succeq 0 \quad-\left(\mathbf{L}^{\top} \mathbf{P}+\mathbf{P L}\right)-\varepsilon \mathbf{I} \succeq 0, \quad \operatorname{tr}(\mathbf{P})=m,
$$

where $\operatorname{tr}(\mathbf{P})$ is the usual trace of a matrix. The last condition simply ensures the scaling of $\mathbf{P}$ is fixed, and also has the nice property that $|\mathbf{a}|^{2}$ also satisfies it. The maximization of $\varepsilon$ intuitively ensures that $\Phi_{T}(\mathbf{a})=\mathbf{a}^{\top} \mathbf{P a}$ is as far away from zero as possible, and thus more likely to have better conditional stability behavior.

However, the role of $q^{2}$ must also be added. We decided to do this a posteriori by simply considering,

$$
\Phi\left(\mathbf{a}, q^{2}\right)=\mathbf{a}^{\top} \mathbf{P a}+\alpha_{\Phi} q^{2},
$$

where $\alpha_{\Phi}>0$ is a constant to be chosen. Due to the constraint that $\operatorname{tr}(\mathbf{P})=m$, it makes sense (in terms of order of magnitude) to choose $\alpha_{\Phi}=1$, but other possibilities include $\alpha_{\Phi}=\min _{i} P_{i i}$ or $\alpha_{\Phi}=\max _{i} P_{i i}$. In this work we chose $\alpha_{\Phi}=1$.

\section{D.2 Step 2}

Here, we proceed as described in [6], where it was shown that for bounded trajectories (in a fluid system all trajectories are bounded) and for any storage function $V\left(\mathbf{a}, q^{2}\right)$,

$$
\frac{\mathrm{d} V\left(\mathbf{a}(t), q^{2}(t)\right)}{\mathrm{d} t}+\Phi\left(\mathbf{a}, q^{2}\right) \leq U \quad \Rightarrow \quad \overline{\Phi\left(\mathbf{a}, q^{2}\right)} \leq U .
$$

Looking at (2.9), it is clear that it suffices to show that

$$
\tilde{G}+\Xi+\Phi \leq U
$$

Here, once again $\Xi$ is simply a bound in terms of $\left(\mathbf{a}, q^{2}\right)$, and there are several ways to obtain a valid $\Xi$, as we have shown throughout this document. We chose the one from Method 1, as it is the one of the tightest bounds leading to a problem of reasonable size and which does not use the "Peter-Paul" inequality. Thus, choose $\Xi$ as in (B.10), so that the SDP becomes

$$
\min U, \quad \text { subject to } \frac{\partial V}{\partial q^{2}} \in \operatorname{SOS}(\mathbf{a}, q) \text {, (B.5), (B.9) \& } U-\tilde{G}-\Xi-\Phi \in \operatorname{SOS}(\mathbf{a}, q) \text {. }
$$


The main difference in these constraints and derivation (with respect to the other methods) is the presence of $U$, that $V$ no longer has to be positive definite (and is no longer free of scaling) and more importantly that $V(\mathbf{0}, 0)$ and $\Xi(\mathbf{0}, 0)$ no longer have to vanish. Therefore, the ansatzes (A.1) and (B.3) should be modified to

$$
\begin{aligned}
V\left(\mathbf{a}, q^{2}\right) & =\sum_{0 \leq \operatorname{deg}\left(\operatorname{mon}_{\iota}\right) \leq \operatorname{deg}(V)-1} c_{\iota} \operatorname{mon}_{\iota}\left(\mathbf{a}, q^{2}\right)+c_{E}\left(|\mathbf{a}|^{2}+q^{2}\right)^{\operatorname{deg}(V) / 2}, \\
r_{i}\left(\mathbf{a}, q^{2}\right) & =\sum_{0 \leq \operatorname{deg}\left(\operatorname{mon}_{\iota}\right) \leq \operatorname{deg}(V)} c_{\iota}^{r} \operatorname{mon}_{\iota}\left(\mathbf{a}, q^{2}\right) .
\end{aligned}
$$

\section{D.3 Step 3}

We know that $\Phi(\mathbf{0}, 0)=0$ and that $\frac{\mathrm{d} \Phi}{\mathrm{d} t}(\mathbf{0}, 0)=0$, so when $\left(\mathbf{a}, q^{2}\right) \neq(\mathbf{0}, 0)$, the final step is to prove that $\Phi<C=U+\bar{\varepsilon}$ implies that $\frac{\mathrm{d} \Phi}{\mathrm{d} t}<0$, where $\bar{\varepsilon}$ is a small positive number. Using the $S$-procedure, it is sufficient to satisfy the condition

$$
\frac{\mathrm{d} \Phi}{\mathrm{d} t} \leq-(C-\Phi) S
$$

where $S$ is a positive definite function satisfying that $S(\mathbf{0}, 0)=0$ (so that $\frac{\mathrm{d} \Phi}{\mathrm{d} t}(\mathbf{0}, 0)=0$ does not violate the inequality). Note, all the focus here is the behavior near the origin $(\mathbf{0}, 0)$ since far away, where $-(C-\Phi) S$ is very positive, it does not really matter.

Next, simply treat $\Phi$ as $V$ in (2.9) and proceed analogously to note that it is sufficient to prove that

$$
\tilde{G}_{\Phi}+\Xi_{\Phi} \leq-(C-\Phi) S,
$$

where $\tilde{G}_{\Phi}=\frac{\partial \Phi}{\partial \mathbf{a}} \cdot \mathbf{f}(\mathbf{a})+2 \frac{\partial \Phi}{\partial q^{2}} \kappa q^{2}$ and where $\mathbf{M}_{\Phi} \cdot\left(\boldsymbol{\Theta}_{\mathbf{a b}}+\boldsymbol{\Theta}_{\mathbf{c}}\right) \leq \Xi_{\Phi}$ with $\mathbf{M}_{\Phi}=\frac{\partial \Phi}{\partial \mathbf{a}}-2 \frac{\partial \Phi}{\partial q^{2}} \mathbf{a}$ and $\Xi_{\Phi}(\mathbf{0}, 0)=0$. Note that the inequality is sufficient since we already know that $\frac{\partial \Phi}{\partial q^{2}}=\alpha_{\Phi}>0$ by construction (see (D.3)). Once again, $\Xi_{\Phi}$ can be estimated in different ways, and for much the same reasons described in step 2 we chose the technique from Method 1.

Since $S$ is unknown, expected to be an SOS polynomial and with $S(\mathbf{0}, 0)=0$, consider the ansatz

$$
S\left(\mathbf{a}, q^{2}\right)=\sum_{2 \leq \operatorname{deg}\left(\operatorname{mon}_{\iota}\right) \leq \operatorname{deg}(S)} c_{\iota}^{S} \operatorname{mon}_{\iota}\left(\mathbf{a}, q^{2}\right),
$$

where $\operatorname{deg}(S)$ is an even number to be chosen freely. Therefore, the final SDP is a feasibility test of the following conditions,

$$
S-\overline{\bar{\varepsilon}}\left(|\mathbf{a}|^{2}+q^{2}\right) \in \operatorname{SOS}(\mathbf{a}, q),(\mathrm{B} .5)_{\Phi}, \quad(\mathrm{B} .9)_{\Phi} \quad \&-\tilde{G}_{\Phi}-\Xi_{\Phi}-(C-\Phi) S \in \operatorname{SOS}(\mathbf{a}, q),
$$

where $\overline{\bar{\varepsilon}}$ is another small positive number. One could attempt to maximize $\overline{\bar{\varepsilon}}$, but in this particular case does not yield interesting insight and seems to add cost. Here, $(\text { B.5 })_{\Phi}$ and $(\mathrm{B} .9)_{\Phi}$ are simply (B.5) and (B.9) but with $\mathbf{M}$ replaced by $\mathbf{M}_{\Phi}$ instead. Meanwhile in the ansatzes (B.3) and (B.7), $\operatorname{deg}(V)$ should be replaced by 4, since $\operatorname{deg}\left(\mathbf{M}_{\Phi}\right)$ is not $\operatorname{deg}(\Phi)-2=0(\operatorname{deg}(\mathbf{M})=\operatorname{deg}(V)-2$ only happens due to the ansatz for $V$ in (B.3)), but rather $\operatorname{deg}\left(\mathbf{M}_{\Phi}\right)=1$ so the $r_{i}$ and $s_{i}$ are chosen to be the smallest even degree which makes sense, i.e. $\operatorname{deg}\left(r_{i}\right)=4$ and $\operatorname{deg}\left(s_{i}\right)=2$. 


\section{E Computational Results of Different Approaches}

The results shown here are for two-dimensional plane Couette flow with a length $L_{\text {dom }}=\frac{2 \pi}{3.75}$ (where the height is unity), so that the principal wavenumber is $\alpha_{1}=3.75$. This is very close to the critical wavenumber obtained via the energy stability limit, and the energy stability limit in this domain is $\operatorname{Re}_{E}=177.3$.

The idea is to compare the different methods described (at least those that were implemented). With this in mind, we first show results at $R e=179.5$ which is above the energy stability limit. We do this using six eigenmodes: the eigenmodes corresponding to the two largest eigenvalues at $\alpha_{0}=0$ and the same with $\alpha_{1}=3.75$. There are six, because for each eigenvalue associated to $\alpha \neq 0$ there are two eigenmodes: horizontal translation invariance requires choosing two linearly independent eigenmodes and we choose them to be $\frac{\pi}{2}$ out of phase to ensure orthogonality. We refer to this set of six eigenmodes as "Modes I." Additionally, "Modes II" has all the modes in "Modes I" with two additional associated to the highest eigenvalue of $\alpha_{2}=7.5$, for a total of eight modes. All experiments were performed with $\operatorname{deg}(V)=4$. A value of $\delta_{i}=1$ was used at every point where the "Peter-Paul" inequality was utilized. Lastly, in the Bounding method ( $(\mathrm{D})$ it was used that $\bar{\varepsilon}=10^{-8}$ and $\overline{\bar{\varepsilon}}=10^{-4}$. To compare the results and computational performance of each algorithm, it is useful to show the final value of $\varepsilon$, as this gives a rough idea of how effective the algorithm is (the higher the value of $\varepsilon$ the better), and we also are showing the time inside the SDP solver to give a rough estimate of the computational costs, which are typically high. Having said that, the total computation time consists of: (i) the time taken to solve the eigenvalue problem and compute the relevant tensors $\left(\mathbf{L}, \mathcal{N}, \mathbf{R}_{i}\right.$ and $C_{i}$ in (2.6) and (A.3)), (ii) the time to setup the SDP (i.e., computing the dynamical system itself along with all the SDP constraints), (iii) the preprocessing time to find symmetries and parse to the appropriate format compatible with a given SDP solver, and (iv) the SDP solver time. The results are shown in Table 1.

\begin{tabular}{c|c|c|c|c|}
\multirow{2}{*}{ SDP } & \multicolumn{4}{|c|}{ Re $=179.5$} \\
& $\varepsilon$ & Sodes I & \multicolumn{2}{c|}{ Modes II } \\
& $\varepsilon$ SD solver time (s) & $\varepsilon$ & SDP solver time (s) \\
\hline Original 1 & $2.54 \times 10^{-6}$ & 48 & - & - \\
Original 2 & 0.0030 & 48 & - & - \\
Method 1 & 0.0175 & 210 & 0.1495 & 3462 \\
Method 2 & 0.0170 & 235 & 0.1512 & 8931 \\
Bounding & - & 339 & - & 5195
\end{tabular}

Table 1: Performance of the methods at a fixed $\mathrm{Re}=179.5$ and for two different sets of modes.

In terms of $\varepsilon$, the two methods that seem to be the most robust are Method 1 and Method 2, but for some reason, the computational cost of Method 2 is actually higher and does not scale well as more modes are added, so Method 1 is preferred. Also, Original 1 and Original 2 behave very similarly, as expected, but Original 1 produces a value of $\varepsilon$ which is safer to trust. Unfortunately, large memory requirements did not allow for simulations to be completed with Modes II via Original 1 and Original 2. Thus, these methods do not scale well as more modes are added, and to overcome this, probably an alternative implementation that does not use auxiliary variables is required (see Section A.3 for more 
details). Lastly, since the Bounding method is based on Method 1, it was to be expected that the cost was higher than that of Method 1, which indeed is the case.

Next, the results are shown for both sets of modes, but this time showing the highest Reynolds number at $L_{\mathrm{dom}}=\frac{2 \pi}{3.75}$ for which each method was found to be stable. These are presented in Table 2.

\begin{tabular}{c|c|c|c|c|}
\multirow{2}{*}{ SDP } & \multicolumn{3}{|c|}{ Best Re attaining global stability } \\
& Re Modes I & $\varepsilon$ & Re & $\varepsilon$ \\
\hline Original 1 & 179.5 & $2.54 \times 10^{-6}$ & - & - \\
Original 2 & 179.5 & 0.0030 & - & - \\
Method 1 & 194 & $1.60 \times 10^{-4}$ & 230 & $1.07 \times 10^{-5}$ \\
Method 2 & 195 & $2.97 \times 10^{-5}$ & 228 & $9.16 \times 10^{-5}$ \\
Bounding & 190 & - & 214 & -
\end{tabular}

Table 2: Best Re resulting in global stability for each method and for two different sets of modes.

Clearly, Method 1 seems to be the best in terms of obtaining the largest value of Re (combined with a better computational performance over Method 2). Method 2 and the Bounding method perform quite well but are more expensive than Method 1. Meanwhile the Original 1 and Original 2 methods perform almost the same and do not go very high. When adding more modes, the question was if higher Re could be attained or if the bounds would degenerate to the point where it was not viable to add more modes. Thankfully, it seems, up to the precision of the solvers, that for Method 1 and Method 2 the performance does improve (Method 2 is much more expensive). In any case, the results are satisfactory, since the highest value one could hope for with these sets of modes (i.e. where the truncated system becomes unstable with both Modes I and Modes II) was $\operatorname{Re}=266$, and Method 1 resulted in $\operatorname{Re}=230$, which is not very far. Experimenting with different $\delta_{i}$ might improve the results of Method 2 even further. In the future, Method 3 could provide significant advantages in terms of speed, and is certainly worth looking into. On the other hand, the method using much better bounds for $\mathbf{M} \cdot \boldsymbol{\Theta}_{\mathbf{a b}}$ seems very expensive but it could be useful to try out eventually, as it may improve the global stability results.

Note that all results reported here were implemented using MATLAB via YALMIP. The SDP solver used was MOSEK. To cement the confidence in the results, they could potentially be verified with multiple-precision (using the solver SDPA-GMP) or with interval arithmetic (using the solver VSDP which relies on INTLAB), but such verification has not been done.

\section{References}

[1] F. H. Busse, A property of the energy stability limit for plane parallel shear flow, Archive for Rational Mechanics and Analysis, 47 (1972), pp. 28-35.

[2] S. I. Chernyshenko, 11th informal note on stability and bounds by SOS: speed up and better $\Theta$ estimates, Informal note: private communication, (2017).

[3] O. Dauchot and F. Daviaud, Finite-amplitude perturbation in plane Couette flow, Europhysics Letters, 28 (1994), p. 225. 
[4] P. G. Drazin and W. H. Reid, Hydrodynamic Stability, Cambridge University Press, Cambridge, UK, second ed., 2004.

[5] G. Fantuzzi, Comments on Sergei's informal note \#11, Informal note: private communication, (2017).

[6] G. Fantuzzi, D. Goluskin, D. Huang, and S. I. Chernyshenko, Bounds for deterministic and stochastic dynamical systems using sum-of-squares optimization, SIAM Journal on Applied Dynamical Systems, 15 (2016), pp. 1962-1988.

[7] P. J. Goulart and S. I. Chernyshenko, Global stability analysis of fluid flows using sum-of-squares, Physica D: Nonlinear Phenomena, 241 (2012), pp. 692-704.

[8] S. Grossmann, The onset of shear flow turbulence, Reviews of Modern Physics, 72 (2000), pp. 603-618.

[9] D. Huang, S. Chernyshenko, P. Goulart, D. Lasagna, O. Tutty, and F. Fuentes, Sum-of-squares of polynomials approach to nonlinear stability of fluid flows: an example of application, Proceedings of the Royal Society of London A: Mathematical, Physical and Engineering Sciences, 471 (2015), pp. 1364-5021.

[10] D. D. Joseph, Nonlinear stability of the Boussinesq equations by the method of energy, Archive for Rational Mechanics and Analysis, 22 (1966), pp. 163-184.

[11] _ Stability of Fluid Motions I, vol. 27 of Springer Tracts in Natural Philosophy, Springer-Verlag, Berlin, Germany, 1976.

[12] M. NAGATA, Three-dimensional finite-amplitude solutions in plane couette flow: bifurcation from infinity, Journal of Fluid Mechanics, 217 (1990), pp. 519-527.

[13] W. M. OrR, The stability or instability of the steady motions of a perfect liquid and of a viscous liquid. Part II: A viscous liquid, Proceedings of the Royal Irish Academy. Section A: Mathematical and Physical Sciences, 27 (1907), pp. 69-138.

[14] S. A. Orszag And L. C. Kells, Transition to turbulence in plane poiseuille and plane couette flow, Journal of Fluid Mechanics, 96 (1980), pp. 159-205.

[15] V. A. Romanov, Stability of plane-parallel Couette flow, Functional Analysis and Its Applications, 7 (1973), pp. 137-146.

[16] J. SERrin, On the stability of viscous fluid motions, Archive for Rational Mechanics and Analysis, 3 (1959), pp. 1-13.

[17] G. I. TAYLOR, Stability of a viscous liquid contained between two rotating cylinders, Philosophical Transactions of the Royal Society of London A: Mathematical, Physical and Engineering Sciences, 223 (1923), pp. 289-343.

[18] N. Tillmark and P. H. Alfredsson, Experiments on transition in plane Couette flow, Journal of Fluid Mechanics, 235 (1992), pp. 89-102. 


\title{
Indentation of Deformable Plastic Layers
}

\author{
Thomasina Ball
}

December 10, 2017

\section{Introduction}

The indentation of deformable plastic layers has been studied extensively for determining the vertical bearing capacity of rigid strip footings. The solutions to this classic problem $[14 ; 9]$ use the method of characteristics to determine an upper bound on the pressure underneath a vertically loaded indenter placed onto an idealised semi-inifinite rigid-plastic foundation. These studies for a homogeneous half-space have been extended to consider non-homogeneous plastic solids [17; 15]; irregular shaped bodies [18]; rolling contacts [11; 4] and axisymmetric geometries $[16 ; 6 ; 3]$. The connection to a finite layer has also been considered for the plastic flow between two rough parallel plates being forced together $[14 ; 9]$, with application to the flow near the end of a glacier [13]. However, apart from a few exceptions, most problems require numerical integration along characteristic curves to calculate the pressure.

More recently, the behaviour of a finite plastic layer has been explored through the use of viscoplastic fluids. Viscoplastic lubrication theory has been used to model the final shape of a two-dimensional slump [5] and the confined flow of viscoplastic fluid between rigid moving boundaries [8], where asymptotic expansion is based on the small aspect ratio $\epsilon$. This formulation provides a more simple approach to calculating the plastic deformation of a shallow layer.

One application of studying the indentation of deformable plastic layers is in understanding the formation of footprints. Tracks and traces are of particular interest when studying extinct animals as they provide evidence for behaviour, paleoecology and evolution [7]. A number of studies have investigated the relationship between the indenter shape, the indentation left behind and the rheology of the substrate to try and deduce what characteristics can be determined from tracks and traces of distinct animals. Simple indentation models [1] have been used when looking at subfossil mammalian tracks in the Severn estuary. These experiments consisted of indenting an axisymmetric indenter into a layered plasticine structure and showed qualitative agreement with the essential features of track formation. Other experimental work has focused on practical field studies such [12] looking at live emus running across a range of substrate rheologies, from dry to damp sand and firm to fluid mud or clay. They found that footprints from the same trackmaker can appear morphologically different according to the properties of the sediment, and hence shows that care needs to be taken to understand the original rheological conditions of the substrate at the time of track making. More recently, numerical studies have been used to simulate the indentation of a deformable layer [2]. They used a finite-element simulation to study the depth versus 
pressure exerted due to a rigid human foot indenting an elastic-plastic substrate with and without a firm subsurface layer. They found that depending on the depth of the initial deformable layer, regions either indented deeper or shallower than expected from the peak pressure in that region. This work demonstrated the importance of underfoot consolidation in altering when sediment is able to resist deformation under load and support larger pressures. These laboratory and numerical studies described above demonstrate the complexity of understanding the formation of footprints and the leading order effects substrate rheology and foot morphology can have. However, a simple quantitative approach to understanding the characteristics of footprints has yet to be taken.

In this study, we will explore the indentation of deformable plastic layers by building on the current advances in viscoplastic lubrication theory. In particular, we will consider the vertical indentation of a shallow viscoplastic layer by a flat-based and cylindrical indenter. We hope this will give insight the dominant balances in plastic deformation with application to footprint formation amongst many other interesting problems.

\section{Theoretical Model: Loading}

We wish to understand the deformation of a plastic layer by a rigid indenter. To do so we consider two stages: i) loading stage, where indentation is due to a given force, and ii) lift off stage, where the indenter lifts off the deformed substrate at a given speed. In all of the analysis to follow, we assume a two-dimensional geometry and that the motion of the indenter is always perpendicular to the substrate.

To model the substrate, we consider two different rheologies: a purely cohesive model with cohesion $\tau_{Y}$ to describe a mud-like substrate, and secondly a non-cohesive model with angle of friction $\phi$ to describe a granular substrate such as dry sand. During the loading stage, a rigid indenter deforms the substrate due to a given force $F$. We consider firstly the indentation of a shallow viscoplastic layer and then outline current theory for the indentation of a deep plastic layer.

\subsection{Shallow layer}

We use viscoplastic lubrication theory with a Bingham rheology as a vehicle for understanding a shallow plastic layer [8]. We consider the deformation of a uniform layer of viscoplastic fluid of height $h_{0}$, density $\rho$, viscosity $\mu$ with an indenter of geometry $\eta(x)$, and characteristic length scale $x_{0}$. The height of the indenter is given by $h(x, t)=\delta(t)+\eta(x)$, where $\delta(0)=h_{0}$, for $-L<x<L$ where $L$ is the contact point on the indenter. The free-surface height of the fluid outside of this region $x>L$ is also defined as $h(x, t)$. We consider two particular cases: (a) when the the contact point $L$ is fixed for example a flat indenter with corners, $\eta(x)=0$, and (b) when the contact point $L$ is moving and the indenter has some geometry $\eta(x)$ for example a cylindrical indenter or a flat indenter with rounded edges. In case (a) we would like to solve for the height the free-surface reaches up the side of the indenter $h(L, t)=h_{L}(t)$, whereas in case (b) we would like to solve for the contact point $L(t)$ and hence calculate $h_{L}(t)=\delta(t)+\eta(L)$, see figure 1 .

The shallow-layer approximation can be used when the characteristic length scales are much greater than the characteristic height scales, $x_{0} \gg h_{0}$. 
(a)

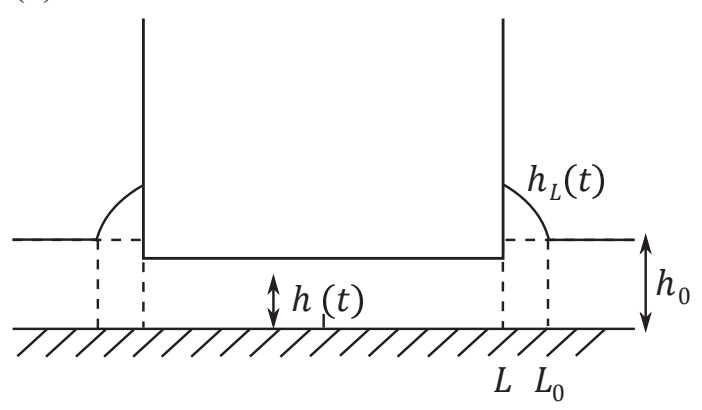

(b)

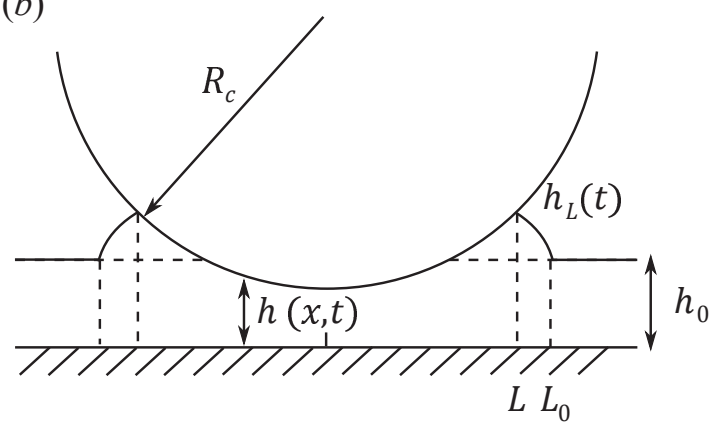

Figure 1: Schematic of (a) flat-based indenter, $\eta(x)=0$ and (b) cylinder, $\eta(x)=\frac{1}{2 R_{c}} x^{2}$.

\subsection{Bingham rheology}

To parametrise the viscoplastic layer we consider a Bingham rheology. A Bingham rheology is one in which the stress tensor varies linearly with strain rate provided the stress is above a given yield stress. Below this yield stress the strain rate is zero. This can be summarised as follows

$$
\begin{aligned}
\tau_{i j}=\left(\frac{\tau_{Y}}{\dot{\gamma}}+\mu\right) \dot{\gamma}_{i j} & |\tau|>\tau_{Y} \\
\dot{\gamma}_{i j}=0 & \text { otherwise }
\end{aligned}
$$

where $\dot{\gamma}=\sqrt{\frac{1}{2} \dot{\gamma}_{i j} \dot{\gamma}_{i j}}$ and $\tau=\sqrt{\frac{1}{2} \tau_{i j} \tau_{i j}}$. The form of the yield stress $\tau_{Y}$ depends on the rheology of the substrate. For the purely cohesive case, the yield stress is constant and for the non-cohesive case the yield stress varies linearly with pressure, $\tau_{Y}=p \tan \phi$, where $\phi$ is the angle of friction.

Assuming horizontal scales $x_{0}$ are much larger than vertical scales $h_{0}$, the thin film approximation can be used such that

$$
u \gg w \text { and } \frac{\partial}{\partial x} \ll \frac{\partial}{\partial z} .
$$

These scales allow $O\left(\epsilon=h_{0} / x_{0}\right)$ terms to be identified in the momentum equation and the strain rate tensor, and hence gives

$$
\begin{aligned}
\frac{\partial}{\partial z} \tau_{x z} & =\frac{\partial p}{\partial x} \\
0 & =\frac{\partial p}{\partial z}+\rho g .
\end{aligned}
$$

The pressure can then be integrated to give

$$
p=P+\rho g(h-z),
$$

where $P$ is the pressure at $z=h$. Substituting into the momentum equation then gives the stress as a function of $x$ and $z$,

$$
\tau_{x z}=T_{x z}+\left(P_{x}+\rho g h_{x}\right)(z-h) \equiv\left(\frac{\tau_{Y}}{\dot{\gamma}}+\mu\right) u_{z} \quad \text { for } \quad\left|\tau_{x z}\right|>\tau_{Y}
$$


where $T_{x z}$ is the stress at $z=h$. The model can be split into two regions: region 1 below the indenter $-L \leq x \leq L$; and region 2 outside the indenter $x \geq L$. In region 1 there are no-slip conditions on the top and bottom boundaries generating a squeeze flow beneath the indenter. In region 2 there is a free-surface on the top boundary giving a zero stress boundary condition there.

\subsection{Purely cohesive, $\tau_{Y}=$ constant}

\subsubsection{Free-surface flow}

For the free-surface flow $T_{x z}=0$ and $P=0$ due to the zero normal stress condition on the top surface. From equation (7) in section 2.2 we then have

$$
u_{z}=\frac{\left(P_{x}+\rho g h_{x}\right)}{\mu}(z-h)-\frac{\operatorname{sgn}\left(u_{z}\right)}{\mu} \tau_{Y} .
$$

The $\operatorname{sgn}\left(u_{z}\right)$ is required because the fluid is yielded when $|\tau|>\tau_{Y}$. Rearranging the equation for the velocity gradient gives an expression for the yield surface $Y$ when $\dot{\gamma} \equiv\left|u_{z}\right|=0$,

$$
u_{z}=-\frac{\rho g h_{x}}{\mu}(Y-z) \quad \text { where } \quad Y=h+\frac{\tau_{Y}}{\rho g h_{x}} .
$$

Integrating and using the no-slip boundary condition at $z=0$ gives velocity field

$$
\begin{array}{cc}
u=-\frac{\rho g h_{x}}{2 \mu}(2 Y-z) z & 0 \leq z \leq Y, \\
u=u_{p}=-\frac{\rho g h_{x}}{2 \mu} Y^{2} & Y \leq z \leq h,
\end{array}
$$

where $u_{p}$ is the plug velocity. Integrating the free-surface flux and then applying local mass conservation gives a governing equation for the evolution of the free surface

$$
\frac{\partial h}{\partial t}=\frac{\rho g}{6 \mu} \frac{\partial}{\partial x}\left(h_{x} Y^{2}(3 h-Y)\right), \quad F^{F S}=-\frac{\rho g h_{x}}{6 \mu} Y^{2}(3 h-Y),
$$

where $F^{F S}$ is the net horizontal flux.

\subsubsection{Squeeze flow}

Underneath the indenter there is a squeeze flow as fluid is pushed out the way by the indenter. The no-slip boundaries conditions give a parabolic profile suggesting there is a plug region in the centre at the turning point where $u_{z}=0$. We can also assume symmetry about the centre line $z=h / 2$ and hence apply boundary conditions

$$
u=0 \quad \text { at } \quad z=0, \quad u_{z}=0 \Rightarrow \tau_{x z}=0 \quad \text { at } \quad z=\frac{h}{2} .
$$

The stress can the be written as

$$
\tau_{x z}=\left(P_{x}+\rho g h_{x}\right)\left(z-\frac{h}{2}\right) \equiv\left(\frac{\tau_{Y}}{\dot{\gamma}}+\mu\right) u_{z} .
$$


As in the free-surface case, this can be rearranged to give the velocity gradient in terms of the yield surfaces $Y_{ \pm}$for $z<Y_{-}$and $z>Y_{+}$,

$$
u_{z}=-\frac{\Gamma}{\mu}\left(Y_{ \pm}-z\right) \quad \text { where } \quad Y_{ \pm}=\left(\frac{h}{2} \pm \frac{\tau_{Y}}{|\Gamma|}\right)
$$

and $\Gamma=P_{x}+\rho g h_{x}$ is the reduced pressure gradient. The squeeze flow flux can be found by integrating the velocity gradient

$$
F_{S F}=2 \int_{0}^{\frac{h}{2}} u \mathrm{~d} z \equiv 2 \int_{0}^{Y_{-}}\left(\frac{h}{2}-z\right) u_{z} \mathrm{~d} z=-\frac{\Gamma}{3 \mu} Y_{-}^{2}\left(\frac{3 h}{2}-Y_{-}\right) .
$$

Hence, applying local mass conservation with $h(x, t)=\delta(t)+\eta(x)$, the governing equation for the pressure gradient can be written as

$$
x \dot{\delta}=\frac{\Gamma}{3 \mu}\left(\frac{h}{2}-\frac{\tau_{Y}}{|\Gamma|}\right)^{2}\left(h+\frac{\tau_{Y}}{|\Gamma|}\right) .
$$

\subsubsection{Global mass conservation and equation of motion for $\delta(t)$}

We consider global conservation of mass to relate the depth $\delta$ to contact length $L$. By considering the area underneath the indenter, global conservation of mass can be written as a sum of integrals in the two regions,

$$
h_{0} L_{0}=\delta L+\int_{0}^{L} \eta \mathrm{d} x+\int_{L}^{L_{0}} h^{F S} \mathrm{~d} x
$$

where $L_{0}$ is the half-length of the deforming substrate, see figure 1. To close the system, the equation of motion for $\delta(t)$ can be written as,

$$
m \ddot{\delta}=-m g+2 \int_{0}^{L} P \mathrm{~d} x
$$

where $m$ is the mass per unit width of the indenter and $g$ is the acceleration due to gravity.

\subsubsection{Non-dimensionalisation}

We non-dimensionalise vertical and horizontal lengthscales by the initial depth of fluid $h_{0}$ and characteristic lengthscale of the geometry $x_{0}$ respectively. A scale for the pressure is defined by balancing the normal force due to the fluid with the weight of the indenter. Finally, a timescale is defined by balancing the inertial forcing with the weight of the indenter. Hence, non-dimensional variables can be defined as,

$$
\hat{x}=\frac{x}{x_{0}}, \quad \hat{h}=\frac{h}{h_{0}}, \quad \hat{P}=\frac{P}{P_{0}}=\frac{x_{0}}{m g} P, \quad \hat{t}=\frac{t}{t_{0}}=\left(\frac{g}{h_{0}}\right)^{1 / 2} t .
$$


Dropping the hat decoration, the governing equations for the free-surface flow, the squeeze flow and the evolution of $\delta(t)$ then reduce to

$$
\begin{aligned}
V \frac{\partial h}{\partial t} & =\frac{R}{6} \frac{\partial}{\partial x}\left(h_{x} Y^{2}(3 h-Y)\right) \quad \text { with } Y=h+\frac{B}{R h_{x}} \\
V x \dot{\delta} & =\frac{\Gamma}{3}\left(\frac{h}{2}-\frac{B}{|\Gamma|}\right)^{2}\left(h+\frac{B}{|\Gamma|}\right) \\
L_{0} & =\delta L+\int_{0}^{L} \eta \mathrm{d} x+\int_{L}^{L_{0}} h^{F S} \mathrm{~d} x \\
\ddot{\delta} & =-1+2 \int_{0}^{L} P \mathrm{~d} x,
\end{aligned}
$$

with parameters

$$
B=\tau_{Y} x_{0}^{2} / m g h_{0}, \quad R=\rho x_{0} h_{0} / m, \quad \text { and } \quad V=\mu x_{0}^{3} / m h_{0}^{2} \sqrt{g h_{0}} .
$$

The Bingham number $B$ characterises the strength of the substrate, $R$ controls the influence of gravity and $V$ the influence of viscosity. This model is valid until $\dot{\delta}=0$ at which point the squeeze flow turns off and the layer plugs up.

\subsubsection{Plastic limit, free-surface}

In the plastic limit, as $V \rightarrow 0$, the terms involving time dependence in the free-surface and squeeze evolution equations are small and hence the flow becomes quasi-static. For the free-surface flow this implies $Y=h+B / R h_{x} \rightarrow 0$, hence

$$
h h_{x}=-\frac{B}{R} \Rightarrow h=\left(h_{L}^{2}+\frac{2 B}{R}(L-x)\right)^{1 / 2} .
$$

2.3.6 Plastic limit, squeeze flow case (a): flat-based i ndenter, $\eta(x)=0, x_{0}$ $=L$

In the plastic 1 imit, the yield surfaces $Y_{ \pm}=h / 2 \mp B /\left(P_{x}+R h_{x}\right)$ in the squeeze flow also tend to the boundaries, $Y_{-} \rightarrow 0, Y_{+} \rightarrow h$ implying

$$
\frac{h}{2}=-\frac{B}{P_{x}+R h_{x}}
$$

Assuming further that $R \ll 1$ so the hydrostatic pressure is small, the pressure in the squeeze flow can be solved for with boundary condition $P(x=1)=R\left(h_{L}-\delta\right) \ll 1$,

$$
P_{x}=-\frac{2 B}{\delta} \quad \Rightarrow \quad P=\frac{2 B}{\delta}(1-x)
$$

Substituting the pressure into the evolution equation for $\delta$ we have,

$$
\ddot{\delta}=-1+\frac{4 B}{\delta} \int_{0}^{1} 1-x \mathrm{~d} x=-1+\frac{2 B}{\delta},
$$




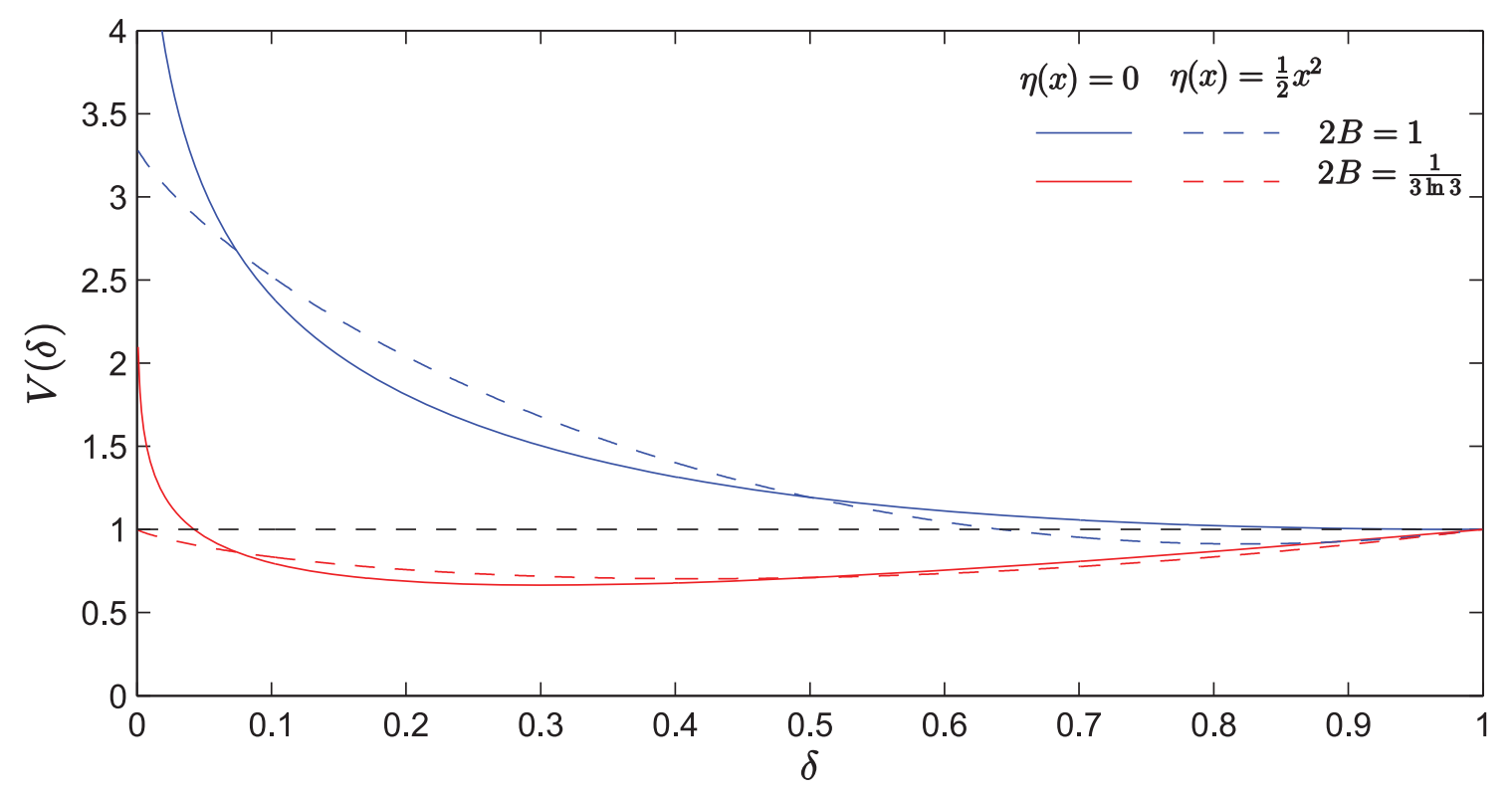

Figure 2: Potential energy $V(\delta)$ against depth $\delta$ for the flat-based indenter (solid lines) and the parabolic indenter (dashed lines) and two values of the Bingham number $2 B=1,1 / 3 \ln 3$ given by the blue and red lines, respectively.

which defines equilibrium depth $\delta_{e q}=2 B$ where the normal force due to the fluid balances the weight of the object. Multiplying by $\dot{\delta}$ the energy equation can be derived, and hence an expression for the final depth of the indenter $\delta_{f}$,

$$
1+\frac{\dot{\delta}_{0}^{2}}{2}=V\left(\delta_{f}\right)=\delta_{f}-2 B \ln \delta_{f}
$$

The minimum value of $V(\delta)$ is attained at the equilibrium depth $\delta=\delta_{e q}=2 B$, with value $V\left(\delta_{e q}\right)=2 B-2 B \ln 2 B$. When $\dot{\delta}_{0}=0$ and $2 B<1\left(\delta_{e q}<1\right)$, the indenter sinks to a height $\delta_{f}$. However, when $\dot{\delta}_{0}=0$ and $2 B>1$, the equilibrium depth is above the height of the layer. This is because the layer is not sufficiently stressed to deform so the indenter remains on the surface with $\delta_{f}=1$, see figure 2 .

\subsubsection{Plastic limit, squeeze flow case (b): parabola $\eta(x)=\frac{1}{2} x^{2}, x_{0}=\left(R_{c} h_{0}\right)^{1 / 2}$}

In the case of a parabolic indenter (local approximation for a cylinder), the pressure gradient can be written as,

$$
P_{x}=-R x-\frac{2 B}{\delta\left(1+\frac{1}{2 \delta} x^{2}\right)} .
$$

The normal force due to the fluid is given by

$2 \int_{0}^{L} P \mathrm{~d} x=-2 \int_{0}^{L} x P_{x} \mathrm{~d} x=2 \int_{0}^{L} R x^{2}+\frac{2 B x}{\delta\left(1+\frac{1}{2 \delta} x^{2}\right)} \mathrm{d} x=\frac{2 R L^{3}}{3}+4 B \ln \left(1+\frac{1}{2 \delta} L^{2}\right)$. 
Unlike the flat-based indenter with fixed $L$, for the parabola the evolution of $\delta(t)$ is coupled to the evolution of $L(t)$. Global conservation of mass gives

$$
\frac{L^{3}}{6}-L(1-\delta)+\frac{R}{6 B}\left(2 h_{L}+1\right)\left(h_{L}-1\right)^{2}=0 .
$$

In the limit of $R \ll 1$, the conservation of volume reduces to $L^{2}=6(1-\delta)$, hence the energy equation for $\delta$ can be written as

$$
\frac{\dot{\delta}_{0}^{2}}{2}+1=V(\delta)=\delta_{f}+4 B\left(\frac{1}{2}\left(3-2 \delta_{f}\right) \ln \left(3-2 \delta_{f}\right)+\delta_{f} \ln \delta_{f}\right) .
$$

Figure 2 plots the potential energy $V(\delta)$ against the depth of the indenter. The minimum point is given by $\delta=\delta_{e q}=3 /(\exp (1 / 4 B)+2)$ which tends to 1 as the Bingham number diverges. For $\dot{\delta}_{0}=0$, the parabola appears to reach the bottom provided $2 B<1 / 3 \ln 3$. In general, equation (34) suggests the parabola reaches the bottom of the initial layer provided the initial speed of the indenter is sufficiently large,

$$
\dot{\delta}_{0}^{2}>2(6 B \ln 3-1)
$$

However, in the plastic limit there will always be a thin viscous layer between the parabola and the base of the substrate. This is violated in this case because the assumption that the yield surfaces tend towards the boundaries, $Y_{-} \rightarrow 0, Y_{+} \rightarrow h$, breaks down as $\delta \rightarrow 0$. If we rescale the variables in terms of $\delta, \hat{x}=x / \delta^{1 / 2}, \hat{h}=h / \delta$, for $\eta(x)=\eta_{0} x^{n}$, then we have

$$
V \delta^{\frac{1}{n}-\frac{3}{2}} \dot{\delta}=\frac{\Gamma}{3}\left(\frac{h}{2}-\frac{B}{|\Gamma|}\right)^{2}\left(h+\frac{B}{|\Gamma|}\right) .
$$

For $n>0$ the left hand side diverges as $\delta \rightarrow 0$ suggesting we are no longer in the quasistatic limit so the approximation of $Y_{-} \rightarrow 0, Y_{+} \rightarrow h$ is not valid. Hence, equation (34) does not hold for small $\delta$.

\subsection{Numerical solution}

The full system described by equations (21-24) can be solved numerically using MATLAB's in-built solver ODE15s. Figure 3 shows the numerical solution for parameters $V=1, B=1$ and $R=1$ with initial condition $\dot{\delta}_{0}=-2$. Figure $3($ a-c) shows the position of the cylinder, yield surfaces and free-surface profile at time intervals $t=0.02,0.14$ and 0.26 . Figure $3(\mathrm{~d})$ plots depth of the cylinder $\delta(t)$ with time to its stopping position at $\delta_{f}=0.71, t=0.29$. In figure $3(\mathrm{a}-\mathrm{c})$ there appears to be a discontinuity between the squeeze flow and free-surface flow as indicated by the transition from two yield surfaces to one. This is because there is an $O(1)$ aspect ratio region at the contact line $x=L$ which is not captured by the lubrication model, where the yielded and plug regions smoothly transition. Instead the lubrication model matches the two regions by taking the horizontal volume flux to be continuous at $x=L$. 
(a)
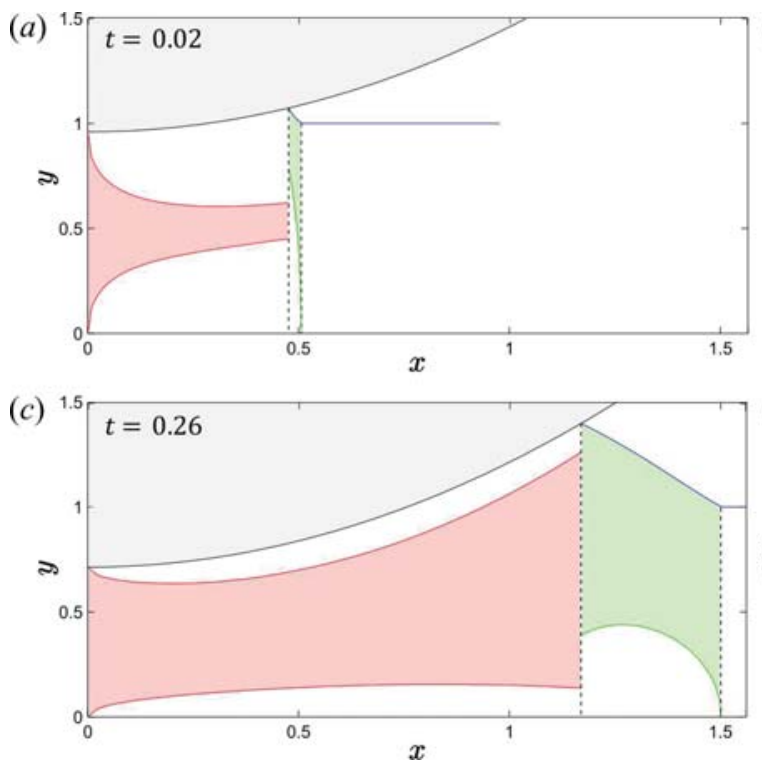
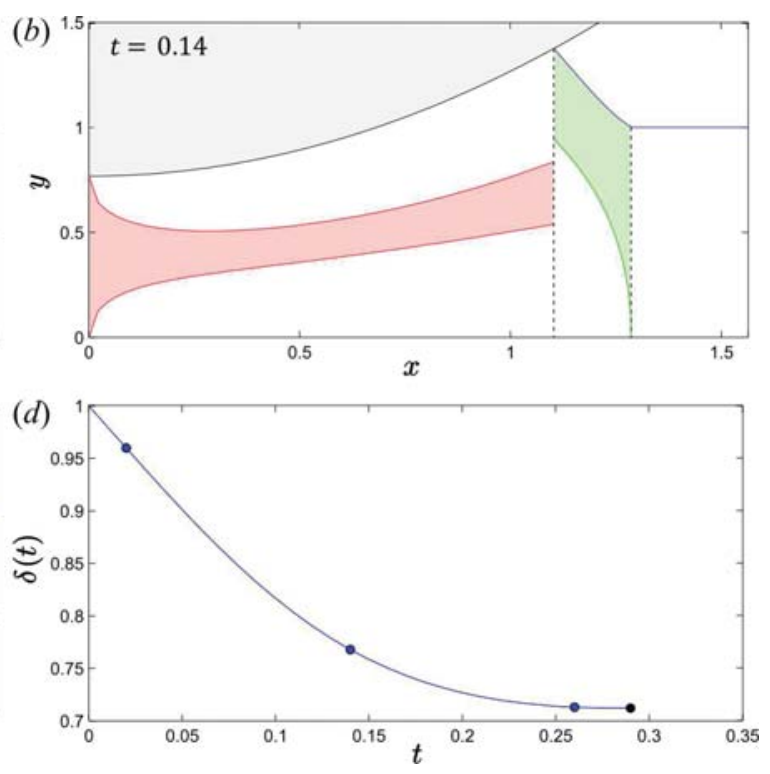

Figure 3: Full numerical solution for a parabolic indenter dropped onto a pure cohesive substrate with $\dot{\delta}_{0}=-2, V=1, B=1$ and $R=1$ plotted at (a) $t=0.02$, (b) $t=0.14$, and (c) $t=0.26$. The coloured lines indicate the edge of cylinder (black), free surface (blue), yield surfaces in the squeeze flow (red), and yield surfaces in free surface flow (green), with black dashed lines separating the squeeze flow and the free-surface flow. The squeeze flow plug, free-surface plug and cylinder are shaded in red, green and grey, respectively. (d) Height of the cylinder with time. Blue dots are plots are $t=0.02,0.14,0.26$ and the black dot is final resting place of cylinder when $\dot{\delta}=0$ at $\delta_{f}=0.71, t=0.29$. 


\subsection{Mohr-Coulomb $\tau_{Y}=p \tan \phi$}

In a Mohr-Coulomb model, the stress tensor can be written as

$$
\tau_{i j}=\tau_{Y} \frac{\dot{\gamma}_{i j}}{\dot{\gamma}} \quad \text { where } \quad \tau_{Y}=c+p \tan \phi
$$

where $c$ is the cohesion and $\phi$ is the internal friction angle. For generality, we will consider $c \neq 0$ to begin with and then take $c=0$ for the non-cohesive case. As in the Bingham model, we regularise by adding a viscous term in order to solve for the flow field. This gives

$$
\tau_{i j}=\left(\frac{c+p \tan \phi}{\dot{\gamma}}+\mu\right) \dot{\gamma}_{i j}
$$

\subsubsection{Free-surface flow}

For the free surface flow $p(z=h)=0$, which gives

$$
\mu u=\left(-\rho g h h_{x}-c-\rho g h \tan \phi\right) z+\frac{1}{2}\left(\rho g h_{x}+\rho g \tan \phi\right) z^{2} .
$$

Setting $u_{z}=0$ defines the yield surfaces

$$
Y=h-\frac{c}{\rho g\left(\left|h_{x}\right|-\tan \phi\right)} .
$$

As in the pure cohesive case, the governing equation for $h$ can be found by considering local mass conservation to give

$$
\frac{\partial h}{\partial t}=\frac{\partial}{\partial x}\left(\frac{\rho g\left(h_{x}+\tan \phi\right)}{6 \mu} Y^{2}(3 h-Y)\right) .
$$

\subsubsection{Squeeze flow}

The squeeze flow is treated in a similar manner to the pure cohesive case. In the bottom yielded region, the velocity gradient can be integrated to give the velocity for $0<z<Y_{-}$

$$
\mu u_{z}=\Gamma\left(z-Y_{-}\right)+\rho g \tan \phi\left(z-Y_{-}\right) \quad \Rightarrow \quad \mu u_{-}=(\Gamma+\rho g \tan \phi) z\left(\frac{z}{2}-Y_{-}\right),
$$

where $\Gamma=P_{x}+\rho g h_{x}$. Similarly, the velocity in the top yielded region for $Y_{+}<z<h$ can be written as

$$
\mu u_{+}=(\Gamma-\rho g \tan \phi)\left[z\left(\frac{z}{2}-Y_{+}\right)-h\left(\frac{h}{2}-Y_{+}\right)\right] .
$$

One equation for the yield surfaces is given by matching the plug speeds, $u_{-}\left(Y_{-}\right)=u_{+}\left(Y_{+}\right)$,

$$
h-\left(\frac{-\Gamma-\rho g \tan \phi}{-\Gamma+\rho g \tan \phi}\right)^{1 / 2} Y_{-}=Y_{+} .
$$

Another equation is given by setting the top and bottom boundaries to be at the yield stress

$$
-\Gamma\left(Y_{+}-Y_{-}\right)=2 c+2 P \tan \phi+\rho g \tan \phi\left(2 h-Y_{-}-Y_{+}\right) .
$$

Depth integrating the velocity and applying local mass conservation an ODE for the pressure $P$ can be found,

$$
-x \dot{\delta}=\frac{(\Gamma+\rho g \tan \phi)}{6 \mu} Y_{-}^{3}-\frac{(\Gamma-\rho g \tan \phi)}{6 \mu}\left(Y_{+}^{3}-3 Y_{+} h^{2}+2 h^{3}\right) .
$$




\subsubsection{Non-dimensionalisation}

We non-dimensionalise as in section 2.3.4 which gives the additional non-dimensional parameter $\Phi=\frac{x_{0}}{h_{0}} \tan \phi$ which controls the influence of the angle of friction $\phi$. The Bingham number is now written as $B=c x_{0}^{2} / m g h_{0}$.

\subsubsection{Plastic limit, free-surface}

In the plastic limit, $Y \rightarrow 0$, and equation for the quasistatic free surface $h(x, t)$ can be found

$$
\frac{h h_{x}}{\frac{B}{R \Phi}+h}=-\Phi
$$

Using boundary condition $h(L, t)=h_{L}$, this can be integrated to give

$$
h-h_{L}-\frac{B}{R \Phi} \ln \left(\frac{h+\frac{B}{R \Phi}}{h_{L}+\frac{B}{R \Phi}}\right)=(L-x) \Phi .
$$

In the non-cohesive case, $B=0$, the free surface profile in the plastic limit reduces to the linear profile with gradient given by the angle of friction $\phi$,

$$
h-h_{L}=(L-x) \Phi .
$$

\subsubsection{Plastic limit, squeeze flow case (a): flat-based indenter $\eta(x)=0, x_{0}=L$}

In the plastic limit, the yield surfaces tend to the boundaries, $Y_{-} \rightarrow 0, Y_{+} \rightarrow h=\delta(t)$. Hence, this gives

$$
-P_{x} \delta=2 B+2 \Phi P+R \Phi \delta .
$$

Multiplying by an integrating factor and using boundary condition $P(x=1)=R\left(h_{L}-\delta\right)$, an expression for the pressure can be found

$$
P=-\left(\frac{B}{\Phi}+\frac{R \delta}{2}\right)+\left(R h_{L}-\frac{R \delta}{2}+\frac{B}{\Phi}\right) \exp \left(\frac{2 \Phi}{\delta}(1-x)\right)
$$

The equation of motion for the depth of the indenter in the non-cohesive case is then given by

$$
\ddot{\delta}=-1-R \delta-\frac{\delta}{\Phi}\left(R h_{L}-\frac{R \delta}{2}\right)\left(1-\exp \left(\frac{2 \Phi}{\delta}\right)\right) .
$$

A relationship between $h_{L}$ and $\delta$ is found by considering global mass conservation

$$
1-\delta=\frac{1}{2}\left(h_{L}-1\right)\left(L_{0}-1\right) \quad \Rightarrow \quad h_{L}=1+(2 \Phi(1-\delta))^{1 / 2} .
$$

The force balance equation can then be integrated to give an expression for $\delta_{f}$ in terms of initial condition $\dot{\delta}_{0}$ and parameters $\Phi$ and $R$,

$$
1+\frac{\dot{\delta}_{0}^{2}}{2}=\delta_{f}+R \int_{\delta_{0}}^{\delta_{f}}\left(\delta+\delta\left(\left(\frac{2(1-\delta)}{\Phi}\right)^{1 / 2}-\frac{\delta}{2 \Phi}\right)\left(1-\exp \left(\frac{2 \Phi}{\delta}\right)\right)\right) \mathrm{d} \delta .
$$

Figure 4 plots the final depth $\delta_{f}$ as a function of $\Phi$ and $R$ (blue and red solid lines). For small $1 / R$, the flat-based indenter sits on the surface of the plastic layer with $\delta_{f}=1$. As in the pure cohesive case, this defines a yield criterion as a function of parameters $\Phi$ and $R$ where the layer is not sufficiently stressed to deform. 


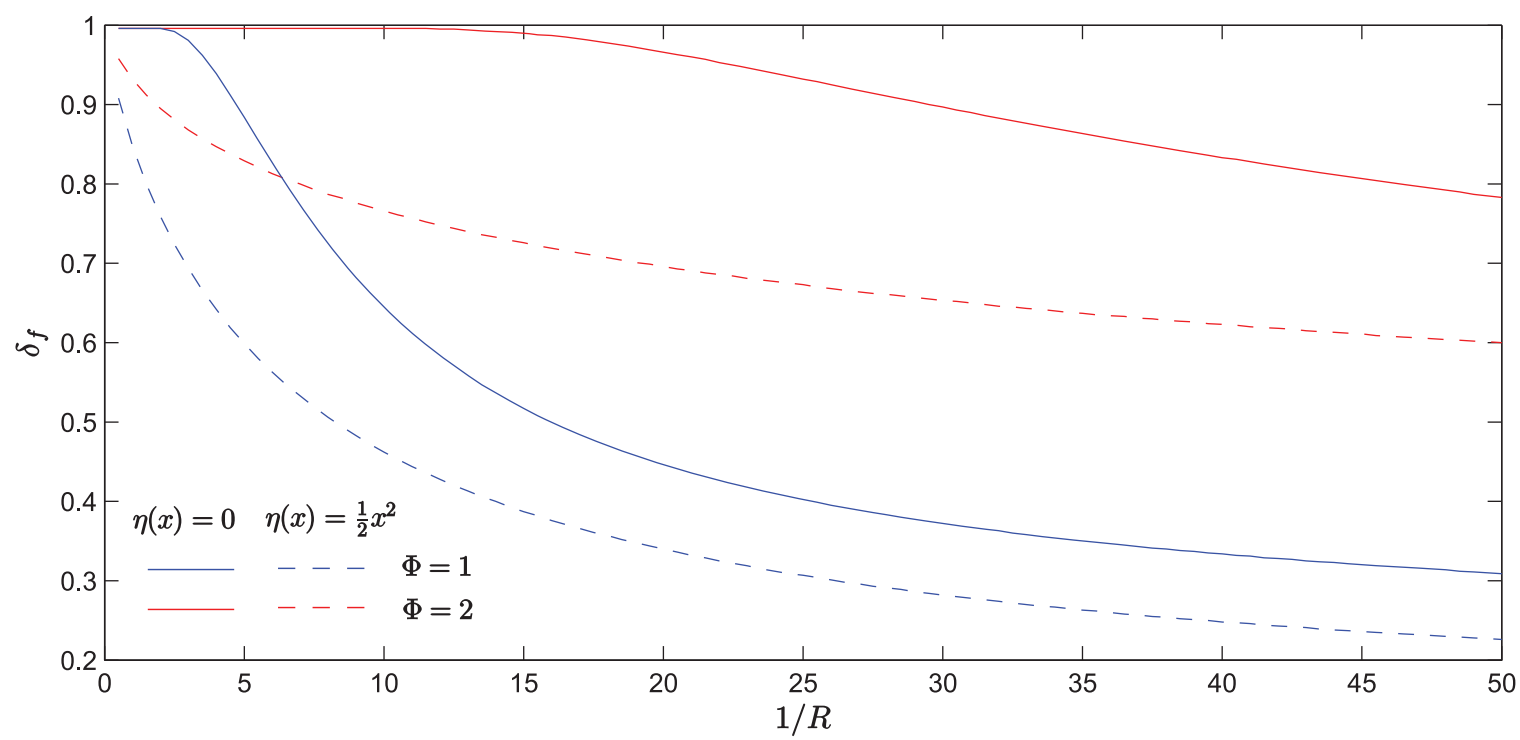

Figure 4: Plot of the final depth $\delta_{f}$ as a function of $1 / R$ for a flat-based indenter (solid lines) and parabolic i ndenter ( dashed 1 i nes) with $\Phi \neq, 2$.

2.5.6 Plastic limit, squeeze flow case (b): parabola $\eta(x)=\frac{1}{2} x^{2}, x_{0}=\left(R_{c} h_{0}\right)^{1 / 2}$

For a parabolic indenter, $h=\delta(t)+\frac{1}{2} x^{2}$, the pressure in the squeeze flow is given by $\Gamma=P_{x}+R x$. Hence, in the plastic limit

$$
-\left(P_{x}+R x\right)\left(\delta+\frac{1}{2} x^{2}\right)=2 B+2 \Phi P+R \Phi\left(\delta+\frac{1}{2} x^{2}\right) .
$$

In the non-cohesive case,

$$
P_{x}+\frac{2 \Phi P}{\delta+\frac{1}{2} x^{2}}=-R(x+\Phi) .
$$

This can be integrated with boundary condition $P(x=L)=R\left(h_{L}-\delta\right)$. Substituting the pressure into the force balance equation, together with global mass conservation

$$
\left(\delta+\frac{1}{2} L^{2}-1\right)^{2}=2 \Phi\left(L(1-\delta)-\frac{1}{6} L^{3}\right),
$$

and integrating twice, an expression for $\delta_{f}$ can be found in terms of $\dot{\delta}_{0}$ and parameters $\Phi$ and $R$, see section 2.5.5. Figure 4 plots the final depth $\delta_{f}$ as a function of $\Phi$ and $R$ (blue and red dashed lines).

\subsection{Deep plastic layer}

Thus far we have considered a shallow layer of viscoplastic fluid in order to understand the deformation of a shallow plastic layer. The case of a deep layer has been studied extensively using the method of characteristics $[14,9]$. These studies calculate the pressure 
underneath a flat-based indenter placed onto a rigid-plastic half-space by the construction of sliplines. We outline here the method for constructing sliplines and state two key results for a purely cohesive and a non-cohesive substrate. We then describe three possibilities for the free-surface displacement.

In the deep layer limit, we require the full force-balance equations

$$
\frac{\partial \sigma_{z}}{\partial x}+\frac{\partial \tau_{x z}}{\partial z}=0, \quad \frac{\partial \sigma_{x}}{\partial z}+\frac{\partial \tau_{x z}}{\partial x}=0
$$

where $\sigma_{x}$ and $\sigma_{z}$ are the normal stress components in the $x$ and $z$ directions respectively and $\tau_{x z}$ is the shear stress. Requiring yield condition $\left(\sigma_{x}-\sigma_{z}\right)^{2}+4 \tau_{x z}^{2}=4 \tau_{Y}^{2}$, the stress components can then be written in terms of new parameter $\theta$,

$$
\sigma_{x}=-p+\tau_{Y} \sin 2 \theta, \quad \sigma_{z}=-p=-\tau_{Y} \sin 2 \theta \quad \text { and } \quad \tau_{x z}=\tau_{Y} \cos 2 \theta .
$$

Substituting the parametrisation (59) into the full force-balance equations (58) and differentiating, equations for $p$ and $\theta$ can be found

$$
\left(\cos \theta \frac{\partial}{\partial x}+\sin \theta \frac{\partial}{\partial z}\right)\left(p+2 \tau_{Y} \theta\right)=\left(\sin \theta \frac{\partial}{\partial x}-\cos \theta \frac{\partial}{\partial z}\right)\left(p-2 \tau_{Y} \theta\right)=0 .
$$

These define two sets of characteristics ( $\alpha$ and $\beta$ characteristics) along which quantities $p \pm 2 \tau_{Y} \theta$ are conserved. To solve for the slipline field the quantities $p$ and $\theta$ first need to be defined along some boundary. The values of $p$ and $\theta$ are calculated elsewhere by integrating along the characteristics equations (60) from a region of known information.

For the case of a pure cohesive plastic layer where $\tau_{Y}=$ constant, the pressure underneath an indenter of contact length $a=2 L$ can be calculated analytically and is given by

$$
p=\tau_{Y}(2+\pi)
$$

[14]. The force per unit length is therefore $F=\tau_{Y}(2+\pi) a$. For the case of a non-cohesive material such that $\tau_{Y}=p \tan \phi$, the pressure is given by

$$
p=\frac{1}{2} \rho g N_{\gamma} a,
$$

where $N_{\gamma}=6.5$ is a Terzaghi coefficient $[19,3]$ calculated numerically, with force per unit length $F=\frac{1}{2} \rho g N_{\gamma} a^{2}$. These expressions for the force $F$ describe the initial condition where a flat-based indenter of contact length $a$ is placed onto the flat surface of a deep plastic layer. Hence, this theory can only be extended to small deformations of the layer where the surface can be approximated as horizontal.

For a flat-based indenter, comparing the weight of the indenter with the force exerted by the plastic layer gives a yield criterion for when the indenter can deform the substrate. For example in the pure cohesive case, the indenter will deform the substrate provided

$$
\tau_{Y} \leq \frac{m g}{(2+\pi) w a},
$$

where $m$ and $w$ are the mass and length of the indenter, respectively. To determine the equilibrium depth of the indenter, the velocity field must be calculated to update the free 
surface, and hence calculate the new force exerted by the plastic layer. An iterative numerical approach can then be used to determine the depth at which the forces are in balance.

For the case of a parabolic indenter, the increasing contact length with depth of indentation means more progress can be made analytically. Again, for the pure cohesive case, equating the force exerted by the plastic layer with the weight of the indenter gives a contact length $a=m g / \tau_{Y}(2+\pi) w$. The contact length must now be related to the depth of the indentation. Figure 5 shows possible relationships between contact length $a$ and depth of indentation $d$. Figure 5(a) shows when the layer is allowed to compact such that there is no free-surface deformation, in contrast to the previous formulation where we assumed incompressibility. As a result the free surface remains horizontal with $d=\frac{1}{2 R_{c}}(a / 2)^{2}$ for shallow depths $d$. In figure $5(\mathrm{c})$ we have considered when gravity is neglected giving a vertical free-surface. Conservation of mass then implies $d=\frac{1}{6 R_{c}}(a / 2)^{2}$. We anticipate the free-surface to be in between these two end-members such as figure 5(b). For the pure cohesive case, the free-surface displacement for shallow indentations can be found using the slipline calculations [18]. For small deformations due to the curved surface of a cylinder, the velocity on the surface of the plastic layer is shown to be

$$
v(x, t)=\left\{\begin{array}{cc}
-V & 0 \leq x \leq a / 2 \\
V & a / 2 \leq x \leq a,
\end{array}\right.
$$

where the length of the deformed region outside is the same as the half-length of the indenter due to the symmetry of the slipline field. And hence, the deformed region outside has profile [18]

$$
h=d-\frac{x^{2}}{14 R_{c}} \quad \text { where } \quad d=\frac{2}{7 R_{c}}\left(\frac{a}{2}\right)^{2} .
$$

For the parabolic indenter, the contact length and depth as function of the mass of the indenter are therefore given by

$$
a=\frac{m g}{\tau_{Y}(2+\pi) w}, \quad d=\frac{1}{14 R_{c}}\left(\frac{m g}{\tau_{Y}(2+\pi) w}\right)^{2},
$$

for a pure cohesive substrate, and

$$
a=\left(\frac{2 m}{\rho N_{\gamma} w}\right)^{1 / 2}, \quad d=\frac{2 m}{14 R_{c} \rho N_{\gamma} w},
$$

for a Mohr-Coulomb substrate.

In this section we have described a theoretical model for the loading stage of indentation where either a flat-based or parabolic indenter is placed onto a shallow plastic layer. We have also outlined the current theory for indentation of a deep plastic layer using the method of characteristics to build up a slipline field. In the next section we will describe experiments of indentation into shallow and deep layers with the aim of making comparisons with the theory described. 
(a)

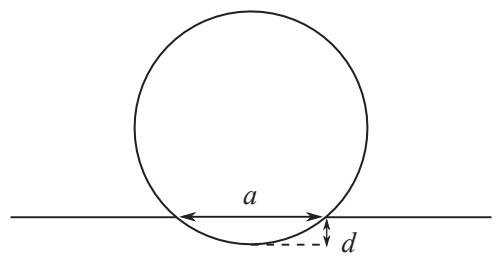

(b)

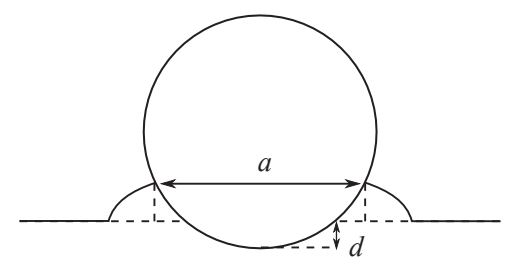

(c)

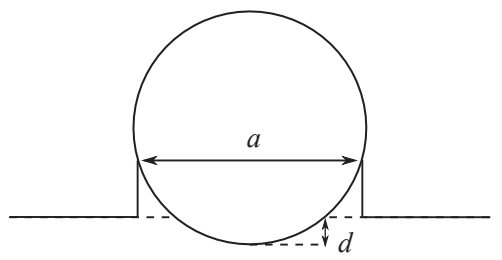

Figure 5: Schematic showing possible relationships between contact length $a$ and depth indented into the plastic layer $d$. (a) Pure compaction $d=\frac{1}{2 R_{c}}(a / 2)^{2}$, (b) some deformation outside $\frac{1}{6 R_{c}}(a / 2)^{2}<d<\frac{1}{2 R_{c}}(a / 2)^{2}$, and (c) vertical free surface $d=\frac{1}{6 R_{c}}(a / 2)^{2}$.

\section{$3 \quad$ Experiments}

\subsection{Setup}

Experiments were conducted to investigate the relationship between depth, contact length and mass for a cylinder indenting a deformable substrate. Joint compound was used as a Bingham rheology and two sizes of ballotini $(0.2 \mathrm{~mm}$ and $1 \mathrm{~mm})$ were used as Mohr-Coulomb rheologies, both with angle of friction $\phi=24.9 \pm 0.7$. The experimental setup is shown in figure 6. A clear perspex cylinder of radius $R_{c}=0.076 \mathrm{~m}$ was attached to the end of a lever that was allowed to rotate about a pivot. Weights were placed on the lever and incrementally moved along to increase the moment, and hence mass on the cylinder. A scale placed on the inside of the cylinder allowed the contact arc length to be measured by eye. Two cameras were also set up to record the displacement of the cylinder. The first camera took photos parallel to the substrate surface to measure the depth of the cylinder, figure 6(b). The second camera was placed at a known oblique angle to take photos of a laser line shone through the cylinder, figure 6(c). This gave a second measurement of the contact arc length and depth as well as a profile of the free surface outside.

\subsection{Image processing}

Photographs taken parallel to the substrate were analysed to determine the depth of the indentation. A blue strip on the top of the cylinder was used to track the displacement between images. We found that the cylinder compressed slightly due to the weight placed on top. As a result, a compliance test was carried out to measure the deformation of the cylinder under a given load when placed on a rigid surface. This is then subtracted from the measured displacements.

Photographs of the laser line taken at known oblique angle were analysed to deduce the profiles of the indentations, see figure 7(a). Firstly, the red filter of the image is taken to get an intensity plot figure 7(b). A moving average is then used to smooth the profiles, with a lower threshold chosen to eliminate noise. The profile is then determined by calculating the weighted average along each vertical strip of pixels, figure 7 (c) (blue solid line). The laser line was imaged through the bottom half of the cylinder causing some distortion of the profile to take place. This is corrected for by subtracting off a reference profile of the 
(a)

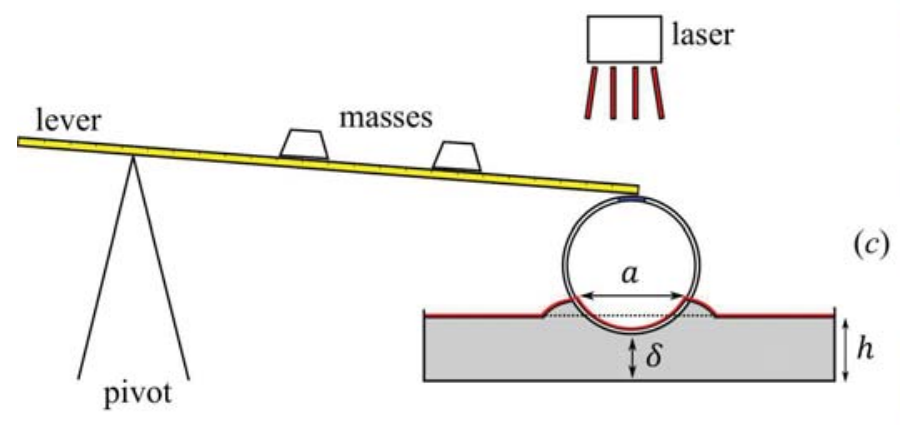

(b)

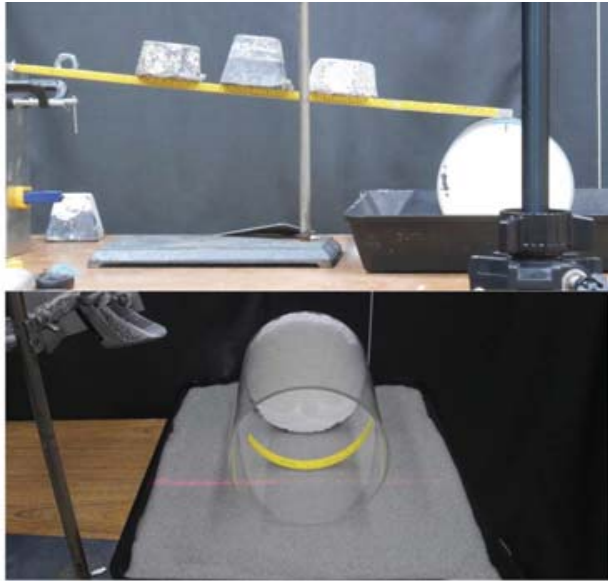

Figure 6: Experimental setup. (a) Schematic of the experimental setup. (b) Sample photograph taken from the first camera parallel to the substrate, and (c) taken from the second camera at known oblique angle.
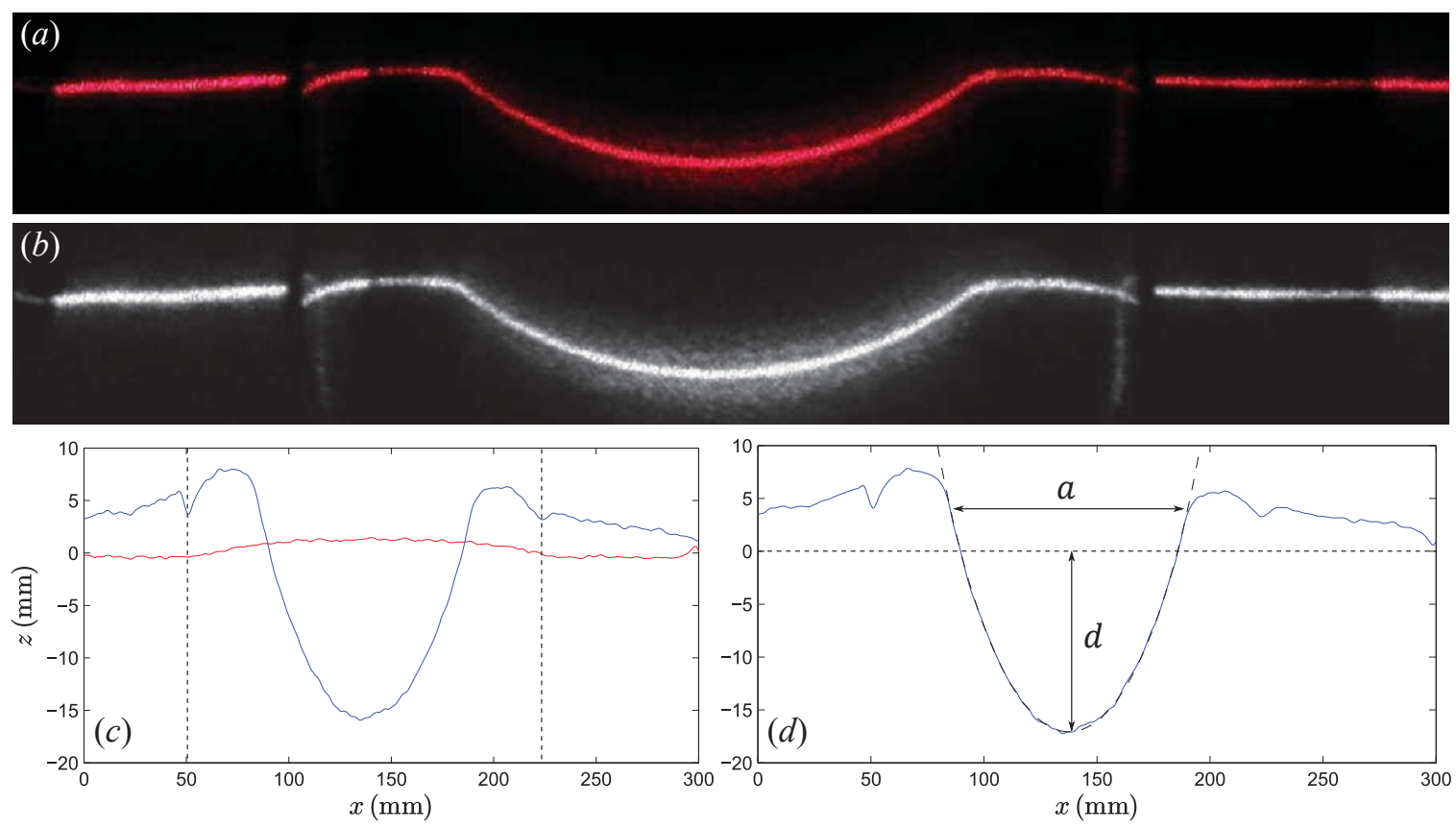

Figure 7: Image processing for joint compound experiment with $h_{0}=4 \mathrm{~cm}$. (a) Image of laser line, and (b) red filtered image of laser line. (c) Detected displacement of joint compound (blue solid line) and reference profile of laser line projected through the perspex cylinder (red solid line), edge of the cylinder indicated by vertical black-dashed lines. (d) Final profile of displacement. 
cylinder resting on the surface of the joint compound with no mass, figure 7(c) (red solid line), where the black-dashed lines indicate the edge of the cylinder. The final displacement profile is given in figure $7(\mathrm{~d})$ with the contact length $a$ and the depth $d$ indicated and superimposed cylinder outline given by the black dashed line.

\subsection{Joint Compound}

\subsubsection{Yield stress}

A slump test can be used to measure the yield stress of the joint compound. From section 2.3.5, the final profile of a shallow slump of plastic fluid is given by

$$
h(x)=\left(\frac{2 \tau_{Y}}{\rho g}\left(L_{s}-x\right)\right)^{1 / 2}
$$

where $L_{s}$ is the radius of the slump, [10]. By measuring the radius and central height of a slump of joint compound, the yield stress can be measured, $\tau_{Y}=\rho g h_{0}^{2} / 2 L_{s}$. In our experiments, the yield stress is measured to be $\tau_{Y}=35 \pm 5 \mathrm{~Pa}$.

\subsubsection{Loading experiments}

For the joint compound, we carried out a series of loading experiments where the mass on the cylinder was gradually increased by moving masses along the pivot lever. The depth, $\delta_{f}$, and contact length, $a=2 L$, where measured using the methods described in section 3.1. Figure 8 plots the depth and contact length against the applied mass for four different substrate heights $h_{0}=1,2,3$ and $4 \mathrm{~cm}$. Figure 8(b) shows excellent agreement between the contact length measured from the profiles (filled circles) and the contact length measured by eye (empty squares). As the depth of the substrate increases, the contact lengths begin to collapse onto a universal curve, seen by the close agreement between the contact lengths for $h_{0}=3 \mathrm{~cm}$ (orange points) and $h_{0}=4 \mathrm{~cm}$ (purple points). This is to be expected since as $h_{0}$ increases there is a transition from indenting a shallow layer where the depth $\delta_{f}$ is proportional to $h_{0}$ to indenting a deep layer where the depth $\delta_{f}$ is independent of $h_{0}$. Figure $8(\mathrm{a})$ plots the depth against applied mass and shows there is a discrepancy between the depth measured from the profiles (filled circles) and the depth measured from tracking the side view of the cylinder (empty squares). The depth measured from the profiles show a collapse of the data for larger substrate depths onto a universal curve (orange and purple filled circles), consistent with the measured contact length, whereas the depth measured from the side profiles show a continued increase in depth. In addition, the profiles seen in figures 7, 9 are in excellent agreement with the theoretical cylinder shape with measured radius $R_{c}=0.076 \mathrm{~m}$ (black dashed lines) suggesting there is no error in converting the profiles to depths. And hence, the laser line gives a more accurate measure of the depth of the indentation. On possible reason the depth calculated from the side images disagrees could be that the compliance of the cylinder is not properly accounted for. In future experiments, more tests need to be carried out to characterise the compliance of the indenter.

Figure 8 also plots the theoretical curves for the deep plastic layer (black dashed lines) and the viscoplastic layer (black dot-dashed lines). The deep theory is given by equation (66) 

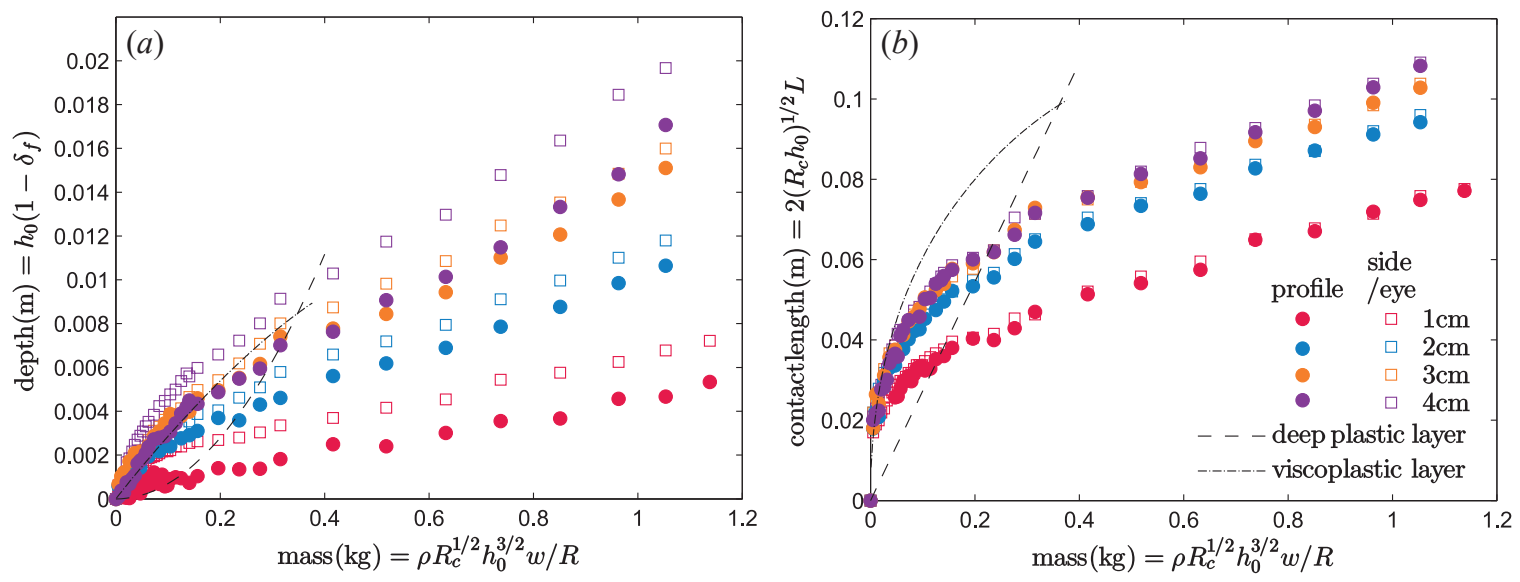

Figure 8: Loading experiments for a cylinder indenting a layer of joint compound. (a) Depth with mass measured using the detected profiles (filled circles) and the photographs tracking the cylinder from side view (empty squares). (b) Contact length with mass measured using the detected profiles (filled circles) and by eye (empty squares). The theoretical curves for the deep plastic layer (black dashed lines) and the viscoplastic layer (black dot-dashed lines) are plotted for parameters $\tau_{Y}=35 \mathrm{~Pa}, \rho=1517 \mathrm{kgm}^{-3}, h_{0}=0.01 \mathrm{~m}, R_{c}=0.076 \mathrm{~m}$ and $w=0.2 \mathrm{~m}$.

and the viscoplastic theory by numerically solving the force balance outlined in section 2.3.7 with $R$ non-negligible. Qualitatively, the experimental data has the same characteristic shape as suggested by the shallow viscoplastic theory but appears to disagree quantitatively by a scale factor. In terms of the deep plastic theory, initially the contact length appears to grow linearly as suggested by the theory but then quickly diverges. This may be because the surface of the layer can no longer be approximated as horizontal.

\subsubsection{Profiles}

The detected free-surface profiles can also be used to compare with the theoretical models. Figure 9(a-d) plots the profiles for layer depths $h_{0}=1,2,3$ and $4 \mathrm{~cm}$ due to applied loads $m=0.24,1.05 \mathrm{~kg}$. For the smallest depth, figure 9(a), the theoretical curve (26) is plotted for two values of the yield stress $\tau_{Y}=20$ and $35 \mathrm{~Pa}$ and suggests a smaller yield stress than measured from the slump test is required to fit the experimental results. As the layer depth increases, the region over which deformation occurs increases. For the largest layer depth, figure 9 (d), the deformation begins to reach the edge of the containing box. As a result, the flow can feel the influence of the side walls and hence mobilises a larger region of the layer than suggested by the theoretical free-surface for the deep plastic layer (green solid line). In addition, the profiles of layer depth $h_{0}=3$ and $4 \mathrm{~cm}$ suggest that mass is not conserved. This could be due to an error zeroing the profiles with the reference image or fluid escaping the test region. To experimentally increase the height of the layer depth, blocks were added into the box to dam a smaller region. Any fluid escaping this region would cause an apparent loss of mass with indentation. 

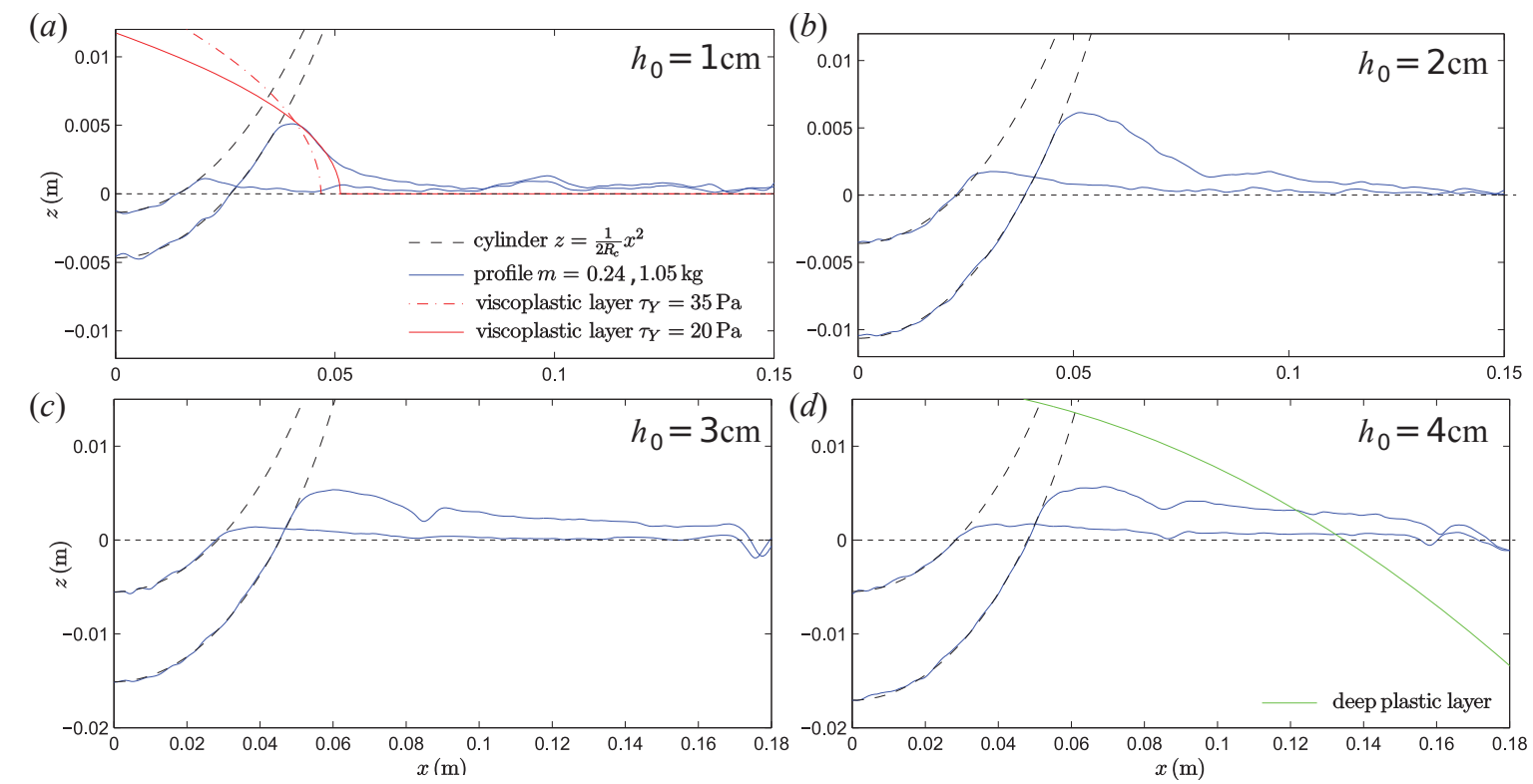

Figure 9: Profiles of loading experiments for a cylinder indenting joint compound for layer depths $h_{0}=1,2,3$ and $4 \mathrm{~cm}$, (a-d) respectively, and applied load $m=0.24,1.05 \mathrm{~kg}$ (blue lines). (Note change of axes.) Position of the cylinder given by the black dashed lines.(a) Theoretical curves for a viscoplastic free-surface flow with yield stress $\tau_{Y}=35$ $\mathrm{Pa}$ (red dot-dashed line) and $\tau_{Y}=20 \mathrm{~Pa}$ (red solid line). (d) Theoretical curve for a deep plastic layer free-surface profile (green solid line). 


\subsection{Ballotini}

\subsubsection{Loading experiments}

For the ballotini we again carried out a series of loading experiments for a range of substrate heights $h_{0}=1-5 \mathrm{~cm}$ with ballotini diameters $0.2 \mathrm{~mm}$ (small) and $1 \mathrm{~mm}$ (large). Figure 10 plots the depth and contact length against the applied mass, where filled data points indicate large ballotini and empty data points small ballotini. As in the joint compound case, there is a clear difference between between shallow and deep substrate depths with the depth of indentation and contact length smaller for $h_{0}=1 \mathrm{~cm}$ (purple points). This again highlights the transition from indenting a shallow layer to a deep layer. There also appears to be a discrepancy between the small and large ballotini with the same angle of friction $\phi$. At the start of each experiment the ballotini substrate was mixed around then levelled once to give a uniform $h_{0}$ with a loose structure. This may have led to a different packing density for the two ballotini sizes. For example, if the small ballotini had a tighter packing density we would anticipate the depth of indentation to be less for a given mass. This could explain the difference between the two ballotini sizes however further experiments need to be carried out to rule out other possibilities.

The theoretical curves for the deep plastic layer (black dashed lines) and the viscoplastic layer (black dot-dashed lines) are also plotted on figure 10. The deep plastic layer, see equation (67), does a good job at replicating the linear and square root structure of the depth and contact length for larger substrate depths, whilst the theory for a viscoplastic layer, numerical solution in section 2.5.6, as in the joint compound case, over predicts the depth and contact length for a given mass quantitatively by a scale factor.

\subsubsection{Dropping experiments}

In addition to the static loading experiments, we carried out a series of dropping experiments to investigate the effect the initial speed of the indenter on the surface of the substrate $\dot{\delta_{0}}$ has on the final depth $\delta_{f}$. To do so we dropped the cylinder from a range of increasing heights with the same loading mass each time onto a substrate of $1 \mathrm{~mm}$ ballotini of depth $h_{0}=5 \mathrm{~cm}$. Figure 11 shows the final profile for four different heights. This demonstrates that the final depth $\delta_{f}$ increases with initial speed, as anticipated from our formulation in section 2.1 .

In this section we have described some preliminary experiments conducted to investigate the indentation of two substrate rheologies with a clear transition between shallow layers, where the indentation is predicted to be proportional to $h_{0}$, and deep layers, where the indentation is predicted to be independent of $h_{0}$. In addition, for the experiments with ballotini, we saw a difference with diameter size suggesting the experiments were very sensitive to initial conditions. For both the ballotini and the joint compound, there is good agreement with the overall shape suggested by the theoretical models however there appears to be a scale factor discrepancy between experimental results and the viscoplastic model. The profile shapes show promising comparisons with the viscoplastic model for the shallowest depth $h_{0}=1 \mathrm{~cm}$, however highlight the need to measure more accurately the rheology of the substrate. The dropping experiments also nicely demonstrate the relationship between initial speed $\dot{\delta_{0}}$ and the final depth of indentation $\delta_{f}$. 


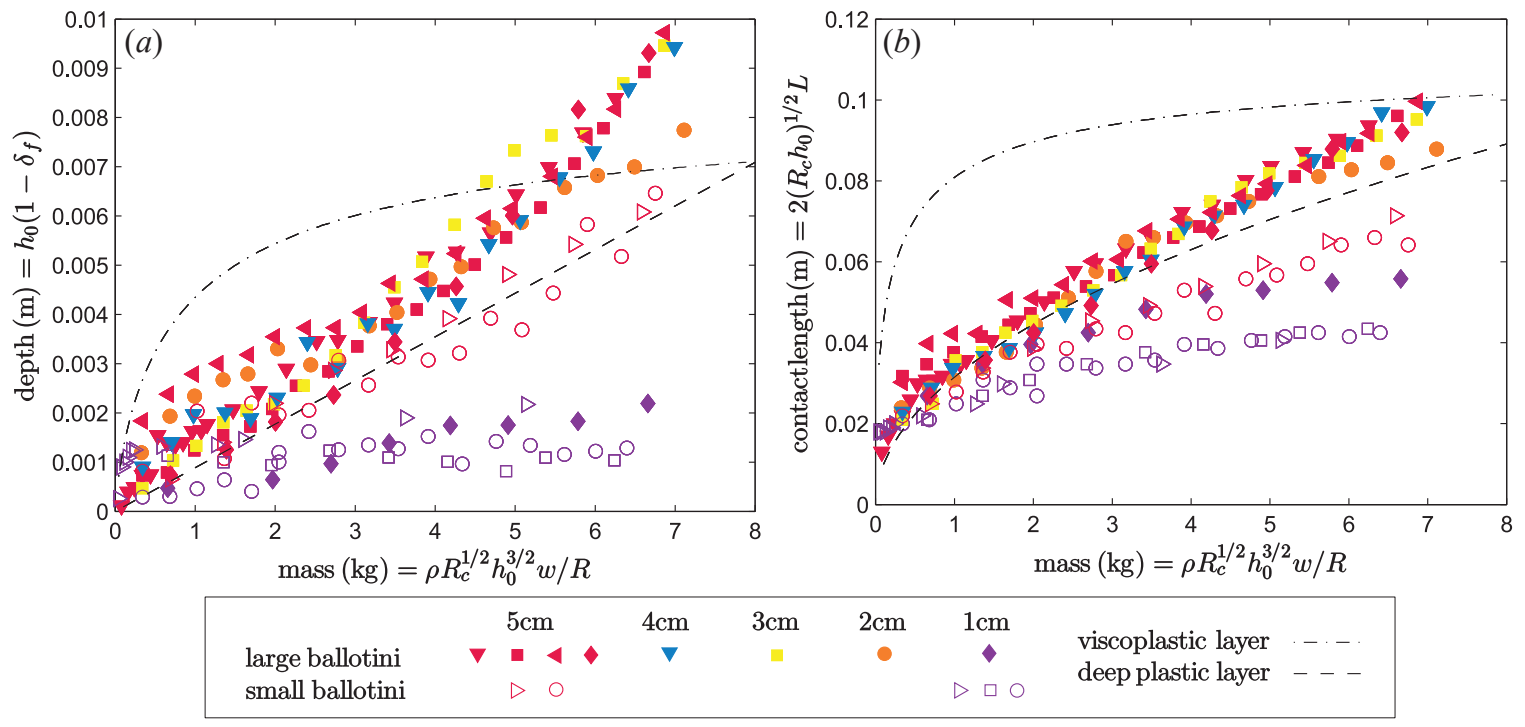

Figure 10: Loading experiments for a cylinder indenting a layer of ballotini of height $h_{0}=$ $1-5 \mathrm{~cm}$. (a) Depth and (b) contact length with mass for small $0.2 \mathrm{~mm}$ diameter (empty points) and large $1 \mathrm{~mm}$ diameter (filled points) ballotini. Theoretical curves for the deep plastic layer (black dashed lines) and viscoplastic layer (black dot-dashed lines) are plotted for parameters $\phi=24.9, \rho=1550 \mathrm{kgm}^{-3}, N_{\gamma}=6.5, h_{0}=0.01 \mathrm{~m}, R_{c}=0.076 \mathrm{~m}$ and $w=0.2 \mathrm{~m}$.

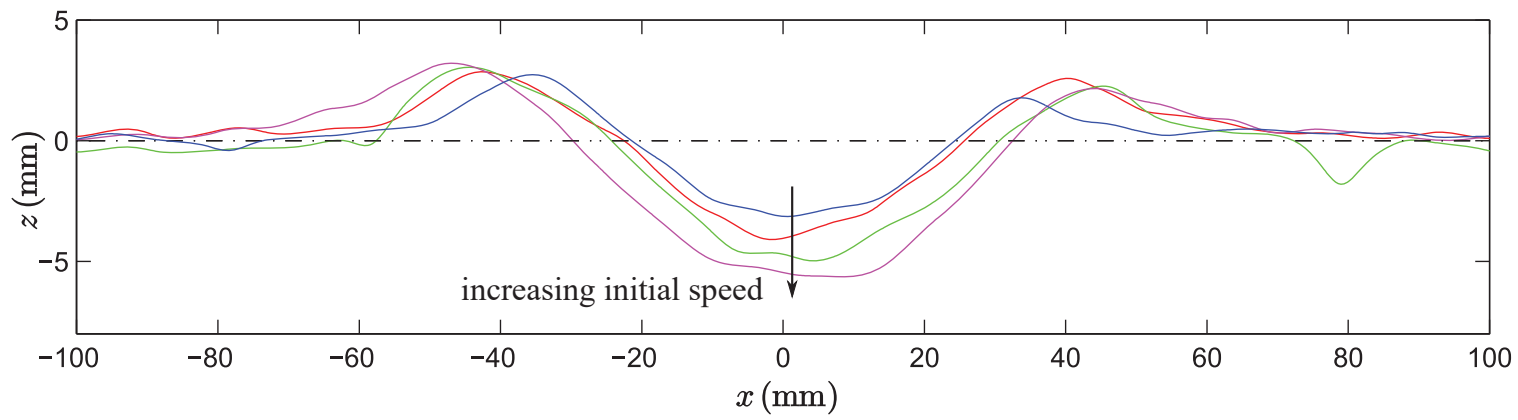

Figure 11: Profiles of dropping experiments for a substrate of $1 \mathrm{~mm}$ ballotini with depth $h_{0}=5 \mathrm{~cm}$. Coloured lines indicate different initial heights of the cylinder above the surface, and hence different initial speeds $\dot{\delta_{0}}$. 

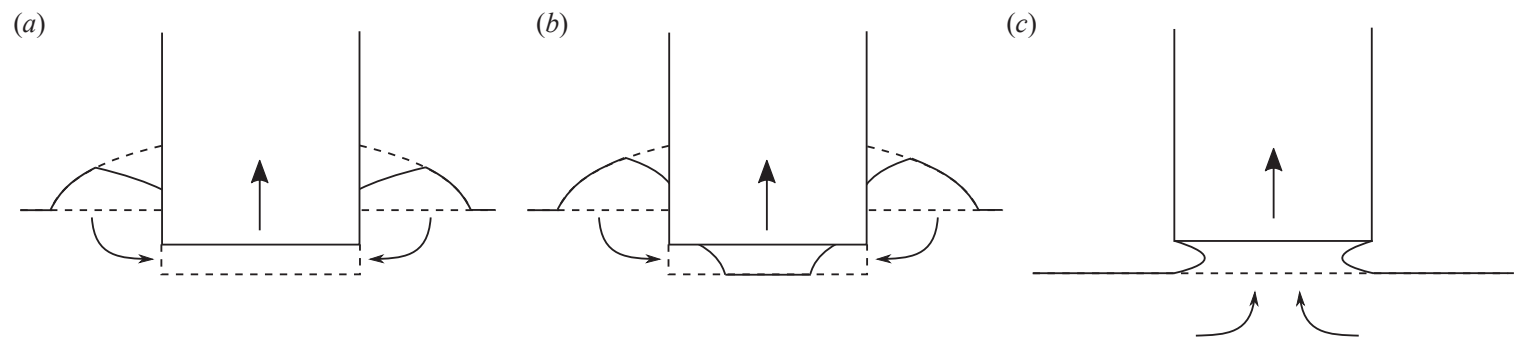

Figure 12: Schematic of methods of lift off: (a) reverse squeeze flow, (b) avalanching, and (c) adhesion.

\section{Theoretical Model: Lift Off}

We now consider the second stage of our theoretical model: lift off, where the indenter lifts off the deformed substrate at a given speed. This stage describes the final indentation left after the indenter has been removed. We consider three methods of lift off: (a) reverse squeeze flow, (b) avalanching and (c) adhesion, see figure 12. We will primarily consider the lift off of a flat-based indenter; however, the methods used could easily be applied to more complicated geometries such as a parabola as discussed previously.

\subsection{Reverse squeeze flow}

The first method of lift off considers when no air can get underneath the indenter. Instead, as the indenter is lifted above its final resting depth $\delta_{f}$ a reverse squeeze flow is generated in which material from the outside free-surface flow is pulled underneath to fill the gap. We assume final lift off can occur when the free surface outside meets the corner of the indenter i.e. pressure is atmospheric. Figure 13(a) shows the final stage of lift off when the indenter can detach from the substrate. At this point the depth of the indentation has raised from the $\delta_{f}$ to $\delta_{l}$ with the excavated mound of material outside meeting the flat base at $x=1$.

\subsubsection{Purely cohesive, $\tau_{Y}=$ constant}

From section 2.3.5, the free-surface before lift off is given by

$$
h=\left(h_{L}^{2}+\frac{2 B}{R}(1-x)\right)^{1 / 2} .
$$

Provided the speed of lift off is sufficiently slow that the free-surface flow remains in the plastic limit, the free-surface profile steps through a series of static shapes with the final profile given by

$$
h=\left\{\begin{array}{lc}
\left(h_{L}^{2}+\frac{2 B}{R}(1-x)\right)^{1 / 2} & 1<x<L_{f}, \\
\left(\delta_{L}^{2}+\frac{2 B}{R}(x-1)\right)^{1 / 2} & L_{f}<x<L_{0},
\end{array}\right.
$$

see figure $13(\mathrm{a})$. To find $\delta_{L}$ mass conservation is used, which in the plastic limit is an equal areas construction given the quasistatic free-surface profiles. Hence by global conservation 
(a)

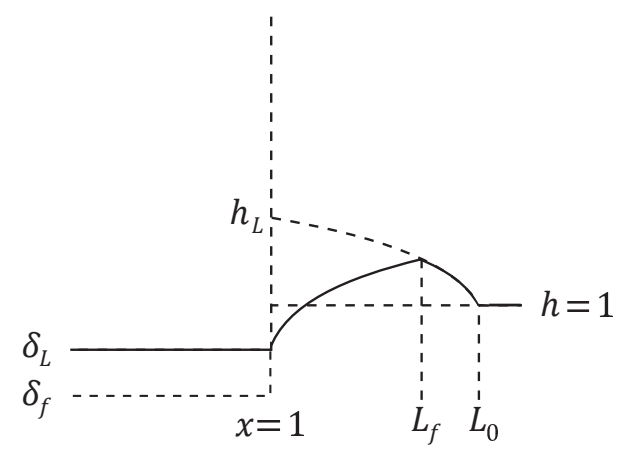

(b)

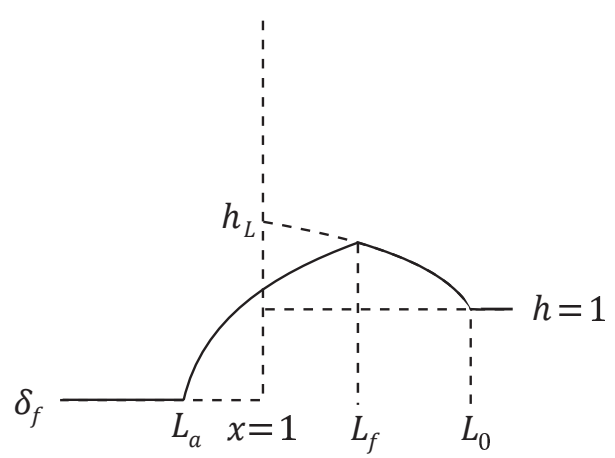

Figure 13: Schematic of the parameters for (a) the reverse squeeze flow, and (b) the avalanching method.

of mass, the free-surface profile can be integrated to give

$$
\delta_{L}-\delta_{f}=\frac{R}{3 B}\left(h_{L}^{3}+\delta_{L}^{3}-2\left(\frac{1}{2}\left(h_{L}^{2}+\delta_{L}^{2}\right)\right)^{3 / 2}\right),
$$

using $h_{L}^{2}+\frac{2 B}{R}\left(1-L_{f}\right)=\delta_{L}^{2}+\frac{2 B}{R}\left(L_{f}-1\right)$. Calculating $\delta_{f}$ and $h_{L}$ from section 2 , the depth $\delta_{L}$ can be determined as a function of $B$ and $R$. Figure 14(a) plots the final profile for two sets of parameters, $B=2, R=1$ (red lines) and $B=1, R=2$ (blue lines).

\subsubsection{Mohr-Coulomb, $\tau_{Y}=p \tan \phi$}

For the Mohr-Coulomb case, the free-surface profile before lift off is given by

$$
h=h_{L}+\Phi(1-x),
$$

see section 2.5.4. As in the pure cohesion case, for a sufficiently slow lift off speed the free-surface flow remains in the plastic limit and hence can be written as (figure 13(a)).

$$
h=\left\{\begin{array}{cc}
h_{L}+\Phi(1-x) & 1<x<L_{f} \\
\delta_{L}+\Phi(x-1) & L_{f}<x<L_{0}
\end{array}\right.
$$

Integrating the free-surface profile after lift off gives an equation relating $\delta_{L}, \delta_{f}$ and $h_{L}$,

$$
\delta_{L}-\delta_{f}=\frac{\left(h_{L}-\delta_{L}\right)^{2}}{4 \Phi} .
$$

As in the pure cohesion case, together with the depth $\delta_{f}$ and height $h_{L}$ from section 2 , the depth $\delta_{L}$ can be determined as a function of $\Phi$ and $R$.

\subsection{Avalanching}

The second method of lift off considers when air can get underneath the indenter. When the indenter is lifted off the substrate, the gap between the indenter and the final depth $\delta_{f}$ is filled with air in the interior with 'avalanching' regions near the edge where there is a squeeze flow and free surface flow, see figure 12(b). When the indenter is removed, the base of the indentation remains at $\delta_{f}$ with the mound of material outside now meeting the base at $x=L_{a}<1$ (figure $13(\mathrm{~b})$ ). 

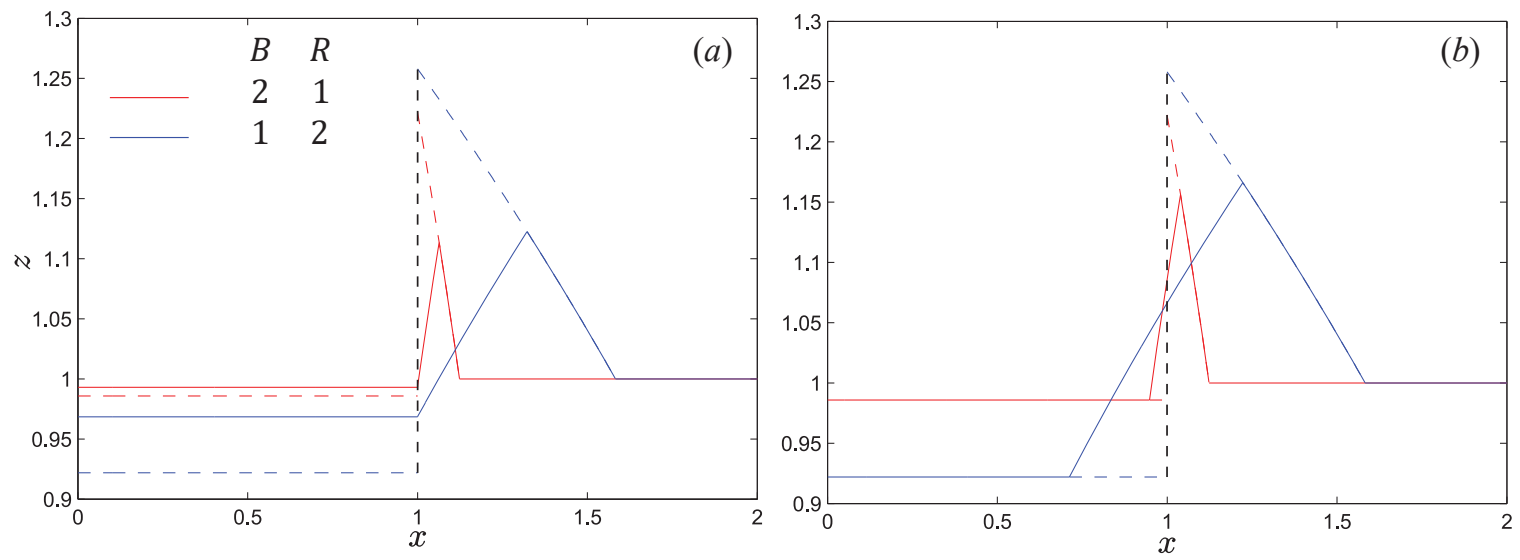

Figure 14: Plot of final indentation for the pure cohesion model after lift off for (a) squeeze flow method, and (b) avalanching method for parameters $B=2, R=1$ (red lines) and $B=1, R=2$ (blue lines). The profiles before and after lift off are given by the dashed and solid lines respectively.

\subsubsection{Purely cohesive, $\tau_{Y}=$ constant}

In the plastic limit, the profile of the free-surface can be written as

$$
h=\left\{\begin{array}{cc}
\left(\delta_{f}^{2}+\frac{2 B}{R}\left(x-L_{a}\right)\right)^{1 / 2} & L_{a}<x<L_{f}, \\
\left(h_{L}^{2}+\frac{2 B}{R}(1-x)\right)^{1 / 2} & L_{f}<x<L_{0} .
\end{array}\right.
$$

Using global mass conservation to match the areas of slumped material then gives

$$
\frac{R}{3 B}\left(h_{L}^{3}+\delta_{f}^{3}\right)+\left(1-L_{a}\right)=\frac{2 R}{3 B}\left[\frac{h_{L}^{2}+\delta_{f}^{2}}{2}+\frac{B}{R}\left(1-L_{a}\right)\right]^{3 / 2} .
$$

And hence, using $\delta_{f}$ and $h_{L}$ calculated in section $2, L_{a}$ can be found as a function of $B$ and $R$. Figure 14(b) plots the final profile for the two sets of parameters $B=2, R=1$ (red lines) and $B=1 R=2$ (blue lines).

\subsubsection{Mohr-Coulomb, $\tau_{Y}=p \tan \phi$}

The profile of the free-surface can be written as

$$
h=\left\{\begin{array}{cc}
h_{L}+\Phi(1-x) & L_{a}<x<L_{f} \\
\delta_{f}+\Phi\left(x-L_{a}\right) & L_{f}<x<L_{0}
\end{array}\right.
$$

Using global mass conservation to match the areas of slumped material then gives

$$
\left(h_{L}-\delta_{f}\right)\left(h_{L}-h_{f}\right)-\frac{1}{2}\left(h_{L}-h_{f}\right)^{2}-\frac{1}{2}\left(h_{f}-\delta_{f}\right)^{2}=0,
$$

where $h_{f}=h_{L}+\Phi\left(1-L_{f}\right)=\delta_{f}+\Phi\left(L_{f}-L_{a}\right)$. Together with $\delta_{f}$ and $h_{L}$ calculated in section $2, L_{a}$ can be found as a function of parameters $\Phi$ and $R$. 


\subsection{Adhesion}

In both the reverse squeeze flow and the avalanching method, we have assumed that lift off happens completely when the pressure at the edge of the indenter $x=1$ is atmospheric i.e. when the free-surface reaches the corner. A non-cohesive material has no strength in tension, therefore you would expect this to be the case. For a cohesive material however, there would be some adhesion to the indenter. When thinking about track making, this adhesion must be able to be characterised in the form of a pressure or stress condition on the base allowing lift off at some point. In the experiments described in the next section we have only considered a non-cohesive material,therefore adhesion is not applicable.

\subsection{Experiments}

To investigate the lift off stage experimentally we used the setup as described in section 3 with a layer of $1 \mathrm{~mm}$ diameter ballotini of depth $h_{0}=5 \mathrm{~cm}$. As the ballotini is dry, we would anticipate that air can get in between the particles and underneath the indenter during lift off. And hence, we expect the method of lift off to be as described in section 4.2 with the bottom of the indentation remaining the same while avalanching occurs on the sides.

Figure 15 shows two lift off experiments with different initial depths for a cylindrical indenter. Figure 15(a,b) plot the detected profiles before (blue solid line) and after (red solid line) lift off together with the free-surface profile for a non-cohesive material with angle of friction $\phi=24.9 \pm 0.7$ (black dot-dashed line), see equation (49). The corresponding photographs, figure $15(\mathrm{c}, \mathrm{d})$ are taken after lift off to demonstrate the final profile. In the first experiment, figure 15(a), the indentation is sufficiently shallow that the sides of the indentation remain roughly at or below the angle of friction. As a result, almost no avalanching occurs as seen by the negligible difference between the before and after profiles. When the indentation depth is increased, the walls become steeper than the angle of friction at the contact points, see figure 15(b). In this case, when lift off occurs, the ballotini avalanches down into the interior of the indentation with the bottom of the indentation remaining fixed. Figure 15(b) suggests that the final profile then sits at an angle less than the angle of friction. This may be due to any added inertia caused by the process of lift off.

In this section we have considered a simple theoretical model to describe the process of lift off for a flat-based indenter on either a purely cohesive or Mohr-Coulomb substrate. We have looked at three different methods for lift off: (a) reverse squeeze flow, (b) avalanching and (c) adhesion. Our preliminary experiments with dry ballotini demonstrate the method of avalanching when air can get underneath the indenter. To explore to process of lift off further we need to consider the problem of adhesion and the point at which completed detachment from the substrate can occur.

\section{Conclusion}

The aim of this work was to understand the indentation of deformable plastic layers theoretically and experimentally. Our theoretical model using viscoplastic lubrication theory describes the indentation of a shallow plastic layer. We have shown that the plastic limit reduces to a force balance and equal areas construction given the quasistatic free-surface 

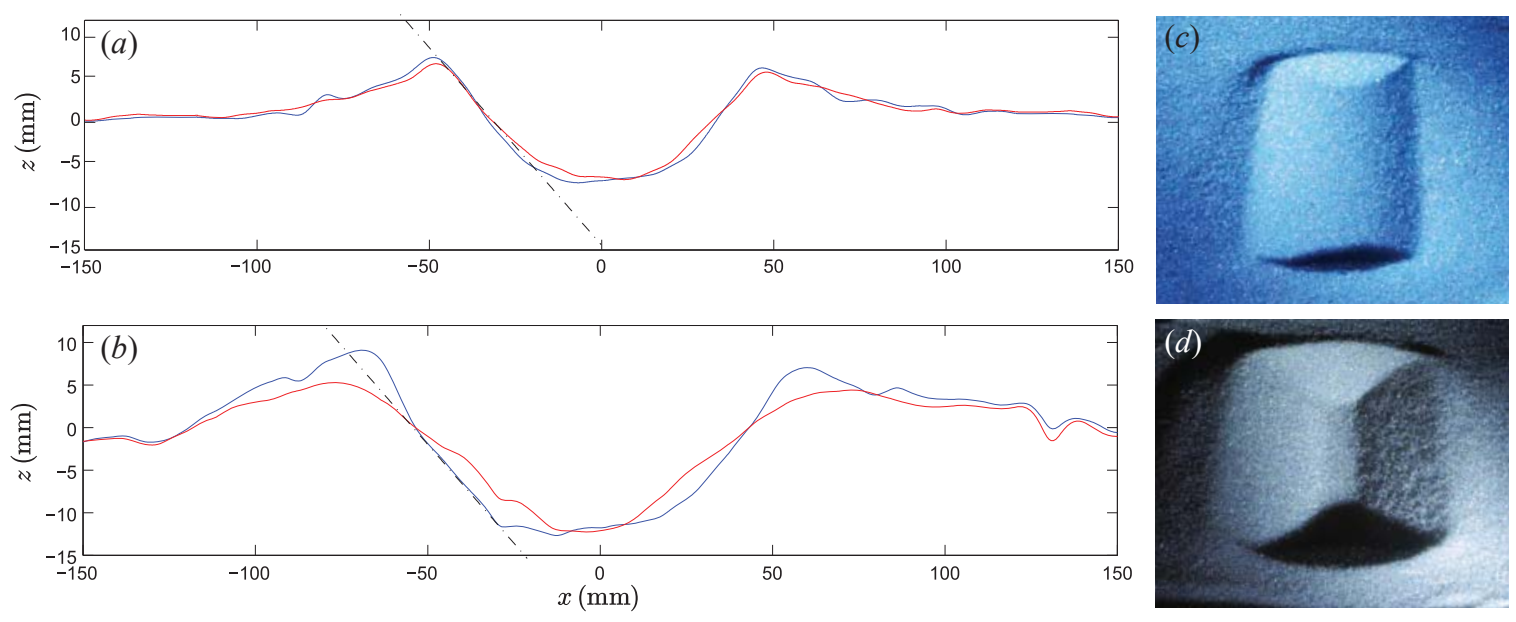

Figure 15: Lift off experiments with a layer of $1 \mathrm{~mm}$ ballotini of depth $h_{0}=5 \mathrm{~cm}$. $(\mathrm{a}, \mathrm{b})$ Depth profiles for increasing depths of indentation for before (blue solid line) and after (red solid line) lift off. Black dot-dashed line indicates theoretical angle of friction $\phi=24.9 \pm 0.7$. $(\mathrm{c}, \mathrm{d})$ Photographs of final profiles for experiments shown in $(\mathrm{a}, \mathrm{b})$, respectively.

profiles. The simple experimental setup used has produced promising preliminary results for the indentation of a pure cohesive (joint compound) and non-cohesive (ballotini) substrate with some qualitative comparisons with our theoretical model.

To extend this work in future, we plan on conducting further experiments to investigate the indentation of deformable plastic layers. In terms of a pure cohesive material we would like to better characterise the yield stress of the substrate and confirm that a Bingham rheology is an appropriate approximation opposed to a more complicated Herschel-Bulkley model. In addition, we would like to characterise the discrepancy between ballotini sizes to check initial conditions resetting the surface are not playing a role. In terms of experimental setup, we would like to improve the simple system to reduce the compliance of the indenter, for example by clamping the sizes of half cylinder, and extending to axisymmetric geometries such a hemisphere. Finally, when discussing the problem of lift off, we touched on the case of adhesion. This needs to be explored further both theoretically and experimentally as the process of lift off ultimately determines the final indentation left behind.

\section{Acknowledgments}

I would like to thank Neil Balmforth and Ian Hewitt for their thorough supervision throughout the summer and tireless enthusiasm to find some 'good mud'! 


\section{References}

[1] J. R. L. Allen, Subfossil mammalian tracks (Flandrian) in the Severn Estuary, S. W. Britain: mechanics of formation, preservation and distribution, Phi. Trans. R. Soc. Lond., 352 (1997), pp. 481-518.

[2] K. T. Bates, R. Savage, T. C. Pataky, S. A. Morse, E. Webster, P. L. Falkingham, L. Ren, Z. Qian, D. Collins, M. R. Bennett, J. Mcclymont, R. H. Crompton, M. Sa, E. Webster, F. Pl, Z. Qian, D. Collins, and J. McCLYMONT, Does footprint depth correlate with foot motion and pressure?, J. R. Soc. Interface, (2013).

[3] M. D. Bolton And C. K. LaU, Vertical bearing capacity factors for circular and strip footings on Mohr-Coulomb soil, Can. Geotech. J., 30 (1993), pp. 1024-1033.

[4] I. F. Collins, A Simplified Analysis of the Rolling of a Cylinder on a Rigid/Perfectly Plastic Half-Space, Int. J. Mech. Sci., 14 (1972), pp. 1-14.

[5] N. Dubash, N. Balmforth, A. Slim, and S. Cochard, What is the final shape of a viscoplastic slump?, J. Nonnewton. Fluid Mech., 158 (2009), pp. 91-100.

[6] G. Eason And R. T. Shield, The plastic indentation of a semi-infinite solid by a perfectly rough circular punch, Z. Angew. Math. Phys., 11 (1960), pp. 33-43.

[7] D. D. Gillette And M. G. Lockley, Dinosaur tracks and traces, Cambridge University Press, 1991.

[8] I. J. Hewitt and N. J. Balmforth, Viscoplastic lubrication theory with application to bearings and the washboard instability of a planing plate, J. Nonnewton. Fluid Mech., (2012), pp. 1-17.

[9] R. HiLl, The mathematical theory of plasticity, vol. 11, Oxford university press, 1950.

[10] K. LiU And C. MeI, Approximate equations for the slow spreading of a thin sheet of bingham plastic fluid, Phys. Fluids A, 2 (1990), pp. 30-36.

[11] E. A. Marshall, Rolling contact with Plastic Deformation, J. Mech. Phys. Solids., 16 (1968), pp. 243-254.

[12] J. Milàn, Variations in the Morphology of Emu (Dromaius Novaehollandiae) Tracks Reflecting Differences in Walking Pattern and Substrate Consistency: Ichnotaxonomic Implications, Palaeontology, 49 (2006), pp. 405-420.

[13] J. NyE, Plasticity solution for a glacier snout, J. Glaciol., 6 (1967), pp. 695-715.

[14] L. PRAndtL, Uber die harte plastischer korper, nachrichten von der koniglichen gesellschaft der wissenschaften zu gottingen, Math. Phys. KI, 12 (1920), pp. 74-85.

[15] J. SAlençon, Bearing capacity of a footing on an angle of shearing resistance $=0$ soil with linearly varying shear strength, Geotechnique, 24 (1974). 
[16] R. T. SHIELD. On the plastic flow of metals under conditions of axial symmetry. Proc. Royal Soc. A, 233 (1955), pp. 267-287.

[17] A. J. M. SPENCER, Perturbation methods in plasticity - I plane strain of non-homogenous plastic solids, J. Mech. Phys. Solids, 9 (1961), pp. 279-288.

[18] _ Pertubation Methods in Plasticity - II Plane Strain of Slightly Irregular Bodies, Mech. Phys. Solids, 10 (1962), pp. 17-26.

[19] K. Terzaghi, Theoretical Soil Mechanics, John Wiley \& Sons, Inc., 1943. 


\section{A Simple Adiabatic Model for Vertical Variation of Halocline Slope in the Beaufort Gyre}

Jessica Kenigson

January 13, 2018

\section{Introduction}

\subsection{Forcing, circulation, and freshwater content of the Beaufort Gyre}

The Beaufort Gyre is a large wind-driven circulation in the Canada Basin of the Western Arctic Ocean between approximately $120^{\circ}-180^{\circ} \mathrm{W}$ and $70^{\circ}-85^{\circ} \mathrm{N}$. Figure 1 shows the bathymetry and spatial pattern of salinity at $200 \mathrm{~m}$ in the Beaufort Gyre. The gyre is a persistent feature in the upper $\sim 300 \mathrm{~m}$ of the ocean, driven primarily by the climatological anticyclonic winds associated with the wintertime Beaufort High sea level pressure system. The winds cause Ekman downwelling, which pumps low-salinity surface water into the gyre interior, deepens the halocline, and sets up the resulting anticyclonic circulation. Although the climatological mean winds are anticyclonic, there is considerable seasonal variability; the winds are strongly anticyclonic during the winter and weakest (potentially even cyclonic) during the summer. Nevertheless, the upper-ocean circulation is persistently anticyclonic due to the bowl-shaped deformation of the isopycnals associated with the freshwater storage [9]. The surface waters of the Arctic are relatively fresh due to the excess of precipitation over evaporation in the basin and due to its drainage of numerous large river systems and low-salinity Pacific inflow.

The freshwater content $(\mathrm{m})$ is defined as

$$
F W C=\int_{D}^{\eta} \frac{S_{r e f}-S}{S_{r e f}} d z
$$

where $S$ is measured in practical salinity units, $S_{\text {ref }}$ is a reference salinity (typically 34.80 ), $\eta$ is the sea surface $(\mathrm{m})$, and $D(\mathrm{~m})$ is the depth of the isohaline of the reference salinity [4]. $S_{r e f}$ is chosen to be near the mean salinity, and therefore $F W C$ represents the amount of freshwater that would need to be introduced in order to obtain the observed salinity (beginning from $S_{r e f}$ ). Equation 1 can be integrated over an area of interest to obtain the volumetric fresh water content [4]. The total Arctic freshwater content has been estimated to be $93,000 \mathrm{~km}^{3}$ over $1980-2000$, with $18,500 \mathrm{~km}^{3}$ being stored in the Beaufort Gyre; over 2000-2010, the Arctic freshwater content increased to 101,000 km $\mathrm{km}^{3}$ and the Beaufort Gyre freshwater content increased to $23,500 \mathrm{~km}^{3}$ [9]. In addition, the freshwater content of the Beaufort Gyre has been estimated to have increased by $8000 \pm 2000 \mathrm{~km}^{3}$ from $1995-2010$ 


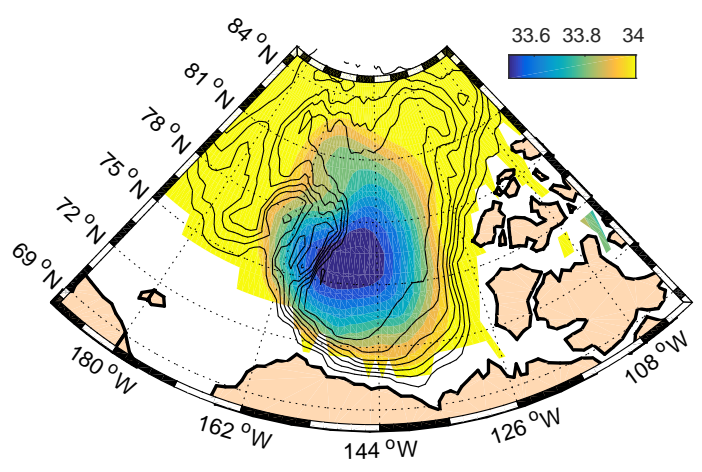

Figure 1: Salinity at $200 \mathrm{~m}$ (colormap) from the PHC climatology (winter mean) and bathymetry (black contours). Note the pronounced salinity minimum within the Beaufort Gyre, which is a major freshwater reservoir in the Arctic Ocean.

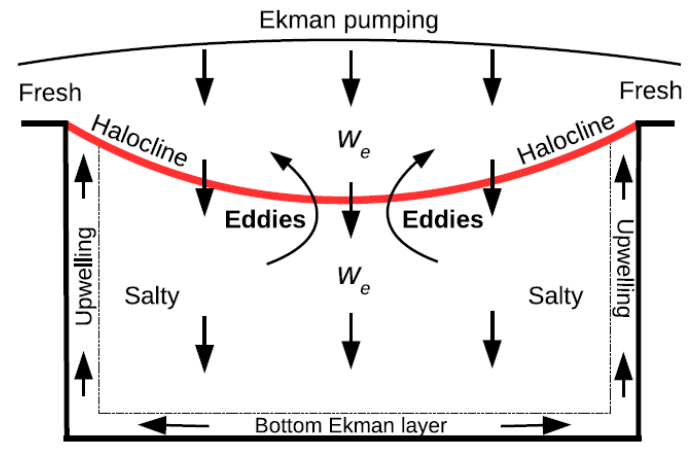

Figure 2: Schematic diagram of the halocline model in [7]. The halocline is represented as an interface that is deepened by Ekman pumping. Mesoscale eddies (baroclinic instability) form along the interface to counteract the deepening. The halocline is modeled to be at a fixed depth at the gyre boundaries. Figure from [7].

due predominantly to the spin-up of the gyre by the increasing trend of the anticyclonic wind stress curl [3].

Wind stress variability over the Beaufort Gyre modulates the storage and release of freshwater, which has implications for freshwater and ice exchanges with the North Atlantic Ocean and the Atlantic Meridional Overturning Circulation (AMOC). It has been proposed that when high sea level pressure and anticyclonic wind anomalies prevail in the Arctic, freshwater accumulates through various mechanisms [9]. River runoff into the Arctic Ocean is enhanced due to shifts in storm tracks. Arctic sea ice also grows during anticyclonic wind regimes due to the cooler temperatures that tend to predominate. Convergence of Ekman transport pumps freshwater into the gyre, which also causes convergence and ridging of sea ice. Finally, export of freshwater through Fram Strait declines, which increases the surface salinity in the Greenland Sea, destabilizes the stratification of the water column and favors deep water formation. As freshwater storage in the Beaufort Gyre is associated with doming of the sea surface, eventually, an anomalous dynamic height gradient between the Beaufort Gyre and the North Atlantic develops, which induces anomalous flow through Fram Strait and the Canadian Arctic Archipelago and sets the stage for a transition to the cyclonic circulation regime. During cyclonic circulation regimes, these processes are reversed.

The halocline in the Canada Basin is subdivided into a "warm halocline" centered around $50 \mathrm{~m}$ depth with salinity from $\sim 30-32$ and temperature $\sim 0{ }^{\circ} \mathrm{C}$, and a "cold halocline" centered around $150 \mathrm{~m}$ depth with salinity from $\sim 32-33$ and temperature $\sim-1.5^{\circ} \mathrm{C}$ [10]. There is considerable interannual temperature variability and no significant seasonal variability in the warm halocline. The warm halocline is a persistent year-round feature and is sustained by summertime subduction of Chukchi Shelf Waters. The salinity minimum in 
the central Beaufort Gyre that is set up by the Ekman pumping prevents strong wintertime mixing that would expose the overlying sea ice to the warm halocline; entrainment of this heat could cause approximately $1 \mathrm{~m}$ of sea ice melt [10].

The storage and release of freshwater in the Beaufort Gyre thus has a broad range of implications for the global climate. However, the dynamics of the Beaufort Gyre and the processes that determine the halocline structure are uncertain.

\subsection{Beaufort Gyre dynamics and simple model}

The Beaufort Gyre is a persistent feature in the Canada Basin [9]. However, until recently the basic dynamical balance of the mean circulation has been unclear. Specifically, what processes oppose the deepening of the halocline due to Ekman pumping, allowing a steadystate circulation to develop?

It has been proposed that mesoscale eddy fluxes are capable of balancing the Ekman pumping in steady state [6]. The bowl-shaped deformation of the isopycnals in the halocline is associated with the buildup of gravitational potential energy, which is a baroclinically unstable configuration that leads to the formation of mesoscale eddies. These eddies act to oppose the steepening of the halocline slope. Experiments with the MITgcm as well as simple scaling arguments [6] suggest that the timescale of adjustment of the gyre to changes in wind forcing (i.e., the time required for the halocline to reach its equilibrium depth) is

$$
T \sim \frac{R^{2}}{K}
$$

where $R$ represents the radius of the gyre and $K$ represents the eddy diffusivity. Therefore, for a significant release of freshwater from the Beaufort Gyre to occur, it is necessary for wind forcing anomalies to persist longer than the equilibration timescale, which was estimated to be $\sim 6$ years.

The following theory is developed in [7]. In this framework, the halocline is represented as an isopycnal interface that is deepened by Ekman pumping; the deepening of the halocline is opposed by the activity of mesoscale eddies (Figure 2). Assume that

$$
\frac{D b}{D t}=S
$$

where $b$ represents buoyancy and $S$ is a source term. Here we model the gyre in cylindrical coordinates. Expanding and taking the Reynolds average of both sides yields

$$
\bar{b}_{t}+\bar{v} \bar{b}_{r}+\bar{w} \bar{b}_{z}+\overline{v^{\prime} b_{r}^{\prime}}+\overline{w^{\prime} b_{z}^{\prime}}=\bar{S}
$$

Here $v$ represents a velocity in the $r$-direction, $w$ represents a velocity in the $z$-direction, and $\overline{v^{\prime} b_{r}^{\prime}}$ and $\overline{w^{\prime} b_{z}^{\prime}}$ are interpreted as eddy fluxes of buoyancy. It is desirable to re-write the eddy fluxes in Equation 3 as advection of mean buoyancy gradients by eddy velocities $v^{*}$ and $w^{*}$, i.e., 


$$
\bar{b}_{t}+\left(\bar{v}+v^{*}\right) \bar{b}_{r}+\left(\bar{w}+w^{*}\right) \bar{b}_{z}=\bar{S} .
$$

This condition requires that

$$
\overline{v^{\prime} b_{r}^{\prime}}+\overline{w^{\prime} b_{z}^{\prime}}=v^{*} \bar{b}_{r}+w^{*} \bar{b}_{z}
$$

Using the Transformed Eulerian Mean framework [1], there exists a mean streamfunction $\bar{\Psi}$ such that

$$
\bar{v}=-\bar{\Psi}_{z}, \bar{w}=\frac{1}{r}(r \bar{\Psi})_{r}
$$

which satisfies

$$
\bar{\Psi}=\frac{\bar{\tau}}{\rho_{0} f}
$$

where $\tau(r, t)$ represents the (known) azimuthal surface wind forcing of the gyre, $\rho_{0}$ is a reference density, and $f$ is the coriolis parameter. In addition, there is an eddy streamfunction $\Psi^{*}$ with associated eddy velocities

$$
v^{*}=-\Psi_{z}^{*}, w^{*}=\frac{1}{r}\left(r \Psi^{*}\right)_{r}
$$

which is given by

$$
\Psi^{*}=-\frac{\overline{w^{\prime} b^{\prime}}}{\bar{b}_{r}}=\frac{\overline{v^{\prime} b^{\prime}}}{\bar{b}_{z}} .
$$

This represents the so-called "adiabatic limit" in which eddy fluxes are assumed to be along-isopycnal, i.e., perpendicular to gradients of buoyancy. In this formulation, eddies "re-arrange" existing water masses but do not create "new" water masses (new density classes). This condition can be observed by defining the eddy buoyancy flux as

$$
F_{b}=\left(\begin{array}{c}
\overline{v^{\prime} b^{\prime}} \\
0 \\
\overline{w^{\prime} b^{\prime}}
\end{array}\right)
$$

and noting that

$$
\begin{aligned}
F_{b} \cdot \nabla b & =\overline{v^{\prime} b^{\prime}} \cdot \bar{b}_{r}+\overline{w^{\prime} b^{\prime}} \cdot \bar{b}_{z} \\
& =\bar{b}_{r} \bar{b}_{z}\left(\frac{\overline{v^{\prime} b^{\prime}}}{\bar{b}_{z}}+\frac{\overline{w^{\prime} b^{\prime}}}{\bar{b}_{r}}\right) \\
& =0
\end{aligned}
$$


by Equation 9 . Using the condition $\nabla \cdot\left(v^{*}, 0, w^{*}\right)=0$, which follows from Equation 8 , Equation 5 is satisfied. A mesoscale eddy parameterization is needed for the eddy fluxes in Equation 9 and the Gent-McWilliams parameterization [2],

$$
\overline{v^{\prime} b^{\prime}}=-K \bar{b}_{r},
$$

is used. Here $K\left(\mathrm{~m}^{2} \mathrm{~s}^{-1}\right)$ represents the eddy diffusivity and

$$
\Psi^{*}=K s
$$

by Equation 9, where $s=\left|\bar{b}_{r} / \bar{b}_{z}\right|$. (For the bowl-shaped deformation of the halocline, it follows that $\bar{b}_{r} / \bar{b}_{z}<0$.)

It is the residual circulation, $\tilde{\Psi}=\bar{\Psi}+\Psi^{*}$, that drives changes in the halocline depth. Substituting Equations 6 and 8 into Equation 4, it follows that

$$
\bar{b}_{t}+\frac{1}{r}(r \tilde{\Psi})_{r} \bar{b}_{z}-\tilde{\Psi}_{z} \bar{b}_{r}=\bar{S} .
$$

In ocean models (e.g., [12]), it is typical for the eddy diffusivity $K$ to be parameterized to be proportional to the isopycnal slope, i.e.,

$$
K=k s^{n-1}
$$

where $k$ represents the eddy efficiency and $n$ is a small positive integer. This parameterization is used in [7] with $n=2$. Along with Equations 7 and 12, this implies that

$$
\tilde{\Psi}=\frac{\bar{\tau}}{\rho_{0} f}+k\left(-\frac{\bar{b}_{r}}{\bar{b}_{z}}\right)^{2} .
$$

Equations 13 and 14 are accompanied by the boundary conditions

$$
\left.\bar{b}\right|_{r=R}=b_{R}(z),\left.\quad \bar{b}_{r}\right|_{r=0}=0,\left.\quad \bar{b}_{z}\right|_{z=0, H}=0,\left.\quad \bar{b}\right|_{t=0}=b_{0}(r, z) .
$$

(From now on, the overbar over Reynolds averaged quantities will be dropped.) Linearizing Equations 13 - 14 about the long-term time mean yields

$$
\begin{array}{r}
b_{t}+\frac{1}{r}(\tilde{\Psi} r)_{r} b_{0 z}-\tilde{\Psi}_{z} b_{0 r}=S \\
\tilde{\Psi}=\frac{\tau}{\rho_{0} f}-n \frac{\tau_{0}}{\rho_{0} f} \frac{s}{s_{0}} \\
\frac{s}{s_{0}}=\left(\frac{b_{r}}{b_{0 r}}-\frac{b_{z}}{b_{0 z}}\right) .
\end{array}
$$


Here subscripts "0" indicate the basic state and other variables indicate perturbations about the basic state. Defining $h=b / b_{0 z}$, where $h$ represents the isopycnal depth perturbation, these equations imply

$$
h_{t}=\frac{1}{r}\left(n K_{0} r h_{r}\right)_{r}+\frac{1}{r}\left(r \frac{-\tau}{\rho_{0} f}\right)_{r}
$$

where

$$
K_{0}=k s_{0}^{n-1}=k\left(\frac{-\tau_{0}}{\rho_{0} f k}\right)^{(n-1) / n},
$$

and $n=2$ is, again, a reasonable choice. The boundary conditions for the linearized depth perturbation equation are given by

$$
\left.h\right|_{r=R}=0,\left.\quad h_{r}\right|_{r=0}=0 .
$$

Equations 16 - 18 are derived in the appendix of [7]. A gyre adjustment timescale is also derived in [7] and is obtained by decomposing the (nondimensionalized) eddy diffusion operator

$$
L:=\frac{1}{r}\left(K_{0} r h_{r}\right)_{r}
$$

into orthogonal eigenmodes $h_{i}^{*}$ and expressing

$$
h=\sum_{i=1}^{\infty} a_{i}(t) h_{i}^{*}
$$

where $a_{i}(t)$ is an amplitude function that is exponentially decaying on a timescale $T_{i}$. The equilibration timescale of the gyre is set by the slowest timescale

$$
T_{0}=\frac{1}{n \lambda} \frac{R^{2}}{K_{0}(R)}
$$

where the eigenvectors $h_{i}$ and eigenvalues $\lambda$ are shown in Figure 6 of [7].

\section{Extension of the Simple Model}

\subsection{Rationale for a modified model}

The boundary conditions of the existing model assume that the isopycnal interface representing the halocline is at a fixed depth at the boundary of the gyre (see Figure 2). However, 
observations suggest that there is considerable seasonal variability in isopycnal outcropping location [10].

The Polar Science Center Hydrographic Climatology $(\mathrm{PHC})$ provides gridded $\left(1^{\circ} \times 1^{\circ}\right)$ means of summer (July, August, September) and winter (March, April, May) temperature and salinity at a sequence of depths between 0 and $5500 \mathrm{~m}$ (Figure 3, left and central columns). We use the Thermodynamic Equation of SeaWater 2010 (TEOS-10) equations [5] to estimate the winter and summer climatological density and potential density (Figure 3 , right column).

Figure 3 reveals considerable seasonal variability of buoyancy, particularly within the upper $\sim 50 \mathrm{~m}$ of the gyre. This variability is predominantly associated with the seasonal variability of salinity rather than temperature, and there is seasonal outcropping of isopycnals (compare the top and bottom rows of Figure 3). Since this variability in the isopycnal outcropping location cannot be captured by the existing model, we modify the model to accommodate it.

Specifically, we consider Equation 16 with the modified boundary conditions

$$
\left.h_{r}\right|_{r=0, R}=0
$$

i.e., no flux is permitted through the gyre boundary.

\subsection{Solution of linearized equations in an idealized case}

We first consider a simplified case of Equation 16 with boundary conditions given by Equation 19 in which $K_{0}$ is constant with respect to $r$ and $\tau=0$. In this case Equation 16 simplifies to

$$
h_{t}=\frac{n K_{0}}{r}\left(h_{r}+r h_{r r}\right)
$$

Suppose that there is a solution of the form

$$
h=a(t) b(r) .
$$

Then

$$
a^{\prime}(t) b(r)=n K_{0}\left(\frac{1}{r} a(t) b^{\prime}(r)+a(t) b^{\prime \prime}(r)\right) .
$$

Separating variables,

$$
\frac{a^{\prime}(t)}{a(t)}=n K_{0}\left(\frac{1}{r} \frac{b^{\prime}(r)}{b(r)}+\frac{b^{\prime \prime}(r)}{b(r)}\right)
$$

Then the LHS implies 

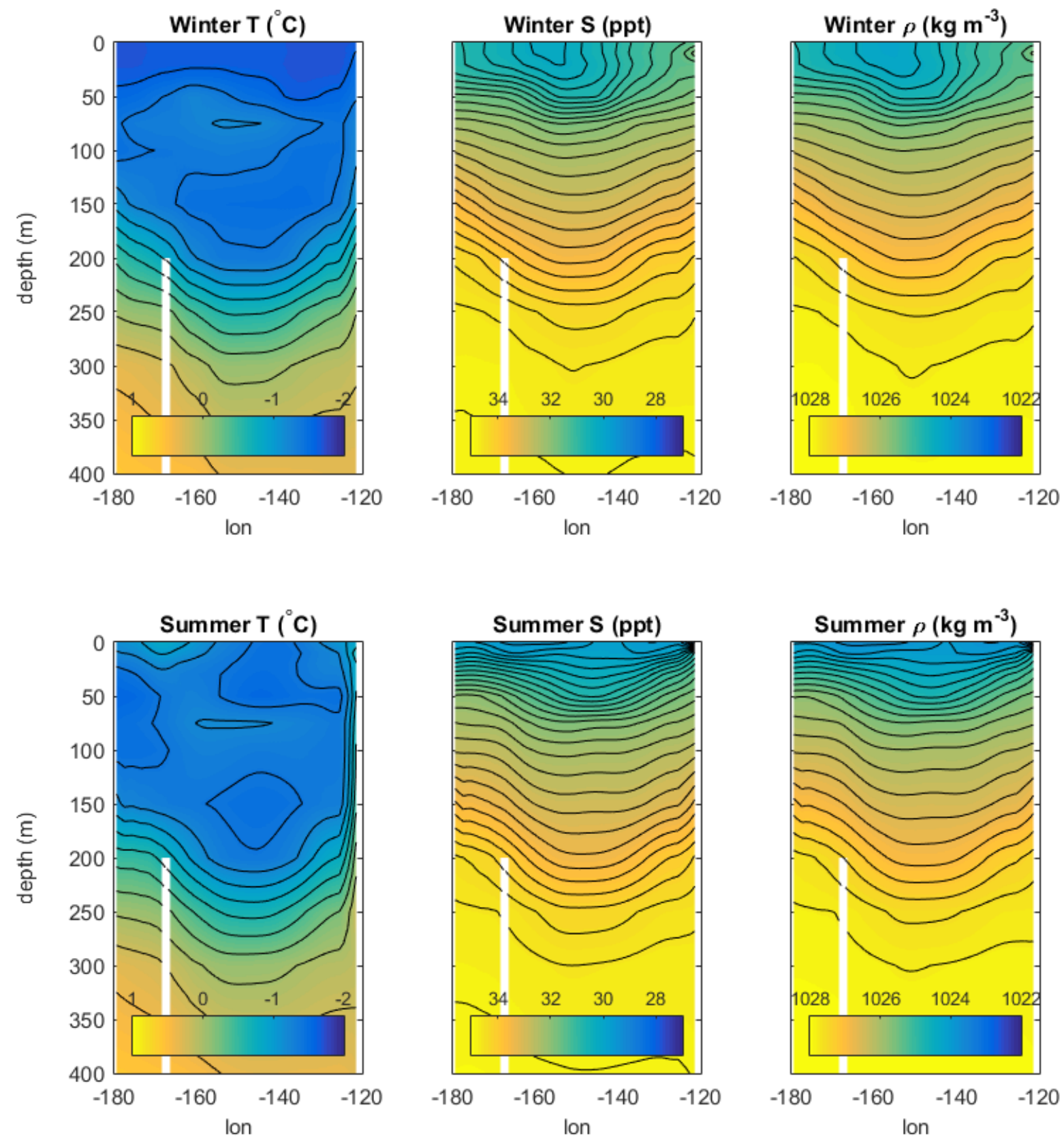

Figure 3: Longitude-depth transect of winter (March, April, May) and summer (June, July, August) mean temperature and salinity from the PHC climatology in the upper $400 \mathrm{~m}$ of the Beaufort Gyre at $75.5^{\circ} \mathrm{N}$ (left and central columns). Potential density is estimated from the TEOS-10 equations using the temperature and salinity profiles (right column). 


$$
\frac{a^{\prime}(t)}{a(t)}=-\lambda
$$

for some $\lambda \geq 0$, and therefore

$$
a(t)=c_{0} e^{-\lambda t}
$$

for any constant $c_{0}$. The RHS implies

$$
n K_{0} b^{\prime \prime}(r)+\frac{n K_{0}}{r} b^{\prime}(r)+\lambda b(r)=0
$$

or equivalently

$$
r^{2} b^{\prime \prime}(r)+r b^{\prime}(r)+\frac{\lambda}{n K_{0}} r^{2} b(r)=0 .
$$

With the substitution

$$
c^{2}=\frac{\lambda}{n K_{0}}
$$

the equation is seen to have solutions $J_{0}(c r)$ (Bessel function of the first kind) and $Y_{0}(c r)$ (Bessel function of the second kind). The general solution to the separable PDE is then

$$
h(r, t)=c_{0} e^{-\lambda t}\left(c_{1} J_{0}\left(\sqrt{\frac{\lambda}{n K_{0}}} r\right)+c_{2} Y_{0}\left(\sqrt{\frac{\lambda}{n K_{0}}} r\right)\right)
$$

for $\lambda \geq 0$. The values of $\lambda$ for which nontrivial solutions exist are constrained by the boundary conditions. In the case of the simple halocline model in [7] with boundary conditions given by Equation 19, the condition $\left.h_{r}\right|_{r=0}=0$ requires $c_{2}=0$. The condition $\left.h_{r}\right|_{r=R}=0$ requires

$$
\lambda_{m}=\frac{n K_{0}}{R^{2}} \alpha_{1 m}^{2}, m=0,1, \ldots
$$

where $\alpha_{1 m}$ is the $m$-th zero of the Bessel function $J_{1}$. In that case

$$
h(r, t)=\sum_{m=0}^{\infty} c_{m} e^{-\lambda_{m} t} J_{0}\left(\sqrt{\frac{\lambda_{m}}{n K_{0}}} r\right)
$$




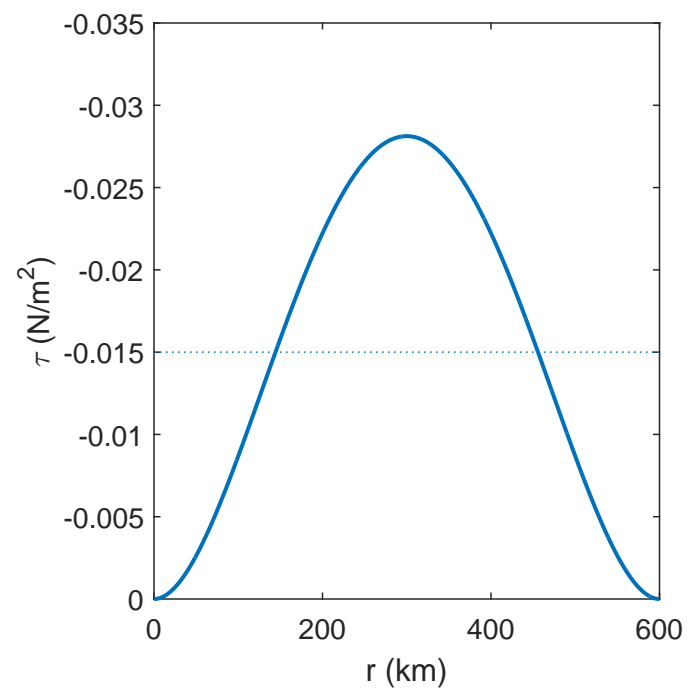

Figure 4: Diagram of the basic state wind forcing given by Equation 20.

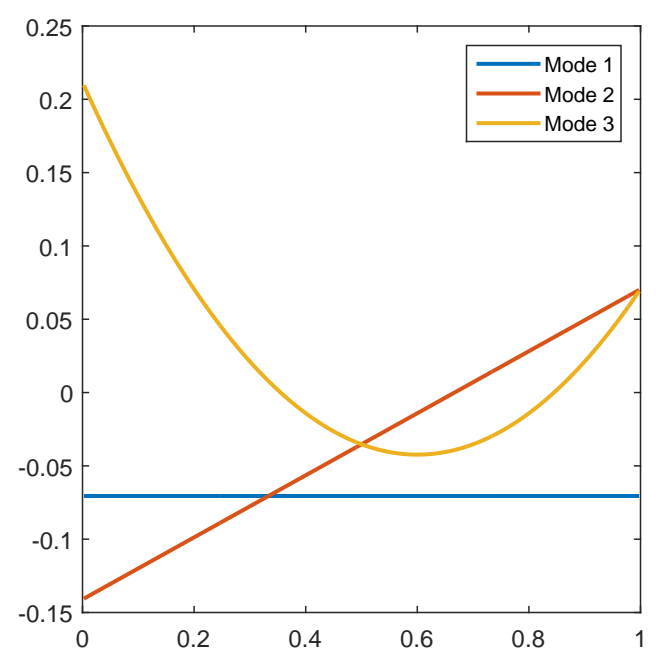

Figure 5: Eigenfunctions of Equation 24 corresponding to the first three eigenvalues $\hat{\lambda}=$ $0,3,8$. (The eigenfunctions are not normalized to be orthonormal.)

\subsection{Eigenanalysis of linearized equation in the absence of wind forcing perturbation}

In reality, $K_{0}$ varies spatially, in balance with the basic state wind forcing $\tau_{0}$ (Equation 17). Therefore, Equation 16 does not lend itself to a simple analytical solution.

\subsubsection{Basic state wind forcing}

The wind forcing $\tau$ is assumed to be a known function. We assume that it has the form

$$
\tau_{0}(r)=-30 \tau_{M}\left(\frac{r}{R}\left(1-\frac{r}{R}\right)\right)^{2}
$$

where $\tau_{M}$ represents the magnitude of the mean value of $\tau_{0}$ over the gyre. That is,

$$
\tau_{M}=\left|\frac{1}{A} \int_{0}^{2 \pi} \int_{0}^{R} \tau_{0}(r, \theta) r d r d \theta\right|
$$

where $A=\pi R^{2}$ is the area of the gyre. Here we take $\tau_{M}=0.015 \mathrm{~N} \mathrm{~m}^{-2}$, which is chosen to facilitate comparison with [7]. Figure 4 shows the basic state wind forcing.

\subsubsection{Eigenanalysis}

Suppose that $\mathcal{L}[h]$ is a self-adjoint linear operator on a space of real-valued functions $\mathcal{F}$ defined on $[0, R]$ which are integrable with respect to an $r$-weighted inner product 


$$
\langle f, g\rangle=\int_{0}^{R} r f(r) g(r) d r .
$$

Then the eigenfunctions of $\mathcal{L}$ form a complete orthonormal basis $h_{i}^{*}$ such that for any function $h$ in $\mathcal{F}$,

$$
h=\sum_{i=0}^{\infty} c_{i}(t) h_{i}^{*}
$$

where $c_{i}=\left\langle h, h_{i}^{*}\right\rangle$. We examine the eigenfunctions of the linear operator

$$
\mathcal{L}[h]=\frac{1}{r}\left(K_{0}(r) r h_{r}\right)_{r}, \quad h_{r}(0)=0, \quad h_{r}(R)=0 .
$$

Lemma 2.1 The linear operator defined by Equation 22 is self-adjoint with respect to the inner product given by Equation 21.

\section{Proof}

$$
\begin{aligned}
\langle\mathcal{L}[f], g\rangle & =\int_{0}^{R}\left(K_{0} r f_{r}\right)_{r} g d r \\
& =\int_{0}^{R}\left(K_{0} r f_{r} g\right)_{r} d r-\int_{0}^{R}\left(K_{0} r g_{r}\right) f_{r} d r \\
& =\left(\left.K_{0} r f_{r} g\right|_{0} ^{R}-\int_{0}^{R}\left(K_{0} r g_{r} f\right)_{r} d r+\int_{0}^{R}\left(K_{0} r g_{r}\right)_{r} f d r\right. \\
& =\left(\left.K_{0} r g_{r} f\right|_{0} ^{R}+\langle f, \mathcal{L}[g]\rangle\right. \\
& =\langle f, \mathcal{L}[g]\rangle .
\end{aligned}
$$

Although we cannot solve Equation 16 analytically, as in [7] the solution $h$ can be decomposed into spatially-varying orthogonal modes (eigenvectors) $h_{i}$ with amplitudes $c_{i}(t)$ which are exponentially decaying on a timescale $T_{i}$ (inversely proportional to eigenvalues $\left.\lambda_{i}\right)$. Therefore, we consider the eigenvalue problem

$$
\frac{n}{r}\left[r K_{0}(r) h_{r}\right]_{r}=-\lambda h
$$

subject to the boundary conditions (Equation 19). It is considerably more convenient to solve the nondimensionalized problem that results from choosing

$$
\hat{r}=r / R, \quad \hat{h}=h / R, \quad \hat{K}_{0}=K_{0}(r) / K_{0}(R / 2), \quad \hat{T}=\left(n T K_{0}(R / 2)\right) / R^{2} .
$$

The resulting problem is given by 


$$
\left[\hat{r} \hat{K}_{0} \hat{h}_{\hat{r}}\right]_{\hat{r}}=-\frac{\hat{r} \hat{h}}{\hat{T}}=-\hat{\lambda} \hat{r} \hat{h}
$$

where $\lambda:=1 / T$ and $\hat{K}_{0}=4 \hat{r}(1-\hat{r})$ by Equation 17 and Equation 20. The nondimensionalized boundary conditions become

$$
\left.\hat{h}_{\hat{r}}\right|_{\hat{r}=0,1}=0 .
$$

Then the timescale of equilibration of the gyre is given by

$$
T_{0}=\frac{1}{\lambda_{0}}=\frac{R^{2} \hat{T}_{0}}{n K_{0}(R / 2)}=\frac{R^{2}}{n \hat{\lambda_{0}} K_{0}(R / 2)}
$$

where the eigenfunctions are assumed to be sorted such that $\lambda_{0}$ is smallest, i.e., $T_{0}$ is longest. Thus the equilibration is controlled by the slowest eigenfunction.

We solve Equation 24 numerically. The first three eigenvalues are $\hat{\lambda}=0,12,32$ and the eigenfunctions are shown in Figure 5.

\subsection{The nonlinear problem}

\subsubsection{Steady state solution}

In steady state, the residual circulation vanishes, i.e., $\tilde{\Psi}=0$. It follows from Equation 14 that

$$
h(r)=-\int_{0}^{r}\left(\frac{-\tau\left(r^{\prime}\right)}{\rho_{0} f k}\right)^{\frac{1}{2}} d r^{\prime}+h(0) .
$$

Assume that the surface wind stress is given by Equation 20. Then Equation 26 implies

$$
h(r)=-\left(\frac{30 \tau_{M}}{\rho_{0} f k}\right)^{\frac{1}{2}}\left(\frac{r^{2}}{2 R}-\frac{r^{3}}{3 R^{2}}\right)+h(0) .
$$

There is no particular reason that $h(r)$, as given by Equation 27, must be positive. Given that

$$
-\left(\frac{30 \tau_{M}}{\rho_{0} f k}\right)^{\frac{1}{2}}\left(\frac{r^{2}}{2 R}-\frac{r^{3}}{3 R^{2}}\right)=-\left(\frac{30 \tau_{M}}{\rho_{0} f k}\right)^{\frac{1}{2}} \frac{r^{2}}{R}\left(\frac{1}{2}-\frac{r}{3 R}\right)<0
$$

for $0 \leq r \leq R$, the sign of $h(r)$ depends upon the choice of $h(0)$. If $h(0)$ is sufficiently large, then $h(r)>0$ for all $r$, i.e., the isopycnal does not "outcrop." There is a relationship between the location at which the steady state solution outcrops and the volume $V$ that is bounded between the isopycnal and the surface. If the isopycnal does not outcrop, then 


$$
V=2 \pi \int_{0}^{R} r h(r) d r=-\frac{70 \pi C R^{3}}{60}+h(0) \pi R^{2}
$$

where

$$
C=\left(\frac{30 \tau_{M}}{\rho_{0} f k}\right)^{\frac{1}{2}}
$$

Otherwise,

$$
V=2 \pi \int_{0}^{y_{0}} r h(r) d r=-\frac{\pi C y_{0}^{4}}{4 R}+\frac{2 \pi C y_{0}^{5}}{15 R^{2}}+\pi y_{0}^{2} h(0)
$$

where $r=y_{0}$ is the outcropping location (i.e., the location at which $h\left(y_{0}\right)=0$ ). Substituting $h(0)$ from Equation 27 into Equation 30 (with $h\left(y_{0}\right)=0$ ), it follows that

$$
\frac{V}{2 \pi C}=-\frac{1}{10 R^{2}} y_{0}^{5}+\frac{1}{8 R} y_{0}^{4}
$$

Let

$$
q\left(y_{0}\right):=-\frac{1}{10 R^{2}} y_{0}^{5}+\frac{1}{8 R} y_{0}^{4}-\frac{V}{2 \pi C} .
$$

Suppose that $0 \leq y_{0} \leq R$. Then

$$
q^{\prime}\left(y_{0}\right)=-\frac{1}{2 R^{2}} y_{0}^{4}+\frac{1}{2 R} y_{0}^{3}=\frac{y_{0}^{3}}{2 R}\left(1-\frac{y_{0}}{R}\right) \geq 0
$$

while

$$
q(0)=-\frac{V}{2 \pi C}<0 .
$$

Therefore, for the existence of a single root $0 \leq y_{0} \leq R$ of Equation 31, it is necessary and sufficient that

$$
q(R)=\frac{R^{3}}{40}-\frac{V}{2 \pi C} \geq 0
$$

or equivalently

$$
V \leq \frac{C \pi R^{3}}{20}
$$


Define

$$
V_{c}=\frac{C \pi R^{3}}{20}=\left(\frac{30 \tau_{M}}{\rho_{0} f k}\right)^{\frac{1}{2}} \frac{\pi R^{3}}{20} .
$$

Then $V_{c}$ represents the "critical volume" such that if $V<V_{c}$, then the isopycnal will outcrop; if $V>V_{c}$, then the isopycnal will not outcrop. If $V=V_{c}$, then outcropping will occur at $y_{0}=R$. A contour plot of $V_{c}$ as a function of the wind stress $\tau_{M}$ and eddy efficiency $k$ is given in Figure 6.

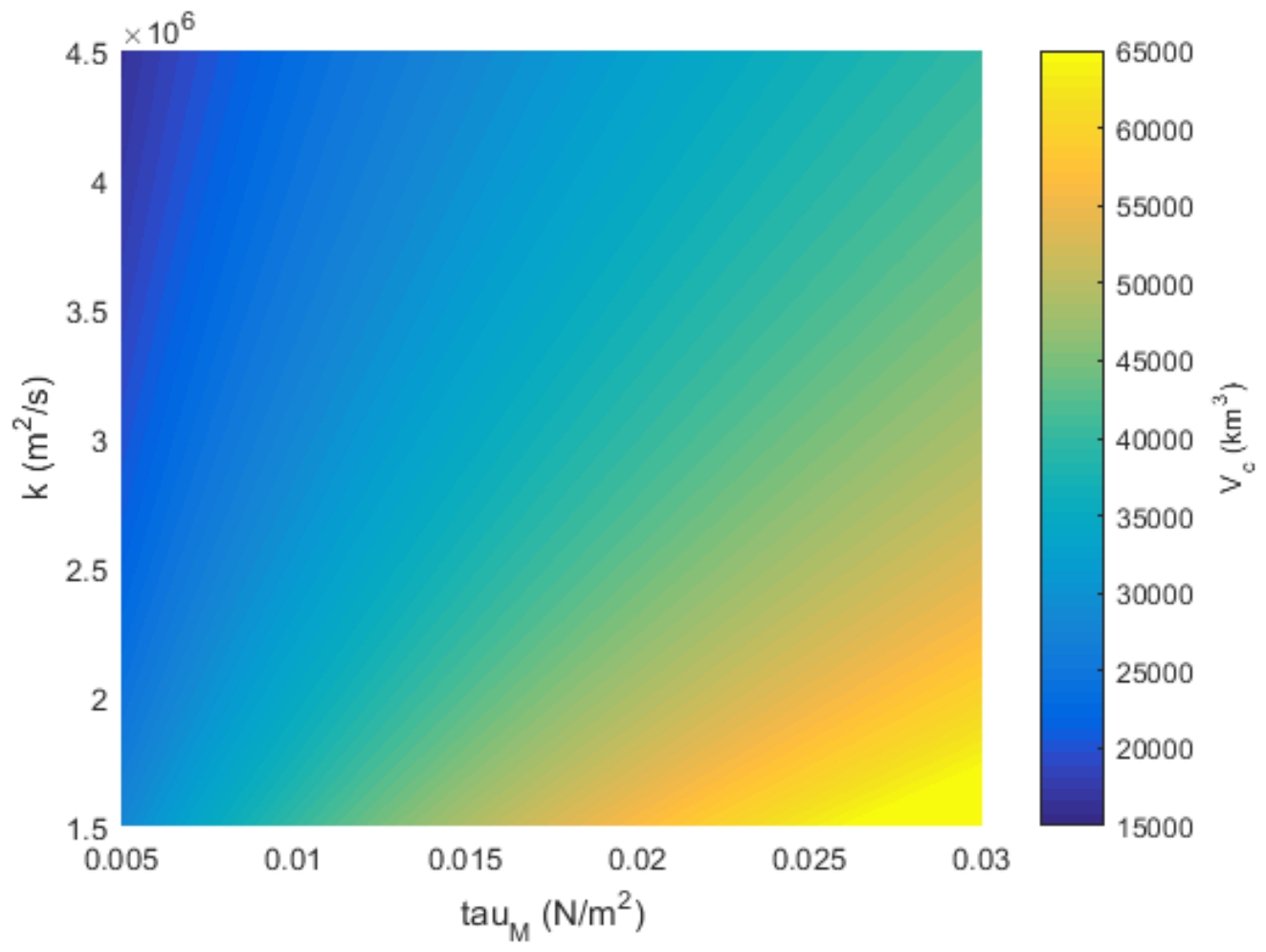

Figure 6: Plot of the critical volume $V_{c}$ defined in Equation 32 as a function of the mean surface wind stress $\tau_{M}$ and the eddy efficiency $k$. Reference values for the model are $\tau_{M}=0.015 \mathrm{~N} \mathrm{~m}^{-2}$ and $k=3 \cdot 10^{6} \mathrm{~m}^{2} \mathrm{~s}^{-1}$.

\subsection{Numerical solution of the nonlinear problem}

Consider the nonlinear problem

$$
h_{t}=\frac{1}{r}\left(r K h_{r}\right)_{r}+\frac{1}{r}\left(r \frac{-\tau}{\rho_{0} f}\right)_{r}
$$

with 


$$
K=k s=k\left|h_{r}\right|
$$

and

$$
\left.h_{r}\right|_{r=0, R}=0 .
$$

We assume that $\tau$ is a known function given by Equation 20. As previously noted, we wish to relax the assumption of a fixed isopycnal depth at the gyre boundary in [7]. Using these boundary conditions and the choice of $\tau$ according to Equation 20 (specifically, $\tau(0)=$ $\tau(R)=0$ ), it follows from Equation 33 that

$$
V_{t}=2 \pi \int_{0}^{R} r h_{t} d r=0
$$

i.e., the gyre volume is conserved. However, if $h(r, t)$ changes sign on $0 \leq r \leq R$, then the positive volume bounded between the isopycnal and the surface is not conserved. In addition, a constraint is needed to prevent $h<0$, which is physically unrealistic. Thus, a modification of Equation 33 is needed and we introduce a regularization factor $F$ given by

$$
F(h)=100 \exp (-10 h)+1 .
$$

The modified halocline depth evolution equation is given by

$$
h_{t}=\frac{1}{r}\left(r K F h_{r}\right)_{r}+\frac{1}{r}\left(r \frac{-\tau}{\rho_{0} f}\right)_{r} .
$$

Now if $h \leq 0$, then $F(h)$ is very large, i.e., the diffusion term is strong. However, if $h \geq 0$, then $F(h) \approx 1$, so that $F(h)$ does not modify the solution. An added benefit is that if $h \geq 0$ for all $r$ and $t$, then the positive volume bounded between the isopycnal and the surface will be conserved.

We implement a numerical scheme using the "pdepe" package in Matlab to solve Equation 37 to steady state $\left(\sim 3\right.$ years) for three initial volumes $\left(0.5 V_{c}, V_{c}\right.$, and $\left.1.5 V_{c}\right)$. The results are compared in Figure 7 with the theoretical steady state solution given by Equation 26 and reveal that the model closely approaches the theoretical steady state with minimal volume "loss" (i.e., negative signed volume).

In addition, we explore the steady state solution as a function of the wind stress and initial volume of the gyre (Figures 8 and 9). Strong mean surface wind stress deepens the halocline near the gyre interior and shoals it near the boundary (Figure 8). Figure 9 shows the steady state solution for the critical volume with $\tau_{M}=0.015 \mathrm{~N} \mathrm{~m}^{-2}$ (yellow curve) and for various fractions of the critical volume. For $\tau_{M}=0.015 \mathrm{~N} \mathrm{~m}^{-2}$, it turns out that $V_{c} \approx 34,600 \mathrm{~km}^{3}$. 


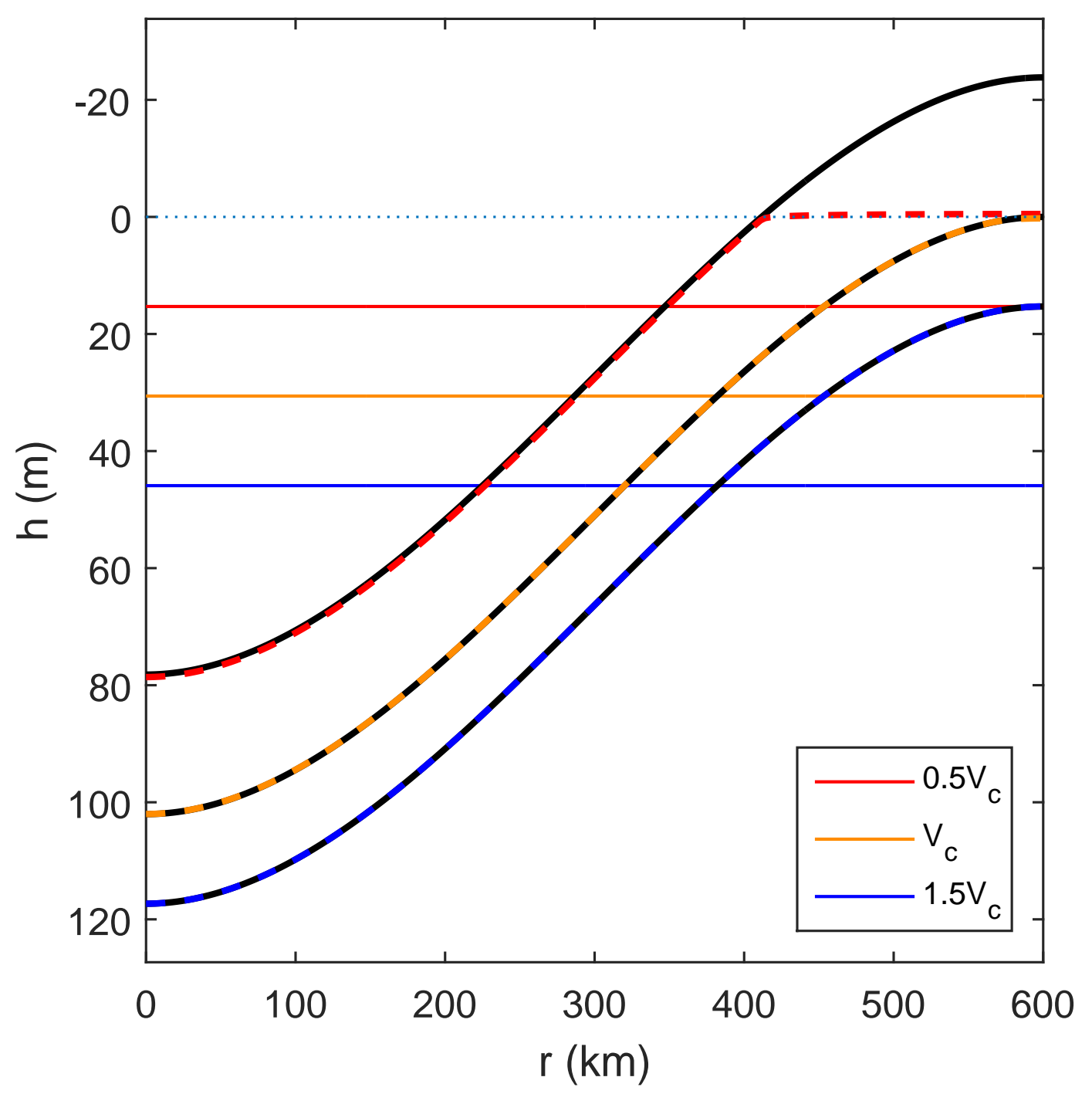

Figure 7: Initial (flat colored lines) and steady state (dashed colored lines) $h$ as obtained from Equation 37 with initial conditions chosen such that the initial volumes are $0.5 V_{c}, V_{c}$, and $1.5 V_{c}$; theoretical solution of Equation 26 for corresponding volumes (black curves). 


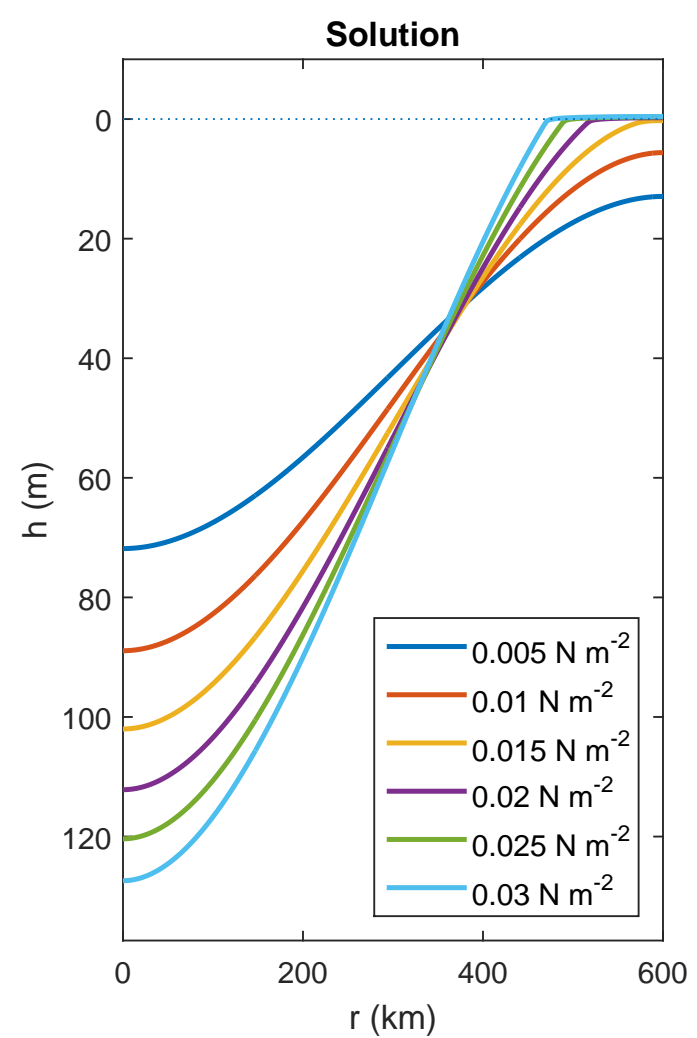

Figure 8: Steady state solutions of Equation 37 for varying choices of $\tau_{M}$. Each equation is initialized from a constant state $h_{0}(r)=V_{c} / \pi R^{2}$, where $V_{c}$ is the critical volume for $\tau_{M}=0.015 \mathrm{~N}$ $\mathrm{m}^{-2}$. (Thus, $V_{c}$ is the volume bounded between the yellow curve and the surface.)

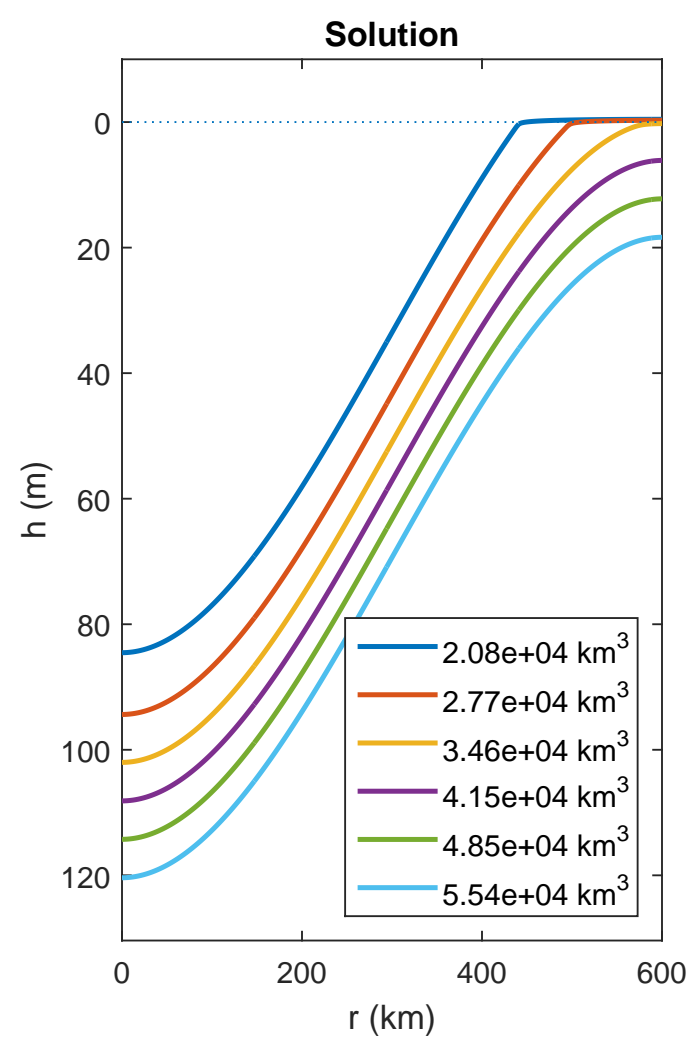

Figure 9: Steady state solutions of Equation 37 for varying initial volume $V$. Each equation is initialized from a constant state $h_{0}(r)=V / \pi R^{2}$. The yellow curve shows the solution for the critical volume $V_{c}$ with $\tau_{M}=0.015 \mathrm{~N} \mathrm{~m}^{-2}$. 


\section{Multi-layer Model}

Integrating Equation 33 with $h_{t}=0$ reveals that in steady state,

$$
h_{r}=\frac{\tau}{\rho_{0} f K}
$$

or with the assumption $K=k\left|h_{r}\right|$,

$$
h_{r}=-\left(-\frac{\tau}{\rho_{0} f k}\right)^{\frac{1}{2}} .
$$

In any case, in steady state, the slope $h_{r}$ is directly proportional to the surface wind stress and inversely proportional to the eddy diffusivity/eddy efficiency. However, Figure 3 reveals considerable vertical variation of the winter and summer mean halocline slope. Specifically, between about $\sim 50-200 \mathrm{~m}$, the isopycnal slope rapidly increases with depth. Evidently, the single-layer halocline model cannot capture this vertical variation.

In addition, estimates of the along-isopycnal eddy diffusivity have recently been derived from observations at four moorings in the Beaufort Gyre using the scaling law

$$
K \sim(E K E)^{\frac{1}{2}} \cdot l
$$

where $E K E$ represents the eddy kinetic energy and $l$ represents a mixing length scale [8]. Figure 11 reveals that the eddy diffusivity decreases with depth, which is not immediately consistent with the parameterization of the eddy diffusivity $K$ as directly proportional to the isopycnal slope $h_{r}$.

\subsection{Multi-layer uncoupled model with observed eddy diffusivity}

In order to attempt to capture the observed vertical variation of the halocline slope, we consider a multi-layer model in which each isopycnal has a depth evolution equation given by

$$
h_{i t}=\frac{1}{r}\left(r F K_{i} h_{i r}\right)_{r}+\frac{1}{r}\left(r \frac{-\tau}{\rho_{0} f}\right)_{r}
$$

for $1 \leq i \leq N$, where $N$ represents the number of interfaces. The boundary conditions for each interface are given by Equation 35 and the surface wind stress $\tau$ is given by Equation 20. Here $K_{i}$ is a constant with respect to $r$ and $t$. Note that in this model, the isopycnal depths evolve independently of each other (i.e., the interfaces are uncoupled).

As for the nonlinear single-layer halocline model, we solve Equation 39 numerically to steady state ( $\sim 10$ years), initializing each equation from a state of constant depth $z_{i}$ (Figure 10). Here $K_{i}$ is estimated from the mean diffusivity observed in the Beaufort Gyre at a sequence of depths $z_{i}=\{30,80,130,180,230,280\} \mathrm{m}$ (see Figure 11, red line). Since the observed diffusivity is noisy, we fit a cubic interpolant to a series of points from the observed 


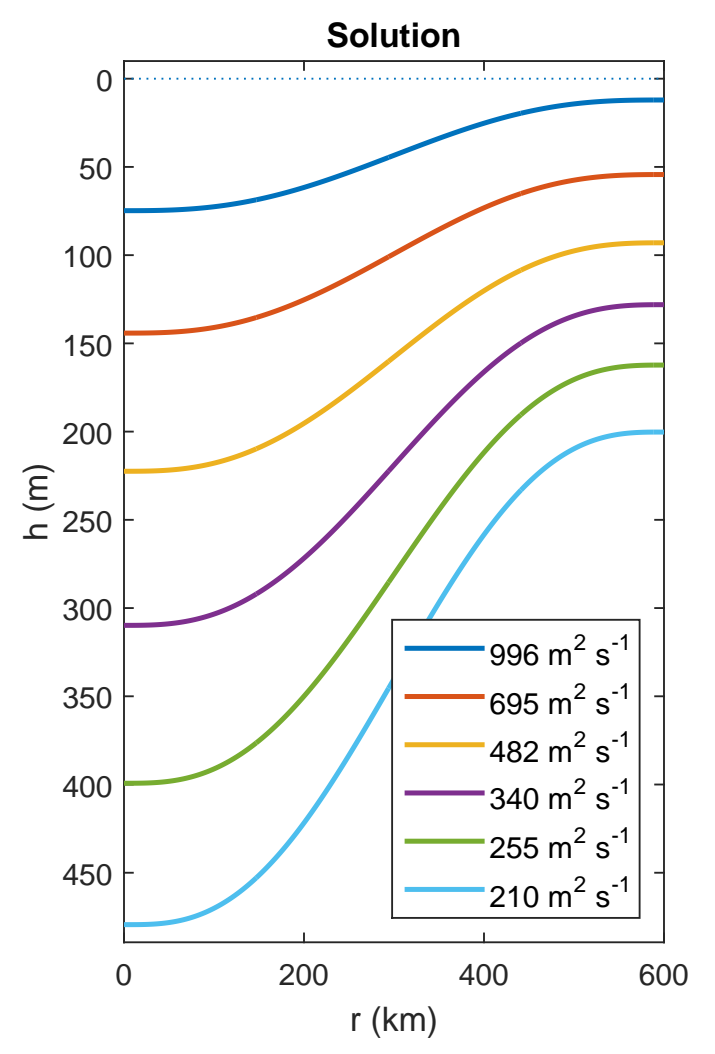

Figure 10: Steady state solutions of Equation 39 for each isopycnal interface with constant eddy diffusivity $K_{i}$ indicated in the legend.

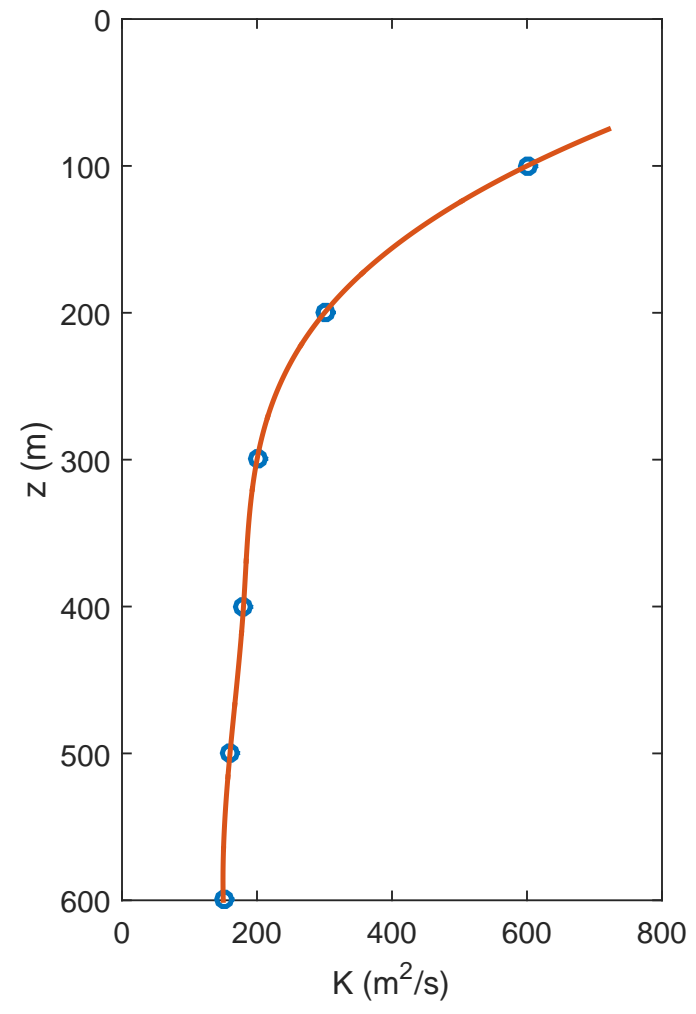

Figure 11: Vertical profile of the estimated along-isopycnal eddy diffusivity $\left(K_{\lambda}\right)$ in the Beaufort Gyre. Estimates from mean of four moorings in [8] (blue circles) and interpolated profile (red curve). 
data and use it to estimate $K_{i}$ for each depth $z_{i}$. The applied diffusivities and steady state layer depths are shown in Figure 10.

Thus, this simple model is able to capture the observed mean vertical structure of the halocline, and in particular, the increase of isopycnal slope with depth beneath the mixed layer (Figure 3, right column). However, the model does not explain what processes determine the observed vertical profile of the eddy diffusivity in the first place.

\subsection{Multi-layer coupled model}

By Equation 38, which was derived in the absence of boundary fluxes, it follows that a necessary condition for vertical variation of the isopycnal slope is vertical variation of the eddy diffusivity. Specifically, in steady state the ratio of the interface slopes satisfies

$$
\frac{H_{1 r}}{H_{2 r}}=\frac{K_{2}}{K_{1}} .
$$

(From now on, $h$ will denote a layer thickness and $H$ will denote the depth of a particular isopycnal interface, a distinction that will become relevant in the following derivation.)

A multi-layer coupled model should be capable of capturing the observed variation of the halocline slope and eddy diffusivity with depth. As has been demonstrated, the existing model with a given interface-dependent eddy diffusivity can capture the observed halocline slope in steady state. However, it cannot explain how the gyre equilibrated to the observed state with the observed vertical variation of eddy diffusivity. Baroclinic instability, and thus the eddy diffusivity at any point in time, should depend not only upon the local interface slope $H_{i r}$, but upon the stability characteristics of the entire water column. Therefore, we construct a multi-layer coupled model in which each interface depth $H_{i}$ evolves according to

$$
H_{i t}=\frac{1}{r}\left(r F K_{i} H_{i r}\right)_{r}+\frac{1}{r}\left(r \frac{-\tau}{\rho_{0} f}\right)_{r}
$$

for $i=\{1,2\}$. This equation is accompanied by the boundary condition

$$
\left.H_{i r}\right|_{r=0, R}=0,
$$

i.e., there is no flux of height (equivalently, volume) through the boundary. We consider a three-layer model, which represents a compromise between the need to accurately capture instability that arises from interactions between layers and the desire for simplicity and to keep computational demands low.

Equation 40 is solved through the iterative process illustrated in Figure 12. First, the time interval $t_{0}, \ldots t_{N}$ on which the solution is desired is subdivided into a number of equally-spaced segments $t_{j}, \ldots, t_{j+M}$. Beginning at time $t_{0}$, the initial layer slopes and densities are used to calculate the geostrophic velocities in the $\theta$-direction in each layer from the multi-layer shallow water equations. The resulting profile of the water column can be baroclinically unstable, i.e., the tilt of the isopycnals relative to the isobars can represent a 


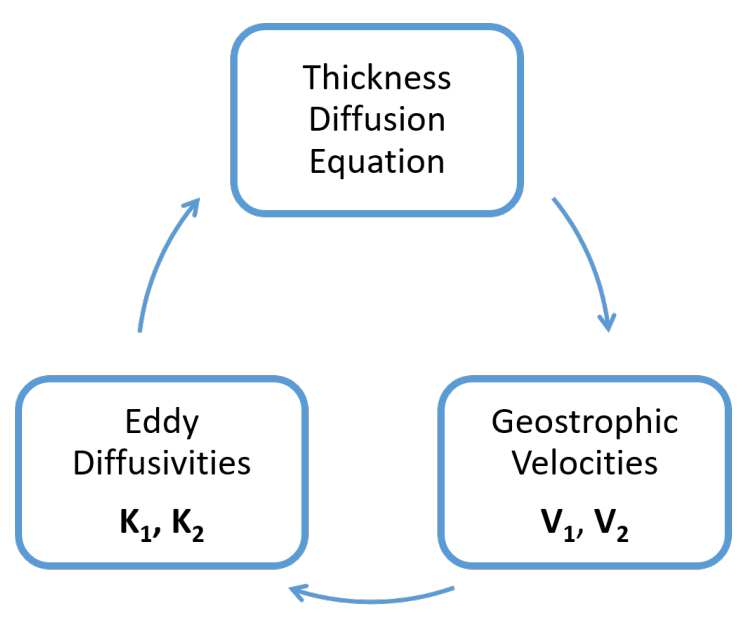

Figure 12: Process model of the solution method of the three-layer coupled model.

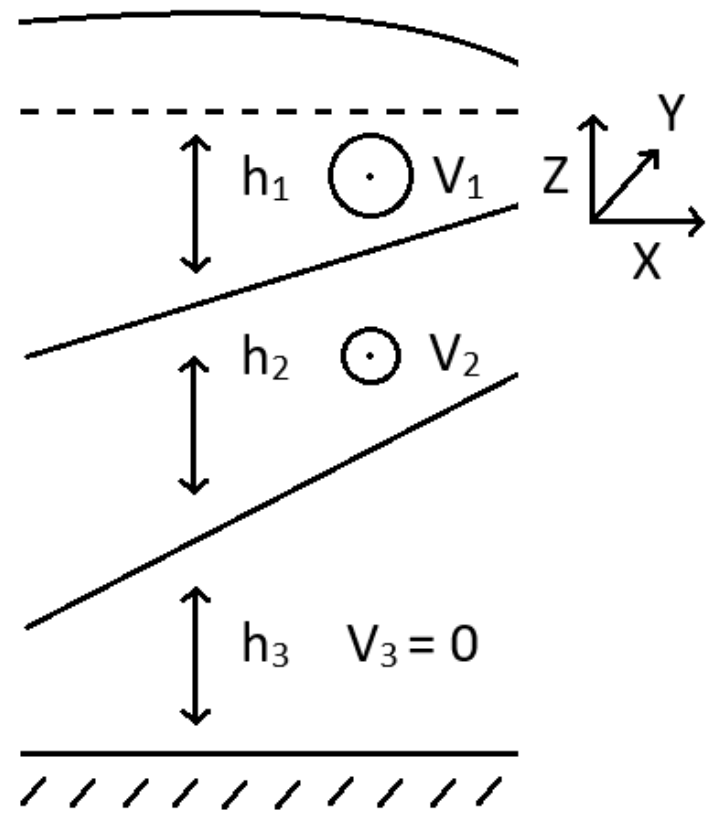

Figure 13: Schematic diagram of the layer thicknesses $h_{i}$ and layer geostrophic velocities $V_{i}$ in the three-layer model.

source of potential energy that is converted into kinetic energy as the baroclinic instability (eddy) grows. Therefore, a stability analysis is performed in the setting of the multi-layer shallow water quasi-geostrophic potential vorticity equations and the eddy diffusivity $K_{i}$ for each layer interface is estimated. Crucially, the eddy diffusivity depends upon the vertical profile of the isopycnal slope and geostrophic current such that Equation 40 represents a multi-layer coupled model in which the interfaces interact through the depth-varying eddy diffusivity $K_{i}$. The eddy diffusivities $K_{i}$ are then used to integrate Equation 40 forward to time $t_{M}$, and the process of obtaining the geostrophic velocities and eddy diffusivities is repeated for the next segment beginning with the model state at time $t_{M}$. Equation 40 is thus integrated forward until the solution is obtained on the entire interval $t_{0}, \ldots t_{N}$. Due to the computational demands of this algorithm, we currently calculate $K_{i}$ at a single spatial location and update the eddy diffusivity every $M$ timesteps. More detailed derivations of these calculations follow.

\subsubsection{Geostrophic velocities}

The geostrophic velocities are obtained from the interface slopes and densities in the setting of the multi-layer shallow water approximation via the geostrophic relations. Using the hydrostatic relation and the simplified geometry in Figure 13, it follows that the pressure $P_{i}$ in the $i$ th layer is given by 


$$
\begin{aligned}
& P_{1}=\rho_{1} g(\eta-z) \\
& P_{2}=\rho_{1} g \eta+\rho_{1} g h_{1}-\rho_{2} g\left(z+h_{1}\right) \\
& P_{3}=\rho_{1} g \eta+\rho_{1} g h_{1}+\rho_{2} g h_{2}-\rho_{3} g\left(z+h_{1}+h_{2}\right),
\end{aligned}
$$

where $\eta$ represents the sea surface height perturbation. Differentiating with respect to $r$ yields

$$
\begin{aligned}
& \frac{\partial P_{1}}{\partial r}=\rho_{1} g \frac{\partial \eta}{\partial r} \\
& \frac{\partial P_{2}}{\partial r}=\left(\rho_{2}-\rho_{1}\right) g \frac{\partial h_{1}}{\partial r}+\rho_{1} g \frac{\partial \eta}{\partial r} \\
& \frac{\partial P_{3}}{\partial r}=\left(\rho_{3}-\rho_{1}\right) g \frac{\partial h_{1}}{\partial r}+\left(\rho_{3}-\rho_{2}\right) g \frac{\partial h_{2}}{\partial r}+\rho_{1} g \frac{\partial \eta}{\partial r} .
\end{aligned}
$$

The system can be closed by imposing $V_{3}=0$, i.e., the assumption of no motion in the deep bottom layer. This then implies that

$$
\begin{aligned}
& V_{1}=\frac{g}{\rho_{0} f}\left[\left(\rho_{3}-\rho_{1}\right) \frac{\partial h_{1}}{\partial r}+\left(\rho_{3}-\rho_{2}\right) \frac{\partial h_{2}}{\partial r}\right] \\
& V_{2}=\frac{g}{\rho_{0} f}\left[\left(\rho_{3}-\rho_{2}\right) \frac{\partial h_{1}}{\partial r}+\left(\rho_{3}-\rho_{2}\right) \frac{\partial h_{2}}{\partial r}\right] .
\end{aligned}
$$

Now letting $\rho_{0}=\rho_{1}$ and defining the reduced gravity $g_{i}^{\prime}$ as

$$
g_{i}^{\prime}=\frac{\rho_{i+1}-\rho_{i}}{\rho_{1}} g
$$

it follows that

$$
\begin{aligned}
& V_{1}=\frac{1}{f}\left[\left(g_{2}^{\prime}+g_{1}^{\prime}\right) \frac{\partial h_{1}}{\partial r}+g_{2}^{\prime} \frac{\partial h_{2}}{\partial r}\right] \\
& V_{2}=\frac{1}{f}\left[g_{2}^{\prime} \frac{\partial h_{1}}{\partial r}+g_{2}^{\prime} \frac{\partial h_{2}}{\partial r}\right] .
\end{aligned}
$$

\subsection{Stability analysis}

The following theory is developed in [11]. Given the geostrophic velocities, we perform a stability analysis on the linearized multi-layer shallow water quasi-geostrophic potential vorticity equations. Given the shallow water approximation, in each layer the potential vorticity $Q_{i}$ satisfies

$$
\frac{D Q_{i}}{D t}=0, \quad Q_{i}=\frac{\zeta_{i}+f}{h_{i}}
$$


where $\zeta_{i}$ is the relative vorticity of the fluid, $f$ is the planetary vorticity, and $h_{i}$ is the layer thickness. In other words, the potential vorticity is a conserved quantity. Assuming that 1) variations in layer thickness are small relative to the total layer thickness; 2) the Rossby number is small; and 3) variations in the Coriolis parameter are small, then this equation can be linearized to give the so-called quasi-geostrophic potential vorticity in each layer,

$$
q_{i}=\left(\beta y+\zeta_{i}-f_{0} \frac{h_{i}^{\prime}}{H_{i}}\right)
$$

which is also conserved. Here $H_{i}$ represents the basic state layer thickness and $h_{i}^{\prime}$ represents a layer thickness perturbation. For simplicity, we assume that $\beta=0$.

The $N$-layer multi-layer shallow water equations may be obtained by introducing a streamfunction $\psi_{i}$ for each layer. Then the potential vorticity $q_{i}$ in each layer $1 \leq i \leq N$ can be expressed as

$$
\begin{aligned}
q_{1} & =\nabla^{2} \psi_{1}+\frac{f_{0}^{2}}{H_{1}}\left(\frac{\psi_{2}-\psi_{1}}{g_{1}^{\prime}}-\frac{f_{0}^{2}}{g H_{1}} \psi_{1}\right) \\
q_{i} & =\nabla^{2} \psi_{i}+\frac{f_{0}^{2}}{H_{i}}\left(\frac{\psi_{i-1}-\psi_{i}}{g_{i-1}^{\prime}}-\frac{\psi_{i}-\psi_{i+1}}{g_{i}^{\prime}}\right) \\
q_{N} & =\nabla^{2} \psi_{N}+\frac{f_{0}^{2}}{H_{N}}\left(\frac{\psi_{N-1}-\psi_{N}}{g_{N-1}^{\prime}}+\frac{f_{0}}{H_{n}} \eta_{b}\right),
\end{aligned}
$$

and evolves according to

$$
\frac{\partial q_{i}}{\partial t}+J\left(\psi_{i}, q_{i}\right)=0
$$

Here we assume that the bottom topography $\eta_{b}=0$. We now investigate this system for the presence of baroclinic instability by linearizing Equations 44-46 and 47 (with $N=3$ ) about a basic state layer potential vorticity $Q_{i}$, velocity $V_{i}$ and streamfunction $\Psi_{i}$. Here $V_{i}$ is constant in each layer and represents the velocity in the $y$-direction (see Figure 13). Then Equations 44-46 reduce to

$$
\left[\begin{array}{l}
q_{1}^{\prime} \\
q_{2}^{\prime} \\
q_{3}^{\prime}
\end{array}\right]=\left[\begin{array}{c}
\nabla^{2} \psi_{1}^{\prime} \\
\nabla^{2} \psi_{2}^{\prime} \\
\nabla^{2} \psi_{3}^{\prime}
\end{array}\right]+f_{0}^{2}\left[\begin{array}{ccc}
-\frac{1}{g H_{1}}-\frac{1}{g_{1}^{\prime} H_{1}} & \frac{1}{g_{1}^{\prime} H_{1}} & 0 \\
\frac{1}{g_{1}^{\prime} H_{2}} & -\frac{1}{g_{1}^{\prime} H_{2}}-\frac{1}{g_{2}^{\prime} H_{2}} & \frac{1}{g_{2}^{\prime} H_{2}} \\
0 & \frac{1}{g_{2}^{\prime} H_{3}} & -\frac{1}{g_{2}^{\prime} H_{3}}
\end{array}\right]\left[\begin{array}{c}
\psi_{1}^{\prime} \\
\psi_{2}^{\prime} \\
\psi_{3}^{\prime}
\end{array}\right]
$$

Separating the terms in Equation 47 into sums of basic state variable and perturbations (indicated by primes) yields

$$
\begin{aligned}
\frac{\partial q_{i}^{\prime}}{\partial t}+\frac{\partial Q_{i}}{\partial t} & +\frac{\partial \psi_{i}^{\prime}}{\partial x} \frac{\partial q_{i}^{\prime}}{\partial y}+\frac{\partial \psi_{i}^{\prime}}{\partial x} \frac{\partial Q_{i}}{\partial y}+\frac{\partial \Psi_{i}}{\partial x} \frac{\partial q_{i}^{\prime}}{\partial y}+\frac{\partial \Psi_{i}}{\partial x} \frac{\partial Q_{i}}{\partial y} \\
& -\frac{\partial \psi_{i}^{\prime}}{\partial y} \frac{\partial Q_{i}}{\partial x}-\frac{\partial \psi_{i}^{\prime}}{\partial y} \frac{\partial q_{i}^{\prime}}{\partial x}-\frac{\partial \Psi_{i}}{\partial y} \frac{\partial Q_{i}}{\partial x}-\frac{\partial \Psi_{i}}{\partial y} \frac{\partial q_{i}^{\prime}}{\partial x}=0
\end{aligned}
$$


Subtracting the equation satisfied by the basic state variables and assuming that products of perturbations are small, the linearized potential vorticity evolution equation reduces to

$$
\frac{\partial q_{i}^{\prime}}{\partial t}+\frac{\partial \psi_{i}^{\prime}}{\partial x} \frac{\partial Q_{i}}{\partial y}+\frac{\partial \Psi_{i}}{\partial x} \frac{\partial q_{i}^{\prime}}{\partial y}-\frac{\partial \psi_{i}^{\prime}}{\partial y} \frac{\partial Q_{i}}{\partial x}-\frac{\partial \Psi_{i}}{\partial y} \frac{\partial q_{i}^{\prime}}{\partial x}=0
$$

Now by assumption, there are no gradients of basic state variables in the $y$-direction, so this equation further reduces to

$$
\frac{\partial q_{i}^{\prime}}{\partial t}+V_{i} \frac{\partial q_{i}^{\prime}}{\partial y}+u_{i}^{\prime} \frac{\partial Q_{i}}{\partial x}=0
$$

after making use of the streamfunction relations. Here

$$
\left[\begin{array}{l}
\frac{\partial Q_{1}}{\partial x} \\
\frac{\partial Q_{2}}{\partial x} \\
\frac{\partial Q_{3}}{\partial x}
\end{array}\right]=f_{0}^{2}\left[\begin{array}{ccc}
-\frac{1}{g H_{1}}-\frac{1}{g_{1}^{\prime} H_{1}} & \frac{1}{g_{1}^{\prime} H_{1}} & 0 \\
\frac{1}{g_{1}^{\prime} H_{2}} & -\frac{1}{g_{1}^{\prime} H_{2}}-\frac{1}{g_{2}^{\prime} H_{2}} & \frac{1}{g_{2}^{\prime} H_{2}} \\
0 & \frac{1}{g_{2}^{\prime} H_{3}} & -\frac{1}{g_{2}^{\prime} H_{3}}
\end{array}\right]\left[\begin{array}{l}
V_{1} \\
V_{2} \\
V_{3}
\end{array}\right]
$$

Now define

$$
L_{z}:=f_{0}^{2}\left[\begin{array}{ccc}
-\frac{1}{g H_{1}}-\frac{1}{g_{1}^{\prime} H_{1}} & \frac{1}{g_{1}^{\prime} H_{1}} & 0 \\
\frac{1}{g_{1}^{\prime} H_{2}} & -\frac{1}{g_{1}^{\prime} H_{2}}-\frac{1}{g_{2}^{\prime} H_{2}} & \frac{1}{g_{2}^{\prime} H_{2}} \\
0 & \frac{1}{g_{2}^{\prime} H_{3}} & -\frac{1}{g_{2}^{\prime} H_{3}}
\end{array}\right] .
$$

We search for potential vorticity perturbations with a wave-like structure in the $y$-direction that are growing in time. Specifically, assuming the ansatz

$$
q_{i}^{\prime}=\Re\left[\tilde{q}_{i} e^{i k(y-c t)}\right], \quad{ }_{i}^{\prime}=\Re\left[\tilde{\psi}_{i} e^{i k(y-c t)}\right],
$$

and substituting Equations 50 and 51 into Equation 49 yields

$$
V_{i} \tilde{q}_{i}-\tilde{\psi}_{i} \frac{\partial Q_{i}}{\partial x}=c \tilde{q}_{i}
$$

where, by Equation 48,

$$
\tilde{q}_{i}=\left(L_{z}-k^{2} I\right) \tilde{\psi}_{i}
$$

Therefore, Equation 52 is equivalent to a matrix eigenvalue problem that can be solved numerically for $c$ and $\tilde{q}_{i}$. For every wavenumber $k$, there are three eigenvalues $c_{1}, c_{2}, c_{3}$ and corresponding eigenvectors $\tilde{q_{i 1}}, \tilde{q_{i 2}}, \tilde{q_{i 3}}$ that solve the system. By Equation 51, the fastestgrowing perturbation is represented by the eigenvector whose corresponding eigenvalue has 
maximum imaginary part. Therefore, we search numerically over a range of wavenumbers spanning the baroclinic deformation radii for the maximum baroclinic instability growth rate given by

$$
\lambda=\max _{k} k \Im[c]
$$

and therefore

$$
\begin{aligned}
q_{i}^{\prime} & =e^{\lambda t}\left(\Re\left[\tilde{q}_{i}\right] \cos (k y)+i \Im\left[\tilde{q}_{i}\right] \sin (k y)\right) \\
u_{i}^{\prime} & =e^{\lambda t}\left(\Re\left[\tilde{u}_{i}\right] \cos (k y)+i \Im\left[\tilde{u}_{i}\right] \sin (k y)\right)
\end{aligned}
$$

and

$$
\begin{aligned}
q_{i}^{\prime} u_{i}^{\prime} & =e^{2 \lambda t}\left(\Re\left[\tilde{q}_{i}\right] \Re\left[\tilde{u}_{i}\right] \cos ^{2}(k y)+\Im\left[\tilde{q}_{i}\right] \Im\left[\tilde{u}_{i}\right] \sin ^{2}(k y)\right. \\
& \left.-\Re\left[\tilde{q}_{i}\right] \Im\left[\tilde{u}_{i}\right] \sin (k y) \cos (k y)-\Im\left[\tilde{q}_{i}\right] \Re\left[\tilde{u}_{i}\right] \sin (k y) \cos (k y)\right) .
\end{aligned}
$$

Averaging meridionally,

$$
\overline{q_{i}^{\prime} u_{i}^{\prime}} \sim\left(\Re\left[\tilde{q}_{i}\right] \Re\left[\tilde{u}_{i}\right]+\Im\left[\tilde{q}_{i}\right] \Im\left[\tilde{u}_{i}\right]\right) .
$$

Now the layer eddy diffusivity $\kappa_{i}$ that we seek is given by

$$
\kappa_{i}=-\frac{\overline{q_{i}^{\prime} u_{i}^{\prime}}}{Q_{i x}}
$$

However, $\overline{q_{i}^{\prime} u_{i}^{\prime}}$ is known only up to a constant factor. Therefore, we choose the parameterization

$$
\hat{\kappa}_{i}=-\frac{\Re\left[\tilde{q}_{i}\right] \Re\left[\tilde{u}_{i}\right]+\Im\left[\tilde{q}_{i}\right] \Im\left[\tilde{u}_{i}\right]}{Q_{i x}}, \quad \kappa_{i}=k_{c} \lambda \frac{\hat{\kappa}_{i}}{\max _{i} \hat{\kappa}_{i}}
$$

where $k_{c}=10^{9}$ is chosen such that $k_{i}$ has the observed order of magnitude for eddy diffusivity in the Beaufort Gyre halocline (i.e., $100-1000 \mathrm{~m}^{2} \mathrm{~s}^{-1}$ ). Thus, $\kappa_{i}$ is proportional to the baroclinic instability growth rate $\lambda$ and comparing $\hat{\kappa}_{i} / \hat{\kappa}_{j}$ for $i \neq j$ reflects the ratio of the eddy diffusivities between the layers.

Notably, $\kappa_{i}$ represents the eddy diffusivity of the $i$-th layer, rather than the $i$-th interface. However, the geometry of the three-layer model (Figure 13) suggests that $K_{1}=\kappa_{1}$ and $K_{2}=\kappa_{3}$, where $K_{i}$ represents the eddy diffusivity coefficient for the $i$-th interface. 


\subsection{Results}

Using this algorithm, we investigate the solution of Equation 40 in steady state. Specifically, we initialize the model from numerous configurations of initial isopycnal slopes (Figure 15, red dots) and integrate forward until the model reaches steady state (typically $\sim 5$ years). In all cases, the model evolves to a steady state in which the isopycnals are approximately parallel (Figure 15, black dots), i.e., $S_{1} \approx S_{2}$ and $K_{1} \approx K_{2}$. Figure 14 illustrates the evolution to steady state for a representative case.

Figure 15 reveals that there are two configurations for the vertical profile of the eddy diffusivity; these configurations correspond to the two baroclinic modes in the three-layer problem (Figure 16). A sign change of the basic state potential vorticity gradient between layers is a necessary condition for baroclinic instability. If this sign change occurs between the second and third layers, then the baroclinic instability can be dominated by the first baroclinic mode and $K_{1} \approx K_{2}$. On the other hand, if the sign change occurs between the first and second layers, then the instability can be dominated by the second baroclinic mode and $K_{2}<<K_{1}$.

Regardless of the initial conditions, the model evolves to a steady state in which $S_{1} \approx S_{2}$, i.e., the line $S_{1}=S_{2}$ is an attractor (Figure 15). The magnitude of the final slopes is determined by the wind stress $\tau$.

However, the observed winter and summer mean isopycnal slopes in the Beaufort Gyre are depth-dependent (Figure 3, right column). In order to obtain depth-dependent isopycnal slopes in steady state, it is necessary to introduce fluxes of potential vorticity (equivalently, volume) at the gyre boundary. The following is a simplified proof-of-concept that is solved analytically.

Suppose that a flux of volume $Q$ enters the second layer only, and that no flux enters the first layer, through the gyre boundary. Specifically, the boundary conditions for the second layer thickness are

$$
\left.h_{2 r}\right|_{r=0}=0,\left.\quad K h_{2 r}\right|_{r=R}=\frac{Q}{2 \pi R} .
$$

In order to preserve the gyre volume, it is necessary to remove an equivalent volume from the gyre interior, distributed across the entire gyre. Specifically, the thickness evolution equation is given by

$$
h_{2 t}=\frac{1}{r}\left(r K h_{2 r}\right)_{r}-\frac{Q}{\pi R^{2}} .
$$

(Here we are assuming that $K=K_{1}=K_{2}$.) This equation satisfies

$$
2 \pi \int_{0}^{R} r h_{2 t}=0
$$

i.e., the volume bounded between the first and second interfaces is constant with respect to time. The steady-state solution of this equation is given by 


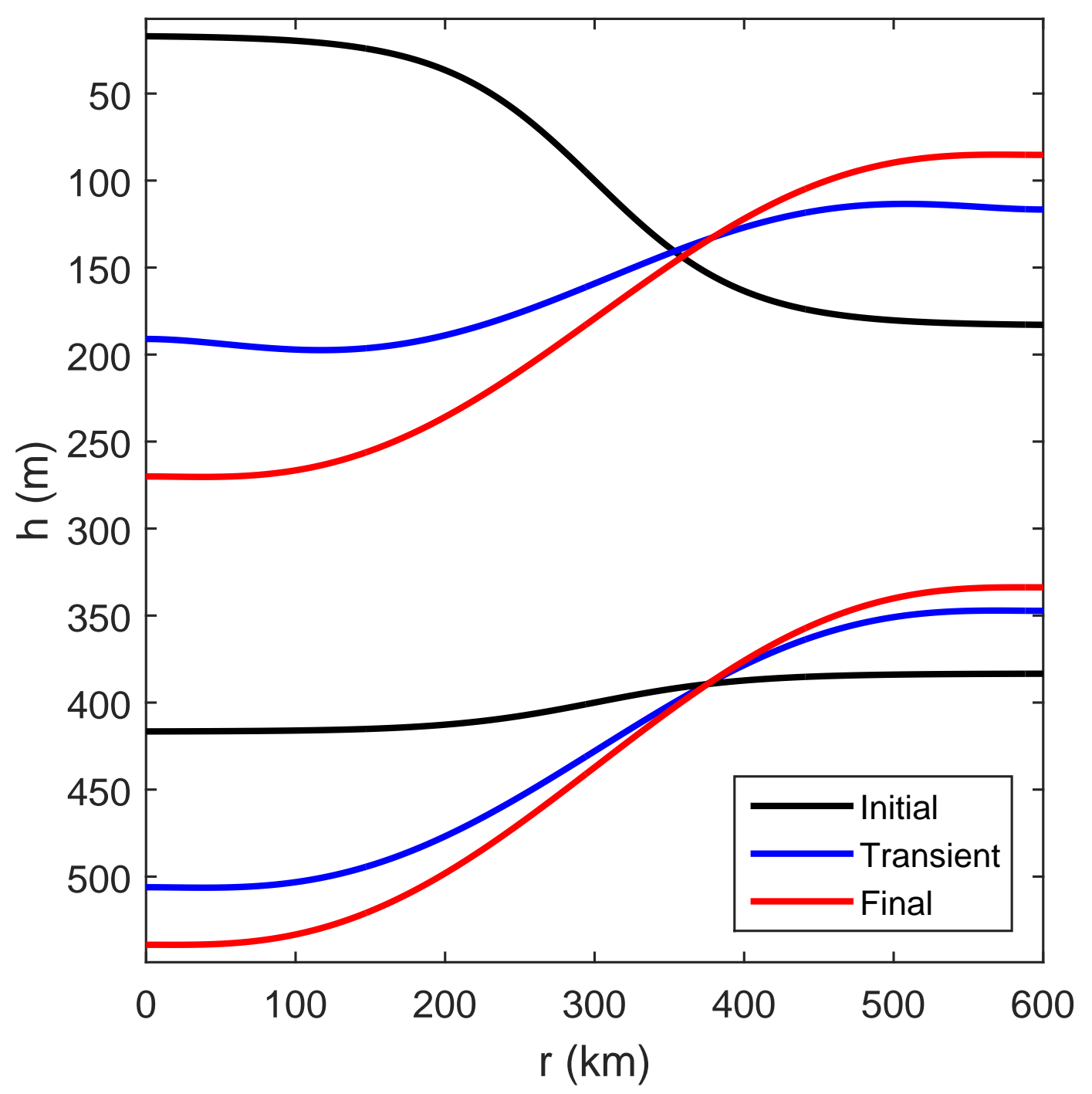

Figure 14: Example of the evolution of the halocline depth in the multi-layer model to steady state (red curves; $\sim 5$ years) from the indicated initial state (black curves). The model state after $\sim 2.5$ years is also indicated (blue curves). 


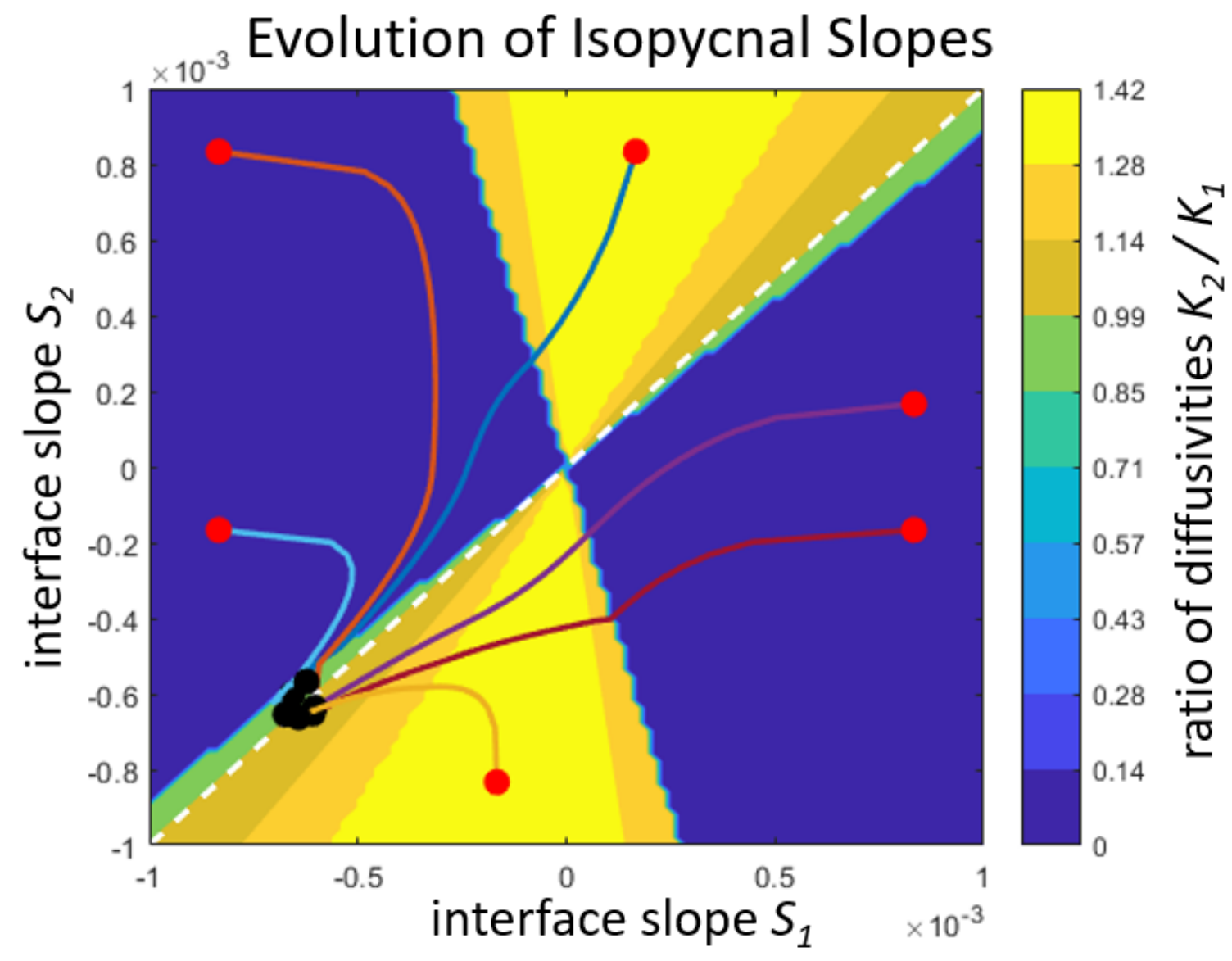

Figure 15: Ratio of diffusivities $K_{2}$ and $K_{1}$ as a function of the isopycnal slopes $S_{1}$ and $S_{2}$ (colormap). Slope trajectories from the three-layer coupled model (colored lines) for various choices of the initial slopes (red dots), and resulting steady-state slopes (black dots). The gyre attracts to a steady state of parallel slopes (i.e., $S_{1}=S_{2}$; dashed white line). The wind stress $\tau_{M}$ determines the magnitude of the final slopes. 
$K_{1} \approx K_{2}$

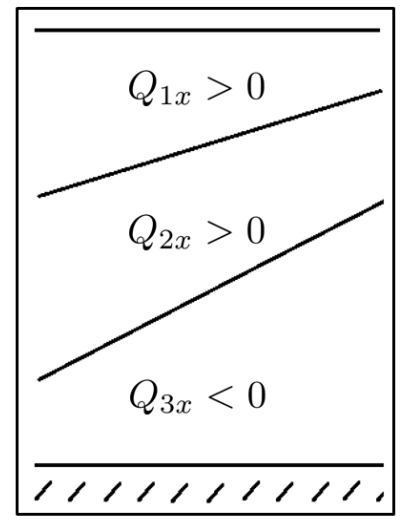

Wavenumber vs. Instability Max Growth Rate

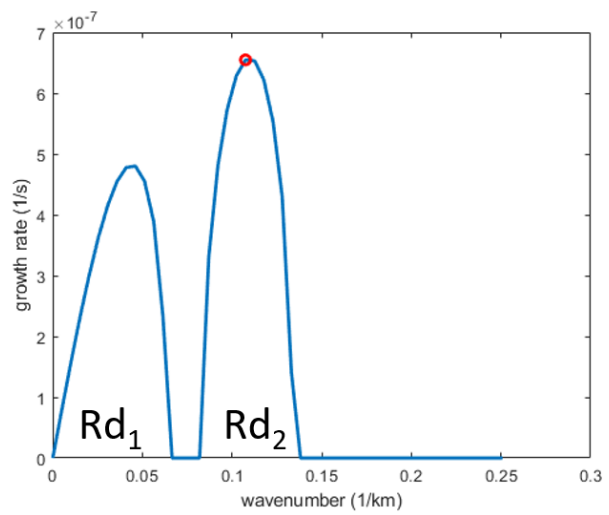

$K_{1}>>K_{2}$

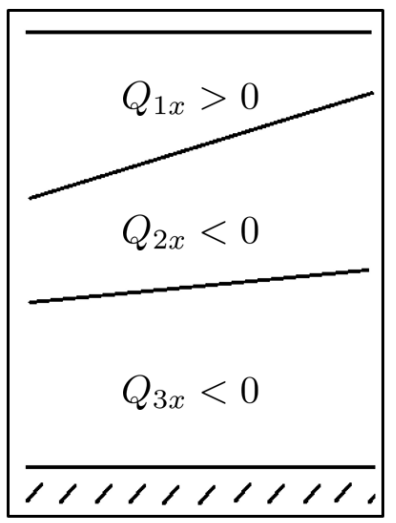

Figure 16: (Center) Example of maximum growth rate $k c$ for various wavenumbers $k$ and wavenumber corresponding to the maximum growth rate (red circle). In this case the instability is dominated by the second baroclinic mode. Sign change of PV gradient $Q_{i x}$ is a necessary condition for baroclinic instability. (Left) Schematic diagram of water column profile and eddy diffusivities when the instability is dominated by the first baroclinic mode. (Right) Schematic diagram of water column profile and eddy diffusivities when the instability is dominated by the second baroclinic mode.

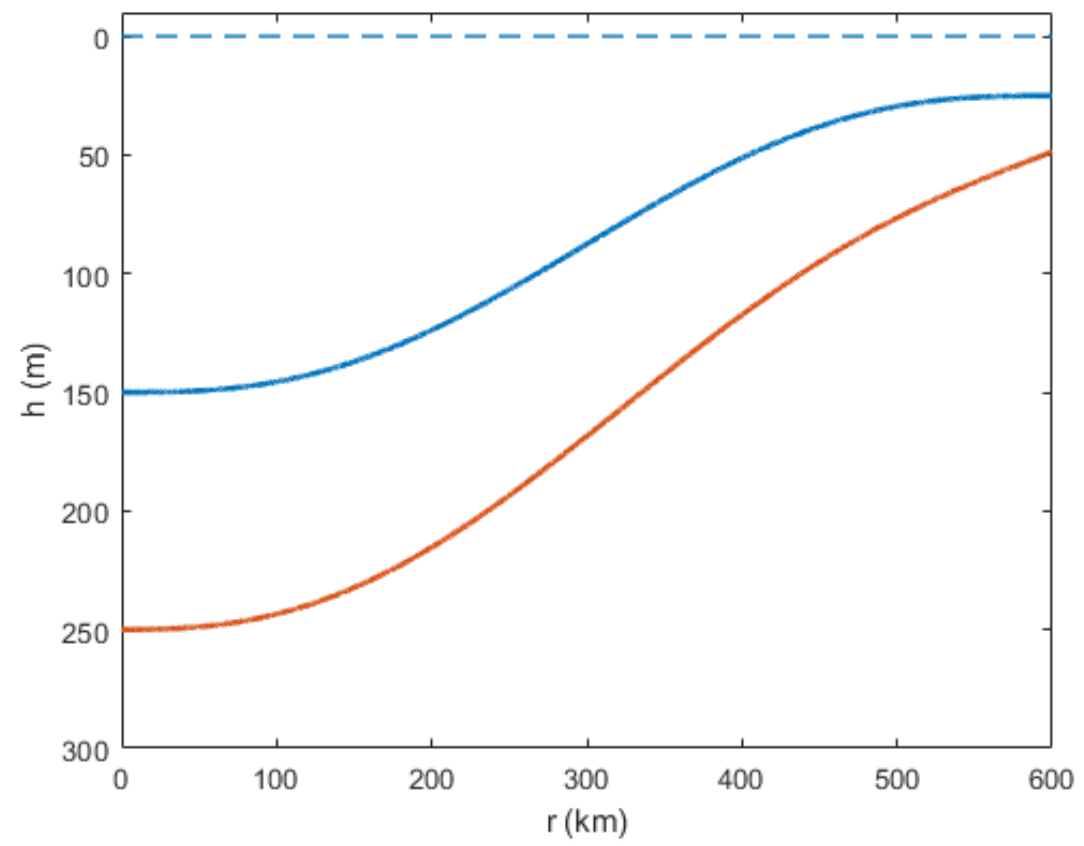

Figure 17: Solution of Equations 55 and 56 with $h_{1}(0)=150 \mathrm{~m}, h_{2}(0)=100 \mathrm{~m}$, and $Q=-15,000$ $\mathrm{km}^{3} \mathrm{yr}^{-1}$. 


$$
h_{2}(r)=h_{2}(0)+\frac{Q r^{2}}{4 \pi R^{2} K} .
$$

As before, the evolution equation for the first layer depth is given by

$$
h_{1 t}=\frac{1}{r}\left(r K h_{1 r}\right)_{r}+\frac{1}{r}\left(r \frac{-\tau}{\rho_{0} f}\right)_{r}
$$

with boundary conditions

$$
\left.h_{1 r}\right|_{r=0, R}=0 .
$$

In steady state, the solution of this equation is given by

$$
h_{1}(r)=h_{1}(0)-\frac{30 \tau_{M}}{\rho_{0} f K}\left(\frac{r^{3}}{3 R^{2}}-\frac{r^{4}}{2 R^{3}}+\frac{r^{5}}{5 R^{4}}\right) .
$$

The solution of Equations 55 and 56 is shown in Figure 17 with some representative choices of the unspecified parameters $\left(h_{1}(0)=150 \mathrm{~m}, h_{2}(0)=100 \mathrm{~m}, Q=-15,000 \mathrm{~km}^{3} \mathrm{yr}^{-1}\right.$. Here the boundary flux is exaggerated to show effect.) It can be seen that the isopycnal slope increases with depth.

\section{Conclusions}

Current adiabatic models of the Beaufort Gyre halocline represent it as a buoyant interface with a depth that is that is increased by Ekman pumping; the deepening is opposed by mesoscale eddy transport. In steady state, the isopycnal slope is predicted to be proportional to the strength of the surface wind stress and inversely proportional to the eddy diffusivity. Eddy diffusivity is often parameterized to be proportional to isopycnal slope, yet observations from the PHC climatology suggest that the isopycnal slope increases with depth, while mooring-derived along-isopycnal eddy diffusivity decreases with depth. This suggests that the current theory should be reconsidered.

First, we have improved the single-layer halocline model from [7]. Specifically, seasonal outcropping of isopycnals is observed in the Beaufort Gyre, a phenomenon that the modified single-layer model can now capture. In this setting, we have also derived an expression for a so-called "critical volume" $V_{c}$ that depends upon the wind stress and eddy diffusivity. The volume of the gyre in relation to the critical volume determines whether the halocline outcrops.

In addition, we have developed a multi-layer model in which the isopycnal interfaces are coupled with each other through the depth-dependent eddy diffusivity. The strength and vertical profile of the eddy diffusivity is determined from the baroclinic instability characteristics of the geostrophic currents, which are derived from the isopycnal slope via thermal wind balance. Using this simple model of eddy-mean flow interactions, we have identified 
the critical processes that determine the vertical structure of the halocline. Specifically, we have shown that potential vorticity sources at the gyre boundary (over continental slopes) are key to setting up a realistic depth-varying distribution of the isopycnal slope in steady state. In the absence of the boundary fluxes, the gyre attracts to a steady state with depthindependent isopycnal slope, regardless of the initial conditions. These findings further justify the need for observational constraints on boundary fluxes and eddy diffusivity.

\section{Acknowledgements}

I am grateful to have taken part in the WHOI GFD program and particularly appreciate the mentorship of Renske Gelderloos and Georgy Manucharyan. I also thank the program directors, Claudia Cenedese and Mary-Louise Timmermans, as well as the numerous scientists with whom we had productive conversations about the project: Sam Pegler, Joe Pedlosky, Jack Whitehead, Mary-Louise Timmermans, Glenn Flierl and others.

\section{References}

[1] D. Andrews And M. E. McIntyre, Planetary waves in horizontal and vertical shear: The generalized Eliassen-Palm relation and the mean zonal acceleration, Journal of the Atmospheric Sciences, 33 (1976), pp. 2031-2048.

[2] P. R. Gent and J. C. Mcwilliams, Isopycnal mixing in ocean circulation models, Journal of Physical Oceanography, 20 (1990), pp. 150-155.

[3] K. A. Giles, S. W. Laxon, A. L. Ridout, D. J. Wingham, and S. Bacon, Western arctic ocean freshwater storage increased by wind-driven spin-up of the Beaufort Gyre, Nature Geoscience, 5 (2012), pp. 194-197.

[4] T. W. Haine, B. Curry, R. Gerdes, E. Hansen, M. Karcher, C. Lee, B. Rudels, G. Spreen, L. De Steur, K. D. Stewart, et Al., Arctic freshwater export: Status, mechanisms, and prospects, Global and Planetary Change, 125 (2015), pp. 13-35.

[5] IOC, SCOR, AND IAPSO, The international thermodynamic equation of seawater 2010: Calculation and use of thermodynamic properties., 2010. 196 pp., Intergovernmental Oceanographic Commission, Manuals and Guides No. 56, UNESCO (English).

[6] G. E. Manucharyan And M. A. Spall, Wind-driven freshwater buildup and release in the Beaufort Gyre constrained by mesoscale eddies, Geophysical Research Letters, 43 (2016), pp. 273-282.

[7] G. E. Manucharyan, M. A. Spall, and A. F. Thompson, A theory of the wind-driven Beaufort Gyre variability, Journal of Physical Oceanography, 46 (2016), pp. 3263-3278.

[8] G. Meneghello, J. Marshall, S. T. Cole, and M.-L. Timmermans, Observational inferences of lateral eddy diffusivity in the halocline of the Beaufort Gyre. (manuscript in review), 2017. 
[9] A. Proshutinsky, R. Bourke, and F. McLaughlin, The role of the Beaufort Gyre in Arctic climate variability: Seasonal to decadal climate scales, Geophysical Research Letters, 29 (2002).

[10] M.-L. Timmermans, J. Marshall, A. Proshutinsky, and J. Scott, Seasonally derived components of the Canada Basin halocline, Geophysical Research Letters, 44 (2017), pp. 5008-5015.

[11] G. K. VAllis, Atmospheric and oceanic fluid dynamics, Cambridge University Press, 2017.

[12] M. Visbeck, J. Marshall, T. Haine, and M. Spall, Specification of eddy transfer coefficients in coarse-resolution ocean circulation models, Journal of Physical Oceanography, 27 (1997), pp. 381-402. 


\title{
Investigating the Effect of Iceberg Aspect Ratio on Submarine Melting
}

\author{
Eric William Hester
}

May 10, 2018

\begin{abstract}
Icebergs play many roles in the dynamics of polar climates. Their geographical, and geometric, distributions have important consequences for shipping, polar ecosystems, and ice sheet-ocean modelling. However, current parameterizations of iceberg deterioration largely ignore their geometry. We examine the influence of aspect ratio and ambient relative velocity on iceberg melting in a series of novel experiments. We find that aspect ratio is an important control on iceberg melting, with lateral melt rates typically exceeding basal melt rates. The standard parameterizations of Weeks and Campbell [37] and the Jenkins three equation model [16] could not reproduce this geometry dependent melting, and underestimate the melt rate. We emphasise that $f$ urther investigation into the influence of geometry on melting is necessary.
\end{abstract}

\section{An introduction to icebergs}

Icebergs are generated by calving at the margins of ice shelves and glaciers, and constitute a large component of the freshwater output from ice sheets, making up $45 \%$ of Antarctic freshwater loss [27].

The most obvious impact of icebergs is on shipping. In the aftermath of the sinking of the Titanic, the International Ice Patrol was set up to monitor iceberg locations in the North Atlantic [29]. With projected increases in Arctic shipping, it is crucial to understand the expected distributions of icebergs. Icebergs also have important impacts on ecosystems; nutrient release during melting can boost biological productivity in an area up to 10 times their actual size [33], while bed scouring can have devastating effects on seabed biology [8]. At the largest scales, icebergs can be a dominant component of the freshwater flux from land ice to the ocean in the Greenland and Antarctic ice shelves [11, 23, 31]. As such, understanding their subsequent evolution is key to modelling the interaction between ice sheets and the ocean.

A central factor in modelling icebergs is their size, which determines iceberg evolution and geographical distribution [31]. Many studies have examined iceberg size distribution, in both Greenland [5, 35] and Antarctica [2, 9, 34, 36]. Enormous variation in sizes are observed, from the smallest growlers at several meters wide, to iceberg B-15, the largest iceberg ever recorded, at $300 \mathrm{~km} \times 40 \mathrm{~km}$. Importantly, there is a large range of iceberg aspect ratios observed in nature, where the aspect ratio is defined as the ratio of iceberg length $L$ to submerged iceberg depth $H$. Yet there has been very little investigation on the impact of aspect ratio on iceberg melting - typically being ignored in melting parameterizations.

The current treatment of aspect ratio focusses on its impact on iceberg stability $[4,38]$. Below a certain length $L$ to total depth $D$ ratio, the iceberg becomes unstable, overturning when 


$$
\frac{L}{D}<\sqrt{0.92+\frac{58.32 \mathrm{~m}}{D}}
$$

\subsection{Our investigation}

The goal of this summer project is to investigate specifically the effect of aspect ratio on iceberg melting in a series of novel laboratory experiments.

We compare our experimental results with two common melting parameterizations, and suggest directions for future investigation, with the ultimate goal to develop improved parameterizations of iceberg deterioration which take into account their geometry and aspect ratio.

\section{Models of iceberg melt}

The problem of modelling ice melting has been seriously considered for well over one and a half centuries. The formalisation of the moving boundary interface condition was first established by Josef Stefan in his 1889 paper (as cited by [6]).

\subsection{Weeks and Campbell 1973 [37]}

The first serious attempt to model iceberg deterioration was made by Weeks and Campbell in 1973 [37]. Their goal was to investigate the feasibility of towed icebergs as a fresh water source for arid climates. Surprisingly, they found it was both technologically and economically feasible. In doing so they had to account for the many sources of iceberg deterioration; melting, wave erosion, calving, insolation, and others. We focus on their widely adopted parameterization of iceberg melting.

Weeks and Campbell modelled the melting using empirical relations for turbulent heat transfer over a flat plate [10], resulting from relative motion between the iceberg and water. They saw this motion as a result of towing, but it can arise in nature. Any force other than water drag (air drag, Coriolis force, wave induced motion) will lead to a velocity difference between the ice and water. Furthermore, a sheared water column can result in substantial increases in relative velocities [13]. We now examine Weeks and Campbell's derivation.

The model relies on an empirical relation for the average Nusselt number $\overline{\mathrm{Nu}}$ (a nondimensional ratio of convective to conductive heat transfer) in turbulent convection [10],

$$
\overline{\mathrm{Nu}}=\frac{\bar{h} L}{k},
$$

where $\bar{h}$ is the averaged convective heat transfer coefficient, $L$ is a characteristic length, and $k$ is the thermal conductivity of the fluid.

The turbulent heat transfer relation for the Nusselt number in flow past a heated plate is given in term of the non-dimensional Reynolds, Re, and Prandtl, Pr, numbers,

$$
\overline{\mathrm{Nu}}=0.037 \operatorname{Re}^{0.8} \operatorname{Pr}^{1 / 3},
$$

where Re is the ratio of inertial to viscous forces, and Pr is the ratio of heat and momentum transfer, 


$$
\operatorname{Re} \equiv \frac{U L}{\nu}, \quad \operatorname{Pr} \equiv \frac{\nu}{\kappa} .
$$

Here, $U=\left|v_{i}-v_{w}\right|$ is the relative velocity between the ice and water, $\nu$ is the momentum diffusivity, and $\kappa$ is the thermal diffusivity, related to the thermal conductivity $k$ by $\kappa=k / \rho_{w} c_{p}$, where $\rho_{w}$ and $c_{p}$ are the density and heat capacity of seawater.

To determine the melt rate of a given submerged area, $A$, (either single or multiple faces), we consider the total heat transfer into the ice $q$, given by

$$
q=\bar{h} A \Delta T,
$$

where $\Delta T$ is the temperature difference between the ice and water. We can then relate the heat absorption of the ice face $q$ to a melt rate $u$ (in units of velocity) through the latent heat $\Lambda$

$$
u=\frac{q}{A \Lambda \rho_{i}} .
$$

Hence, we recover Weeks and Campbell's relation [37]

$$
u=\left(0.037 \frac{\rho_{w}}{\rho_{i}} \nu^{-7 / 15} \kappa^{2 / 3} \frac{c_{w}}{\Lambda}\right) \frac{U^{0.8} \Delta T}{L^{0.2}} .
$$

It is important to note that this parametrisation implies the melt rate will go to zero as the velocity goes to zero (though turbulence will cease at this point, invalidating the relation).

This issue is addressed by the improved parameterization of FitzMaurice et al. [12]. FitzMaurice et al. found that there are three regimes of iceberg melting, dependent on the behaviour of meltwater plumes [12]. These plumes rise along the iceberg sides, driven by the buoyancy of the fresh meltwater. As they rise they become turbulent, entraining the warmer ambient water. Thus, even when the iceberg is stationary relative to the ocean, there is a buoyancy driven flow up the side walls, leading to increased melting. Once the relative velocity of the iceberg becomes comparable to the plume velocity, the upstream plume is detached and swept away. FitzMaurice et al. model this regime by substituting the plume velocity into Weeks and Campbell's parameterization (slightly modified to account for increasing entrainment as the ambient velocity increases, and the appropriate plume temperature). Only at higher velocities, when both the front and rear plumes are detached, is Weeks and Campbell's original parameterization correctly recovered.

\section{$2.2 \quad H o l l a n d$ and Jenkins $1999[16]$}

A second widely used parameterization of ice melting was derived by Holland and Jenkins [16]. Their parameterization uses the three thermodynamic equations for interfacial temperature $T_{b}$ and salinity $S_{b}$ that must be satisfied at the ice-water interface, which in general differ from the far-field values of these quantities, in the ice $\left(T_{i}, S_{i}\right)$ and water $\left(T_{w}, S_{w}\right)$.

The temperature at the interface lies on the liquidus line, where the temperature, $T_{b}$, is salinity and pressure dependent, and approximated by a linear relation,

$$
T_{b}\left(S_{b}\right) \approx a+b S_{b}+c p,
$$

where $p$ is the pressure and $a, b$, and $c$ are constants. Heat is conserved at the interface, so that the absorption of latent heat during melting is provided by heat transfer from the ice and water

$$
Q_{\text {latent }}^{T}=Q_{\text {ice }}^{T}+Q_{w a t e r}^{T} .
$$


A similar relation holds for salinity, though now there is no release of salt during melting of fresh ice, nor any diffusion of salt through the ice.

$$
Q_{\text {brine }}^{S}=Q_{\text {ice }}^{S}+Q_{\text {water }}^{S}
$$

Here $Q_{\text {latent }}^{T}$ and $Q_{\text {brine }}^{S}$ are given in terms of the latent heat, $\Lambda$, density of ice $\rho_{i}$, the melt rate of the ice $u$, and the interface salinity $S_{b}$

$$
Q_{\text {latent }}^{T}=-\rho_{i} u \Lambda, \quad Q_{b r i n e}^{S}=-\rho u S_{b} .
$$

The heat flux from the ice is a simple diffusive solution, while the salt flux from the ice is zero. The remaining components, $Q_{\text {water }}^{T / S}$, are obtained using a parameterization of turbulent heat and salt transfer. This is the primary development of Holland and Jenkins' work.

In it, the heat and salt transfer are parameterised in terms of transfer coefficients $\Gamma^{T}$ and $\Gamma^{S}$, and a drag coefficient $C_{d}$

$$
Q_{\text {water }}^{T / S}=\rho c_{p} C_{d}^{1 / 2} \Gamma^{T / S} U\left(T_{b}-T_{w}\right),
$$

where $U$ is the relative iceberg-ocean velocity. The heat diffusion is modelled by simply using the temperature difference between the ice and interface. Putting these requirements together, the ablation velocity $u$ (and interfacial temperature $T_{b}$ and salinity $S_{b}$ ) are found by solving the following system of equations

$$
\begin{aligned}
u\left(\rho_{i} \Lambda+\rho_{i} c_{i}\left(T_{b}-T_{i}\right)\right) & =\rho_{w} c_{w} C_{d}^{1 / 2} U \Gamma^{T}\left(T_{w}-T_{b}\right), \\
u \rho_{i} S_{b} & =\rho_{w} C_{d}^{1 / 2} U \Gamma^{S}\left(S_{w}-S_{b}\right), \\
T_{b} & =\lambda_{1} S_{b}+\lambda_{2} .
\end{aligned}
$$

However, this parameterization is now completely independent of iceberg geometry, predicting the same melt rate for all sides of an iceberg in uniform ambient flow.

\section{Experimental Method}

\subsection{Measuring a melt rate}

We first define a measure of the overall melt rate for an ice block with differing melting between and within each immersed face. There is otherwise no way to compare the melting of different shaped ice blocks. Ideally, we would weight the rate of volume loss by the current submersed surface area, to give a melt rate velocity

$$
\frac{1}{A(t)} \frac{d V}{d t}
$$

However as written above it is an instantaneous measure, which is difficult to obtain experimentally. Given that we only have access to the initial and final ice bass values during the experiment, we assume the melt rate velocity $u$ is constant in time, approximating it as

$$
u \equiv \frac{2}{A(t)+A(0)} \frac{V(t)-V(0)}{t} .
$$

The volume change can be inferred from the mass loss, assuming the density of ice, and the initial area is easily calculated from the dimensions of the block. It is the final area estimation that requires the most effort, and will require several image processing techniques, discussed later. 


\subsection{Ice preparation}

Having defined a metric for the melt rate, we now consider the manufacture of the ice blocks. To minimise bubble formation, fresh water that had been still for at least two days was siphoned into an ice block mold. Unfortunately, a small amount of bubble formation was still observed despite these precautions, though they are not expected to be of relevance. Two mold types were used, with (interior) dimensions $10 \times 15 \times 30 \mathrm{~cm}$, and $32.5 \times 22.5 \times 6 \mathrm{~cm}$. These molds were kept in an industrial refrigerator at -30 to $-25{ }^{\circ} \mathrm{C}$ for one to two days, to allow the entire block to fully freeze and equilibrate to fridge temperature. A wooden handle was frozen into the block using several clamps during the freezing process. The deeper mold was used to generate arbitrary length blocks. This was done by filling up to the desired length, freezing, removing the block, and finally refreezing the handle in the side of now tilted ice block. To visualise the melting of the block, several $\mathrm{ml}$ of blue food dye were injected in the water before freezing.

\subsection{The experiment}

Before each experiment, the mold was removed from the freezer, and dipped in a bucket of room temperature water to release the ice block. Once removed, pictures were taken of the ice using a Nikon Coolpix P7000 camera from approximately $1 \mathrm{~m}$ distance. A ruler was included in the picture for distance calibration. The mass of the combined ice and mold was measured before each experiment using a scale with $2 \mathrm{~g}$ precision. The temperature of the tank water was measured using a thermometer with $0.1{ }^{\circ} \mathrm{C}$ precision before each experiment, and the density at $20.000^{\circ} \mathrm{C}$ was measured using an Anton Paar 5000M Density Meter.

The desired immersed depth of the ice block was scored on the side before each experiment, and the block was subsequently immersed in the tank to approximately this depth. Later experiments calibrated the immersed depth by measurement of the dimensions of the handle, iceblock, mold, and tank mount beforehand.

The tank (Fig. 1) was filled with oceanic saltwater of salinity 30 to $31 \mathrm{~g} / \mathrm{kg}$, and temperature 18 to $21^{\circ} \mathrm{C}$, which could be pumped through the tank and recirculated at three speeds, $0,1.5$, and $3.5 \mathrm{~cm} \mathrm{~s}^{-1} .40 \mathrm{~cm}$ of plastic mesh and a $10 \mathrm{~cm}$ honeycomb grating were used to laminarise the incident flow. However, surface tension effects prevented a completely uniform velocity. Instead, the upper surface was stationary, with a roughly $1 \mathrm{~cm}$ shear layer below the surface, below which the velocity was approximately uniform. Adding surfactant reduced the pinning, but the effect was only temporary, (and potentially hazardous when reacting with bleach used to suppress algae growth).

The velocities of the tank were previously calibrated using multiple runs of large 'floaters' polystyrene blocks weighted down by a horizontal plastic cross (of diameter $4 \mathrm{~cm}$ and depth 2 $\mathrm{cm}$ ) located a given distance below the surface. The high drag of the cross forces the floater to move with the average velocity of the fluid at that depth. The time for the floater to traverse $40 \mathrm{~cm}$ down the tank was calculated 10 times for each floater, giving the velocity measurement estimate.

The experiments were recorded for 10 minutes using the P7000 camera, after which the blocks were removed from the tank, weighed and photographed from each side to compare the ice shape to the initial dimensions.

\subsection{Post-processing}

We analyse these post-experiment images to detect the final shape of the iceberg using opencv for Python (Fig. 2). First, the image is cropped to contain only the melted part of the ice block. Then the red channel is used to $\operatorname{detec}^{\perp}$ the ice block, as the blue dye absorbs red. A 


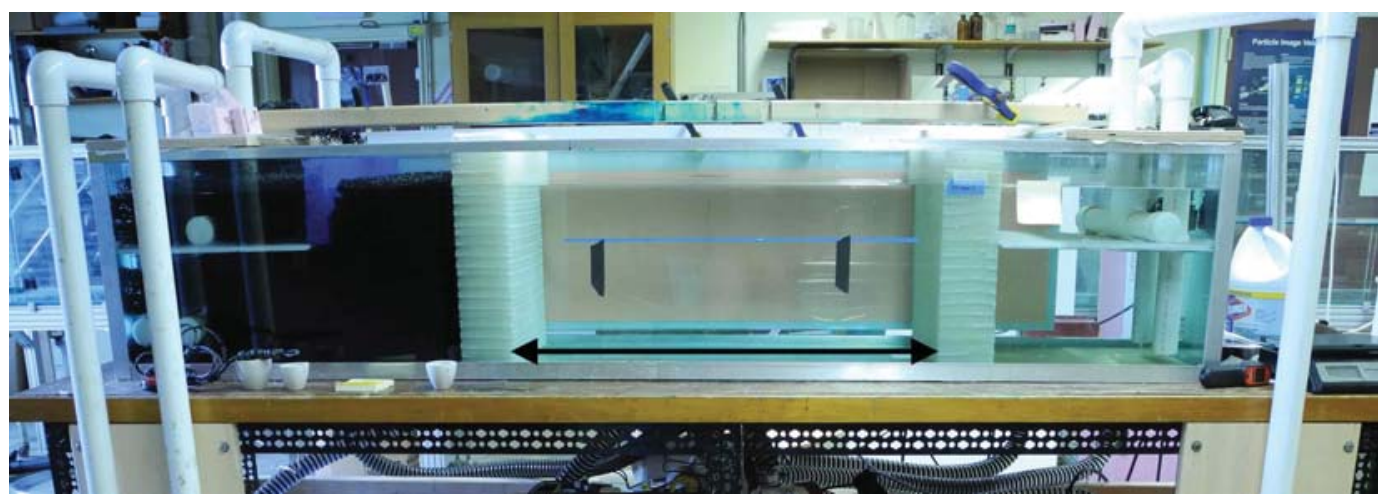

Figure 1: Photo of experimental tank. The central region into which the block is immersed (see black arrow) measures $76.5 \mathrm{~cm}$ long, $42 \mathrm{~cm}$ wide, and $33.5 \mathrm{~cm}$ deep.
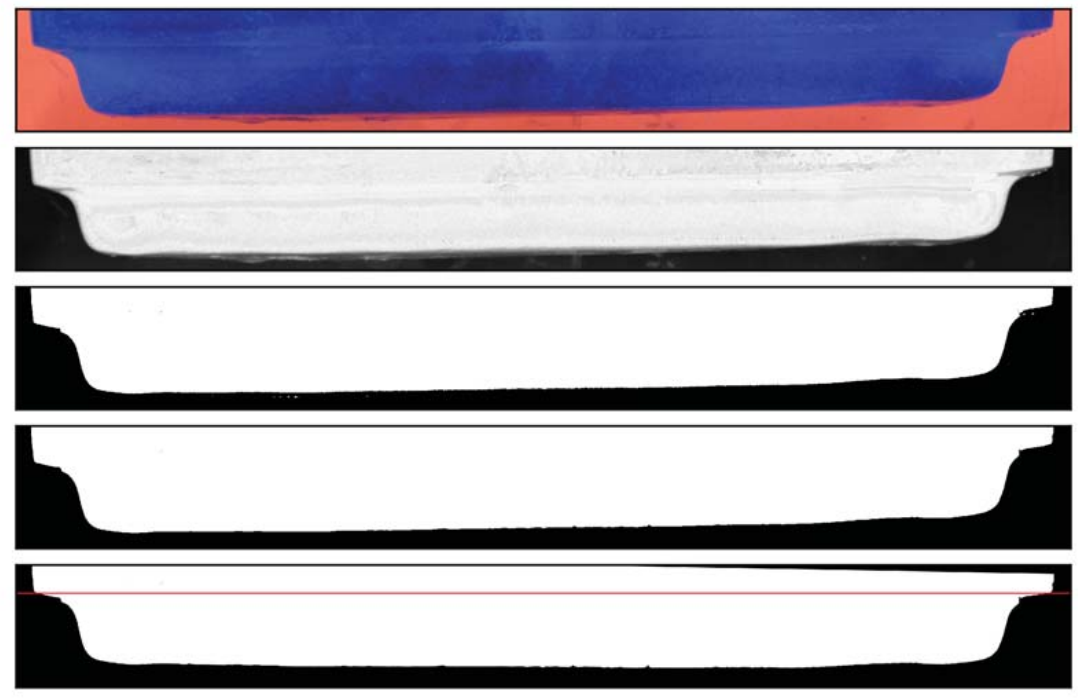

Figure 2: Stages of image processing. The red channel of the image is taken, then a uniform threshold applied to obtain a binary image. An opening morphological transform is used to eliminate noise, followed by corner detection and rotation. The red line is an estimate of the waterline.

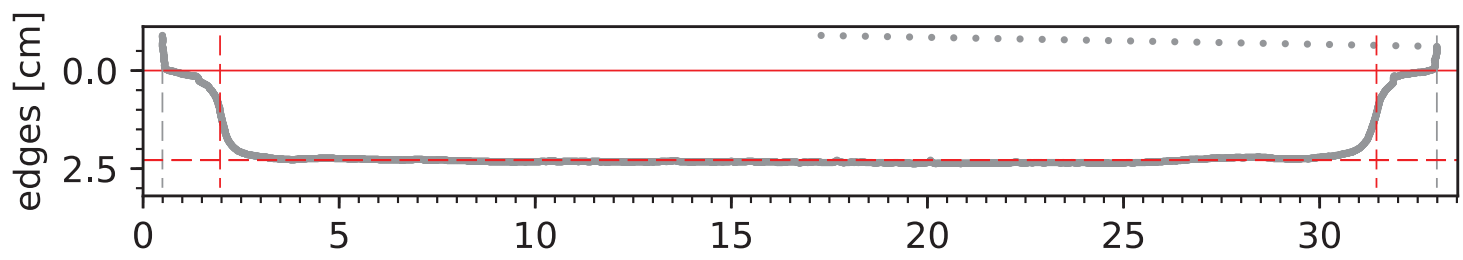

Figure 3: After processing the image, the edges can be detected by looking at leftmost, bottommost, and rightmost white pixels. The initial side edges are shown in dashed gray, the initial bottom edge is the bottom of the graph, and the final shape of the block is given by the solid gray line. The top solid red line is an estimate of the waterline, while the dashed lines are mean values of left, bottom, and right melting. 
uniform threshold can then be applied to give a binary image, and inversion applied to give a white block on a black background. This threshold was chosen manually for each image, as different experiments had different lighting conditions. Spurious noise (small numbers of isolated white pixels) is minimised by applying an erosion filter (white pixels near black pixels are switched to black). The resultant shrinking of the ice block pixels is undone by applying a subsequent dilation filter (black pixels adjacent to white are switched to white). This removes small regions of noise, but preserves large features in the image. This process is known as opening the image.

After this process, we detect the left, bottom, and right edges of the image by measuring the leftmost, bottommost, and rightmost white pixels. Using this outline we can then determine final dimensions of the block. To determine the amount of melting we attempted to infer the waterline (the red line in figures 2 and 3). This was the uppermost point at which the ice block had noticeable melting. Specifically, the highest point along the block more than a certain number of pixels to the right/left of the leftmost/rightmost white pixel.

We then estimate an average depth and width from the final ice shape by taking the mean depth between the bottom corners, and the mean width above the highest corner. The distance from the waterline to the mean of the bottom is compared to the initial immersed depth of the ice block to infer a mean basal melt. The side melts are estimated from the difference between the rightmost and leftmost points (of the unmelted portion), and the average right and left sides (Fig. 3).

The conversion ratio of pixel to $\mathrm{cm}$ for each photo is assumed to be uniform in each direction, and at each point throughout the image. In reality this is not the case, however several pictures with rulers in both orientations were taken, and differences are typically less than 1 percent.

\subsubsection{Experimental video profiles}

By subtracting the initial frame from subsequent video frames, and applying thresholding to the result, we estimated side profiles of the ice blocks during the course of the experiment. Example melting profiles for these experiments are shown in figure 6. Unfortunately, the video processing cannot distinguish between the blue of the ice block and the blue of the melt water, leading to poor shape detection for our melting experiments. However, we can discern that the leading edge retreats at a roughly constant rate in time. Owing to these issues the main results use pictures of the final profiles of the ice blocks.

\section{Results}

Over 50 experiments were performed, with aspect ratios from 0.4 to 13 , at three ambient velocities $U$ of $0,1.5$, and $3.5 \mathrm{~cm} \mathrm{~s}^{-1}$. The ambient fluid temperature was kept as constant as possible at around $19{ }^{\circ} \mathrm{C} \pm 2{ }^{\circ} \mathrm{C}$, and the salinity of the water at $30 \mathrm{~g} / \mathrm{kg}$.

The main series of experiments examined the influence of aspect ratio using a constant immersed depth of $H=3 \mathrm{~cm}$, where the streamwise length $L$ varied from $10 \mathrm{~cm}$ to $32.5 \mathrm{~cm}$, in approximate increments of $5 \mathrm{~cm}$.

\subsection{Qualitative observations}

\subsubsection{High flow velocity}

A time series of two experiments at $U=3.5 \mathrm{~cm} \mathrm{~s}^{-1}$ is shown in Figure 4, confirming the highly nonuniform nature of ice block melting, both within and between experiments. The frontal melt rate is much larger than that of the bast and sides; the incoming flow is at the highest 
temperature at the front of the block, and cools as it loses heat into the ice, thereby reducing the melt rate with distance.

The frontal melt is itself also nonuniform with depth, leading to an increasing slope on the leading edge during the experiment. Part of this is expected from the nonuniform velocity profile of the tank mentioned in the methods section, though this is only expected to be a small effect. Further, the fluid speed would increase going toward the bottom edge of the face, as is typical for any flow around a bluff body, enhancing the melt rate.

We also observe a striking non-uniformity in the basal melt, which reaches its maximum away from the leading edge. Proceeding downstream along the bottom of the ice, we first see a somewhat turbulent region of increased dye concentration, as implied by our Reynolds number $\operatorname{Re}=U H / \nu \approx 1000$. This increased concentration suggests recirculation, typical of flow separation problems. This pooling of cold meltwater reduces heat replenishment (and hence melting) from the ambient water. Turbulence would still cause some mixing with the ambient water however, entraining heat and mitigating the insulating effect of the region.

Behind this recirculation region, we see a clear maximum in the basal melt. This region moves downstream during the experiment, and has a measurably increased melt rate. Interestingly, the general characteristics of the leading profiles of the two blocks are quite similar, despite their different aspect ratios. The key difference is that the longer ice block extends beyond the local maximum in basal melt, beyond which the melting is lower, and much more uniform. In fact (though difficult to see from the time series in figure 4), the dye pattern has changed from turbulent eddies to steady, straight streaks, suggesting a return to laminar flow after the reattachment region.

This configuration is essentially that of flow past a forward-facing step, which while studied previously $[1,18,22,26,30]$, has not been thoroughly investigated from the perspective of heat transfer $[1,18]$. The general properties of turbulent flow past a forward facing step can be seen in our experiment - flow separation after the leading edge, leading to a region of turbulent recirculation, and subsequent reattachment of the flow. Furthermore, experiments on heat transfer have also found a maximum in the Nusselt number at the point of reattachment [1]. This intuitively makes sense - the fluid at the reattachment point has not been cooled by the ice, so should lead to an increased melt rate. The velocity in this region also has a nonzero component normal to the ice face, which (as with the front face), has been observed to increase the melt rate [21].

Understanding the scaling of this reattachment region is key to predicting whether this local enhancement of the melting observed in the laboratory will be geophysically relevant. A range of values have been found by different authors (summarised in [30]), but for Reynolds

Table 1: Experimental data.

\begin{tabular}{l|l|l}
$L$ & Initial Ice block streamwise length & $10,15,20,25,32.5 \mathrm{~cm}$ \\
$W$ & Initial Ice block transverse width & $10-22.5 \mathrm{~cm}$ \\
$H$ & Initial Ice block immersed depth & $3-20 \mathrm{~cm}$ \\
$T_{i}$ & Ice block Temperature & -30 to $-4^{\circ} \mathrm{C}$ \\
$T_{w}$ & Ambient water temperature & $18-21^{\circ} \mathrm{C}$ \\
$S_{w}$ & Ambient water salinity & $30-31 \mathrm{~g} / \mathrm{kg}$ \\
$t$ & Experiment duration & $10 \mathrm{~min}$ \\
$U$ & Ambient water velocity & $0,1.5,3.5 \mathrm{~cm} \mathrm{~s}^{-1}$ \\
$\rho_{i}$ & Ice density & $0.92 \mathrm{~g} / \mathrm{cm}^{3}$
\end{tabular}


numbers $\operatorname{Re}=10^{3}$ to $10^{5}$, the reattachment length is found to be a small multiple of the step height, ranging from roughly 1 to 5 . Our experiments with different relative velocities and iceberg lengths (but identical depths) all reveal a similar location (relative to the leading edge) of the melting region - in agreement with previous studies indicating a depth dependence of the recirculation region length. Should this dependence hold for higher Reynolds numbers $\left(10^{7}\right.$ to $10^{9}$ ), we can expect the increased melting at the reattachment point to have measurable consequences for real icebergs. If instead the reattachment length is limited to some smaller scale, it may be also related to the scalloping of iceberg undersides (though our experiment did not show evidence of further local maxima in the melting behind the first one).

It is important to note that past experiments of flow past a step [30] wereperformed for two dimensional blocks, in which the step extended the entire length of the channel. In our case (and for that of icebergs), we expect the transverse width to become relevant when it approaches the same order of magnitude as the depth. Indeed, the shorter ice block was also less wide than the longer one (Fig. 4) $(10 \mathrm{~cm}$ to $22.5 \mathrm{~cm})$. These turbulent reattachment regions were also found on the sides of the ice block, leading to similar regions of increased melt on the transverse sides of the block.

The complex shape evolution of a melting ice block echoes previous investigations on the influence of flow and obstacle geometry on melting [15], dissolution [17], and erosion [24]. Higher velocities will unsurprisingly increase melting, but that melting can be highly nonuniform emphasising the difficulties of applying current parameterizations to iceberg melting.

The suppressed turbulence observed downstream of the larger block also affects the distribution of meltwater in the water column. The water near the free surface behind the longer ice block is much darker than for the short block, suggesting that a larger proportion of meltwater is reaching the surface - with important consequences for the biological environment surrounding an iceberg, as well as the vertical distribution of the freshwater flux due to iceberg melting.

\subsubsection{Low flow velocity}

Experiments were also performed for the same dimension ice blocks at the lower velocity of $1.5 \mathrm{~cm} \mathrm{~s}^{-1}$. The same trends can be identified in the $1.5 \mathrm{~cm} \mathrm{~s}^{-1}$ experiments, with a local maximum in the melt rate behind the leading edge, followed by a return to laminar flow, and more uniform lower melt rate, behind this maximum. The length scale of this recirculation region is similar to that observed in the higher velocity experiments.

The main differences are the lower melt rate, and the reduced turbulence of the flow, where the dye streaks appear mostly laminar throughout the experiment. This means meltwater is no longer mixed throughout the depth of the iceberg, and instead pools near the free surface of the water.

\subsubsection{Zero flow velocity}

The zero velocity experiments unsurprisingly lack any local increases in the melt, unlike the experiments with a relative velocity. However, these experiments also exhibit sinking plumes of dyed water. Though the vast majority of melt water does appear to rise to the surface, these plumes remain unexpected as fresh melt water should be far less dense than ambient salt water, despite the temperature difference. It is believed this stems from the neglect of latent heat in such an assertion. The melting of ice in salt water is a very different process to that of mixing cold fresh water with salt water [14, 19]; during the melting, the latent heat is absorbed from the adjacent salt water. As there is no replenishing of the ambient water from continual circulation, this process can cool the salt water sufficiently that it will sink, entraining the dyed meltwater along with it. 

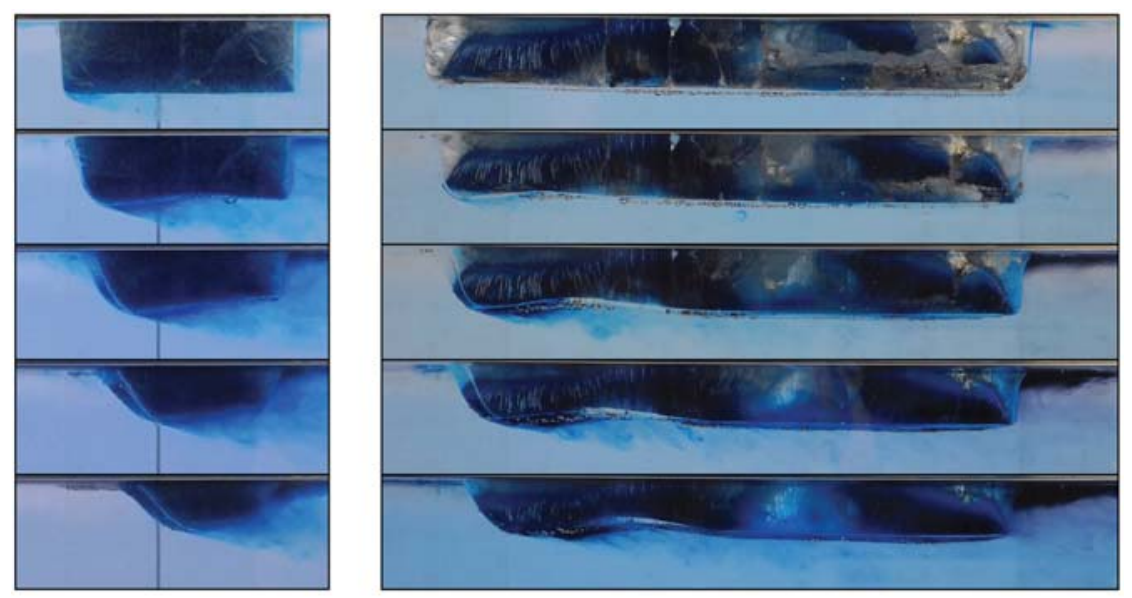

Figure 4: Time series of two experiments with $L=10 \mathrm{~cm}$ (left) and $32.5 \mathrm{~cm}$ (right). Each ice block was immersed up to $3 \mathrm{~cm}$, in ambient water at $20^{\circ} \mathrm{C}$, moving at $U=3.5 \mathrm{~cm} \mathrm{~s}-1$. Frames are shown every two minutes.
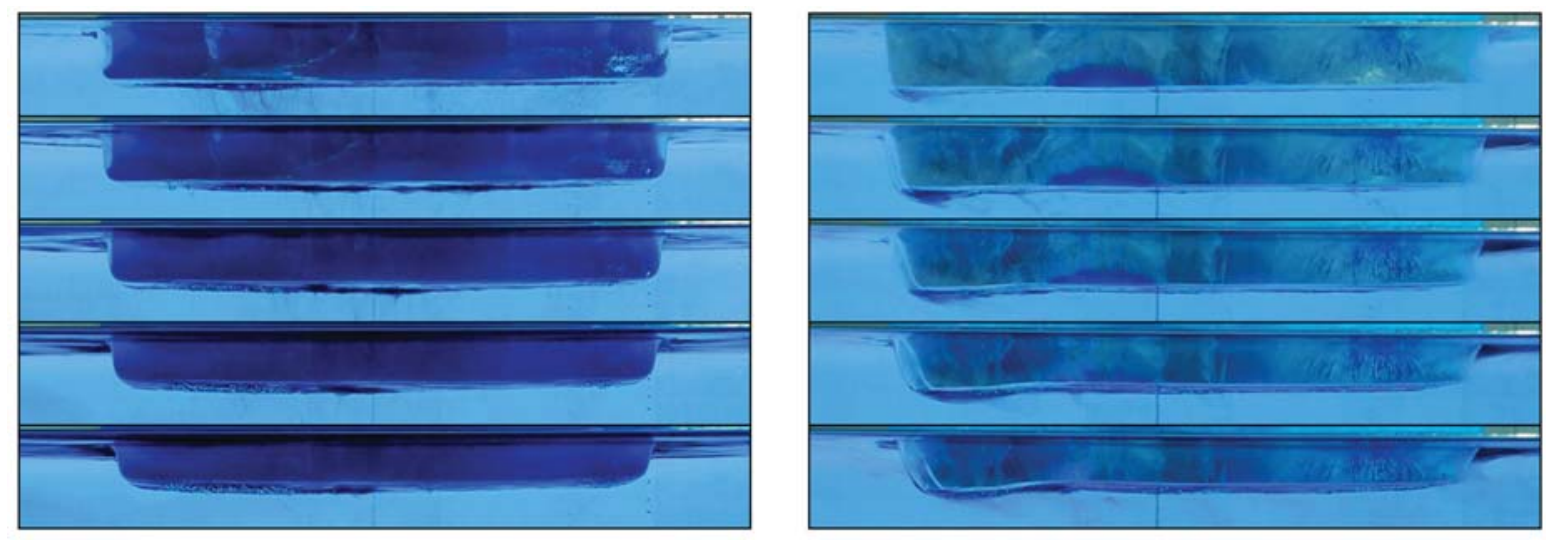

Figure 5: Time series of two experiments with $L=32.5 \mathrm{~cm}$ (left), for $U=0$ and $1.5 \mathrm{~cm} \mathrm{~s}^{-1}$ (right).

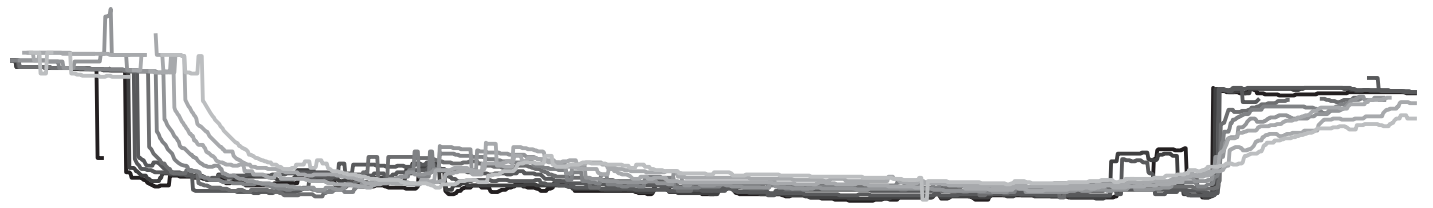

Figure 6: Measured side profiles of $32.5 \mathrm{~cm}$ long i ce block i mmersed in fluid moving at $U=3.5 \mathrm{~cm} \mathrm{~s}-1$. The edge detection works poorly as a result of the similar colour of the ice block and the dyed melt water. Contours are taken at one minute intervals. 


\subsection{Quantitative results}

\subsubsection{Post-experiment picture profiles}

Using the image processing techniques described in section 3.4, the final profiles of the melted ice blocks were extracted from the photos taken immediately after each experiment. In figure 7 , the average melted profiles of the ice blocks are plotted. The average profile is taken by averaging the profiles from the left and right side of the melted ice block, after aligning at the upstream edge. For each relative fluid velocity, all experimental average melting profiles are plotted on the same graph, revealing broadly similar trends. The profiles are positioned so that the origin corresponds to the location of the front corner of the unmelted ice block. Averaging the left and right profiles is done to compensate for possible asymmetries in the melting and picture processing.

A final melting curve (dashed) was then constructed from the average of the individual experiments. The downstream faces of the ice blocks are omitted from the average calculation, and the most downstream points of the bases for each ice block length are shown with black circles. The grey error bars around the average profile are twice the average of the standard deviations of the individual profiles at each point. The reduced number of profiles at higher lengths affects the standard deviation calculation, but acts as a sensible first estimate of the variability of the melting profiles.

From this averaged profile we can estimate basal and frontal melt. The frontal melt is estimated from the average of the curve between two points identified as the top and bottom of the front face, and average basal melt as the average value of the dashed curve between the start and end of the base. These profiles reveal the disparity between frontal and basal melt for each velocity, highlighting the geometric dependence of iceberg melt (table 2).

As well as the non uniform melting between faces, there exist large variations within each face. Figure 8 examines the nonuniform basal melt, showing the localised and cumulatively averaged basal melt rate for each of the fluid velocities. Basal melt rates at fluid velocities of $0 \mathrm{~cm} \mathrm{~s}^{-1}$ and $1.5 \mathrm{~cm} \mathrm{~s}^{-1}$ are comparable, though the more turbulent $3.5 \mathrm{~cm} \mathrm{~s}^{-1}$ experiment is markedly increased. This is in agreement with the findings of FitzMaurice et al. [13], who observed a roughly constant melt rate below a threshold fluid velocity - attributed to the influence of rising meltwater plumes. The melt rate was observed to be controlled by the maximum of the velocity of the meltwater plumes and the ambient fluid velocity. FitzMaurice's findings neglected the basal melt however, so her model is not directly applicable to the present experiments.

Instead, the basal melting of our experiments would naturally give rise to a gravity current, as the melt water spreads along the iceberg base to then rise to the surface. The Froude number of gravity currents is approximately unity, and the thickness of the meltwater layer $h$ was observed to be approximately 2 to $4 \mathrm{~mm}$, giving a gravity current velocity of around $\sqrt{g h} \approx 1.4$ to $2.0 \mathrm{~cm} \mathrm{~s}^{-1}$. As such, we expect a weak dependence on the ambient fluid velocity when it is below this gravity current speed. We note however that this is not a confined channel gravity current, and also that some small amount of sinking of the dye was observed. Waves were also observed on the interface which propagated toward the center.

It is worth noting that the average melt rates in table 2 are of the entire dashed lines in figure 7. The average basal melt of a shorter block should be larger, as a larger proportion of the base is in the enhanced melting region. To understand the change in average basal melt with length, we plot the cumulatively averaged basal melt in figure 8 . The averaging procedure will naturally reduce the influence of localised features, but we still see a noticeable variation in the average basal melt rate for fluid velocity $3.5 \mathrm{~cm} \mathrm{~s}^{-1}$ as a function of length. This variation in basal melt with length is less pronounced than that between the average frontal and basal melt, and would likely be a secondary effect in the real world, though it is still detectable, with 


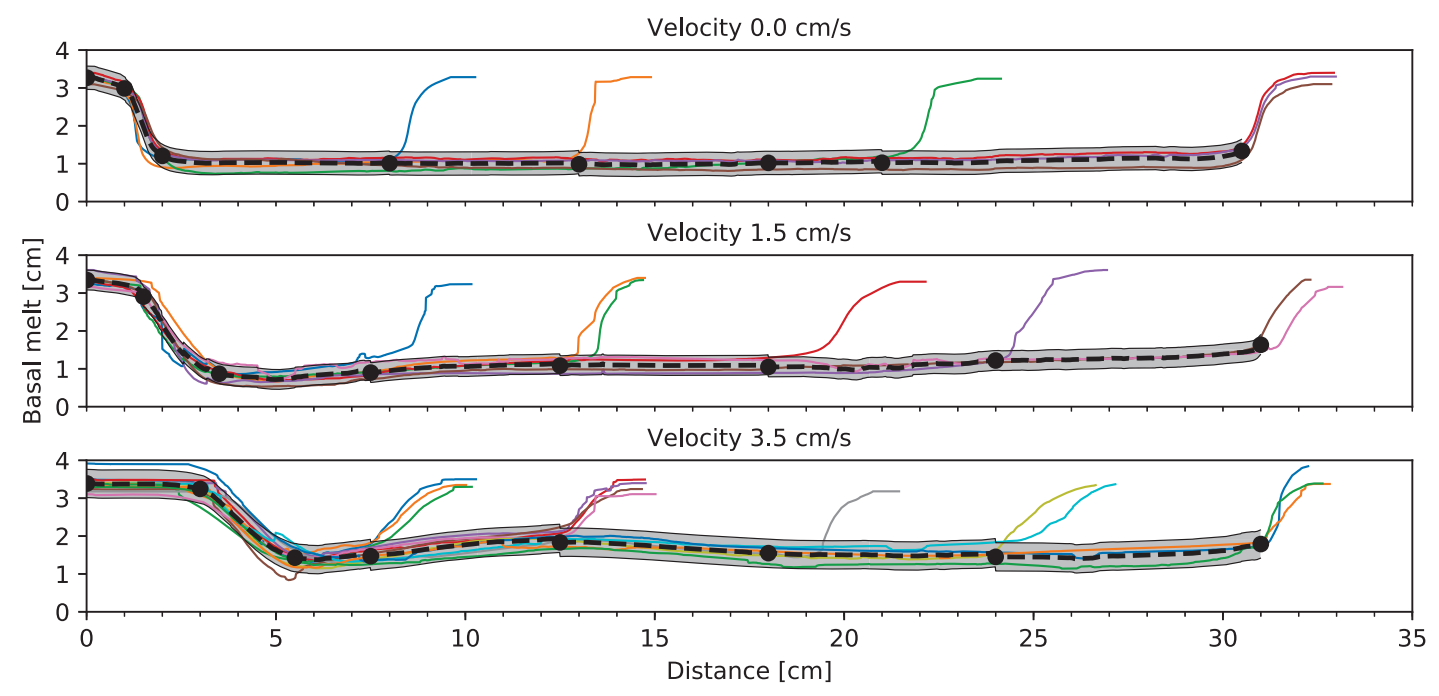

Figure 7: Final profiles of experiments. The profiles are aligned at their edge. We observe highly nonuniform melting between and within each face of the block.

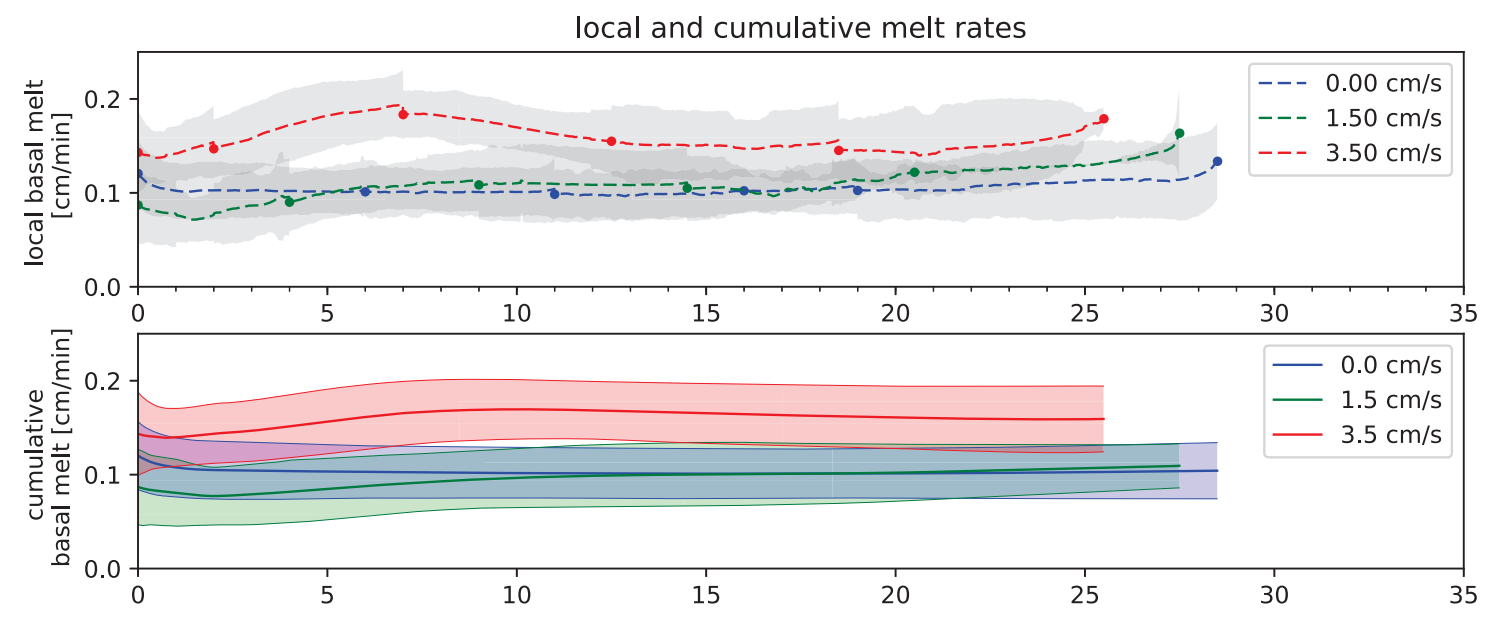

Figure 8: Local and cumulative basal melt rates at $0 \mathrm{~cm} \mathrm{~s}^{-1}$ (blue), $1.5 \mathrm{~cm} \mathrm{~s}^{-1}$ (green), and $3.5 \mathrm{~cm} \mathrm{~s}^{-1}$ (red).

Table 2: Table of average melting rates $\left(\mathrm{cm} \mathrm{min}^{-1}\right)$ of each face.

\begin{tabular}{l|lll} 
Face & $U=0 \mathrm{~cm} \mathrm{~s}^{-1}$ & $U=1.5 \mathrm{~cm} \mathrm{~s}^{-1}$ & $U=3.5 \mathrm{~cm} \mathrm{~s}^{-1}$ \\
\hline Front & $0.15 \pm 0.03$ & $0.23 \pm 0.05$ & $0.41 \pm 0.06$ \\
Sides & $0.13 \pm 0.02$ & $0.14 \pm 0.03$ & $0.19 \pm 0.06$ \\
Rear & $0.15 \pm 0.05$ & $0.13 \pm 0.10$ & $0.15 \pm 0.11$ \\
Base & $0.09 \pm 0.04$ & $0.10 \pm 0.04$ & $0.15 \pm 0.04$
\end{tabular}


up to $20 \%$ variation in the cumulative basal melt rate.

Figure 8 shows that the $1.5 \mathrm{~cm} \mathrm{~s}^{-1}$ velocity experiments have a slightly increasing basal melt rate with length. This is unfortunately a result of imperfect methodology in deriving melt rates from the final ice block photos. The top corners of the ice blocks, where the melting begins, are actually located somewhat above the water line of the tank. This is thought to result from a positive meniscus forming at the air-water interface on the ice block. The typical amount of above water melting was estimated as $3 \mathrm{~mm}$, however this varied between velocities, experiments, and faces (for $1.5 \mathrm{~cm} \mathrm{~s}^{-1}$ ). This can be exacerbated by tilting of the ice block when placed in the water. During the freezing process, the wooden handle would sometimes rotate and tilt in the ice, leading to a slight rotation when placed in the tank.

To assess the accuracy of our averaged profile, we also compare it with an automated procedure for calculating the dimensions of individual profile in figure 9 , in which the width is calculated from the average of the middle third horizontal section of the block, and the depth is the average of the lowest third of the block. We see broad agreement between the automated calculations, and the dimensions of the averaged profile, with the spread of values typically lying within the uncertainty bars of the averaged melt rate. And again, we see a large difference between typical melt rates observed for each face of the ice block; for non-zero fluid velocity, frontal melting can be two to three times larger than that of the other faces. The basal melt is observed to always be lower than side melting - implying that tabular icebergs with large aspect ratios should melt at a lower rate than smaller aspect ratio icebergs. We additionally observe a slight decrease in the basal melt rate as a function of length at the highest velocities, in accord with the cumulative melt rate calculations discussed earlier.

As a test of our method, we compare estimates of volume loss obtained from the final profiles, and the averaged profile, to the actual volume loss measured from the change in mass over the experiment (Fig. 10). The estimates of volume loss assume that the melting of each face occurs while maintaining a constant rectangular shape, at the rates estimated by the respective method. The uncertainties of the volume loss are half the range of volume losses calculated using upper and lower estimates for each the melt rates of each face. Albeit with some scatter, we see clear agreement between the estimates and direct measurements of volume loss.

Next, we use these estimates of melting to determine an overall melt rate for each experiment (from section 3.1) in figure 11. The volume loss is measured from the change in mass, and the average area inferred using the melt rate estimates from the averaged profiles in table 2 , assuming a rectangular shape is conserved during the melt. We note that an additional set of experiments is also used in figures 10 and 11, in which the ice block depth was varied from $5 \mathrm{~cm}$ to $20 \mathrm{~cm}$, in increments of $5 \mathrm{~cm}$. We compare our experimental measurements to typically used parameterizations of Weeks and Campbell [37], and the three equation parameterization of Holland and Jenkins [16] (with parameters used in table 3).

We observe a strong decrease in the overall melt rate as aspect ratio is increased, almost halving from tall icebergs (aspect ratio less than unity), to the largest icebergs used (aspect ratio 13). This variation is primarily due to the different relative side areas of each aspect ratio. A longer ice block has a much larger basal area compared to a tall block of the same total area. As such, the overall melting tends to the lower basal melt rate. Shorter blocks are instead more affected by their side melt.

This geometric dependence in the melt is completely absent in the Jenkins' three equation parameterization, and only weakly accounted for in Weeks and Campbell parameterization, each of which underestimate the melting for the parameters used in table 3 . This failure stresses the need to account for different side melt rates and side areas in iceberg modelling. 

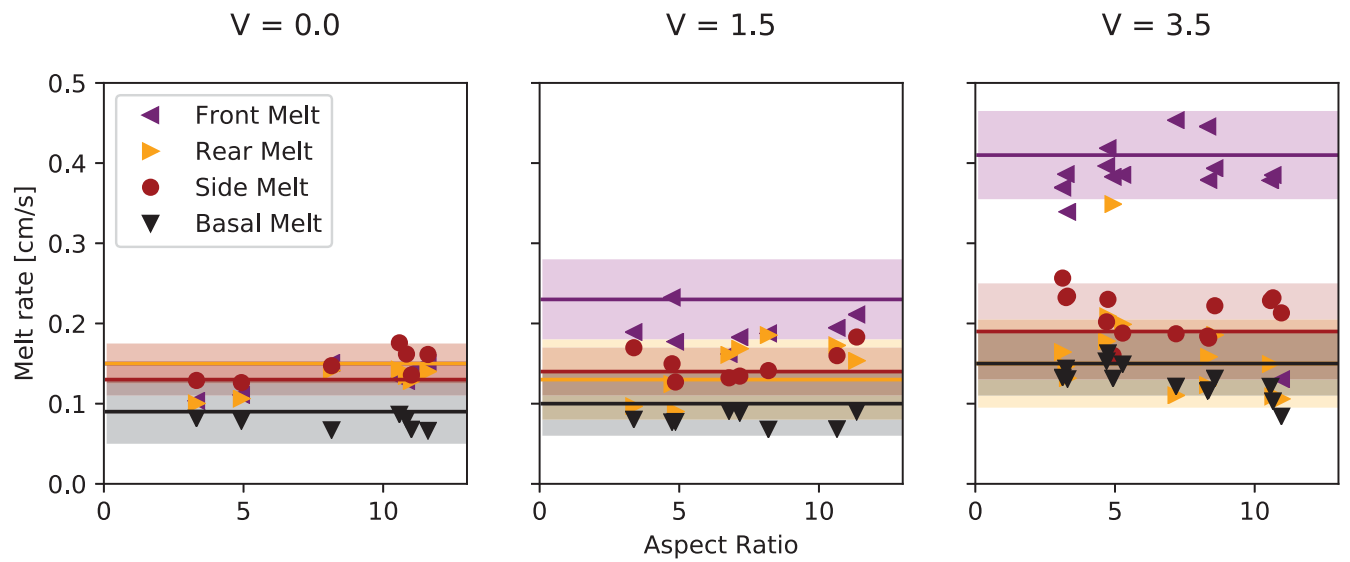

Figure 9: Individual automated side melt calculations (points) and the estimates $\mathrm{f}$ rom the averaged profiles (coloured bars) as a function of aspect ratio. We see agreement between individual and averaged calculations, and strong differences between melt $r$ ates on each face of the block.
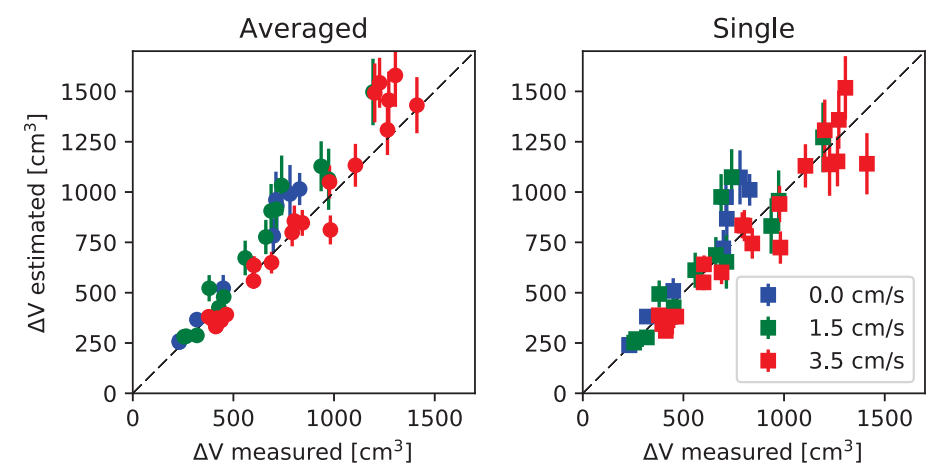

Figure 10: Comparisons of estimated volume loss to measured volume loss, using average (left) and individual (right) profiles, assuming uniform melt on each $\mathrm{f}$ ace (but still varying melt $\mathrm{r}$ ates between faces). This plot includes additional experiments with greater depths. Uncertainties are estimated as half the r ange of volumes using high and low estimates f or the melt $r$ ates.
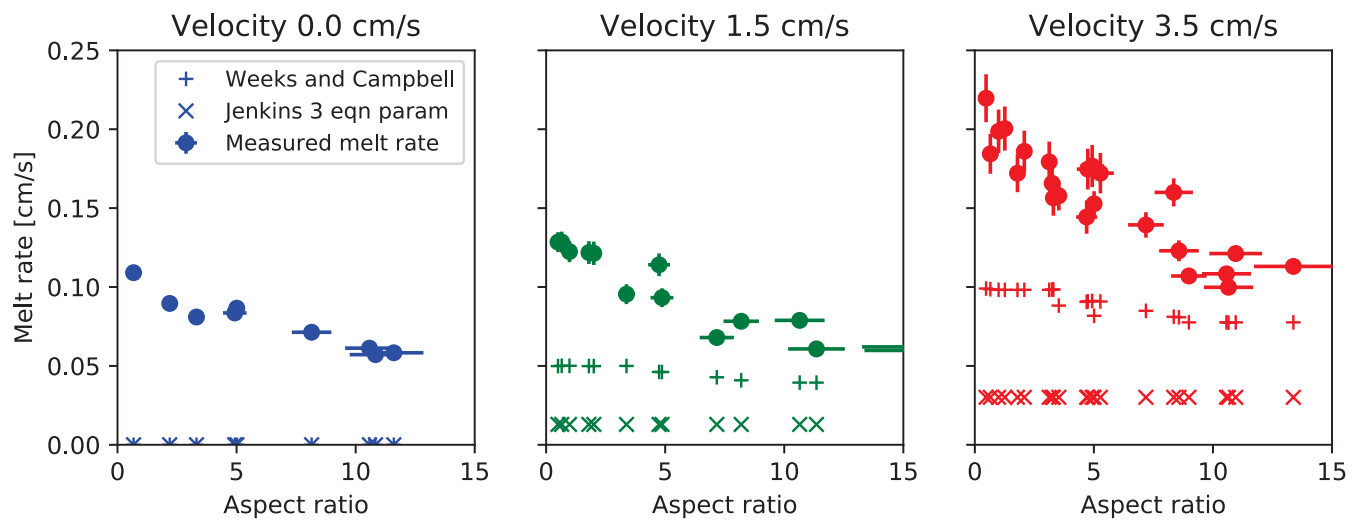

Figure 11: Averaged melt $r$ ates of different velocity experiments, as $\mathrm{f}$ unction of aspect $\mathrm{r}$ atio, for all sets of experiments. Both the Weeks and Campbell $(+)$ and Jenkins three equation $(x)$ parameterizations are also shown for comparison, using values from table 3 . Neither parameterization f ully captures the geometric dependence of the i ce block melting. 
Table 3: Table of values of physical constants, from [3], and [20].

$\begin{array}{cll}\text { Symbol } & \text { Name } & \text { Value } \\ T_{i} & \text { Ice temperature } & -4{ }^{\circ} \mathrm{C} \\ T_{w} & \text { Water temperature } & 19^{\circ} \mathrm{C} \\ S_{w} & \text { Water salinity } & 30.5 \mathrm{~g} \mathrm{~kg}^{-1} \\ \rho_{w} & \text { Water density } & 1021 \mathrm{~kg} \mathrm{~m}^{-3} \\ \rho_{i} & \text { Ice density } & 920 \mathrm{~kg} \mathrm{~m}^{-3} \\ \nu & \text { Water momentum diffusivity } & 1.304 \times 10^{-6} \mathrm{~m}^{2} \mathrm{~s}^{-1} \\ \kappa & \text { Water thermal diffusivity } & 1.38 \times 10^{-7} \mathrm{~m}^{2} \mathrm{~s}^{-1} \\ c_{w} & \text { Water heat capacity } & 4192 \mathrm{~J} \mathrm{~kg}^{-1}{ }^{\circ} \mathrm{C}^{-1} \\ c_{i} & \text { Ice heat capacity } & 2108 \mathrm{~J} \mathrm{~kg}^{-1} \mathrm{\circ}^{-1} \\ \Lambda & \text { Ice Latent heat } & 3.34 \times 10^{5} \mathrm{~J} \mathrm{~kg}^{-1} \\ \lambda_{1} & \text { Liquidus slope } & -0.057^{\circ} \mathrm{C} \mathrm{kg} \mathrm{g}^{-1} \\ \lambda_{2} & \text { Liquidus intercept } & 0.083{ }^{\circ} \mathrm{C} \\ C_{d} & \text { Drag coefficient } & 0.0025 \\ \Gamma_{T} & \text { Temperature transfer coefficient } & 0.011 \\ \Gamma_{S} & \text { Salinity transfer coefficient } & 3.1 \times 10^{-4}\end{array}$

\section{Conclusion}

Typical existing parameterizations of iceberg melting ignore the possible influence of the iceberg's aspect ratio $[16,37]$. To test these models, we ran a series of laboratory experiments, examining the dependence of the melt rate on iceberg size and shape for three different ambient relative velocities. We find that iceberg geometry has a strong effect on the melt rate of icebergs.

Melt rates are highest on the upstream facing side (with respect to the ambient flow), followed by the remaining lateral sides, with lowest melting occurring at the base of the iceberg. Changing the relative area of each face will thus change the overall melt rate.

Furthermore, the melt rate of each face is itself non uniform, with localised regions of increased melt rate of over $50 \%$ observed. These regions are believed to correspond to the reattachment zones of separated flow around the ice block [1]. The extent of these regions have been observed to scale with the obstacle height [30], and as such may be geophysically relevant.

To improve melting estimates, we emphasise that melt rates must depend both on the aspect ratio and orientation of the iceberg, in addition to the fluid velocity used by current parameterizations.

\section{Acknowledgements}

I firstly wish to thank my supervisors Claudia Cenedese and Craig McConnochie for their help throughout this project, whose many thoughts and advice I am deeply grateful for. I greatly appreciate the expertise and help provided by Anders Jensen in building (and fixing) the experimental apparatus. I also thank my f ellow GFD scholars for vastly improving an already excellent experience with your friendship, humour, and support. Finally, to all other GFD staff members and visiting guests, I have truly enjoyed my experience of the culture of inquiry (and fun) you have fostered over the years. I will always remember this summer fondly and look forward to meeting many of you in the future. 


\section{References}

1. Abu-Mulaweh, H. Turbulent mixed convection flow over a forward-facing step - the effect of step heights. International Journal of Thermal Sciences 44, 155-162 (2005).

2. Andres, M., Silvano, A., Straneo, F. \& Watts, D. R. Icebergs and Sea Ice Detected with Inverted Echo Sounders. Journal of Atmospheric and Oceanic Technology 32, 1042-1057 (2015).

3. Batchelor, G. An Introduction to Fluid Dynamics (Cambridge University Press, 2000).

4. Bigg, G. R., Wadley, M. R., Stevens, D. P. \& Johnson, J. A. Modelling the dynamics and thermodynamics of icebergs. Cold Regions Science and Technology 26, 113135 (1997).

5. Budd, W. F., Jacka, T. H. \& Morgan, V. I. Antarctic Iceberg Melt Rates Derived from Size Distributions and Movement Rates. Annals of Glaciology 1, 103-112 (1980).

6. Crepeau, J. Josef Stefan: His life and legacy in the thermal sciences. Experimental Thermal and Fluid Science 31, 795-803 (2007).

7. Dinniman, M. S., Asay-Davis, X. S., Galton-Fenzi, B. K., Holland, P. R., Jenkins, A. \& Timmermann, R. Modeling Ice Shelf/Ocean Interaction in Antarctica: A Review. Oceanography 29, 144-153 (2016).

8. Dowdeswell, J. \& Bamber, J. Keel depths of modern Antarctic icebergs and implications for sea-floor scouring in the geological record. Marine Geology 243, 120131 (2007).

9. Dowdeswell, J. A., Whittington, R. J. \& Hodgkins, R. The sizes, frequencies, and freeboards of East Greenland icebergs observed using ship radar and sextant. Journal of Geophysical Research: Oceans 97, 3515-3528 (1992).

10. Eckert, E. \& Drake, R. Heat and Mass Transfer (R.E. Krieger Publishing Company, 1959).

11. Enderlin, E. M., Hamilton, G. S., Straneo, F. \& Sutherland, D. A. Iceberg meltwater fluxes dominate the freshwater budget in Greenland's iceberg-congested glacial fjords. Geophysical Research Letters 43. 2016GL070718, 11, 287-11, 294 (2016).

12. FitzMaurice, A., Cenedese, C. \& Straneo, F. Nonlinear response of iceberg side melting to ocean currents. Geophysical Research Letters 44. 2017GL073585, 56375644 (2017).

13. FitzMaurice, A., Straneo, F., Cenedese, C. \& Andres, M. Effect of a sheared flow on iceberg motion and melting. Geophysical Research Letters 43. 2016GL071602, 12, 520-12, 527 (2016). 
14. Gade, H. G. Melting of Ice in Sea Water: A Primitive Model with Application to the Antarctic Ice Shelf and Icebergs. Journal of Physical Oceanography 9, 189198 (1979).

15. Hao, Y. L. \& Tao, Y.-X. Heat Transfer Characteristics of Melting Ice Spheres Under Forced and Mixed Convection. Journal of Heat Transfer 124, 891-903 (Sept. 2002).

16. Holland, D. M. \& Jenkins, A. Modeling Thermodynamic Ice--Ocean Interactions at the Base of an Ice Shelf. Journal of Physical Oceanography 29, 1787-1800 (1999).

17. Huang, J. M., Moore, M. N. J. \& Ristroph, L. Shape dynamics and scaling laws for a body dissolving in fluid flow. Journal of Fluid Mechanics 765 (2015).

18. Jayakumar, J. S., Kumar, I. \& Eswaran, V. Hybrid mesh finite volume CFD code for studying heat transfer in a forward-facing step. Physica Scripta 2010, 014060 (2010).

19. Jenkins, A. The Impact of Melting Ice on Ocean Waters. Journal of Physical Oceanography 29, 2370-2381 (1999).

20. Jenkins, A., Nicholls, K. W. \& Corr, H. F. J. Observation and Parameterization of Ablation at the Base of Ronne Ice Shelf, Antarctica. Journal of Physical Oceanography 40, 2298-2312 (2010).

21. Josberger, E. G. \& Martin, S. A laboratory and theoretical study of the boundary layer adjacent to a vertical melting ice wall in salt water. Journal of Fluid Mechanics 111, 439-473 (1981).

22. Largeau, J. F. \& Moriniere, V. Wall pressure fluctuations and topology in separated flows over a forward-facing step. Experiments in Fluids 42, 21 (Nov. 2006).

23. Martin, T. \& Adcroft, A. Parameterizing the fresh-water flux from land ice to ocean with interactive icebergs in a coupled climate model. Ocean Modelling 34, 111-124 (2010).

24. Moore, M. N. J., Ristroph, L., Childress, S., Zhang, J. \& Shelley, M. J. Self-similar evolution of a body eroding in a fluid flow. Physics of Fluids 25, 116602 (2013).

25. Neshyba, S. \& Josberger, E. G. On the Estimation of Antarctic Iceberg Melt Rate. Journal of Physical Oceanography 10, 1681-1685 (1980).

26. Pearson, D. S., Goulart, P. J. \& Ganapathisubramani, B. Investigation of turbulent separation in a forward-facing step flow. Journal of Physics: Conference Series $\mathbf{3 1 8}$, 022031 (2011).

27. Rignot, E., Jacobs, S., Mouginot, J. \& Scheuchl, B. Ice-Shelf Melting Around Antarctica. Science 341, 266-270 (2013).

28. Russell-Head, D. S. The Melting of Free-Drifting Icebergs. Annals of Glaciology 1, 119122 (1980).

29. Savage, S. in Geomorphological Fluid Mechanics (eds Balmforth, N. J. \& Provenzale, A.) 279-318 (Springer Berlin Heidelberg, Berlin, Heidelberg, 2001).

30. Sherry, M. J., Jacono, D. L., Sheridan, J., Mathis, R. \& Marusic, I. Flow separation characterisation of a forward facing step immersed in a turbulent boundary layer. 
Sixth International Symposium on Turbulence and Shear Flow Phenomena, 1325 (2009).

31. Silva, T. A. M., Bigg, G. R. \& Nicholls, K. W. Contribution of giant icebergs to the Southern Ocean freshwater flux. Journal of Geophysical Research: Oceans 111. C03004, n/a-n/a (2006).

32. Smith, F. T. Laminar flow of an incompressible fluid past a bluff body: the separation, reattachment, eddy properties and drag. Journal of Fluid Mechanics 92, 171-205 (1979).

33. Smith, K. L., Robison, B. H., Helly, J. J., Kaufmann, R. S., Ruhl, H. A., Shaw, T. J., Twining, B. S. \& Vernet, M. Free-Drifting Icebergs: Hot Spots of Chemical and Biological Enrichment in the Weddell Sea. Science 317, 478-482 (2007).

34. El-Tahan, M., Venkatesh, S. \& El-Tahan, H. Validation and Quantitative Assessment of the Deterioration Mechanisms of Arctic Icebergs. Journal of Offshore Mechanics and Arctic Engineering 109, 102-108 (Feb. 1987).

35. Tournadre, J., Bouhier, N., Girard-Ardhuin, F. \& Remy, F. Large icebergs characteristics from altimeter waveforms analysis. Journal of Geophysical Research: Oceans 120, 1954-1974 (2015).

36. Venkatesh, S. \& El-Tahan, M. Iceberg life expectancies in the Grand Banks and Labrador Sea. Cold Regions Science and Technology 15, 1-11 (1988).

37. Weeks, W. F. \& Campbell, W. J. Icebergs as a Fresh-Water Source: An Appraisal. Journal of Glaciology 12, 207-233 (1973).

38. Weeks, W. \& Mellor, M. Some elements of iceberg technology. English (1978). 


\title{
Nonlinear Influence of Background Rotation on Iceberg Melting
}

\author{
Agostino N. Meroni
}

December 17, 2017

\section{Introduction}

Iceberg dynamics and thermodynamics still include processes that are poorly understood. Indeed, there is lot of interest in comprehending the complex interactions between icebergs and their surrounding, both in terms of how the environment influences the iceberg and vice versa. In particular, the meltwater produced by icebergs themselves has been observed to modify the local ocean physical and chemical properties, affecting both the dynamical $[17,9]$ and the biogeochemical response [16, 4]. Moreover, icebergs are well known to pose a hazard for human activities such as oil platforms, submarine pipelines and, of course, navigation [3].

Recent numerical works have underlined the importance of correctly describing the iceberg size distribution in order to get the right climatology for sea-ice, ocean temperature and salinity $[18,14]$. In particular around Antarctica, where large tabular icebergs with areas that can reach values up to $O\left(10^{3} \mathrm{~km}^{2}\right)$ exist, the incorrect size distribution representation, for example by neglecting these giant icebergs, can lead to a bias towards the South in the freshwater input [14].

For a comprehensive review of the mechanisms that control icebergs dynamics and melting, the reader is referred to $[15,1]$. Contributions to the melting come both from surface processes and from subsurface ones, as depicted in figure 1. In particular, above the air-sea interface, solar radiation, forced convection due to the winds and sublimation take place, but they represent the least important mechanisms. At the interface, instead, the dominant mechanism, wave erosion, continuously acts and reduces the iceberg volume both by directly transferring heat from the seawater through the periodic wave motion and by inducing calving of the iceberg fraction above the sea level that is left because of the melting itself. Below the sea level then, buoyant and forced convection significantly contribute to the submarine melting by entraining relatively warmer oceanic water in the turbulent layer attached to the iceberg. The former is due to the vertical motion associated to the positively buoyant meltwater, while the latter is due to the relative motion of the water masses, as explained more in detail below [15].

Forced convection at the base of the iceberg is the mechanism studied in the present work. It refers to the transfer of heat between a fluid and a submerged body through the turbulent boundary layer that develops at the interface due to the relative fluid flow [5]. In the case of icebergs, the relative fluid motion is provided by the fact that their displacement is not always controlled by vertically uniform flow. Thus, for example, if there is a vertical shear in the current or if the wind drives the iceberg or if the iceberg 


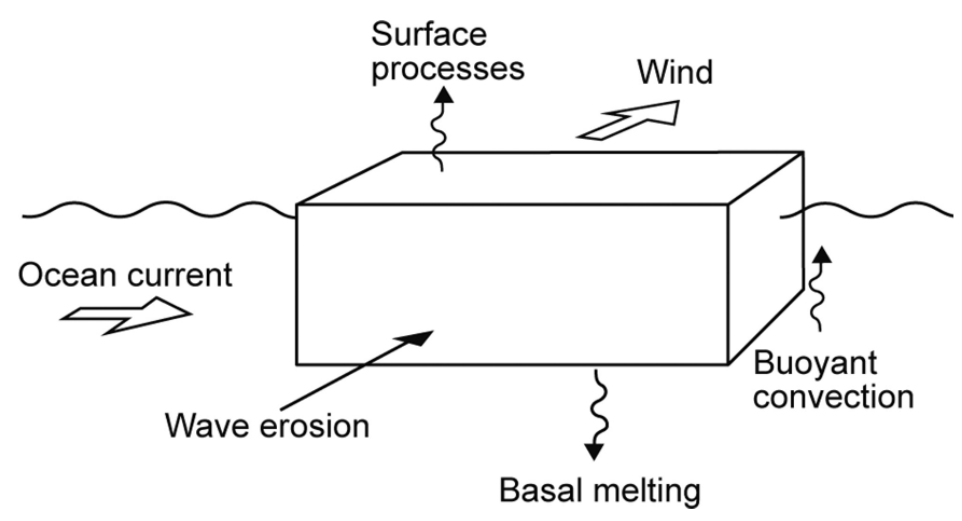

Figure 1: Schematic of the iceberg deterioration processes, adapted from [1].

gets stuck by some bathymetric feature, it is easy to imagine the presence of a relative flow with respect to the iceberg $[1,7]$.

Various efforts have been done to represent this kind of melting as a function of the fluid and ice properties (relative flow speed, fluid temperature and salinity, ice temperature), to better understand the relevant physical quantities involved in the process and, as an application, to capture this submarine melting in general circulation models (GCM). The two most widespread parameterizations are Weeks and Campbell (WC) [21] and the three equations [10], first developed for the basal ice shelf melting. Examples of the use of both schemes in GCM are [2] for the former and [20, 14] for the latter, among others. In general, though, there is evidence that the iceberg melting representation in GCMs is over-simplified and active research brings new insights in how such mechanisms work [6].

The goal of the present study is to quantify the effects of a background rotation on iceberg melting, if any. In section 2 some background information about the current iceberg melting parameterizations and the analytical solutions of the flow below a solid obstacle in a rotating frame of reference in simple configurations, leading to the socalled Taylor columns, is given. Section 3 is devoted to describing the experimental methods and setup to tackle the problem, the results of which are presented in section 4 . Some remaining open issues and conclusions are given in section 5 .

\section{Background}

\subsection{Submarine melting parameterization}

Following a similar experimental procedure to previous work [6], the focus is on the WC submarine melting parameterization [21] that describes the submarine melt rate per unit area SMR as a function of the relative flow speed $U$, the driving temperature $\Delta T=T_{w}$ $-T_{i}>0$ (with $T_{w}$ denoting the water temperature and $T_{i}$ denoting the ice temperature) 
and the length scale of the iceberg in the direction of the flow $\ell$ as

$$
\mathrm{SMR}=K \Delta T \frac{U^{0.8}}{\ell^{0.2}},
$$

where $K$ is a constant of proportionality that contains various physical constants, as described in the following derivation [21].

The SMR is defined as the iceberg volume loss per unit area and per unit time. It can be written as

$$
\mathrm{SMR}=\frac{\Delta V}{A \Delta t},
$$

where $\Delta V$ is the volume loss, $A$ is the area of the iceberg in contact with the fluid and $\Delta t$ is the time interval considered. If the iceberg is already at its melting temperature and thus all the heat transferred from the fluid to the iceberg $Q$ contributes to its melting, then with $\rho_{i}$ denoting the ice density and $L_{i}$ its latent heat of fusion, one can write

$$
\mathrm{SMR}=\frac{Q}{L_{i} \rho_{i} A \Delta t} .
$$

At this point, to generalize this relationship, one introduces the Nusselt number $\mathrm{Nu}$ that, over a length scale $\ell$ and for a fluid with thermal conductivity $\kappa$, is the nondimensional version of the heat per unit time, per unit area and per unit driving temperature transferred between the body and the fluid

$$
q=\frac{Q}{A \Delta t \Delta T}
$$

namely

$$
\mathrm{Nu}=\frac{q \ell}{\kappa} .
$$

This gives that the SMR scales with the Nusselt number as

$$
\mathrm{SMR}=\frac{\Delta T \kappa}{L_{i} \rho_{i} \ell} \mathrm{Nu}
$$

But the scaling of the $\mathrm{Nu}$ with respect to the Reynolds number, $\operatorname{Re}=U \ell / \nu$, with $\nu$ being the fluid viscosity, and the Prandtl number, $\operatorname{Pr}=c_{p} \rho_{w} \nu / \kappa$, with $c_{p}$ being the fluid specific heat and $\rho_{w}$ its density, are known from past experimental works in the form

$$
\mathrm{Nu} \simeq C \operatorname{Re}^{m} \operatorname{Pr}^{n}
$$

where the coefficients $C, m, n$ depend on the geometry of the body and on the Re of the flow [5]. In this work, two geometrical configurations are considered: a flat plate, which has been used to derive the WC parameterization [21], and an infinite cylinder, for reasons that will be clarified in section 3. Table 1 contains the values of $C, m, n$ for $\operatorname{Re} \sim 10^{4}$, which is consistent with the experimental setup described below. With the values for the flow past a flat plate, it is now clear that by using equation (4) in equation (3), the final form of the WC parameterization (1) is obtained.

As already mentioned, the WC parameterization has been applied both to numerical works [2] and experimental ones [6]. In particular, in the laboratory it has been possible 


\begin{tabular}{cccc}
\hline Geometry & $C$ & $m$ & $n$ \\
\hline Flat plate & 0.037 & 0.8 & 0.33 \\
Infinite cylinder & 0.193 & 0.618 & 0.31 \\
\hline
\end{tabular}

Table 1: Coefficients of the scaling of $\mathrm{Nu}$ as a function of Re and $\mathrm{Pr}$ as in equation (4) for Re $\sim 10^{4}$ and for two geometrical configurations [5].

to test its behavior for vanishing relative speed $U$, which is a limit where the free stream forced convection becomes a second order process and the melting is controlled by other mechanisms. For the lateral melting, it has been shown that the forced convection due to the buoyant plumes is the major process for low $U[6]$. This happens because the meltwater produced by the ice block itself, being buoyant due to its lower salinity content, moves upwards forming plumes along the sides of the block. Figure 2 shows that, for low relative velocity $\left(u<w_{p}\right.$, where $w_{p} \simeq 2.5 \mathrm{~cm} \mathrm{~s}^{-1}$ is the characteristic vertical velocity of the plume), the melting is correctly parameterized using the buoyant plume temperature and velocity in the $\mathrm{WC}$ expression. While for higher velocity, $u>w_{p}$, the $\mathrm{WC}$ parameterization with the free stream quantities fits the data well. The schemes in the right panel of the same figure show the different behavior of the vertical plumes in the two regimes (A) and $(\mathrm{C})$. In the former, the meltwater produced by the ice block at the ice-water interface forms buoyant plumes that remain attached to the ice block, which is thus unaware of the free stream flow. In the latter, instead, due to the higher free stream velocity, the plumes are swept away and the free stream velocity and temperature control the forced convection that melts the ice block. There is also an intermediate regime, marked with (B) in the left panel of the figure, that is intermediate between the two.

\subsection{Taylor column dynamics}

Since the goal of the work is to study the effects of background rotation on iceberg melting, the fluid dynamics in a rotating frame of reference past an obstacle is here revised in some simple configurations. In particular, it is well known that in a barotropic, inviscid, fast-rotating flow (in the sense of low Rossby number, as explained more in detail below), the velocity field is independent of the coordinate in the direction of the axis of rotation. In a system where the axis of rotation is vertical, this can be written as $\frac{\partial}{\partial z} \mathbf{u}=0$

and implies that, if an obstacle is placed somewhere in the domain, the flow is forced to go around it not only at the depth where the obstacle is physically present, but also everywhere on top of (or below) it. It is as if the obstacle was virtually extended throughout the entire fluid column. The fluid that occupies this virtual volume remains stagnant and is a so-called Taylor column (TC) [19].

But this is the extreme case where the Coriolis time scale is much smaller than the advective one. In the range between the non-rotating case and this extreme one, the analytical solution for a cylindrical obstacle is now revised following $[12,13]$. Consider a 

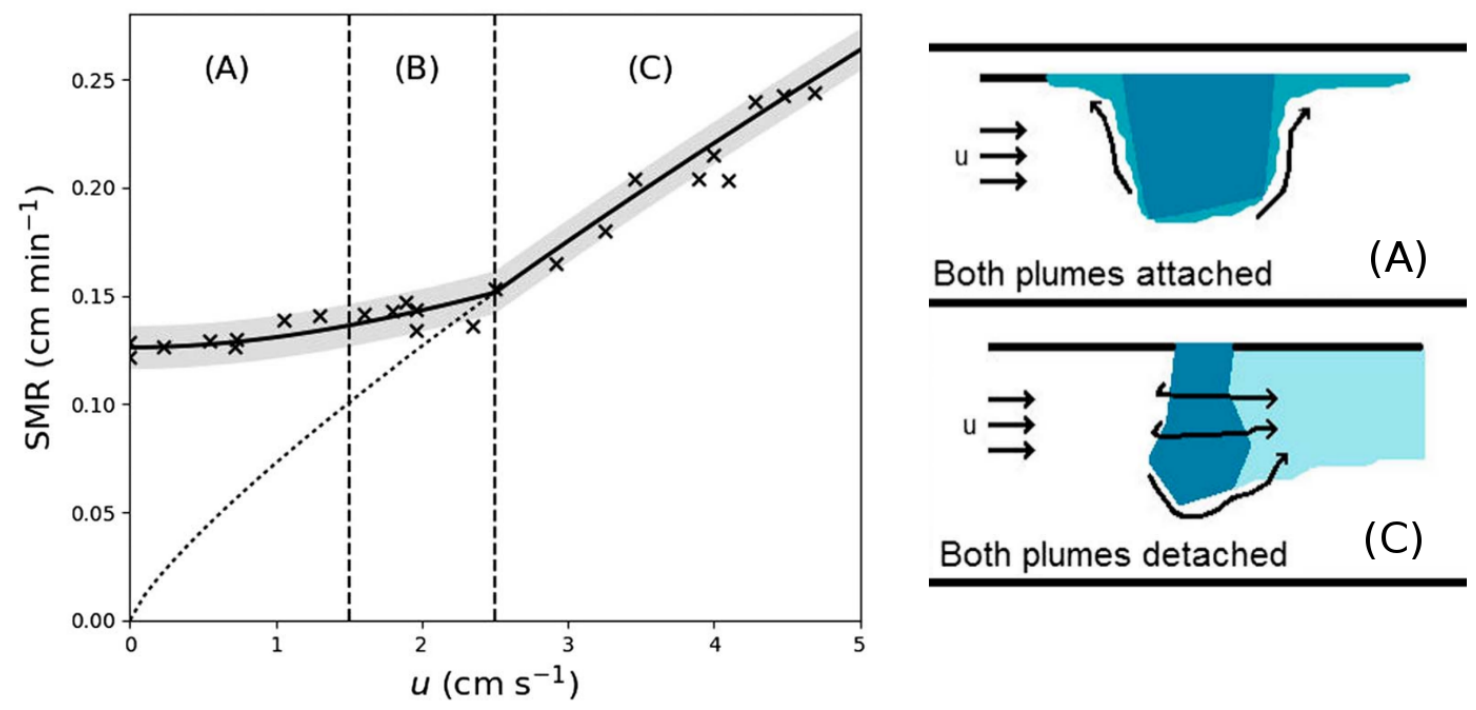

(A)

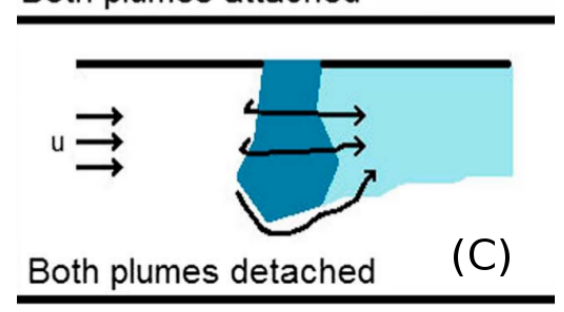

Figure 2: Left panel: lateral SMR as a function of the free stream velocity. The WC parameterization in regimes (A) and (B) is calculated with the plume temperature and velocity, while in regime $(\mathrm{C})$ it uses the free stream ones. Right panel: schemes of the two regimes. (A) shows that for low relative speed the buoyant plumes shield the ice block from the fluid flow and the buoyant convection controls the lateral melting. (C) shows that for high relative speed the plumes are swept away and the forced convection controls the melting. Regime (B) is intermediate and the figure is adapted from [6]. 
homogeneous and constant fluid flow confined between two flat plates distant $H$ from each other and rotating at a constant rate $\Omega$, which sets a background constant absolute vorticity $f=2 \Omega$. Let $U$ be the fluid velocity and choose a frame of reference with the $x$ axis along the direction of the flow, with no loss of generality. If a flat-topped cylindrical obstacle of radius $L$ and height $h \ll H$ is attached to one of the plates, defining the Rossby number as $\mathrm{Ro}=U / f L$ and the nondimensional height of the obstacle as $h_{0}=h / H$, the steady state flow dynamics at low Ro is determined only by the parameter

$$
\alpha=\frac{\operatorname{Ro}}{h_{0}}=\frac{U}{f L} \frac{H}{h}
$$

[12]. In particular, starting from a relatively large $\alpha$, the presence of the obstacle affects the flow by bending the streamlines, following potential vorticity (PV) conservation. As an example, consider that the obstacle is attached to the upper plate. Then, by writing down the nondimensional linearized PV conservation equation as

$$
\frac{D}{D t}\left(\nabla^{2} \Psi+h_{0}\right)=0
$$

where $\Psi$ is the streamfunction, together with the far field conditions

$$
u=-\partial \Psi / \partial y=\alpha \quad \text { and } \quad v=\partial \Psi / \partial x=0 \quad \text { for } \quad x^{2}+y^{2} \rightarrow \infty,
$$

it is easy to understand that if the background vorticity is positive $(f>0)$, the squeezing due to the obstacle $\left(h_{0}>0\right)$ induces a negative (anticyclonic) component in the relative vorticity $\left(\nabla^{2} \Psi<0\right)$. As $\alpha$ increases, a stagnation point appears in the velocity field. It has been shown that the critical value for which this condition is reached depends on the geometry of the obstacle and on the stratification of the fluid [11]. For even lower values of $\alpha$, then, a closed circular streamline below the obstacle appears. Its radius increases for decreasing $\alpha$ and it delimits a region of zero motion, the so-called Taylor column. The panels of the top row of figure 3 show these solutions for three different values of $\alpha$.

Since, as clarified in the following, in the experimental setup there is a horizontal shear in the far field flow, the solution of the problem of the above is obtained in a very similar way for the case of horizontally sheared flow, by adding a second parameter, $\beta$, that controls the horizontal shear [13]. In particular, if the horizontal velocity changes by an amount $\Delta U$ over a cross-flow distance $\Delta y$, the new parameter is defined as

$$
\beta=\frac{\Delta U / \Delta y}{f} \frac{H}{h}
$$

and modifies the $u$ velocity far field condition of equation (7) as

$$
u=-\partial \Psi / \partial y=\alpha+\beta y .
$$

For the rest, the problem is the same and it can be shown that the solutions differ whether the vorticity added by the horizontal shear has the same sign of the vorticity anomaly induced by the squeezing below the obstacle or the opposite. In the former case, the stagnation region is generated for values of $\alpha$ higher than the zero-shear critical one $\alpha_{c}$, while in the latter, the Taylor column occurs for $\alpha<\alpha_{c}$. 
Figure 3 shows the analytical steady solution of the TC problem outlined above, both for zero horizontal shear (top row) and for a positive one (bottom row). The parameters used to plot this figure are taken from the experimental setup as described in section 3 . In the case of positive background rotation $(f>0)$, the direction of the flow along the streamlines (solid thin black) is from left to right, while for $f<0$ it is from right to left. The top row is obtained for a uniform far field velocity $U=4 \mathrm{~cm} \mathrm{~s}^{-1}$, while the bottom row for a positively sheared flow $U=U_{0}+y \Delta U / \Delta y$, with $U_{0}=4 \mathrm{~cm} \mathrm{~s}^{-1}$ and $\Delta U / \Delta y=0.1 \mathrm{~s}^{-1}$. The different columns correspond to different values of $\alpha=\mathrm{Ro}_{0} / h_{0}$ and for the geometry considered, the critical value at which the stagnation point in the zero-shear case appears is $\alpha_{c}=0.5$ [11]. Panels (A), (D) have $\alpha=1>\alpha_{c}$ and do not have any TC, because the background rotation has a rather small effect on the flow and thus the difference between the velocity magnitude of the flow with respect to the far field (denoted with the color shading) is small. Panels (B), (E) have $\alpha=0.5=\alpha_{c}$ and in the zero-shear case, panel (B), the stagnation point at the lower end of the obstacle is visible in correspondence of the cusp in the appropriate streamline. Panel E shows that the presence of a positive shear makes it easier to have a region of no motion (delimited by the black dashed line), because the added background velocity due to the positive $\beta$ is of the same sign of the vorticity anomaly induced by the squeezing of the fluid below the obstacle. Panels (C) and $(\mathrm{F})$, then, have $\alpha=0.25<\alpha_{c}$ and they both show a region of zero motion, which has been shown to grow bigger for lower values of $\alpha$ [12]. It is interesting to notice that while on the lower side of the obstacle the region of zero motion appears, on the upper side there is a relative increase in velocity with respect to the far field profile.

To have a sense of the importance of such dynamics in the real oceans, the following typical values of the quantities defining $\alpha$ are considered. Take a relative speed of $U \sim 10$

$\mathrm{cm} \mathrm{s}^{-1}$, a Coriolis parameter of $f \sim 10^{-4} \mathrm{~s}^{-1}$, a horizontal length scale of the iceberg of $L \sim 20 \mathrm{~km}$, a depth of the water of $H_{w} \sim 10^{3} \mathrm{~m}$ and a draft of the iceberg of $h_{i} \sim 500 \mathrm{~m}$. This leads to

$$
\alpha=\frac{U}{f L} \frac{H_{w}}{h_{i}} \sim 10^{-1}
$$

which is of the same order of magnitude of the critical value $\alpha_{c}=0.5$ considered above, which motivates the current investigation because it shows that rotation can be important in the dynamics in the vicinity of an iceberg and, thus, can impact its melting.

\section{Methods}

The experiments were conducted in a rotating tank with a diameter of $210 \mathrm{~cm}$ and filled with seawater with salinity of roughly $33 \mathrm{~g} \mathrm{~kg}^{-1} \mathrm{kept}$ at room temperature, $18-20^{\circ} \mathrm{C}$. At least 30 minutes before the beginning of each experiment, the rotating tank was turned on to set the fluid in solid body rotation with angular velocity $\Omega_{0}$ and corresponding absolute vorticity $f_{0}=2 \Omega_{0}$. The spin-up time that characterizes this transient fluid acceleration has been largely studied in the past [8] and is given by the expression

$$
\tau_{E}=\frac{H}{\left(2 \nu f_{0}\right)^{1 / 2}},
$$



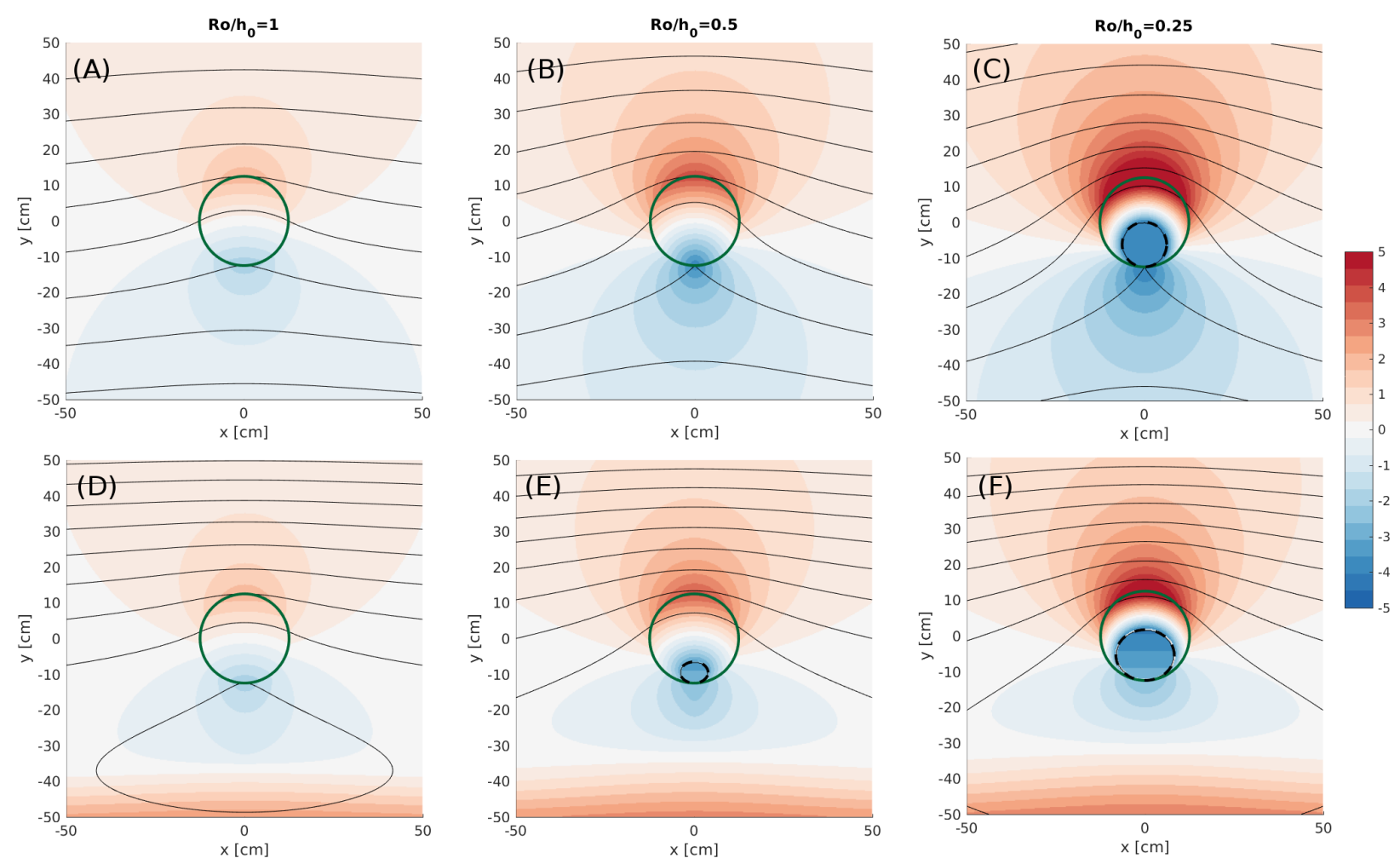

Figure 3: Top row, panels (A), (B), (C): analytical steady solution of the TC problem with zero horizontal shear for different values of $\alpha=\mathrm{Ro}_{0} / h_{0}$ [12]. Bottom row, panels (D), (E), (F): solution of the same problem and for the same values of $\alpha$, but with positive horizontal shear with $\Delta U / \Delta y=0.1 \mathrm{~s}^{-1}$ [13]. The black solid thin lines are streamlines, while the color shading indicates the difference between the flow velocity magnitude and the far field velocity profile: constant and equal to $4 \mathrm{~cm} \mathrm{~s}^{-1}$ in the top row and increasing with $y$ with $U(y=0)=4 \mathrm{~cm} \mathrm{~s}^{-1}$ in the bottom row. The green solid line is the section of the cylindrical obstacle and the black dashed line in panels $(\mathrm{C}),(\mathrm{E})$ and $(\mathrm{F})$ delimits the region of no motion predicted by the analytical solutions. 
with $H$ being the fluid column depth and $\nu$ its viscosity. The physics that controls this spinup process involves the Ekman boundary layer at the bottom of the tank, which pushes the fluid far from the axis of rotation and, by mass conservation, brings fluid parcels with higher angular momentum, that accelerate the fluid throughout the tank, inwards.

Once the fluid was in solid body rotation, a cylindrical ice block of radius $L$ was suspended in the water with a wooden support at a distance $R$ from the axis of rotation and with a submerged part of height $h$ (see figures 4 and 5 ). At this point, since the ice block was fixed in the frame of reference of the tank, two options were available to set a relative flow between the seawater and the ice block. The first consisted of increasing the rotation rate of the tank by a certain amount $\Delta \Omega$ at the beginning of the experiment, so that the relative flow speed at the center of the obstacle was initially $U_{0}=R \Delta \Omega$. This resulted, according to the spin-up dynamics described before [8], in a relative flow decaying almost exponentially

$$
U(t) \simeq U_{0} \mathrm{e}^{-t / \tau_{E}}
$$

in the frame of reference of the tank and the ice block. The second option, instead, consisted in increasing the rotation rate of the tank by a smaller amount $\delta \Omega<\Delta \Omega$ at regular intervals $\delta t$, calculated using the exponential decay above, so that the relative flow speed would be constant. In particular, using the equivalent of equation (11) for the angular velocity together with the expression of the spin-up time (10), the interval $\delta t$ at which the tank acceleration is needed to balance the relative velocity, decay was found by inverting

$$
\delta \Omega=\Delta \Omega\left(1-\mathrm{e}^{-t / \tau_{E}}\right)
$$

after choosing $\delta \Omega$ to be some fraction of $\Delta \Omega$. The choice for the experiments was $\delta \Omega$ $=\Delta \Omega / 10$, so that the values of $\delta t$ were between 15 to $40 \mathrm{~s}$, as a function of the initial background rotation, $f_{0}$. This procedure was tested for different values of $f_{0}, R$, $\Delta \Omega$ by measuring the fluid angular velocity with floating tracers and after a few trial and error tests, it was possible to keep the relative flow speed constant, with fluctuations of the order of $5 \%$ (not shown). Despite some experiments that were carried in the first configuration (constant $f$ decaying $U$ ), all the data analyzed and shown in what follows come from the series of experiments with constant $U$ and increasing $f$.

The ice blocks were made in stainless steel cylindrical molds with radius $L=12.5$ $\mathrm{cm}$ and were roughly $5 \mathrm{~cm}$ tall, so that they could be half submerged during the experiments, resulting in values of $h \simeq 2$ or $3 \mathrm{~cm}$. The water used to make the ice blocks was deaired and dyed with $2 \mathrm{ml}$ blue food colorant in order to be able to distinguish the meltwater from the seawater of the tank. There are two reasons why the ice blocks were cylindrical. The former is because the focus of this work was on the basal iceberg melting rather than the lateral one. Thus, since the circle is the figure that maximizes the area for a given perimeter, the cylindrical shape has the highest basal-to-lateral area ratio for a given height. This means that it is the optimal choice to study the melting coming from the base and for the values of the experiments, $h \sim 2 \mathrm{~cm}$ and $L=12.5 \mathrm{~cm}$, the area of the base $\pi L^{2}$ is roughly three times larger than the area of the side $2 \pi L h$. The latter reason is because the analytical solutions for the TC problem, described in section 2.2, has been developed only for flat-topped cylindrical obstacles $[12,13]$. 

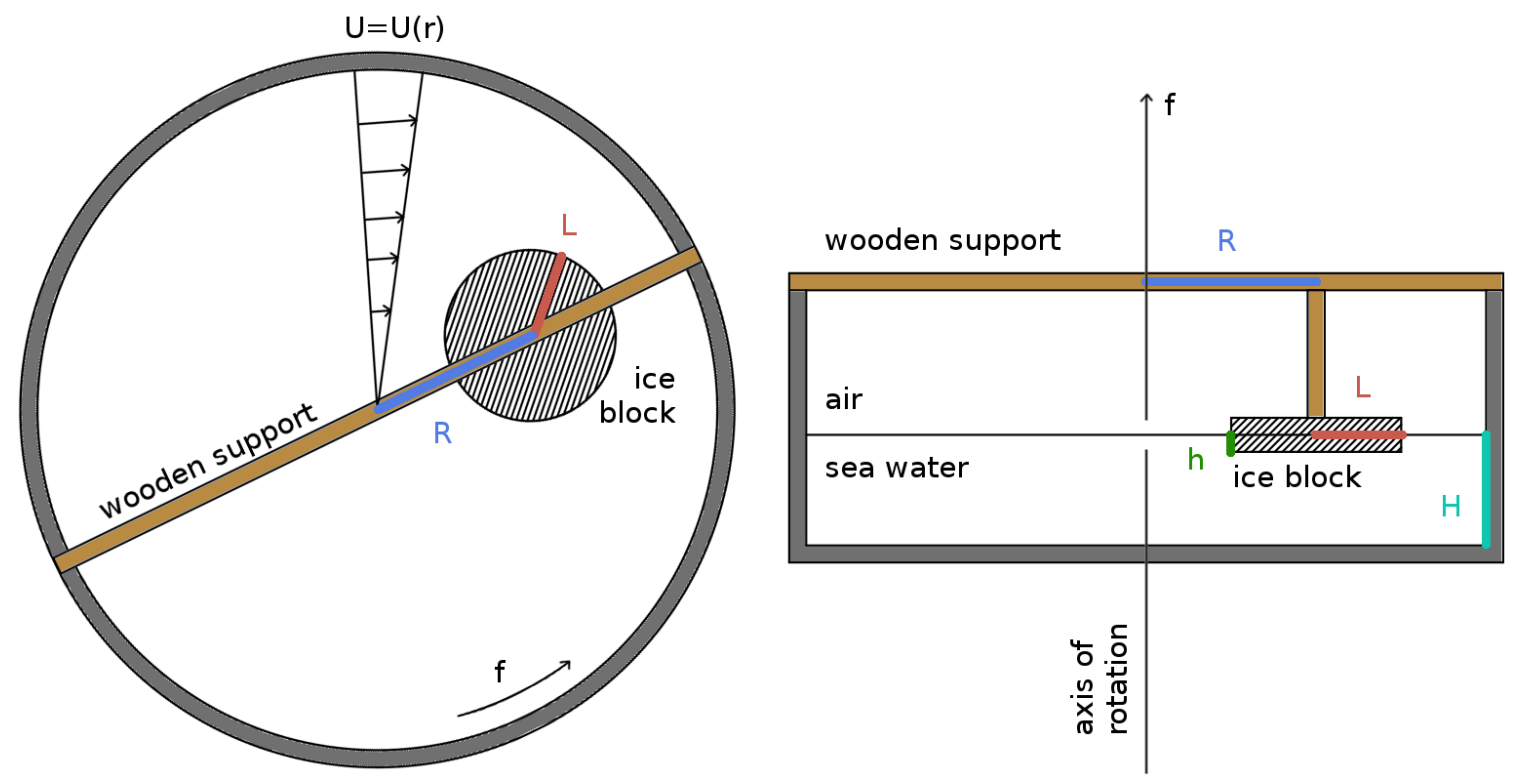

Figure 4: On the left, a top view schematic of the apparatus (not to scale), where the radially increasing relative velocity profile $U=U(r)$ in the frame of reference of the ice block is drawn. The distance of the center of the ice block (hatched) from the axis of rotation $R$, the radius of the ice block $L$ and the direction of the rotation in the frame of reference of the laboratory $f$ are marked, as well. On the right, a side view of the same apparatus is sketched with also the draft of the ice block $h$ and the depth of the seawater $H$. 


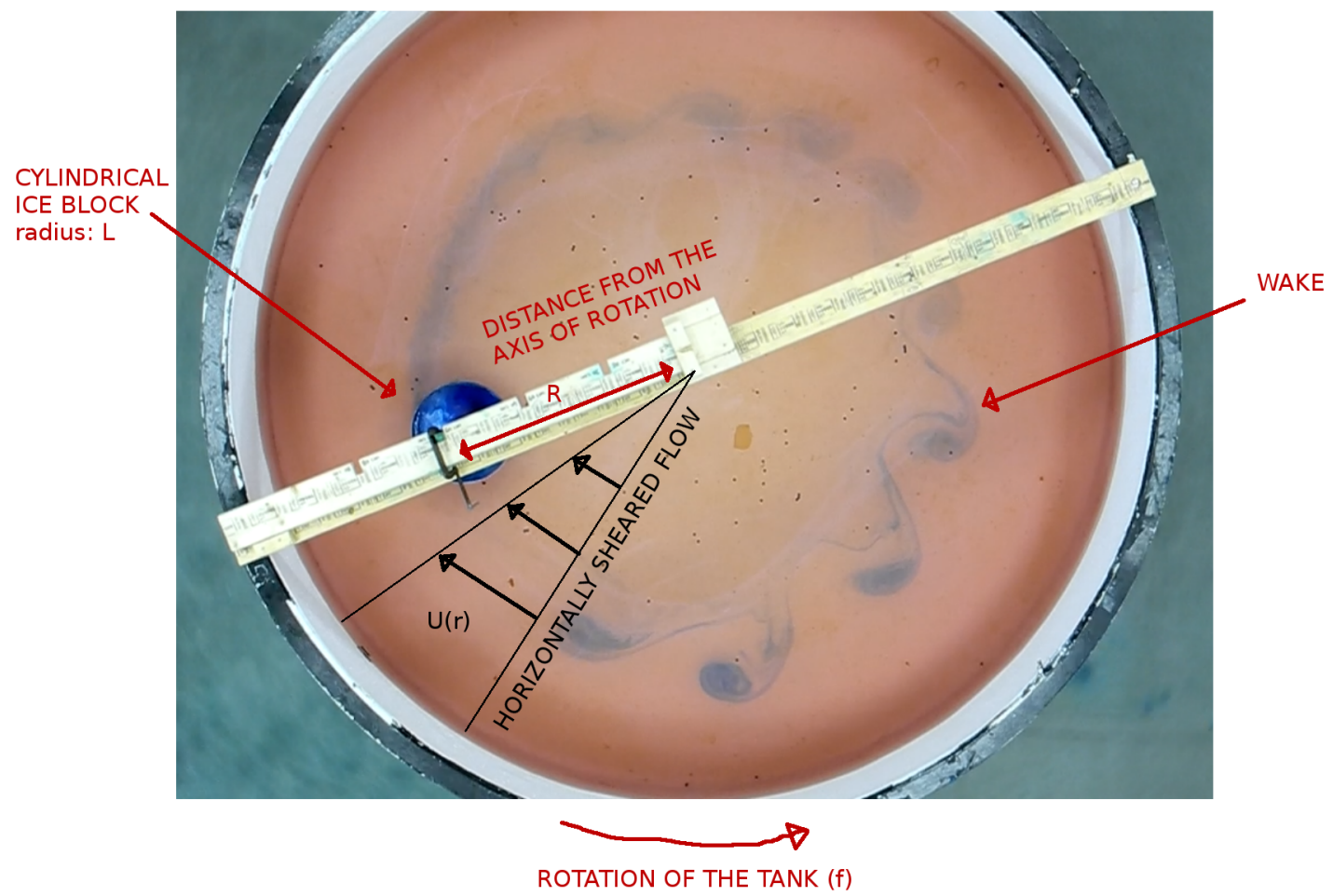

Figure 5: Top view picture of the apparatus during an experiment that shows how all the elements sketched in the previous schematic looked like in the laboratory. 
Special attention was paid during the experiments with high background rotation rate because the water surface was free to adjust to the well known paraboloid shape reached at the balance between the centrifugal acceleration and the hydrostatic pressure gradient. Denoting with $H_{\text {eff }}$ the effective height of the water as a function of the radial distance $r$ from the axis of rotation and the rotation rate itself $\Omega$, one can write

$$
H_{e f f}(r, \Omega)=H+\frac{\Omega^{2}}{4 g}\left(2 r^{2}-D^{2}\right)
$$

where $g$ is the acceleration due to gravity, $D=105 \mathrm{~cm}$ is the radius of the rotating tank and $H$, the non-rotating value, was chosen to be equal to $12 \mathrm{~cm}$. All the experiments, even those with the most tilted free surface, were conducted making sure that the upper side of the ice block was never submerged in order to avoid the introduction of an extra melting source.

For each experiment, two quantities were measured: the mass loss $\Delta m$ and the initial submerged draft $h_{0}$. To understand how the SMR was obtained, let us consider a reasonable scenario in which the average radius of the cylindrical ice block $L=L(t)$ decreases linearly in time as

$$
L(t)=L_{0}-\dot{\delta} t
$$

and the average submerged height as

$$
h(t)=h_{0}-\dot{\epsilon} t
$$

The lateral and basal ice block melt rates are thus

$$
\frac{d L}{d t}=-\dot{\delta}, \quad \frac{d h}{d t}=-\dot{\epsilon}, \quad \text { with } \quad \dot{\delta}, \dot{\epsilon}>0
$$

and supposing that they are constant one can write that over a time interval $\Delta t$ the radius and the height decrease by an amount $\delta=\dot{\delta} \Delta t$ and $\epsilon=\dot{\epsilon} \Delta t$, respectively. Given the expressions (14) and (15), the submerged instantaneous area is

$$
A(t)=\pi L(t)[L(t)+2 h(t)]
$$

and the corresponding volume is

$$
V(t)=\pi L^{2}(t) h(t)
$$

which decreases in time with a rate

$$
\frac{d V}{d t}=-\pi L(t)[L(t) \dot{\epsilon}+2 h(t) \dot{\delta}] .
$$

This enables us to write the instantaneous SMR as

$$
\operatorname{SMR}(t)=-\frac{1}{A(t)} \frac{d V}{d t}=\frac{L(t) \dot{\epsilon}+2 h(t) \dot{\delta}}{L(t)+2 h(t)}>0 .
$$

It is now interesting to have a sense of how the side melt rate and the basal melt rate compare. To do so, the two different geometrical configurations (flat plate and infinite 
cylinder) discussed in section 2.1 for the heat turbulent transfer are considered. If the side melt rate is taken to scale with the Re as if the flow was around an infinite cylinder and if the basal melt rate scales as if flow was past a flat plate, the ratio $\dot{\delta} / \dot{\epsilon}$ is

$$
\frac{\dot{\delta}}{\dot{\epsilon}} \simeq 0.81
$$

using the coefficients of table 1 , the typical velocity of the experiments $U=4 \mathrm{~cm} \mathrm{~s}^{-1}$ and the radius of the cylinder $L=12.5 \mathrm{~cm}$ as a length scale. This justifies the assumption

$$
\dot{\delta}=\dot{\epsilon}
$$

which, if replaced in the expression (20), shows immediately that the SMR is constant and equal to

$$
\mathrm{SMR}=\dot{\epsilon}=\dot{\delta}
$$

Over a time interval $\Delta t$, the ice block volume loss with the assumption $\delta=\epsilon$ is simply

$$
|\Delta V|=\int_{0}^{\Delta t} \mathrm{~d} t\left|\frac{d V}{d t}\right|=\pi\left[L_{0}^{2} h_{0}-\left(L_{0}-\epsilon\right)^{2}\left(h_{0}-\epsilon\right)\right] .
$$

Thus, the correct value of the area $A^{*}$ to be used when calculating the SMR of the experiments in the form

$$
\mathrm{SMR}_{A^{*}} \overline{\frac{|\Delta V|}{t}}
$$

can be inferred by inverting the above expression to get to the first order in $\epsilon$

$$
A^{*}=\pi\left[L_{0}\left(L_{0}+2 h_{0}\right)-\epsilon\left(2 L_{0}+h_{0}\right)\right]
$$

This, to same order in $\epsilon$, can be shown with little algebra to be equal to the average area

$$
A_{a v}=\frac{1}{2}\left(A_{i n}+A_{f}\right),
$$

where

$$
A_{\text {in }}=\pi L_{0}\left(L_{0}+2 h_{0}\right) \quad \text { and } \quad A_{f}=\pi\left(L_{0}-\epsilon\right)\left[\left(L_{0}-\epsilon\right)+2\left(h_{0}-\epsilon\right)\right]
$$

are simply the initial and the final submerged ice block areas. Since from the measurements $\Delta V=\Delta m / \rho_{i}, h_{0}$ and $L_{0}$ are known, the two equivalent ways of obtaining the SMR from the experiments are to invert equation (24) to get $\epsilon$ and then find SMR as in equation (23) dividing $\epsilon$ by the duration of the experiment, or to calculate the average area of equation (27) imposing the same volume integral of equation (24) and then apply the definition (25) with $A^{*}=A_{a v}$. Note that the duration of the experiments, $\Delta t=3$ min, was chosen so that the final shape was still a quite regular cylinder and in the case of high background rotation rate, where the free surface was significantly tilted, the mean value of the submerged height of the cylinder was used to calculate the geometrical properties of the block. The time interval was short enough to linearize the evolution of the submerged area in time and, thus, to justify the truncation to the first order in $\epsilon$ of the expressions (26) and (27). 


\section{Results}

In this section, the experimental data obtained from four series of experiments with different values of free stream velocity, $U \in\{0,2,4,6\} \mathrm{cm} \mathrm{s}^{-1}$, are described.

Figure 6 shows the SMR of all the experiments as a function of $U$. The data points are color-coded with the value of $\alpha=\mathrm{Ro} / h_{0}$ (except for $U=0 \mathrm{~cm} \mathrm{~s}^{-1}$, where $\alpha=0$ because $\mathrm{Ro}=0$ ), that controls the TC dynamics as described in section 2.2. The solid line shows the WC parameterization, as in equation (1), with $K=0.052, \Delta T=18^{\circ} \mathrm{C}$ and $\ell=2 L=25 \mathrm{~cm}$ (note that here the diameter of the ice block is used). The value of $K$, as discussed in 2.1, contains several physical constants of the system and it is here chosen so that all the inputs of the parameterization are taken in S.I. units and the SMR is measured in $\mathrm{cm} \mathrm{min}^{-1}$. As for $\Delta T$, it is taken as the difference between the seawater room temperature, $18^{\circ} \mathrm{C}$, and the ice freezing temperature in freshwater, $0^{\circ} \mathrm{C}$, because the temperature gradient between ice and seawater is much larger than their salinity gradient and thus, the ice is in a condition of pure melting with no dissolution. Physically, it means that a thin layer of freshwater insulates the ice block from the seawater, so that the salinity at the interface is zero and the freezing temperature is, accordingly, $0^{\circ} \mathrm{C}$.

Back to figure 6 , the experimental error is shaded along the parameterization to have an estimate of the uncertainty in the matching between the data and the parameterized line. The first evident feature is that for $U=0 \mathrm{~cm} \mathrm{~s}^{-1}$ and $U=2 \mathrm{~cm} \mathrm{~s}^{-1}$, the SMR is much higher than the parameterized one, which is indicative of another process controlling the melting for low free stream velocity, as found in previous works [6]. The hypothesis is that at low $U$, the positively buoyant meltwater formed at the base of the ice block will flow as a gravity current faster than the free stream velocity. Unfortunately, due to the configuration of the experimental setup, it has not been possible to observe this processes at the base of the ice block accurately. Thus, further experiments with submerged cameras and/or in a non-rotating transparent tank are encouraged to better observe and understand what happens at the base of the block.

As a first simple attempt to include this basal meltwater pool process in the SMR dependence on the free stream velocity, a constant SMR below a certain threshold velocity $U_{t h r}$ was suggested. In particular, $U_{t h r}$ was calculated inverting the same $\mathrm{WC}$ parameterization as before,

$$
\mathrm{SMR}_{U=0}=K \Delta T \frac{U_{t h r}^{0.8}}{\ell^{0.2}}
$$

assuming the SMR to be equal to the value obtained from the experiments at zero relative flow velocity, $\mathrm{SMR}_{U=0}=0.09 \pm 0.01 \mathrm{~cm} \mathrm{~min}^{-1}$. The value obtained is $U_{t h r}=3.8 \pm 0.5 \mathrm{~cm}$ $\mathrm{s}^{-1}$ and figure 7 shows the just mentioned constant SMR behavior below this threshold. Data points at high $\alpha$ (which means low rotation rate) of both the $4 \mathrm{~cm} \mathrm{~s}^{-1}$ and $2 \mathrm{~cm} \mathrm{~s}^{-1}$ series agree quite well with this constant value.

Let us now focus on a single series of experiment with constant non-zero free stream velocity. A common feature that the three series with $U \in\{2,4,6\} \mathrm{cm} \mathrm{s}^{-1}$ share is that as $\alpha$ decreases, the SMR increases. The SMR as a function of $\alpha$ for $U=4 \mathrm{~cm} \mathrm{~s}^{-1}$ is shown in figure 8. The data points, together with their uncertainty, are shown as green dots. The

uncertainty on the SMR, $\sigma_{\mathrm{SMR}}=0.01 \mathrm{~cm} \mathrm{~min}^{-1}$, is obtained from few repetitions of the same experiment and has then been extended to all the data points. While the uncertainty 


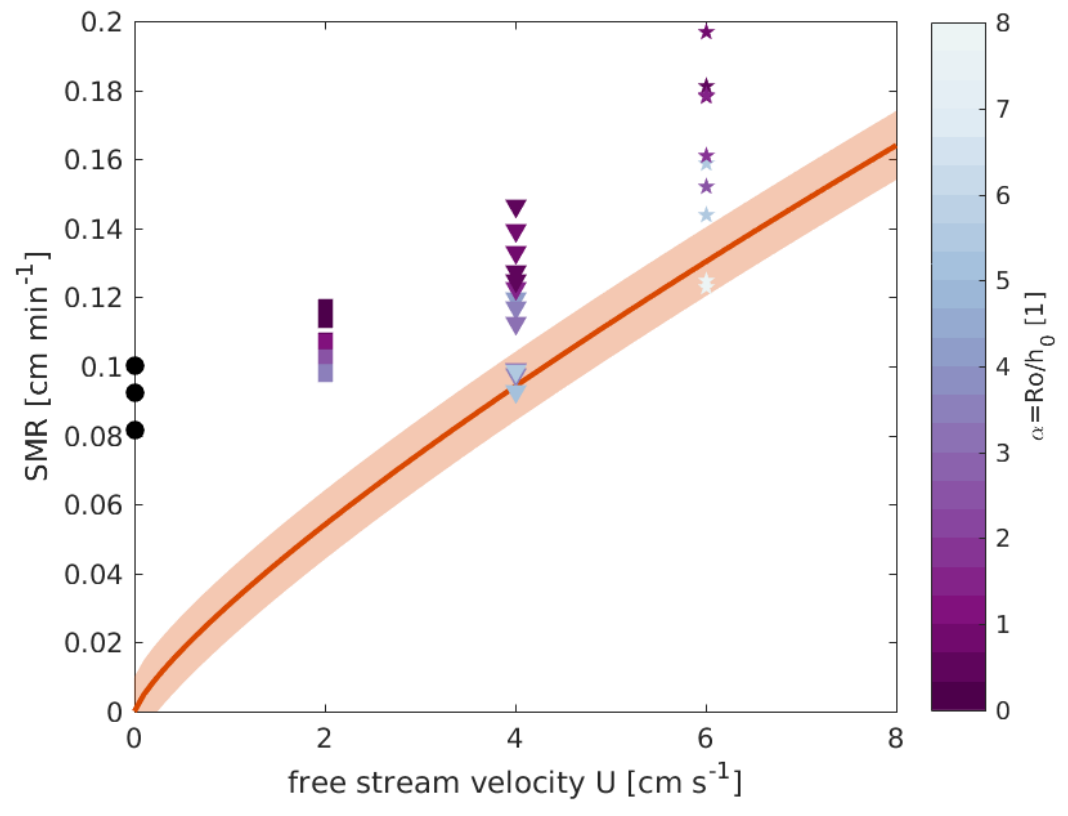

Figure 6: SMR as a function of the free stream velocity $U$ for all the experiments considered. The WC parameterization of equation (1) is shown as a solid line with the experimental error shaded. The color of the data points is chosen according to the value of $\alpha$ of each experiment. 


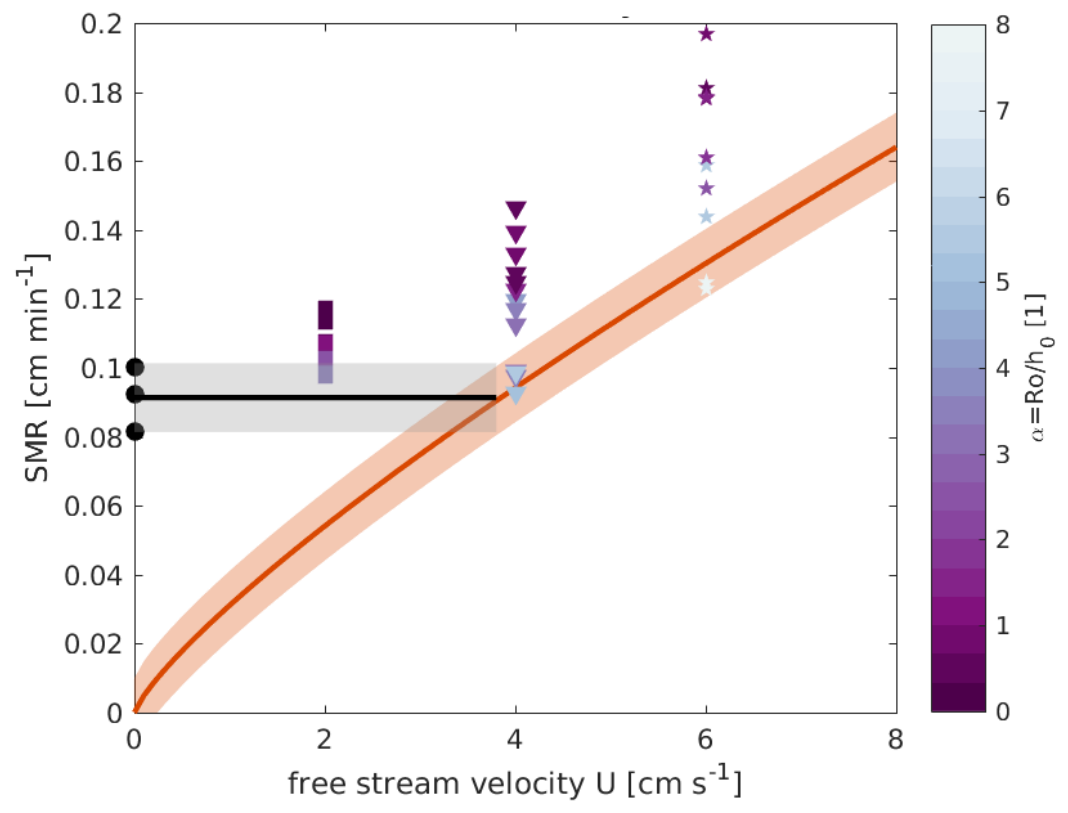

Figure 7: With respect to figure 6, a constant SMR line is added (black) in correspondence of the experimental value obtained with no relative flow, $\mathrm{SMR}_{U=0}=0.09 \pm 0.01 \mathrm{~cm} \mathrm{~min}^{-1}$, for relative velocity below the threshold $U_{t h r}=3.8 \pm 0.5 \mathrm{~cm} \mathrm{~s}^{-1}$. See the text for further details. 
on $\alpha$ comes both from the experimental uncertainty on $h$, the draft of the ice block, and from the fact that to maintain the relative velocity $U$ constant, the rate of rotation had to increase throughout the experiment. In this figure, the significant increasing trend as $\alpha$ decreases, i.e. the background rotation increases, is visible. The black solid line denotes the value of the $\mathrm{WC}$ parameterization calculated as before, for $U=4 \mathrm{~cm} \mathrm{~s}^{-1}$, which is constant because it does not depend anyhow on the background rotation rate. The first step to try to understand the increasing trend of melt rate for increasing background rotation was to include the TC dynamics in the WC parameterization. To do so, instead of using the free stream velocity $U$, the area average velocity over the base of the cylindrical obstacle of the analytical steady solutions of the TC problem of section $2.2, \bar{U}$, is used to calculate the parameterized SMR shown with the red line. Going from right to left, namely going towards higher $f$, there is a small peak in the melt rate, due to the relative increase in velocity on one of the side of the obstacle (see figure 3), but then, as the TC grows bigger, the average velocity at the base of the obstacle decreases, determining a relatively fast decrease in the melt rate, as well. The problem of such approach is that it neglects the well-known fact that the parameterization underestimates the melting as the velocity vanishes. To avoid that, the value of the threshold velocity $U_{t h r}$ determined above is replaced in the analytical solution pointwise wherever the velocity magnitude drops below the value $U_{t h r}$ itself and then the area average of this effective field is found, $U^{*}$. The effect of such correction on the SMR is shown by the blue line, together with the uncertainty associated to the $U_{t h r}$ itself. It still underestimates the experimental data, but the increasing trend as $\alpha$ decreses is captured. It is important to underline that this last threshold correction is done only in terms of the melt rate and there is no claim on the description of the effective velocity field due to the meltwater pool that accumulates at the base of the obstacle.

Figures 9 and 10 show the data points of the series of experiments with $U=2 \mathrm{~cm}$ $\mathrm{s}^{-1}$ and $U=6 \mathrm{~cm} \mathrm{~s}^{-1}$, respectively, together with the same curves introduced in figure 8 . While for $U=2 \mathrm{~cm} \mathrm{~s}^{-1}$, the velocity is always below threshold and thus there is not a strong dependence on the rotation rate neither in the experimental data points nor in the corrected velocity $U^{*}$ parameterization, which agree quite well, for $U=6 \mathrm{~cm} \mathrm{~s}^{-1}$ the trend in the data points as a function of $\alpha$ is stronger and the $U^{*}$ parameterized curve is further below the data with respect to the case with $U=4 \mathrm{~cm} \mathrm{~s}^{-1}$.

This is an indication that some other mechanism is happening at the base of the ice block and it is still not described in the parameterization. A possible explanation for that comes by looking a figure 11, which shows two pictures of the bottom of the ice blocks after two different experiments with the same $U=4 \mathrm{~cm} \mathrm{~s}^{-1}$, but different background rotation: the left panel shows the ice block after an experiment with low $f$ and the right panel with high $f$. One has to imagine that the flow was coming from the top of the picture, with the velocity radially increasing in the tank from right to left. Even if no quantitative measurements were done, this picture clearly shows that with the same relative speed $U$, the melting at the base of the ice block was higher for the experiment with high $f$ with respect to the one with low $f$. In particular, the melting was enhanced on the outer edge of the block, because of the relative increase in velocity on one side of the cylinder at low $\alpha$, as shown in figure 3. This physically explains in general the trend of increasing melt rate as the background rotation increases for the various $U$. But by taking a closer look to the pictures, one might notice that in the case of high background rotation rate, the melting 


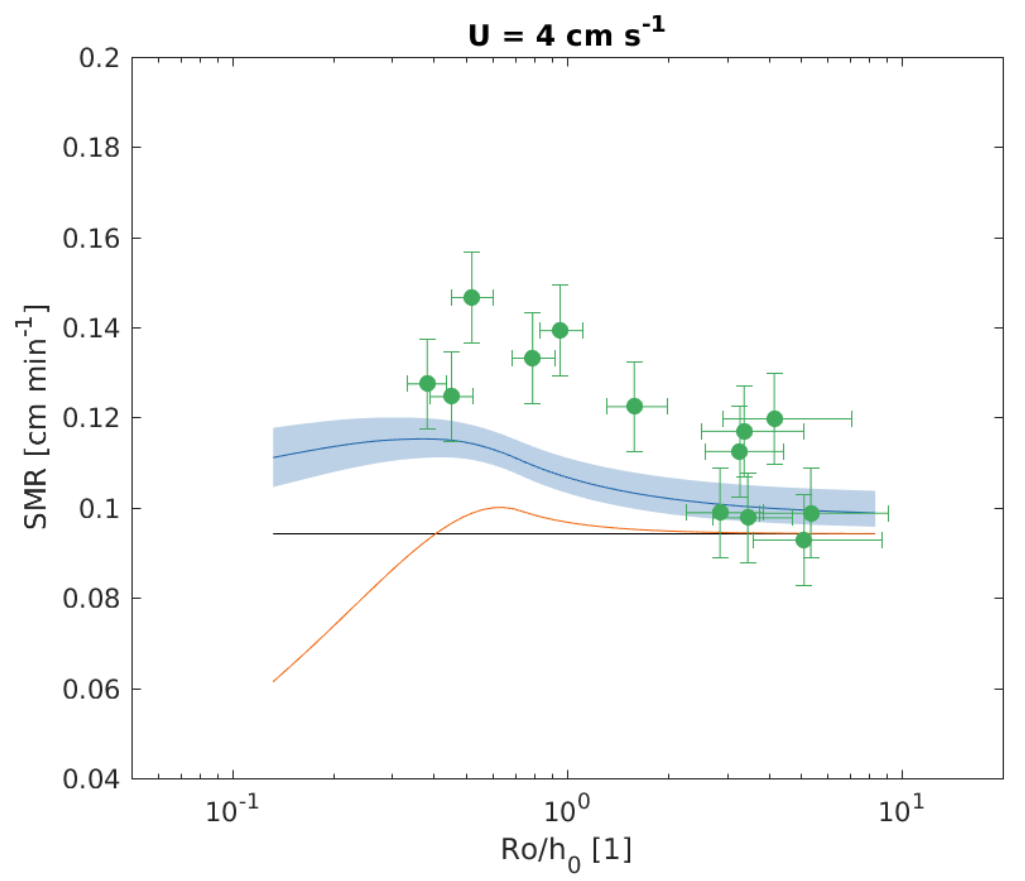

Figure 8: SMR as a function of $\alpha$ for $U=4 \mathrm{~cm} \mathrm{~s}^{-1}$. The black constant line is the WC parameterization; the red line includes the TC dynamics using the parameterization the area average velocity magnitude of the analytical solution of the TC problem, $\bar{U}$; and the blue line includes both the TC dynamics and the threshold behavior observed in figure 7 applied pointwise before taking the velocity area average to find the corrected velocity $U^{*}$. See the text for further details. 


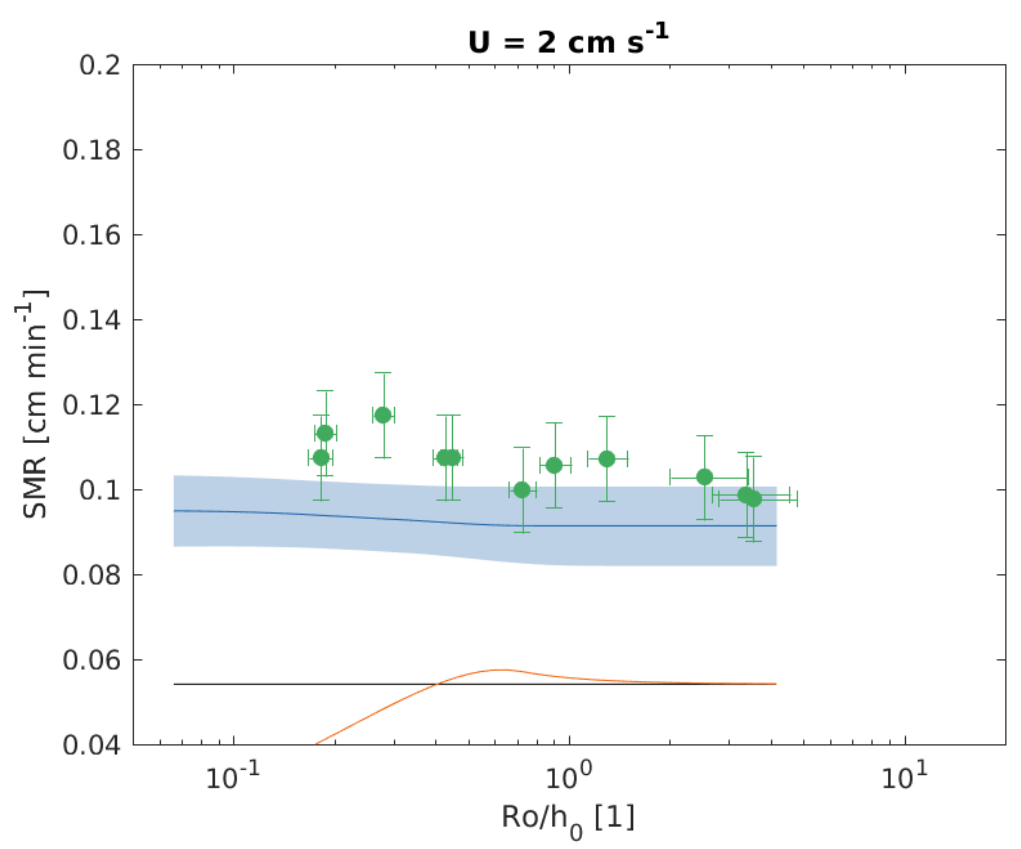

Figure 9: As in figure 8 but for $U=2 \mathrm{~cm} \mathrm{~s}^{-1}$.

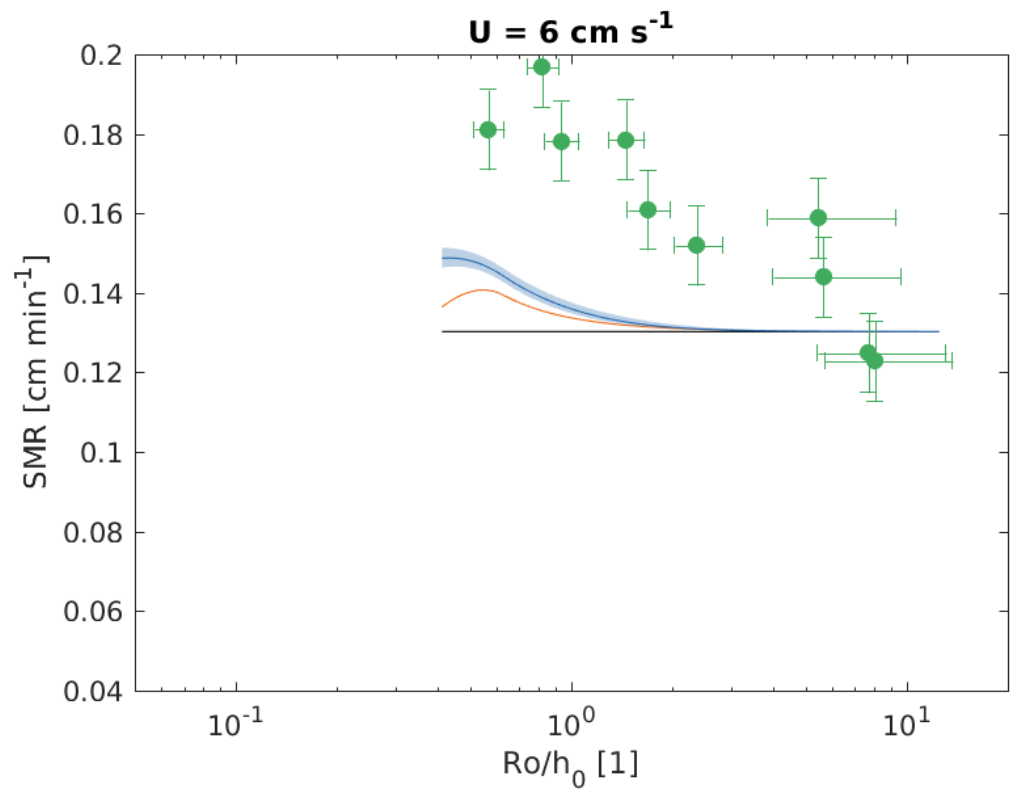

Figure 10: As in figure 8 but for $U=6 \mathrm{~cm} \mathrm{~s}^{-1}$. 


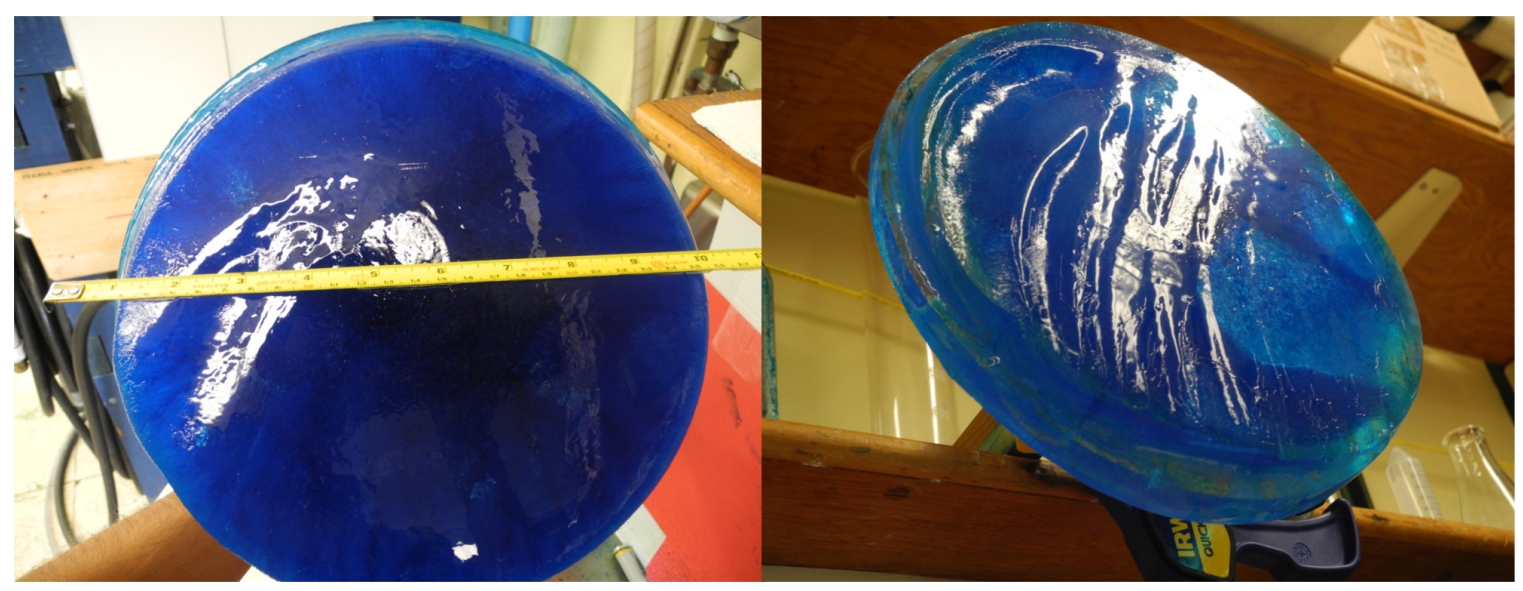

Figure 11: Two pictures of the bottom of the ice blocks after two experiments with the same free stream velocity $U=4 \mathrm{~cm} \mathrm{~s}^{-1}$. In the left panel the rotation rate was lower than the one in the right panel and, in particular, a partial TC was observed in the experiment of the right picture, which was not the case in the experiment of the left one. Wider and deeper elongated melted stripes are visible in the right panel, due to the increased velocity at the base of the ice block determined by the TC dynamics.

happens along some elongated stripes, which are more numerous, deeper and wider than in the case of low background rotation. This suggests that some form of instability growth linked to this little channel might explain the extra melting that the parameterization is not able to explain.

Finally, figure 12 is the same as figure 6 but with the corrected velocity $U^{*}$, which takes into account both the TC dynamics and the threshold behavior observed in figure 7 , instead of the free stream one, $U$. The data points have different shape to distinguish their free stream velocity and one can see that, although the agreement is still not very good, especially for the very low $\alpha$ high free stream velocity $U$ data, the WC parameterization calculated with the corrected velocity $U^{*}$ captures the increased melting at high rotation rate and the threshold behavior better than the same parameterization with the free stream velocity $U$.

\section{Conclusions}

Through laboratory experiments in a rotating tank with cylindrical ice blocks mimicking Antarctica tabular icebergs, the effects of background rotation on iceberg melting were investigated. Preliminary results show that at high rotation rate, the base of the ice block melts at a higher rate because of an increased average basal velocity below the block itself. This results from the enhanced relative vorticity below the block following the squeezing of the fluid column in the rotating system. In particular, at low background rotation, the fluid flow is almost unaffected. When increasing the rotation, i.e. lowering $\alpha$, the ratio of the Rossby number and the non-dimensional height of the obstacle, the TC starts forming on one side of the obstacle and partially covers its base. In this condition, where the TC 


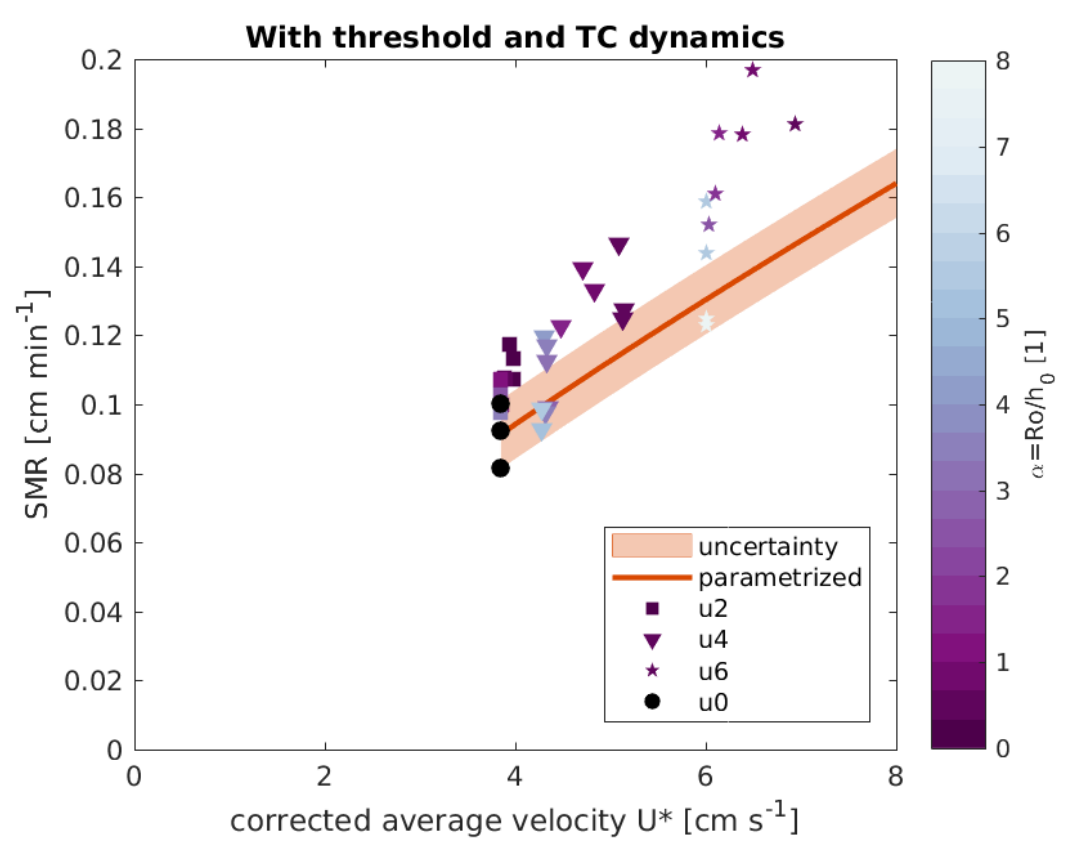

Figure 12: Similarly to figure 6, the SMR is shown as a typical velocity, which here is the corrected velocity $U^{*}$ which takes into account both the TC dynamics and the threshold behavior observed above, instead of the free stream velocity $U$, as was in figure 6 .

is not present, the flow velocity is larger than the far-field value, which is thought to be responsible for the observed higher melting. At even higher background rotation, i.e. lower $\alpha$, the TC extends its area until it covers the entire ice block base. It is expected, then, that the melt rate is no longer dependent on the rotation rate, because the $\mathrm{TC}$ dynamics do not modify the flow structure anymore.

The WC parameterization [21] is then applied to describe the trend of the SMR as a function of the free stream velocity $U$. It is observed that for low relative velocity, below a certain threshold $U_{t h r}$, the basal melt is not controlled by the forced convection due to $U$, but it is probably controlled by a form of upside-down gravity current formed by the buoyant meltwater pool that shields the ice block from the external fluid flow. More work is needed to better understand what is happening in this region, both in a non-rotating environment and in a rotating one. Two corrections have been done to the $\mathrm{WC}$ parameterization to include the TC dynamics and the observed change in regime at low relative free stream velocity. Given the analytical steady solution for the velocity magnitude below a cylindrical obstacle in a rotating frame of reference $u_{T C}(x, y)[12,13]$, wherever its value falls below the threshold velocity $U_{t h r}$, it is replaced by $U_{t h r}$ itself. Then, the area average of this corrected velocity field over the base of the obstacle $U^{*}$ is used as effective velocity in the WC parameterization, resulting in a better agreement between data and theory.

However, some aspects of the dynamics that are thought to be important are yet to be included in the description of the process. Among the others, there are: the effects of the meltwater on the TC dynamics through changes in the stratification, which are known to introduce a dependence of the velocity on the height [11]; the curvature of 
the streamlines due to the geometrical shape of the tank; the time transient features of the flow response and the turbulence added in the flow by the finiteness of the cylindrical obstacle. The possibility of an unstable growth of the melted channels at the base of the block (see figure 11) might partially explain the higher melting measured in the laboratory with respect to the parameterization, as well.

Despite a few issues that still need to be considered, the present work shows that the back-ground rotation can increase the basal melting of the large tabular icebergs due to modifi-cations of the flow typical of a rotating system and that for low relative velocity the basal melt is not controlled by the free-stream velocity.

\section{Acknowledgments}

Thanks Craig, Claudia, Bruce for the good work done together. I really learnt a lot and had a good time in the lab. Thanks Kate for the very good idea. I found it fascinating from the very beginning. Thanks Anders for the extremely precious help in building the apparatus and the ice block molds. Thanks to the lab crew, Madi, Eric, Thomasina and Earle. Thanks to the Barn crew, Robert, Federico, Guillaume and all the visitors. Thanks Margaret for the eclipse sun glasses. Thanks to the special environment of the school and to all the people that made it possible. And thanks Claudia for the suggestion of applying to the program and the continuous support.

\section{References}

[1] G. R. BIGG, Icebergs: Their science and links to global change, Cambridge University Press, 2015.

[2] G. R. Bigg, M. R. Wadley, D. P. Stevens, and J. A. Johnson, Modelling dynamics and thermodynamics of icebergs, Cold Reg. Sci. Technol., 26 (1997), pp. 113135 .

[3] G. R. Bigg And D. J. Wilton, Iceberg risk in the Titanic year of 1912: Was it exceptional?, Weather, 69 (2014), pp. 100-104.

[4] L. P. A. Duprat, G. R. BigG, And D. J. Wilton, Enhanced Southern Ocean marine productivity due to fertilization by giant icebergs, Nat. Geosci., 9 (2016), pp. 219-221.

[5] E. R. G. Eckert and R. M. Drake, Heat and mass transfer, McGraw-Hill Book Co., 1959.

[6] A. Fitzmaurice, C. Cenedese, and F. Straneo, Nonlinear response of iceberg side melting to ocean currents, Geophys. Res. Lett., 44 (2017).

[7] A. FitzMaurice, F. Straneo, C. Cenedese, and M. Andres, Effect of a sheared flow on iceberg motion and melting, Geophys. Res. Lett., 43 (2016), pp. 12520-12527.

[8] H. P. Greenspan and L. N. Howard, On a time-dependent motion of a rotating fluid, J. Fluid Mech., 17 (1963), pp. 385-404. 
[9] J. J. Helly, R. S. Kaufmann, G. R. Stephenson, and M. Vernet, Cooling, dilution and mixing of ocean water by free-drifting icebergs in the Weddell Sea, DeepSea Res. II, 58 (2011), pp. 1346-1363.

[10] D. M. Holland And A. Jenkins, Modeling thermodynamic ice-ocean interactions at the base of an ice shelf, J. Phys. Oceanogr., 29 (1999), pp. 1787-1800.

[11] H. E. Huppert, Some remarks on the initiation of inertial Taylor columns, J. Fluid Mech., 67 (1975), pp. 397-412.

[12] A. P. Ingersoll, Inertial Taylor columns and Jupiter's Great Red Spot, J. Atmos. Sci., 26 (1969), pp. 744-752.

[13] E. R. Johnson, Taylor columns in horizontally sheared flow, Geophys. Astrophys. Fluid Dynamics, 24 (1983), pp. 143-164.

[14] T. Rackow, C. Wesche, R. Timmermann, H. H. Hellmer, S. Juricke, and T. Jung, A simulation of small to giant Antarctic iceberg evolution: Differential impact on climatology estimates, J. Geophys. Res. Oceans, 122 (2017), pp. 3170-3190.

[15] S. B. Savage, Aspects of iceberg deterioration and drift, in LNP 582: Geomorphological fluid mechanics, N. J. Balmforth and A. Provenzale, eds., Springer-Verlag Berlin Heidelberg, 2001, pp. 279-318.

[16] K. L. Smith, B. H. Robison, J. J. Helly, R. S. Kaufmann, H. A. Ruhl, T. J. Shaw, B. S. Twining, And M. Vernet, Free-drifting icebergs: Hot spots of chemical and biological erichment in the Weddell Sea, Science, 317 (2007), pp. 478-482.

[17] G. R. Stephenson, J. Sprintall, S. T. Gille, M. Vernet, J. J. Helly, and R. S. Kaufmann, Subsurface melting of a free-floating Antarctic iceberg, Deep-Sea Res. II, 58 (2015), pp. 1336-1345.

[18] A. A. Stern, A. Adcroft, and O. Sergienko, The effects of Antarctic iceberg calving-size distribution in a global climate model, J. Geophys. Res. Oceans, 121 (2016), pp. $5773-5788$.

[19] G. I. TAYLOR, Experiments on the motion of solid bodies in rotating fluids, Proc. Royal Soc. A, 104 (1923), pp. 213-220.

[20] R. Timmermann, Q. Wang, and H. H. Hellmer, Ice shelf basal melting in a global finite-element sea ice-ice shelf-ocean model, Ann. Glaciol., 53 (2012), pp. 303-314.

[21] W. F. Weeks And W. J. CAmpBell, Icebergs as a fresh-water source: an appraisal, J. Glaciol., 12 (1973), pp. 207-233. 


\title{
Cooling via Baroclinic Acoustic Streaming
}

\author{
Guillaume Michel
}

March 20, 2018

\section{Introduction}

As most waves, sound waves have the faculty to drive steady Eulerian flows. This effect, called acoustic streaming, can be sensed as a consequence of the conservation of momentum. Indeed, consider a progressive acoustic wave subject to an attenuation mechanism, e.g. viscosity or thermal conductivity: whereas the energy of the wave shall be mostly transferred to internal energy (and result in a local heating), a mean flow must be generated in order to conserve momentum. Such non-zero Eulerian mean motions play a crucial role in mass and heat transport. This has been recognized for water waves by Longuet-Higgins [1], where it is of the same order as the Stokes drift (the difference between Lagrangian and Eulerian mean velocities). For sound waves, acoustic streaming is by far the main transport mechanism [2]. Given that powerful ultrasonic sources are nowadays of common use, acoustic streaming can be considered a simple and low-cost way to enhance heat transfer [3]. In this study, we investigate the effect of acoustic waves on a strong, stably stratified medium, namely a fluid in between two parallel plates of very different temperatures.

In the absence of thermal driving, the streaming flow generated by plane standing waves has been worked out by Rayleigh [4] in the limit $H_{*} \gg \delta_{B L}$, where $H_{*}$ is the width of the system and $\delta_{B L}$ is the width of the boundary layers (for a more general study, see [5]). For a plane wave of the form $U_{*} \cos \left(k_{*} \tilde{x}\right) \vec{f}_{x}$, it consists of two series of vortices located symmetrically about the median plane, of typical velocity $3 U^{2} /\left(16 a_{*}\right)$, where $a_{*}$ is the speed of sound and $k_{*}$ the wavenumber. Note that, even though acoustic streaming results in this case from viscous dissipation in the Stokes boundary layers, the mean flow does not depend on the viscosity, and therefore does not vanish in the limit of infinitely small dissipation. Assuming that both the mean flow and the acoustic waves are not affected by heat, i.e. that the density does not depend on temperature, the additional heat flux associated with this streaming flow has been computed for small aspect ratio [6]. Experiments have confirmed that acoustic streaming enhances heat transfers [7]. Direct numerical simulations have also been performed for relatively small aspect ratio, in the absence of gravity [8, 9], and show that moderate thermal driving results in a vertical merging of these stack cells and in an increase of the velocity of the streaming flow. This solution strongly contrasts with the one theoretically considered for the computation of the heat flux [6].

This discrepancy between full DNS and previous theoretical studies results from the assumption that the mean flow is driven by acoustic streaming taking place in the viscous boundary layers. However, it has been recently recognized that in the presence of a 
stratification, the dominant driving force takes place in the bulk and results from baroclinic production of vorticity of acoustic waves [10]. Acknowledging this mechanism as the leading one, Chini et al. were able to obtain the correct order of magnitude for the mean velocity in a strongly stratified system driven by acoustic streaming (a stabilized HIV lamp [11]), whereas usual Rayleigh streaming would lie two orders of magnitude below. Along the same lines of these authors, we demonstrate in this report that a similar description can be applied to a gas between two plates subjected to a intense temperature difference.

We perform a multi-scale analysis of this problem and obtain governing equations for the mean flow and for the acoustic waves. This reduced model emphasizes the complex dynamics of the system, as waves and streaming flow present a two-way coupling: acoustic waves drive a mean flow, that in return modifies the density field and thus affects the wave field. We derive an approximate solution in some range of parameters, that provides an accurate model for the previously mentioned direct numerical simulation of the full system. We also present numerical simulations of this reduced set of equations, that can describe regimes with strong coupling.

This report is organized as follows: we first review the basic mechanisms of acoustic streaming, then describe the system and the multiple scale analysis. In section 3 , we show that the acoustic wave field can be obtained as the solution of a one-dimensional eigenvalue problem, and describe how its amplitude evolves. In section 4 , we then compute an approximate solution of this system and evaluate the associated heat flux and efficiency. This is compared to previous direct numerical simulations of the full problem. Finally, in section 6 , we present the results of numerical simulations, then draw our conclusion.

\section{Basic Mechanisms of Acoustic Streaming}

\subsection{The role of vorticity}

In order to emphasize the role of stratification in acoustic streaming, we first review some very basic facts about nonlinearities in acoustics. In all the following, acoustic fields will be assumed to be of small amplitudes compared to the speed of sound $a_{*}$. As a result, nonlinear terms in the Navier-Stokes equation do not much affect the waves to a first approximation. Indeed, this governing equation reads, for a Newtonian compressible fluid without second viscosity,

$$
\rho\left[\partial_{t} \vec{v}+(\vec{v} \cdot \vec{\nabla}) \vec{v}\right]=-\vec{\nabla} P+\vec{f}+\eta\left(\triangle \vec{v}+\frac{1}{3} \vec{\nabla}(\vec{\nabla} \cdot \vec{v})\right)
$$

and, with $\omega$ the angular frequency of the wave, $k$ its wavenumber and $U$ the wave amplitude,

$$
\left|\frac{(\vec{v} \cdot \vec{\nabla}) \vec{v}}{\partial_{t} \vec{v}}\right| \sim \frac{U^{2} k}{\omega U} \sim \frac{U}{a_{*}} \ll 1 .
$$

Therefore, nonlinearities appear as a small correction for the waves dynamics. However, it would be unfortunate to disregard their consequences based on this fact since, although small, such effects may be cumulative and affect the long-time evolution of the wave field. Given the time-dependence of the waves, the nonlinear term will contain high frequencies: this results in the apparition of harmonics in the signal, and may also lead to shock waves 
or transfers of energy between wave trains (acoustic waves undergo three-waves or more interactions). Such high frequencies can still be regarded as waves, and will not be further discussed.

In the present work, we are interested in the low-frequencies that may be driven by this nonlinear term. For this purpose, it is useful to cast it as

$$
(\vec{v} \cdot \vec{\nabla}) \vec{v}=\vec{\nabla}\left(\frac{\vec{v}^{2}}{2}\right)+(\vec{\nabla} \times \vec{v}) \times \vec{v}
$$

This evidences that, although constant terms shall always result from quadratic nonlinearities of an oscillating field, part of them are balanced by a pressure variation ${ }^{1}$. Therefore, in order to drive a mean flow, acoustic waves must have some vorticity.

It is then natural to wonder the conditions necessary for an acoustic field to acquire vorticity. This has been so far mostly discussed for fluids with uniform density background. In this case vorticity is generated in an irrotational flow by viscosity [12, 13], although external forces or moving boundary can also be considered. We shall see that another strong source of vorticity resides in an inhomogeneous background density $\rho_{0}$. This can be evidenced by taking the curl of the linear Euler equation, that describes inviscid and linear acoustics,

$$
\vec{\nabla} \times\left(\rho_{0} \partial_{t} \vec{v}=-\vec{\nabla} P\right) \Longrightarrow \partial_{t}(\vec{\nabla} \times \vec{v})=\frac{(\vec{\nabla} \rho) \times(\vec{\nabla} P)}{\rho_{0}^{2}} .
$$

The left-hand side of this equation is the so-called "baroclinic contribution", and is non-zero when isobars and isopycnals differ.

\subsection{Acoustic streaming in a horizontal cavity}

As a first approach of acoustic streaming, we review the theory in a channel with an uniform density background, mostly done by Rayleigh [4]. It results from vorticity being generated in the thin boundary layers, contrary to "quartz wind", in which viscosity acts in the bulk during the propagation of a wave train. We consider two parallel boundaries separated by a height $H_{*}$, of same temperature, and describe the steady state associated with a plane standing wave in the $x$ direction, of wavenumber $k_{*}$. In all the following, tildes and stars refer to dimensional quantities, boldfaces to vectors, bars to time-averaged quantities and primes to oscillating fields. The setup is sketched in Fig. 1, and we assume that a steadystate is reached. To derive the streaming flow, we proceed as follows:

1. We assume that an operator drives an acoustic wave along the $x$ direction in the bulk, then compute the corrections caused by the presence of boundary layers.

2. In the boundary layers, we define and compute the "Reynolds stress", i.e. the mean force density that acts on the streaming flow.

3. We balance this force with viscosity in the boundary layers, and show that it results in an effective slip velocity for the streaming flow.

\footnotetext{
${ }^{1}$ Because acoustic waves are compressible flows, one should not forget about the term $\left(\rho-\rho_{0}\right) \partial_{t} \vec{v}$, where $\rho_{0}$ is a background density field that may, however, often be expressed as a gradient.
} 


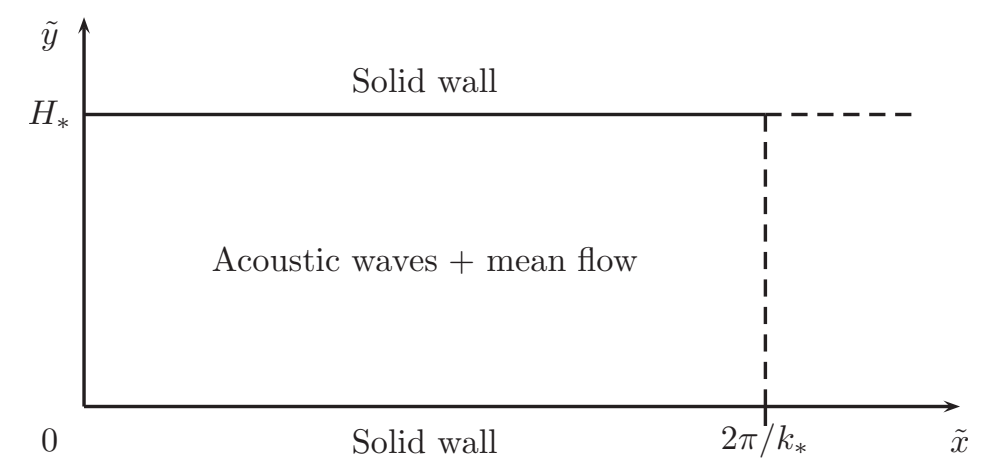

Figure 1: Rayleigh problem of acoustic streaming

\begin{tabular}{|c|c|c|}
\hline Quantity & Expansion or scaling & Parameters involved \\
\hline$x$ velocity $\tilde{u}(\tilde{x}, \tilde{y}, \tilde{t})$ & $\epsilon a_{*}\left(u_{1}(x, y, t)+\epsilon u_{2}(x, y, t)+\ldots\right)$ & The speed of sound $a_{*}$ \\
$y$ velocity $\tilde{v}(\tilde{x}, \tilde{y}, \tilde{t})$ & $\epsilon^{2} a_{*}\left(v_{1}(x, y, t)+\epsilon v_{2}(x, y, t)+\ldots\right)$ & The speed of sound $a_{*}$ \\
Density $\tilde{\rho}(\tilde{x}, \tilde{y}, \tilde{t})$ & $\epsilon \rho_{*}\left(1+\epsilon \rho_{1}(x, y, t)+\ldots\right)$ & The background density $\rho_{*}$ \\
Pressure $\tilde{p}(\tilde{x}, \tilde{y}, \tilde{t})$ & $p_{*}+\rho_{*} a_{*}^{2}\left(\epsilon p_{1}(x, y, t)+\ldots\right)$ & The background pressure $p_{*}$ \\
$\tilde{t}$ & $\omega_{*}^{-1} t$ & The angular frequency $\omega_{*}$ \\
$\tilde{x}$ & $k_{*}^{-1} x$ & The wavenumber $k_{*}=\omega_{*} / a_{*}$ \\
$\tilde{y}$ & $\delta y$, where $\delta_{B L}=\sqrt{2 \nu / \omega_{*}}$ & The B.L. thickness $\delta_{B L}$ \\
Small parameter $\epsilon$ & $\epsilon=k_{*} \delta$ & $\epsilon$ is dimensionless and small \\
\hline
\end{tabular}

Table 1: Scaling of the variables for the Rayleigh streaming

4. We compute the bulk flow by balancing this driving with viscosity, and compare the result to numerical simulations that include inertia.

\subsubsection{Effect of the boundary layers on the acoustic field}

The presence of solid boundaries in a fluid imposes no-slip boundary conditions, that can not be handled by potential flows. It thus generates both vorticity and strong velocity gradients, that are often the dominant damping mechanism (a typical example being sloshing). Quite surprisingly, the wave field actually undergoes changes everywhere, even far from the boundary layer. Here we derive the acoustic wave field at the leading order in the bottom boundary layer, as well as the small correction that affects the bulk flow.

In this problem, we have several small dimensionless parameters. The first one, previously mentioned, is the ratio of the wave amplitude to the speed of sound. Other ones compare the boundary layer thickness $\delta_{B L}=\sqrt{2 \nu / \omega}$, of the order of a few microns for ultrasounds in air, to the acoustic wavelength and to the width of the system. For simplicity, these numbers are chosen equal and small, which results in the expansions reported in Table 1. This problem has four governing equations:

1. The continuity equation,

$$
\partial_{\tilde{t}} \tilde{\rho}+\partial_{\tilde{x}}(\tilde{\rho} \tilde{u})+\partial_{\tilde{y}}(\tilde{\rho} \tilde{v})=0
$$


that reads with our scalings and at the leading order

$$
\partial_{t} \rho_{1}+\partial_{x} u_{1}+\partial_{y} v_{1}=0 \text {. }
$$

2. The equation of state for an isentropic evolution,

$$
\beta_{s}=\frac{1}{\tilde{\rho}}\left(\frac{\partial \tilde{\rho}}{\partial \tilde{p}}\right)_{S},
$$

with $a_{*}=1 / \sqrt{\beta_{s} \rho_{*}}$. At the leading order, it yields $p_{1}=\rho_{1}$.

3. The Navier-Stokes equation along the $y$ direction,

$$
\tilde{\rho}\left(\partial_{\tilde{t}} \tilde{v}+\tilde{u} \partial_{\tilde{x}} \tilde{v}+\tilde{v} \partial_{\tilde{y}} \tilde{v}\right)=-\partial_{\tilde{y}} \tilde{p}+\rho_{*} \nu\left(\partial_{\tilde{x} \tilde{x}} \tilde{v}+\partial_{\tilde{y} \tilde{y}} \tilde{v}+\frac{1}{3} \partial_{\tilde{y}}\left(\partial_{\tilde{x}} \tilde{u}+\partial_{\tilde{y}} \tilde{v}\right)\right),
$$

that reduces at the leading order to

$$
\partial_{y} p_{1}=0
$$

4. The Navier-Stokes equation along the $x$ direction,

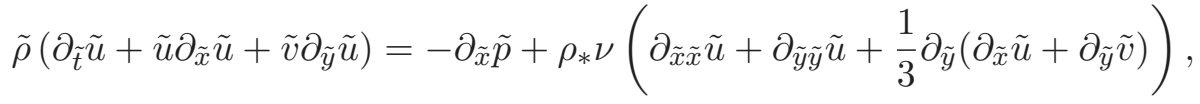

that is at order $O(\epsilon)$

$$
\partial_{t} u_{1}=-\partial_{x} p_{1}+\frac{\partial_{y y} u_{1}}{2} .
$$

This set of equations has to be solved with a no-slip boundary condition at $y=0$ and with the far-field assumed to be an acoustic standing wave of the form

$$
u(x, y=\infty, t)=\cos (x) \cos (t) .
$$

This boundary condition (12) with (9) and (11) gives the order one pressure,

$$
p_{1}(x, y, t)=\sin (x) \sin (t) .
$$

We can then get a close equation for $u_{1}$,

$$
\partial_{t} u_{1}=-\cos (x) \sin (t)+\frac{\partial_{y y} u_{1}}{2}
$$

that describes a Stokes boundary layer and is straightforward to solve,

$$
u_{1}(x, y, t)=\cos (x)\left[\cos (t)\left(1-\cos (y) e^{-y}\right)+\sin (t) \sin (y) e^{-y}\right] .
$$

Now that we know both $\rho_{1}$ (from $p_{1}$ and the equation of state) and $u_{1}$, the continuity equation (6) with the boundary condition $v(x, y=0, t)=0$ result in

$$
v_{1}=\frac{\sin (x)}{2}\left[\cos (t)\left(-1-\sin (y) e^{-y}+\cos (y) e^{-y}\right)+\sin (t)\left(1-\sin (y) e^{-y}-\cos (y) e^{-y}\right)\right] .
$$

Note that $v_{1}$ does not vanish in the limit $y \rightarrow \infty$, i.e. that the effect of the solid boundary is not restricted to the boundary layer. However, $v$ is scaled as a small quantity compared to $u$, so that this velocity field remains a correction. 


\subsubsection{Definition and computation of the Reynolds stress}

The streaming flow is a second order quantity, and we therefore need to consider (10) at order $O\left(\epsilon^{2}\right)$,

$$
\partial_{t} u_{2}+\rho_{1} \partial_{t} u_{1}+u_{1} \partial_{x} u_{1}+v_{1} \partial_{y} u_{1}=-\partial_{x} p_{2}+\frac{\partial_{y y} u_{2}}{2}+\frac{1}{3} \partial_{y}\left(\partial_{x} u_{1}+\partial_{y} v_{1}\right) .
$$

The left hand side can be modified with the continuity equation (6),

$$
\partial_{t} u_{2}+\partial_{t}\left(\rho_{1} u_{1}\right)=-\partial_{x} p_{2}-\partial_{x} u_{1}^{2}-\partial_{y}\left(u_{1} v_{1}\right)+\frac{\partial_{y y} u_{2}}{2}+\frac{1}{3} \partial_{y}\left(\partial_{x} u_{1}+\partial_{y} v_{1}\right) .
$$

We then take the time-average of this equation,

$$
0=-\partial_{x} \bar{p}_{2}-\overline{\partial_{x} u_{1}^{2}}-\overline{\partial_{y}\left(u_{1} v_{1}\right)}+\frac{\partial_{y y} \bar{u}_{2}}{2} .
$$

We evidence on this simple system a general feature of acoustic streaming that consists of an effective force on the second order mean velocity field coming from inertial leading order terms. Generally speaking, this force $\mathbf{F}=F_{j} \mathbf{e}_{j}$ can be written as the divergence of the Reynolds stress, and is

$$
F_{j}=-\frac{\partial\left(\overline{\rho u_{i} u_{j}}\right)}{\partial x_{i}}
$$

where the repeated suffix $i$ is summed over one to three. With (15) and (16), we obtain

$$
F_{x}=\frac{\sin (2 x)}{4}\left(2+e^{-2 y}+\sin (y) e^{-y}-3 \cos (y) e^{-y}\right) .
$$

Whereas the constant term along the $y$ direction can be handled by the pressure field $\bar{p}_{2}$, the other ones cannot.

\subsubsection{Effective slip velocity}

Most of the Reynolds stress divergence has to be balanced with viscosity, i.e. with the term $\partial_{y y} \bar{u}_{2}$, that reads

$$
\partial_{y y} \bar{u}_{2}=-\frac{\sin (2 x)}{2}\left(\sin (y) e^{-y}-3 \cos (y) e^{-y}+e^{-2 y}\right) .
$$

With the boundary conditions $\left(\partial_{y} \bar{u}_{2}\right)(x, y=\infty, t)=0$ and $\bar{u}_{2}(x, y=0, t)=0$, it provides

$$
\bar{u}_{2}(x, y, t)=\frac{\sin (2 x)}{8} e^{-y}(-3 \sin (t)-\cos (y)+2 \sinh (y)+\cosh (y)) .
$$

In particular, the mean second order flow does not vanish far away from the boundary layer, where it takes the value

$$
\bar{u}_{2}(x, y=\infty, t)=\frac{3 \sin (2 x)}{8}
$$

This limit velocity is, with matched asymptotic expansion, a boundary condition for the mean flow. In particular, we emphasize that this quantity does not depend on the value of the viscosity, and is a small fraction of the acoustic wave amplitude. More precisely, if we denote by $U_{*}=\epsilon a_{*}$ the dimensional amplitude of the acoustic wave,

$$
\overline{\tilde{u}}(\tilde{x}, \tilde{y}=0, \tilde{t})=\frac{3 U_{*}^{2}}{8 a_{*}} \sin \left(2 k_{*} \tilde{x}\right) .
$$




\subsubsection{Mean flow in the bulk}

If the driving imposed by acoustic streaming is balanced by viscosity (this regime being called "Rayleigh streaming"), we have to solve in the entire domain

$$
\mathbf{0}=-\nabla \tilde{p}+\eta \triangle \overline{\tilde{\mathbf{u}}}_{2}, \quad \overline{\tilde{u}}\left(\tilde{x}, \tilde{y}=0 \text { or } H_{*}, \tilde{t}\right)=\frac{3 U_{*}^{2}}{8 a_{*}} \sin \left(2 k_{*} \tilde{x}\right) .
$$

The steady-state is found with the use of a stream function $\psi\left(\overline{\tilde{u}}_{2}=\partial_{\tilde{y}} \psi, \overline{\tilde{v}}_{2}=-\partial_{\tilde{x}} \psi\right)$, that has to be a solution of $\nabla^{4} \psi=0$. Note that

$$
\sinh (n y) \sin (n x) \quad \text { and } \quad y \cosh (n y) \sin (n x)
$$

are solutions of $\nabla^{4} \psi=0$, so we can look for $\psi$ of the form

$$
\psi(\tilde{x}, \tilde{y})=\left[A \sinh \left(n\left(\tilde{y}-H_{*} / 2\right)\right)+B n\left(\tilde{y}-H_{*} / 2\right) \cosh \left(n\left(\tilde{y}-H_{*} / 2\right)\right)\right] \sin (n \tilde{x}) .
$$

We still have to enforce the boundary conditions. Canceling $\tilde{v}$ at the solid boundaries gives $B=-A\left(n H_{*} / 2\right)^{-1} \tanh \left(n H_{*} / 2\right)$. The effective slip condition fixes $n=2 \tilde{k}$ and $B$, so that

$$
(\tilde{x}, \tilde{y})=A \Psi(y) \sin (2 \tilde{k} \tilde{x})
$$

with

$$
A=\left(\frac{3 U_{*}^{2}}{16 A_{*} k_{*}}\right) \times\left(\operatorname{sech}\left(\tilde{k} H_{*}\right)-\sinh \left(\tilde{k} H_{*}\right) /\left(\tilde{k} H_{*}\right)\right)^{-1}
$$

and

$$
\Psi(y)=\sinh \left(2 \tilde{k}\left(\tilde{y}-\frac{H_{*}}{2}\right)\right)-\frac{\tanh \left(\tilde{k} H_{*}\right)}{\tilde{k} H_{*}}\left(2 \tilde{k}\left(\tilde{y}-\frac{H_{*}}{2}\right)\right) \cosh \left(2 \tilde{k}\left(\tilde{y}-\frac{H_{*}}{2}\right)\right) .
$$

This solution describes a set four vortices per acoustic wavelength, two in the horizontal direction, and two in the vertical one. Their energies are localized at a distance $\sim \tilde{k}^{-1}$ of the boundaries, so that the streaming velocity at the center of the cell becomes very weak if the aspect ratio $\tilde{k} H_{*}$ is large, see Fig. 2 . This is the reason why this regime of acoustic streaming is usually described in the limit $\delta \ll H \ll k_{*}^{-1}$, where the stream function then becomes

$$
\psi(\tilde{x}, \tilde{y}) \simeq \frac{3 U_{*}^{2} H_{*}}{4 a_{*}}\left[\left(\frac{\tilde{y}}{H_{*}}-\frac{1}{2}\right)^{3}-\frac{1}{4}\left(\frac{\tilde{y}}{H_{*}}-\frac{1}{2}\right)\right] \sin \left(2 k_{*} \tilde{x}\right),
$$

and the velocities are

$$
\begin{gathered}
\overline{\tilde{u}}_{2}(\tilde{x}, \tilde{y}) \simeq-\frac{3 U_{*}^{2}}{4 a_{*}}\left[\frac{1}{4}-3\left(\frac{\tilde{y}}{H_{*}}-\frac{1}{2}\right)^{2}\right] \sin \left(2 k_{*} \tilde{x}\right) \\
\overline{\tilde{v}}_{2}(\tilde{x}, \tilde{y}) \simeq-\frac{3 U_{*}^{2} k_{*} H_{*}}{2 a_{*}}\left[\left(\frac{\tilde{y}}{H_{*}}-\frac{1}{2}\right)^{3}-\frac{1}{4}\left(\frac{\tilde{y}}{H_{*}}-\frac{1}{2}\right)\right] \cos \left(2 k_{*} \tilde{x}\right) .
\end{gathered}
$$

A typical velocity induced in this system if often defined as the $x$ velocity in the mid-plane, that is $3 U_{*}^{2} /\left(16 a_{*}\right)$. 

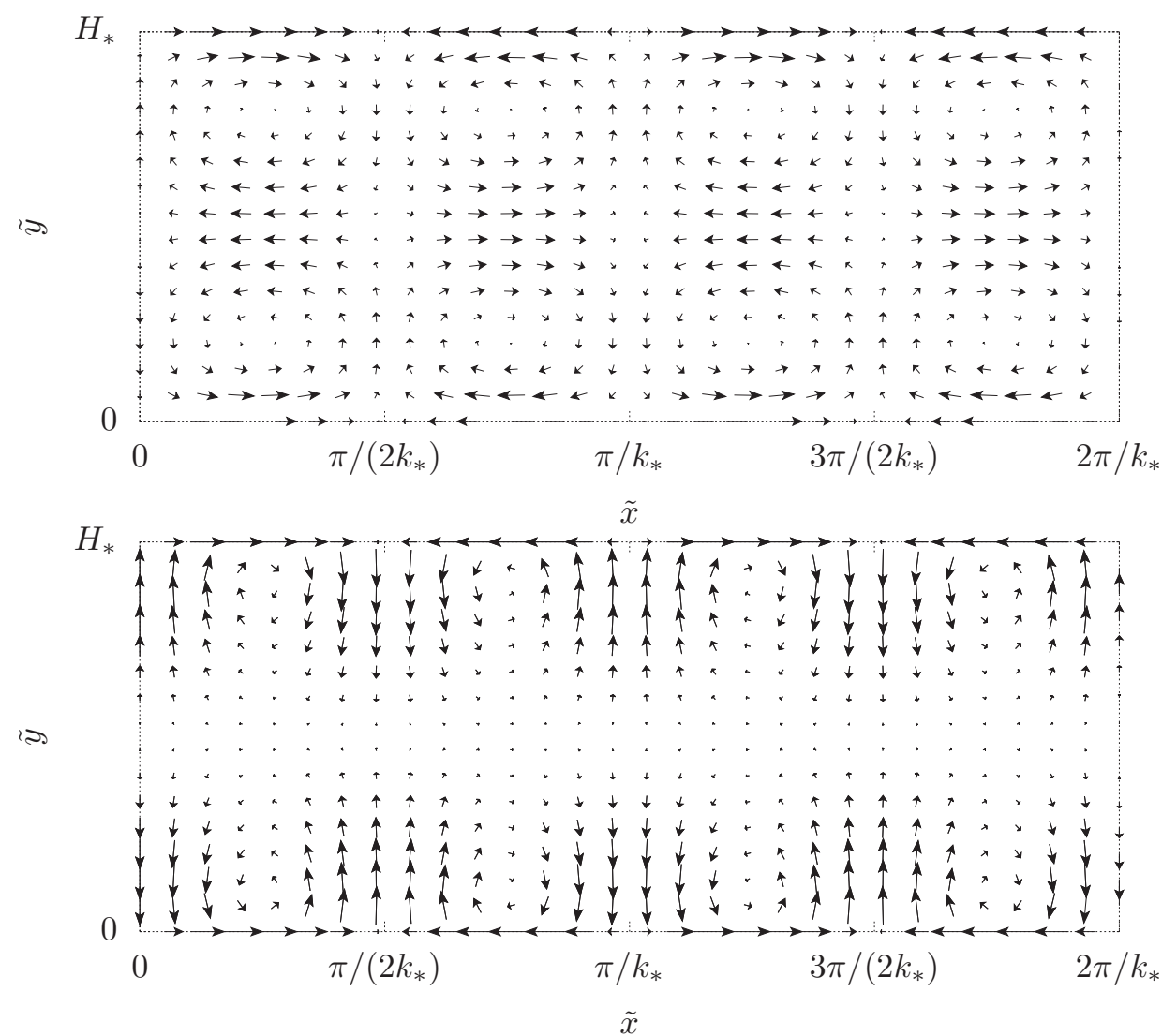

Figure 2: Rayleigh streaming for aspect ratios $k_{*} H_{*}=0.5$ (top) and $k_{*} H_{*}=5$ (bottom).

\subsubsection{Effect of inertia ("Stuart streaming" or "Eckart streaming")}

Up to now, we have considered that the flow in the bulk is fully balanced by viscosity, which may not always be valid. In order to discuss this assumption, we have to evaluate the streaming Reynolds number $R_{s}$, defined by

$$
R_{s}=\frac{U_{*}^{2} \ell}{a_{*} \nu}
$$

where $\ell$ is the relevant length-scale. We thereafter consider large and small aspect ratios.

Large aspect ratios If the aspect ratio is large, then the system does not depend on $H_{*}$ anymore and $\ell=k_{*}^{-1}$, so that

$$
R_{s}=\left(\frac{U_{*}}{a_{*}}\right)^{2} \times\left(\frac{a_{*}}{k_{*} \nu}\right) .
$$


For an ideal gas (and, generally speaking, for most gas), the order of magnitude of the kinematic viscosity is the product of the speed of sound to the mean free-path $\ell_{p}$, so that

$$
R_{s} \sim\left(\frac{U_{*}}{a_{*}}\right)^{2} \times\left(\frac{\lambda_{*}}{\ell_{p}}\right)
$$

where $\lambda_{*}=2 \pi / k_{*}$. Therefore, although the first term is small, the streaming Reynolds number can still be large (for air in usual conditions, $\ell_{p} \sim 10^{-7} \mathrm{~m}$ ). Inertia results in a jet-like flow where the velocities are concentrated in a second boundary layer, whose length $\delta_{B L, M F}$ lies between the one of the acoustic waves $\delta_{B L} \sim \sqrt{\nu / \omega}$ and the wavelength $\lambda_{*}[14]$. It can be estimated based on the velocity $U_{*}^{2} / a_{*}$ and the characteristic length $k_{*}^{-1}$,

$$
\delta_{B L, M F} \sim \sqrt{\frac{\nu}{k_{*}^{-1} \times\left(U_{*}^{2} / a_{*}\right)}} \sim\left(\frac{a_{*}}{U_{*}}\right) \times \delta_{B L} \gg \delta_{B L}
$$

To illustrate this, we report in Fig. 3 the velocity field obtained with a direct numerical simulation of the bulk flow with $R_{s}=100$ and $k_{*} H_{*}=5$. This has been obtained with Dedalus [15]. Compared to Fig. 2, this clearly evidences this jet-like structure.

Small aspect ratios Similarly, if the aspect ratio is small, we rather define a streaming Reynolds number based on $H_{*}$,

$$
R_{h}=\frac{U_{*}^{2} H_{*}}{a_{*} \nu}=R_{s} \times\left(k_{*} H_{*}\right),
$$

where $R_{s}$ is defined in (36). The jet-like structures are less pronounced (see Fig. 3), but the effect of inertia can still be observed for large values of $R_{h}$.

\subsection{Main features of Rayleigh streaming}

The main features of this streaming flow can be summarized as:

1. The amplitude of the streaming flow is quadratic in the amplitude of the acoustic wave (see, e.g., (25)).

2. In this setup, it results in stacked vortices of energy localized within a distance $k_{*}^{-1}$ from the walls.

3. The streaming flow close to the boundary layer is directed toward the velocity nodes of the acoustic wave, i.e. away from the pressure nodes.

\section{Governing Equations for the Waves and the Mean Flow}

\subsection{Notations and dimensional equations}

The problem we consider is sketched in Fig. 4 and consists of a thin layer of an ideal gas in between two horizontal boundaries that drive the system toward a stably stratified steady state. Compared to before, we add this temperature difference and our claim is that the 

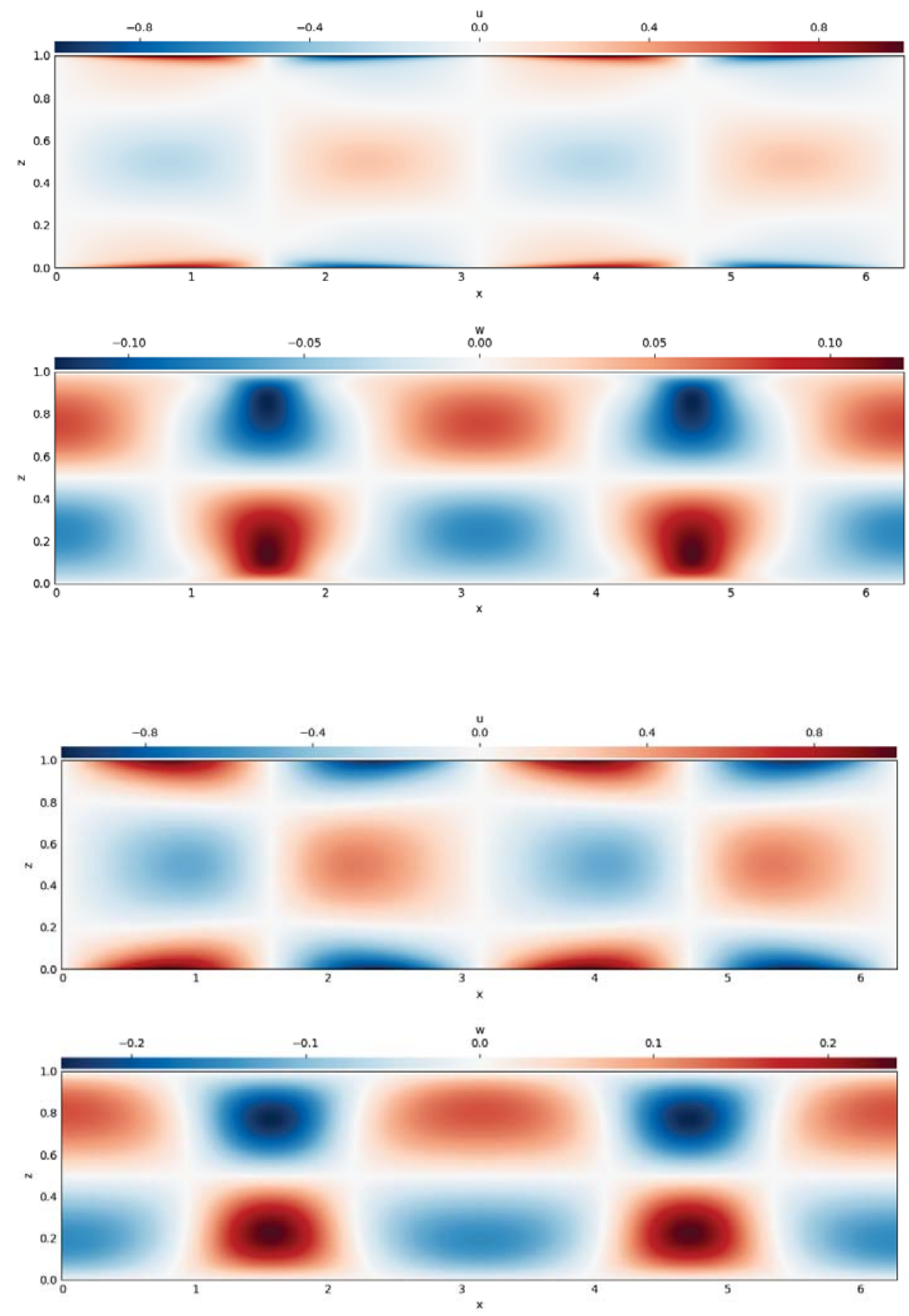

Figure 3: Rayleigh streaming for $k_{*} H_{*}=5, R_{s}=100$ (top two plots) and $k_{*} H_{*}=0.5, R_{h}=$ 100 (bottom two plots). $u$ and $w$ are the horizontal and vertical velocity fields, scaled with $3 U_{*}^{2} /\left(8 a_{*}\right), z$ is scaled with $H_{*}$, and $x$ with $k_{*}^{-1}$. 


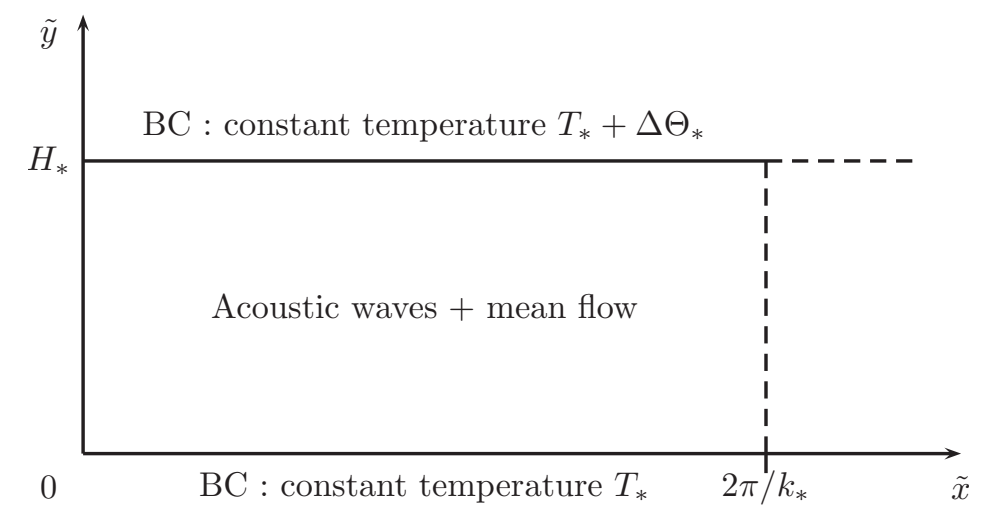

Figure 4: Schematic of the problem: acoustic waves interact with a mean flow in a thin layer of an ideal gas subject to a vertical thermal driving.

streaming flow will be driven by baroclinic vorticity in the bulk rather than viscosity in the boundary layers. For simplicity, we still consider two-dimensional flows and we neglect the effect of gravity ${ }^{2}$. The dimensional parameters and variables are defined in Table 2.

The kinematic boundary conditions are no-slip boundary conditions at $\tilde{y}=0$ and $\tilde{y}=H_{*}$, and periodicity in the $\tilde{x}$ direction of period $2 \pi / k_{*}$. Moreover, we fix $\tilde{u}(x=0, y, t)=0$ so that there is no exchange of mass between nearby cells. The thermal boundary conditions also consist of periodicity in $\tilde{x}$, and we require a constant temperature at the bottom, $\tilde{T}(\tilde{y}=0)=T_{*}$, and at the top, $\tilde{T}\left(\tilde{y}=H_{*}\right)=T_{*}+\Delta \Theta_{*}$. We then define $\Gamma=\Delta \Theta_{*} / T_{*}$ as the dimensionless strength of this thermal driving. In the absence of flow, the dimensional temperature therefore reads

$$
\tilde{T}_{B}(\tilde{y})=T_{*}\left(1+\Gamma \frac{\tilde{y}}{H_{*}}\right) .
$$

The dimensional equations are the same as in [10], excepted that we consider viscous heating. They are reported below, with $\nabla=\left(\partial_{x}, \partial_{y}\right)$.

$$
\left\{\begin{array}{l}
\tilde{\rho}\left[\partial_{\tilde{t}} \tilde{\mathbf{u}}+(\tilde{\mathbf{u}} \cdot \tilde{\nabla}) \tilde{\mathbf{u}}\right]=-\tilde{\nabla} \tilde{p}+\mu\left[\tilde{\nabla}^{2} \tilde{\mathbf{u}}+\frac{1}{3} \tilde{\nabla}(\tilde{\nabla} \cdot \tilde{\mathbf{u}})\right] \\
\partial_{\tilde{t} \tilde{\rho}}+\tilde{\nabla} \cdot(\tilde{\rho} \tilde{\mathbf{u}})=0 \\
\tilde{\rho} c_{v}\left[\partial_{\tilde{t}} \tilde{T}+(\tilde{\mathbf{u}} \cdot \tilde{\nabla}) \tilde{T}\right]=-\tilde{p}(\tilde{\nabla} \cdot \tilde{u})+\kappa \tilde{\nabla} \tilde{T}^{2} \tilde{T}-\Phi, \\
\tilde{p}=\tilde{\rho} R_{s} \tilde{T}
\end{array}\right.
$$

We consider the dynamic viscosity $\mu$ and the thermal conductivity $\kappa$ to be independent of the temperature. The dissipation function $\Phi$ describes viscous heating and is

$$
\Phi=2 \mu\left[\left(\partial_{\tilde{x}} \tilde{u}\right)^{2}+\left(\partial_{\tilde{y}} \tilde{v}\right)^{2}-\frac{\left(\partial_{\tilde{x}} \tilde{u}+\left(\partial_{\tilde{y}} \tilde{v}\right)^{2}\right.}{3}\right]+\mu\left(\partial_{\tilde{y}} \tilde{u}+\partial_{\tilde{x}} \tilde{v}\right)^{2}
$$

\footnotetext{
${ }^{2}$ Gravity would affect the equations at the leading order if the Richardson number $R i=g_{*} /\left(k_{*} a_{*}^{2}\right)$ is scaled as $\epsilon^{3 / 2}$, which corresponds to a typical value of this number.
} 


\begin{tabular}{|c|c|}
\hline Notation & Definition \\
\hline$(\tilde{x}, \tilde{y})$ & (Horizontal, vertical) coordinate \\
$H_{*}$ & Height of the channel \\
$k_{*}$ & Horizontal wavenumber of the acoustic waves \\
$\kappa$ & Thermal conductivity \\
$\tilde{\rho}$ & Gas density \\
$\tilde{\mathbf{u}}=(\tilde{u}, \tilde{v})$ & Gas velocity \\
$\tilde{p}$ & Pressure \\
$\tilde{T}$ & Temperature \\
$\mu$ & Dynamic viscosity \\
$R_{s}$ & Specific gas constant \\
$\left(c_{v}, c_{p}\right)$ & Specific coefficient at constant (volume, pressure) \\
$\left.a_{*}=c_{p} / c_{v}\right) R_{s} T_{*}$ & Speed of sound \\
$p_{*}$ & Equilibrium pressure at $\tilde{y}=0$ if $\tilde{T}(\tilde{x}, \tilde{y})=T_{*}$ \\
$U_{*}$ & Typical amplitude of the acoustic wave (velocity) \\
\hline
\end{tabular}

Table 2: Definitions of the dimensional parameters

Since viscous heating results from the viscous term in the Navier-Stokes equation, we have

$$
\iint d x d y \Phi=\iint d x d y \mu \mathbf{u} \cdot\left[\tilde{\nabla}^{2} \tilde{\mathbf{u}}+\frac{1}{3} \tilde{\nabla}(\tilde{\nabla} \cdot \tilde{\mathbf{u}})\right] .
$$

\subsection{Dimensionless equations}

We now turn to dimensionless variables and equations. The scalings and dimensionless parameters used for this purpose are reported in Table 3 (for clarity, we define $\epsilon=S^{-1}$ ). Note that the aspect ratio has been chosen small. Even though the heat flux is not expected to be maximal in this regime, since the flow will mainly be along the $x$ direction, this is motivated by the following facts. First, we expect the analysis of the acoustic wave-field to be simple in a domain thin compared to the wavelength. We shall see that it constrains the acoustic field to stay in the first mode along the vertical direction. Second, most theoretical and analytical studies have been performed in this regime, so that comparisons to previous works can be done. Third, at the leading order, the pressure gradient will be found orthogonal to the background density gradient, resulting in an important baroclinic contribution in the vorticity equation.

To illustrate these scalings, a setup in which $\epsilon=10^{-3}$, and other parameters are perfectly scaled $\left(h=\Gamma=R e_{s}=P e_{s}=1, \gamma=7 / 5\right)$, would consist in a transducer sending a powerful audible sound $(f \simeq 600 \mathrm{~Hz})$ in a strongly stratified long and thin layer $\left(H_{*} \simeq 3 \mathrm{~mm}\right.$, $2 \pi / k_{*} \simeq 60 \mathrm{~cm}, \Delta \Theta \simeq T_{*} \simeq 300 \mathrm{~K}$ ). Since $\epsilon$ is very small, $h$ and $\Delta \Theta$ can be tuned so that a height of $3 \mathrm{~cm}$ and a temperature difference of $30 \mathrm{~K}$ can also be described by this system. The total temperature and pressure are written as a background profile plus a perturbation,

$$
T(x, y, t)=T_{B}(y)+\Theta(x, y, t), \quad P(x, y, t)=P_{B}(y)+\pi(x, y, t) .
$$

We report below the dimensionless set of equations. 


\begin{tabular}{|c|c||c|c|c|}
\hline Variable & Scale & Parameter & Definition & Scaling \\
\hline$x$ & $k_{*}^{-1}$ & Strouhal number $S$ & $a_{*} / U_{*}$ & $S=1 / \epsilon$ \\
$y$ & $H_{*}$ & Aspect ratio $\delta$ & $k_{*} H_{*}$ & $\delta=\sqrt{\epsilon} h$ \\
$t$ & $\left(a_{*} k_{*}\right)^{-1}$ & Temperature gradient $\Gamma$ & $\Delta \Theta_{*} / T_{*}$ & $\Gamma=O(1)$ \\
$u$ & $a_{*}$ & Reynolds number $R e$ & $\rho_{*} U_{*} /\left(k_{*} \mu\right)$ & $R e=R e_{s} / \epsilon$ \\
$v$ & $\left(k_{*} H_{*}\right) a_{*}$ & Péclet number $P e$ & $\rho_{*} c_{p} U_{*} /\left(k_{*} \kappa\right)$ & $P e=P e_{s} / \epsilon$ \\
$\rho$ & $\rho_{*} \equiv p_{*} /\left(R_{s} T_{*}\right)$ & Specific heat ratio $\gamma$ & $c_{p} / c_{v}$ & $\gamma=O(1)$ \\
$T$ & $T_{*}$ & & & \\
$P$ & $p_{*}$ & & & \\
\hline
\end{tabular}

Table 3: Definitions and scalings of the dimensionless parameters and variables

$$
\begin{gathered}
\rho\left[\partial_{t} u+(\mathbf{u} \cdot \nabla) u\right]=-\frac{1}{\gamma} \partial_{x} \pi+\frac{\epsilon^{2}}{R e_{s}}\left[\left(\partial_{x x}+\frac{1}{\epsilon h^{2}} \partial_{y y}\right) u+\frac{1}{3} \partial_{x}(\nabla \cdot \mathbf{u})\right] \\
\rho\left[\partial_{t} v+(\mathbf{u} \cdot \nabla) v\right]=-\frac{1}{\epsilon \gamma h^{2}} \partial_{y} \pi+\frac{\epsilon^{2}}{R e_{s}}\left[\left(\partial_{x x}+\frac{1}{\epsilon h^{2}} \partial_{y y}\right) v+\frac{1}{3 \epsilon h^{2}} \partial_{y}(\nabla \cdot \mathbf{u})\right] \\
\partial_{t} \Theta+(\mathbf{u} \cdot \nabla) \Theta+v \frac{d T_{B}}{d y}=(1-\gamma)\left(T_{B}+\Theta\right)(\nabla \cdot \mathbf{u})+\frac{\epsilon^{2} \gamma}{\rho P e_{s}}\left(\partial_{x x}+\frac{1}{\epsilon h^{2}} \partial_{y y}\right) \Theta+O\left(\epsilon^{3}\right), \\
\partial_{t} \rho+\partial_{x}(\rho u)+\partial_{y}(\rho v)=0 \\
\rho=\frac{1+\pi}{T_{B}+\Theta}
\end{gathered}
$$

\subsection{Expansion with respect to $\epsilon$}

To describe an acoustic field that evolves rapidly in time and whose properties depend on a slow modification of the density field, we introduce a slow time scale $T=\epsilon t$ and use the WKB approximation. Therefore, a function $f(x, y, t)$ becomes $f(x, y, \phi, T)$, where $\phi$ and $T$ are independent variables. $\phi$ stands for the rapidly evolving phase, and may be written as

$$
\phi(t)=\frac{\Phi(T)}{\epsilon},
$$

where $d \Phi / d T$ is of order one. We define the instantaneous angular frequency by

$$
\omega(T)=\frac{d \phi}{d t}=\frac{d \Phi}{d T} .
$$

In this framework, the time-derivative of $f$ reads

$$
\partial_{t} f \rightarrow \omega \partial_{\phi} f+\epsilon \partial_{T} f
$$


The fast time average of a function $f$ is

$$
\bar{f}(x, y, T)=\frac{1}{2 n \pi} \int_{\phi}^{\phi+2 n \pi} f(x, y, s, T) d s,
$$

for sufficiently large positive integer $n$, so that any function can be split according to

$$
f(x, y, \phi, T)=\bar{f}(x, y, T)+f^{\prime}(x, y, \phi, T), \quad \bar{f}^{\prime}=0 .
$$

We then express all the fields as series of $\epsilon$ :

- $(u, v, \pi)=\epsilon\left(u_{1}, v_{1}, \pi_{1}\right)+\epsilon^{2}\left(u_{2}, v_{2}, \pi_{2}\right)+\ldots$

- $(\Theta, \rho)=\left(\Theta_{0}, \rho_{0}\right)+\epsilon\left(\Theta_{1}, \rho_{1}\right)+\ldots$

- $\Phi=\Phi_{0}+\epsilon \Phi_{1}+\ldots$, so that $\omega=\omega_{0}+\epsilon \omega_{1}+\ldots$

The derivation of governing equations for the streaming flow at the leading order can be found in [10], and are reproduced below,

$$
\left\{\begin{array}{l}
\bar{\rho}_{0}\left(\partial_{T} \bar{u}_{1}+\bar{u}_{1} \partial_{x} \bar{u}_{1}+\bar{v}_{1} \partial_{y} \bar{u}_{1}\right)=-\frac{\partial_{x} \bar{\pi}_{2}}{\gamma}-\partial_{x}\left(\bar{\rho}_{0} \overline{u_{1}^{\prime 2}}\right)-\partial_{y}\left(\bar{\rho}_{0} \overline{u_{1}^{\prime} v_{1}^{\prime}}\right)+\frac{\partial_{y y} \bar{u}_{1}}{R e_{s} h^{2}} \\
\partial_{y} \bar{\pi}_{2}=0 \\
\partial_{T} \bar{\rho}_{0}+\partial_{x}\left(\bar{\rho}_{0} \bar{u}_{1}\right)+\partial_{y}\left(\bar{\rho}_{0} \bar{v}_{1}\right)=0 \\
\partial_{T} \bar{\Theta}_{0}+\bar{u}_{1} \partial_{x} \bar{\Theta}_{0}+\bar{v}_{1} \partial_{y}\left(\bar{\Theta}_{0}+T_{B}\right)=(1-\gamma)\left(\bar{\Theta}_{0}+T_{B}\right)\left(\partial_{x} \bar{u}_{1}+\partial_{y} \bar{v}_{1}\right)+\frac{\gamma \partial_{y y} \bar{\Theta}_{0}}{P e_{s} h^{2} \bar{\rho}_{0}}(61) \\
\bar{\rho}_{0}=\frac{1}{\bar{\Theta}_{0}+T_{B}}
\end{array}\right.
$$

Substituting the density $\bar{\rho}_{0}$ with $(62)$, it also reads

$$
\left\{\begin{array}{l}
\frac{\partial_{T} \bar{u}_{1}+\bar{u}_{1} \partial_{x} \bar{u}_{1}+\bar{v}_{1} \partial_{y} \bar{u}_{1}}{\bar{\Theta}_{0}+T_{B}}=-\frac{\partial_{x} \bar{\pi}_{2}}{\gamma}-\partial_{x}\left(\frac{\overline{u_{1}^{\prime 2}}}{\bar{\Theta}_{0}+T_{B}}\right)-\partial_{y}\left(\frac{\overline{u_{1}^{\prime} v_{1}^{\prime}}}{\bar{\Theta}_{0}+T_{B}}\right)+\frac{\partial_{y y} \bar{u}_{1}}{R e_{s} h^{2}}(63) \\
\partial_{y} \bar{\pi}_{2}=0 \\
\partial_{x} \bar{u}_{1}+\partial_{y} \bar{v}_{1}=\frac{\partial_{y y} \bar{\Theta}_{0}}{P e_{s} h^{2}} \\
\partial_{T} \bar{\Theta}_{0}+\bar{u}_{1} \partial_{x} \bar{\Theta}_{0}+\bar{v}_{1} \partial_{y}\left(\bar{\Theta}_{0}+T_{B}\right)=\left(\bar{\Theta}_{0}+T_{B}\right) \frac{\partial_{y y} \bar{\Theta}_{0}}{P e_{s} h^{2}} \\
\bar{\rho}_{0}=\frac{1}{\bar{\Theta}_{0}+T_{B}}
\end{array}\right.
$$

On the other hand, the equations describing the acoustic waves are

$$
\left\{\begin{array}{l}
\omega_{0} \bar{\rho}_{0} \partial_{\phi} u_{1}^{\prime}+\frac{1}{\gamma} \partial_{x} \pi_{1}^{\prime}=0 \\
\partial_{y} \pi_{1}^{\prime}=0 \\
\omega_{0} \partial_{\phi} \rho_{1}^{\prime}+\partial_{x}\left(\bar{\rho}_{0} u_{1}^{\prime}\right)+\partial_{y}\left(\bar{\rho}_{0} v_{1}^{\prime}\right)=0 \\
\omega_{0} \partial_{\phi} \Theta_{1}^{\prime}+u_{1}^{\prime} \partial_{x} \bar{\Theta}_{0}+v_{1}^{\prime} \partial_{y}\left(\bar{\Theta}_{0}+T_{B}\right)+(\gamma-1)\left(\bar{\Theta}_{0}+T_{B}\right)\left(\partial_{x} u_{1}^{\prime}+\partial_{y} v_{1}^{\prime}\right)=0 \\
\pi_{1}^{\prime}-\rho_{1}^{\prime}\left(\bar{\Theta}_{0}+T_{B}\right)-\bar{\rho}_{0} \Theta_{1}^{\prime}=0
\end{array}\right.
$$


We obtain a close set of equations that describes the coupled dynamics of a wave field and a streaming flow. On a fast time-scale, the acoustic waves are not affected by attenuation mechanisms (viscosity and thermal diffusivity). They are expected to affect the waves on the slow time-scale, and this effect is not taken into account by this set of equations. We shall see later on that such effects, among which are damping and energy transfer with the mean flow, are part of a second order solvability condition.

\subsection{Energy balance}

\subsubsection{Dimensional energy balance}

Although not essential for the rest of the study, it it interesting to have a look at the energy balance. For this system, it is (see Appendix A for more details),

$$
\frac{\mathrm{d} E_{c}}{\mathrm{~d} t}=\dot{Q}
$$

where $\dot{Q}$ is the total heat flux received by the gas, defined by

$$
\dot{Q}=\kappa \int d x\left[\left(\partial_{\tilde{y}} \tilde{T}\right)(\tilde{x}, \tilde{y}=0, \tilde{t})-\left(\partial_{\tilde{y}} \tilde{T}\right)\left(\tilde{x}, \tilde{y}=H_{*}, \tilde{t}\right)\right]
$$

and $E_{c}$ the kinetic energy, defined by

$$
E_{c}=\iint d x d y\left(\frac{\tilde{\rho} \tilde{\mathbf{u}}^{2}}{2}+\frac{\tilde{p}}{\gamma-1}\right)
$$

In (75), we recognize the macroscopic kinetic energy density and the microscopic kinetic energy density of an ideal gas, i.e. the internal energy of an ideal gas ${ }^{3}$.

\subsubsection{Dimensionless energy balance expended with respect to $\epsilon$}

With dimensionless quantities, (73) becomes

$$
\begin{aligned}
\frac{\mathrm{d}}{\mathrm{d} t} \iint d x d y\left[\frac{p}{\gamma(\gamma-1)}\right. & \left.+\frac{\rho}{2}(u+\sqrt{\epsilon} h v)^{2}\right] \\
= & \frac{\epsilon}{h^{2} P e_{s}(\gamma-1)} \int d x\left[\left(\partial_{y} T\right)(x, y=0, t)-\left(\partial_{y} T\right)(x, y=1, t)\right] .
\end{aligned}
$$

That gives, at order $\epsilon$ and with fast-time averaging,

$$
0=\int d x\left[\left(\partial_{y} \bar{\Theta}_{0}\right)(x, y=0, t)-\left(\partial_{y} \bar{\Theta}_{0}\right)(x, y=1, t)\right]
$$

that can be also derived from the initial set of equations. This states that the instantaneous heat fluxes at the top and at the bottom are equal.

\footnotetext{
${ }^{3}$ Since we assume that $C_{v}$ does not depend on $T, U(T)=C_{v} T=\frac{n R T}{\gamma-1}=\frac{P V}{\gamma-1}$ for an ideal gas.
} 
At order $\epsilon$, for the fast time scale, we get

$$
\iint d x d y\left(\partial_{\phi} \pi_{1}^{\prime}\right)=0
$$

given that $\Theta_{0}$ does not depend on the fast time. This equality also results from the study of the acoustic modes $\left(\pi_{1}^{\prime} \propto g(x)\right.$, see next section).

At order $\epsilon^{2}$, with fast-time averaging,

$$
\iint d x d y\left(\partial_{T} \bar{\pi}_{1}\right)=\frac{\gamma}{h^{2} P e_{s}} \int d x\left[\left(\partial_{y} \bar{\Theta}_{1}\right)(x, y=0, t)-\left(\partial_{y} \bar{\Theta}_{1}\right)(x, y=1, t)\right] .
$$

This balances the internal energy variation of the gas with the instantaneous heat flux. Given that $\bar{\pi}_{1}(x, y, T)=\bar{\pi}_{1}(T)$, this equation gives access to $\bar{\pi}_{1}$, via

$$
\frac{d \bar{\pi}_{1}}{d T}=\frac{\gamma}{2 \pi h^{2} P e_{s}} \int d x\left[\left(\partial_{y} \bar{\Theta}_{1}\right)(x, y=0, t)-\left(\partial_{y} \bar{\Theta}_{1}\right)(x, y=1, t)\right] .
$$

\section{Properties of the Acoustic Waves}

We now focus on the acoustic waves. Their evolution only depends on one slow variable, the first order density $\bar{\rho}_{0}\left(\bar{\Theta}_{0}+T_{B}=\bar{\rho}_{0}^{-1}\right)$. This quantity is considered as a given function in this section. Therefore, the set of governing equations is linear, and we thereafter consider only one eigenvector. All the fields can then be expressed as

$$
f_{1}^{\prime}(x, y, \phi, T)=\frac{1}{2}\left(A(T) \hat{f}_{1}(x, y, T) e^{i \phi}+\text { c.c. }\right),
$$

where $f$ stands for any variable $(u, v, \rho, \pi, \Theta), A(T)$ is a slowly evolving amplitude, and $\hat{f}_{1}$, a complex function, describes the geometry of the mode. For this decomposition to be unique, we need a normalization condition for $\hat{f}$, that we shall derive later on. The slow evolution of $A$ cannot be obtained from the first order set of equations, and will be found as a solvability condition for the waves at the next order.

\subsection{Geometry of the mode}

\subsubsection{Reduction to a single ode}

Given the decomposition (81), the governing equations are

$$
\left\{\begin{array}{l}
i \omega_{0} \bar{\rho}_{0} \hat{u}_{1}+\frac{1}{\gamma} \partial_{x} \hat{\pi}_{1}=0 \\
\partial_{y} \hat{\pi}_{1}=0 \\
i \omega_{0} \hat{\rho}_{1}+\partial_{x}\left(\bar{\rho}_{0} \hat{u}_{1}\right)+\partial_{y}\left(\bar{\rho}_{0} \hat{v}_{1}\right)=0 \\
i \omega_{0} \hat{\Theta}_{1}+\hat{u}_{1} \partial_{x} \bar{\Theta}_{0}+\hat{v}_{1} \partial_{y}\left(\bar{\Theta}_{0}+T_{B}\right)=(1-\gamma)\left(\bar{\Theta}_{0}+T_{B}\right)\left(\partial_{x} \hat{u}_{1}+\partial_{y} \hat{v}_{1}\right) \\
\hat{\pi}_{1}=\hat{\rho}_{1}\left(\bar{\Theta}_{0}+T_{B}\right)+\bar{\rho}_{0} \hat{\Theta}_{1}
\end{array}\right.
$$


This system becomes, with $\bar{\Theta}_{0}(x, y)+T_{B}(y)=1 / \bar{\rho}_{0}$,

$$
\left\{\begin{array}{l}
i \omega_{0} \bar{\rho}_{0} \hat{u}_{1}+\frac{1}{\gamma} \partial_{x} \hat{\pi}_{1}=0 \\
\partial_{y} \hat{\pi}_{1}=0 \\
\hat{\rho}_{1}=\frac{i}{\omega_{0}}\left[\partial_{x}\left(\bar{\rho}_{0} \hat{u}_{1}\right)+\partial_{y}\left(\bar{\rho}_{0} \hat{v}_{1}\right)\right] \\
\hat{\Theta}_{1}=\frac{i}{\omega_{0}}\left[\hat{u}_{1} \partial_{x} \bar{\rho}_{0}^{-1}+\hat{v}_{1} \partial_{y} \bar{\rho}_{0}^{-1}-\frac{1-\gamma}{\bar{\rho}_{0}}\left(\partial_{x} \hat{u}_{1}+\partial_{y} \hat{v}_{1}\right)\right] \\
\hat{\pi}_{1}=\frac{\hat{\rho}_{1}}{\bar{\rho}_{0}}+\bar{\rho}_{0} \hat{\Theta}_{1}
\end{array}\right.
$$

Combining (89), (90) and (91), $\hat{\pi}_{1}$ can then be expressed as a function of $\hat{u}_{1}$ and $\hat{v}_{1}$,

$$
\hat{\pi}_{1}=\frac{i \gamma}{\omega_{0}}\left(\partial_{x} \hat{u}_{1}+\partial_{y} \hat{v}_{1}\right)
$$

Therefore, the initial set of equations reduces to two coupled partial differential equations,

$$
\left\{\begin{array}{l}
\partial_{x}\left(\partial_{x} \hat{u}_{1}+\partial_{y} \hat{v}_{1}\right)=-\omega_{0}^{2} \bar{\rho}_{0} \hat{u}_{1} \\
\partial_{y}\left(\partial_{x} \hat{u}_{1}+\partial_{y} \hat{v}_{1}\right)=0
\end{array}\right.
$$

We can go further and obtain a single ordinary differential equation. To this end, we formally integrate a combination of these equations,

$$
\partial_{y}(93)+\partial_{x}(94) \Longrightarrow \partial_{y}\left(\bar{\rho} \hat{u}_{1}\right)=0 \Longrightarrow \hat{u}_{1}=\frac{f(x)}{\bar{\rho}_{0}}
$$

where $f$ is an unknown function of $x$ only. (94) then becomes

$$
\partial_{x}\left(\frac{f(x)}{\bar{\rho}_{0}}\right)+\partial_{y} \hat{v}_{1}=g(x),
$$

where $g$ is another unknown function. (93) finally reads

$$
g^{\prime}=-\omega_{0}^{2} f \Longrightarrow f(x)=-\frac{g^{\prime}(x)}{\omega_{0}^{2}} .
$$

Equations (95) and (96) provide expressions for $\hat{u}_{1}$ and $\hat{v}_{1}$ as a function of this unknown function $g$ only (remember that the bottom boundary condition is $\hat{v}_{1}(x, y=0, T)=0$ ):

$$
\left\{\begin{array}{l}
\hat{u}_{1}(x, y, T)=-\frac{g^{\prime}(x)}{\omega_{0}^{2} \bar{\rho}_{0}(x, y)} \\
\hat{v}_{1}(x, y, T)=y g(x)+\partial_{x}\left(\frac{g^{\prime}(x)}{\omega_{0}^{2}} \int_{0}^{y} \frac{d y}{\bar{\rho}_{0}}\right)
\end{array}\right.
$$

The upper boundary condition $\hat{v}_{1}(x, y=1, T)=0$ provides a differential equation for $g$,

$$
g(x)=-\frac{d}{d x}\left(\frac{g^{\prime}(x) \alpha(x)}{\omega_{0}^{2}}\right) \Longleftrightarrow g^{\prime \prime}(x)+\frac{\alpha^{\prime}}{\alpha} g^{\prime}(x)+\frac{\omega_{0}^{2}}{\alpha} g(x)=0
$$


where $\alpha(x)$ is defined by

$$
\alpha(x)=\int_{0}^{1} \frac{d y}{\bar{\rho}_{0}(x, y)} .
$$

The ability to describe a two-dimensional acoustic field with a single ordinary differential equation is a result of the thin layer approximation: in any container of aspect ratio of order unity, the frequencies of the modes have to be found through a two-dimensional eigenvalue problem. This provides a huge simplification to the analysis of these acoustic modes, and we thereafter characterize this function $g$.

\subsubsection{General features of $g$}

Real-valued function We impose $\hat{u}_{1}$ to be a real field. Equations (93) and (94) thus imply that $\hat{v}_{1}$ is also a real field, (92) that $\hat{\pi}_{1}$ is a pure imaginary one, and so on. Given that $\hat{u}_{1}$ and $\hat{v}_{1}$ are directly related to $g$ (see (98) and (99)), we deduce that $g$ is real-valued.

Orthogonality (100) is a second order differential equation in the "Sturm-Liouville form" (or "self-adjoint form"), and cannot be solved explicitly. Its mechanical equivalent is the motion of a mass attached to a spring of variable stiffness (note that $\alpha>0$ ), and driven or damped by a linear friction force. We therefore expect that $g$ is a function "that oscillates". This is confirmed by the following integral, computed with the $2 \pi$ periodicity in $x$ :

$$
\int_{0}^{2 \pi} g(x) d x=-\left[\frac{g^{\prime}(x) \alpha(x)}{\omega_{0}^{2}}\right]_{0}^{2 \pi}=0 .
$$

Thus, there must exist one or more $x_{0}$ such that $g^{\prime}\left(x_{0}\right)=0$, which correspond to nodes for the $x$-velocity: $\hat{u}_{1}\left(x_{0}, y, T\right)=0$. To enforce the zero mass exchange at $x=0$, the boundary conditions of (100) must then be $g^{\prime}(0)=g^{\prime}(1)=0^{4}$. We can also show that the eigenvectors of this ode are orthogonal. Let $\left(g_{A}, g_{B}\right)$ be two eigenvectors and $\left(\omega_{A}, \omega_{B}\right)$ their angular eigenfrequencies,

$$
\begin{aligned}
\left(\omega_{A}^{2}-\omega_{B}^{2}\right) \int_{0}^{2 \pi} g_{A}(x) g_{B}(x) d x & =\int_{0}^{2 \pi}\left[\left(\omega_{A}^{2} g_{A}\right) g_{B}-g_{A}\left(\omega_{B}^{2} g_{B}\right)\right] d x \\
& =\int_{0}^{2 \pi}\left[g_{A} \frac{d}{d x}\left(g_{B}^{\prime} \alpha\right)-g_{B} \frac{d}{d x}\left(g_{A}^{\prime} \alpha\right)\right] d x \\
& =\left[\alpha\left(g_{A} g_{B}^{\prime}-g_{B} g_{A}^{\prime}\right)\right]_{0}^{2 \pi}=0
\end{aligned}
$$

This provides a scalar product on eigenvectors, and we therefore require them to be normalized, i.e.

$$
\int_{0}^{2 \pi} g(x)^{2} d x=1
$$

\footnotetext{
${ }^{4}$ With (58), this implies that $\bar{u}(x=0, t, y)$ is always zero.
} 
Vertically averaged velocities We define $\left(\hat{U}_{1}, \hat{V}_{1}\right)$ as the vertically averaged $x$ and $y$ velocity:

$$
\begin{aligned}
\hat{V}_{1}(x) & =\int_{0}^{1} \hat{v}_{1}(x, y) d y=\frac{g(x)}{2}+\frac{d}{d x}\left(\frac{g^{\prime}(x)}{\omega_{0}^{2}} \int_{0}^{1} d y \int_{0}^{y} \frac{d y^{\prime}}{\bar{\rho}_{0}\left(x, y^{\prime}, T\right)}\right) \\
& =\frac{g(x)}{2}+\frac{d}{d x}\left(\frac{g^{\prime}(x)}{2 \omega_{0}^{2}} \int_{0}^{1} d y \int_{0}^{1} \frac{d y^{\prime}}{\bar{\rho}_{0}\left(x, y^{\prime}, T\right)}\right) \\
& =\frac{g(x)}{2}+\frac{d}{d x}\left(\frac{g^{\prime}(x) \alpha(x)}{2 \omega_{0}^{2}}\right)=\frac{g(x)}{2}-\frac{g(x)}{2}=0 .
\end{aligned}
$$

Therefore, the acoustic field has a zero vertical mean. For the horizontal velocity, we get

$$
\hat{U}_{1}(x)=\int_{0}^{1} \hat{u}_{1}(x, y) d y=-\frac{g^{\prime}(x)}{\omega_{0}^{2}} \int_{0}^{1} \frac{d y}{\bar{\rho}_{0}(x, y)}=-\frac{g^{\prime}(x) \alpha(x)}{\omega_{0}^{2}} .
$$

Similarly, we can derive some kind of orthogonality condition for these mean $x$ velocities. Let $\left(\hat{U}_{A}, \hat{U}_{B}\right)$ be two eigenvectors, associated with the angular eigenfrequencies $\left(\omega_{A}, \omega_{B}\right)$ and the functions $\left(g_{A}, g_{B}\right)$ :

$$
\begin{aligned}
\int_{0}^{2 \pi} \hat{U}_{A}(x) g_{B}^{\prime}(x) d x & =-\int_{0}^{2 \pi} \frac{g_{A}^{\prime}(x) \alpha(x)}{\omega_{A}^{2}} g_{B}^{\prime}(x) d x \\
& =-\left[\frac{g_{A}^{\prime}(x) \alpha(x)}{\omega_{A}^{2}} g_{B}(x)\right]_{0}^{2 \pi}-\int_{0}^{2 \pi} g_{A}(x) g_{B}(x) d x=-\delta_{A}^{B} .
\end{aligned}
$$

These equalities result from periodicity in $x$ and from the differential equation (100) for $g_{A}$. $\delta_{A}^{B}$ is the Kronecker delta, equal to unity if $A=B, 0$ otherwise.

\subsection{Slow evolution of the amplitude}

With the previous system, we can at every time find the shape and the frequency of any acoustic mode. However, the evolution of $A(T)$ is not constrained. Since we expect several physical effects that are not described at this order to be involved (as damping or energy transfer to the mean flow), we have to derive equations for the waves at the next order (see Appendix B). The following amplitude equation can then be obtained (see Appendix C):

$$
\begin{aligned}
& \frac{2}{A \omega_{0}^{-1}} \frac{\mathrm{d}\left(A \omega_{0}^{-1}\right)}{\mathrm{d} T}=-\frac{i \omega_{0}}{P e_{s} h^{2}} \iint d x d y g(x) \partial_{y y} \hat{\Theta}_{1} \\
& +\iint d x d y\left(\partial_{x} \bar{u}_{1}+\partial_{y} \bar{v}_{1}\right)\left[(1-\gamma) g(x)^{2}+\frac{g^{\prime}(x)^{2}}{\omega_{0}^{2} \bar{\rho}_{0}}\left(\frac{P e_{s}}{R e_{s}}-\frac{1}{2}\right)\right] \\
& -\frac{1}{2 \omega_{0}^{2}} \iint d x d y g^{\prime}(x)^{2}\left(\bar{u}_{1} \partial_{x} \bar{\rho}_{0}^{-1}+\bar{v}_{1} \partial_{y} \bar{\rho}_{0}^{-1}\right) .
\end{aligned}
$$

The terms in the right-hand side of this equation describe thermal damping, energy exchange with the mean flow, and heat transfer at the boundaries. The quantity on the left-hand side, $A \omega_{0}^{-1}$, is related to the energy of the wave-field, that is at the leading order

$$
\iint d x d y \bar{\rho}_{0} \frac{A(T)^{2}}{2} \times \frac{\hat{u}_{1}^{2}}{2}=\frac{A(T)^{2}}{4 \omega_{0}^{4}} \int d x g^{\prime}(x)^{2} \alpha(x)=\left(\frac{A(T)}{2 \omega_{0}}\right)^{2} .
$$


We emphasize that, in order to describe phenomena that fully rely on baroclinic driving, we have disregarded in this problem dissipation in the boundary layers. Although the associated effective slip boundary condition would be of higher order in the reduced system we consider, dissipation in the boundary layer would however dominate all the terms in (113). Indeed, an order of magnitude of the dimensional time-scale $\tau_{B L}$ over which the dissipation in the boundary layer damps the wave can be found by

$$
\tau_{B L} \sim \frac{\iint d x d y \rho_{*}\left(\tilde{u}^{\prime}\right)^{2}}{\iint d x d y \rho_{*} \nu\left(\tilde{u}^{\prime} / \delta_{B L}\right)^{2}} \sim \frac{h \sqrt{R_{s}}}{\omega_{*} \sqrt{\epsilon}} .
$$

Therefore, this time-scale is in between the fast one and the slow one. If we were to consider boundary layers, the input power would have to strictly balance such dissipation by viscosity at this time-scale, and this power would at order $T$ result in an additional heat input at $y=0$ and $y=1$ as a consequence of viscous heating.

\section{Linear response}

We now consider the simplest regime of this system, in which an acoustic mode of given amplitude and geometry drives a laminar mean flow. For consistency, we hope that this flow will, in return, not much affect the geometry of the acoustic mode.

\subsection{Reynolds stress}

For this problem, $\bar{\rho}_{0}=T_{B}^{-1}=(1+\Gamma y)^{-1}$, so that

$$
\alpha=1+\frac{\Gamma}{2}
$$

does not depend on $x$. We can therefore find an explicit expression for the function $g$,

$$
g(x)=C_{1} \cos \left(\frac{\omega_{0}}{\sqrt{\alpha}} x\right)+C_{2} \sin \left(\frac{\omega_{0}}{\sqrt{\alpha}} x\right)
$$

where $C_{1}$ and $C_{2}$ are constants that have to be determined. The boundary conditions on $g, g^{\prime}(0)=g^{\prime}(2 \pi)=0$, fix $C_{2}=0$. Moreover, periodicity requires

$$
\omega_{0}=n \sqrt{\alpha},
$$

and, with the normalization condition, we end up with

$$
g(x)=\frac{\cos (n x)}{\sqrt{\pi}} .
$$

We can then compute $\hat{u}_{1}$ and $\hat{v}_{1} \cdot \hat{u}_{1}$ is

$$
\hat{u}_{1}=-\frac{g^{\prime}}{\omega_{0}^{2} \bar{\rho}_{0}}=(1+\Gamma y) \frac{\sin (n x)}{n \alpha \sqrt{\pi}},
$$




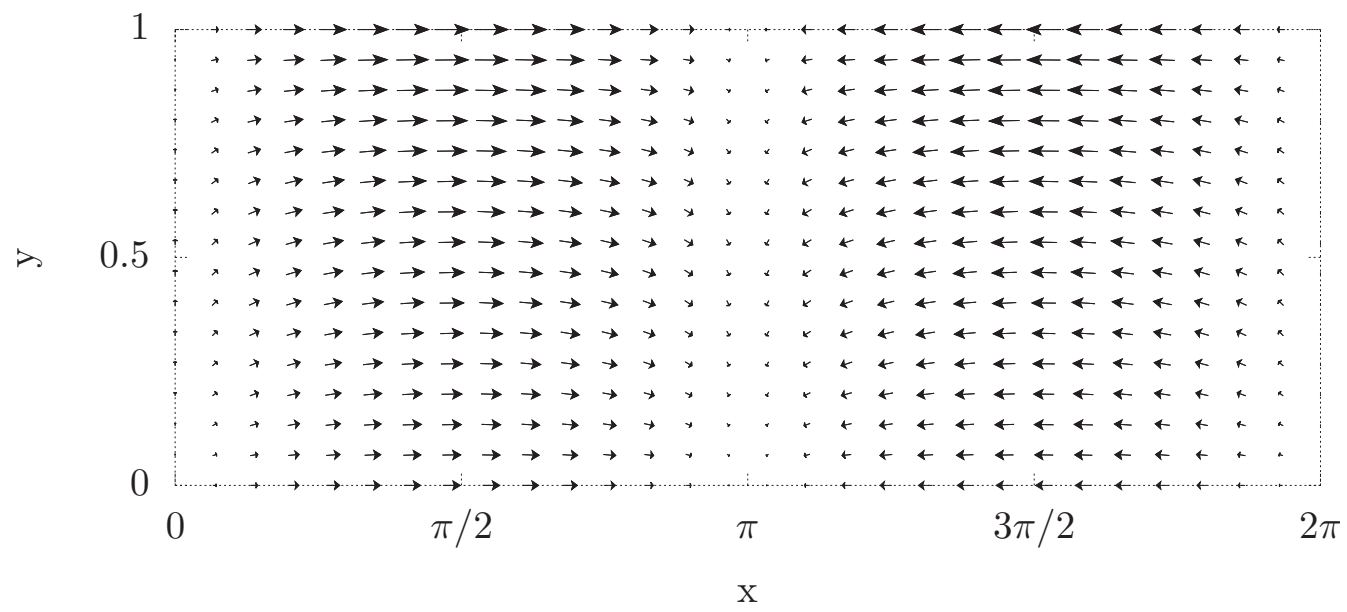

Figure 5: Geometry of the acoustic velocity fields for the mode $n=1$.

and $\hat{v}_{1}$ is

$$
\hat{v}_{1}=y \frac{\cos (n x)}{\sqrt{\pi}}-\partial_{x}\left(\frac{n \sin (n x)}{n^{2} \alpha \sqrt{\pi}} \int_{0}^{y}(1+\Gamma y) d y\right)=\frac{y}{\sqrt{\pi}}\left(1-\frac{(1+\Gamma y / 2)}{\alpha}\right) \cos (n x) .
$$

According to (122) and (123), the vertical velocity field does not depend on $n$, contrary to the horizontal one that decays with $n$. The velocity field for $n=1$ is sketched in Fig. 5 , and reveals the presence of vorticity. We then evaluate the first contribution to the Reynolds stress,

$$
-\partial_{x}\left(\bar{\rho}_{0} \overline{u_{1}^{\prime 2}}\right)=-\partial_{x}\left(\frac{1}{1+\Gamma y} \frac{A(T)^{2}}{2} \hat{u}_{1}^{2}\right)=-A(T)^{2} \frac{(1+\Gamma y)}{2 n \alpha^{2} \pi} \sin (2 n x),
$$

the second one,

$$
-\partial_{y}\left(\bar{\rho}_{0} \overline{u_{1}^{\prime} v_{1}^{\prime}}\right)=-\partial_{y}\left(\frac{1}{1+\Gamma y} \frac{A(T)^{2}}{2} \hat{u}_{1} \hat{v}_{1}\right)=\frac{A(T)^{2}}{4 \pi n \alpha^{2}} \Gamma\left(y-\frac{1}{2}\right) \sin (2 n x),
$$

so that the full Reynolds stress reads

$$
R(x, y)=-\frac{A(T)^{2}}{2 \pi n \alpha^{2}}\left(1+\frac{\Gamma}{4}+\frac{\Gamma}{2} y\right) \sin (2 n x)
$$

\subsection{Laminar mean flow}

We then assume that the steady state mean flow is small enough so that equations can be linearized. Contrary to the usual analysis of Rayleigh streaming, we cannot use a stream function because, in general, this flow is compressible (even though the Mach number is 
negligible). The governing equations (63-67) read

$$
\left\{\begin{array}{l}
0=-\frac{1}{\gamma} \partial_{x} \delta \bar{\pi}+R(x, y)+\frac{\partial_{y y} \delta \bar{u}}{R e_{s} h^{2}} \\
\partial_{y} \delta \bar{\pi}=0 \\
\partial_{x} \delta \bar{u}+\partial_{y} \delta \bar{v}=\frac{\partial_{y y} \delta \bar{\Theta}_{0}}{P e_{s} h^{2}} \\
\delta \bar{v}=(1+\Gamma y) \frac{\partial_{y y} \delta \bar{\Theta}}{\Gamma P e_{s} h^{2}} \\
\delta \bar{\rho}=-\frac{\delta \Theta}{(1+\Gamma y)^{2}}
\end{array}\right.
$$

We can remove $\bar{\pi}$ by combining (127) and (128),

$$
\partial_{x y} R=-\frac{\partial_{y y y x} \delta \bar{u}}{R e_{s} h^{2}} .
$$

The conservation of mass (129) and (130) also imply

$$
\partial_{x} \delta \bar{u}=-\frac{(1+\Gamma y)}{\Gamma P e_{s} h^{2}} \partial_{y y y} \delta \bar{\Theta} .
$$

Finally, we obtain a close partial differential equation for $\bar{\Theta}$,

$$
\partial_{x y} R=\frac{(1+\Gamma y) \partial_{\text {yyyyyy }} \delta \bar{\Theta}+3 \Gamma \partial_{y y y y y} \delta \bar{\Theta}}{\Gamma R e_{s} P e_{s} h^{4}},
$$

that is,

$$
\frac{(1+\Gamma y) \partial_{y y y y y y} \delta \bar{\Theta}+3 \Gamma \partial_{y y y y y} \delta \bar{\Theta}}{\Gamma R e_{s} P e_{s} h^{4}}=-\frac{2 A^{2} \Gamma}{\pi(2+\Gamma)^{2}} \cos (2 n x)
$$

This equation has to be solved with the following boundary conditions

1. $\delta \bar{\Theta}(y=0)=\delta \bar{\Theta}(y=0)=0$ (fixed temperatures at the boundaries)

2. $\delta \bar{\Theta}^{\prime \prime}(y=0)=\delta \bar{\Theta}^{\prime \prime}(y=1)=0($ from $(130))$

3. $\delta \bar{\Theta}^{\prime \prime \prime}(y=0)=\delta \bar{\Theta}^{\prime \prime \prime}(y=1)=0($ from $(133))$

4. $2 \pi$ periodicity in $x$

With these boundary conditions, a unique solution can be found. Since it is quite lengthy, the general solution is postponed to Appendix D and we thereafter focus on $\Gamma=1$, in which case

$$
\delta \bar{\Theta}(x, y)=-\frac{2 A^{2} R e_{s} P e_{s} h^{4}}{9 \pi} \cos (2 n x) G(y),
$$

with

$$
\begin{aligned}
G(y)= & \frac{1}{1080(-3+\ln (16))} \times\left[60(1+y)^{2} \ln (1+y)\right. \\
& \left.+y\left(94-90 y-20 y^{2}-222 \ln (2)+3 y^{4}(-3+\ln (16))-5 y^{3}(-5+\ln (64))\right)\right] .
\end{aligned}
$$




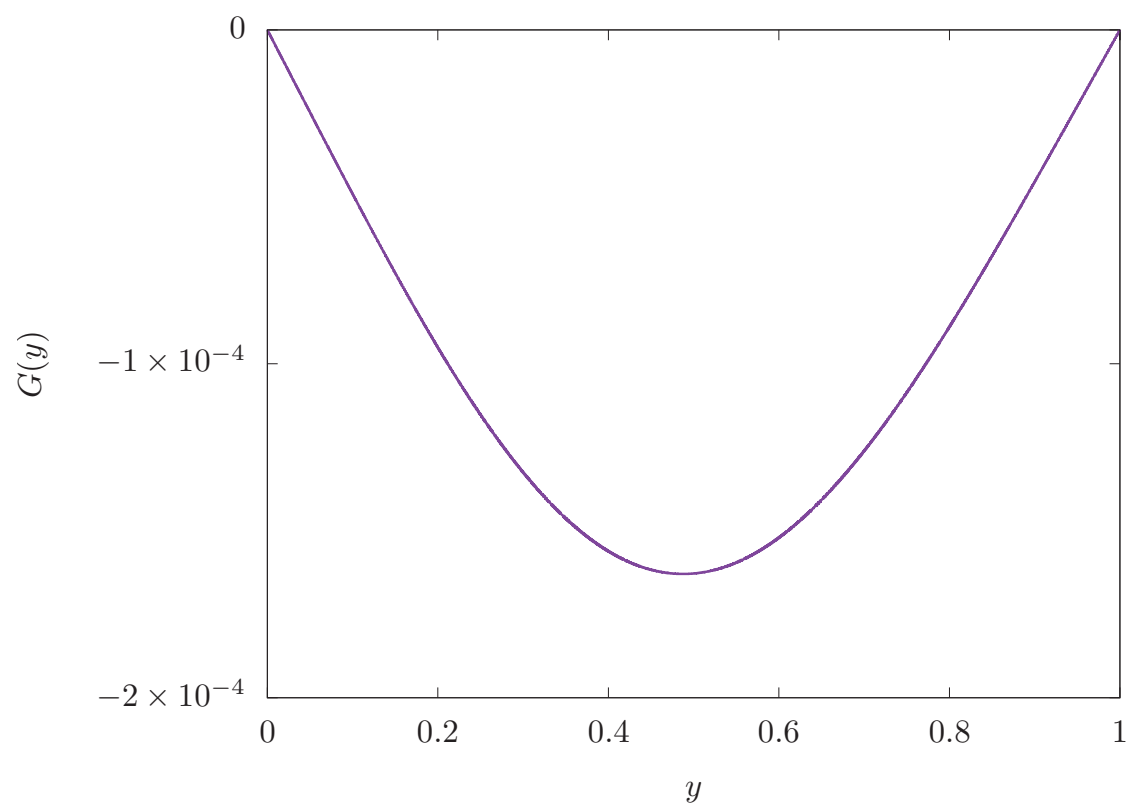

Figure 6: Vertical structure of the temperature perturbation.

This function is always negative, and reaches its minimal value in the range $[0,1]$ at $y=$ 0.488 , where $G \simeq-1.63 \times 10^{-4}$. It turns out to be quite similar to a sine function, see Fig. 6 . The very small values taken by this function in this range may seem surprising given that it is the solution of an ordinary differential equation with coefficients of order unity. However, similar features often arise when high order derivatives cap the variation speed of a function with boundary condition zero at both sides (see, for instance, the linear regime of convection between two vertical walls of different temperatures).

With this result, we can compare the amplitude of the velocity fields balanced by viscosity caused by dissipation in the boundary layers (Rayleigh streaming) to the one driven by a background density gradient. The dimensional maximal velocities of these flows along the $x$ direction are respectively ${ }^{5}$

$$
\tilde{u}_{R}=\frac{3 U_{*}^{2}}{8 a_{*}}=\left(\frac{3 \epsilon}{8}\right) U_{*} \quad \text { and } \quad \tilde{u}_{B}=\left(3.2 \times 10^{-4} A^{2} R e_{s} h^{2}\right) U_{*} .
$$

Therefore, for small values of $\epsilon$, the baroclinic forcing dominates the one caused by dissipation in the boundary layers.

\subsection{Comparison with previous work}

This streaming flow can be compared to the study of Lin and Farouk [8], in which direct numerical simulations of the full set of equations have been performed. The system considered is similar to ours and consists in a thin channel in which a temperature difference

\footnotetext{
${ }^{5}$ Numerically, $\max _{y \in[0,1]}\left|(1+y) G^{\prime \prime \prime}(y)\right|=8.99 \times 10^{-3}$ (reached for $\left.y=0.777\right)$.
} 
can be applied between the two horizontal walls. With no thermal driving, the response is found close to the one described in Sec. 1 and consists of stack cells. When a temperature difference is applied, the vertical cells merge.

For their case 1C, corresponding to the highest temperature difference, the dimensionless parameters are:

$$
\epsilon=10^{-2}, \quad \gamma=1.4, \quad \Gamma=0.2, \quad h=2.3, \quad R e_{s}=5.7, \quad P e_{s}=4.1 .
$$

The amplitude $A$ of the wave field is not reported, but it can be reasonably assumed to be the one in the absence of temperature difference, in which case $A \simeq 6$.

For these parameters, the $x$ (resp. $y$ ) component of the streaming velocity at $x=3 \pi / 4$ (resp. $\pi / 2$ ) are found to be in our model

$$
\delta \bar{u}\left(x=\frac{3 \pi}{4}, y\right)=-\frac{(1+\Gamma y)}{\pi(2+\Gamma)^{2}} A^{2} \operatorname{Re}_{s} h^{2} \Gamma G_{\Gamma}^{\prime \prime \prime}(y),
$$

and

$$
\delta \bar{v}\left(x=\frac{\pi}{2}, y\right)=\frac{(1+\Gamma y)}{\pi(2+\Gamma)^{2}} 2 A^{2} R e_{s} h^{2} \Gamma G_{\Gamma}^{\prime \prime}(y) .
$$

where the function $G$ now reads

$$
\begin{aligned}
G_{0.2}(y) & =47.664\left(7.19999866 y-0.1575906 y^{2}+0.096 y^{3}-0.0047285 y^{4}\right. \\
& \left.+0.0002914 y^{5}-1.44 y^{2} \ln (0.2)-\left(36+14.4 y+1.44 y^{2}\right) \ln (1+0.2 y)\right)
\end{aligned}
$$

The data of [8] are not dimensionless, and we then consider the dimensional streaming velocities, obtained by multiplying $\delta \bar{u}$ by $\epsilon a_{*}$, and $\delta \bar{v}$ by $\epsilon^{3 / 2} a_{*} h$, with $a_{*}=353 \mathrm{~m} \cdot \mathrm{s}^{-1}$ here. The comparison is reported in Fig. 7, and shows a quantitative agreement (no adjustable parameter), although the dynamics in [8] involves several effects not taken into account by the linear response model, as boundary layers, viscous heating, inertia, and evolution of the viscosity and diffusivity with temperature. This confirms that our model captures the main features of this dynamics.

\subsection{Additional heat flux}

Fig. 8 shows the streaming velocity field together with the temperature perturbation: we can clearly infer an increase in the heat flux, that we want to compute. A practical issue we face is that the first order additional heat flux vanishes, since the temperature disturbance is of zero mean in the $x$ direction:

$$
\kappa \int_{0}^{2 \pi} d x\left(\partial_{y} \delta \bar{\Theta}\right)(x, y=0) \propto \int_{0}^{2 \pi} d x \cos (2 n x)=0 .
$$

Instead of computing the next order temperature perturbation, we will show that the integral of $(\tilde{\nabla} \tilde{T})^{2}$ contains the information we are looking for. To prove this, we go back to the dimensionless quantities and the expansion in power of $\epsilon$. On one hand, integration by part of this integral yields

$$
\begin{aligned}
\kappa \iint(\tilde{\nabla} \tilde{T})^{2} d x d y & =\kappa \iint d x d y\left(\tilde{\nabla} \tilde{T}_{B}+\tilde{\nabla} \tilde{\Theta}_{0}+\epsilon \tilde{\nabla} \tilde{\Theta}_{1}+\ldots\right)^{2} \\
& =\kappa \iint d x d y\left(\left(\tilde{\nabla} \tilde{T}_{B}\right)^{2}+\left(\tilde{\nabla} \tilde{\Theta}_{0}\right)^{2}+\epsilon^{2}\left(\tilde{\nabla} \tilde{\Theta}_{1}\right)^{2}+\ldots\right) .
\end{aligned}
$$




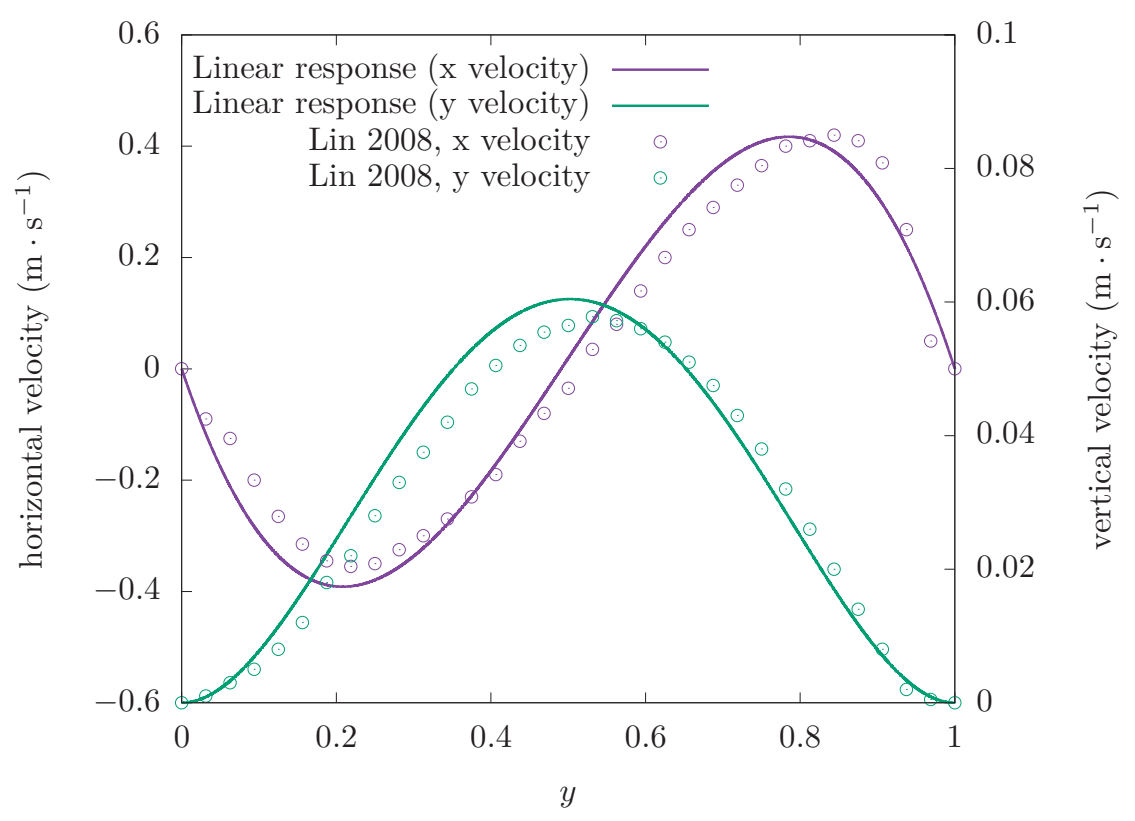

Figure 7: Comparison between the numerical data of [8] (case 1C) and the linear response.

On the other hand, we also have

$$
\kappa \iint(\tilde{\nabla} \tilde{T})^{2} d x d y=\kappa \int d x\left[\tilde{T} \partial_{\tilde{y}} \tilde{T}\right]_{0}^{H_{*}}-\kappa \iint d x d y \tilde{T} \tilde{\nabla}^{2} \tilde{T}=\dot{Q} \Delta \Theta_{*}-\kappa \iint d x d y \tilde{T} \tilde{\nabla}^{2} \tilde{T},
$$

where $\Delta \Theta_{*}=\tilde{T}\left(\tilde{y}=H_{*}\right)-\tilde{T}(\tilde{y}=0)$ is the imposed temperature difference and $\dot{Q}>0$ the heat flux that goes through this system (see (74)). We then work out the last term with the heat equation, with viscous heating denoted as $\Phi$, as in (43).

$$
\begin{aligned}
& \kappa \iint d x d y \tilde{T} \tilde{\nabla} 2 \tilde{T}=\iint d x d y \tilde{T}\left(\tilde{\rho} c_{v} \frac{D \tilde{T}}{D \tilde{t}}+\tilde{p}(\tilde{\nabla} \cdot \tilde{\mathbf{u}})+\Phi\right) \\
& =\frac{c_{v}}{2} \iint d x d y \tilde{\rho}(\tilde{\mathbf{u}} \cdot \tilde{\nabla}) \tilde{T}^{2}-\iint \tilde{T} \tilde{p} \frac{(\tilde{\mathbf{u}} \cdot \tilde{\nabla}) \tilde{\rho}}{\tilde{\rho}} d x d y+\iint d x d y \Phi T \\
& =0-\iint \frac{\tilde{p}}{\tilde{\rho}}((\tilde{\mathbf{u}} \cdot \tilde{\nabla})(\tilde{T} \tilde{\rho})-\tilde{\rho}(\tilde{\mathbf{u}} \cdot \tilde{\nabla}) \tilde{T}) d x d y+\iint d x d y \Phi \tilde{T}
\end{aligned}
$$

To derive these equations, we explicitly state that we consider a steady-state, for which $\tilde{\nabla} \cdot(\tilde{\rho} \tilde{\mathbf{u}})=0$. Using the equality $\iint d x d y \tilde{\rho} \tilde{T}(\tilde{\mathbf{u}} \cdot \tilde{\nabla}) \tilde{T}=0$ derived in this set of equations, we obtain

$$
\begin{aligned}
\kappa \iint d x d y \tilde{T} \tilde{\nabla} 2 \tilde{T} & =-\iint d x d y \tilde{T} \tilde{\mathbf{u}} \cdot \tilde{\nabla} \tilde{p}+\iint d x d y \tilde{p}(\tilde{\mathbf{u}} \cdot \tilde{\nabla}) \tilde{T}+\iint d x d y \Phi \tilde{T} \\
& =-\iint d x d y \tilde{T} \tilde{\mathbf{u}} \cdot \tilde{\nabla} \tilde{p}+\iint d x d y \Phi \tilde{T}
\end{aligned}
$$




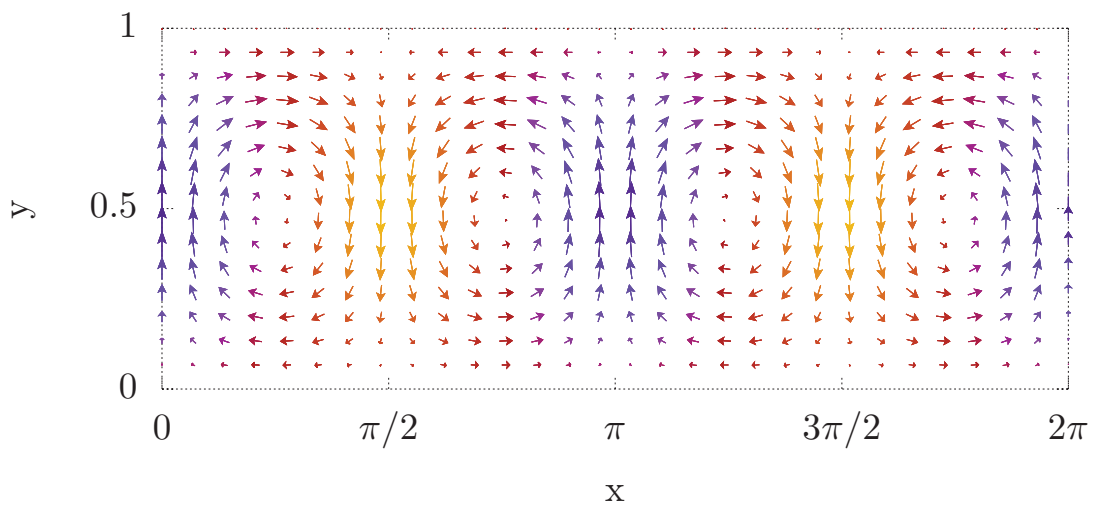

Figure 8: Mean velocity field forced by the mode $n=1$. Color is the supplementary temperature perturbation (blue is cold): remember that the bottom boundary is cold in this setup.

We recognize the pressure gradient of the momentum equation, so that

$$
\begin{aligned}
-\kappa \iint d x d y \tilde{T} \tilde{\nabla}^{2} \tilde{T} & =\iint d x d y \tilde{T}(\tilde{\mathbf{u}} \cdot(\tilde{\nabla} \tilde{p})-\Phi) \\
& =\iint d x d y \tilde{T}\left(\tilde{\mathbf{u}} \cdot \mu\left[\tilde{\nabla}^{2} \tilde{\mathbf{u}}+\frac{1}{3} \tilde{\nabla}(\tilde{\nabla} \cdot \tilde{\mathbf{u}})\right]-\tilde{\mathbf{u}} \cdot(\tilde{\mathbf{u}} \cdot \tilde{\nabla}) \tilde{\mathbf{u}}-\Phi\right) .
\end{aligned}
$$

If the temperature were constant, viscous heating would cancel out the viscous term, see (46). For the given study, all these terms are of high order in $\epsilon$, and can then be neglected. Therefore, at the leading order, the heat flux in a steady state is given by

$$
\dot{Q} \Delta \Theta_{*}=\kappa \iint(\tilde{\nabla} \tilde{T})^{2} d x d y .
$$

To deal with dimensionless quantities, we introduce the Nusselt number $N u$ for the heat flux, defined by

$$
N u=\frac{\dot{Q}}{2 \pi \kappa \Delta \Theta_{*}\left(k_{*} H_{*}\right)^{-1}}=1+\frac{1}{2 \pi \Gamma} \int_{0}^{2 \pi} d x\left(\partial_{y}\left(\Theta_{0}+\epsilon \Theta_{1}+\ldots\right)\right)(y=0) .
$$

From (155) and (156), we obtain at the leading order in $\epsilon$

$$
N u-1=\frac{1}{2 \pi \Gamma} \int_{0}^{2 \pi} d x\left(\partial_{y} \bar{\Theta}_{0}\right)(y=0)=\frac{1}{2 \pi \Gamma^{2}} \iint d x d y\left(\partial_{y} \bar{\Theta}_{0}\right)^{2}
$$

In this section, $\bar{\Theta}_{0}$ has been computed for $\Gamma=1$ as a linear response at the leading order in the wave amplitude $A(T)$, see (136). Thus, ${ }^{6}$

$$
N u-1 \underset{\Gamma=1}{=} \frac{2 A^{4} R e_{s}^{2} P e_{s}^{2} h^{8}}{81 \pi^{2}} \int_{0}^{1} d y G_{B C 1}^{\prime}(y)^{2} \underset{\Gamma=1}{\simeq} 3.2 A^{4} R e_{s}^{2} P e_{s}^{2} h^{8} 10^{-10} .
$$

\footnotetext{
${ }^{6}$ Numerics give $\int_{0}^{1} d y G_{B C 1}^{\prime}(y)^{2} \simeq 1.276 \times 10^{-7}$.
} 


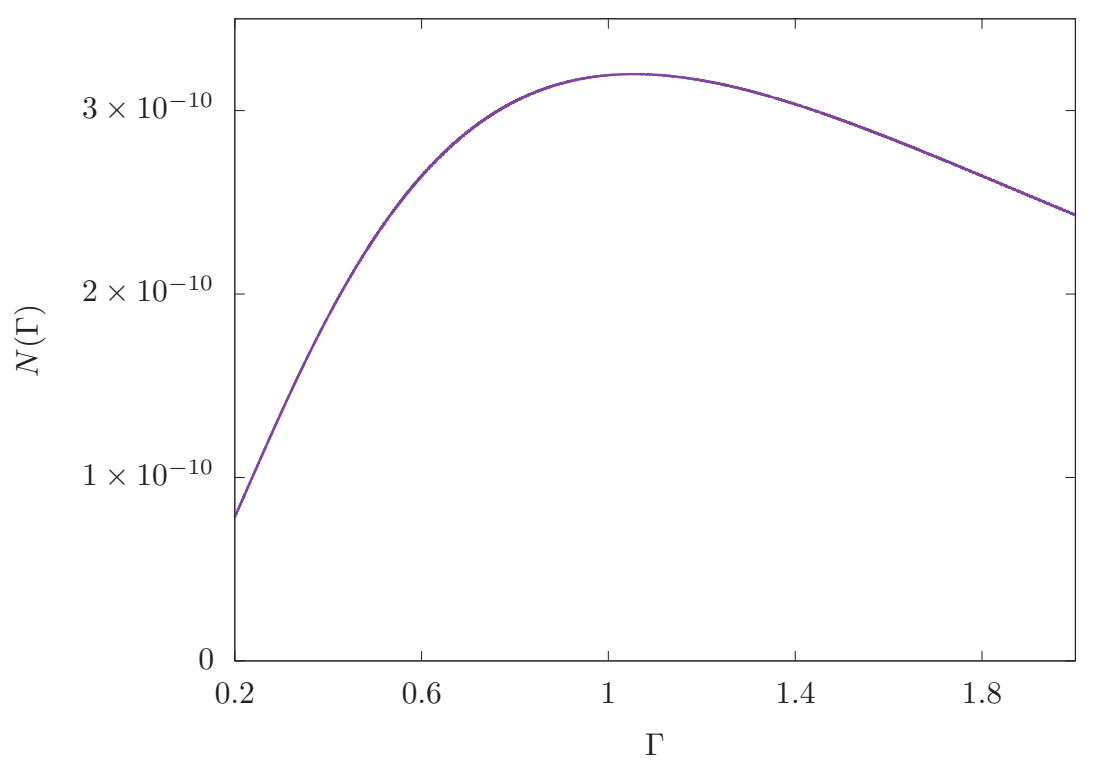

Figure 9: Prefactor of the Nusselt number

Generally speaking, it can be cast under the form

$$
N u-1=N(\Gamma) A^{4} R e_{s}^{2} P e_{s}^{2} h^{8},
$$

where $N(\Gamma)$ is reported in Fig. 9. This has to be compared to the result obtained for streaming based on dissipation in the boundary layers, worked out in [6], that reads ${ }^{7}$

$$
(N u-1)_{B L}=6.2 P e_{s}^{2} h^{4} \epsilon^{2} 10^{-6} .
$$

This demonstrates that, although the Nusselt number we just derived is very small, it still lies several order of magnitude above the one resulting from acoustic streaming in the boundary layers. Our very small Nusselt number is a consequence of the fact that the Reynolds stress is balanced for this linear solution by viscosity, and not by inertia. This second regime is associated with larger velocities, hence larger heat conduction.

We emphasize, again, that the present study neglects dissipation in the boundary layers. Would it be considered, the additional heat flux would be dominated by the power required to sustain the wave.

\subsection{Efficiency of this heat pump}

We look for the power required to sustain the linear steady state. This can be done with the amplitude equation. In the limit of small amplitude, the dominant term is the one that does not vanish for $\bar{u}_{1}=\bar{v}_{1}=\bar{\Theta}_{1}=0$. For $\Gamma=1$, we find

$$
\hat{\Theta}_{1}=\frac{i \cos (x)}{\omega_{0} \sqrt{\pi}}\left((\gamma-1)+y\left(\gamma-\frac{2}{3}\right)-\frac{y^{2}}{3}\right),
$$

\footnotetext{
${ }^{7}$ The Péclet number $P E$ defined in [6] is in our notations $P E=3 P e_{s} h^{2} \epsilon / 32$.
} 
and then

$$
-\frac{i \omega_{0}}{P e_{s} h^{2}} \iint d x d y g(x) \partial_{y y} \hat{\Theta}_{1}=-\frac{2}{3 P e_{s} h^{2}} .
$$

This stands for dissipation caused by thermal diffusion. In order to observe a steady-state, this has to be balanced by an input power $\mathcal{P}$, so that the amplitude equation finally reads

$$
\frac{1}{E} \frac{\mathrm{d} E}{\mathrm{~d} T}=-\frac{2}{3 P e_{s} h^{2}}+\frac{\mathcal{P}}{E},
$$

where $E=A^{2} /\left(2 \omega_{0}^{2}\right)$ is the mean energy of the acoustic waves. This yields in a steady state

$$
P=\frac{2 A^{2}}{9 P e_{s} h^{2}}
$$

Finally, the efficiency $\mathcal{E}$ is given by

$$
\mathcal{E}=\frac{2 \pi \Gamma(N u-1)}{\mathcal{P}} \underset{\Gamma=1}{=} 0.9 \times 10^{-8} A^{2} \operatorname{Re}_{s}^{2} P e_{s}^{3} h^{8}
$$

The dependence on $A$ comes from the fact that most of the injected power is, for small $A$, actually used to balance linear damping of the waves (and not energy transfer from the waves to the mean flow). Although definitely small, this efficiency presents a huge dependence on $h$ that emphasizes the importance on cells of aspect ratio of order one.

\section{$5 \quad$ Numerical Simulations}

The linear response solution previously derived, and upon which the additional heat flux and Nusselt number have been computed, does not hold when there is inertia or feedback to the acoustic wave field. In order to describe the dynamics of the system in this regime, we performed numerical simulations of the reduced set of equations (63 - 66), where the Reynolds stress is computed at each time step by solving the eigenvalue problem (100). This has been done with Dedalus [15].

For parameters of order unity, the solution obtained is a steady-state very similar to the linear response. This has been used to check the correctness of our theoretical computa-tions. On the other hand, when these dimensionless parameters are increased, the numerical solution differs from the linear response, and states with strong feedback can be described. The characterization of this regime is still under progress, but as a preliminary result we report the solution obtained for the parameters of Lin et al. in Fig. 10.

\section{Conclusion}

One main conclusion of this work is that acknowledging the presence of a density gradient as the main driving mechanism of acoustic streaming is crucial to get the correct dynamics in stratified flows. In particular, baroclinic acoustic streaming has been shown to result in velocity fields much bigger than the ones obtained from the usual boundary layer theory. This mechanism is especially favorable for enhancing heat transfers with a good efficiency: 


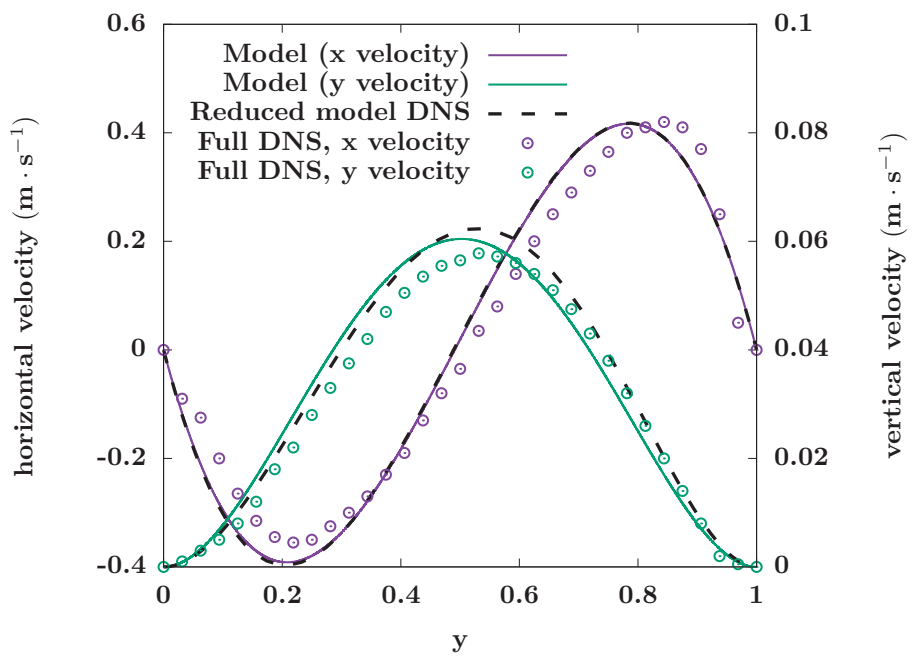

Figure 10: Numerical solution for the parameters of [8] and comparison with the linear model.

whereas the power used to excite the acoustic waves is mainly converted to heat within the boundary layers in the absence of temperature driving, it can a priori be fully transferred to the streaming flow with the baroclinic mechanism.

Because streaming motions are important in this regime, the temperature disturbance is also large, which results in a two-way coupling between the waves and the mean flow [10]. For a strongly stratified thin layer, the dynamics involve both a fast time (associated with the period of the waves) and a slow one (on which the mean quantities evolve). With a solvability condition, we have completely eliminated the fast time scale, and found that both the geometry and the amplitude of the waves can be explicitly solved on the slow time scale. We have also obtained a first order solution for the steady-state that compares well with the previous numerical study of the full system with moderate aspect ratio. Numerical simulations can be used to obtain the solution for any range of parameters. This has been done to check that our theoretical computations were correct, and to compare them to the DNS of Lin et al. [8].

Moreover, the computation of the heat flux and of the efficiency clearly points out the relevance of aspect ratios of order one. In such limit, the flow associated with acoustic streaming caused by boundary layers is restricted to a small portion of the domain, whereas the one resulting from baroclinic acoustic streaming, acting as a bulk force, would probably not. More generally, such quasi-linear systems in which fast waves are coupled with the mean flow they drive, can be found in other domains of fluid mechanics. For instance, it describes zonal jets (see [16] and references therein) and strongly stratified turbulence [17].

\section{Acknowledgment}

I would like to thank Greg Chini for his thorough supervision and the constant support he provided (although he may use a new computer). I am also grateful to Keaton J. Burns for 
introducing me to Dedalus, and for fruitful discussions about physics and numerics. Finally, I would like to thank all the GFD staff members and fellows for this nice summer.

\section{References}

[1] M.S. Longuet-Higgins, Mass transport in water waves, Phil. Trans. A 245, 535 (1953)

[2] J. Lighthill, Acoustic streaming, J. Sound Vib. 61, 391 (1978)

[3] M. Legay et al., Enhancement of Heat Transfer by Ultrasound: Review and Recent Advances, Int. J. Chem. Eng. 2011, 670108 (2011)

[4] L. Rayleigh, On the circulation of air observed in Kundts tubes, and on some allied acoustical problems, Philos. Trans. R. Soc. London 175, 1 (1884).

[5] M. F. Hamilton, Y. A. Ilinskii, and E. A. Zabolotskaya, Acoustic streaming generated by standing waves in two-dimensional channels of arbitrary width, J. Acoust. Soc. Am. 113, $153(2003)$

[6] P. Vainshtein, M. Fishman and C. Gutfinger, Acoustic enhancement of heat transfer between two parallel plates, Int. J. Heat Mass Transf. 38, 1893 (1995)

[7] B-G. Loh, S. Huan, P. I. Ro and C. Kleinstreuer, Acoustic streaming induced by ultrasonic flexural vibrations and associated enhancement of convective heat transfer, $J$. Acoust. Soc. Am. 111, 875 (2002)

[8] Y. Lin and B. Farouk, Heat transfer in a rectangular chamber with differentially heated horizontal walls: Effects of a vibrating sidewall, Int. J. Heat Mass Transf. 51, 3179 (2008)

[9] M. K. Atkas and T. Ozgumus, The effects of acoustic streaming on thermal convection in an enclosure with differentially heated horizontal walls, Int. J. Heat Mass Transf. 53, $5289(2010)$

[10] G. P. Chini, Z. Malecha and T. D. Dreeben, Large-amplitude acoustic streaming, J. Fluid Mech. 744, 329 (2014)

[11] T. D. Dreeben and G. P. Chini, Two-dimensional streaming flows in high-intensity discharge lamps, Phys. Fluids 23, 056101 (2011)

[12] C. Eckart, Vortices and streams caused by sound waves, Phys. Rev. 73, 68 (1948)

[13] H. Medwin and I. Rudnick, Surface and volume sources of vorticity in acoustic fields, J. Acoust. Soc. Am. 25, 538 (1953)

[14] J. T. Stuart, Double boundary layers in oscillatory viscous flow, J. Fluid Mech. 24, $673(1966)$ 
[15] K. J. Burns, G. M. Vasil, J. S. Oishi, D. Lecoanet, B. P. Brown, and E. Quataert, Dedalus: A Flexible Framework for Spectrally Solving Differential Equations (in preparation)

[16] J. B. Marston, G. P. Chini and S. M. Tobias, Generalized Quasilinear Approximation: Application to Zonal Jets, Phys. Rev. Lett. 116, 214501 (2016)

[17] G. P. Chini, K. Julien, C. Rocha, C.-C. Caulfield, Coupled reduced equations for strongly stratified flows (in preparation)

\section{A Derivation of the energy equation}

The energy balance can be obtained from the initial set of equations (41 - 44), starting from

$$
0=\int_{\mathcal{C}} \mathrm{d} \ell(\tilde{p} \tilde{\mathbf{u}}) \mathrm{d} \vec{S}=\iint d x d y \tilde{\nabla} \cdot(\tilde{p} \tilde{\mathbf{u}}),
$$

and using the fact that for any function $f$, our boundary conditions lead to

$$
\iint d x d y \frac{D f}{D t}=\iint d x d y\left(\partial_{t} f+(\tilde{\mathbf{u}} \cdot \tilde{\nabla}) f\right)=\frac{\mathrm{d}}{\mathrm{d} t} \iint d x d y f-\iint d x d y f(\tilde{\nabla} \cdot \tilde{\mathbf{u}}) .
$$

The main steps of the computation are:

1. Split the divergence in (166) and use (41) and (43) to obtain

$$
0=-\iint \frac{\tilde{p}}{\tilde{\rho}} \frac{D \tilde{\rho}}{D \tilde{t}}-\iint d x d y \tilde{\mathbf{u}} \tilde{\rho} \frac{D \tilde{\mathbf{u}}}{D \tilde{t}}+\iint d x d y \Phi .
$$

2. With (42), find

$$
\iint d x d y \tilde{\mathbf{u}} \tilde{\rho} \frac{D \tilde{\mathbf{u}}}{D \tilde{t}}=\frac{d}{d \tilde{t}} \iint \frac{\tilde{\rho} \tilde{\mathbf{u}}^{2}}{2} .
$$

3. Using (42), (43) and $c_{v}(\gamma-1)=R_{s}$ for an ideal gas, derive

$$
-\iint \frac{\tilde{p}}{\tilde{\rho}} \frac{D \tilde{\rho}}{D \tilde{t}}=-\frac{d}{d \tilde{t}} \iint d x d y \frac{\tilde{p}}{\gamma-1}+\dot{Q}+\iint d x d y \Phi
$$

and finally obtain (73).

\section{B Set of equations for the waves at the next order}

NS in the $x$ direction. We look for (68) at the next order. Eq. (48) at order $\epsilon^{2}$ is

$$
\rho_{1}\left[\omega_{0} \partial_{\phi} u_{1}^{\prime}\right]+\bar{\rho}_{0}\left[\omega_{0} \partial_{\phi} u_{2}^{\prime}+\omega_{1} \partial_{\phi} u_{1}^{\prime}+\partial_{T} u_{1}+u_{1} \partial_{x} u_{1}+v_{1} \partial_{y} u_{1}\right]=-\frac{1}{\gamma} \partial_{x} \pi_{2}+\frac{1}{R e_{s} h^{2}} \partial_{y y} u_{1} .
$$

Fast-time averaging this equation yields

$$
\omega_{0} \overline{\rho_{1}^{\prime} \partial_{\phi} u_{1}^{\prime}}+\bar{\rho}_{0}\left[\partial_{T} \bar{u}_{1}+\overline{u_{1} \partial_{x} u_{1}}+\overline{v_{1} \partial_{y} u_{1}}\right]=-\frac{1}{\gamma} \partial_{x} \bar{\pi}_{2}+\frac{1}{R e_{s} h^{2}} \partial_{y y} \bar{u}_{1}
$$


that eventually becomes (58) with the use of (70). To get an equation for $u_{2}^{\prime}$, we subtract equation (172) to (171),

$$
\begin{aligned}
\omega_{0} \bar{\rho}_{0} \partial_{\phi} u_{2}^{\prime}+\frac{1}{\gamma} \partial_{x} \pi_{2}^{\prime}=-\omega_{0}\left[\rho_{1} \partial_{\phi} u_{1}^{\prime}-\overline{\rho_{1}^{\prime} \partial_{\phi} u_{1}^{\prime}}\right]-\omega_{1} \bar{\rho}_{0} \partial_{\phi} u_{1}^{\prime} \\
\quad-\bar{\rho}_{0}\left[\partial_{T} u_{1}^{\prime}+u_{1} \partial_{x} u_{1}-\overline{u_{1} \partial_{x} u_{1}}+v_{1} \partial_{y} u_{1}-\overline{v_{1} \partial_{y} u_{1}}\right]+\frac{1}{R e_{s} h^{2}} \partial_{y y} u_{1}^{\prime} .
\end{aligned}
$$

NS in the $y$ direction. We look for (69) at the next order. Eq. (49) at order $\epsilon$ is

$$
\frac{\partial_{y} \pi_{2}}{\gamma h^{2}}=-\bar{\rho}_{0} \omega_{0} \partial_{\phi} v_{1} \Longrightarrow \partial_{y} \pi_{2}^{\prime}=-\gamma h^{2} \bar{\rho}_{0} \omega_{0} \partial_{\phi} v_{1}^{\prime} \text {. }
$$

Conservation of mass. We look for (70) at the next order. Eq. (51) at order $\epsilon^{2}$ is

$$
\left(\omega_{0} \partial_{\phi} \rho_{2}+\omega_{1} \partial_{\phi} \rho_{1}+\partial_{T} \rho_{1}\right)+\partial_{x}\left(\bar{\rho}_{0} u_{2}+\rho_{1} u_{1}\right)+\partial_{y}\left(\bar{\rho}_{0} v_{2}+\rho_{1} v_{1}\right)=0
$$

Fast time average yields

$$
\partial_{T} \bar{\rho}_{1}+\partial_{x}\left(\bar{\rho}_{0} \bar{u}_{2}+\overline{\rho_{1} u_{1}}\right)+\partial_{y}\left(\bar{\rho}_{0} \bar{v}_{2}+\overline{\rho_{1} v_{1}}\right)=0,
$$

And then,

$$
\omega_{0} \partial_{\phi} \rho_{2}^{\prime}+\partial_{x}\left(\bar{\rho}_{0} u_{2}^{\prime}\right)+\partial_{y}\left(\bar{\rho}_{0} v_{2}^{\prime}\right)=-\omega_{1} \partial_{\phi} \rho_{1}^{\prime}-\partial_{T} \rho_{1}^{\prime}-\partial_{x}\left(\rho_{1} u_{1}-\overline{\rho_{1} u_{1}}\right)-\partial_{y}\left(\rho_{1} v_{1}-\overline{\rho_{1} v_{1}}\right) .
$$

Internal energy balance We look for (71) at the next order. Eq. (50) at order $\epsilon^{2}$ is

$$
\begin{gathered}
\omega_{0} \partial_{\phi} \Theta_{2}+\omega_{1} \partial_{\phi} \Theta_{1}+\partial_{T} \Theta_{1}+u_{2} \partial_{x} \bar{\Theta}_{0}+u_{1} \partial_{x} \Theta_{1}+v_{2} \partial_{y} \bar{\Theta}_{0}+v_{1} \partial_{y} \Theta_{1}+v_{2} \frac{d T_{B}}{d y}=(1-\gamma) \\
{\left[\left(T_{B}+\bar{\Theta}_{0}\right)\left(\partial_{x} u_{2}+\partial_{y} v_{2}\right)+\Theta_{1}\left(\partial_{x} u_{1}+\partial_{y} v_{1}\right)\right]+\frac{\gamma}{\bar{\rho}_{0} P e_{s}}\left(\partial_{x x} \bar{\Theta}_{0}+\frac{\partial_{y y} \Theta_{1}}{h^{2}}-\frac{\rho_{1} \partial_{y y} \bar{\Theta}_{0}}{\bar{\rho}_{0} h^{2}}\right),}
\end{gathered}
$$

Fast time average yields

$$
\begin{aligned}
& \partial_{T} \bar{\Theta}_{1}+\bar{u}_{2} \partial_{x} \bar{\Theta}_{0}+\overline{u_{1} \partial_{x} \Theta_{1}}+\bar{v}_{2} \partial_{y} \bar{\Theta}_{0}+\overline{v_{1} \partial_{y} \Theta_{1}}+\bar{v}_{2} \frac{d T_{B}}{d y}=(1-\gamma) \\
& {\left[\left(T_{B}+\bar{\Theta}_{0}\right)\left(\partial_{x} \bar{u}_{2}+\partial_{y} \bar{v}_{2}\right)+\overline{\Theta_{1}\left(\partial_{x} u_{1}+\partial_{y} v_{1}\right)}\right]+\frac{\gamma}{\bar{\rho}_{0} P e_{s}}\left(\partial_{x x} \bar{\Theta}_{0}+\frac{\partial_{y y} \bar{\Theta}_{1}}{h^{2}}-\frac{\bar{\rho}_{1} \partial_{y y} \bar{\Theta}_{0}}{\bar{\rho}_{0} h^{2}}\right),}
\end{aligned}
$$

And then

$$
\begin{aligned}
& \omega_{0} \partial_{\phi} \Theta_{2}^{\prime}+\omega_{1} \partial_{\phi} \Theta_{1}^{\prime}+\partial_{T} \Theta_{1}^{\prime}+u_{2}^{\prime} \partial_{x} \bar{\Theta}_{0}+u_{1} \partial_{x} \Theta_{1}-\overline{u_{1} \partial_{x} \Theta_{1}}+v_{2}^{\prime} \partial_{y} \bar{\Theta}_{0}+v_{1} \partial_{y} \Theta_{1}-\overline{v_{1} \partial_{y} \Theta_{1}} \\
& +v_{2}^{\prime} \frac{d T_{B}}{d y}=(1-\gamma)\left[\left(T_{B}+\bar{\Theta}_{0}\right)\left(\partial_{x} u_{2}^{\prime}+\partial_{y} v_{2}^{\prime}\right)+\Theta_{1}\left(\partial_{x} u_{1}+\partial_{y} v_{1}\right)-\overline{\Theta_{1}\left(\partial_{x} u_{1}+\partial_{y} v_{1}\right)}\right] \\
& +\frac{\gamma}{P e_{s}}\left(\frac{\partial_{y y} \Theta_{1}^{\prime}}{\bar{\rho}_{0} h^{2}}-\frac{\rho_{1}^{\prime} \partial_{y y} \bar{\Theta}_{0}}{\bar{\rho}_{0}^{2} h^{2}}\right),
\end{aligned}
$$

that also reads

$$
\begin{aligned}
& \omega_{0} \partial_{\phi} \Theta_{2}^{\prime}+u_{2}^{\prime} \partial_{x} \bar{\Theta}_{0}+v_{2}^{\prime} \partial_{y}\left(\bar{\Theta}_{0}+T_{B}\right)+(\gamma-1)\left[\left(T_{B}+\bar{\Theta}_{0}\right)\left(\partial_{x} u_{2}^{\prime}+\partial_{y} v_{2}^{\prime}\right)\right] \\
& =-\omega_{1} \partial_{\phi} \Theta_{1}^{\prime}-\partial_{T} \Theta_{1}^{\prime}-u_{1} \partial_{x} \Theta_{1}+\overline{u_{1} \partial_{x} \Theta_{1}}-v_{1} \partial_{y} \Theta_{1}+\overline{v_{1} \partial_{y} \Theta_{1}} \\
& +(1-\gamma)\left[\Theta_{1}\left(\partial_{x} u_{1}+\partial_{y} v_{1}\right)-\overline{\Theta_{1}\left(\partial_{x} u_{1}+\partial_{y} v_{1}\right)}\right]+\frac{\gamma}{\bar{\rho}_{0} P e_{s} h^{2}}\left(\partial_{y y} \Theta_{1}^{\prime}-\frac{\rho_{1}^{\prime} \partial_{y y} \bar{\Theta}_{0}}{\bar{\rho}_{0}}\right) .
\end{aligned}
$$


Equation of state. We look for (72) at the next order. Eq. (52) at order $\epsilon^{2}$ is

$$
\pi_{2}=\rho_{2}\left(T_{B}+\bar{\Theta}_{0}\right)+\rho_{1} \Theta_{1}+\bar{\rho}_{0} \Theta_{2},
$$

Fast time average yields

$$
\bar{\pi}_{2}=\bar{\rho}_{2}\left(T_{B}+\bar{\Theta}_{0}\right)+\overline{\rho_{1} \Theta_{1}}+\bar{\rho}_{0} \bar{\Theta}_{2},
$$

And then

$$
\pi_{2}^{\prime}-\rho_{2}^{\prime}\left(T_{B}+\bar{\Theta}_{0}\right)-\bar{\rho}_{0} \Theta_{2}^{\prime}=\rho_{1} \Theta_{1}-\overline{\rho_{1} \Theta_{1}} .
$$

\section{Slow evolution of the amplitude}

We wish to derive an equation for the evolution of $A(T)$. To do so, we have to find a solvability condition. We proceed as follows:

1. We write all the variables at the next order as

$$
f(x, y, T, \phi)_{2}^{\prime}=B(T) / 2\left(e^{i \phi} \hat{f}_{2}(x, y, T)+\text { c.c. }\right) .
$$

We keep the previous form for the variables at order one, and we discard all the terms that do not go as $e^{ \pm i \phi}$.

2. Using the set of equations at the next order, we reduce this system of five variables $\left(\hat{u}_{2}, \hat{v}_{2}, \hat{\pi}_{2}, \hat{\rho}_{2}, \hat{\Theta}_{2}\right)$ to a system of two variables $\left(\hat{u}_{2}\right.$ and $\left.\hat{v}_{2}\right)$.

3. We find the adjoint of this linear system and the general form of the solvability condition.

4. We enforce it and get a close equation for $d A(T) / d T$.

\section{C.1 Step 2, part 1: get an expression for $\hat{\rho}_{2}$}

We recall the conservation of mass at the next order, equation (177):

$$
\omega_{0} \partial_{\phi} \rho_{2}^{\prime}+\partial_{x}\left(\bar{\rho}_{0} u_{2}^{\prime}\right)+\partial_{y}\left(\bar{\rho}_{0} v_{2}^{\prime}\right)=-\omega_{1} \partial_{\phi} \rho_{1}^{\prime}-\partial_{T} \rho_{1}^{\prime}-\partial_{x}\left(\rho_{1} u_{1}-\overline{\rho_{1} u_{1}}\right)-\partial_{y}\left(\rho_{1} v_{1}-\overline{\rho_{1} v_{1}}\right) .
$$

It becomes

$$
\begin{aligned}
& B(T)\left(\omega_{0} i \hat{\rho}_{2}+\partial_{x}\left(\bar{\rho}_{0} \hat{u}_{2}\right)+\partial_{y}\left(\bar{\rho}_{0} \hat{v}_{2}\right)\right)= \\
& -\frac{d A}{d T} \hat{\rho}_{1}-A(T)\left[\omega_{1} i \hat{\rho}_{1}+\frac{\delta \hat{\rho}_{1}}{\delta T}+\partial_{x}\left(\bar{\rho}_{1} \hat{u}_{1}+\hat{\rho}_{1} \bar{u}_{1}\right)+\partial_{y}\left(\bar{\rho}_{1} \hat{v}_{1}+\hat{\rho}_{1} \bar{v}_{1}\right)\right],
\end{aligned}
$$

where $\delta \hat{f} / \delta T$ is the functional derivative of $\hat{f}$ with respect to any dependence it may have on slow time $T$. For a later use, we rewrite this equation as

$$
\begin{aligned}
\frac{B(T) \hat{\rho}_{2}}{\bar{\rho}_{0}} & =\frac{i B(T)}{\omega_{0} \bar{\rho}_{0}}\left(\partial_{x}\left(\bar{\rho}_{0} \hat{u}_{2}\right)+\partial_{y}\left(\bar{\rho}_{0} \hat{v}_{2}\right)\right)+\frac{i}{\omega_{0} \bar{\rho}_{0}} \frac{d A}{d T} \hat{\rho}_{1} \\
& +\frac{i}{\omega_{0} \bar{\rho}_{0}} A(T)\left[\frac{\delta \hat{\rho}_{1}}{\delta T}+i \omega_{1} \hat{\rho}_{1}+\partial_{x}\left(\bar{\rho}_{1} \hat{u}_{1}+\hat{\rho}_{1} \bar{u}_{1}\right)+\partial_{y}\left(\bar{\rho}_{1} \hat{v}_{1}+\hat{\rho}_{1} \bar{v}_{1}\right)\right] .
\end{aligned}
$$




\section{C.2 Step 2, part 2: get an expression for $\hat{\Theta}_{2}$}

We perform the same simplification for the internal energy balance at order two (179),

$$
\begin{aligned}
& \frac{B(T)}{A(T)}\left(i \omega_{0} \hat{\Theta}_{2}+\hat{u}_{2} \partial_{x} \bar{\Theta}_{0}+\hat{v}_{2} \partial_{y}\left(\bar{\Theta}_{0}+T_{B}\right)+(\gamma-1)\left[\left(T_{B}+\bar{\Theta}_{0}\right)\left(\partial_{x} \hat{u}_{2}+\partial_{y} \hat{v}_{2}\right)\right]\right) \\
& =-\omega_{1} i \hat{\Theta}_{1}-\frac{1}{A(T)} \frac{d A}{d T} \hat{\Theta}_{1}-\frac{\delta \Theta}{\delta T}-\hat{u}_{1} \partial_{x} \bar{\Theta}_{1}-\bar{u}_{1} \partial_{x} \hat{\Theta}_{1}-\hat{v}_{1} \partial_{y} \bar{\Theta}_{1}-\bar{v}_{1} \partial_{y} \hat{\Theta}_{1} \\
& +(1-\gamma)\left[\hat{\Theta}_{1}\left(\partial_{x} \bar{u}_{1}+\partial_{y} \bar{v}_{1}\right)+\bar{\Theta}_{1}\left(\partial_{x} \hat{u}_{1}+\partial_{y} \hat{v}_{1}\right)\right]+\frac{\gamma}{\bar{\rho}_{0} P e_{s} h^{2}}\left(\partial_{y y} \hat{\Theta}_{1}-\frac{\hat{\rho}_{1}}{\bar{\rho}_{0}} \partial_{y y} \bar{\Theta}_{0}\right),
\end{aligned}
$$

that can also be written

$$
\begin{aligned}
& B(T) \hat{\Theta}_{2}=\frac{B(T) i}{\omega_{0}}\left(\hat{u}_{2} \partial_{x} \bar{\Theta}_{0}+\hat{v}_{2} \partial_{y}\left(\bar{\Theta}_{0}+T_{B}\right)+(\gamma-1)\left[\left(T_{B}+\bar{\Theta}_{0}\right)\left(\partial_{x} \hat{u}_{2}+\partial_{y} \hat{v}_{2}\right)\right]\right) \\
& +\frac{i A(T)}{\omega_{0}}\left(\omega_{1} i \hat{\Theta}_{1}+\frac{1}{A(T)} \frac{d A}{d T} \hat{\Theta}_{1}+\frac{\delta \Theta}{\delta T}+\hat{u}_{1} \partial_{x} \bar{\Theta}_{1}+\bar{u}_{1} \partial_{x} \hat{\Theta}_{1}+\hat{v}_{1} \partial_{y} \bar{\Theta}_{1}+\bar{v}_{1} \partial_{y} \hat{\Theta}_{1}\right) \\
& +\frac{i A(T)}{\omega_{0}}(\gamma-1)\left[\hat{\Theta}_{1}\left(\partial_{x} \bar{u}_{1}+\partial_{y} \bar{v}_{1}\right)+\bar{\Theta}_{1}\left(\partial_{x} \hat{u}_{1}+\partial_{y} \hat{v}_{1}\right)\right] \\
& -\frac{i A(T)}{\omega_{0}} \frac{\gamma}{\bar{\rho}_{0} P e_{s} h^{2}}\left(\partial_{y y} \hat{\Theta}_{1}-\frac{\hat{\rho}_{1}}{\bar{\rho}_{0}} \partial_{y y} \bar{\Theta}_{0}\right) .
\end{aligned}
$$

\section{C.3 Step 2, part 3: get an expression for $\hat{\pi}_{2}$}

We recall the equation of state (182),

$$
\pi_{2}^{\prime}-\rho_{2}^{\prime}\left(T_{B}+\bar{\Theta}_{0}\right)-\bar{\rho}_{0} \Theta_{2}^{\prime}=\rho_{1} \Theta_{1}-\overline{\rho_{1} \Theta_{1}} .
$$

Now that we know $\hat{\rho}_{2}$ and $\hat{\Theta}_{2}$, we can find $\hat{\pi}_{2}$. First, with our notations and $\left(T_{B}+\bar{\Theta}_{0}\right)=\bar{\rho}_{0}^{-1}$,

$$
B(T) \hat{\pi}_{2}=B(T) \frac{\hat{\rho}_{2}}{\bar{\rho}_{0}}+B(T) \bar{\rho}_{0} \hat{\Theta}_{2}+A(T) \hat{\rho}_{1} \bar{\Theta}_{1}+A(T) \bar{\rho}_{1} \hat{\Theta}_{1} .
$$

If $A(T)=0$, this equation would be the same as at order one and read

$$
\hat{\pi}_{2}=\frac{i \gamma}{\omega_{0}}\left(\partial_{x} \hat{u}_{2}+\partial_{y} \hat{v}_{2}\right) .
$$

We have to complete this equation with forcing terms. We get:

$$
\begin{aligned}
& B(T)\left(\hat{\pi}_{2}-\frac{i \gamma}{\omega_{0}}\left(\partial_{x} \hat{u}_{2}+\partial_{y} \hat{v}_{2}\right)\right)= \\
& \frac{i}{\omega_{0} \bar{\rho}_{0}} \frac{d A}{d T} \hat{\rho}_{1}+\frac{i}{\omega_{0} \bar{\rho}_{0}} A(T)\left[\frac{\delta \hat{\rho}_{1}}{\delta T}+i \hat{\rho}_{1} \omega_{1}+\partial_{x}\left(\bar{\rho}_{1} \hat{u}_{1}+\hat{\rho}_{1} \bar{u}_{1}\right)+\partial_{y}\left(\bar{\rho}_{1} \hat{v}_{1}+\hat{\rho}_{1} \bar{v}_{1}\right)\right] \\
& +\frac{i A(T) \bar{\rho}_{0}}{\omega_{0}}\left(\omega_{1} i \hat{\Theta}_{1}+\frac{1}{A(T)} \frac{d A}{d T} \hat{\Theta}_{1}+\frac{\delta \hat{\Theta}_{1}}{\delta T}+\hat{u}_{1} \partial_{x} \bar{\Theta}_{1}+\bar{u}_{1} \partial_{x} \hat{\Theta}_{1}+\hat{v}_{1} \partial_{y} \bar{\Theta}_{1}+\bar{v}_{1} \partial_{y} \hat{\Theta}_{1}\right) \\
& +\frac{i A(T) \bar{\rho}_{0}}{\omega_{0}}(\gamma-1)\left[\hat{\Theta}_{1}\left(\partial_{x} \bar{u}_{1}+\partial_{y} \bar{v}_{1}\right)+\bar{\Theta}_{1}\left(\partial_{x} \hat{u}_{1}+\partial_{y} \hat{v}_{1}\right)\right] \\
& -\frac{i A(T)}{\omega_{0}} \frac{\gamma}{P e_{s} h^{2}}\left(\partial_{y y} \hat{\Theta}_{1}-\frac{\hat{\rho}_{1}}{\bar{\rho}_{0}} \partial_{y y} \bar{\Theta}_{0}\right)+A(T) \hat{\rho}_{1} \bar{\Theta}_{1}+A(T) \bar{\rho}_{1} \hat{\Theta}_{1} .
\end{aligned}
$$


We write this equation as

$$
\hat{\pi}_{2}=\frac{i \gamma}{\omega_{0}}\left(\partial_{x} \hat{u}_{2}+\partial_{y} \hat{v}_{2}\right)+\frac{\mathcal{H}}{B(T)}
$$

where $\mathcal{H}$ is a known complex-valued function.

\section{C.4 Step 2, part 4: get a system of equations for $\hat{u}_{2}$ and $\hat{v}_{2}$}

We start with the Navier-Stokes equation on the $y$ direction at the second order (174),

$$
B(T) \partial_{y} \hat{\pi}_{2}=-i \gamma h^{2} \bar{\rho}_{0} \omega_{0} A(T) \hat{v}_{1} \Longrightarrow B(T) \partial_{y}\left(\partial_{x} \hat{u}_{2}+\partial_{y} \hat{v}_{2}\right)=\frac{i \partial_{y} \mathcal{H} \omega_{0}}{\gamma}-h^{2} \bar{\rho}_{0} \omega_{0}^{2} A(T) \hat{v}_{1} .
$$

Similarly, the Navier-Stokes equation on the $x$ direction at the second order (173) is

$$
\begin{aligned}
& \omega_{0} \bar{\rho}_{0} i B(T) \hat{u}_{2}+\frac{i B(T)}{\omega_{0}} \partial_{x}\left(\partial_{x} \hat{u}_{2}+\partial_{y} \hat{v}_{2}\right)+\frac{\partial_{x} \mathcal{H}}{\gamma}= \\
& -i \omega_{0} \bar{\rho}_{1} A(T) \hat{u}_{1}-i \omega_{1} \bar{\rho}_{0} A(T) \hat{u}_{1}+\frac{A(T)}{R e_{s} h^{2}} \partial_{y y} \hat{u}_{1} \\
& -\bar{\rho}_{0}\left(\frac{d A}{d T} \hat{u}_{1}+A(T) \frac{\delta \hat{u}_{1}}{\delta T}+A(T) \hat{u}_{1} \partial_{x} \bar{u}_{1}+A(T) \bar{u}_{1} \partial_{x} \hat{u}_{1}+A(T) \hat{v}_{1} \partial_{y} \bar{u}_{1}+A(T) \bar{v}_{1} \partial_{y} \hat{u}_{1}\right),
\end{aligned}
$$

that is,

$$
\begin{aligned}
& B(T)\left(\partial_{x}\left(\partial_{x} \hat{u_{2}}+\partial_{y} \hat{v_{2}}\right)+\omega_{0}^{2} \bar{\rho}_{0} \hat{u}_{2}\right)=\frac{i \omega_{0} \partial_{x} \mathcal{H}}{\gamma} \\
& -\omega_{0}^{2} \bar{\rho}_{1} A(T) \hat{u}_{1}-\omega_{0} \omega_{1} \bar{\rho}_{0} A(T) \hat{u}_{1}-i \omega_{0} \frac{A(T)}{R e_{s} h^{2}} \partial_{y y} \hat{u}_{1} \\
& +i \omega_{0} \bar{\rho}_{0}\left(\frac{d A}{d T} \hat{u}_{1}+A(T) \frac{\delta \hat{u}_{1}}{\delta T}+A(T) \hat{u}_{1} \partial_{x} \bar{u}_{1}+A(T) \bar{u}_{1} \partial_{x} \hat{u}_{1}+A(T) \hat{v}_{1} \partial_{y} \bar{u}_{1}+A(T) \bar{v}_{1} \partial_{y} \hat{u}_{1}\right) .
\end{aligned}
$$

\section{C.5 Step 3: general solvability condition}

The previous system is of the form

$$
\left\{\begin{array}{l}
\partial_{x}\left(\partial_{x} \hat{u}_{2}+\partial_{y} \hat{v}_{2}\right)+\omega_{0}^{2} \overline{\rho_{0}} \hat{u}_{2}=\mathcal{F} \\
\partial_{y}\left(\partial_{x} \hat{u}_{2}+\partial_{y} \hat{v}_{2}\right)=\mathcal{G}
\end{array}\right.
$$

where $\mathcal{F}$ and $\mathcal{G}$ are complex-valued functions. In this vector space $\left(\mathbb{R}^{2} \rightarrow \mathbb{C}\right)^{2}$, a vector writes

$$
\mathbf{V}=\left(\begin{array}{l}
\hat{u} \\
\hat{v}
\end{array}\right)
$$

The linear operator we consider is

$$
\mathcal{L}=\left(\begin{array}{cc}
\partial_{x x}+\omega_{0}^{2} \bar{\rho}_{0} & \partial_{x y} \\
\partial_{x y} & \partial_{y y}
\end{array}\right),
$$


so that the above system is simply $\mathcal{L} \mathbf{V}=\mathbf{F}$. We define a scalar product $(\cdot \mid \cdot)$ as

$$
\left(\mathbf{V}_{A} \mid \mathbf{V}_{B}\right)=\int_{0}^{2 \pi} d x \int_{0}^{1} d y\left(\mathbf{V}_{A}^{\mathrm{T}} \cdot \mathbf{V}_{B}^{*}\right)=\int_{0}^{2 \pi} d x \int_{0}^{1} d y\left(\hat{u}_{A} \hat{u}_{B}^{*}+\hat{v}_{A} \hat{v}_{B}^{*}\right),
$$

where ${ }^{*}$ stands for the conjugate. We can easily check that $\mathcal{L}$ is self-adjoint $\left(\mathcal{L}=\mathcal{L}^{\dagger}\right)$, given the $2 \pi$ periodicity in $x$ and the kinematic boundary conditions in $y(v(x, y=0)=v(x, y=$ 1) $=0)$ :

$$
\begin{aligned}
\left(\mathcal{L} \mathbf{V}_{A} \mid \mathbf{V}_{B}\right) & =\iint d x d y\left[\left(\partial_{x x} \hat{u}_{A}+\omega_{0}^{2} \bar{\rho}_{0} \hat{u}_{A}+\partial_{x y} \hat{v}_{A}\right) \hat{u}_{B}^{*}+\left(\partial_{x y} \hat{u}_{A}+\partial_{y y} \hat{v}_{A}\right) \hat{v}_{B}^{*}\right] \\
& =\iint d x d y\left[\left(\partial_{x x} \hat{u}_{B}^{*}+\omega_{0}^{2} \bar{\rho}_{0} \hat{u}_{B}^{*}+\partial_{x y} \hat{v}_{B}^{*}\right) \hat{u}_{A}+\left(\partial_{x y} \hat{u}_{B}^{*}+\partial_{y y} \hat{v}_{B}^{*}\right) \hat{v}_{A}\right] \\
& =\left(\mathbf{V}_{A} \mid \mathcal{L} \mathbf{V}_{B}\right) .
\end{aligned}
$$

We also know what vectors are in the kernel of $\mathcal{L}$ : it consists of the first order acoustic modes already described, one of them being $\mathbf{V}_{1}=\left(\hat{u}_{1}, \hat{v}_{1}\right)$. Therefore, we must have

$$
\left(\mathbf{F} \mid \mathbf{V}_{1}\right)=0
$$

that also reads,

$$
\iint\left(\mathcal{F}(x, y) \hat{u}_{1}^{*}(x, y)+\mathcal{G}(x, y) \hat{v}_{1}^{*}(x, y)\right) d x d y=0
$$

\section{C.6 Step 4, part 1: first approach of the solvability condition}

Given that $\hat{u}_{1}$ and $\hat{v}_{1}$ are real-valued fields, the solvability condition (204) can be easily decomposed in a real and imaginary one. The crucial one concerns the imaginary part of $\mathcal{F}=\mathcal{F}_{r}+i \mathcal{F}_{i}$ and $\mathcal{G}=\mathcal{G}_{r}+i \mathcal{G}_{i}$, that are respectively

$$
\begin{aligned}
\mathcal{F}_{i} & =\frac{\omega_{0}}{\gamma} \partial_{x} \mathcal{H}_{r}-\frac{\omega_{0} A(T)}{R e_{s} h^{2}} \partial_{y y} \hat{u}_{1} \\
& +\omega_{0} \bar{\rho}_{0}\left(\frac{d A}{d T} \hat{u}_{1}+A(T) \frac{\delta \hat{u}_{1}}{\delta T}+A(T) \hat{u}_{1} \partial_{x} \bar{u}_{1}+A(T) \bar{u}_{1} \partial_{x} \hat{u}_{1}+A(T) \hat{v}_{1} \partial_{y} \bar{u}_{1}+A(T) \bar{v}_{1} \partial_{y} \hat{u}_{1}\right),
\end{aligned}
$$

and,

$$
\mathcal{G}_{i}=\frac{\partial_{y} \mathcal{H}_{r} \omega_{0}}{\gamma}
$$

The terms involving $\mathcal{H}_{r}$ in the solvability conditions are

$\frac{\omega_{0}}{\gamma} \iint d x d y\left(\hat{u}_{1} \partial_{x} \mathcal{H}_{r}+\hat{v}_{1} \partial_{y} \mathcal{H}_{r}\right)=-\frac{\omega_{0}}{\gamma} \iint d x d y \mathcal{H}_{r}\left(\partial_{x} \hat{u}_{1}+\partial_{y} \hat{v}_{1}\right)=-\frac{\omega_{0}}{\gamma} \iint d x d y g(x) \mathcal{H}_{r}$, 
where the real part of $\mathcal{H}$ results from (191). For this purpose, remember that both $\hat{\rho}_{1}, \hat{\Theta}_{1}$ and $\hat{\pi}_{1}$ are pure imaginary fields. Thus,

$$
\begin{aligned}
& \mathcal{H}_{r}=\frac{i}{\omega_{0} \bar{\rho}_{0}} \frac{d A}{d T} \hat{\rho}_{1}+\frac{i}{\omega_{0} \bar{\rho}_{0}} A(T)\left[\frac{\delta \hat{\rho}_{1}}{\delta T}+\partial_{x}\left(\hat{\rho}_{1} \bar{u}_{1}\right)+\partial_{y}\left(\hat{\rho}_{1} \bar{v}_{1}\right)\right] \\
& +\frac{i A(T) \bar{\rho}_{0}}{\omega_{0}}\left(\frac{1}{A(T)} \frac{d A}{d T} \hat{\Theta}_{1}+\frac{\delta \hat{\Theta}_{1}}{\delta T}+\bar{u}_{1} \partial_{x} \hat{\Theta}_{1}+\bar{v}_{1} \partial_{y} \hat{\Theta}_{1}\right) \\
& +\frac{i A(T) \bar{\rho}_{0}}{\omega_{0}}(\gamma-1)\left[\hat{\Theta}_{1}\left(\partial_{x} \bar{u}_{1}+\partial_{y} \bar{v}_{1}\right)\right]-\frac{i A(T)}{\omega_{0}} \frac{\gamma}{P e_{s} h^{2}}\left(\partial_{y y} \hat{\Theta}_{1}-\frac{\hat{\rho}_{1}}{\bar{\rho}_{0}} \partial_{y y} \bar{\Theta}_{0}\right) .
\end{aligned}
$$

Note that this procedure has removed all the higher order mean-flow terms $\left(\bar{\Theta}_{1}, \bar{\rho}_{1}\right)$. This has also removed $\omega_{1}$, and if we keep to this part of the solvability condition we will not be able to get the slow evolution of the phase. Now, we are going to simplify $\mathcal{H}_{r}$ with the expressions of $\hat{\rho}_{1}$ and $\hat{\Theta}_{1}$. For instance, with (91) and (92),

$$
\frac{i}{\omega_{0} \bar{\rho}_{0}} \frac{d A}{d T} \hat{\rho}_{1}+\frac{i \bar{\rho}_{0}}{\omega_{0}} \frac{d A}{d T} \hat{\Theta}_{1}=\frac{i}{\omega_{0}} \frac{d A}{d T}\left(\frac{\hat{\rho}_{1}}{\bar{\rho}_{0}}+\bar{\rho}_{0} \hat{\Theta}_{1}\right)=\frac{i}{\omega_{0}} \frac{d A}{d T} \hat{\pi}_{1}=-\frac{\gamma}{\omega_{0}^{2}} \frac{d A}{d T} g(x) .
$$

\section{C.7 Step 4, part 2: a closer look at the functional derivatives}

For further simplifications, we have to give a formal definition of the functional derivative $\delta / \delta T$. For a given real-valued functional $F$ defined by

$$
F\left\{\begin{array}{l}
\text { function space } \rightarrow \mathbb{R} \\
f \rightarrow F(f)
\end{array}\right.
$$

the functional derivative $\frac{\delta F}{\delta f}(h)$ describes how $F(f)$ evolves when $f \rightarrow f+h$, with $|h| \rightarrow 0$ :

$$
\frac{\delta F}{\delta f}(h)=\lim _{\varepsilon \rightarrow 0}\left(\frac{F(f+\varepsilon h)-F(f)}{\varepsilon}\right) .
$$

In the present work, we consider functions $f$ that have a functional dependence on $\bar{\rho}_{0}$ and also depend on the real parameters $x$ and $y$. In all the previous calculations, we were interested in how the real number $f\left(x, y ;\left[\bar{\rho}_{0}\right]\right)$ evolves for fixed $x$ and $y$, while $\bar{\rho}_{0}$ evolves on the slow time. Formally, we should therefore define a functional $f_{x, y}$, such that

$$
f_{x, y}\left\{\begin{array}{l}
([0,2 \pi] \times[0,1] \rightarrow \mathbb{R}) \rightarrow \mathbb{R} \\
\bar{\rho}_{0} \rightarrow f\left(x, y ;\left[\bar{\rho}_{0}\right]\right)
\end{array}\right.
$$

and the shorthand $\frac{\delta f}{\delta T}$ should therefore be understood as

$$
\left(\frac{\delta f}{\delta T}\right) \rightarrow \frac{\delta f_{x, y}}{\delta \bar{\rho}_{0}}\left(\partial_{T} \bar{\rho}_{0}\right)
$$

This term is the increment in $f\left(x, y ;\left[\bar{\rho}_{0}\right]\right)$ at fixed $x$ and $y$ for an infinitely small slow-time increase $\left(\bar{\rho}_{0} \rightarrow \bar{\rho}_{0}+\varepsilon \partial_{T} \bar{\rho}_{0}\right)$. We can perform basic operations on these functional derivatives. Let us for instance take the example of one showing up in (208):

$$
\left(\frac{\delta \hat{\rho}_{1}}{\delta T}\right) \rightarrow \frac{\delta \hat{\rho}_{1, x, y}}{\delta \bar{\rho}_{0}}\left(\partial_{T} \bar{\rho}_{0}\right)
$$


with similar notations as above. Moreover, equations (91), (92) and (96) give

$$
\hat{\rho}_{1}=\frac{i \gamma \bar{\rho}_{0} g}{\omega_{0}}-\bar{\rho}_{0}^{2} \hat{\Theta}_{1}
$$

In this equation, both $\bar{\rho}_{0}, g, \hat{\Theta}_{1}$ and $\omega_{0}$ have a functional dependence on $\bar{\rho}_{0}$. Functional derivatives obey linearity and product rule, so that (216) becomes

$$
\left(\frac{\delta \hat{\rho}_{1}}{\delta T}\right)=i \gamma\left(\frac{\bar{\rho}_{0}}{\omega_{0}} \frac{\delta g}{\delta T}+\frac{g}{\omega_{0}} \frac{\delta \bar{\rho}_{0}}{\delta T}+\bar{\rho}_{0} g \frac{\delta \omega_{0}^{-1}}{\delta T}\right)-\bar{\rho}_{0}^{2} \frac{\delta \hat{\Theta}_{1}}{\delta T}-\hat{\Theta}_{1} \frac{\delta \bar{\rho}_{0}^{2}}{\delta T} .
$$

We then detail each of these terms successively.

$\frac{\delta g}{\delta T}$ : since $g$ does only depend on $\alpha$, this term is formally defined as

$$
\frac{\delta g}{\delta T}=\frac{\delta g_{x}}{\delta \alpha}\left(\partial_{T} \alpha\right)
$$

$\frac{\delta \bar{\rho}_{0}}{\delta T}$ : it stands for the evolution of $\bar{\rho}_{0}$ at given $x$ and $y$ as time goes by, i.e.

$$
\frac{\delta \bar{\rho}_{0}}{\delta T}=\frac{\delta \bar{\rho}_{0, x, y}}{\delta \bar{\rho}_{0}}\left(\partial_{T} \bar{\rho}_{0}\right)=\partial_{T} \bar{\rho}_{0}(x, y, T) .
$$

$\frac{\delta \omega_{0}^{-1}}{\delta T}: \omega_{0}$ is also a functional of $\bar{\rho}_{0}$ (or rather $\alpha$ ), because it is defined as an eigenvalue of an ode involving $\alpha$. This term cannot be changed much,

$$
\frac{\delta \omega_{0}^{-1}}{\delta T}=-\frac{1}{\omega_{0}^{2}} \frac{\delta \omega_{0}}{\delta \alpha}\left(\partial_{T} \alpha\right)=-\frac{\partial_{T} \omega_{0}}{\omega_{0}^{2}} .
$$

$\frac{\delta \hat{\Theta}_{1}}{\delta T}$ : this term fortunately cancels out with another one in the expression of $\mathcal{H}_{r}$.

$\frac{\delta \bar{\rho}_{0}^{2}}{\delta T}:$ similarly to $(220)$,

$$
\frac{\delta \bar{\rho}_{0}^{2}}{\delta T}=2 \bar{\rho}_{0} \partial_{T} \bar{\rho}_{0}(x, y, T)
$$

Therefore, $\frac{\delta \hat{\rho}_{1}}{\delta T}$ can be written as

$$
\frac{\delta \hat{\rho}_{1}}{\delta T}=i \gamma\left(\frac{\bar{\rho}_{0}}{\omega_{0}} \frac{\delta g}{\delta T}+\frac{g \partial_{T} \bar{\rho}_{0}}{\omega_{0}}-\frac{\bar{\rho}_{0} g \partial_{T} \omega_{0}}{\omega_{0}^{2}}\right)-\bar{\rho}_{0}^{2} \frac{\delta \hat{\Theta}_{1}}{\delta T}-2 \bar{\rho}_{0} \partial_{T} \bar{\rho}_{0} \hat{\Theta}_{1} .
$$

The term $\frac{\delta \hat{\Theta}_{1}}{\delta T}$ being canceled by a similar one in (208), we end up with only one functional derivative left in $\mathcal{H}_{r}$, that reads in the solvability condition

$$
X=-\frac{\omega_{0}}{\gamma} \iint d x d y g(x) \times \frac{i A(T)}{\omega_{0} \bar{\rho}_{0}} \times\left(i \gamma \frac{\bar{\rho}_{0}}{\omega_{0}} \frac{\delta g}{\delta T}\right)=\frac{A(T)}{\omega_{0}} \iint d x d y g(x) \frac{\delta g}{\delta T} .
$$


The integral over $y$ is immediately computed, since nothing depends on $y$. Going back to more formal notations, we have

$$
X=\frac{A(T)}{\omega_{0}} \int_{0}^{2 \pi} d x g(x) \frac{\delta g_{x}}{\delta \alpha}\left(\partial_{T} \alpha\right)=\frac{A(T)}{2 \omega_{0}} \int_{0}^{2 \pi} d x \frac{\delta g_{x}^{2}}{\delta \alpha}\left(\partial_{T} \alpha\right)
$$

We then use the formal definition of the functional derivative (215) together with the normalization condition (106):

$$
X=\frac{A(T)}{2 \omega_{0}} \lim _{\varepsilon \rightarrow 0}\left(\frac{\int_{0}^{2 \pi} d x g_{x}^{2}\left(\alpha+\varepsilon \partial_{T} \alpha\right)-\int_{0}^{2 \pi} d x g_{x}^{2}(\alpha)}{\varepsilon}\right)=\frac{A(T)}{2 \omega_{0}} \lim _{\varepsilon \rightarrow 0}\left(\frac{1-1}{\varepsilon}\right)=0 .
$$

Finally, we end up with no functional derivative left from $\mathcal{H}_{r}$ in the solvability condition. Let us have a look at the other contribution, i.e. the term $\frac{\delta \hat{u}_{1}}{\delta T}$ in $\mathcal{F}_{i}$ (see (205)). It reads

$$
Y=\iint d x d y \hat{u}_{1} \times \omega_{0} \bar{\rho}_{0} A(T) \frac{\delta \hat{u}_{1}}{\delta T} .
$$

According to $(98), \hat{u}_{1}=-g^{\prime} /\left(\omega_{0}^{2} \bar{\rho}_{0}\right)$, and then

$$
Y=\frac{A(T)}{\omega_{0}} \iint d x d y g^{\prime}(x) \frac{\delta}{\delta T}\left(\frac{g^{\prime}}{\omega_{0}^{2} \bar{\rho}_{0}}\right) .
$$

As previously, we simplify the functional derivative,

$$
\begin{aligned}
Y & =\frac{A(T)}{\omega_{0}} \iint d x d y g^{\prime}(x)\left(\frac{1}{\omega_{0}^{2} \bar{\rho}_{0}} \frac{\delta g^{\prime}}{\delta T}-\frac{2 g^{\prime} \partial_{T} \omega_{0}}{\bar{\rho}_{0} \omega_{0}^{3}}-\frac{g^{\prime} \partial_{T} \bar{\rho}_{0}}{\omega_{0}^{2} \bar{\rho}_{0}^{2}}\right) \\
& =\frac{A(T)}{\omega_{0}^{3}} \int d x \alpha g^{\prime} \frac{\delta g^{\prime}}{\delta T}-\frac{2 A(T) \partial_{T} \omega_{0}}{\omega_{0}^{4}} \int d x \alpha g^{\prime 2}-\frac{A(T)}{\omega_{0}^{3}} \int d x g^{\prime 2}(x) \int d y \frac{\partial_{T} \bar{\rho}_{0}}{\bar{\rho}_{0}^{2}} .
\end{aligned}
$$

The normalization condition (106) with the constitutive relation on $g$ (100) gives

$$
\int_{0}^{2 \pi} d x g^{\prime}(x)^{2} \alpha(x)=\left[g(x) \alpha(x) g^{\prime}(x)\right]_{0}^{2 \pi}+\int_{0}^{2 \pi} d x g(x) \times\left(\omega_{0}^{2} g(x)\right)=\omega_{0}^{2} .
$$

The second term of $Y$, involving $\partial_{T} \omega_{0}$, can then be immediately computed. The functional derivative becomes

$$
\begin{aligned}
& \frac{A(T)}{\omega_{0}^{3}} \int d x \alpha g^{\prime} \frac{\delta g^{\prime}}{\delta T}=\frac{A(T)}{2 \omega_{0}^{3}} \int d x \alpha \frac{\delta g^{\prime 2}}{\delta T}=\frac{A(T)}{2 \omega_{0}^{3}} \int d x\left(\frac{\delta\left(\alpha g^{\prime 2}\right)}{\delta T}-g^{\prime 2} \frac{\delta \alpha}{\delta T}\right) \\
& =\frac{A(T)}{2 \omega_{0}^{3}}\left[\lim _{\varepsilon \rightarrow 0}\left(\frac{\int d x\left[\alpha g^{\prime 2}\right]\left[\alpha+\varepsilon \partial_{T} \alpha\right]-\int d x\left[\alpha g^{\prime 2}\right][\alpha]}{\varepsilon}\right)-\int d x g^{\prime}(x)^{2} \partial_{T} \alpha\right] \\
& =\frac{A(T)}{2 \omega_{0}^{3}}\left[2 \omega_{0} \partial_{T} \omega_{0}-\int d x g^{\prime}(x)^{2} \partial_{T} \alpha\right]=\frac{A(T) \partial_{T} \omega_{0}}{\omega_{0}^{2}}-\frac{A(T)}{2 \omega_{0}^{3}} \int d x g^{\prime}(x)^{2} \partial_{T} \alpha .
\end{aligned}
$$

Coming back to $Y$, we get,

$$
Y=-\frac{A(T) \partial_{T} \omega_{0}}{\omega_{0}^{2}}-\frac{A(T)}{2 \omega_{0}^{3}} \int d x g^{\prime}(x)^{2} \partial_{T} \alpha-\frac{A(T)}{\omega_{0}^{3}} \int d x g^{\prime 2}(x) \int d y \frac{\partial_{T} \bar{\rho}_{0}}{\bar{\rho}_{0}^{2}}
$$


and finally, with the definition of $\alpha$,

$$
Y=-\frac{A(T) \partial_{T} \omega_{0}}{\omega_{0}^{2}}+\frac{A(T)}{2 \omega_{0}^{3}} \int d x g^{\prime}(x)^{2} \partial_{T} \alpha
$$

Again, the functional derivative is simplified with the use of the normalization condition: finally, we no longer have them in the solvability condition.

\section{C.8 Step 4, part 3: simplifying the first part of the solvability condition}

According to (207), the solvability condition can be written as

$$
\underbrace{\iint d x d y \hat{u}_{1}\left(\mathcal{F}_{i}-\frac{\omega_{0}}{\gamma} \partial_{x} \mathcal{H}_{r}\right)}_{S_{1}}-\underbrace{\frac{\omega_{0}}{\gamma} \iint d x d y g(x) \mathcal{H}_{r}}_{S_{2}}=0,
$$

and we are in this section interested in simplifying $S_{1}$. With (205), we have

$$
\begin{aligned}
S_{1}= & \iint d x d y \hat{u}_{1} \omega_{0} \bar{\rho}_{0} \frac{d A}{d T} \hat{u}_{1}-\iint d x d y \hat{u}_{1} \frac{\omega_{0} A(T)}{R e_{s} h^{2}} \partial_{y y} \hat{u}_{1} \\
& +A(T) \iint d x d y \hat{u}_{1} \omega_{0} \bar{\rho}_{0}\left(\frac{\delta \hat{u}_{1}}{\delta T}+\hat{u}_{1} \partial_{x} \bar{u}_{1}+\bar{u}_{1} \partial_{x} \hat{u}_{1}+\hat{v}_{1} \partial_{y} \bar{u}_{1}+\bar{v}_{1} \partial_{y} \hat{u}_{1}\right) .
\end{aligned}
$$

We detail all these terms successively:

- Combining (231) and (98), the first term is found to be $\omega_{0}^{-1} \frac{d A}{d T}$.

- The second term can be written as

$$
-\iint d x d y \hat{u}_{1} \frac{\omega_{0} A(T)}{R e_{s} h^{2}} \partial_{y y} \hat{u}_{1}=-\frac{A(T)}{R e_{s} h^{2} \omega_{0}^{3}} \int d x g^{\prime}(x)^{2} \int d y \frac{1}{\bar{\rho}_{0}} \partial_{y y} \frac{1}{\bar{\rho}_{0}} .
$$

Integrating by part this integral splits it into a sign-definite bulk dissipation and a sign-indefinite power exchanges at the solid boundaries.

- The third term involves the functional derivative of $\hat{u}_{1}$ and has already been worked out, see (236). It reads

$$
A(T) \omega_{0} \iint d x d y \hat{u}_{1} \bar{\rho}_{0} \frac{\delta \hat{u}_{1}}{\delta T}=-\frac{A(T) \partial_{T} \omega_{0}}{\omega_{0}^{2}}+\frac{A(T)}{2 \omega_{0}^{3}} \int d x g^{\prime}(x)^{2} \partial_{T} \alpha .
$$

- The fourth term is:

$$
\begin{aligned}
& A(T) \iint d x d y \hat{u}_{1} \omega_{0} \bar{\rho}_{0} \hat{u}_{1} \partial_{x} \bar{u}_{1}=\frac{A(T)}{\omega_{0}^{3}} \iint d x d y \frac{g^{\prime}(x)^{2}}{\bar{\rho}_{0}} \partial_{x} \bar{u}_{1} \\
& =-\frac{A(T)}{\omega_{0}^{3}} \iint d x d y \bar{u}_{1}\left(\frac{2 g^{\prime}(x) g^{\prime \prime}(x)}{\bar{\rho}_{0}}+g^{\prime}(x)^{2} \partial_{x} \bar{\rho}_{0}^{-1}\right) .
\end{aligned}
$$


- The fifth term is

$$
\begin{aligned}
& A(T) \iint d x d y \hat{u}_{1} \omega_{0} \bar{\rho}_{0} \bar{u}_{1} \partial_{x} \hat{u}_{1}=\frac{A(T)}{\omega_{0}^{3}} \iint d x d y g^{\prime}(x) \bar{u}_{1} \partial_{x}\left(\frac{g^{\prime}(x)}{\bar{\rho}_{0}}\right) \\
& =\frac{A(T)}{\omega_{0}^{3}} \iint d x d y \bar{u}_{1}\left(g^{\prime}(x)^{2} \partial_{x} \bar{\rho}_{0}^{-1}+\frac{g^{\prime}(x) g^{\prime \prime}(x)}{\bar{\rho}_{0}}\right) .
\end{aligned}
$$

- The sixth term is

$$
\begin{aligned}
& A(T) \iint d x d y \hat{u}_{1} \omega_{0} \bar{\rho}_{0} \hat{v}_{1} \partial_{y} \bar{u}_{1}=-\frac{A(T)}{\omega_{0}} \iint d x d y g^{\prime}(x) \hat{v}_{1} \partial_{y} \bar{u}_{1} \\
& =\frac{A(T)}{\omega_{0}} \iint d x d y g^{\prime}(x) \bar{u}_{1} \partial_{y} \hat{v}_{1} \\
& =\frac{A(T)}{\omega_{0}} \iint d x d y g^{\prime}(x) \bar{u}_{1}\left(g(x)+\frac{g^{\prime}(x)}{\omega_{0}^{2}} \partial_{x} \bar{\rho}_{0}^{-1}+\frac{g^{\prime \prime}(x)}{\omega_{0}^{2} \bar{\rho}_{0}}\right) .
\end{aligned}
$$

- The last term is

$$
A(T) \iint d x d y \hat{u}_{1} \omega_{0} \bar{\rho}_{0} \bar{v}_{1} \partial_{y} \hat{u}_{1}=\frac{A(T)}{\omega_{0}^{3}} \iint d x d y g^{\prime}(x)^{2} \bar{v}_{1} \partial_{y} \bar{\rho}_{0}^{-1}
$$

Putting all this together, we get

$$
\begin{aligned}
S_{1}= & \frac{1}{\omega_{0}} \frac{d A}{d T}-\frac{A(T)}{R e_{s} h^{2} \omega_{0}^{3}} \iint d x d y \frac{g^{\prime}(x)^{2}}{\bar{\rho}_{0}} \partial_{y y} \bar{\rho}_{0}^{-1}-\frac{A(T) \partial_{T} \omega_{0}}{\omega_{0}^{2}}+\frac{A(T)}{2 \omega_{0}^{3}} \int d x g^{\prime}(x)^{2} \partial_{T} \alpha \\
& +\frac{A(T)}{\omega_{0}^{3}} \iint d x d y \bar{u}_{1}\left(g^{\prime}(x)^{2} \partial_{x} \bar{\rho}_{0}^{-1}+\omega_{0}^{2} g(x) g^{\prime}(x)\right)+\frac{A(T)}{\omega_{0}^{3}} \iint d x d y g^{\prime}(x)^{2} \bar{v}_{1} \partial_{y} \bar{\rho}_{0}^{-1} .
\end{aligned}
$$

\section{C.9 Step 4, part 4: simplifying the second part of the solvability condition}

We are interested in simplifying $S_{2}$, that is defined as

$$
S_{2}=\frac{\omega_{0}}{\gamma} \iint d x d y g(x) \mathcal{H}_{r},
$$

with $\mathcal{H}_{r}$ given by (208) simplified with (209),

$$
\begin{aligned}
& \mathcal{H}_{r}=-\frac{\gamma g(x)}{\omega_{0}^{2}} \frac{d A}{d T}+\frac{i}{\omega_{0} \bar{\rho}_{0}} A(T)\left[\frac{\delta \hat{\rho}_{1}}{\delta T}+\partial_{x}\left(\hat{\rho}_{1} \bar{u}_{1}\right)+\partial_{y}\left(\hat{\rho}_{1} \bar{v}_{1}\right)\right] \\
& +\frac{i A(T) \bar{\rho}_{0}}{\omega_{0}}\left(\frac{\delta \hat{\Theta}_{1}}{\delta T}+\bar{u}_{1} \partial_{x} \hat{\Theta}_{1}+\bar{v}_{1} \partial_{y} \hat{\Theta}_{1}\right) \\
& +\frac{i A(T) \bar{\rho}_{0}}{\omega_{0}}(\gamma-1)\left[\hat{\Theta}_{1}\left(\partial_{x} \bar{u}_{1}+\partial_{y} \bar{v}_{1}\right)\right]-\frac{i A(T)}{\omega_{0}} \frac{\gamma}{P e_{s} h^{2}}\left(\partial_{y y} \hat{\Theta}_{1}-\frac{\hat{\rho}_{1}}{\bar{\rho}_{0}} \partial_{y y} \bar{\Theta}_{0}\right) .
\end{aligned}
$$

Again, we compute this term by term:

- The first term is

$$
\frac{\omega_{0}}{\gamma} \iint d x d y g(x) \times\left(-\frac{\gamma g(x)}{\omega_{0}^{2}} \frac{d A}{d T}\right)=-\frac{1}{\omega_{0}} \frac{d A}{d T}
$$


- The two functional derivatives give, according to (223),

$$
\begin{aligned}
& \frac{\omega_{0}}{\gamma} \iint d x d y g(x) \times\left(\frac{i}{\omega_{0} \bar{\rho}_{0}} A(T) \frac{\delta \hat{\rho}_{1}}{\delta T}+\frac{i A(T) \bar{\rho}_{0}}{\omega_{0}} \frac{\delta \hat{\Theta}_{1}}{\delta T}\right) \\
& =\frac{i A(T)}{\gamma} \iint d x d y \frac{g(x)}{\bar{\rho}_{0}} \times\left(-2 \bar{\rho}_{0} \partial_{T} \bar{\rho}_{0} \hat{\Theta}_{1}+i \gamma\left(\frac{\bar{\rho}_{0}}{\omega_{0}} \frac{\delta g}{\delta T}+\frac{g \partial_{T} \bar{\rho}_{0}}{\omega_{0}}-\frac{\bar{\rho}_{0} g \partial_{T} \omega_{0}}{\omega_{0}^{2}}\right)\right) \\
& =-\frac{2 i A(T)}{\gamma} \iint d x d y g(x) \hat{\Theta}_{1} \partial_{T} \bar{\rho}_{0}+\frac{A(T)}{\omega_{0}} \iint d x d y g(x)^{2}\left(\frac{\partial_{T} \omega_{0}}{\omega_{0}}-\frac{\partial_{T} \bar{\rho}_{0}}{\bar{\rho}_{0}}\right) \\
& =-\frac{2 i A(T)}{\gamma} \iint d x d y g(x) \hat{\Theta}_{1} \partial_{T} \bar{\rho}_{0}-\frac{A(T)}{\omega_{0}} \iint d x d y g(x)^{2} \frac{\partial_{T} \bar{\rho}_{0}}{\bar{\rho}_{0}}+\frac{A(T) \partial_{T} \omega_{0}}{\omega_{0}^{2}}
\end{aligned}
$$

- The term involving $\hat{\rho}_{1}$ is,

$$
\begin{aligned}
& \frac{\omega_{0}}{\gamma} \iint d x d y g(x) \times\left(\frac{i}{\omega_{0} \bar{\rho}_{0}} A(T)\left(\partial_{x}\left(\hat{\rho}_{1} \bar{u}_{1}\right)+\partial_{y}\left(\hat{\rho}_{1} \bar{v}_{1}\right)\right)\right) \\
& =-\frac{i A}{\gamma} \iint d x d y\left(-\bar{\rho}_{0}^{2} \hat{\Theta}_{1}+\frac{i \gamma \bar{\rho}_{0} g}{\omega_{0}}\right) \times\left(\bar{u}_{1} \partial_{x}\left(\frac{g}{\bar{\rho}_{0}}\right)+\bar{v}_{1} \partial_{y}\left(\frac{g}{\bar{\rho}_{0}}\right)\right) \\
& =\frac{i A}{\gamma} \iint d x d y \hat{\Theta}_{1}\left(\bar{\rho}_{0} g^{\prime} \bar{u}_{1}-g \bar{u}_{1} \partial_{x} \bar{\rho}_{0}-g \bar{v}_{1} \partial_{y} \bar{\rho}_{0}\right) \\
& +\frac{A}{\omega_{0}} \iint d x d y g(x) \bar{\rho}_{0}\left(\bar{u}_{1} \frac{g^{\prime}(x)}{\bar{\rho}_{0}}+\bar{u}_{1} g(x) \partial_{x} \bar{\rho}_{0}^{-1}+\bar{v}_{1} g(x) \partial_{y} \bar{\rho}_{0}^{-1}\right) .
\end{aligned}
$$

- Other terms read, with the use of (60),

$$
\begin{aligned}
& \frac{\omega_{0}}{\gamma} \iint d x d y g(x) \times \frac{i \bar{\rho}_{0}}{\omega_{0}} A(T)\left(\bar{u}_{1} \partial_{x} \hat{\Theta}_{1}+\bar{v}_{1} \partial_{y} \hat{\Theta}_{1}\right) \\
& =\frac{i A(T)}{\gamma} \iint d x d y g(x) \bar{\rho}_{0}\left(\bar{u}_{1} \partial_{x} \hat{\Theta}_{1}+\bar{v}_{1} \partial_{y} \hat{\Theta}_{1}\right) \\
& =-\frac{i A(T)}{\gamma} \iint d x d y \hat{\Theta}_{1}\left(g^{\prime}(x) \bar{\rho}_{0} \bar{u}_{1}+g(x) \partial_{x}\left[\bar{\rho}_{0} \bar{u}_{1}\right]+g(x) \partial_{y}\left[\bar{\rho}_{0} \bar{v}_{1}\right]\right) \\
& =\frac{i A(T)}{\gamma} \iint d x d y \hat{\Theta}_{1}\left(g(x) \partial_{T} \bar{\rho}_{0}-g^{\prime}(x) \bar{\rho}_{0} \bar{u}_{1}\right) .
\end{aligned}
$$

- We also have

$$
\begin{aligned}
& \frac{\omega_{0}}{\gamma} \iint d x d y g(x) \times \frac{i \bar{\rho}_{0}}{\omega_{0}} A(T)\left((\gamma-1) \hat{\Theta}_{1}\left(\partial_{x} \bar{u}_{1}+\partial_{y} \bar{v}_{1}\right)\right) \\
& =(\gamma-1) \frac{i A(T)}{\gamma} \iint d x d y \hat{\Theta}_{1} g(x) \bar{\rho}_{0}\left(\partial_{x} \bar{u}_{1}+\partial_{y} \bar{v}_{1}\right) \\
& =(1-\gamma) \frac{i A(T)}{\gamma} \iint d x d y \hat{\Theta}_{1} g(x)\left(\partial_{T} \bar{\rho}_{0}+\bar{u}_{1} \partial_{x} \bar{\rho}_{0}+\bar{v}_{1} \partial_{y} \bar{\rho}_{0}\right) .
\end{aligned}
$$

- Finally, the thermal diffusion term reads

$$
\begin{aligned}
& \frac{\omega_{0}}{\gamma} \iint d x d y g(x) \times-\frac{i A(T)}{\omega_{0}} \frac{\gamma}{P e_{s} h^{2}}\left(\partial_{y y} \hat{\Theta}_{1}-\frac{\hat{\rho}_{1}}{\bar{\rho}_{0}} \partial_{y y} \bar{\Theta}_{0}\right) \\
& =-\frac{i A(T)}{P e_{s} h^{2}} \iint d x d y g(x)\left(\partial_{y y} \hat{\Theta}_{1}-\frac{\hat{\rho}_{1}}{\bar{\rho}_{0}} \partial_{y y} \bar{\Theta}_{0}\right) .
\end{aligned}
$$


All these results provide an expression for $S_{2}$ :

$$
\begin{aligned}
S_{2}= & -\frac{1}{\omega_{0}} \frac{d A}{d T}+\frac{A(T) \partial_{T} \omega_{0}}{\omega_{0}^{2}}-\frac{i A(T)}{P e_{s} h^{2}} \iint d x d y g(x)\left(\partial_{y y} \hat{\Theta}_{1}-\frac{\hat{\rho}_{1}}{\bar{\rho}_{0}} \partial_{y y} \bar{\Theta}_{0}\right) \\
& +\frac{A}{\omega_{0}} \iint d x d y g(x) \bar{\rho}_{0}\left(\bar{u}_{1} \frac{g^{\prime}(x)}{\bar{\rho}_{0}}+\bar{u}_{1} g(x) \partial_{x} \bar{\rho}_{0}^{-1}+\bar{v}_{1} g(x) \partial_{y} \bar{\rho}_{0}^{-1}\right) \\
& -\frac{A}{\omega_{0}} \iint g(x)^{2} \frac{\partial_{T} \bar{\rho}_{0}}{\bar{\rho}_{0}}-i A \iint \hat{\Theta}_{1} g(x)\left(\partial_{T} \bar{\rho}_{0}+\bar{u}_{1} \partial_{x} \bar{\rho}_{0}+\bar{v}_{1} \partial_{y} \bar{\rho}_{0}\right) .
\end{aligned}
$$

This can be simplified with the continuity equation,

$$
\begin{aligned}
S_{2}= & -\frac{1}{\omega_{0}} \frac{d A}{d T}+\frac{A(T) \partial_{T} \omega_{0}}{\omega_{0}^{2}}-\frac{i A(T)}{P e_{s} h^{2}} \iint d x d y g(x)\left(\partial_{y y} \hat{\Theta}_{1}-\frac{\hat{\rho}_{1}}{\bar{\rho}_{0}} \partial_{y y} \bar{\Theta}_{0}\right) \\
& +\frac{A}{\omega_{0}} \iint d x d y\left(g(x) g^{\prime}(x) \bar{u}_{1}+g(x)^{2}\left(\partial_{x} \bar{u}_{1}+\partial_{y} \bar{v}_{1}\right)\right) \\
& +i A \iint \hat{\Theta}_{1} g(x) \bar{\rho}_{0}\left(\partial_{x} \bar{u}_{1}+\partial_{y} \bar{v}_{1}\right) .
\end{aligned}
$$

\section{C.10 Step 4, part 5: A final expression for the solvability condition}

The solvability condition $S_{1}=S_{2}$ can finally be expressed, and is after simplification

$$
\begin{aligned}
& \frac{2}{A} \frac{\mathrm{d} A}{\mathrm{~d} T}-\frac{2}{\omega_{0}} \frac{\mathrm{d} \omega_{0}}{\mathrm{~d} T}=-\frac{i \omega_{0}}{P e_{s} h^{2}} \iint d x d y g(x)\left(\partial_{y y} \hat{\Theta}_{1}-\frac{\hat{\rho}_{1}}{\bar{\rho}_{0}} \partial_{y y} \bar{\Theta}_{0}\right) \\
& +\iint d x d y g(x)^{2}\left(\partial_{x} \bar{u}_{1}+\partial_{y} \bar{v}_{1}\right)+i \omega_{0} \iint \hat{\Theta}_{1} g(x) \bar{\rho}_{0}\left(\partial_{x} \bar{u}_{1}+\partial_{y} \bar{v}_{1}\right) \\
& +\frac{1}{R e_{s} h^{2} \omega_{0}^{2}} \iint d x d y \frac{g^{\prime}(x)^{2}}{\bar{\rho}_{0}} \partial_{y y} \bar{\rho}_{0}^{-1}-\frac{1}{2 \omega_{0}^{2}} \int d x g^{\prime}(x)^{2} \partial_{T} \alpha \\
& -\frac{1}{\omega_{0}^{2}} \iint d x d y g^{\prime}(x)^{2}\left(\bar{u}_{1} \partial_{x} \bar{\rho}_{0}^{-1}+\bar{v}_{1} \partial_{y} \bar{\rho}_{0}^{-1}\right) .
\end{aligned}
$$

This can still be simplified:

- Note that

$$
\begin{aligned}
& -\frac{1}{2 \omega_{0}^{2}} \int d x g^{\prime}(x)^{2} \partial_{T} \alpha=\frac{1}{2 \omega_{0}^{2}} \iint d x d y g^{\prime}(x)^{2} \frac{\partial_{T} \bar{\rho}_{0}}{\bar{\rho}_{0}^{2}} \\
& =-\frac{1}{2 \omega_{0}^{2}} \iint d x d y g^{\prime}(x)^{2}\left(\frac{\partial_{x} \bar{u}_{1}+\partial_{y} \bar{v}_{1}}{\bar{\rho}_{0}}-\bar{u}_{1} \partial_{x} \bar{\rho}_{0}^{-1}-\bar{v}_{1} \partial_{y} \bar{\rho}_{0}^{-1}\right) .
\end{aligned}
$$

- Moreover,

$$
\frac{i \omega_{0}}{P e_{s} h^{2}} \iint d x d y g(x)\left(\frac{\hat{\rho}_{1}}{\bar{\rho}_{0}} \partial_{y y} \bar{\Theta}_{0}\right)=i \omega_{0} \iint d x d y g\left(\frac{i \gamma g}{\omega_{0}}-\bar{\rho}_{0} \hat{\Theta}_{1}\right) \times\left(\partial_{x} \bar{u}_{1}+\partial_{y} \bar{v}_{1}\right) .
$$

- The viscous term can also be written, since $\partial_{y y} \bar{\rho}_{0}^{-1}=\partial_{y y} \bar{\Theta}_{0}$,

$$
\iint d x d y \frac{g^{\prime}(x)^{2}}{\bar{\rho}_{0}} \partial_{y y} \bar{\Theta}_{0}=P e_{s} h^{2} \iint d x d y \frac{g^{\prime}(x)^{2}}{\bar{\rho}_{0}}\left(\partial_{x} \bar{u}_{1}+\partial_{y} \bar{v}_{1}\right) .
$$


Therefore, we obtain

$$
\begin{aligned}
& \frac{2}{A \omega_{0}^{-1}} \frac{\mathrm{d}\left(A \omega_{0}^{-1}\right)}{\mathrm{d} T}=-\frac{i \omega_{0}}{P e_{s} h^{2}} \iint d x d y g(x) \partial_{y y} \hat{\Theta}_{1} \\
& +\iint d x d y\left(\partial_{x} \bar{u}_{1}+\partial_{y} \bar{v}_{1}\right)\left[(1-\gamma) g(x)^{2}+\frac{g^{\prime}(x)^{2}}{\omega_{0}^{2} \bar{\rho}_{0}}\left(\frac{P e_{s}}{R e_{s}}-\frac{1}{2}\right)\right] \\
& -\frac{1}{2 \omega_{0}^{2}} \iint d x d y g^{\prime}(x)^{2}\left(\bar{u}_{1} \partial_{x} \bar{\rho}_{0}^{-1}+\bar{v}_{1} \partial_{y} \bar{\rho}_{0}^{-1}\right)
\end{aligned}
$$

\section{Function $G$ for any $\Gamma$}

The solution of (135) for any value of $\Gamma$ is obtained with Mathematica,

$$
G_{\Gamma}(y)=\frac{A+B+C}{D},
$$

where

$$
\begin{aligned}
& A=\Gamma^{2}(y-1) y\left[45+\Gamma\left(46+3 \Gamma+y+3 \Gamma y-(9+7 \Gamma) y^{2}+3(2+\Gamma) y^{3}\right)\right]-30 \Gamma \ln (1+\Gamma y), \\
& B=3 \Gamma^{2}(\Gamma+1) y\left(-7+10 y-5 y^{3}+2 y^{4}\right) \ln (\Gamma)+30(1+\Gamma)(1+2 \Gamma) y \ln (1+\Gamma)-30 \ln (1+\Gamma y), \\
& C=-3 \Gamma(1+\Gamma) y\left(\Gamma\left(-7+y^{3}(-5+2 y)\right) \ln (\Gamma(1+\Gamma))+20 \ln (1+\Gamma y)+10 \Gamma y \ln (\Gamma(1+\Gamma y)),\right. \\
& \text { and } \begin{array}{l}
D=1080 \Gamma^{3}[\Gamma(2+\Gamma)+2(1+\Gamma) \ln (\Gamma)-2(1+\Gamma) \ln (\Gamma(1+\Gamma))] .
\end{array}
\end{aligned}
$$




\title{
The Dynamics of Subglacial Plume Lift-off
}

\author{
Earle Wilson
}

November 27, 2017

\section{Introduction}

The melting of the Greenland and Antarctic ice sheets currently contributes approximately $1.1 \mathrm{~mm}$ per year of the total $3.3 \mathrm{~mm}$ per year rise in global sea-level [1]. Most notably, Greenland's contribution to this rate has increased more than 8-fold between 1994 and 2014 [2]. Recent studies have attributed the accelerated mass loss of the Greenland Ice Sheet to increased melting and ice loss at its marine interface [3-5]. Though much progress has been made to identify the primary mechanisms driving this enhanced mass loss, many aspects of this ice-ocean interaction remain poorly constrained. One such aspect is the impact of subglacial discharge.

Subglacial discharge refers to the release of meltwater from beneath the ice sheet into the ocean. This meltwater enters the ocean from the grounding line and rises along the face of a glacier as a turbulent plume. The turbulent mixing that occurs along the plume's path enhances submarine melting, and such melting may also promote the calving of icebergs [69]. Recent modeling efforts have shown that the submarine melt rate along a glacier's front is sensitive to the spatial distribution of subglacial outlets $[10,11]$. In particular, model results show that a distributed line plume can produce up to five times as much melting as a single point source with the same discharge [10]. Despite its importance, the near-terminus hydrology of marine terminating glaciers remains poorly constrained [12]. This is in no small part due to the immense difficulty of accessing these regions.

The purpose of this project is to further our understanding of near-terminus subglacial discharge by exploring its dynamics with simple models and lab experiments. Here, we focus our attention on the transition that occurs as meltwater exits a subglacial channel and begins to rise as a buoyant plume. We refer to this transition as subglacial plume lift-off. We are specifically interested in where this lift-off occurs as it defines the extent to which relatively warm seawater floods a subglacial channel. This will help us determine the geometry of a subglacial channel's outlet to the ocean.

In this report, we present simple models for subglacial plume lift-off in a rectangular channel. This is accompanied by results from a series of laboratory experiments that attempt to replicate the essential dynamics of plume lift-off. We conclude by using our results to speculate on the extent of seawater intrusion under real-world glaciers. 


\section{Theoretical Model for a Salt Wedge}

\subsection{The estuarine analogy}

In the subglacial plume lift-off zone, cold, fresh subglacial water rises into warm, salty seawater. It is conceivable that, under the right conditions, this lift-off may occur deep inside the subglacial channel. In this scenario, the ocean floods into the subglacial channel and undercuts the glacier. We assume that the dynamics of such an intrusion is fundamentally similar to that of a salt wedge estuary. A salt wedge estuary forms when a fast flowing fresh river discharges into a saline ocean with weak tidal mixing [13]. In this case, the salt wedge refers to the intrusion of seawater inside an estuary and coincides with where the river outflow lifts off the seabed and flows over the ocean [14]. In our case, the salt wedge refers to the section of the subglacial channel that is filled with seawater. This is illustrated in Figure 1.

We note that the dynamics of a salt wedge is somewhat similar to that of a two-layer exchange flow. In a typical two-layer exchange flow, two fluid reservoirs of different densities exchange mass via a narrow channel $[15,16]$. Within the exchange flow's channel, light fluid flows above dense fluid in opposite directions. However, the flow within a salt wedge system is mostly uni-directional as the saline lower layer only partially intrudes the channel and terminates at a front. We believe the dynamics of a subglacial system is more similar to that of a salt wedge than a two-layer exchange flow.

\subsection{An idealized salt-wedge with no entrainment}

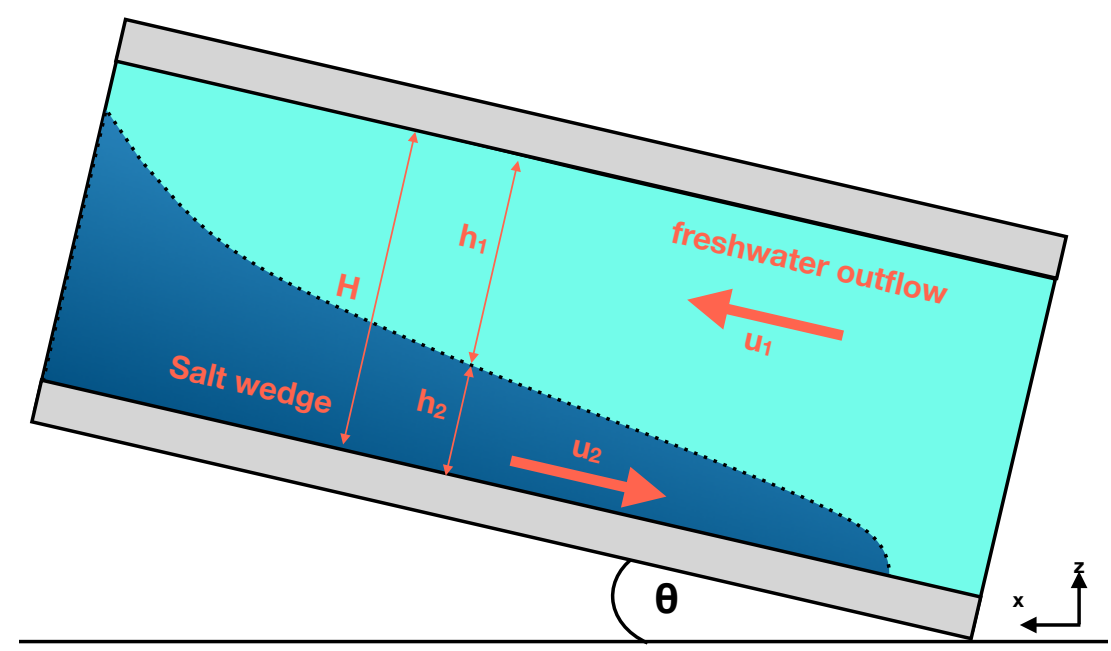

Figure 1: Schematic showing a cross-sectional view of a salt wedge in a rectangular channel

The schematic in Figure 1 illustrates an idealized salt wedge in a rectangular channel. Here, freshwater enters the channel from the right as salt water flows in from the left. The terms $u_{1}$ and $u_{2}$ are the along channel, layer averaged, velocities in the upper and lower layers, respectively. 
To build our mathematical description of a subglacial salt wedge, we adapt the two layer salt wedge model presented in [17]. Like [17], we assume that the freshwater and salt water layers are well mixed and flow at a uniform speed. We likewise assume that the interface between the two layers is infinitely thin and features no interfacial mixing. However, unlike [17], which assumes a free upper surface for the top layer, we assume a rigid upper boundary to better represent the conditions within a subglacial channel. Additionally, we permit the channel to be tilted at a slight angle $\theta$. Lastly, we restrict the height of the channel $H$ to be constant but, for generality, permit its width $w$ to vary.

Given the above assumptions, we can model this system using a pair of shallow water equations. With the stipulation of no entrainment, the continuity equations for the upper and lower layers are

$$
\begin{aligned}
& w \frac{\partial h_{1}}{\partial t}+\frac{\partial Q_{1}}{\partial x}=0, \\
& w \frac{\partial h_{2}}{\partial t}+\frac{\partial Q_{2}}{\partial x}=0 .
\end{aligned}
$$

Here, $h_{1}$ and $h_{2}$ are the thicknesses of the upper and lower layers, respectively. The thickness of the individual layers sum to give the height of the channel, $H$. Similarly, $Q_{1}$ and $Q_{2}$ are the volume fluxes of each layer, which in steady state are conserved along the channel. Continuing with our assumption of no entrainment, the momentum balance for both layers are given by

$$
\begin{aligned}
\frac{\partial u_{1}}{\partial t}+u_{1} \frac{\partial u_{1}}{\partial x}+\frac{\partial P}{\partial x}+\frac{C_{i}\left|u_{1}-u_{2}\right|\left(u_{1}-u_{2}\right)}{h_{1}}+C_{d} u_{1}^{2}\left(\frac{1}{h_{1}}+\frac{2}{w}\right) & =0 \\
\frac{\partial u_{2}}{\partial t}+u_{2} \frac{\partial u_{2}}{\partial x}+\frac{\partial P}{\partial x}+g^{\prime}\left(\frac{\partial h_{2}}{\partial x}+\tan \theta\right)-\frac{C_{i}\left|u_{1}-u_{2}\right|\left(u_{1}-u_{2}\right)}{h_{2}} & +C_{d} u_{2}^{2}\left(\frac{1}{h_{2}}+\frac{2}{w}\right)=0
\end{aligned}
$$

where $C_{i}$ and $C_{d}$ are the coefficients for interfacial and wall drag, $\partial P / \partial x$ is the along channel pressure gradient, and $g^{\prime}$ is reduced gravity. In (3) and (4), we parameterize the interfacial and wall drag forces using the quadratic drag law. For interfacial drag, the transfer of momentum between the two layers is assumed to be proportional to the square of the difference between the layer-averaged velocities. For wall drag, the loss of momentum is assumed to be proportional to the square of the flow velocity that is adjacent to the wall. The $1 / h_{1}$ and $1 / h_{2}$ terms in (3) and (4) represent the scaling for drag along the top and bottom walls of the channel. The $2 / w$ term is the corresponding scaling for drag along both side walls. If the width of the channel is much greater than its height, the effect of side wall drag is negligible.

Reduced gravity is defined as

$$
g^{\prime}=\frac{\Delta \rho}{\rho_{0}} g
$$


where $\Delta \rho$ is the density difference between the two layers, $g$ is the acceleration due to gravity and $\rho_{0}$ is a reference density. If the temperature of the two layers are the same, $\Delta \rho / \rho_{0}$ may be approximated by

$$
\frac{\Delta \rho}{\rho_{0}}=\beta \Delta S,
$$

where $\Delta S$ is the salinity difference between the two layers and $\beta \sim 8 \times 10^{-4} \mathrm{PSU}^{-1}$ is the haline contraction coefficient [17].

The along channel pressure gradient, $\partial P / \partial x$, serves a similar role to the hydrostatic pressure gradient induced by the sea surface slope gradient in a salt wedge estuary. In that system, the sea surface slopes downward towards the ocean and forces flow in the seaward direction. This is distinct from the force represented by $g^{\prime}\left(\frac{\partial h_{2}}{\partial x}\right)$, which acts to drive flow that flattens the interface between the two layers.

To simplify the system, we assume a steady state where the freshwater volume flux, $Q_{1}$, is constant and the lower layer is stagnant $\left(u_{2}=0\right)$. Furthermore, we assume that freshwater flow is always positive $\left(Q_{1}>0\right)$. Additionally, we substitute the upper layer velocity $u_{1}$ in the momentum equations in favor of the upper layer volume flux by using the relationship $u_{1}=Q_{1} / w h_{1}$, to obtain

$$
\begin{array}{r}
\frac{Q_{1}^{2}}{w h_{1}} \frac{\partial}{\partial x}\left(\frac{1}{w h_{1}}\right)+\frac{\partial P}{\partial x}+C_{i}\left(\frac{Q_{1}}{w h_{1}}\right)^{2} \frac{1}{h_{1}} \\
+C_{d}\left(\frac{Q_{1}}{w h_{1}}\right)^{2}\left(\frac{1}{h_{1}}+\frac{2}{w}\right)=0, \\
\frac{\partial P}{\partial x}=C_{i}\left(\frac{Q_{1}^{2}}{w^{2} h_{1}^{2} h_{2}}\right)-g^{\prime}\left(\frac{\partial h_{2}}{\partial x}+\tan \theta\right),
\end{array}
$$

where have used $h_{2}=H-h_{1}$ for constant $H$. Combining the above equations and doing some algebra, we find

$$
\begin{array}{r}
-\frac{Q_{1}^{2}}{g^{\prime} w^{2} h_{1}^{3}}\left(\frac{\partial h_{1}}{\partial x}+\frac{h_{1}}{w} \frac{\partial w}{\partial x}\right)+C_{i} \frac{Q_{1}^{2}}{g^{\prime} w^{2} h_{1}^{2}\left(H-h_{1}\right)}+\frac{\partial h_{1}}{\partial x}-\tan \theta \\
+C_{i} \frac{Q_{1}^{2}}{g^{\prime} w^{2} h_{1}^{3}}+C_{d} \frac{Q_{1}^{2}}{g^{\prime} w^{2} h_{1}^{3}}\left(1+\frac{2 h_{1}}{w}\right)=0
\end{array}
$$

At this stage, we define the non-dimensional upper layer Froude number as

$$
F r_{1}^{2}=\frac{Q_{1}^{2}}{g^{\prime} w^{2} h_{1}^{3}}=\frac{u_{1}^{2}}{g^{\prime} h_{1}}
$$

By this definition, the upper layer Froude number varies in the along channel direction as $h_{1}$ and $u_{1}$ vary. When $F r_{1}=1$, the flow is said to be critical. Flows for which $F r_{1}>1$ and $F r_{1}<1$ are classified as supercritical and subcritical, respectively. We also define the Froude number for the special case where $h_{1}=H$ as

$$
F r_{0}^{2}=\frac{Q_{1}^{2}}{g^{\prime} w^{2} H^{3}}=F r_{1}^{2}\left(\frac{h_{1}}{H}\right)^{3} \text {. }
$$


$F r_{0}$ is known as the freshwater Froude number [17]. Using (10), we re-write the previous equations as

$$
\left(F r_{1}^{2}-1\right) \frac{\partial h_{1}}{\partial x}=F r_{1}^{2}\left[C_{i}\left(\frac{H}{H-h_{1}}\right)+C_{d}\left(1+\frac{2 h_{1}}{w}\right)-\frac{h_{1}}{w} \frac{\partial w}{\partial x}\right]-\tan \theta .
$$

The above equation describes the shape of a steady, non-entraining salt wedge in a rectangular channel, with variable width $w$ and constant tilt $\theta$. To gain some insight, we first consider the simpler case of a horizontal channel $(\theta=0)$ with constant width, for which

$$
\left(F r_{1}^{2}-1\right) \frac{\partial h_{1}}{\partial x}=F r_{1}^{2}\left[C_{i}\left(\frac{H}{H-h_{1}}\right)+C_{d}\left(1+\frac{2 h_{1}}{w}\right)\right] .
$$

The salt wedge is defined as the distance between the freshwater lift-off point (i.e. the nose of the wedge) and the point at which $F r_{1}=1$. For convenience, we designate $x=-L$ as the freshwater lift-off point and $x=0$ as the point where the flow becomes critical. In this framework, the length of wedge is given by $L$. Additionally, $F r_{1}=1$ at $x=0$ and $F r_{1}=F r_{0}$ at $x=-L$. To obtain the full shape of the wedge, we begin our integration of (13) from the point of critical flow, at $x=0$. Since $F r_{1}=1$ at $x=0$, this determines $h_{1}$ at that boundary ${ }^{1}$. The integration is carried out in the negative $\mathrm{x}$-direction (towards the freshwater source) until $h_{1} \approx H$. Through this process, we can determine the freshwater liftoff point and the length of the salt wedge $L$.

Since the terms on the right of (13) are always positive, supercritical flow in the upper layer results in $\partial h_{1} / \partial x$ being positive. This means the freshwater layer will expand upon encountering the salt wedge and continue to do so until it becomes subcritical. However, if $F r_{0}^{2}>1$, subcritical flow cannot occur within the channel. Therefore, a salt wedge cannot exist in a flat, uniform channel where $F r_{0}^{2}>1$. If the upper layer flow is subcritical $\left(F r_{1}^{2}<1\right.$ and thus $\left.F_{0}^{2}<1\right)$, (13) predicts that the upper layer will decrease in height upon encountering the salt water layer. Unlike the supercritical case, this is conducive to a twlayer flow and potentially a salt wedge.

If $h_{1}$ decreases indefinitely in the downstream direction, there must be a point along the channel where $F r_{1}^{2}=1$. However, at this point, (13) becomes singular and thus appears to preclude the existence of such a flow. This apparent paradox can be avoided if we return to the more general case of a channel with variable width. If the width of the channel expands in the downstream direction, it is possible for the right of (12) to sum to zero. If that is case, the flow becomes critical wherever $\left(h_{1} / w\right)(\partial w / \partial x)$ balances the other terms in (12). More importantly, this permits the formation of a salt wedge. Alternatively, we can avoid this breakdown of our model by specifying that $F r_{1}=1$ at the mouth of the channel, thereby establishing a hydraulic control at $x=0$.

To summarize, a steady salt wedge can only occur if the flow in the freshwater layer is subcritical. Furthermore, for a salt wedge to occur in a horizontal rectangular channel with uniform cross-section, critical flow must occur outside the channel. More generally, critical flow can only be supported by a change in channel geometry.

\footnotetext{
${ }^{1}$ For practical purposes, we set $F r_{1}=1-\epsilon$ at $x=0$ to avoid the singularity associated with critical flow. For these simulations, $\epsilon=10^{-4}$.
} 
Figure 2 shows solutions of equation 12 for a $10 \mathrm{~m}$ channel with various freshwater flow velocities. The stated flow velocities are the freshwater flow rates that are imposed at the $x=-L$ boundary, where the plume lifts off the channel. The parameters used for this integration reflect that of a shallow estuary. Here, it is assumed that the width of the channel is much greater than its height and that there is no drag along the upper surface. Following [17], we set $C_{i}=10^{-4}$, which is a value that is typical of salt-wedge estuaries.

Though this example is somewhat contrived, it allows us to visualize some key features of a salt wedge. In Figure 2, we see that the slope of the interface is steepest at both ends where $h_{1} \approx H$ and $h_{1} \approx 0$. This is expected since $h_{1}=0$ and $h_{1}=H$ are singularities in equation 12 . Additionally, Figure $2 \mathrm{~b}$ suggests an inverse relationship between the freshwater flow speed and the equilibrated wedge length.

For the parameters chosen in this example, the wedge length is much longer than its height. However, this aspect ratio is set by the interfacial drag coefficient, which in this case is $10^{-4}$. More generally, the wedge aspect ratio is dependent on some combination of the interfacial and wall drag coefficients, which are generally not known. 

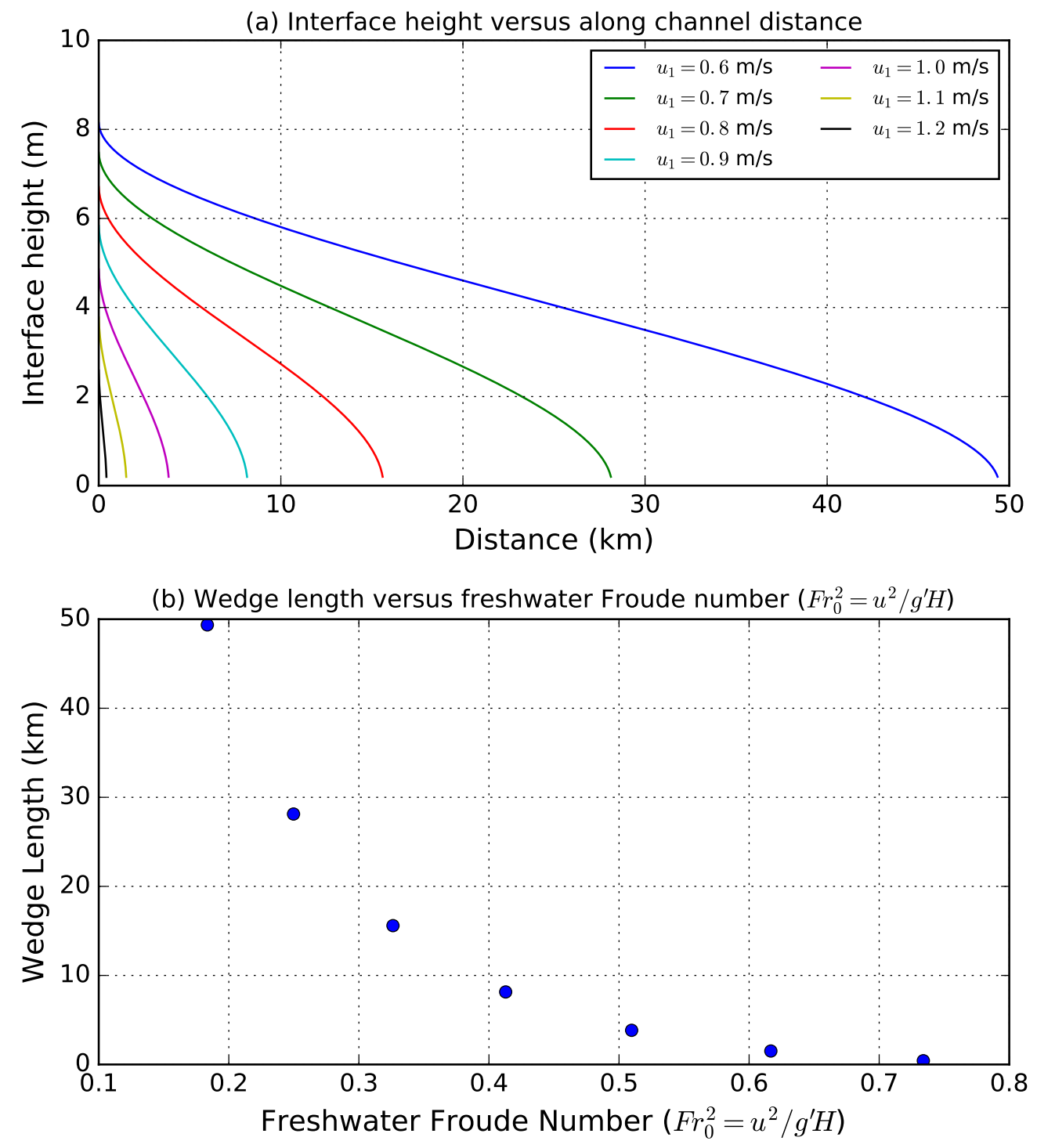

Figure 2: (a) Numerical solutions of equation 12 for a flat estuarine channel with various freshwater flow rates imposed at $x=-L$. For this example, $H=10 \mathrm{~m}, g^{\prime}=0.25 \mathrm{~ms}^{-2}$ and $C_{i}=10^{-4}$. The value $g^{\prime}=0.25$ corresponds to a freshwater and saltwater layer with salinities of 0 PSU and 25 PSU respectively. (b) Wedge length versus freshwater Froude number for the solutions shown in the top plot.

\section{Laboratory Experiments}

\subsection{Experimental set-up}

The main goals of these laboratory experiments are to test the theory outlined in the previous section and to obtain a scaling relationship that allows us to predict the extent of seawater intrusion in a hypothetical subglacial channel. Figure 3 provides a schematic of 
our

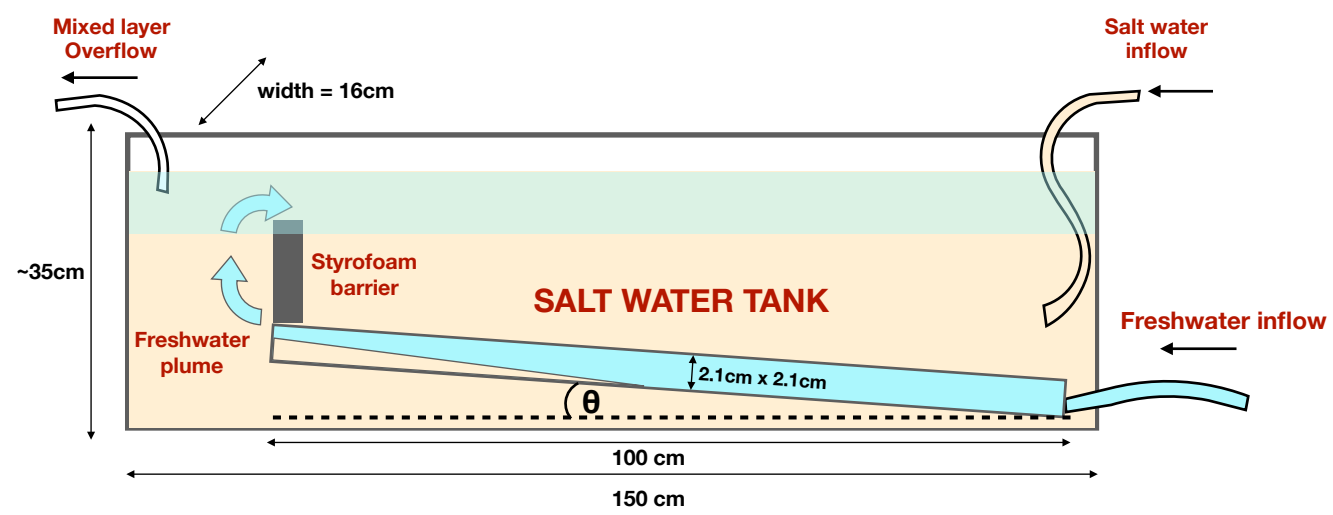

Figure 3: A schematic detailing the salt wedge experiments.

For all experiments, freshwater was pumped through a narrow rectangular duct into a much larger tank filled with saline water. The salt water tank was approximately $150 \mathrm{~cm}$ long, $16 \mathrm{~cm}$ wide and $35 \mathrm{~cm}$ deep The rectangular duct/channel was approximately 100 $\mathrm{cm}$ long and had an inner cross-section of $2.1 \times 2.1 \mathrm{~cm}^{2}$. This experimental configuration chosen so that a steady salt wedge would develop within the rectangular channel for the range of flow rates that could be supplied by the freshwater pump.

For these experiments, there were three main control parameters: the freshwater flow rate, the salinity of the main tank and the slope of the rectangular channel. The freshwater flow rated was varied using an adjustable pump over an approximate range of $5-30 \mathrm{~cm}^{3} / \mathrm{s}$. After careful calibration, this allowed us to precisely set the volume flux of freshwater into the tank. The tank or ambient salinity was varied by mixing local seawater ( $\sim 33 \mathrm{PSU}$ ) with various amounts of freshwater. The tube slope was adjusted via a simple pulley system.

If we define the Reynolds number as

$$
R e=\frac{u H}{\nu},
$$

where $u$ is the imposed freshwater velocity prior to lift-off, $H$ is the height of the channel and $\nu=10^{-6} \mathrm{~m}^{2} / \mathrm{s}$ is the kinematic viscosity. The imposed flow rate of $5-30 \mathrm{~cm}^{3} / \mathrm{s}$ corresponds to a $R e$ range of $250-1400$.

Once set, the freshwater flow rate and tube slope remained fixed during an experiment. However, over the course of an experiment, a mixed layer would form at the top of the tank and grow overtime. To minimize the impact of this dilution, experiments were terminated when the depth of the diluted mixed layer grew to fill the upper two-thirds of the tank. To delay the recirculation of this diluted water to the opening of the rectangular channel, a styrofoam block was inserted above the channel's outlet. This styrofoam block was approximately $10 \mathrm{~cm}$ high and spanned the width of the tank to form a partial dam.

To maintain a constant water level within the tank, a siphon into the mixed layer. In some runs, the salt water within the tank was replenished via a pump that was connected to a larger salt water reservoir. By tuning the siphon's drainage rate and salt water inflow rate, 
it was possible to maintain a quasi-steady mixed layer depth and thus maintain constant ambient salinity at the level of the rectangular channel for an extended period of time.

\subsection{Experimental procedure and data processing}

The first set of experiments were conducted with the tank filled with seawater and the rectangular channel in a horizontal position $(\theta=0)$. With the freshwater pump turned on, the flow rate was held constant until a salt wedge appeared and visibly came to rest within the channel. Once that was realized, the flow rate was adjusted and held constant until the salt wedge evolved to a new equilibrium. This was repeated several times and came to end when the surface mixed layer began to encroach the lower third of the tank. At the end of an experimental run, the tank was drained then refilled with salt water for the next experiment.

A second set of experiments was conducted using seawater mixed with different amounts of freshwater to produce solutions with salinities that range from 16-33 PSU. A third set of experiments was conducted with the channel tilted at small positive angles as indicated in Figure 3.

Each experiment was video recorded with a digital camera that was mounted on a tripod facing the tank. The camera height was adjusted so that it was approximately level with the channel. To highlight the interface between the two layers, the inflowing freshwater was dyed blue and the tank was backlit by a diffuse light source. An image of the salt wedge, as observed during an experiment, is provided in Figure 4.

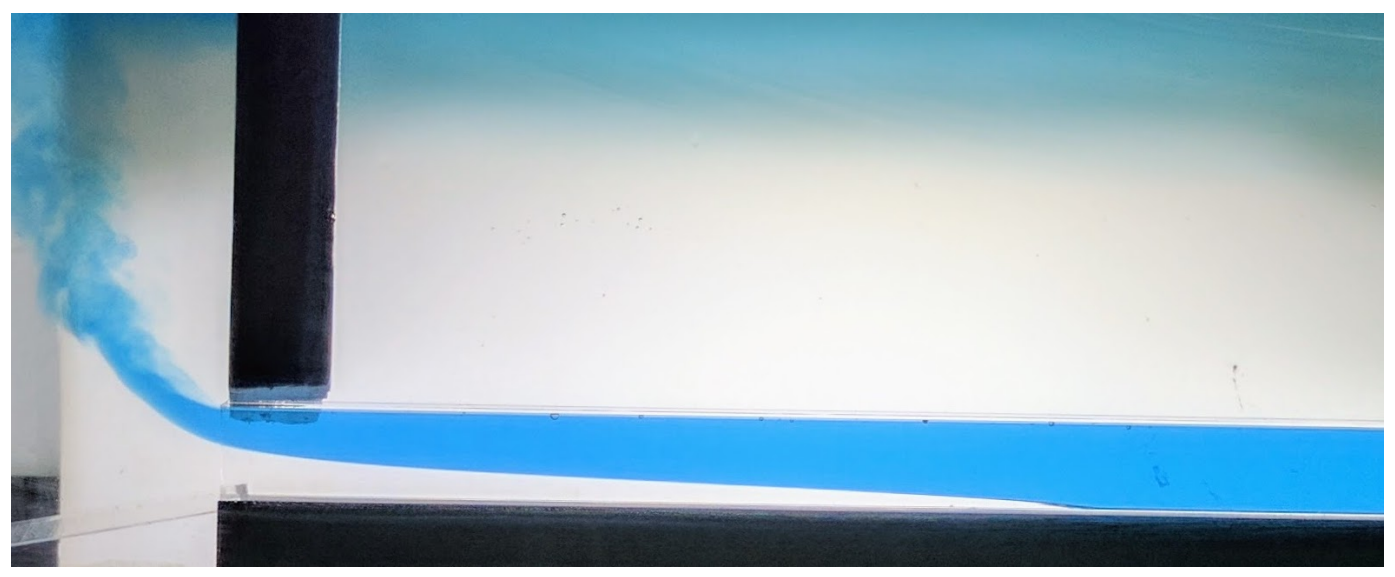

Figure 4: A closeup image of a part of the rectangular channel during an experiment. Here, a salt wedge is observed in the lower half of the tube. For reference, the height of the tube is $2.1 \mathrm{~cm}$

To obtain quantitative data from these experiments, the video recordings were converted to a series of images sampled at every second. To identify the interface between the layers, the images were analyzed through their red channel. Since the dyed water was almost completely opaque to red light, the red channel provided the sharpest contrast between the two layers - much more so than the green and blue channels. This contrast was exploited through the use of a simple red-light intensity threshold. This thresholding was applied to 
vertical sections of the tube in the image. Starting from the saline layer at the base of the tube, the interface was taken to be wherever the red light intensity fell below $10 \%$ of its maximum value. The majority of this process was automated with a set routines written in Python.

\section{Horizontal Channel Experiments}

\subsection{Results summary}

Figures 5 - 6 show example results for a single experimental run. In this run, the freshwater inflow was gradually reduced in five steps. The leftmost, dark blue bold line in Figure 5 indicates the initial equilibrated wedge position. Subsequent bold lines indicate the wedge position just before the pump speed was adjusted. This shows that the image processing algorithm was able to cleanly resolve the shape of the wedge.

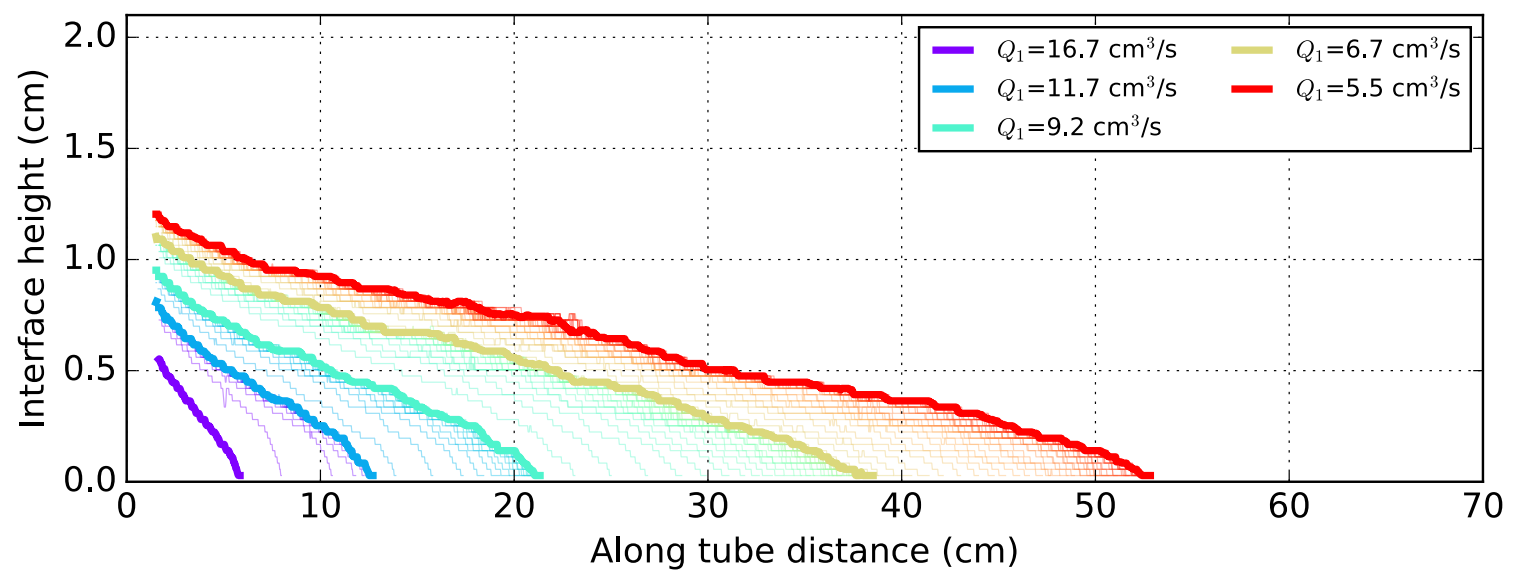

Figure 5: The evolution of a salt wedge for a horizontal channel experiment. Here, the freshwater volume flux was gradually reduced in five steps. Each line represents the position of the salt wedge interface at a 5 second interval. The bold lines indicate the final wedge position just before the freshwater volume was changed.

Figure 6a shows the evolution of the salt wedge length over the duration of the experiment. This plot confirms the salt wedge approached an equilibrium position before each step-change in the freshwater flow rate. In subsequent runs, this experiment was repeated in reverse to ensure that these longer wedge lengths did in fact reach their equilibrium positions. The right plot of Figure 6 shows the wedge length has an apparent inverse relationship with the freshwater volume flux. This relationship bears close resemblance to theoretical curve previously shown in Figure 2. 
(a) Wedge length versus time

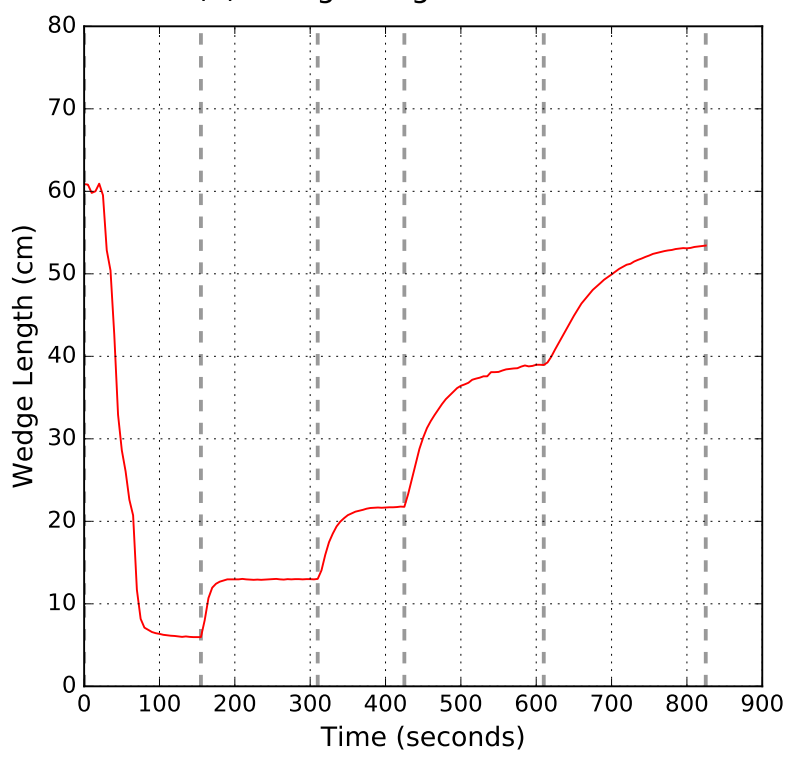

(b) Equilibrium wedge length versus volume flux

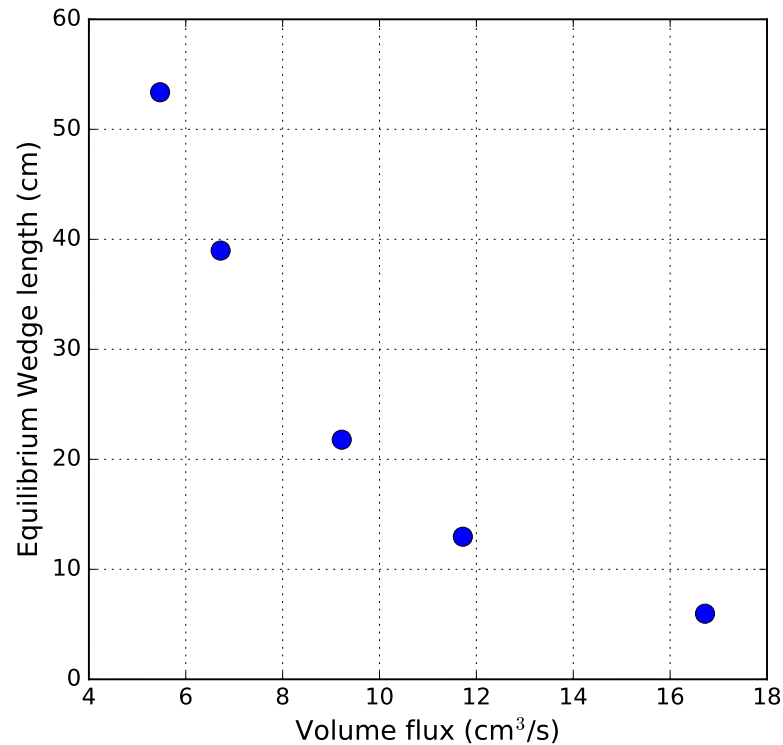

Figure 6: (a) Wedge length versus time for the volume fluxes shown Figure 5. The vertical dashed lines indicate when the freshwater flow rate was ramped down to a new value. (b) Equilibrated wedge length versus the freshwater volume flux. 
(a) Equilibrium wedge length versus volume flux

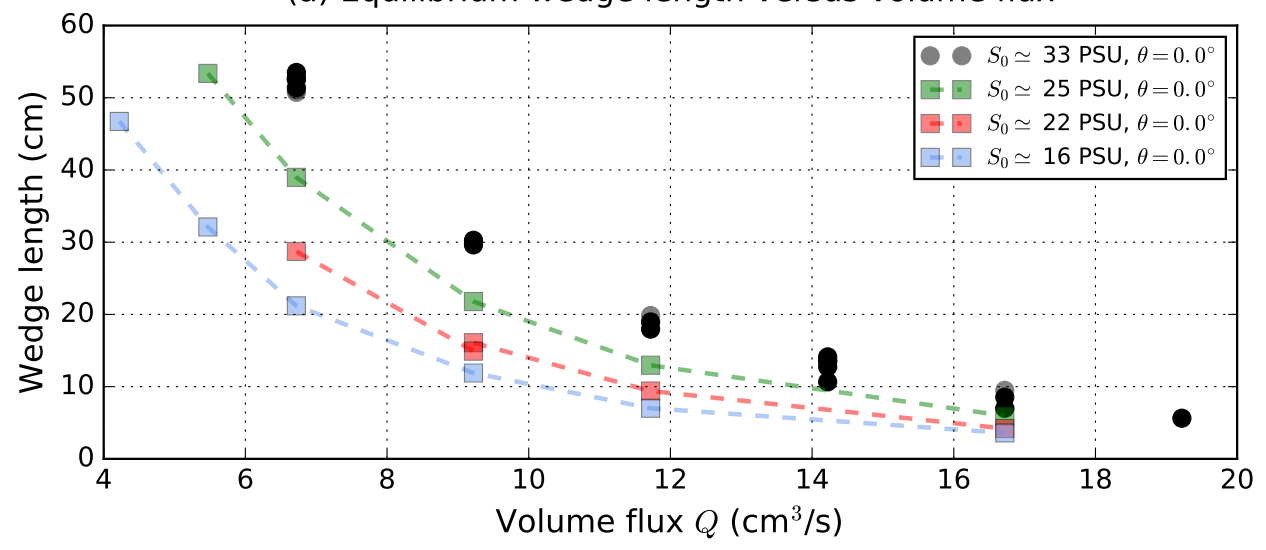

(b) Equilibrium wedge length versus freshwater Froude Number

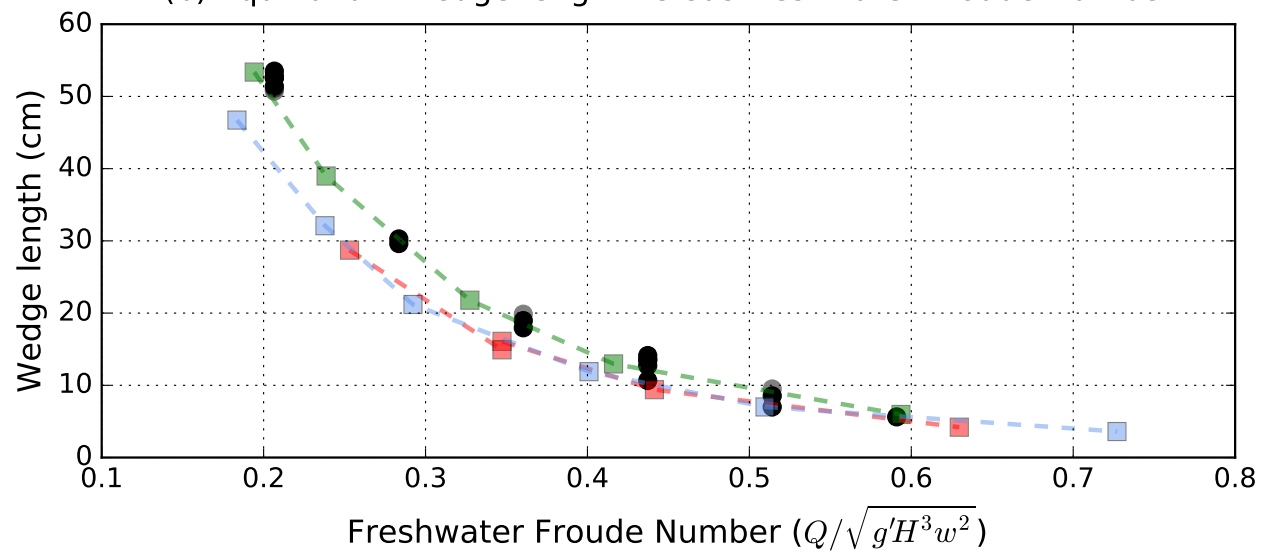

Figure 7: (a) Plot showing results for all horizontal channel experiments. Dark circles represent experiments with pure seawater. Colored squares represent experiments done with diluted seawater. Multiple data points are plotted for repeated runs. The ambient salinity $S_{0}$ for each experiment is provided in the legend. (b) Results for horizontal experiments rescaled in terms of the freshwater Froude number $Q^{2} /\left(g^{\prime} H^{3} w^{2}\right)$.

Figure 7 shows the results for all horizontal channel experiments. Experiments with pure seawater are indicated by black circles. As indicated by the overlapping data points, most of these experiments were repeated several times at the same freshwater flow rate. The colored dots show results for runs done with diluted seawater. Three different seawater to freshwater ratios (3:1, 2:1 and 1:1) were used to produce tank salinities of 24.75 PSU, 22 PSU and 16 PSU. We observe that decreasing the tank salinity, and thus the density difference between the two fluids, reduces the length of the salt wedge for a given flow rate. This is consistent with our theory as reducing the density difference between the two layers is expected to reduce the tendency of the saline layer to slide under the fresh layer.

The effect of varying ambient salinity is mostly accounted for by rescaling the freshwater volume flux by each experiment's reduced gravity $g^{\prime}$. This is shown in the lower plot of Figure 7, which compares the equilibrium wedge length with the non-dimensional freshwa- 
ter Froude number. With this rescaling, the results mostly collapse along a single curve. However, we note that the runs done with the lower ambient salinities are still slightly offset from the other experiments. This slight discrepancy suggests the effect of varying the ambient salinity is not completely captured by $g^{\prime}$.

\subsection{Comparison with theory}

Our next step is to compare the results described in the previous section with our theoretical expectations. The interface slope equation for a flat rectangular duct is given by (13) and is reproduced here for convenience

$$
\left(F r_{1}^{2}-1\right) \frac{\partial h_{1}}{\partial x}=F r_{1}^{2}\left[C_{i}\left(\frac{H}{H-h_{1}}\right)+C_{d}\left(1+\frac{2 h_{1}}{w}\right)\right] .
$$

From our experiment, we have direct measures of all values in the above equation except the wall and interfacial drag coefficients. Though there is a rich literature devoted to the wall drag experienced by single layer pipe flows [18-20], there is very little experimental precedent for the two-layer salt wedge system described here. Moreover, it is not immediately obvious that the wall drag scaling developed for single layer flow is appropriate for this particular two layer flow. Furthermore, we have no a priori knowledge for the value of the interfacial drag coefficient. Our recourse is to approximate these coefficients from the experimental data.

To accomplish this, we first reduce our number of unknowns to one by considering three limiting cases: $C_{i}=C_{d}, C_{i}=0$ and $C_{d}=0$. Next, we rescale the x-coordinate in our model such that $x=C_{o} \hat{x}$, where $C_{o}=C_{i}$ and/or $C_{o}=C_{d}$ depending the limiting case being considered. Doing this eliminates the drag coefficients from our model and gives

$$
\begin{aligned}
& \frac{\partial h_{1}}{\partial \hat{x}}=\frac{F r_{1}^{2}}{\left(F r_{1}^{2}-1\right)}\left(1+\frac{2 h_{1}}{w}\right) \text { for } C_{i}=0, \\
& \frac{\partial h_{1}}{\partial \hat{x}}=\frac{F r_{1}^{2}}{\left(F r_{1}^{2}-1\right)}\left(\frac{H}{H-h_{1}}\right) \text { for } C_{d}=0, \\
& \frac{\partial h_{1}}{\partial \hat{x}}=\frac{F r_{1}^{2}}{\left(F r_{1}^{2}-1\right)}\left[\left(\frac{H}{H-h_{1}}\right)+\left(1+\frac{2 h_{1}}{w}\right)\right] \text { for } C_{d}=C_{i} .
\end{aligned}
$$

For each case, we find solutions for the scaled wedge lengths $\hat{L}$ using the flow parameters from our experiments. With these theoretical wedge lengths, we then find $C_{o}$ such that $L=C_{o} \hat{L}$. We accomplish this by performing a linear least-squares fit for each $\hat{L}$ versus $L$ distribution. The result of this analysis is shown in Figure 8. 


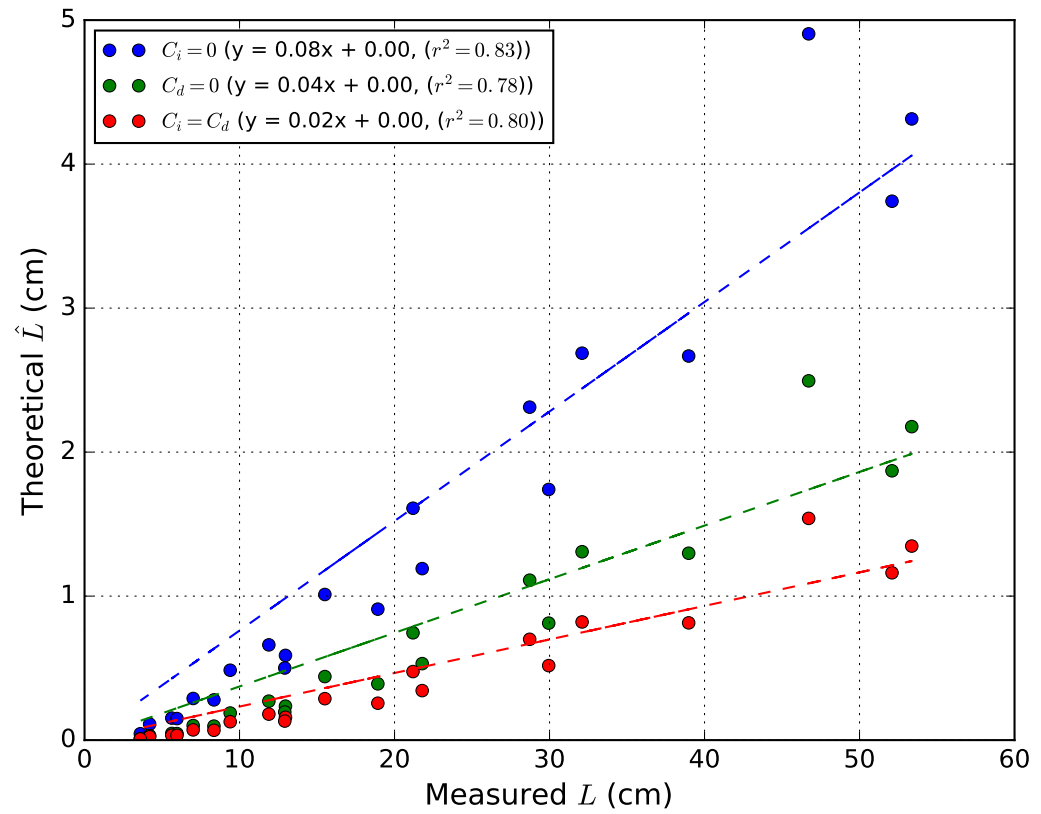

Figure 8: Plot showing scaled theoretical wedge lengths $\hat{L}$ versus experimental wedge lengths $L$ for the three cases described by equations (15)-(17). The blue, green and red dots represent solutions for $\hat{L}$ assuming $C_{i}=0, C_{d}=0$, and $C_{d}=C_{i}$, respectively. The colored dashed lines represent the best fit line for each distribution. Here, the best fit line is forced to pass through $y=0$.

The result of this analysis is shown in Figure 8, which plots the scaled theoretical wedge lengths $\hat{L}$, from equations (15)-(17), against their measured counterparts. Here, we have forced the best fit line to pass through $y=0$. For each case, we see that the relationship between $\hat{L}$ and $L$ is somewhat linear. The R-squared value for each fit is somewhat good $\left(R^{2} \sim 0.8\right)$ but the best fit lines generally lie above the data points associated with shorter wedges. This indicates that assuming $C_{o} \hat{L}$, where $C_{o}$ is the slope of the best fit line, will generally underestimate the salt wedge length for higher flow rates or Froude numbers. Figure 9 shows that this is the case. 


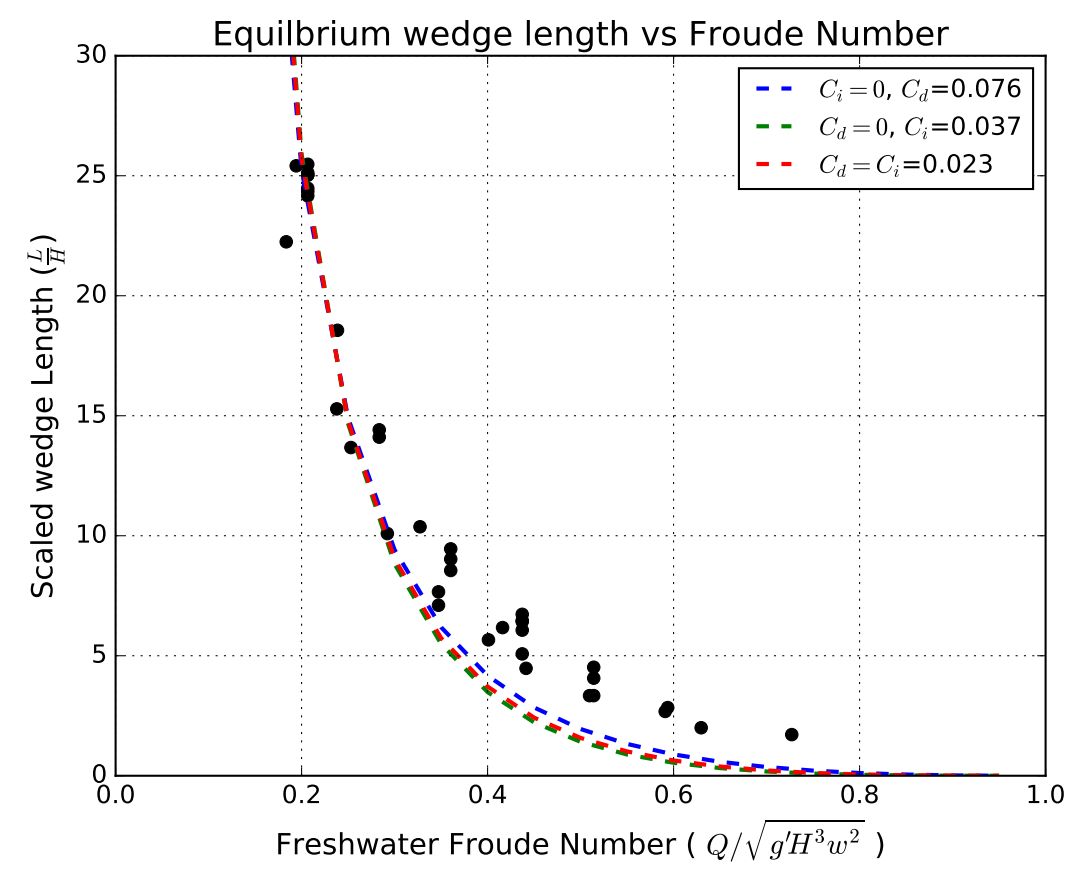

Figure 9: Data from Figure 7 overlain with theoretical curves for the three limiting cases described by (15)-(17). Here, the drag coefficients are assumed to be constant.

This discrepancy suggests that the relationship between $\hat{L}$ and $L$ is not truly linear. A closer examination of Figure 8 reveals that the distribution of data points at shorter wedge lengths have a smaller slope than those at longer wedge lengths. Since shorter wedge lengths are associated with higher flow rates, this result suggest that the drag coefficients have an inverse relationship with the freshwater flow rate. This inverse relationship is wellestablished for the case of single layer pipe flow. For laminar flow pipe flow $(R e<2000)$, the Darcy friction factor $f_{D}$ for wall drag is given by

$$
f_{D}=\frac{64}{R e},
$$

where $f_{D}=8 C_{d}[18,19]$. This formulation suggests we should instead model our drag coefficients as

$$
\begin{aligned}
C_{d} & =\frac{\tilde{C}_{d}}{R e} \\
C_{i} & =\frac{\tilde{C}_{i}}{R e}
\end{aligned}
$$

where is $R e$ is given by (14). With this parameterization for the drag coefficients, we repeat the previous analysis now seeking values of $\tilde{C}_{d}$ and $\tilde{C}_{i}$ that best match our experimental data. For each case, our scaled salt wedge model is given by 


$$
\begin{aligned}
& \frac{\partial h_{1}}{\partial \hat{x}}=\frac{F r_{1}^{2}}{\left(F r_{1}^{2}-1\right)} \frac{1}{R e}\left(1+\frac{2 h_{1}}{w}\right) \quad \text { for } \quad \tilde{C}_{i}=0, \\
& \frac{\partial h_{1}}{\partial \hat{x}}=\frac{F r_{1}^{2}}{\left(F r_{1}^{2}-1\right)} \frac{1}{R e}\left(\frac{H}{H-h_{1}}\right) \text { for } \tilde{C}_{d}=0, \\
& \frac{\partial h_{1}}{\partial \hat{x}}=\frac{F r_{1}^{2}}{\left(F r_{1}^{2}-1\right)} \frac{1}{R e}\left[\left(\frac{H}{H-h_{1}}\right)+\left(1+\frac{2 h_{1}}{w}\right)\right] \text { for } \quad \tilde{C}_{d}=\tilde{C}_{i} .
\end{aligned}
$$

The result of this analysis is shown in Figure 10. In this case, the relationship between the scaled theoretical wedge lengths and the measured wedge lengths are well approximated by a straight line that pass through $y=0$. Additionally, the quality of the line fits are significantly better $\left(R^{2}>0.9\right)$. However, we note that in this case, $C_{i}=0$ assumption produces a linear fit that is notably worse than the others. As expected, we see that the theoretical wedge lengths now have a much better agreement with the data (Figure 11).

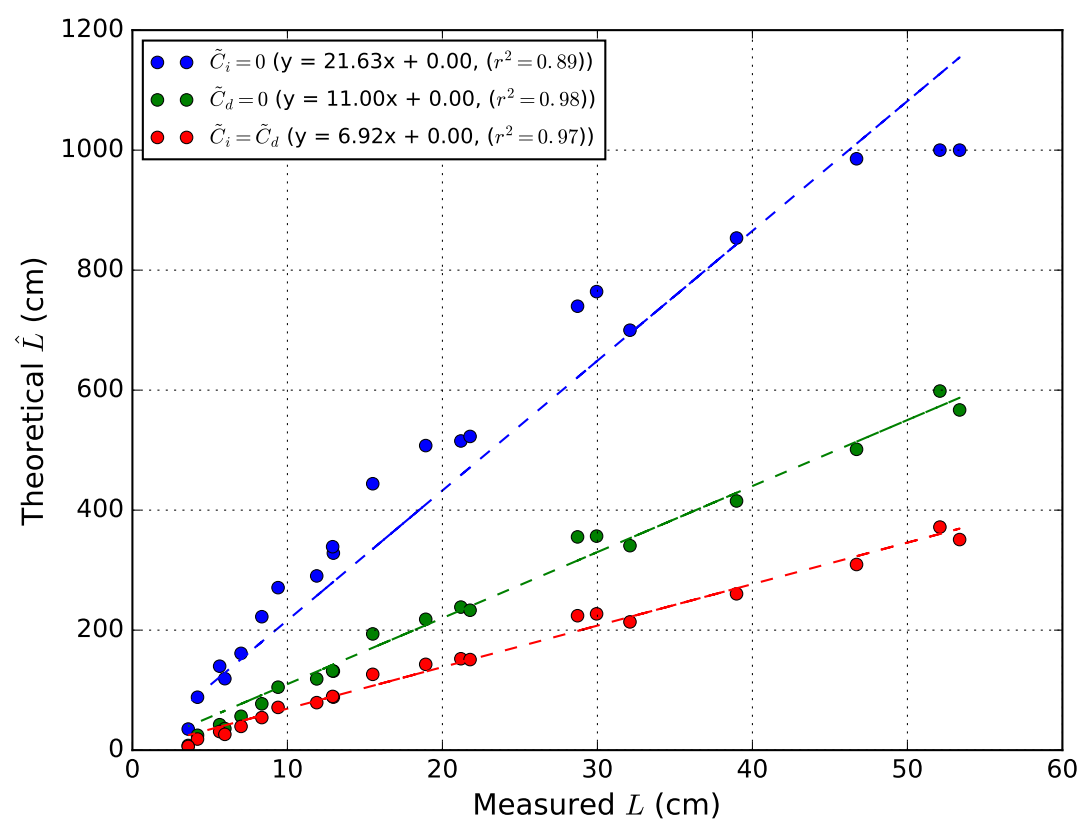

Figure 10: Like Figure 8 but now showing solutions for $\hat{L}$ for $(21)-(23)$, which assume the drag coefficients are inversely proportional to $R e$. 


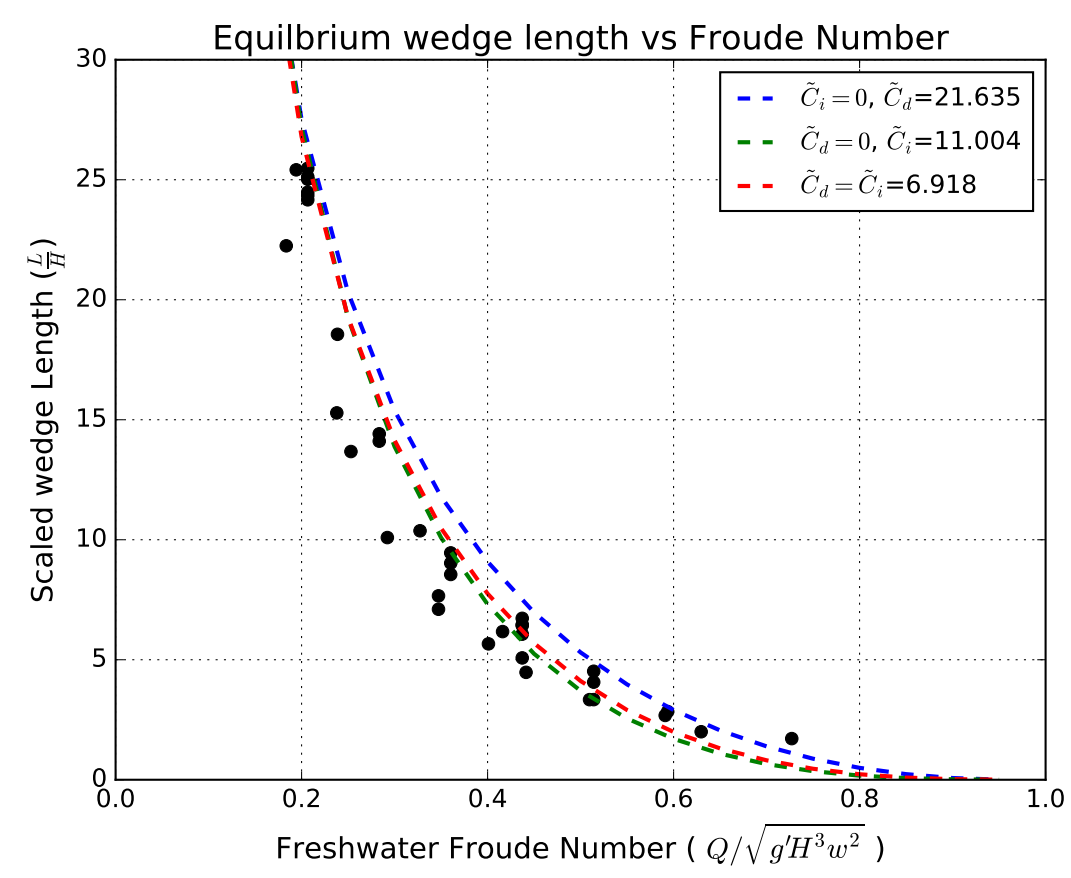

Figure 11: Data from Figure 7 overlain with theoretical curves for the three limiting cases described by (21)-(23). Here, the drag coefficients are assumed to be inversely proportional to $R e$.

From our regression analysis, we find that the $C_{d}=0$ and $C_{i}=C_{d}$ scenarios produce theoretical wedge lengths that have equally good agreement with our data. When $C_{i}=C_{d}$, the best agreement is obtained when

$$
C_{i}=C_{d} \approx \frac{7}{R e}
$$

This result is similar to the drag parameterization for laminar flow in a pipe [18]. We note that this result can only be validated for the range of Froude numbers we tested in the laboratory. 


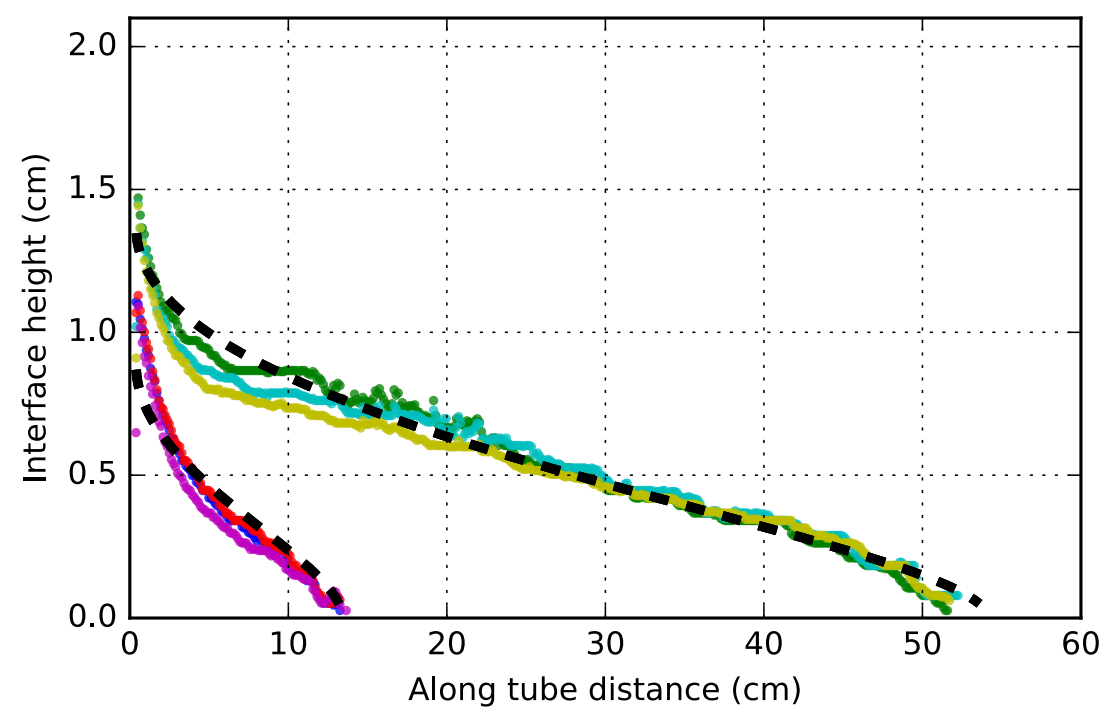

Figure 12: Comparisons between observed and theoretical wedge shapes. The observations are from a single run where the freshwater flow rate was varied back and forth between 6.7 $\mathrm{cm}^{3} / \mathrm{s}$ and $14.2 \mathrm{~cm}^{3} / \mathrm{s}$. Data from the higher freshwater flow rate run are represented by the lower distribution of purple-red dots; the greenish dots represent the data for the higher flow rate. The black dashed lines represent the theoretical solution for each distribution. The theoretical wedge shapes were obtained by assuming $C_{i}=C_{d} \approx 7 / R e$.

Next, we compare the shape of the observed salt wedge to that predicted by theory. Figure 12 shows direct comparisons between theoretical (black dashed lines) and observed (colored dots) interface shapes. The observations chosen here are for a single experimental run where the freshwater flow rate was varied between two flow rates that almost span the range of flow rates used throughout the experiments. In this example, we see that the observed wedge shapes agree reasonably well with our theoretical expectations.

\section{$5 \quad$ Sloped Channel Experiments}

\subsection{Results summary}

The experimental results described thus far have all been in the laminar regime $(R e \sim$ 250-1400). In each case, the interface between the salt and freshwater layers was always well defined and resembled the image shown in Figure 4. While this bodes well for our noentrainment theory, the real subglacial system (with its much larger scales) is well within the turbulent regime. Thus, the final phase of this experiment, was an attempt to observe the salt wedge in a more turbulent state with significant interfacial mixing. 
(a) Interface height

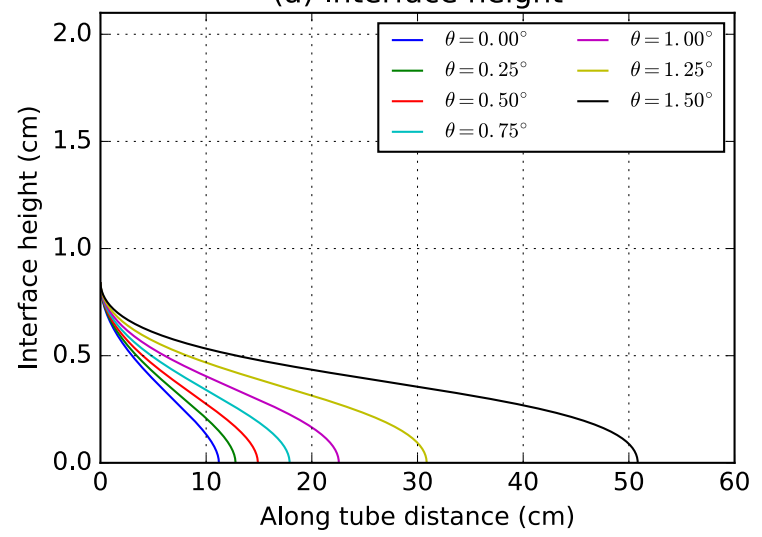

(b) Upper layer velocity

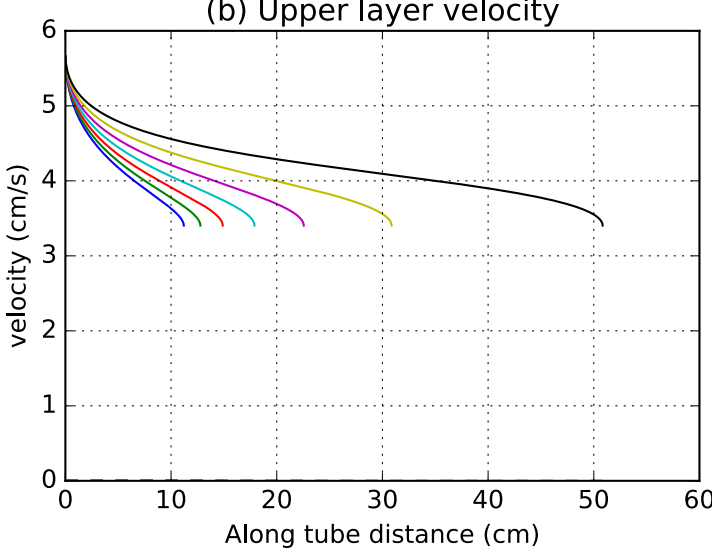

Figure 13: Solutions of (12) for a fixed freshwater flow rate at different channel slopes. For these simulations $Q_{1}=15 \mathrm{~cm}^{3} / \mathrm{s}, H=w=2.1 \mathrm{~cm}, g^{\prime}=$ and $C_{i}=C_{d} \approx 7 / R e$.

Given the narrow constraints of the rectangular channel and the limited range of the pump, the best way to achieve a state of significant interfacial mixing was to tilt the tube so that freshwater layer flowed upwards into the salt water tank. Introducing an upwards tilt allowed the freshwater layer to accelerate under the influence of its own buoyancy. This also introduced an additional tendency for the salt water layer to flow down slope into the channel. Together, both effects act to enhance the velocity shear across the interface $\left(\left(u_{1}-u_{2}\right) / H\right)$. This tendency is captured by the original theory as demonstrated in Figure 13. 
(a) Equilibrium wedge length versus volume flux

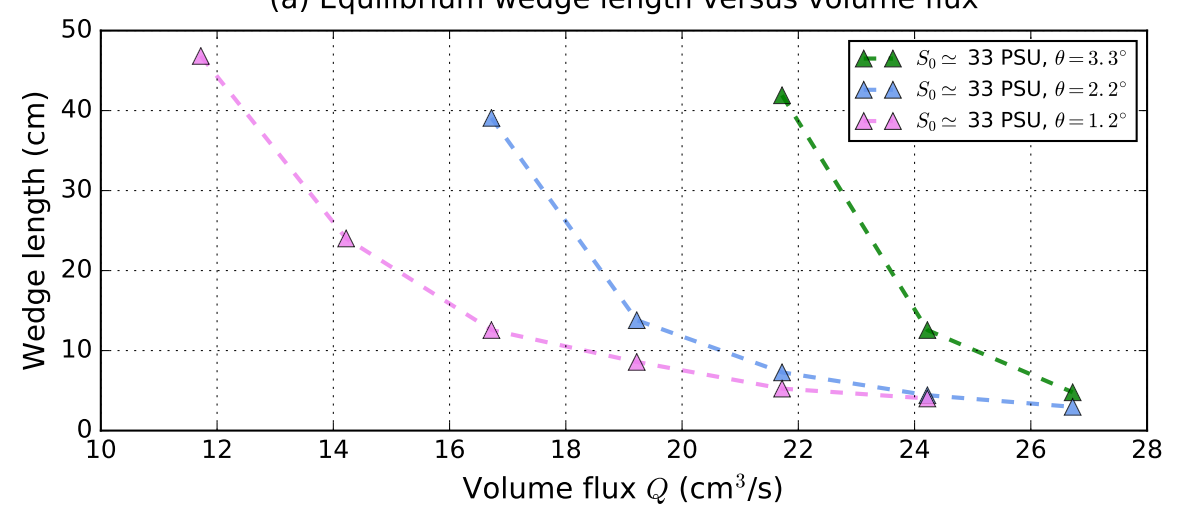

(b) Equilibrium wedge length versus freshwater Froude Number

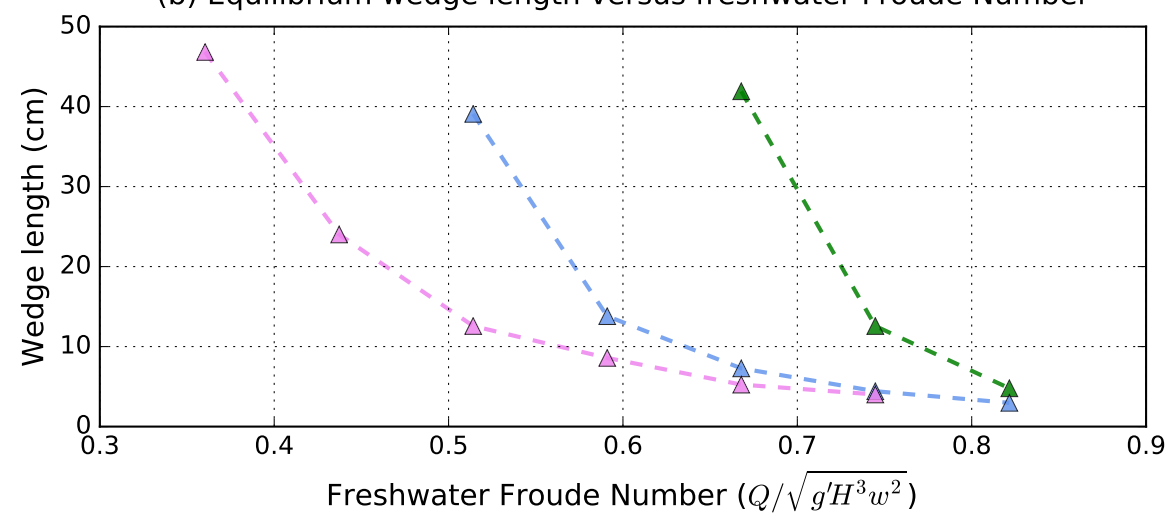

Figure 14: Equilibrium wedge length as in Figure 7, but for sloped channel experiments

Results for the sloped channel experiments are shown in Figure 14. As predicted by (12), increasing the channel slope produces longer salt wedges for a given flow rate. These results are for experiments conducted at relatively shallow channel slopes. At $3.3^{\circ}$, the steepest angle shown in Figure 14, the salt wedge still showed no signs of entrainment.

However, at slightly steeper channel slopes and higher freshwater flow rates, small amplitude interfacial waves began to appear. At even steeper slopes and higher freshwater flow rates, these interfacial waves grew and eventually began to overturn. At the steepest slopes obtained with the tank $\left(\theta \sim 8^{\circ}\right)$, the salt wedge transitioned into what visibly appeared to be an unsteady flow with significant interfacial mixing. 

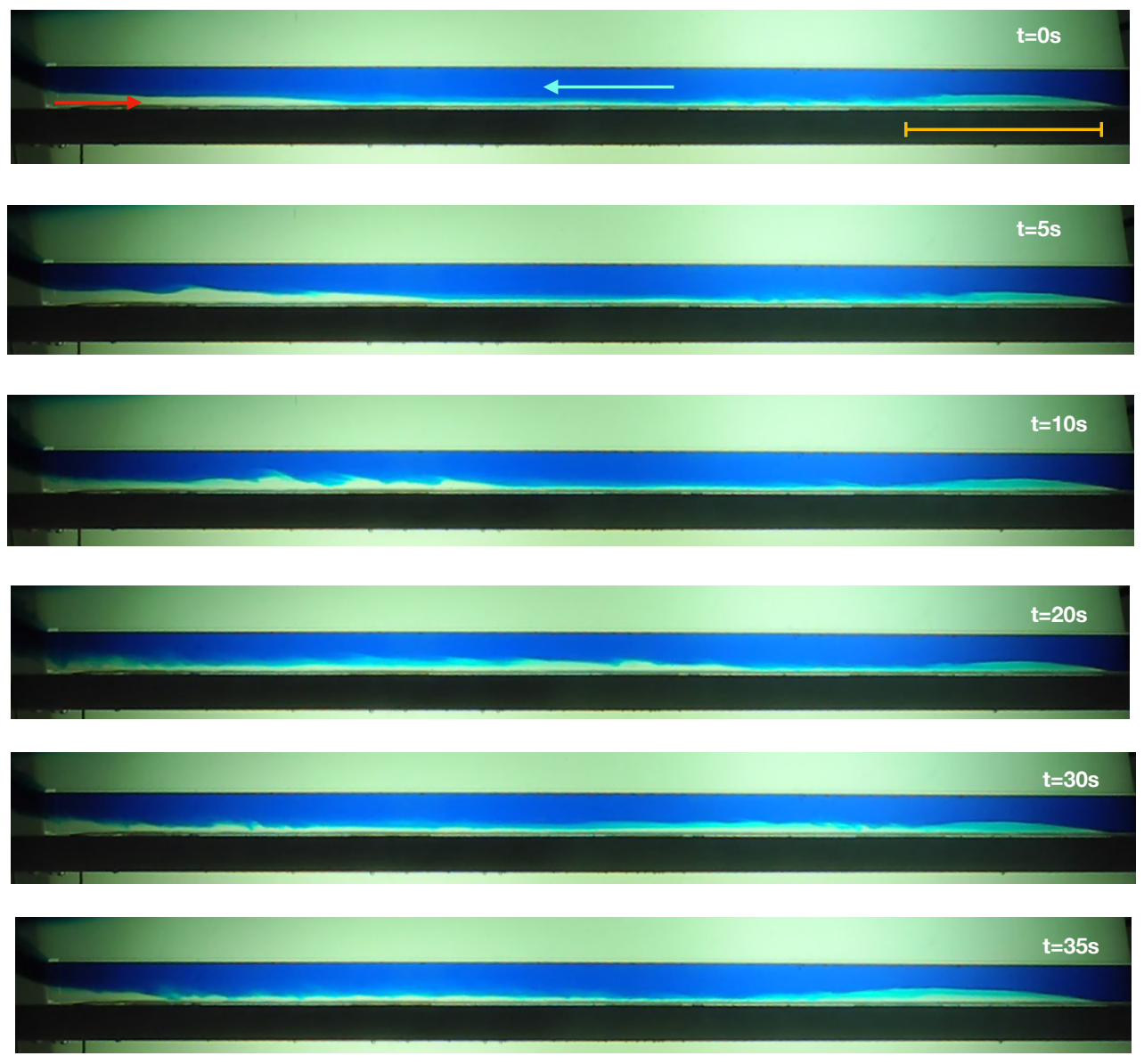

Figure 15: Rotated images showing the time evolution of interfacial waves inside a "steeply" sloped channel. For this experiment, the channel was tilted at $7.8^{\circ}$ and the freshwater flow rate was set to $28 \mathrm{~cm}^{3} / \mathrm{s}$. In the top image, the red and blue arrows indicate the direction of the upper and lower layer flows while the orange bar shows the approximate position of the salt wedge.

Figure 15 shows rotated image snapshots of breaking interfacial waves in the wake of a salt wedge inside a channel tilted at $7.8^{\circ}$. For this run, the freshwater flow rate was held at $28 \mathrm{~cm}^{3} / \mathrm{s}$ for just over 12 minutes. For the latter part of this run, the nose of the salt wedge occupied roughly the same position within the channel. During this period, the flow downstream of the salt wedge displayed episodic bursts of interfacial mixing, an example of which is presented in Figure 15.

At $t=0$ seconds in Figure 15, the region downstream of the salt wedge was in a relatively quiescent state. Approximately five seconds later, a pulse of salt water entered the channel from the left. As this pulse of salt water travelled down the channel, it created wavelike disturbances that overturned and causing mixing across the interface $(t=10-20 \mathrm{~s})$. During this time, the salt wedge gradually contracted in size. The end result of this intense mixing was an intermediate layer of mixed fluid that continued to flow down the tube. At 
$t=35 \mathrm{~s}$, the now diluted pulse of salt water replenished the salt wedge and the two layer flow returned to its initial quiescent state.

Such strong mixing episodes clearly violate the no-entrainment assumption used to build the earlier theory. We must therefore revise our theory to account for this new behavior.

\section{Revised Theory for a Salt Wedge with Entrainment}

\subsection{Derivation}

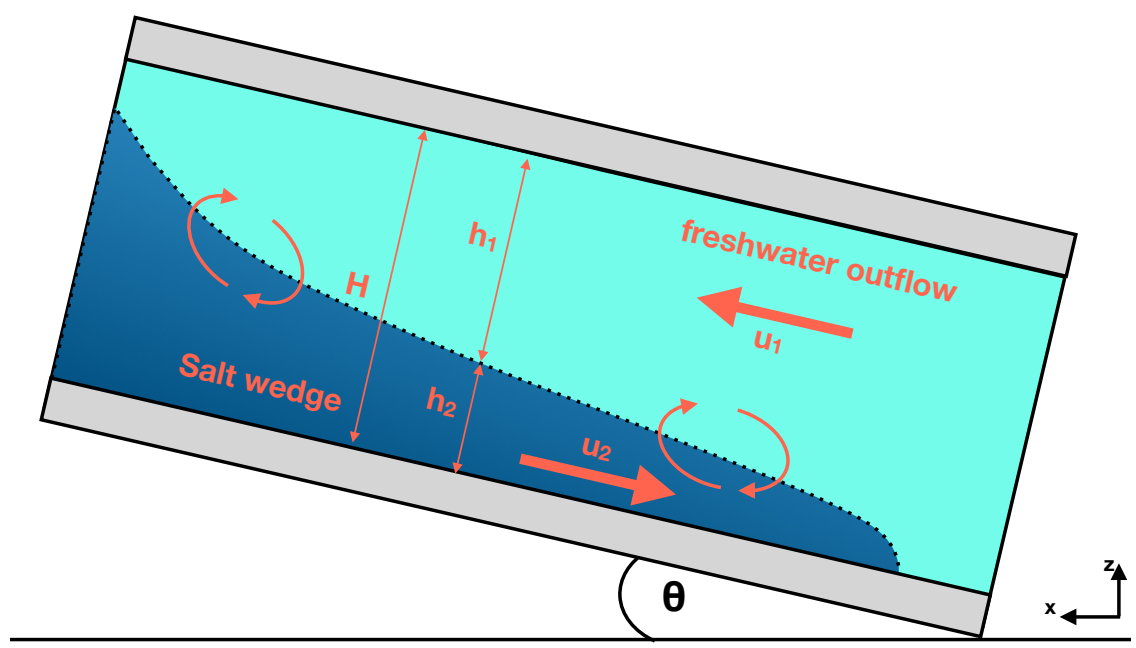

Figure 16: Schematic showing a cross-sectional view of a salt wedge in a sloped rectangular channel - now including entrainment.

With entrainment, the upper and lower layer continuity equations are given by

$$
\begin{aligned}
& \frac{\partial h_{1}}{\partial t}+\frac{\partial q_{1}}{\partial x}=e \\
& \frac{\partial h_{2}}{\partial t}+\frac{\partial q_{2}}{\partial x}=-e,
\end{aligned}
$$

where $e$ is the entrainment rate, $q_{1}=h_{1} u_{1}$ and $q_{2}=h_{2} u_{2}$. The entrainment rate $e$ has units of velocity and is parameterized in terms of the velocity difference between the layers

$$
e=E\left(u_{1}-u_{2}\right) .
$$

where $E$ is an entrainment coefficient. Summing the continuity equations, we see that the total volume flux is conserved along the channel

$$
q_{1}+q_{2}=q_{0}
$$


Likewise, salt conservation is given by

$$
\begin{aligned}
\frac{\partial}{\partial t}\left(S_{1} h_{1}\right)+\frac{\partial}{\partial x}\left(q_{1} S_{1}\right) & =e S_{2}, \\
\frac{\partial}{\partial t}\left(S_{2} h_{2}\right)+\frac{\partial}{\partial x}\left(q_{2} S_{2}\right) & =-e S_{2},
\end{aligned}
$$

where salinities within each layer, $S_{1}$ and $S_{2}$, are assumed to be uniform. Using (25) and (26), the salt conservation equation becomes

$$
\begin{aligned}
& h_{1} \frac{\partial S_{1}}{\partial t}+q_{1} \frac{\partial S_{1}}{\partial x}=e\left(S_{2}-S_{1}\right), \\
& h_{2} \frac{\partial S_{2}}{\partial t}+q_{2} \frac{\partial S_{2}}{\partial x}=0 .
\end{aligned}
$$

During entrainment, the upper layer expands and becomes more saline while the lower shrinks and maintains its salinity. In other words, entrainment is directed from the lower layer to the upper layer. Furthermore, $S_{2}$ is constant and determined by its value at the mouth of the channel.

As before, reduced gravity is given by

$$
g^{\prime}=\frac{\rho_{2}-\rho_{1}}{\rho_{0}} g=\beta\left(S_{2}-S_{1}\right) g
$$

where $\beta$ is the saline contraction coefficient. Using the above relation, we can re-write the upper layer salt budget as an equation for $g^{\prime}$

$$
h_{1} \frac{\partial g^{\prime}}{\partial t}+q_{1} \frac{\partial g^{\prime}}{\partial x}=-e g^{\prime}
$$

The momentum conservation equations are given by

$$
\begin{aligned}
& \frac{\partial u_{1}}{\partial t}+u_{1} \frac{\partial u_{1}}{\partial x}=\frac{G_{1}}{h_{1}}-C_{d} u_{1}^{2}\left(\frac{1}{h_{1}}+\frac{2}{w}\right)-C_{i} \frac{\left(u_{1}-u_{2}\right)^{2}}{h_{1}}+\frac{e\left(u_{2}-u_{1}\right)}{h_{1}} \\
& \frac{\partial u_{2}}{\partial t}+u_{2} \frac{\partial u_{2}}{\partial x}=\frac{G_{2}}{h_{2}}+C_{d} u_{2}^{2}\left(\frac{1}{h_{2}}+\frac{2}{w}\right)+C_{i} \frac{\left(u_{1}-u_{2}\right)^{2}}{h_{2}}
\end{aligned}
$$

where

$$
\begin{aligned}
& G_{1}=-\frac{\partial P}{\partial x}-\rho_{1} g \tan \theta-\frac{1}{2} h_{1} \rho_{1} \frac{\partial g^{\prime}}{\partial x} \\
& G_{2}=-\frac{\partial P}{\partial x}-\rho_{1} g \tan \theta-\rho g^{\prime}\left(\tan \theta-\frac{\partial h_{1}}{\partial x}\right) .
\end{aligned}
$$

These momentum equations are analogous to the ones introduced earlier for the noentrainment case. Here, there are new additions that account for momentum exchange between the two layers due to entrainment and the fact that $g^{\prime}$ can now vary in the along channel direction. 
After subtracting (36) from (35) and doing some simplifications, we arrive at

$$
\begin{array}{r}
\frac{\partial}{\partial t}\left(u_{1}-u_{2}\right)+u_{1} \frac{\partial u_{1}}{\partial x}-u_{2} \frac{\partial u_{2}}{\partial x}=g^{\prime}\left(\tan \theta-\frac{\partial h_{1}}{\partial x}\right)-\frac{1}{2} g_{x}^{\prime} h_{1} \\
-C_{d}\left[\frac{u_{1}^{2}}{h_{1}}\left(1+\frac{2 h_{1}}{w}\right)+\frac{u_{2}^{2}}{h_{2}}\left(1+\frac{2 h_{2}}{w}\right)\right]-C_{i}\left(u_{1}-u_{2}\right)^{2}\left(\frac{1}{h_{1}}+\frac{1}{h_{2}}\right)-E \frac{\left(u_{1}-u_{2}\right)^{2}}{h_{1}} .
\end{array}
$$

The four main variables of interest are $u_{1}, u_{2}, h_{1}$ and $g^{\prime}$. These variables can be evaluated using equations (25), (28), (34) and (39) provided the parameters $q_{0}, \theta, E, C_{d}$ and $C_{i}$ are specified. In steady state, our four independent equations are

$$
\begin{aligned}
q_{1}+q_{2} & =q_{0} \\
\frac{\partial q_{1}}{\partial x} & =E\left(u_{1}-u_{2}\right) \\
q_{1} \frac{\partial g^{\prime}}{\partial x} & =-E\left(u_{1}-u_{2}\right) g^{\prime} \\
u_{1} \frac{\partial u_{1}}{\partial x}-u_{2} \frac{\partial u_{2}}{\partial x}= & g^{\prime}\left(\tan \theta-\frac{\partial h_{1}}{\partial x}\right)-\frac{1}{2} g_{x}^{\prime} h_{1}-C_{d}\left[\frac{u_{1}^{2}}{h_{1}}\left(1+\frac{2 h_{1}}{w}\right)+\frac{u_{2}^{2}}{h_{2}}\left(1+\frac{2 h_{2}}{w}\right)\right] \\
& -C_{i}\left(u_{1}-u_{2}\right)^{2}\left(\frac{1}{h_{1}}+\frac{1}{h_{2}}\right)-E \frac{\left(u_{1}-u_{2}\right)^{2}}{h_{1}} .
\end{aligned}
$$

For the sake of generality, we non-dimensionalize the above system of equations using the following scaling relationships: $x=L \hat{x}, h_{1}=H \hat{h}_{1}, h_{2}=H \hat{h}_{2}, w=H \hat{w}, u_{1}=U \hat{u}_{1}$, $u_{2}=U \hat{u}_{2}, g^{\prime}=g_{o}^{\prime} \hat{g}^{\prime}$ and $q_{1}=H U \hat{q}_{1}$. Here, $H=h_{1}+h_{2}$ is the height of the channel and $U$ is a characteristic velocity scale, which we define as $U=\sqrt{g_{0}^{\prime} H}$. This allows us to state the upper and lower layer Froude numbers as

$$
\begin{aligned}
& F r_{1}^{2}=\frac{u^{2}}{g^{\prime} h_{1}}=\frac{U^{2} \hat{u}^{2}}{g_{0}^{\prime} H \hat{g}^{\prime} \hat{h}_{1}}=\frac{\hat{u}_{1}^{2}}{\hat{g}^{\prime} \hat{h}_{1}}, \\
& F r_{2}^{2}=\frac{\hat{u}_{2}^{2}}{\hat{g}^{\prime} \hat{h}_{2}} .
\end{aligned}
$$

In this non-dimensional framework, our system parameters can be written as

$$
\begin{aligned}
\tan \theta & =\frac{H}{L} \hat{S}, \\
C_{d} & =\frac{H}{L} \hat{C}_{d}, \\
C_{i} & =\frac{H}{L} \hat{C}_{i}, \\
E & =\frac{H}{L} \hat{E}, \\
q_{0} & =\frac{\hat{q_{0}}}{H U} .
\end{aligned}
$$


With these choice of scalings, our non-dimensional conservation equations have the exact form as the original dimensional equations ((40) - (43)). For the sake of convenience and at the risk of confusion, we now drop the hats off the non-dimensional variables.

Using $u_{1}=q_{1} / h_{1}$ and $u_{2}=q_{2} / h_{2}$, we re-write the momentum equations in terms of volume fluxes as

$$
\begin{array}{r}
\left(\frac{q_{1}}{h_{1}^{2}}+\frac{q_{2}}{h_{2}^{2}}\right) \frac{\partial q_{1}}{\partial x}-\left(\frac{q_{1}^{2}}{h_{1}^{3}}+\frac{q_{2}^{2}}{h_{2}^{3}}-g^{\prime}\right) \frac{\partial h_{1}}{\partial x}=g^{\prime} S+\frac{1}{2} h_{1} \frac{q_{0}}{q_{1}^{2}} \frac{\partial q_{1}}{\partial x} \\
-C_{d}\left[\frac{q_{1}^{2}}{h_{1}^{3}}\left(1+\frac{2 h_{1}}{w}\right)+\frac{q_{2}^{2}}{h_{2}^{3}}\left(1+\frac{2 h_{2}}{w}\right)\right]-\left(\frac{C_{i}}{h_{1} h_{2}}+\frac{E}{h_{1}}\right)\left(\frac{q_{1}}{h_{1}}-\frac{q_{2}}{h_{2}}\right)^{2} .
\end{array}
$$

Here, we have made use of the fact that $h_{1}+h_{2}=1$ and $q_{1}+q_{2}=q_{0}$ in our non-dimensional framework. Additionally, by combining (41) and (42), we find

$$
\frac{\partial}{\partial x}\left(q_{1} g^{\prime}\right)=0
$$

This means the along slope buoyancy flux $q_{1} g^{\prime}$ is conserved in this model. Moreover, from our definition of $g^{\prime}$, it follows that $g^{\prime}=1$ upstream of the salt wedge where the freshwater layer fills the entire channel. Since the total volume flux is given by $q_{0}$, it follows that

$$
g^{\prime}=\frac{q_{0}}{q_{1}} .
$$

With some rearrangement to (51), we can now completely outline our model for an entraining salt-wedge in a sloped channel as

$$
\begin{aligned}
h_{1}+h_{2} & =H=1, \\
q_{1}+q_{2} & =q_{0}, \\
\frac{\partial q_{1}}{\partial x} & =E\left(\frac{q_{1}}{h_{1}}-\frac{q_{2}}{u_{2}}\right), \\
\Gamma \frac{\partial q_{1}}{\partial x}-\left[F r_{1}^{2}+F r_{2}^{2}-1\right] \frac{\partial h_{1}}{\partial x} & =S-C_{d}\left[F r_{1}^{2}\left(1+\frac{2 h_{1}}{w}\right)+F r_{2}^{2}\left(1+\frac{2 h_{2}}{w}\right)\right] \\
& -\left(\frac{C_{i}}{g^{\prime} h_{1} h_{2}}+\frac{E}{g^{\prime} h_{1}}\right)\left(\frac{q_{1}}{h_{1}}-\frac{q_{2}}{h_{2}}\right)^{2},
\end{aligned}
$$

where $\Gamma=\frac{1}{g^{\prime}}\left(\frac{q_{1}}{h_{1}^{2}}+\frac{q_{2}}{h_{2}^{2}}-\frac{1}{2} \frac{h_{1} q_{0}}{q_{1}^{2}}\right)$. In the previous non-entraining case, the flow was critical when $F r_{1}=1$. Here, critical flow occurs when the composite Froude number $G^{2}=F r_{1}^{2}+$ $F r_{2}^{2}$ is unity. It should be noted that when $E=0$, equations (55)-(57) reduce to equation 13 - the original salt-wedge model that did not permit entrainment.

As before, $x=0$ is defined to be where the flow becomes critical, which here is where $G^{2}=1$. At the nose of the wedge, where $x=-L, h_{1}=1$ and $q_{1}=q_{0}$. In the non-entraining case, we found solutions for $h_{1}$ by starting our integrations at $x=0$, where $F r_{1}^{2}=1$, and integrating in reverse until $h_{1} \approx H$. In that case, the critical point is associated with a unique value for $h_{1}$. However, in this case, there are many possible combinations of $h_{1}$ and 
$h_{2}$ for which $G^{2}=1$. For this reason, we begin the numerical integrations at $x=-L$ where $h_{1}=H^{2}$ and continue forward until $G^{2}=1$. Once this criteria is met, $L$ can be determined.

\subsection{Simulations}

In this section, we present simulations of our revised salt wedge model that permits entrainment. For these simulations, we assume a rectangular channel with a $2.1 \mathrm{~cm} \times 2.1$ $\mathrm{cm}$ cross-section, similar to the one used in the laboratory experiments. At the boundary $x=-L$, the integrations were initialized with a $15 \mathrm{~cm}^{3} / \mathrm{s}$ freshwater flux, which is again similar to what was used in some of the laboratory experiments. As described earlier, integrations were done in the positive $x$ direction and came to end when $G^{2}=1$.

Following [6], we model the entrainment coefficient $E$ as

$$
E=E_{o} \sin \theta
$$

where $\theta$ is the slope of the channel and $E_{o}$ is some constant. With this parameterization, entrainment is only permitted for non-zero channel slopes.

\footnotetext{
${ }^{2}$ Similar to the method for the non-entraining simulations, we specify $h_{1}=H-\epsilon$ at $x=-L$ to avoid the singularity associated with zero lower layer thickness with $\epsilon=10^{-4}$.
} 

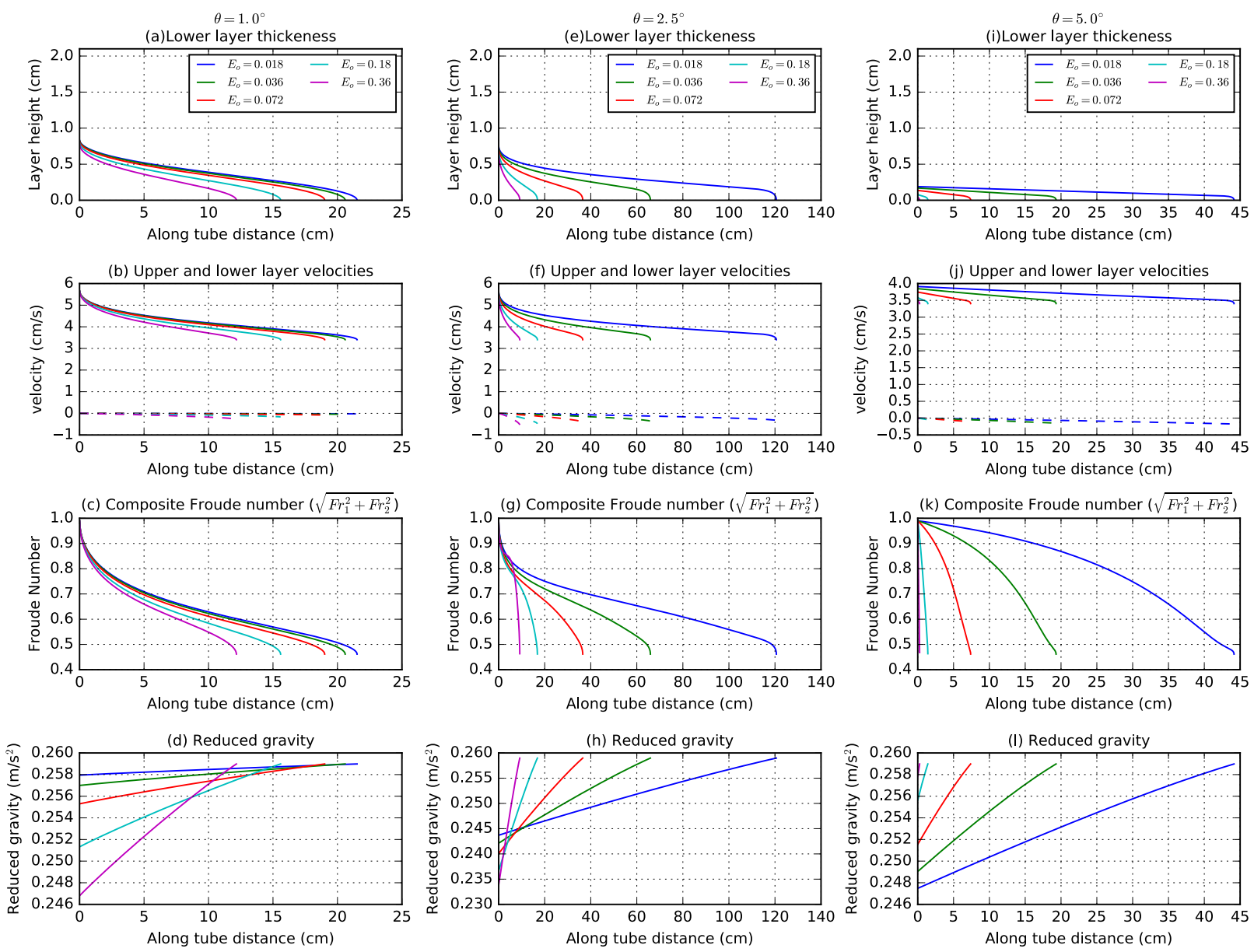

Figure 17: Figure showing solutions for the two-layer salt wedge model with entrainment for various channel slopes and entrainment coefficients. Rows: (From top to bottom) Solutions for along channel interface height, upper (solid lines) and lower (dashed lines) layer velocities, composite Froude number and reduced gravity. Columns: Solutions for various channel slopes $\theta$. From left to right: $\theta=1^{\circ}, \theta=2.5^{\circ}, \theta=5^{\circ}$. These simulations were for a rectangular tube with a $2.1 \mathrm{~cm} \times 2.1 \mathrm{~cm}$ cross-section. The upper layer flow was set to 15 $\mathrm{cm}^{3} / \mathrm{s}$ at $x=-L$. Additionally, $C_{i}=C_{d} \approx 7 / R e$.

Solutions for our two-layer salt wedge model for various channel slopes and entrainment coefficients are shown in Figure 17. Figure 17 a-d show simulations for various entrainment coefficients for a channel with a $1^{\circ}$ slope. The values for the entrainment coefficients range from $E_{o}=0.018$ to $E_{o}=0.36$, corresponding to half and ten times the value used by [6].

In Figure 17a we observe that increasing the entrainment rate reduces the length of the salt wedge. This is because higher entrainment leads to a more rapid thinning and acceleration of the lower layer. Since the Froude number is inversely proportional to layer thickness, the acceleration of the initially thin lower layer drives a quick increase in the composite Froude number and thus a fast approach to critical flow (Figure $17 \mathrm{~b}-\mathrm{c}$ ). As expected, the reduced gravity decreases more rapidly in the along channel direction with 
higher entrainment (Figure 17d).

Figure 17 e-h show similar results for a channel slope of $2.5^{\circ}$. In the non-entraining case, increasing the channel slope increases the length of the salt wedge for a given freshwater flux. Here, we observe that this response depends on the entrainment efficiency. For low entrainment efficiency, a steeper channel slope leads to a longer wedge (for example, the case for $E_{o}=0.018$ in Figure $17 \mathrm{a}, \mathrm{d}$ ), but at higher entrainment rates, a steeper channel can lead to a shorter wedge. The reason for this is two-fold. First, with our parametrization of entrainment (equation 58), increasing the channel slope results in a direct increase in the entrainment rate. Second, increasing the slope of the channel enhances the velocity shear across the two layers, which also enhances entrainment. Both effects tend produce shorter salt wedge intrusions due to their effect on the lower layer thickness and composite Froude number.

At $\theta=5^{\circ}$, we see the dominating effects of entrainment (Figure $17 \mathrm{i}-1$ ). The salt wedges produced by these simulations are all shorter and thinner than the previous cases. This behavior is again dominated by the rapid increase in $F r_{2}^{2}$.

While these simulations are illuminating, they do not reproduce the initial expansion and subsequent contraction of the salt wedge downstream of its nose as shown in Figure 15. This points to several limitations of our salt wedge model. One reason for this discrepancy is that our salt wedge model has no time dependence. The mixing observed in the lab was driven by the intermittent breaking of interfacial waves. Therefore, accurate representation of this process may require a time dependent model. Second, our entrainment model assumes that momentum and mass from the lower is efficiently mixed throughout the upper layer. In contrast, the mixing observed in the laboratory was mostly concentrated near the two-layer interface. One could think of the laboratory observations as a case where the entrainment efficiency is relatively low. However, a more appropriate model for this type of mixing may be a three layer model, where entrainment results in the expansion of an intermediate layer with mixed fluid. Nevertheless, these simulations represent a significant improvement over the non-entraining model and will form the basis for future theoretical development.

\section{Seawater Intrusions in a Realistic Subglacial Channel}

As a final exercise, we use the salt wedge model developed in the previous section to predict the extent of seawater intrusions under real-world glaciers. For this exercise, we will assume a $10 \mathrm{~m}$ by $10 \mathrm{~m}$ channel that is oriented at a very shallow slope $\theta \leq 1$. For this channel, we find a range of subglacial discharge rates that produces freshwater Froude numbers $F r_{0}$ between 0.2 and 0.9 . Here, we note that our empirically derived drag coefficients are only valid for laminar flow and do not apply to this much larger system with $R e \sim 10^{7}$. From pipe flow theory, the wall drag coefficient is expected asymptote to some mean value (which will depend on wall roughness), rather than rapidly approach zero at very large $R e$ [18]. As a matter of convenience, we simply assume $C_{d}=C_{i}=7 / 1000$ - the approximate value for laminar flow when $R e=1000$. 
(a) Seawater intrusion versus subglacial discharge
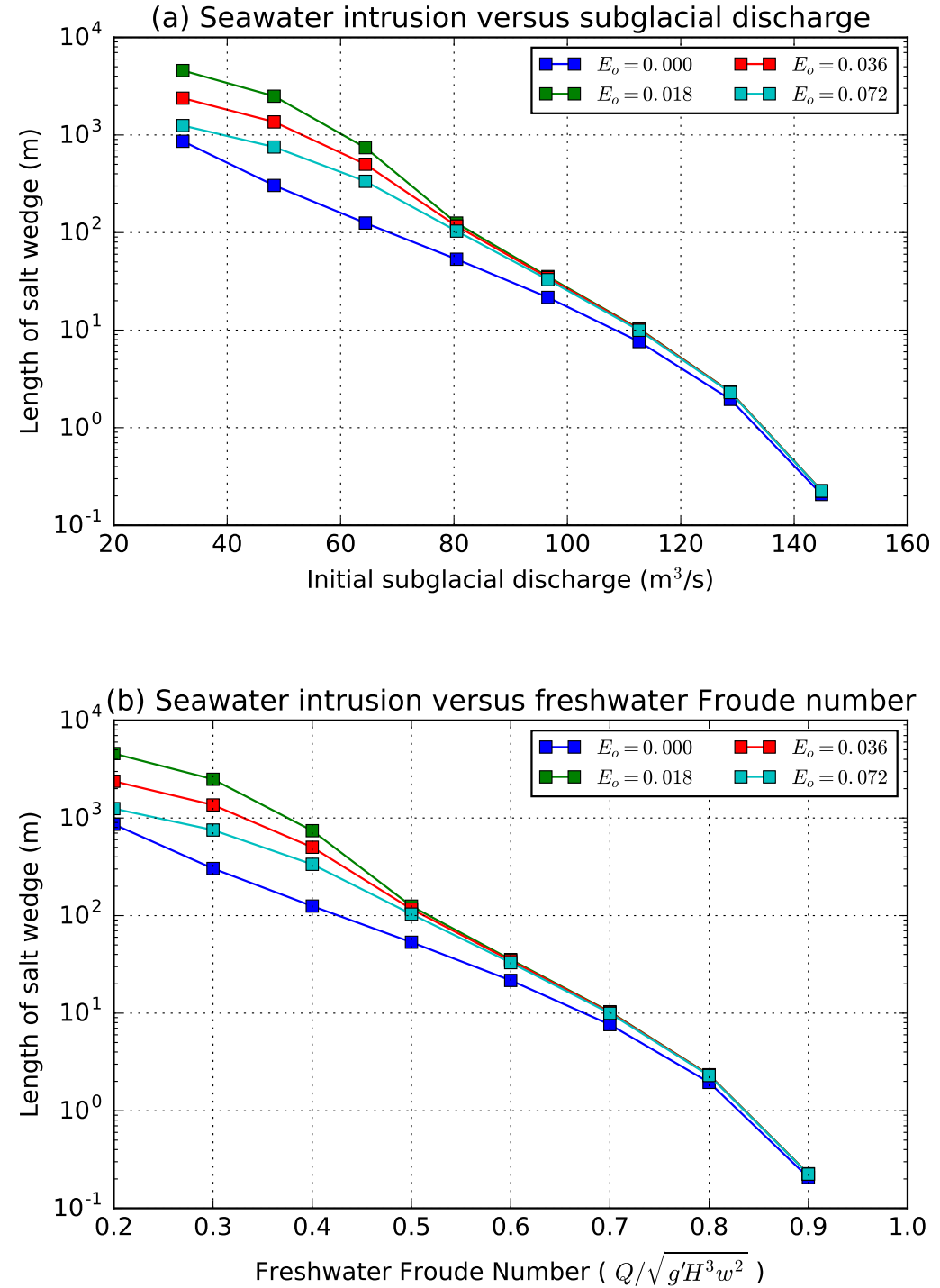

Figure 18: Solutions of salt wedge intrusions using parameters representative of a subglacial outlet. (a) Equilibrium salt wedge length versus subglacial discharge at $x=-L$ for different entrainment coefficients as represented by (58). (b) Equilibrium salt wedge length versus freshwater Froude number $F r_{0}$. For this simulation, we assume a $10 \mathrm{~m}$ by $10 \mathrm{~m}$ subglacial channel and a range of subglacial discharge rates that produce $F r_{0}$ values between 0.2 an 0.9. Since this system is well within the turbulent regime $\left(R e \sim 10^{7}\right)$, we assume constant drag coefficients $C_{d}=C_{i}=0.007$.

The results of this simulation are shown in Figure 18. Here, the equilibrium salt wedge length represents the extent of seawater intrusion into the subglacial channel. For a channel of this size, a $F r_{0}$ range of $0.2-0.9$ corresponds to a subglacial discharge range of approximately $30 \mathrm{~m}^{3} / \mathrm{s}$ to $140 \mathrm{~m}^{3} / \mathrm{s}$. These values are consistent with parameters commonly used in axisymmetric plume models representing subglacial discharge in Greenland [10, 21]. 
For the imposed range of freshwater discharge $Q$, we see that the range of seawater intrusions span several orders of magnitude. For subglacial discharge above $100 \mathrm{~m}^{3} / \mathrm{s}$, the extent of seawater intrusion is minimal (less than a meter). For subglacial discharge below $50 \mathrm{~m}^{3} / \mathrm{s}$, the salt water intrusion extends hundreds of meters into the channel. This result suggests that subglacial channels with weak discharge likely have deep intrusions of seawater. Since the flooding of seawater will likely enhance melting within the subglacial channel, particularly along the lower walls that are in contact with the ocean, our results suggest that these channels will widen over time.

As the channel widens, our theory predicts even deeper seawater intrusions. For example, consider the the case $Q \approx 90 \mathrm{~m}^{3} / \mathrm{s}$. For this channel geometry, $F r_{0}=0.6$. In this state, the extent of seawater intrusion is only a few meters. However, a doubling of the channel's width results in a halving of the $F r_{0}$. Assuming $Q$ remains constant, the expected seawater intrusion is for $F r_{0}=0.3$. From Figure 18b, we see that the length of this intrusion ranges from several hundred to several thousand meters - depending on the entrainment efficiency assumed.

Though this example is very idealized, it clearly illustrates the potential for salt wedges to greatly widen an initially narrow subglacial channel. These results appear to be consistent with recent field observations that suggest that subglacial discharge enters the ocean through broad line plumes rather than concentric point sources $[9,21]$. However, we emphasize that these results are on a preliminary model and hinge on assumptions that are not completely justified. As stated in the previous section, our entraining salt wedge model does not accurately represent entrainment at steep channel relatively steep slopes. Additionally, our empirically derived drag coefficients are only valid for low Reynolds number flows.

\section{Summary}

In this study, we explored the dynamics of subglacial plume lift-off using a combination of idealized models and laboratory experiments. We developed our theoretical framework with the critical assumption that the subglacial flow system is fundamentally similar to that of a salt wedge estuary. With this understanding, we first formulated a theory that describes the steady-state properties of a non-entraining salt wedge confined to a rectangular channel. This theory predicts the shape and length of a salt wedge for a given freshwater volume flux, channel geometry and slope.

In parallel with this theoretical effort, we conducted a series of laboratory experiments to observe the behavior of a salt wedge in a rectangular channel. In these experiments, freshwater was pumped through a narrow rectangular tube into the bottom of tank, filled with water of higher salinity. Experiments were conducted with various freshwater flow rates, tank salinities and channel slopes. With the rectangular tube in a horizontal position, there were no visible signs of mixing across the salt wedge interface.

One key limitation of our salt wedge theory is that it relies on unconstrained coefficients for wall drag, $C_{d}$, and interfacial drag, $C_{i}$. These coefficients were ultimately determined by applying a least squares fit to the experimental data. From this analysis, we found that $C_{d}=C_{i} \approx 7 / R e$ produced the best agreement between theoretical and observed wedge lengths (Figure 11). This result is only valid for the narrow range of laminar flow observed in our laboratory experiments. 
Results from experiments conducted with small positive channel slopes $\left(\theta\right.$ less than $\sim 3^{\circ}$ ) were qualitatively similar to those from the horizontal channel experiments. However, at steeper channel slopes, waves began to appear along the salt wedge interface. At the steepest channel slopes tested, the interfacial waves became unstable and caused mixing between the two layers (Figure 15). Since this observation violated our initial theoretical assumptions, an attempt was made to incorporate entrainment into our salt wedge theory.

This effort led to a revised salt wedge model that permitted the freshwater layer to acquire mass and momentum via entrainment of the more saline lower layer. A key assumption in this model is that both layers remain homogenous during and after entrainment. Following [6], the entrainment rate was assumed to be proportional to the shear across the salt wedge interface and the sine of the channel slope. With this revised salt wedge model, we find that increasing the entrainment rate leads to shorter salt wedges. At high entrainment rates, momentum is efficiently transferred from the upper layer to the lower layer. If the lower layer is initially thin, this results in a rapid increase in the lower layer Froude number and thus the composite Froude number. This was the case in our simulations since we began our integrations at the nose of the salt wedge, where the freshwater begins to lift off the base of the channel.

With our entraining salt wedge model, we speculated on the extent of seawater intrusions for a typical subglacial outlet system. Though these results are preliminary, we find that the extent of seawater intrusion into a subglacial channel can be quite significant and there is a strong tendency for the subglacial channel to expand laterally. This tendency for the subglacial system to form wide discharge outlets would be consistent with recent observations of broad line plumes at tidewater glaciers in Greenland [9, 21].

\section{Future work}

Though insightful, our revised model did not reproduce the observed thinning and subsequent expansion of the freshwater layer as it lifts off the channel and begins to entrain the lower layer (Figure 15). For future work, we propose further developing the two-layer salt wedge model to better represent our experimental results. One possible improvement to our model would be to introduce an intermediate layer along the salt wedge interface. Close observations of experiments done with a steeply sloped channel revealed that the entrainment was mostly confined to the interface. This is similar to what has been observed in exchange flow experiments $[15,16]$. Furthermore, the transient mixing observed in some of our experiments seems to warrant a time-dependent salt wedge model.

A natural extension of our laboratory experiments would be to repeat them at a higher Reynolds number. Achieving full turbulence would almost certainly require a much larger experiment set-up. Doing this would allow for proper determination of the drag coefficients that better represent actual subglacial flow, which is highly turbulent.

\section{Acknowledgements}

First and foremost, I would like to express my deepest gratitude to my advisers Ian Hewitt, Andrew Wells and Claudia Cenedese. Without their expert guidance and countless hours of 
assistance, this project would not have been possible. I would also like to extend a special thanks to Anders Jensen for helping to set up our laboratory experiment and providing technical support throughout the project. Lastly, I would like to thank the many students, fellows and staff of the GFD program who helped to make my time spent at Woods Hole an incredibly fun and intellectually stimulating experience.

\section{References}

[1] E. Rignot, J. Mouginot, and B. Scheuchl. Antarctic grounding line mapping from dif-ferential satellite radar interferometry. Geophysical Research Letters 38.10 (2011), doi: 10.1029/2011GL047109.

[2] X. Chen et al. The increasing rate of global mean sea-level rise during 1993-2014. Nature Climate Change 7.7 (2017), pp. 492-495. doi: 10.1038/nclimate3325.

[3] M. van den Broeke et al. Partitioning recent Greenland mass loss. Science 326.5955 (2009), pp. 984-986. doi: 10.1126/science.1178176.

[4] E. Rignot, M. Koppes, and I. Velicogna. Rapid submarine melting of the calving faces of West Greenland glaciers. Nature Geoscience 3.3 (2010), pp. 187-191. doi: 10.1038/ngeo765.

[5] F. Straneo and C. Cenedese. The dynamics of Greenland's glacial fjords and their role in climate. Annual Review of Marine Science 7.1 (2015), pp. 89-112. doi 10.1146/ annurev-marine-010213-135133.

[6] A. Jenkins. Convection-driven melting near the grounding lines of ice shelves and tidewater glaciers. Journal of Physical Oceanography 41.12 (2011), pp. 2279-2294. doi: 10.1175/JPO-D-11-03.1.

[7] Y. Xu et al. Numerical experiments on subaqueous melting of Greenland tidewater glaciers in response to ocean warming and enhanced subglacial discharge. Annals of Glaciology 53.60 (2012), pp. 229-234. doi: 10.3189/2012AoG60A139.

[8] M. O'Leary and P. Christoffersen. Calving on tidewater glaciers amplified by submarine frontal melting. The Cryosphere 7.1 (2013), pp. 119-128. doi: 10.5194/tc-7-119-2013.

[9] M. J. Fried et al. Distributed subglacial discharge drives significant submarine melt at a Greenland tidewater glacier. Geophys. Res. Lett. 42 (2015), pp. 9328-9366. doi: 10.1002/2015GL065806.

[10] D. A. Slater et al. Effect of near-terminus subglacial hydrology on tidewater glacier submarine melt rates. Geophysical Research Letters 42.8 (2015), pp. 2861-2868. doi: 10.1002/2014GL062494.

[11] D. Carroll et al. The impact of glacier geometry on meltwater plume structure and submarine melt in Greenland fjords. Geophysical Research Letters 43.18 (2016), pp. 9739-9748. doi: 10.1002/2016GL070170.

[12] D. Slater et al. Spatially distributed runoff at the grounding line of a large Green-andic tidewater glacier inferred from plume modelling. Journal of Glaciology 63.238 (2017), pp. 309-323. doi; 10.1017/jog.2016.139. 
[13] D. V. Hansen and M. Rattray. New dimensions in estuary classification. Limnology and Oceanography 11.3 (1966), pp. 319-326. doi 10.4319/lo.1966.11.3.0319.

[14] J. B. Schijf and J. C. Schonfeld. Theoretical considerations on the motion of salt and fresh water.Proceedings Minnesota International Hydraulics Convention, 1953.

[15] C. R. Meyer and P. F. Linden. Stratified shear flow: experiments in an inclined duct. Journal of Fluid Mechanics 753 (2014), pp. 242-253. doi: 10.1017/jfm.2014.358.

[16] A. Lefauve et al. Three-dimensional, time-resolved velocity and density measure-ments of the stratified shear flow in an inclined duct. VIIIth International Symposium on Stratified Flows (2016).

[17] W. Geyer and D. Ralston. The dynamics of strongly stratified estuaries. Treat ise on Estuarine and Coastal Science. Elsevier, 2011. Chap. 2, pp. 37-51. doi: 10. 1016/ B978-0-12-374711-2.00206-0.

[18] L. F. Moody. Friction factors for pipe flow. Trans ASME 66 (1944), pp. 671-684.

[19] N. H. Chen. An explicit equation for friction factor in pipe. Industrial \& Engineering Chemistry Fundamentals 18.3 (1979), pp. 296-297. doi: 10.1021/i160071a019.

[20] B. J. McKeon et al. Friction factors for smooth pipe flow. Journal of Fluid Mechanics 511 (2004), pp. 41-44. doi 10.1017/S0022112004009796.

[21] R. H. Jackson et al. Near-glacier surveying of a subglacial discharge plume: Implications for plume parameterizations. Geophysical Research Letters 44.13 (2017), pp. 6886-6894. doi: 10.1002/2017GL073602. 


\title{
Turbid Tales: \\ Where do Sub-glacial Sediments Go?
}

\author{
Madelaine Gamble Rosevear
}

October 12, 2018

\section{Introduction}

\subsection{Motivation}

At high latitudes, especially around the Antarctic and Greenlandic coastlines, many glaciers terminate in the ocean. Depending on their behaviour sea-ward of the grounding line, these glaciers terminate as either tidewater glaciers, with steep faces, or as ice shelves, with large floating tongues of ice. Water may be present at the bed of a glacier due to in situ basal melting, especially if the ice is very thick, or due to surface melt, which can travel to the bed through englacial conduits.

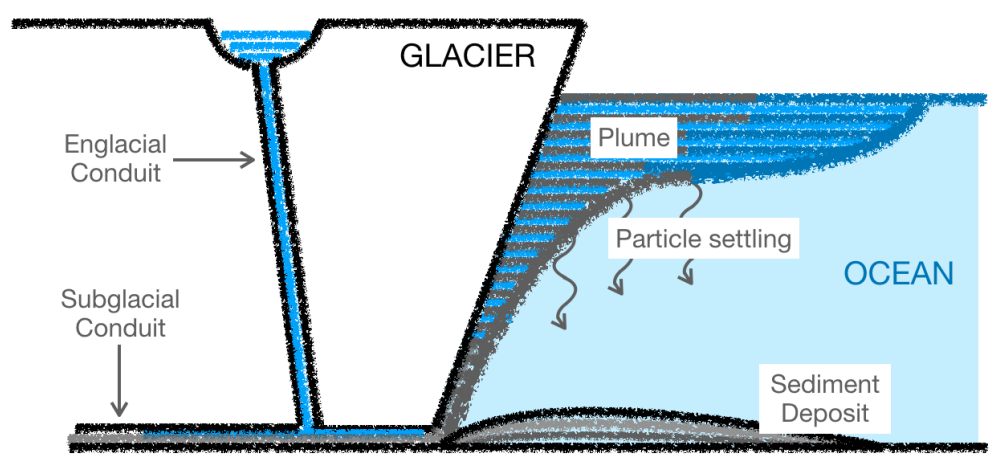

Figure 1: Simple schematic of a glacier terminating in the ocean. Meltwater drains to the bed and enters the ocean at depth, rising to the surface of the ocean as a buoyant plume. Sediments rain out of the plume as it spreads and are deposited on the sea floor.

A considerable amount of glacially eroded sediment is present at the bed, which is entrained into the basal meltwater and transported to the glacier front. The turbid mix of freshwater and sediment that emerges from under the glacier, through one or many channels, is typically less dense than the seawater and rises along the glacier front as a buoyant plume. It may then arrive at the surface (e.g. Fig. 4) or find a level of neutral buoyancy below the surface as its buoyancy evolves with the entrainment of saltwater, the settling of particles, and the addition meltwater from the ice face. Sediments frozen into 
the glacier front may also be released as the ice front melts, providing another source of fresh, particle laden fluid.

The velocity, temperature and salinity of a glacial meltwater plume are used in determining the ablation velocity, or rate of melting, of the glacier face. In models of glacier-ocean systems, the ablation velocity is typically parametrised as being proportional to the plume velocity [6], thus the omission of sediments in plume dynamics could have implications for accurate modelling of ablation and making projections of ice sheet mass balance and sea level. Sedimentary records from fjords and beneath ice shelves contain information about ice sheet history [9], however detailed interpretation of these records requires a model linking sediment deposition to to discharge conditions at the grounding line.

\subsection{Previous studies}

The largest body of relevant literature pertains to turbidity currents, or particle laden gravity currents, which have been studied for their numerous environmental and industrial applications, for example sediment laden rivers entering the ocean, submarine landslides, pyroclastic flows and avalanches. In these instances it is the particles that give the fluid negative buoyancy and drive flow. As the flow evolves, entrainment of ambient fluid and settling of particles will modify the buoyancy of the flow, or remove it alltogether.

Many such studies have considered a particle driven gravity current over a flat bottom $[2,11]$ however some some have investigated the effect of an inclined bottom $[3,10]$ and stratification [10], looking at the evolution of gravity current density due to the competing effects of particle settling and the entrainment of ambient fluid and the eventual separation of the current from the slope and intrusion into the ambient. Other studies have looked at the patterns of sediment deposited from particle laden flows such as gravity currents [3] and particle bearing plumes rising vertically in homogeneous [5] and stratified [13] ambients.

One study has developed a model of sedimentation due to subglacial discharge from beneath a vertical ice face [7]. They considered an outflow with sufficient initial momentum that the plume never attached to the ice face; the initial jet like behaviour of the flow carries it away from the ice before buoyancy takes over and the behaviour becomes closer to that of a pure plume. Once the current hits the surface it flows radially as a surface gravity current.

\subsection{Overview}

The case in which a buoyant, particle bearing plume flows up an inclined ceiling has not been previously studied. Whilst one previous study has investigated sediment deposition due to subglacial discharge [7] they considered distinctly different conditions to those investigated in this study, and have not performed laboratory experiments. Sediment deposition has been measured in some particle laden flows [5, 13], however it has never been studied in this configuration. As such, this study addresses a big gap understanding the effects of slope and particle properties on sediment distribution beneath a sloping "ice" face.

The remainder of the report is arranged as follows. In section 2 we outline the theory developed to explain the observed particle distribution. We then describe the experimental methods used (section 3). In section 4 we show the dependence of particle distribution on the key physical parameters of the study, and apply the theory outlined in section 2 . 
Finally, we discuss the key results and considerations in a geophysical setting (section 5), and outline the conclusions of the study (section 6).

\section{Theory}

Figure 1 illustrates the key processes thought to be taking place in the transport and deposition of sediments by a forced line plume rising beneath a model ice shelf in our experiments. The particle laden plume is a mix of fresh water and particles of diameter $d_{p}$, density $\rho_{p}$ and concentration (by volume) $\phi_{0}$. It enters the domain as a forced line plume, assumed to be uniform in the spanwise direction, characterised by $Q_{0}$, the volume injected per unit time, per unit width of the domain. In all experiments, the density of the injected fluid is less than the density of the saltwater ambient $\rho_{a}$. The line plume enters the domain at a height between $\mathrm{z}=h_{0}$ and $H_{0}$. The domain has a trapezoidal cross-section, with a horizontal lower boundary at $z=0$ and a sloping upper boundary at $z=H(x)=H_{0}+s x$, in which $s=\tan (\theta)$ is the slope corresponding to the upper boundary forming an angle $\theta$ to the horizontal. The forced plume enters the domain at $x=0$ and interacts with the upper and lower boundaries of the tank, forming a recirculating region, which we assume extends to horizontal distance $x_{r}$. From this region a particle-bearing plume emerges, which rises along the upper slope. The plume propagates along the slope, entraining the underlying fluid at a rate proportional to the plume speed, while particles settle within the plume at a rate proportional to the Stokes settling velocity. As particles within the plume rain out from its base, they are advected back towards the source by a return flow that results from entrainment of ambient fluid into the recirculating region. Some of these particles rain out onto the bottom while some are carried into the recirculating region where they are recycled into the plume or settle out. In the following sections we consider these dynamics, assuming

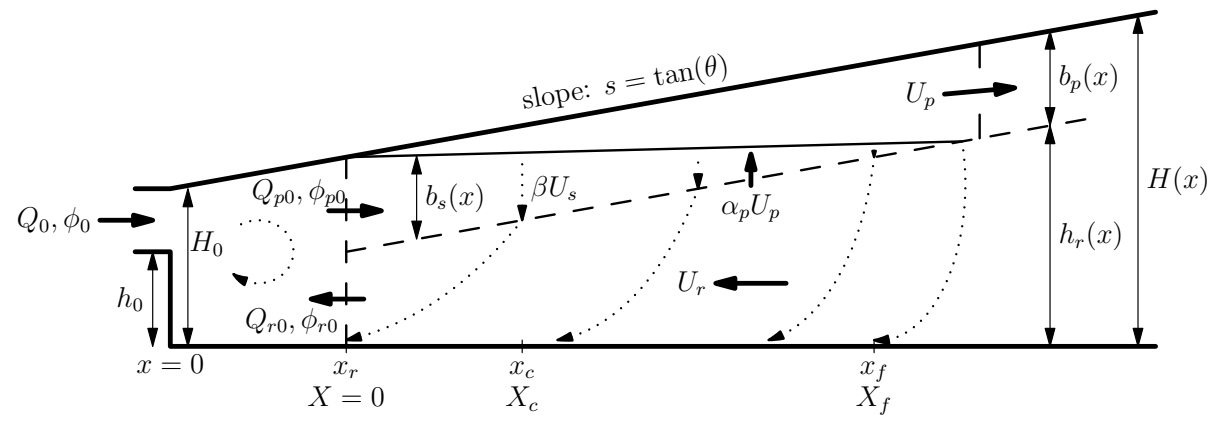

Figure 2: Schematic of the experimental domain with variables defined.

that the system is in steady state. Because the experiments have limited duration, we also consider the effect of particles remaining in the plume after the source has been turned off, and make the assumption that these particles settle vertically onto the bottom of the tank. The mass of settled particles is related to the depth of the particle bed by assuming they settle as loosely packed spheres. 


\subsection{Recirculating region}

The volume flux $Q_{p 0}$ and the reduced gravity $g_{0}^{\prime}=g\left(\rho_{a}-\rho_{p 0}\right) / \rho_{0}$ set the momentum $M_{0}$ and buoyancy $F_{0}$ fluxes, per unit width, at the source:

$$
M_{0} \equiv Q_{0}^{2} / b_{0}, \quad F_{0} \equiv Q_{0} g_{0}^{\prime}
$$

where $b_{0}=H_{0}-h_{0}$ is the vertical extent of the fluid at the source and $\rho_{p 0}$ is the density of the plume. The distance over which our forced plume is expected to behave like a jet is found by dimensional analysis of (1), and we take the length of the recirculating region to be twice this:

$$
x_{r}=2\left(M_{0}^{3} F_{0}^{2}\right)^{1 / 3}
$$

The plume emerges from the recirculating region at $x=x_{r}$ with a volume flux per unit width of $Q_{p 0}$ and a particle concentration $\phi_{p 0}$. These are both determined in part by the properties of the return flow, namely the return flow volume flux $Q_{r 0}$ and return flow particle concentration $\phi_{r 0}$. The corresponding mass fluxes, per unit width per unit time, of particles from the source, into the plume, and from the return flow are

$$
\dot{m}_{0}=\rho_{p} \phi_{0} Q_{0}, \quad \dot{m}_{p 0}=\rho_{p} \phi_{p 0} Q_{p 0}, \quad \dot{m}_{r 0}=\rho_{p} \phi_{r 0} Q_{r 0}
$$

Within the recirculating region a fraction $\Delta$ of particles settle to the base per unit time and are not resuspended. Thus the mass of particles entering the plume is

$$
\dot{m}_{p 0}=(1-\Delta)\left(\dot{m}_{0}+\dot{m}_{r 0}\right) .
$$

Conservation of volume requires that the volume flux into the plume is balanced by the sum of the volume flux into the domain and the return volume flux

$$
Q_{p 0}=Q_{0}-Q_{r 0}
$$

Combining (3), (4) and (5) gives an expression for the particle concentration in the recirculating region

$$
\dot{\phi_{p} 0}=(1-\Delta)\left(\phi_{0} Q_{0}+\phi_{r 0} Q_{r 0}\right) /\left(Q_{0}+Q_{r 0}\right) .
$$

For which estimates of $Q_{r 0}$ and $\phi_{r 0}$ are needed. We assume the former is proportional to $Q_{0}$ such that

$$
Q_{r 0}=\alpha_{r} Q_{0} .
$$

To find $\phi_{r 0}$ we consider how particles that settle out of the plume are advected back by the return flow. These dynamics are considered in the following section. 


\subsection{Sediment transport in the along-slope plume}

We find that the plume propagates along the slope with near-constant speed $U_{p}$, a result that is consistent with [4]. $U_{p}$ is given by

$$
U_{p}=c\left(g_{0}^{\prime} Q_{0}\right)^{1 / 3}
$$

In our experiments, we find that turbulent entrainment into the plume is negligibly small. As such, we ignore the effects of this on particle dynamics. In the absence of entrainment, the volume flux and vertical thickness of the plume are constant along its length and

$$
Q_{p}=Q_{p 0}, \quad b_{p}=b_{p 0}
$$

in which we assume the thickness of the plume emerging from the recirculating region is equal to half the depth of the domain at $x=x_{r}$ :

$$
b_{p 0}=0.5\left(H_{0}+s x_{r}\right) .
$$

Particles were not observed to stay well mixed within the plume as it propagated along the slope. As a result, particles are assumed to settle within the plume at a rate proportional to the stokes settling velocity $U_{s}$

$$
U_{s}=\frac{d^{2}\left(\rho_{p}-\rho_{p 0}\right)}{18 \nu \rho_{p 0}}
$$

Thus, whilst the plume has constant vertical extent $b_{p}=b_{p 0}$, the thickness of the layer within the plume that contains particles decreases along-slope according to

$$
b_{s}=b_{p}-\left(\beta U_{s} / U_{p x}\right) X=b_{p} 0-\beta \gamma X,
$$

in which $X=x-x_{r}$ is the horizontal distance from the recirculating region, $U_{p x}=U_{p} \cos \theta$ is the $x$ projection of the alongslope plume velocity and $0 \leq \beta \leq 1$ is the proportionality constant which is a measure of the turbulent activity within the plume, which determines the capacity for the flow to keep the particles well mixed. If the flow is laminar, we would expect $\beta=1$, corresponding to the particles settling at their stokes settling velocity, whereas if the flow were strongly turbulent we would expect $\beta=0$, corresponding to them remaining well mixed within the plume. The introduction of this parameter allows for the possibility that all the particles will rain out of the flow in finite distance $X_{p f}=b_{p 0} /$ $(\beta \gamma)$ if $\beta>0$. In (12) we have defined $\gamma \equiv U_{s} / U_{p x}$ by analogy with the parameter $\gamma$ defined in $[10]$.

Assuming the concentration of particles is uniform over the thickness $b_{s}$, the mass flux of particles along the plume is found through consideration of the loss of particles $d \dot{m}_{p}$ that rain out from the base of the plume over a distance $d X[11,10]$ :

$$
d \dot{m}_{p}=-U_{s}\left(\dot{m}_{p} / b_{p}\right)\left(d X / U_{p x}\right)
$$

Hence

$$
\dot{m}_{p}=\dot{m}_{p 0}\left[1-\beta \gamma \frac{X}{b_{p 0}}\right]^{1 / \beta}
$$


In the case of well-mixed particles within the plume, so that $\beta \rightarrow 0,(14)$ reduces to the exponential expression $\dot{m}_{p}=\dot{m}_{p 0} \exp \left(-\gamma X / b_{p 0}\right)$, while for the laminar $\beta=1$ case we would obtain a linear relationship: $\dot{m}_{p}=\dot{m}_{p 0}\left(1-\gamma X / b_{p 0}\right)$. The concentration of particles in the plume is given by (9)a and (14):

$$
\phi_{p}=\dot{m}_{p} /\left(\rho_{p} Q_{p}\right)
$$
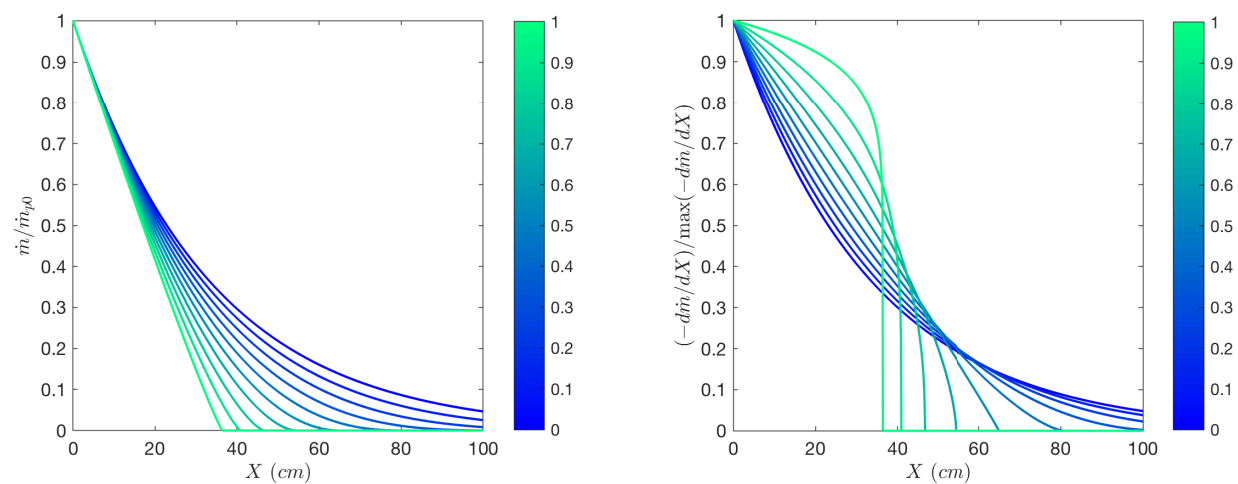

Figure 3: Normalised mass flux profile within the plume, varying beta. For $p_{d}=64$ $\mu \mathrm{m}, \phi_{0}=0.01, Q_{0}=50 \mathrm{~cm}^{3} / \mathrm{s}$.

The height of the sediment bed due to mass lost from the plume is given by:

$$
h=\frac{-T}{\rho_{p} C} \frac{d \dot{m}_{p}}{d X}
$$

where $C$ is the particle packing fraction and $T$ is the total duration of the experiment.

\subsection{Sediment settling in return flow}

Now we have an expression for the mass flux of particles leaving the plume, we consider how they are advected in the underlying ambient before either settling at the base or being re-entrained within the recirculating region. As we have already noted, there is some initial entrainment in the recirculating region with a volume flux given by (4), but negligible entrainment into the plume beyond $x=x_{r}$. Thus, to conserve volume for $x \geq x_{r}$ we require a return flow

$$
U_{r}=Q_{r} / h_{r}=\frac{U_{r 0}}{1+s X / h_{r 0}}
$$

in which $U_{r 0}=Q_{r 0} / h_{r 0}$, and, from (10), $h_{r 0}=H_{0}+s x_{r}-b_{p 0}=0.5\left(H_{0}+s x_{r}\right)$. The path followed by a particle exiting the plume and setting in this flow is given by

$$
\frac{d X}{d t}=-U_{r}, \quad \frac{d Z}{d t}=-U_{s},
$$


Using (17) we obtain an implicit formula for the displacement $\left(\delta_{x}, \delta_{z}\right)$ of a particle originating at $\left(X, h_{r}(X) \equiv h_{r 0}+s X\right)$ :

$$
\delta_{x}^{2} \frac{s}{2 h_{r 0}}+\delta_{x}\left(1+\frac{s}{h_{r 0}} X\right)-\frac{U_{r 0}}{U_{s}} \delta_{z}=0
$$

where of particular interest is the critical distance $X_{c}$ for which all particles that leave the plume for $0 \leq X \leq X_{c}$ pass $X=0$ before touching the bottom of the tank, and are thus entrained back into the recirculating region. Setting $X=X_{c}, \delta_{x}=-X_{c}$ and $\delta_{z}=-h_{r}(X)$ in (19) gives a quadratic expression for for $X_{c}$ :

$$
-X_{c}^{2} \frac{s}{2 h_{r 0}}+X_{c}\left(\frac{U_{r 0}}{U_{s}} s-1\right)+\frac{U_{r 0}}{U_{s}} h_{r 0}=0
$$

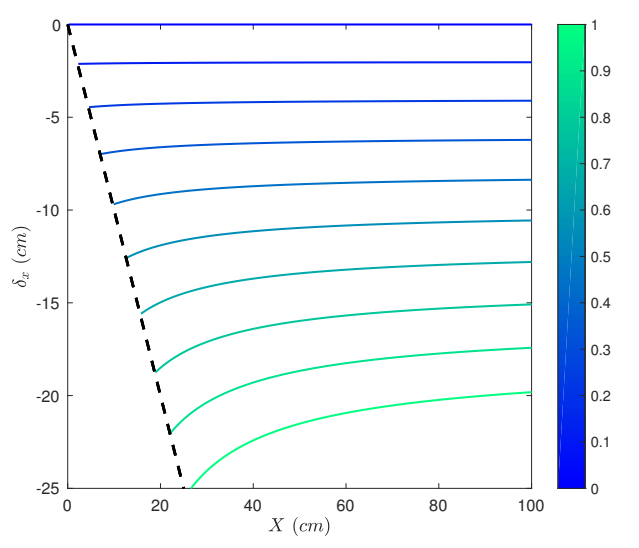

Figure 4: Displacement $\delta_{x}(X)$ due to particle advection in the return flow. Colour-scale indicates entrained volume (as a fraction of input volume flux $Q_{0}$ ) in the recirculating region, a proxy for return flow strength. Critical displacement $\delta_{x}=-X_{c}$ is denoted by the black dashed line. Calculated for $p_{d}=64 \mu \mathrm{m}, \phi_{0}=0.01, Q_{0}=50 \mathrm{~cm}^{3} / \mathrm{s}$.

\subsection{Sediment recycling}

If a particle is lost from the flow at $X \leq X_{c}$, it will return to the recirculating region before being deposited at the base of the tank. The difference in plume particle mass flux at $X=X_{c}$ and $X=0$ gives total mass of particles advected back to the recirculating region:

$$
m_{r 0}=m_{p 0}-m_{p}\left(X_{c}\right)=m_{p 0}\left(1-\left[1-\beta \gamma \frac{X}{b_{p} 0}\right]^{1 / \beta}\right)
$$

We assume that a fraction $\Delta$ is deposited within the recirculating region, and $(1-\Delta) m_{r 0}$ is added to the plume mass flux. This may be solved iteratively until the mass of particles sedimented out is equal to the mass flux into the plume. 


\section{Methods}

\subsection{Apparatus}

The experimental setup is shown in Figs. 5 and 6. The main experimental tank is rectangular with width $W=5 \mathrm{~cm}$, length $L=121 \mathrm{~cm}$ and height $H=18.5 \mathrm{~cm}$. At one end, a row of inlet pipes create an effective line source over the full width of the tank. The source is fed by a bucket of (usually fresh) particle-laden solution, which is kept well mixed with the use of a mechanical stirrer attached to a smart motor. A clear perspex slope is inserted above the source with the desired angle, creating a sloping ceiling up which the plume travels. An electroluminescent light sheet is placed below the tank, covering almost the full length, and a camera is mounted above. A camera is also mounted from the side to track the speed of the current and record the qualitative evolution of the flow.

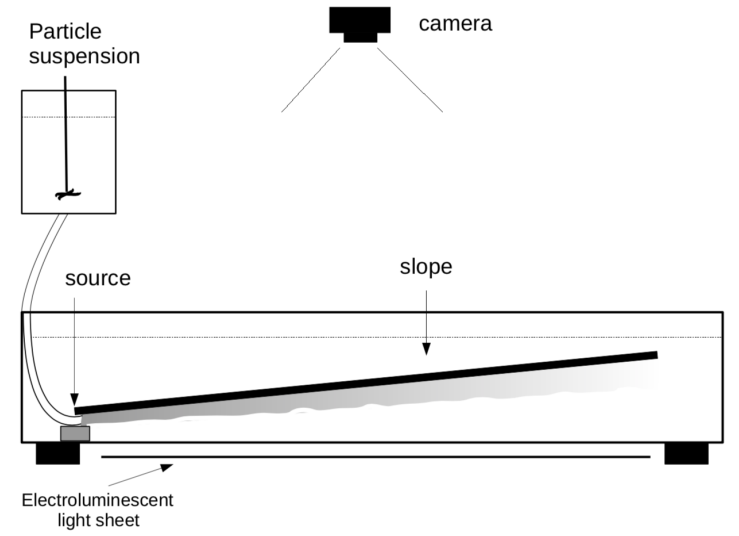

Figure 5: Side view of the experimental apparatus

\subsection{Light attenuation technique and calibration}

A non-intrusive method known as the light attenuation technique uses photographs taken before and after the experiment to measure the thickness of the particle bed. The experimental tank is illuminated from below using an electroluminescent light sheet, which provides a homogeneous light source over the majority of the tank area. The tank is photographed from above before and after each experiment. The light intensity $I$ is related to the particle bed depth $h$ by

$$
I=I_{0}+\left(I_{0}-I_{b}\right) e^{-h / \sigma_{h l}}
$$

where $I_{0}$ is the light intensity with no particles, $I_{b}$ is effective "black" and $\sigma_{b l}$ is the e-folding depth, which is measured. A calibration tank is also placed on the light sheet. Within the tank, a ramp of particles is created with a known bed depth, which is used to calculate $\sigma_{b l}$ (Fig. 7). 


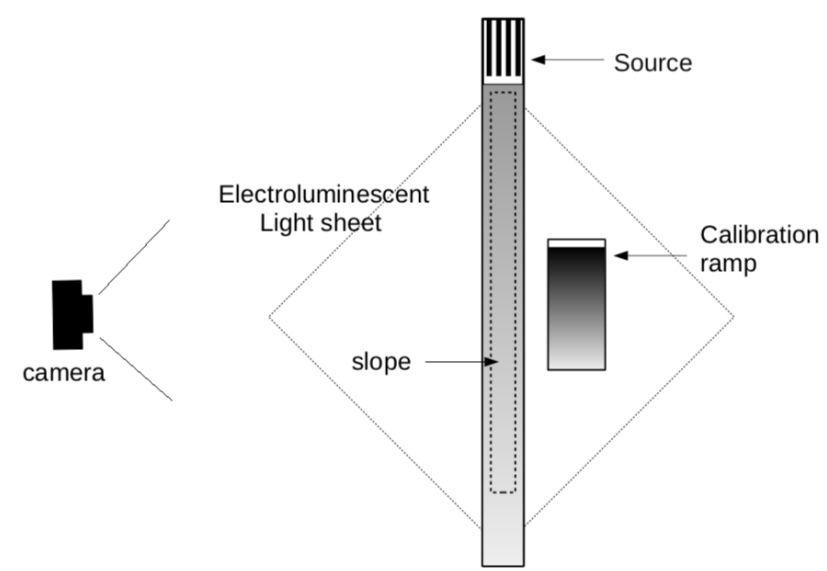

Figure 6: Top view of the experimental aparratus

\subsection{Experimental procedure}

The tank was first filled with room temperature seawater. The source pipes were flushed with fresh water, and put into place in the tank. The whole tank volume was then thoroughly mixed and the tank density $\rho_{t}$ and free surface height $h_{t}$ recorded. Next, freshwater of volume $V_{r}$ and density $\rho_{r}$ was added to the source reservoir, along with mass of particles $m_{p}$, and the solution thoroughly mixed. The first photo of the tank was then taken from above, and the slope inserted into the tank at angle $\theta$. Recording of the experiment was commenced, from above and from the side, and the source was turned on to begin the experiment. Each experiment was run for a time $T$, typically limited by the available volume of the tank which filled over the course of each experiment, and then the source was turned off. Once the particles had settled to the base of the tank, the slope was removed and the final image taken from the above.

\subsection{Experimental parameters}

The key experimental parameters investigated were particle diameter, input particle concentration and slope angle. Particle diameter $p_{d}$ was varied by using different particle size classes: $38-53 \mu \mathrm{m} ; 53-75 \mu \mathrm{m}$ and $63-90 \mu \mathrm{m}$, which will hereafter be reported using the mid-points of the ranges $(45,64$ and $76 \mu \mathrm{m})$. Particle concentration $\phi_{0}$ was set to values of 1,2 and $3 \%$ by varying the mass of particles added to the plume mix, and the slope angle $\theta$ was varied over the range $2 \leq \theta \leq 10^{\circ}$ by manually adjusting the slope insert into the tank. 


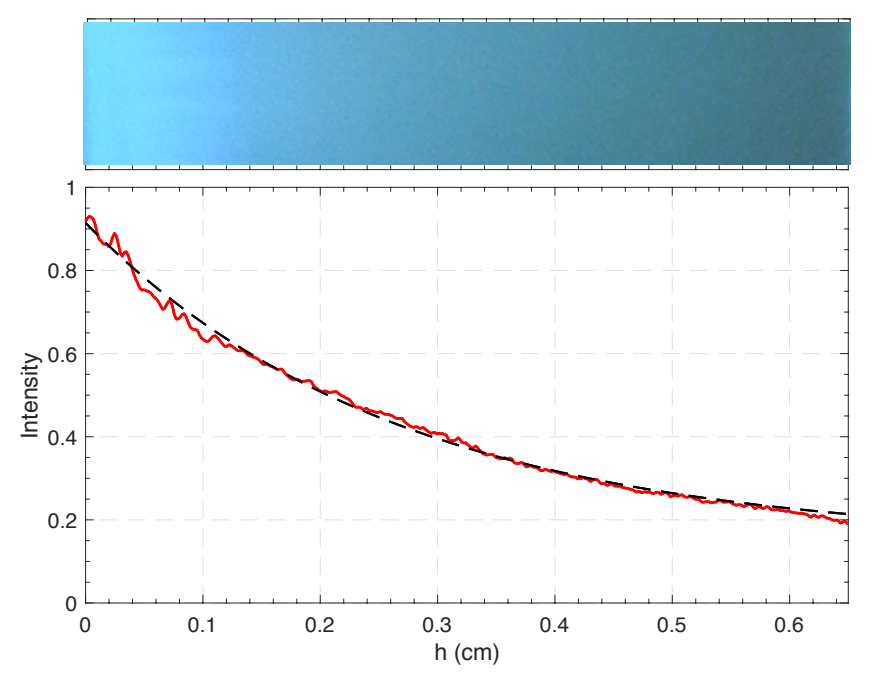

Figure 7: (upper panel) Image of the calibration tank used to calculate intensity for known particle depth. The pixels are averaged in the $y$ axis to obtain the depthintensity relation-ship. (lower panel) Depth-intensity calibration data (red) and fit using (22) (black dashed) for $45 \mu \mathrm{m}$ particles.

\section{Results}

\subsection{Velocity}
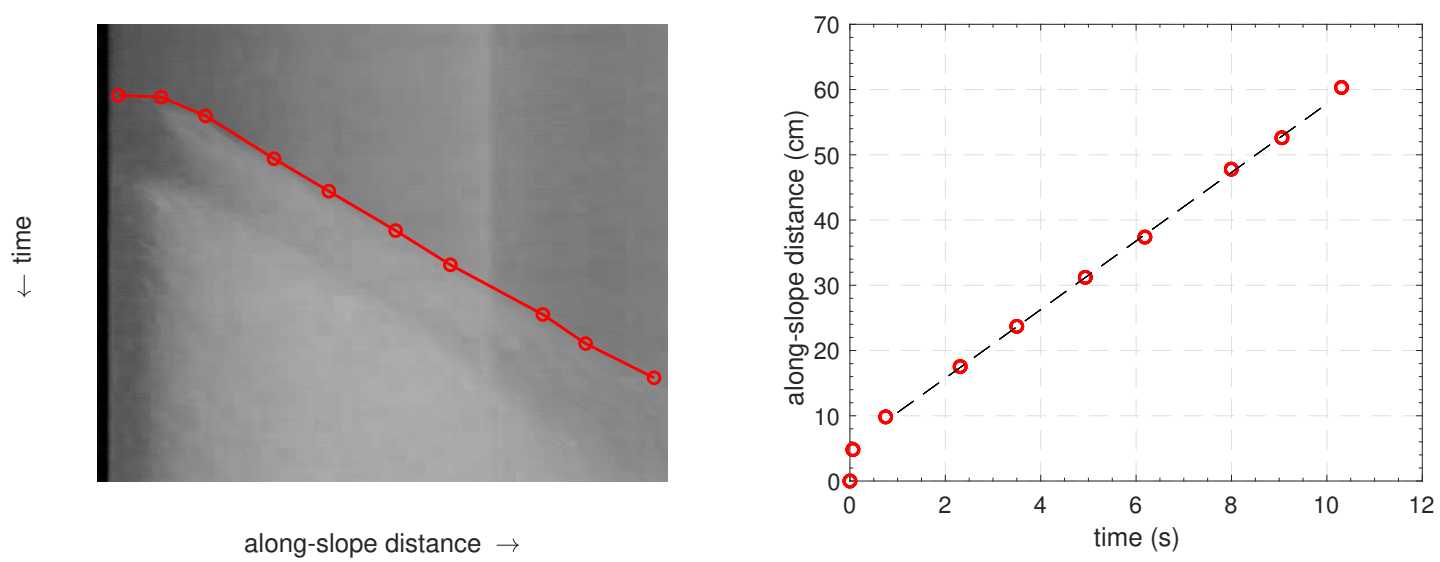

Figure 8: (left) Stacked along-slope segment of pixels from sequential images with front position picked out (red markers). (right) The same data with linear fit $U_{p}$.

The plume front position was measured by taking an along-slope slice through through sequential images of the side view of the tank during the initial phase of the experiment. These slices were stacked, giving a hovmoller-type view of the front position in time and space (Fig. 8a), and used to determine the steady state current speed $U_{p}$ (Fig. 8b). After an initial adjustment period we found that the displacement was well described by a constant 


\begin{tabular}{lcccccccc}
\hline Exp. & $p_{d}(\mu \mathrm{m})$ & $\theta\left(^{\circ}\right)$ & $\phi(\%)$ & $Q_{0}\left(\mathrm{~cm}^{3} / \mathrm{s}\right)$ & $g_{0}^{\prime}\left(\mathrm{m} / \mathrm{s}^{2}\right)$ & $\left(g_{0}^{\prime} Q_{f}\right)^{1 / 3}(\mathrm{~cm} / \mathrm{s})$ & $\mathrm{T}(\mathrm{s})$ & $\tau(\mathrm{s})$ \\
\hline \hline $1^{*}$ & 45 & 6.3 & 0.01 & 52 & 8.8 & 7.7 & 34 & 18 \\
2 & 45 & 4.0 & 0.01 & 37 & 8.4 & 6.7 & 65 & 18 \\
$3^{*}$ & 45 & 3.8 & 0.01 & 33 & 9.3 & 6.7 & 59 & 18 \\
$4^{*}$ & 45 & 3.9 & 0.01 & 41 & 9.2 & 7.2 & 47 & 18 \\
$5^{*}$ & 45 & 2.0 & 0.01 & 53 & 7.8 & 7.4 & 45 & 18 \\
$6^{*}$ & 45 & 2.0 & 0.01 & 28 & 8.2 & 6.1 & 58 & 18 \\
8 & 45 & 4.3 & 0.05 & 27 & 10.2 & 6.5 & 61 & 18 \\
9 & 64 & 4.1 & 0.01 & 45 & 8.4 & 7.2 & 40 & 9 \\
10 & 64 & 6.4 & 0.01 & 29 & 7.5 & 6.0 & 54 & 9 \\
11 & 64 & 4.5 & 0.01 & 31 & 7.3 & 6.1 & 41 & 9 \\
12 & 64 & 2.0 & 0.01 & 41 & 8.7 & 7.1 & 47 & 9 \\
13 & 64 & 5.8 & 0.01 & 31 & 10.1 & 6.8 & 74 & 9 \\
14 & 76 & 4.0 & 0.01 & 51 & 8.2 & 7.5 & 45 & 6 \\
15 & 76 & 6.3 & 0.01 & 32 & 8.6 & 6.5 & 54 & 6 \\
16 & 76 & 2.1 & 0.01 & 26 & 9.2 & 6.2 & 78 & 6 \\
$19^{*}$ & 45 & 6.4 & 0.01 & 54 & 8.8 & 7.8 & 42 & 18 \\
20 & 64 & 4.0 & 0.02 & 45 & 8.0 & 7.1 & 40 & 9 \\
22 & 64 & 3.9 & 0.03 & 55 & 7.9 & 7.5 & 48 & 9 \\
$23^{*}$ & 45 & 10.3 & 0.01 & 50 & 9.0 & 7.7 & 44 & 18 \\
$24^{*}$ & 45 & 4.0 & 0.01 & 59 & 72.8 & 16.3 & 38 & 18 \\
$25^{*}$ & 45 & 4.0 & 0.01 & 33 & 4.6 & 5.3 & 59 & 18 \\
$26^{*}$ & 45 & 4.0 & 0.01 & 53 & 46.9 & 13.5 & 33 & 18 \\
28 & 64 & 4.0 & 0.03 & 56 & 10.1 & 8.3 & 40 & 9 \\
\hline
\end{tabular}

Table 1: Experiments. Asterisk denotes $\tau / T>1 / 3$ indicating results are expected to be strongly influenced by transient behaviour. 
velocity. The initial phase was interpreted as being dominated by the source momentum $M_{0}$ of the flow, and thus $U_{p}$ is given as the best fit to the data after this point.

Fig. 9 plots $U_{p}$ against the velocity scale $\left(g_{0}^{\prime} Q_{f}\right)^{1 / 3}$. Whilst a limited range of source conditions were used, the data show a linear dependence on $\left(g_{0}^{\prime} Q_{f}\right)^{1 / 3}$ with a slope of 1.5 , and do not show slope-dependence, both results of which are consistent with [4].

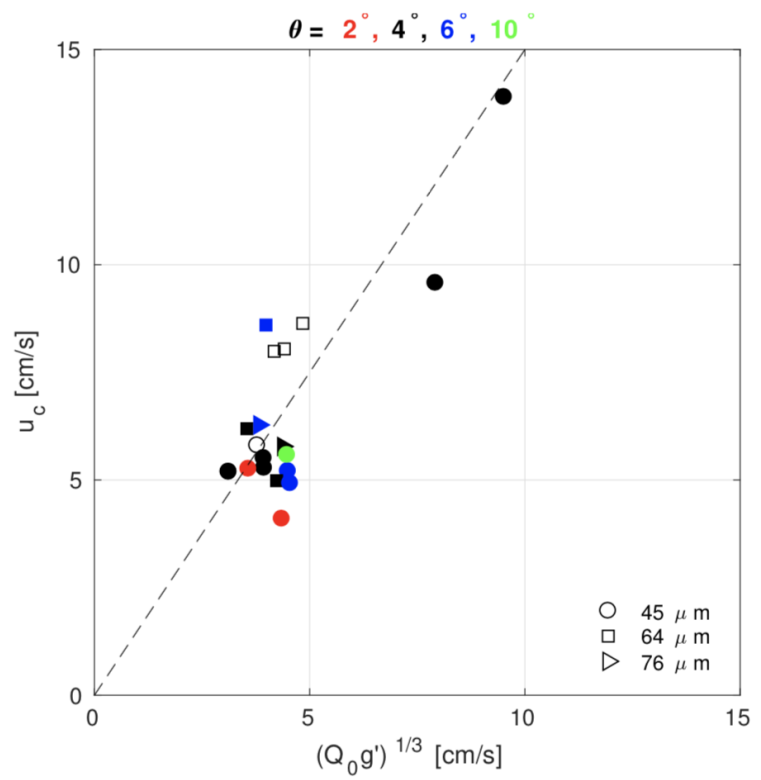

Figure 9: Along-slope front speed $U_{p}$ against velocity scale against $\left(g_{0}^{\prime} Q_{f}\right)^{1 / 3}$ for all experiments. The slope plotted $c=1.5$.

\subsection{Plume evolution}

Fig. 10 shows the temporal evolution of the startup phase of a typical experiment. Once the source is turned on, the forced plume emerges into the experimental tank which is at this point extremely confined, and mixes over the full depth. The buoyant fluid that emerges from this region, which we term the recirculating region, then propagates along-slope. For a small $p_{d}$ experiment such as that in Fig. 10, it takes some time for the particles raining out of the plume to reach the base of the tank, and at later time we observe that the descending particles are being advected back towards the source in the return flow. Finally, we note that some particles can be seen, still in suspension, at the top of the tank.

\subsection{Particle deposition}

Figure 11 illustrates the dependence of the relative particle distributions on key experimental parameters. For fixed particle size and concentration, the shape of the distribution has no observable dependence on the angle of the sloping ceiling (Fig. 11). This is consistent with equation 14, where $\theta$ appears only as a geometric relationship between along slope flow 
speed $U_{p}$ and its $x$ projection $U_{p x}$ : for the range of angles studied here $\left(2 \leq \theta \leq 10^{\circ}\right), \cos \theta$ varies by less than $1.5 \%$.

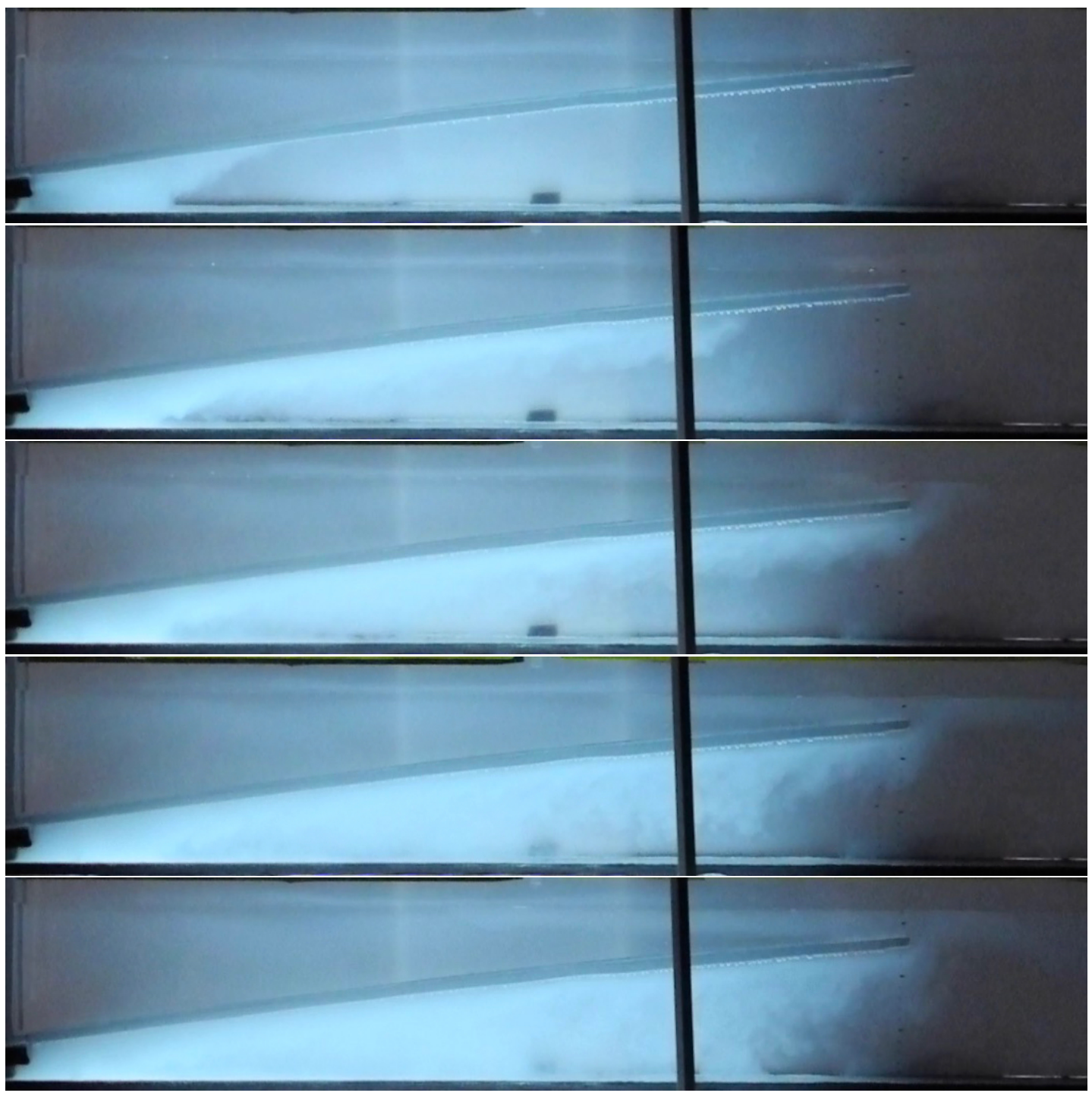

Figure 10: Sequence of images from Exp 2. Frames 1 and 2 are 8 seconds apart, and the subsequent frames are separated by 5 seconds.

The influence of particle diameter is also seen in Fig. 11 where the smaller particle size fraction has considerably shallower distribution of particles, indicating particles are remaining suspended within the flow for longer. This is consistent with expectations based on (14), where smaller particle diameter results in a slower stokes settling velocity. A key observation here is that we have a very "triangular" distribution of particles, especially for larger particle diameters. For $64 \mu \mathrm{m}$ we also observe a cutoff distance $X_{p f}$ beyond which no particles are deposited.

Finally we consider the influence of particle concentration. Few experiments were per- 


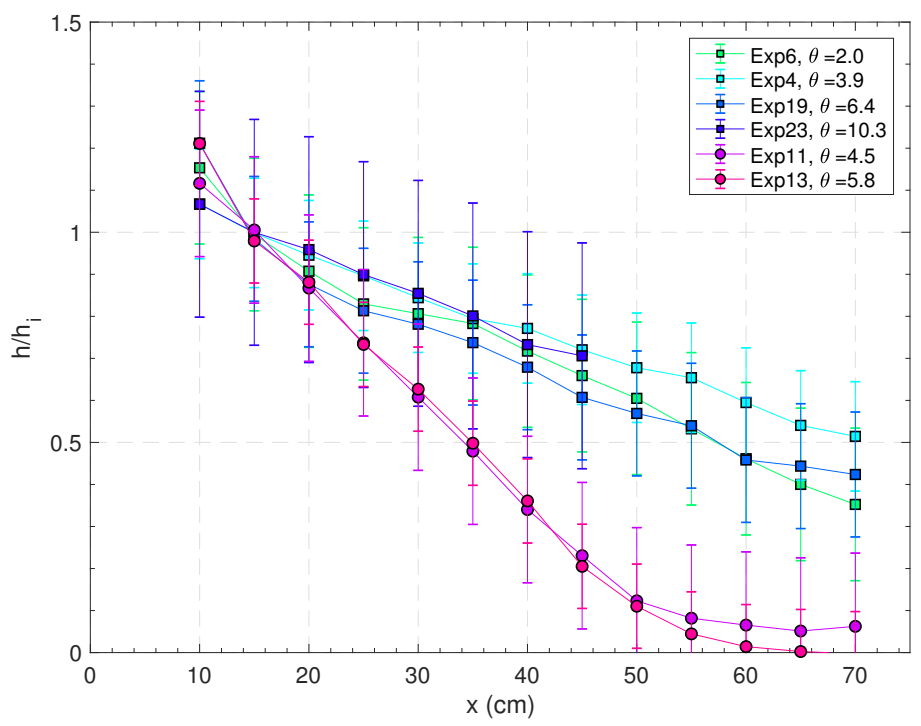

Figure 11: Normalised particle distribution for a suite of experiments varying slope angle for $\phi=0.01 \%$ and $p_{d}=45 \mu \mathrm{m}$ (squares) and $p_{d}=64 \mu$ (circles).

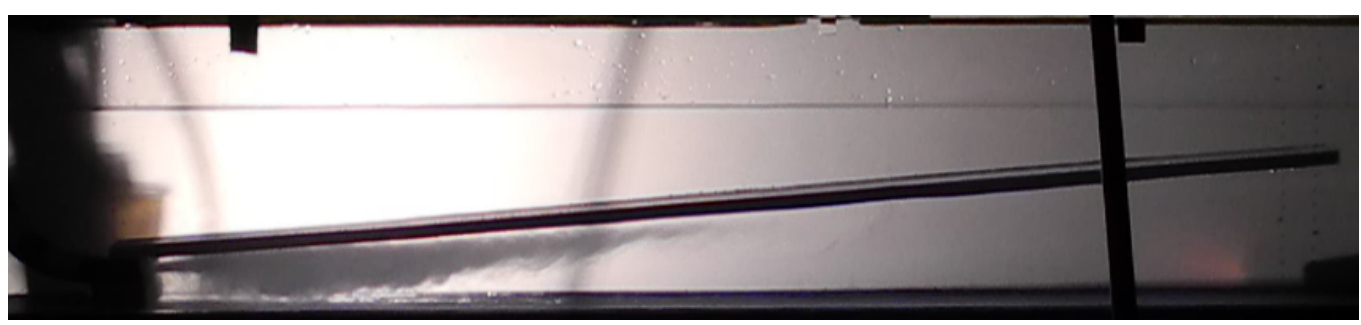

Figure 12: Snapshot of Exp. 20 showing the particle-free layer within the plume, adjacent to the slope and growing with along-slope distance.

formed at concentrations higher than $0.01 \%$ because of the experimental difficulty of keeping so many particles in suspension. Additionally, the high particle deposition rate meant that experiment length was limited by the thickness of the particle bed: too thick a bed absorbed all the source light and made the light attenuation technique impossible. From the experiments that were performed (Figure 13) we see no clear dependence of the normalised particle distribution on $\phi_{0}$.

\subsection{Application of theory}

The theory derived in section 2 applies only to the plume at steady state, thus for it to be applicable we require that the particle deposition due to the startup phase, and due to the particles remaining in the plume once the source has been turned off, is much smaller than that due to the steady portion of the experiment. To test whether or not this is true, we compare the total duration of the experiment $T$ to a timescale based on a particle settling at the stokes settling velocity though a typical plume width $\tau=b_{p 0} / U_{s}$. Table 1 shows these values and highlights the experiments, predominantly those using the smallest particles, 


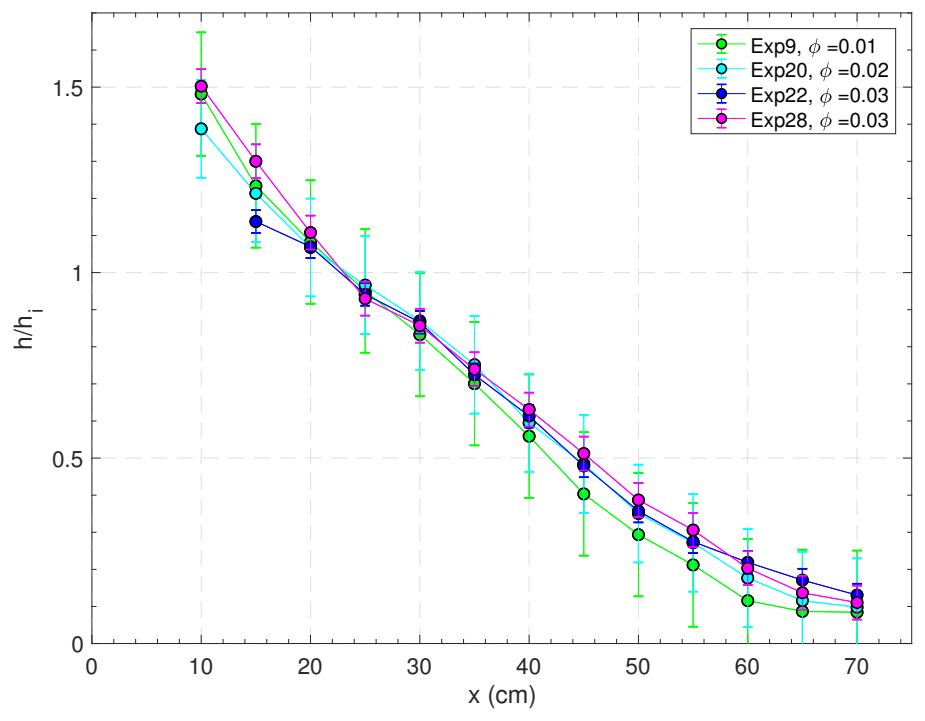

Figure 13: Normalised distributions varying concentration for $64 \mu \mathrm{m}$.

that are expected to be strongly influenced by transient behaviour.

\subsubsection{Steady state}

In order to apply the theory outlined in section 2 we first consider the experiments that are not affected by transient dynamics. There are two main unconstrained parameters in the theory. The first is $\beta$, describing how well mixed the plume is and allowing all the particles to rain out in finite distance. This is a feature of the experiments that is not consistent with a fully turbulent plume, in which the mass loss is exponential in $x$. The observed shape of the particle distribution, especially in the 64 and $76 \mu \mathrm{m}$ experiments places a constraint on the value of $\beta$ : as Fig. 3 shows, we require $\beta \sim 0.5$ to obtain a triangular shaped-distribution.

The other unconstrained quantity is the amount of fluid that the plume entrains in the recirculating region, as this sets the strength of the return flow, and therefore the displacement of particles after they rain out of the plume. This is estimated to be on the order of the plume inflow $Q_{r 0}=$ const. $Q_{p 0}$ where the constant is taken to be 1 for most experiments.

Fig. 14 compares the experimental and model particle bed thicknesses, where the model is applied with and without the advection due to the return flow. The inclusion of the return flow allows the steepness of the particle bed thickness to be explained and greatly improves the model fit to the data. Fig. 15 applies the model to three different $64 \mu \mathrm{m}$ experiments, reproducing the observed distributions extremely closely.

For the $76 \mu \mathrm{m}$ experiments, the model does not reproduce the data closely (Fig. 16). Using $p_{d}=76 \mu \mathrm{m}$, the model produces a particle bed that is far too steep. Fig. 16 shows the expected distributions for the upper and lower bounds of the particle size class: $63-90 \mu \mathrm{m}$. The stokes settling velocity scales with the square of the particle diameter (11), thus the ratio between the settling velocity of the $90 \mu \mathrm{m}$ particles to the $63-90 \mu \mathrm{m}$ particles within 


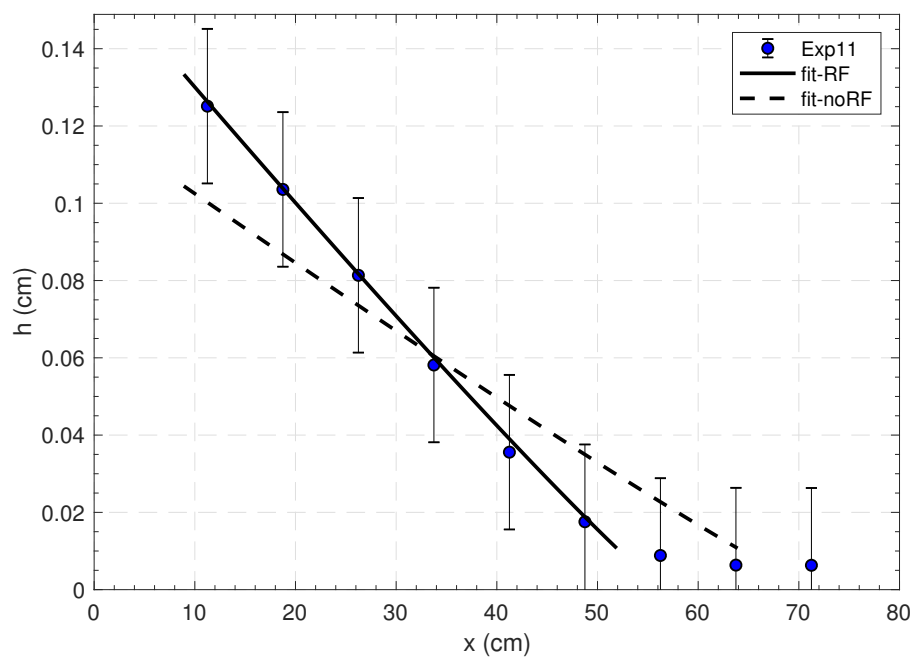

Figure 14: Dimensional particle distribution for experiment 11 and modelled distribution with and without the return flow. For $\beta=0.55, Q_{E}=0.8 Q_{0}$.

the same size class is given by $(90 / 63)^{2} \sim 2$. The inability of the model to reproduce the distribution using $p_{d}=76 \mu \mathrm{m}$ may therefore be explained by the differential settling of particles of different diameter within the same size class. This effect may be highlighted in these experiments as this size class has a larger range than either the 45 or $64 \mu \mathrm{m}$ classes.

\subsubsection{Transient}

For the experiments expected to be strongly influenced by transient behaviour, we also consider the mass of particles remaining within the plume after the source has been turned off. The mass is given by (14) and is assumed to settle directly downwards. Additionally, the steady state mass flux out of the plume is assumed to operate over a restricted interval $T-\tau$ (instead of $T$ as in previous experiments).

For all the $45 \mu \mathrm{m}$ experiments, the inclusion of the mass in the plume was extremely important to capturing the final sediment depth. Fig. 17 shows the total modelled height of the sediment, as well as the component that remained in the plume after the source was turned off. This component alone accounts for the majority of the deposited sediment. Of the sediment deposited during the experiment, it is clear that the return flow has a strong influence. The stepped feature at $x \sim 50 \mathrm{~cm}$ shows the influence of the finite tank length: the model suggests that sediments raining out of the plume at the end of the tank should be advected all the way back to $x \sim 50 \mathrm{~cm}$ before being deposited. Given the small proportion of the sediment bed conforming to the steady state dynamics, it is not possible to ascertain whether the data support this.

While good agreement between the data and the model is found in Fig. 17, the same is not true for many other $45 \mu \mathrm{m}$ experiments. In several cases the model vastly over-predicts the final sediment depth, and in the case of Exp. 6 the shape of the sediment bed is not captured. 


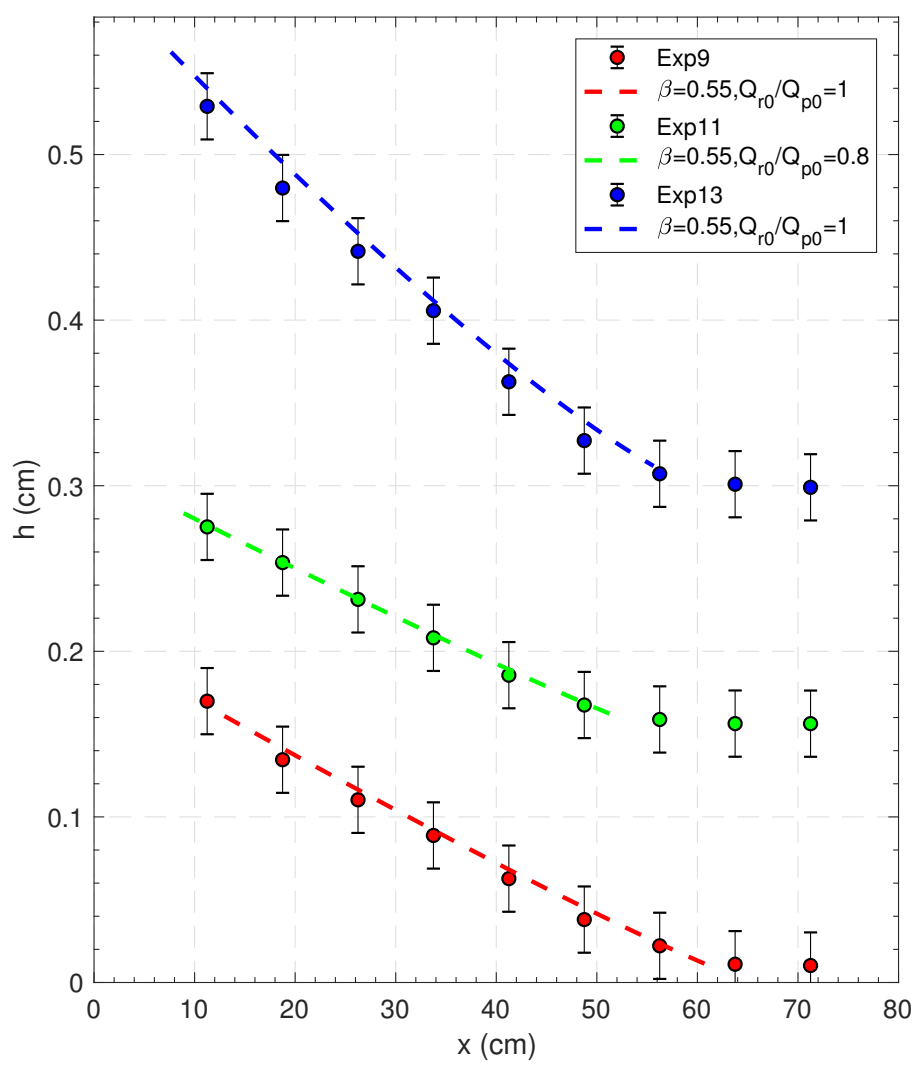

Figure 15: Dimensional particle distribution for several $64 \mu \mathrm{m}$ experiments. Each curve has been offset by $h=0.15 \mathrm{~cm}$

\section{Discussion}

\subsection{Geophysical considerations}

The inclusion of the transient phase of the experiment, i.e. mass left in the plume at final time $T$, allowed us to take steps towards modelling the particle bed depth for the smallest particle class. However, these transient dynamics are unlikely to be important in a geophysical setting, where the plume operation timescale $T$ is on the order of a month rather than $\sim 1$ minute. The fact that the mass deposition from the transient part of the experiment was so much larger than that from the steady start part made it impossible to assess the validity of the steady state theory to these $45 \mu \mathrm{m}$ experiments.

One process that we would expect to occur on geophysical scales, and that we neglected in our experiment, is entrainment into the plume. While this doesn't modify the plume velocity, it would act to increase the width of the plume and therefore modify deposition. In addition, if the plume were sufficiently angled, and entrainment sufficiently strong, particles could be re-entrained into the flow, a process that has been considered in other particle laden flows $[13,5]$.

Another such process is modification of plume buoyancy through particle deposition. We observed $U_{p}$ to be constant, thus we did not need to consider this for our experiments, 


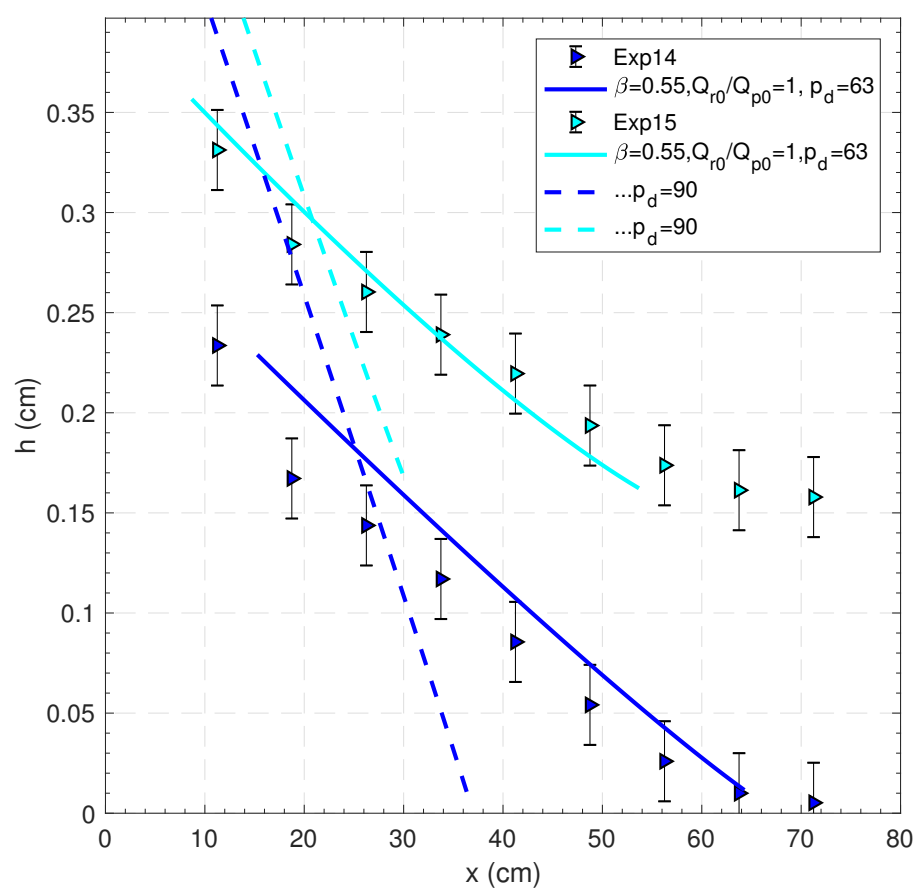

Figure 16: Dimensional particle distribution for experiment for two $76 \mu \mathrm{m}$ experiments. Each curve has been offset by $h=0.15 \mathrm{~cm}$

however in cases where $\phi_{0}$ is a large component of buoyancy and/or $\phi_{p}$ changes significantly as the plume propagates along-slope, modification of buoyancy through particle settling is expected to have a strong effect.

In these experiments our slope, or "ice" was both "non-thermodynamic" and "nonreactive", meaning it did not modify the plume properties by melting, as an ice shelf or tidewater glacier would. In situations where sub-glacial discharge is relatively large as compared to the volume flux from submarine melting, such as Greenland's tidewater glaciers in summer [12], this assumption is valid, however when melting is strong, the feedback between melting and plume buoyancy must also be considered.

Our experiments demonstrated that the return flow generated through entrainment of ambient fluid into the plume is an important process: only through including the advection of particles towards the source in the return flow were we able to capture the observed particle distribution. It is worth noting that this effect was particularly strong due to the line plume geometry that we used. In the case of a more isolated plume in a larger cavity, we expect that this effect would be smaller. However, both these cases (an isolated point source and a more distributed source) may be relevant: a recent study of Kangiata Nunata Sermia in South-west Greenland [8] inferred that a distributed source of buoyancy is the most likely configuration for this particular glacier.

Sediment wedges are commonly found at the grounding lines of both Greenlandic tidewater glaciers and Antarctic Ice Shelves, where they act to stabilise the grounding line [1]. They are thought to be the result of sub-glacially transported sediment. Our results suggest that the advection of sediments back towards the plume source by the return flow is another 


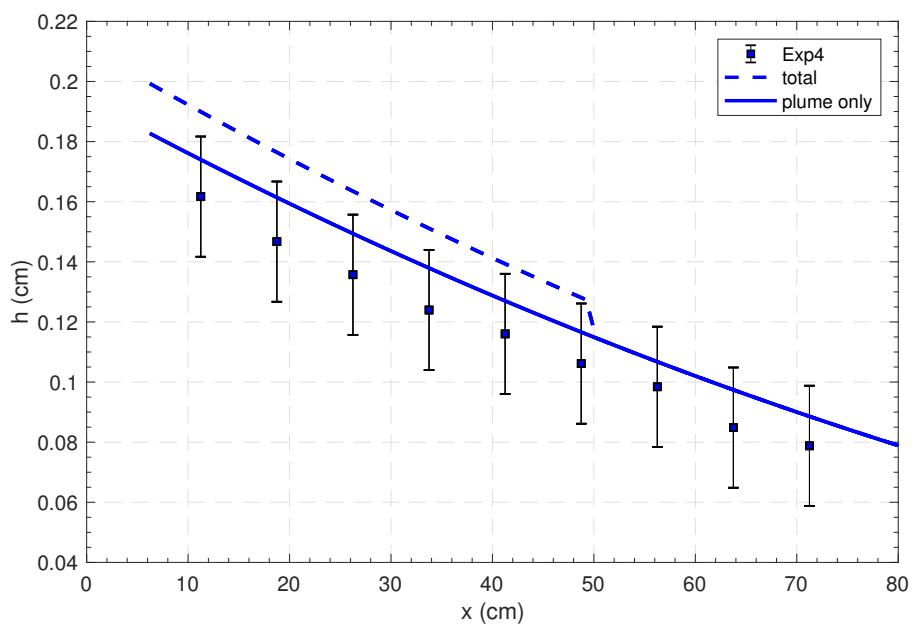

Figure 17: Dimensional particle distribution for Exp. 4 with $p_{d}=45 \mu \mathrm{m}$, showing the total modelled distribution and the contribution from the transient part of the experiment only.

possible mechanism for the creation of these wedges.

\subsection{Settling model}

A key observation from the experiments we performed was that the particles often did not remain well mixed within the plume, suggesting the flow was not sufficiently turbulent to keep them well mixed. The effect of this was accounted for using the parameter $\beta$, which allowed the model to behave either as turbulent (mixing dominated), fully laminar (settling dominated) or somewhere in between.

We can compare the relative importance of settling and mixing by comparing the timescale for settling, $T_{\text {settle }} \sim b_{p 0} / u_{s}$, and the time scale for mixing over the plume width, $T_{K} \sim b_{p 0}^{2} / K$, where $K$ is an eddy viscosity. Using the scale $K \sim u_{\tau} b_{p 0}$, for turbulent channel flow, we find the ratio of time-scales:

$$
\frac{T_{\text {settle }}}{T_{K}}=\frac{b_{p 0} / u_{s}}{b_{p 0}{ }^{2} / K}=\frac{u_{\tau}}{u_{s}}
$$

where $u_{\tau}$ is the friction velocity which may be estimated as a function of the free stream velocity (in this case plume velocity) as $u_{\tau}^{2}=C_{d} u_{p}^{2}$, where an appropriate choice of the drag coefficient $C_{d}$ for a hydraulically smooth surface is $2.5 \times 10^{-3}$. For a typical plume velocity of $6 \mathrm{~cm} / \mathrm{s}$, the friction velocity is $\sim 0.3 \mathrm{~cm} / \mathrm{s}$. For particle diameters $45-76 \mu \mathrm{m}, u_{s}$ ranges from $0.17-0.47 \mathrm{~cm} / \mathrm{s}$ yielding $T_{\text {settle }} / T_{K} \sim 1.8-0.6$. The settling and mixing timescales are therefore of the same order for all experiments, explaining the "mixed" dynamics observed. This ratio also suggests that these experiments are applicable geophysical scenarios in which either the flow is relatively slow or particle diameter, and therefore settling velocity, is large. 


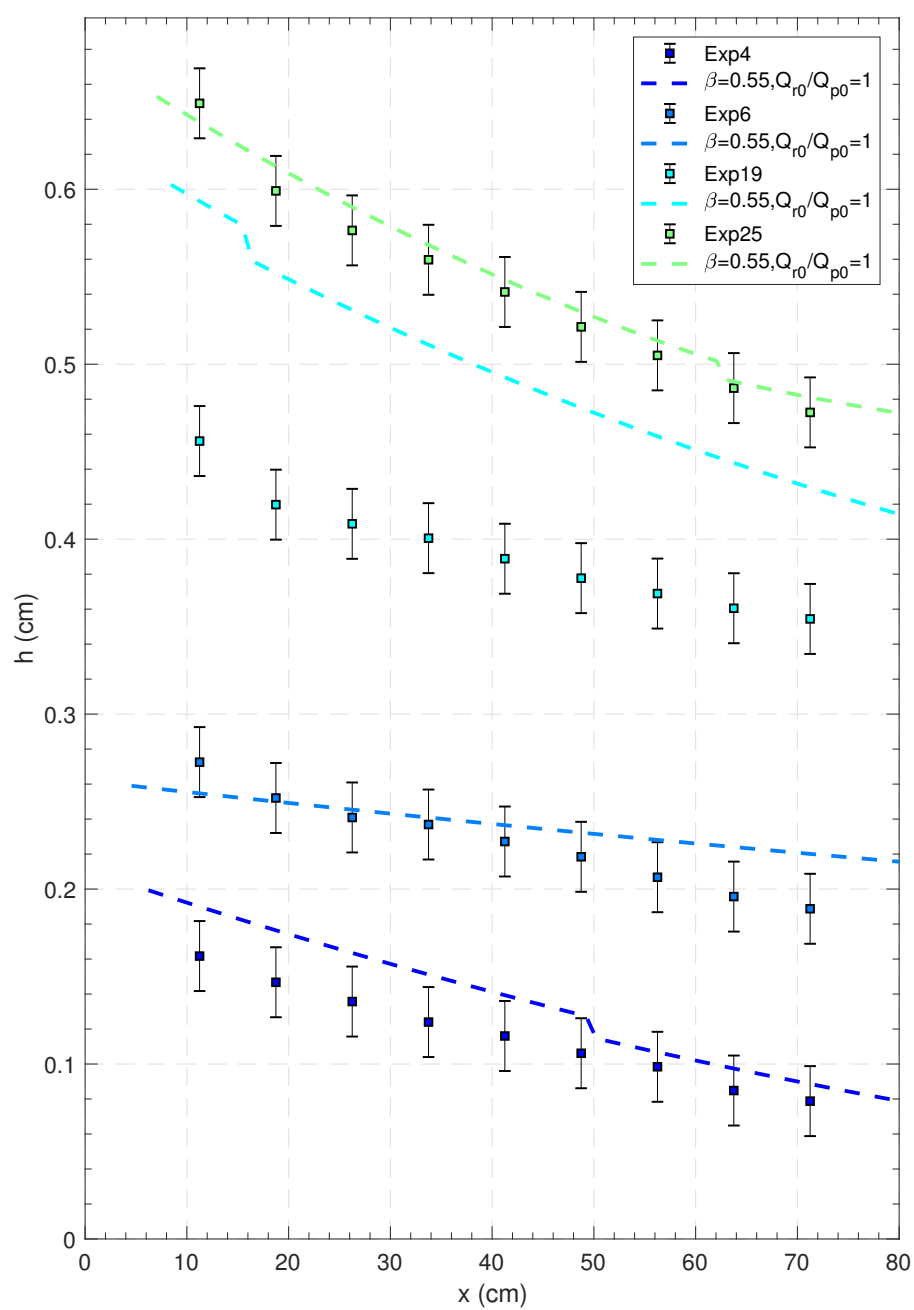

Figure 18: Dimensional particle distribution for several $45 \mu \mathrm{m}$ experiments. Each curve has been offset by $h=0.15 \mathrm{~cm}$

\section{Conclusion}

In this study we performed laboratory experiments to investigate particle laden plumes travelling along an inclined ceiling; specifically the final distribution of particles deposited beneath the plume and how this distribution depended on slope, particle concentration and particle size. For the range of slope angles studied, $2 \leq \theta \leq 10^{\circ}$, the final distribution was not observed to depend on slope. Particle size was shown to control the distribution through parameter $\gamma \equiv U_{s} / U_{p x}$, moreover in order to explain some distributions we had to consider the range of particle diameters within a given particle size-class. The return flow generated by entrainment into the plume was found to advect particles back towards the source, and the inclusion of this process was necessary to capture the final sediment distribution. 


\section{Acknowledgements}

Firstly, thank you to Bruce Sutherland, Claudia Cenedese and Craig McConnochie for sharing their time and invaluable expertise in the lab: it was a real pleasure working with you all! Thanks to Anders Jensen for his technical help and good cheer. Thanks to the lab crew for the swims and solidarity, the rest of the fellows for all the other fun. Finally, thanks to Mary-Louise Timmermans and Claudia Cenedese (this time with her director's hat on) for such an amazing opportunity.

\section{References}

[1] R. B. Alley, S. Anandakrishnan, T. K. Dupont, B. R. Parizek, and D. PolLARD, Effect of sedimentation on ice-sheet grounding-line stability, Science, 315 (2007), pp. 1838-1841.

[2] R. T. Bonnecaze, H. E. Huppert, And J. R. Lister, Particle-driven gravity currents, Journal of Fluid Mechanics, 250 (1993), pp. 339-369.

[3] R. T. Bonnecaze And J. R. Lister, Particle-driven gravity currents down planar slopes, Journal of Fluid Mechanics, 390 (1999), pp. 75-91.

[4] R. E. Britter And P. F. Linden, The motion of the front of a gravity current travelling down an incline, Journal of Fluid Mechanics, 99 (1980), pp. 531-543.

[5] G. G. Ernst, R. S. J. Sparks, S. N. Carey, and M. I. Bursik, Sedimentation from turbulent jets and plumes, Journal of Geophysical Research: Solid Earth, 101 (1996), pp. 5575-5589.

[6] A. Jenkins, A one-dimensional model of ice shelf-ocean interaction, Journal of Geophysical Research: Oceans, 96 (1991), pp. 20671-20677.

[7] R. Mugford And J. Dowdeswell, Modeling glacial meltwater plume dynamics and sedimentation in high-latitude fjords, Journal of Geophysical Research: Earth Surface, 116 (2011).

[8] D. Slater, P. Nienow, A. Sole, T. Cowton, R. Mottram, P. Langen, And D. MAIR, Spatially distributed runoff at the grounding line of a large greenlandic tidewater glacier inferred from plume modelling, Journal of Glaciology, 63 (2017), pp. 309323.

[9] J. Smith, T. J. Andersen, M. Shortt, A. Gaffney, M. Truffer, T. Stanton, R. Bindschadler, P. Dutrieux, A. Jenkins, C.-D. Hillenbrand, et al., Subice-shelf sediments record history of twentieth-century retreat of pine island glacier, Nature, 541 (2017), p. 77. 
[10] K. Snow And B. Sutherland, Particle-laden flow down a slope in uniform stratification, Journal of Fluid Mechanics, 755 (2014), pp. 251-273.

[11] R. S. J. Sparks, R. T. Bonnecaze, H. E. Huppert, J. R. Lister, M. A. HallWOrTh, H. MAder, AND J. Phillips, Sediment-laden gravity currents with reversing buoyancy, Earth and Planetary Science Letters, 114 (1993), pp. 243-257.

[12] F. Straneo and C. Cenedese, The dynamics of greenland's glacial fjords and their role in climate, Annual Review of Marine Science, 7 (2015), pp. 89-112. PMID: 25149564 .

[13] B. R. Sutherland And Y. S. D. Hong, Sedimentation from particle-bearing plumes in a stratified ambient, Physical Review Fluids, 1 (2016). 


\begin{tabular}{|l|l|l|}
\hline $\begin{array}{l}\text { REPORT } \\
\text { DOCUMENTATION PAGE }\end{array}$ & $\begin{array}{l}\text { 1. Report No. } \\
\text { WHOI-2021-05 }\end{array}$ & 3. Recipient's Accession No. \\
\hline $\begin{array}{l}\text { 4. Title and Subtitle } \\
\text { 2017 Program of Study: Ice-Ocean Interactions }\end{array}$ & $\begin{array}{l}\text { 5. Report Date } \\
\text { November } 2018\end{array}$ \\
\cline { 2 - 3 } 7. Author(s) & 6. & 8. Performing Organization Rept. No. \\
Claudia Cenedese and Mary-Louise Timmermans & 10. Project/Task/Work Unit No. \\
\hline 9. Performing Organization Name and Address & $\begin{array}{l}\text { 11. Contract(C) or Grant(G) No. } \\
\text { (C) } \\
\text { Woods Hole Oceanographic Institution }\end{array}$ \\
\hline 12. Sponsoring Organization Name and Address & 13. Type of Report \& Period Covered \\
National Science Foundation & Technical Report \\
\hline
\end{tabular}

15. Supplementary Notes

This report should be cited as: Woods Hole Oceanographic Institution technical Report, WHOI-2021-05

16. Abstract (Limit: 200 words)

The 2017 Geophysical Fluid Dynamics Summer Study Program theme was Ice-Ocean Interactions. Three principal lecturers, Andrew Fowler (Oxford), Adrian Jenkins (British Antarctic Survey) and Fiamma Straneo (WHOI/Scripps Institution of Oceanography) were our expert guides for the fi rst two weeks. Their captivating lectures covered topics ranging from the theoretical underpinnings of ice-sheet dynamics, to models and observations of ice-ocean interactions and high-latitude ocean circulation, to the role of the cryosphere in climate change. These icy topics did not end after the first two weeks. Several of the Fellows' projects related to ice-ocean dynamics and thermodynamics, and many visitors gave talks on these themes. The first ten chapters of this volume document these lectures, each prepared by pairs of the summer's GFD fellows. Following the principal lecture notes are the written reports of the fellows' own research projects.

17. Document Analysis

a. Descriptors Geophysical Fluid Dynamics

Ice-Ocean Interactions

Thermodynamics

b. Identifiers/

Open-Ended Terms

c. COSATI Field/

Group

18. Availability Statement

19. Security Class (This Report)

21. No. of Pages

Approved for public release, distribution unlimited 464 